\title{
EMF RAPID PROGRAM ENGINEERING PROJECTS Project 1 DEVELOPMENT OF RECOMMENDATIONS FOR GUIDELINES FOR FIELD SOURCE MEASUREMENT
}

\section{RECEIVED \\ DEC 211998 \\ OSTI}

\author{
Submitted by: \\ Electric Research and Management, Inc. \\ PO Box 165 \\ State College, PA 16804 \\ Submitted to: \\ Lockheed Martin Energy Research, Inc. \\ PO Box 2008 \\ Bethel Valley Road, \\ Oak Ridge, TN 37831-6070
}

March 11, 1997 


\section{DISCLAIMER}

This report was prepared as an account of work sponsored by an agency of the United States Government. Neither the United States Government nor any agency thereof, nor any of their employees, make any warranty, express or implied, or assumes any legal liability or responsibility for the accuracy, completeness, or usefulness of any information, apparatus, product, or process disclosed, or represents that its use would not infringe privately owned rights. Reference herein to any specific commercial product, process, or service by trade name, trademark, manufacturer, or otherwise does not necessarily constitute or imply its endorsement, recommendation, or favoring by the United States Government or any agency thereof. The views and opinions of authors expressed herein do not necessarily state or reflect those of the United States Government or any agency thereof. 


\section{DISCLAIMER}

Portions of this document may be illegible in electronic image products. Images are produced from the best available original document. 


\title{
EMF RAPID PROGRAM ENGINEERING PROJECTS Project 1 DEVELOPMENT OF RECOMMENDATIONS FOR GUIDELINES FOR FIELD SOURCE MEASUREMENT
}

Task A: Selection of Field Parameters

\author{
Submitted by: \\ Electric Research and Management, Inc. \\ PO Box 165 \\ State College, PA 16804 \\ Submitted to: \\ Lockheed Martin Energy Research, Inc. \\ PO Box 2008 \\ Bethel Valley Road, \\ Oak Ridge, TN 37831-6070
}

October 2, 1997 
1.0 INTRODUCTION $\ldots \ldots \ldots \ldots \ldots \ldots \ldots \ldots \ldots \ldots \ldots \ldots \ldots \ldots \ldots \ldots$

2.0 SCIENCE AND FIELD MEASUREMENTS $\ldots \ldots \ldots \ldots \ldots \ldots \ldots \ldots$

2.1 Biological Guidance in Selecting $N \ldots \ldots \ldots \ldots \ldots \ldots$

2.2 Time Domain Reduction of $n_{t} \ldots \ldots \ldots \ldots \ldots \ldots \ldots$

2.2.1 Sample Theory Limits . . . . . . . . . . . . 7

2.2.2 Reduction Based on Source Knowledge . . . . . . . . . 8

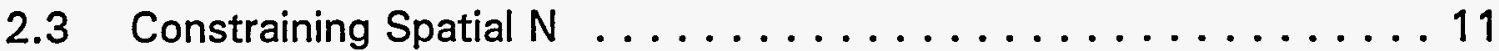

2.3.1 Magnetic Field Spatial Reduction . . . . . . . . . 12

2.3.1.1 Physical Properties of Magnetic Fields . . . . . 12

2.3.1.2 The Magnetic Moment as a Vector ....... 17

2.3.2 Electric Field Spatial Data Reduction . . . . . . . . . 20

2.4 Minimum Data Set . . . . . . . . . . . . . 21

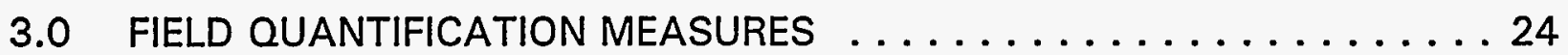

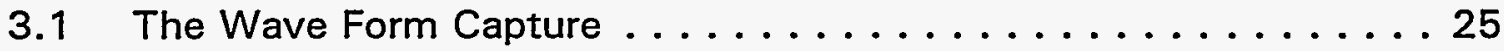

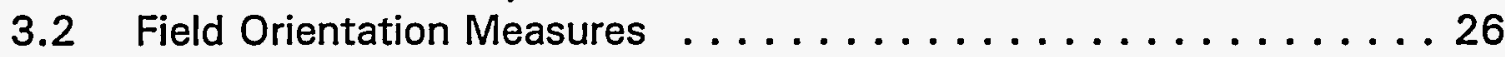

3.2.1 The Resultant ................... 26

3.2.2 The Spatial Components . . . . . . . . . . . 27

3.3 Temporal Measures . . . . . . . . . . . . . . 28

3.3.1 The RMS Calculation . . . . . . . . . . . 28

3.3.2 The DC Calculation .................. 29

3.3.3 The AC RMS Specific Measure ............ 30

3.3.4 The Analog RMS Specific Measure . . . . . . . . . 30

3.4 Specific Measures Related to Force . . . . . . . . . . . 31

3.4.1 Peak and Peak-to-Peak Specific Measures .......... . 31

3.4.2 The Peak Resultant Specific Measure ........... 32

3.5 Uncommon Specific Measures . . . . . . . . . . . . 32

3.5 .1 Relating $A C$ and $D C$ Fields . . . . . . . . . . 32

3.5.2 Coherency and Intermittency ................ 34

3.5.3 Measures of Induction . . . . . . . . . . . . 34

3.5.4 Peak Rate-of-Change of Field . . . . . . . . . . 35

3.5.5 Harmonics ........................ 35

3.6 Measures for Transient Phenomenon ............ 36

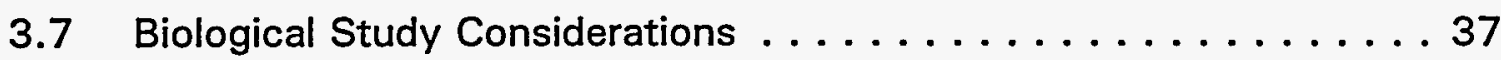

3.8 Specific Measures Conclusion . . . . . . . . . . . . . 40 
4.0 PRESENTLY AVAILABLE INSTRUMENTATION $\ldots \ldots \ldots \ldots \ldots \ldots 44$

4.1 Survey Meters ..................... 44

4.2 Broadband or Narrowband Three-Axis Analog RMS Recorders . . . . 44

4.3 Single-Axis and Three-Axis Wave Capture Recorders ....... 45

4.4 Multi-Sensor Three-Axis Wave Capture Recorders . . . . . . . . . . 47

4.5 Special Purpose Transient Capture Recorders . . . . . . . . . 48

4.6 Electric Field Recorders .....................49

4.7 Confounds to Field Measurements . . . . . . . . . . . . 50

4.8 Conclusion of Measurement Equipment $\ldots \ldots \ldots \ldots \ldots \ldots . \ldots 1$

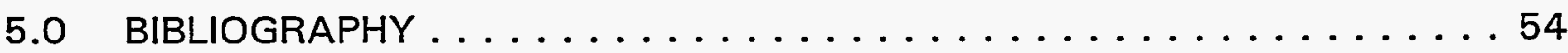

\section{TABLE OF FIGURES AND TABLES}

Figure 2.1 Spatial Location of Sample Points at the Intersection of the Nested Boxes where the Cartesian Origin has been

Selected as the Geometric Center of the Appliance. . . . . . . . 16

Figure 2.2 The Relationships Between the Magnetic Moment and

Distance Vectors and the Current $\ldots \ldots \ldots \ldots \ldots \ldots 18$

Table 2.1 Magnetic Field Parameters Recorded in the EPRI Wire Code Study $\ldots \ldots \ldots \ldots \ldots \ldots \ldots \ldots \ldots$

Table 3.1 Alphabetical Listing of Specific Measures and Related Source Field Attributes $\ldots \ldots \ldots \ldots \ldots \ldots \ldots \ldots \ldots \ldots \ldots$

Table 4.1 Alphabetical Listing of Source Specific Measures With Reference to Biological Group and Measurement Equipment ...................... 52 


\title{
EMF RAPID PROGRAM ENGINEERING PROJECTS \\ Project 1 \\ DEVELOPMENT OF RECOMMENDATIONS FOR GUIDELINES FOR \\ FIELD SOURCE MEASUREMENT
}

\author{
Task A \\ Selection of Field Parameters
}

\subsection{INTRODUCTION}

The goal of this project is to develop a protocol for measuring the electric and magnetic fields around sources. Data from these measurements may help direct future biological effects research by better defining the complexity of magnetic and electric fields to which humanity is exposed, as well as provide the basis for rigorous field exposure analysis and risk assessment once the relationship between field exposure and biological response is better understood. The data base also should have sufficient spatial and temporal characteristics to guide electric and magnetic field management.

The goal of Task $A$ is to construct a set of characteristics that would be ideal to have for guiding and interpreting biological studies and for focusing any future effort at field management. This ideal set will then be quantified and reduced according to the availability (or possible development of) instrumentation to measure the desired characteristics. Factors that also will be used to define pragmatic data sets will be the cost of collecting the data, the cost of developing an adequate data base, and the needed precision in measuring specific characteristics.

A field, electric or magnetic, will always be some function of time and space. The first step in this section of the protocol development will be to determine what span of time and what portion of space are required to quantify the electric and magnetic fields around sources such as appliances and electrical apparatus. Constraints on time will be set by examining measurement limitations and biological data requirements.

Usually, we think of electromagnetics in terms of the frequency spectrum which is classically expressed as ranging from zero cycles per second to $10^{20}$ cycles per second (cycles per second are, by international standard terminology, referred to as hertz and abbreviated by $\mathrm{Hz}$ ). Zero hertz infers time from minus infinity to plus infinity. Except in matters such as geophysics, we rarely concern ourselves with frequencies lower than a few hertz. In fact, the engineering community classifies all frequencies below $3 \mathrm{~Hz}$ as Ultra Low Frequencies (ULF). In this discussion, we will, on a specific 
measurement basis, be estimating the whole ULF range with one value which we will call the "dc" field.

The upper end of the electromagnetic spectrum will not be considered. Frequencies above $300 \mathrm{kHz}$ and below visible light are defined to be radiofrequencies. Radiofrequencies are not within the objective of the RAPID Program. Therefore, the time focus of this protocol can be immediately constrained to the bandwidth from 3 $\mathrm{Hz}$ to $300 \mathrm{kHz}$ with the "dc" value discussed above.

The Institute of Electrical and Electronic Engineers (IEEE) has suggested boundaries to identify various parts of the electromagnetic spectrum ${ }^{1}$. Per these standard definitions, the frequencies that are of interest in this discussion fall into the four following bands: Low Frequency (LF or $30 \mathrm{kHz}$ to $300 \mathrm{kHz}$ ); Very Low Frequency (VLF or $3 \mathrm{kHz}$ to $30 \mathrm{kHz}$ ); Extreme Low Frequency (ELF or $3 \mathrm{~Hz}$ to $3 \mathrm{kHz}$ ); and, as mentioned above, Ultra Low Frequency (ULF or 0 to $3 \mathrm{~Hz}$ ).

The following sections will establish what further constraints are desired and what simplifying techniques can be applied while adequately quantifying electric and magnetic fields. The theory of data reduction will be explored in light of known electromagnetic characteristics. Further reduction of required data will be justified from a cursory review of very recent books and reviews of biological effects of electric and magnetic fields. Then, the list of desired characteristics or parameters will be developed. The final section will evaluate presently used summary measures of these parameters for adequacy in recording all of the parameters that seem to be required. 


\subsection{SCIENCE AND FIELD MEASUREMENTS}

To totally characterize either electric or magnetic fields around any field source, we must describe a vector quantity that varies spatially and changes in time. For the electric field, conventionally, the symbol for the vector is $E$, measured in volts per meter $(\mathrm{V} / \mathrm{m})$. For the magnetic field, the most frequently used quantity is the magnetic flux density which is also a vector and symbolized by the letter $B$. The unit of measure of $B$ is tesla by international standards but in the United States, many still adhere to the more traditional unit of gauss.

These vectors can describe the field at any point in space and time with three signed orthogonal magnitudes or an unsigned magnitude with two angles. The temporal characteristics can be quantified at any point in space by observing the vector over time. Similarly, the spatial characteristics can be quantified at any point in time by observing all points in space around the source. The locations in space can be defined with any coordinate systems desired. The Cartesian coordinates, $X, Y$, and $Z$ relative to a conveniently selected point in space, such as the center of an appliance might be the easiest to visualize. The field can then be totally defined by describing a five-axis array of data containing a number of elements equal to the product of the axis dimensions.

where:

$$
\text { Number of values }=V \bullet n_{t} \bullet n_{x} \bullet n_{y} \bullet n_{z}
$$

Is the Vector, A Magnitude with 3 Components to Represent Direction of the Fit Electric or Magnetic, at the Instant of the Measurement Sample,

$$
\begin{gathered}
n_{x} n_{y} n_{z}=\text { Number of Samples in the } X, Y \text {, and } Z \text { directions } \\
\text { moving away from the field source, and } \\
n_{t}=\text { Number of Time Samples. }
\end{gathered}
$$

These values of $\mathrm{n}$ for both time and space must be infinite to completely define all time and space. This is an extremely important observation for the purposes of measurement. Any reduction in the values of $n$ by placing limits on the number of samples taken is a loss of data.

The primary goal of Task $A$ is to trim the values of $n$ in ways that do not sacrifice necessary data. For this investigation, unnecessary data will be defined as information which is either outside a to-be-determined range of biological interest under the RAPID mission or information that, because of the nature of environmental fields, provides little or no additional information. The first logical step in developing a practical and. 
useful protocol must be to determine the range of $n$ which will help evaluate biological hypotheses and findings.

An important subtlety to maintaining complete generality is that we cannot disregard aspects of the field because we have not anticipated their presence. We must have a system which identifies aspects of the field which fits into our predefined time and space dimensions (range of $\mathrm{n}$ ) and permits a level of measurement sophistication consistent with field complexity.

\subsection{Biological Guidance in Selecting $\mathbf{N}$}

Biological, findings have not yet established the parameter(s) required to quantify environment fields so that a health assessment could be rapidly completed should it ever be deemed necessary. There are some known frequencies and magnitude effects. In the radio frequency bands, the known biological interactions are thermal effects and, therefore, a useful quantification of fields is the root-mean-square (rms) which is a measure of the energy of the field. Because the energy absorption of a biological specimen is dependant on the size of specimen and the wave length of the electromagnetic field at these high frequencies, rms measures in subbands are the most useful quantification of radio frequency electromagnetic fields ${ }^{2}$.

In the extremely low frequency (ELF) band of 3 to $3000 \mathrm{~Hz}$, the electric field or the magnetic field have not yet been widely accepted as having health consequences unless the field strengths are very high. At these high fields, tens of $\mathrm{kV} / \mathrm{m}$ or tens of $\mathrm{mT}$, the frequency of the field and the field strength should be quantified. Generally, sources of very high fields will be dominated by a single frequency and therefore the rms measure is useful. However, the purpose of this study is to quantify much lower intensity fields expected around most electrical apparatus, where single frequency dominance cannot be assumed to be the general case.

For the low level electric and magnetic fields typically found in the environment, tens of volts/meter and much less than tens of microtesla, respectively, there is little generally accepted parameter guidance. Epidemiology studies to date have not correlated with the simple time parameters selected for most past studies which were rms magnitudes collected by field meters with an approximate bandwidth of 40 to $1000 \mathrm{~Hz}$ then averaged over time (Time Weighted Averages, TWA). The time weighted average is a method of pooling these rms data over an arbitrarily selected time interval. Occasionally, the time weighted average also contains a spacial average if more than one measurement is made in the general area of the site of interest. Some studies have explored the impact of the time weighted average assumption. However, any time course portioning that has been tried still does not show significant correlation. 
This lack of correlation has been interpreted by some to demonstrate there are no biological effects at commonly found levels of magnetic fields in our environment. However, one of the motivations for the RAPID research program is that the engineering community may not have evaluated all the aspects of environmentally relevant electric and magnetic field characteristics. More detailed quantification of the time and space characteristics of electric and magnetic fields may offer guidance to what parameters might be useful to record.

Some simple understanding of coupling of electric and magnetic fields with conductive objects is helpful in gaining a feeling for the importance of frequency. Electric fields are effectively terminated at the surface of even poor electrical conductors. While biological systems (bodies) are poor conductors but highly conductive relative to air, electric fields from any nearby source effectively terminate on the surface of the body. For electric field coupling, the body surface acts as one side of the capacitive circuit and the electric field source, e.g., transmission line, acts as the other side of the capacitor. The impedance function of a capacitor is $1 / 2 \pi \mathrm{fC}$, where $\mathrm{C}$ is the capacitance which is a function of surface areas, separation of the surfaces, and the permitivity of air, and $f$ is the frequency of the source. Electric fields become increasingly coupled as frequency increases since the current carried by the body increases with $f$. Conversely, note that as $f$ goes to zero, the impedance function goes to infinity. Therefore, for electric fields, measuring do fields may not be necessary (there are very complex dc electric field phenomena that are beyond the scope of this project because they relate primarily to dc transmission lines ${ }^{3}$ ).

Magnetic fields also couple directly with increasing frequency. The current density in a conducting body increases directly with the rate of change of magnetic flux density normal to a specific plane of the body. Electromagnetic field theory can be used to express this effect as

$$
\boldsymbol{J}=k \sigma f r B
$$

where $J$ is the symbol for current density, $k$ is a constant depending on the system of units selected, $f$ and $B$ are frequency and magnetic flux density, respectively, $\sigma$ is the conductivity of the body, and $\mathrm{r}$ is the radius at which the current density is calculated. Therefore, the frequency of the magnetic field is a major factor in determining coupling. Note; because the geomagnetic (dc) fields at the earth's surface are one to two orders of magnitude above the amplitude of alternating fields that we are interested in measuring, they may need quantification. Random movement in these dc magnetic fields will result in rates of change in the plane of bodies normal to the dc magnetic field vector which will produce current densities at low frequencies. The magnitude of these currents may be in the same order of magnitude as those produced by low level $60 \mathrm{~Hz}$ ac fields. 
To set an upper limit on $n_{t}$, we need to find a physical or biological justification for truncating $f$. The above simple analyses of electric and magnetic field coupling show clearly that frequency has an ever growing importance if the amplitude of the source, electric or magnetic, is held constant. Fortunately, for all sources of $E$ and $B$, the field magnitude rarely remains constant over even a small bandwidth above the source power frequency. For transmission line survey studies, there was a simple justifiable physical reason for truncation at the power frequency. Power system operation criteria have been set that limit the amplitude of harmonics of the power frequency to less than one percent in close proximity to the line. Unfortunately, general apparatus are not limited by these design constraints.

Thus, we must turn back to the biological literature for guidance in limiting $n_{\mathrm{t}}$. Two recent books, "Electromagnetic Fields - Biological Interactions and Mechanisms" 4 and "The CRC Handbook of Biological Effects of Electromagnetic Fields - 2nd Edition"5 discuss very few experiments with exposure frequencies that could not be adequately quantified in the bandwidth of 3 to $3 \mathrm{kHz}$ unless the mission was RF (radiofrequency) research.

The first five chapters of The CRC Handbook are dedicated to explaining known and postulated interactions. For example, on page 129 reference is made to nerve cell firing pulses of .4 milliseconds with .1 millisecond rise times and fall times of .5 milliseconds. This suggests a bandwidth of at least $2.5 \mathrm{kHz}$. On page 199, the author cites experiments involving DNA synthesis which showed effects for frequencies from $15 \mathrm{~Hz}$ to $4 \mathrm{kHz}$. The CRC Handbook cites numerous experiments using frequencies from a few hertz to one kilohertz. It reviews the resonance hypotheses which require concurrent knowledge of $\mathrm{dc}$ and ac magnetic fields. There is a whole chapter dedicated to bone repair by magnetic field exposure. While the repetition rate of the bone repair signals are in the ELF band, there are strong harmonics that cover the VLF band and extend into the LF band. The CRC Handbook does note that outside of the bone repair arena, there are very few positive studies using frequencies in the VLF band. Only by inference from a study cited on page 203 can we find biological effects that may have been related to frequencies in the LF band. (A $20 \mathrm{kHz}$ sawtooth waveform necessarily will have strong harmonics that fall in the LF band, $30 \mathrm{kHz}$ to $300 \mathrm{kHz}$.)

From the excellent reviews presented in The CRC Handbook, relatively strong support for establishing the $n_{t}$ parameter can be drawn. Clearly, $n_{t}$ must be sufficiently large to describe the ELF band. Data must be collected that will at least indicate if magnetic field energy exists in the VLF and LF bands. Finally, the magnetic field data must be collected with a sensor system that can also respond to the dc fields. 


\subsection{Time Domain Reduction of $n_{t}$}

\subsubsection{Sample Theory Limits}

Sampling theorem is introduced into this discussion since virtually all the field measurements are made through a "sampled" process. Some systems utilize computer sampling systems and store data in a digital format. Other systems process the signals with analog, i.e. continuous, circuits before being "sampled". Even measurements made with an analog meter are "sampled" by reading and recording the reading on a measurement log. The number of data samples required to quantify temporal patterns of a field at a point in space is directly proportional to the length of time over which the record is desired and the maximum frequency that is to be identified. From information theory, the Sampling Theorem ${ }^{6}$ states that the sampling frequency $f_{s}$ must be $2 f$ or twice the highest frequency that can completely characterize a band-limited signal which is precisely limited at the frequency $f$. This is often stated as the Nyquist criteria (after $\mathrm{H}$. Nyquist of Bell Laboratories) which requires that the sample rate must be twice the highest frequency to be identified. The sampling theorem also states that there must be $2 \mathrm{fT}$ samples to completely specify a signal over an interval $T$ seconds long.

The Sampling Theorem requires that the signal to be sampled must be band-limited. There must be no frequencies above $f_{s} / 2$ or the Nyquist frequency. If there are frequencies above, these must be filtered out with analog filters since digital filters will not work above the Nyquist frequency. It is essentially impossible to create a perfect filter that allows the Nyquist frequency to pass undistorted and the frequency just above the Nyquist to be reduced to zero. There is a broad selection of various filters that provide various characteristics as the Nyquist frequency is approached. Unfortunately, the more sharply the amplitude of the frequency is reduced, the more phase distortion will be added to the signals below the Nyquist frequency.

If frequencies exist above the Nyquist frequency and the analog filter does not reduce such signals to zero, the signal can be translated from the frequency above the Nyquist to a frequency below the Nyquist and become a part of the signals that are being recorded. This phenomena is known as aliasing since the signal above the Nyqist is "alien" to the signals below the Nyquist. The analog filter is known as the "antialiasing" filter. The characteristics of the anti-aliasing filters used in various systems form an important part of the temporal reduction of data. Frequencies above the antialiasing filter are not recorded and data on such frequencies is lost. Careful consideration of the action of these filters must be given when deciding on the temporal reductions that may be allowed.

This discussion of sampling theory has established a criteria for selecting the lowest upper limit for $n_{r}$. The actual limiting value chosen must be based on either the 
biological considerations discussed above or other electrical characteristics of the source to be measured. So far, we can only state that the maximum sample rate is. equal to $600 \mathrm{kHz}$. Without other knowledge, we might be forced to collect data at that rate.

\subsubsection{Reduction Based on Source Knowledge}

If a measurement of a magnetic field at a single point in space were taken with a bandwidth of 0 to $300 \mathrm{kHz}$ over a period of 24 hours on three axes, the number of "sample points" would be $15.55 \times 10^{10}$.

Clearly, temporal reduction of data either below the $600 \mathrm{kHz}$ sample rate or for significantly shorter periods must be accomplished based on the knowledge of the source. Important source attributes to consider are:

1. The duty cycle of the appliance or electrical apparatus.

2. The power frequency supplying energy to the field source.

3. The method of conversion used by the appliance to transform the energy from one form to another, i.e. from electric power to mechanical power, light, sound, heat, or to another form of electric power.

If a specific duty cycle of an appliance is long, relative to the period of the power source frequency that supplies energy to the appliance, each period may be an exact repeat of the preceding period. If so, then there is a second level of sampling that will greatly reduce $n_{t}$ without the loss of data.

\section{Steady-State and Snapshot Techniques}

Most sources display reasonably consistent temporal behavior over brief periods of time, i.e., the specific function within the duty cycle is constant over a period of time. If the temporal behavior is predictable over a period of time, then the source can be described as being in a "steady state". For temporal reduction, steady state will imply that the source will not change either amplitude or phase of any frequency over the period of measurement.

The analysis of the frequency spectrum of a set of sample data points assumes that steady-state is present over the measurement cycle. The assumption of brief periods of steady-state and specifically over a measurement cycle leads to the introduction of a "snapshot duration" and a "snapshot interval". A snapshot is a record of sample data points taken at a sample frequency $f_{s}$ for $N$ numberof points. The analysis allows frequencies from $f_{s} / 2 \mathrm{~N}$ to ${ }_{s} f / 2$ to be 
characterized. The snapshot duration is $\mathrm{Nf}_{\mathrm{s}}$ seconds (when $\mathrm{f}$ is in $\mathrm{Hz}$ ). The snapshot interval $\lambda$ is the time interval, generally in seconds, from the start of a snapshot to the start of the next snapshot. There is then $\lambda-\mathrm{Nf}_{\mathrm{s}}$ seconds of data which is lost in the measurement if steady state does not exist over the period. If true steady state exists, theoretically $\lambda$ can be infinitely long. Practically information will not be lost if $\lambda$ is limited to the time the source is on without appreciable load or state change, e.g., load or state changes of less than 5 percent change in any variable.

If there are slow changes in the load or operation of a source, the $\lambda$ becomes the sampling frequency of these changes (if $\lambda$ is a constant). Thus, samples taken with a $\lambda$ of 10 seconds would properly record load changes that were slower than $0.05 \mathrm{~Hz}$.

Snapshot sampling techniques can be used if the field source exhibits repeatability. If a field source does not remain on for long periods of time but cycles on and off, e.g., the compressor of a refrigerator, the snapshot sampling techniques can be used because each time the compressor is on the magnetic fields produced are essentially identical. This fact becomes very important when multiple spatial points are to be measured. Measurements made while the compressor is turned off could be suspended and then continued when the compressor turned on again. This technique will be shown to be used frequently in source measurements.

When making measurements of known sources, the conditions of steady-state and/or repeatability can be determined by pre-test to determine what measurements are required for a thorough analysis. Pre-test requires a few measurements to be made over a wide range of frequencies (preferably from 0 to $300 \mathrm{kHz}$ ) and analyzed to determine the steady-state characteristics of the source operation. If no VLF or LF frequencies are noted, then the sample frequency can be set to the ELF range, i.e., $f_{s}$ above $6 \mathrm{kHz}$. If no frequencies below the power frequency except dc are found, then the sample duration $\mathrm{Nf}_{\mathrm{s}}$ can be shortened to match the period of the power frequency.

\section{Snapshot Duration and Maximum $f_{s}$}

Most appliance fields are repetitive at the power frequency of the electrical supply to the appliance, at least within a given duty cycle. Therefore, in the United States, the snapshot duration will normally only need to be 1/60th of a second in duration. The number of data points to be stored within one snapshot interval then is only $\mathrm{f}_{\mathrm{s}} / 60$. If $\mathrm{f}_{\mathrm{s}}$ must go to the limit of $600 \mathrm{kHz}$, then the data for one point in space is still large at $10,000 \times 3$ or 30,000 data samples. 
Thus, knowledge of the appliance conversion systems should be used to reduce $f_{s}$ where possible.

For many source types, such knowledge may be gained from general observations. For example, simple heating appliances would only produce electric and magnetic fields with frequencies which were harmonics present in the power supply voltage. Recent power supply standards, IEEE 5197, constrains the power supply voltage, at least at the point of service, to have less than $3 \%$ total harmonic distortion in the voltage. Further, the magnitude of harmonics usually vary inversely with the harmonic number. Thus, for this simple case, the upper limit of $f_{s}$ would not need to go beyond the ELF band, i.e., $f_{s}=6000 \mathrm{~Hz}$.

Sources which contain motors become more difficult to assess for limiting $f_{s}$. For example, a motor which contains brushes will generate arcs as it operates. These arcs will generate magnetic fields in the VLF and LF bands. ${ }^{8}$. However, other motors which do not have brushes, have very low likelihood of generating magnetic fields outside the ELF band. Appliances that are more sophisticated, such as computers, copying machines, printers, etc., are even more difficult to judge. Electronic switching in power supplies in these appliances may readily produce magnetic fields in the VLF band and occasionally in the LF band.

\section{Transient Temporal Events}

When random events occur in time during the operation of a field source or, quite often at the beginning or end of cyclic operation, the source field must be considered "transient". Simplifying techniques of snapshot duration and snapshot interval no longer directly apply. Frequency limitations from the Nyquist frequency apply but using Fourier analysis techniques of the samples below the Nyquist frequency are not applicable. Waveform analysis will provide information on the transients if the transient response of anti-aliasing filters has not distorted the transient signal. Transient events may involve large rates-ofchange of field levels. Electronic and gap sources may produce rise times in the nano-second range, however major power system transients rarely have rise times less than 1 microsecond.

It should be noted that even if a sampling rate were selected that matched the .1 microsecond rise time, sensors that are band limited for antialiasing above $300 \mathrm{kHz}$ will not pass rise times of less than 2 microseconds. To avoid constant sampling at the $600 \mathrm{kHz}$ rate to capture transient events within the antialiasing limit, triggering on a characteristic of the event such as amplitude or rate of change must be available. Such triggers could record either random or cyclic transients, such as in the starting of a motor. Level triggers (the field 
rises above a pre-set magnitude) may be adequate to record cyclic transients, such as a motor starting transient. However, turn-off transients may also have large rates-of-change but since the levels are falling, a more complex rate-ofchange trigger would be needed to record the transient.

\section{Summary of the Reduction of $n_{t}$}

While transient conditions, as discussed above, may require an $f_{s}$ greater than $600 \mathrm{kHz}$, most of the data required to address biological findings to date can be covered by data collected in the ELF band. When we further consider that the mission of this project is to gather data which characterizes the electric and magnetic field around appliances, the practicality of collecting the large data sets to go beyond the ELF band must be considered. As pointed out above, there will be appliances that produce fields with a significant portion of the total field energy above the ELF band. A pragmatic compromise may be to limit $f_{s}$ to the ELF band, but to record the presence of, and broadband energy levels in, the VLF and/or LF bands. This approach is analogous to the approximation of the ULF band by the dc value. By so doing, we would have recorded the presence of energy in these bands for specific appliances so that, if future research requirements needed more precise parametric information, only select appliances would have to be retested.

If we accept the compromise of limiting $f_{s}$ to the ELF band and measuring for one power frequency cycle is a sufficient snapshot duration with a snapshot interval of one second, then $n_{t} /$ second reduces to 100 , which may be a manageable number. (Note that the use of Fast Fourier transform techniques require a sampling rate at the first sample rate above 100 which is an incremental power of 2 . This rate is 128 samples in the $60 \cdot \mathrm{Hz}$ period.)

Before spending further effort on refining $n_{t}$ we now should turn our efforts to reducing $\mathrm{n}_{\mathrm{x}}, \mathrm{n}_{\mathrm{y}}, \mathrm{n}_{\mathrm{z}}$.

\subsection{Constraining Spatial $\mathbf{N}$.}

Practical measurements of fields require that the number of samples taken are reduced from the infinite number that is required to satisfy Equation 2.1. Reduction which minimizes the loss of data can be based on three factors.

1. Knowledge of the source location relative to perturbing structures.

2. Knowledge of the source construction.

3. Knowledge of the spatial rate of change that can practically occur. 
Each level of knowledge allows the measurements to be further reduced by supplying information sufficient to estimate, if desired, the spatial samples that were not directly measured.

\subsubsection{Magnetic Field Spatial Reduction}

Air, biological systems, and most materials and metals, except those that are ferromagnetic, have essentially the same permeability as free space. This establishes two important considerations for the measurement of magnetic fields.

Magnetic fields do not undergo the rapid spatial changes unless they are associated with the boundaries of ferromagnetic materials or highly conductive metals. For this study, large spatial rates-of-change are defined as $1 \mu \mathrm{T} / \mathrm{cm}$. Large, highly conductive metal ( $\sigma \geq 10^{6}$ seimens) objects can impact the magnetic fields at the perimeter of such objects. Ferromagnetic metals, principally nickel and iron and their alloys, have permeabilities much greater than free space. Consideration must be given to the possibility that there may be highly conductive structures or ferromagnetic materials in the area where the measurements are made. The fact that the magnetic field being measured is in the air implies that a large air-gap exist between the measurement point, the magnetic field source, and any ferromagnetic object. The field, remote from extreme curvatures of the conductive or ferromagnetic object, will not have extreme spacial change patterns. The size of the magnetic measurement device and the number of measurements that must be made to determine the spacial changes in the magnetic field density must be adjusted to match these conditions.

As noted above, the permeability in humans is that of free space and the fact that humans have relatively poor conductivity ( $\sigma z .5$ seimens, as compared to metal at $\sigma \geq 10^{6}$ seimens) allows magnetic field to be measured by a human operator without regard for position of the human below radio frequencies. In fact, the magnetic field distortions by the human body is very minimal even at frequencies as high as $300 \mathrm{kHz}$. By applying Equation 2.2 with the conductivity and maximum radial dimension of the human body, it can be shown that distortion of the spatial magnetic field density at the surface of the body for magnetic field density below $300 \mathrm{kHz}$ is less than $1 \%$. This fact will be used in the development of protocols in Task C.

\subsubsection{Physical Properties of Magnetic Fields}

Before moving forward in developing analytic techniques that will help in the reduction of spatial $n$, it is important to be mindful of a general observation. The magnetic flux density external to an appliance or piece of electrical apparatus is seldom present as the principal function of the source. All magnetic fields normally experienced during human activity are from the stray fields from devices which require magnetic fields to function. There may be little relationship between the current supplied to a device and 
the fields that will be measured in the environment. As an example, the magnetic fields in a motor are generated for the purpose of turning the motor shaft. Those magnetic fields external to the motor housing are stray fields. One manufacturer may construct the motor to minimize the stray fields, while another manufacturer may allow high stray fields as a consequence of cost reduction. Motors with the same ratings may have stray fields that are orders of magnitude stronger in one case over the other. Design features that limit the prediction of magnetic field density from the currents drawn by the source will be dealt with in Task B.

However, as will be shown, pseudo-current dependence can be assumed for the sake of developing spatial models. Gross spatial characteristics of magnetic fields may suggest a means for reducing $n_{x}, n_{y}$ and $n_{z}$ For a simple loop of wire of radius $R$, with a current I produces a magnetic field vector at the center of the loop which is perpendicular to the plane of the loop. The direction of the vector is defined by the right-hand rule, i.e. if the fingers of the right hand curl in the direction of the current in the loop, the thumb will point in the direction of the magnetic field. The magnetic flux density produced by such a loop current source takes on the characteristic of a dipole field.

If the distance from the center of the loop is much larger than the distance across the loop, the $B$ changes as approximately $1 / r^{3}$ where $r$ is the distance from the measurement point to the center of the source. The simplified expression for $B$ where $r>R$ is

$$
B=\frac{\mu_{0} R^{2} l}{2 r^{3}}
$$

where: $R$ is the effective loop radius, $I$ is the single-turn loop current, and $\mu$ is the permeability of free space.

Field theory texts quite frequently develop a term referred to as the magnetic field moment which is symbolically denoted as $\mathrm{m}$. Mathematically, the magnetic moment is defined as

$$
m=\Pi R^{2} l
$$

Thus, the dipole relationship may be used even for sources that do not exhibit a clear current dependent magnetic field relationship. The confusion of effective dipole 
currents, l, can be eliminated by thinking in terms of the dipole moment. In terms of the dipole moment, the magnitude of the magnetic flux density takes the form

$$
B=\frac{\mu_{0} m}{2 \pi r^{3}}
$$

It will be shown in Task $C$ with magnetic fields recorded around appliances that many appliance or apparatus sources have at least the gross characteristics of a dipole. Therefore, appliances which can be effectively modeled as a dipole may only require enough spatial measurements to verify that or where the dipole model fits. Further, if the single dipole model is valid, one parameter, $m$, describes all space!

Some appliances or at least some regions near an appliance are better characterized as a line source. The power cord to some appliances, such as a simple lamp, may be the dominant source of the magnetic field. Field theory shows that for a long, straight wire, i.e. mathematically infinite length, the magnitude of the magnetic flux density is computed simply as

$$
B=\frac{\mu_{0} l}{2 \pi r}
$$

The field near two wires carrying current in opposite directions can be derived from Equation 1.6. Thus, field from most power leads can be computed from the equation

$$
B=\frac{\mu_{0} l d}{2 \pi r^{2}}
$$

for $r$ much larger than $d$, where $d$ is the distance between the wires and $r$ is the closest distance from the center of the wire pair to the point of measurement. The direction of $B$ depends on the vector summation of $B$ field contribution from each individual wire. If $r$ is much larger than $d$, then the $B$ changes as approximately $1 / r^{2}$. If $r$ is less than five times $d$, then Equation 2.6 should be used to compute both the magnitude and direction of $\mathbf{B}$.

In the above discussion, it was shown that conceptually, the above three equations $(2.5,2.6$, and 2.7$)$ can be used to greatly reduce $n_{x}, n_{y}$, and $n_{z}$. At this point, the reasoning and general approach will be demonstrated with simple examples. The 
discussion on Task $C$ will demonstrate, with examples of comprehensive measurement, how effective the concepts are in actual practice.

Although magnetic field sources can be extremely small, most sources which interact with human activity have an object dimension of $5 \mathrm{~cm}$ ( 2 inches) or greater. For such a small source, the deviation of Equation 2.3 suggests that at distances of $15 \mathrm{~cm} / 6$ inches) or greater from such a source the magnetic field spatial characteristics to be modeled by Equation 2.5. If spatial measurements are made between 15 and $30 \mathrm{~cm}$ (12 inches), the effective magnetic moment of this source can be computed. The dipole moment estimation can be utilized as a method for reducing the spatial domain measurements as described in Equation 2.1. To test this estimate, another measurement should be made at a radial distance twice that of the first. If the measured value is $1 / 8$ th of the value used to compute $m$, then the dipole moment of Equation 2.5 is confirmed. (In general, much beyond 10 times the dipole radius, other ambient sources might interfere with the rapidly diminishing dipole field.) This hypothetical example illustrates a conceptual point that can be used with caution as sources rarely produce perfectly symmetric dipoles. The larger the appliance, the more unlikely that simple models will adequately describe the space of interest.

If mathematical assumptions that are made concerning the source are valid, data is reduced but no information is lost. However, the complex nature of the fields caused by the physical construction of the sources and the conductivity, permeability or permitivity of materials in or near a source introduces deviations from even sophisticated models. There are rare cases where manufacturing models of a specific source exist. Unfortunately, these models are usually considered "trade secrets". As such, they are almost never available in accessible data files. In general, there are few devices with known external field "profiles".

Consider a real example of a microwave oven. The example oven is a box $58 \mathrm{~cm}$ wide by $38 \mathrm{~cm}$ deep by $43 \mathrm{~cm}$ high ( 23 inches wide by 15 inches deep by 17 inches high). Without any knowledge or assumption about the oven's source characteristics, the spatial characteristics of a field external to the oven to $1 \mathrm{~cm}$ resolution within $300 \mathrm{~cm}$ of the center of the oven, requires a Cartesian grid with about 215.9 million measurement points. However, if the size of the dominant source in the oven is the magnetron creating the microwaves which is typically $5 \mathrm{~cm}$ across in all three directions, then measurements between 15 and $30 \mathrm{~cm}$ would be a first guess at spatial sample reduction. Using $30 \mathrm{~cm}$ sample separation would reduce the spatial data to 9260 measurements (this excludes measurements inside the oven). To determine the adequacy of the $30 \mathrm{~cm}$ spatial assumption, measurements in cube arrays around the oven in nested cubes will be required. For example, select the first interior nested cube at $30 \mathrm{~cm}$ away from the oven center on all sides. To check for corner effects, there would be three evenly spaced positions on the horizontal, the vertical and the depth grids. The spacing would be $30 \mathrm{~cm}$ apart with a total of 26 
measurements. The next nested cube could be a convenient dimension such as 100 $\mathrm{cm}$ from the center of the oven with three measurement positions across the horizontal, the vertical and the depth grids. Again, there would be 26 measurements. Figure 2.1 shows the spatial location of sample points where the Cartesian origin has been selected as the geometric center of the appliance.

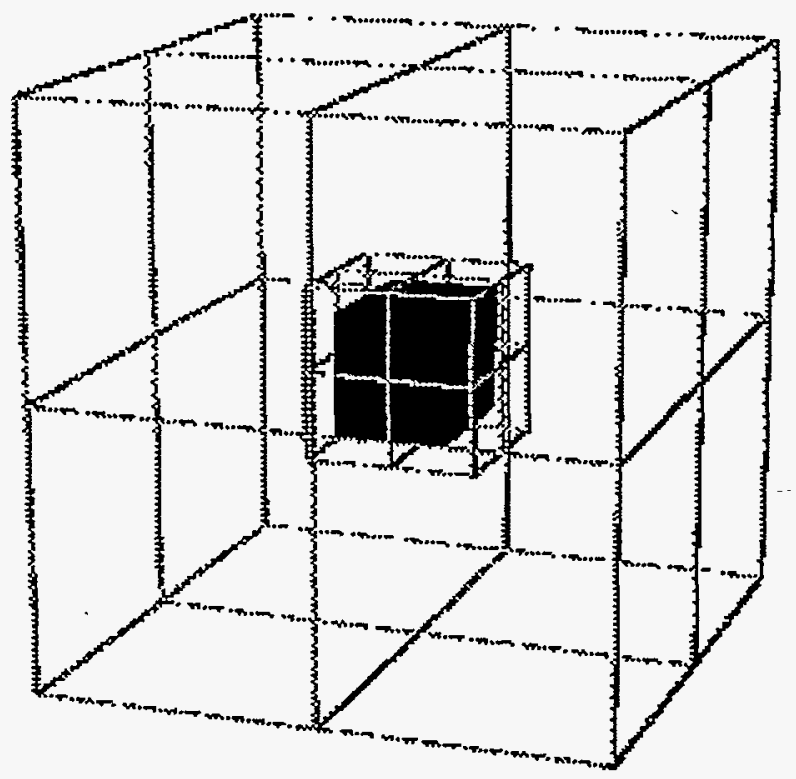

Figure 2.1 Spatial Location of Sample Points at the Intersection of the Nested Boxes where the Cartesian Origin has been Selected as the Geometric Center of the Appliance.

The first set of data from the interior nested cube would be examined to see if an electrical center for the appliance is apparent. The electrical center may be offset from the geometric center by $\left(x_{e}, y_{e}, z_{e}\right)$. If so, the magnetic field at Location 1 will equal

$$
B_{1}=\frac{\mu_{0} m}{2 \pi\left[\sqrt{\left(x_{1}-x_{\theta}\right)^{2}+\left(y_{1}-y_{\theta}\right)^{2}+\left(z_{1}-z_{\theta}\right)^{2}}\right]^{3}}
$$

Similarly, 


$$
B_{n}=\frac{\frac{\mu_{0} m}{2 \pi}}{\left[\sqrt{\left(x_{n}-x_{\theta}\right)^{2}+\left(y_{n}-y_{\theta}\right)^{2}+\left(z_{n}-z_{\theta}\right)^{2}}\right]^{3}}
$$

Using a least squares solution will give the values $x_{e}, y_{e}, z_{e}$ and a measure of the "goodness" of the fit. The second set of nested data will only be necessary if there is a poor fit. By looking only at the locations on a given side using spatial points from both nested cubes, the possibility of multiple electrical centers can be examined. If multiple electrical centers cannot be clearly defined, then a third nested cube at 200 $\mathrm{cm}$ to $300 \mathrm{~cm}$ may be necessary to map the spatial characteristic of the magnetic field around the appliance.

Many of the measurements would be too near to the source for the dipole approximation but would provide additional information about the center of the source. The combination of the data can be used to locate the loci of the gross dipole center. The maximum field recorded would establish the maximum field level in distances too close to the oven for the dipole algorithms.

This example demonstrates a concept for reducing the number of spatial measurements based on limited knowledge of magnetic fields from typical sources. The data not recorded because measurements were not taken at possible spatial points of interest can be readily regenerated.

The use of dipoles as an approximation for appliance measurements utilizes the concept of a point dipole or a vanishing spherical dipole. Deriving the magnetic moment from actual measured data is difficult since both the coordinates and the plane of the magnetic moment had to be known ahead of time. Research is underway by the Electric Power Research Institute to develop equations to determine the value and location of the moment based on magnetic field readings. Such software, designed for magnetic survey and shielding work, may allow ready characterization of an appliance by moments.

\subsubsection{The Magnetic Moment as a Vector}

The magnetic moment vector $\boldsymbol{m}$ is defined as

$$
\boldsymbol{m}=N / A
$$


where $N$ is the number of turns in the planer coil, $/$ is the current in the coil, and $A$ is the area enclosed by the coil. The units for $\boldsymbol{m}$ is Ampere-turn-meter ${ }^{2}$ and the direction of $\boldsymbol{m}$ is normal to the plane of the coil pointing upward for current circulating in a counter-clockwise direction. This is the same as the right-hand rule. Figure 2.2 shows these relationships.

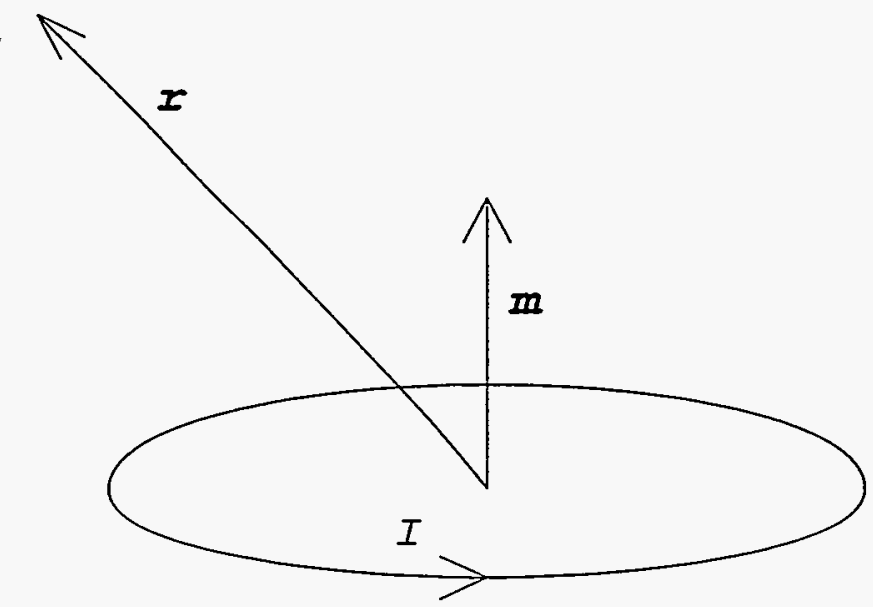

Figure 2.2 The Relationships Between the Magnetic Moment and Distance Vectors, and the Current.

The magnetic flux density in Tesla for this coil is calculated from the traditional equations as

$$
B=\frac{\mu_{0}}{4 \pi}\left[\frac{-m}{r^{3}}+\frac{3(m \cdot r) r}{r^{5}}\right] \text {. }
$$

where $r$ is the distance vector to the point where $B$ is to be calculated. The $r$ is the magnitude of the distance vector in meters. The $\mu_{0}$ is the permeability of air with the value of $4 \pi(10)^{-7}$ Tesla-meter per ampere.

The complexity of this equation tends to limit consideration of the moment as a useful characterization of appliances. Working the equation to solve $\boldsymbol{m}$ from $\boldsymbol{B}$ as would be needed to match magnetic field measurements to a moment would be difficult. The moment depended on the size of the coil and the number of turns as well as knowledge of the coil plane. However a technique has been developed to provide this reverse calculation process by fitting the data. This method assumes the moment is created from a point magnetic moment with a coil of infinitely small size. The data 
must all be relatively distant field measurements since the fields increase to infinity as the point source is approached.

The technique to determine the dipole is to assume an infinitely small spherical magnetic dipole with an assumed spatial location for the point source. The data is fitted to provide the moment based on all the available data. This greatly reduces the significance of errors in specific readings. When the error of the fit has met predetermined criteria, the magnetic moment data is provided as an output. The moment $m$ consists of six numbers, a real and an imaginary component for the $x$, the $y$ and the $z$ axis. The real and imaginary components form the time phase information for the moment. The moment can then be used to reconstruct the field at any location given the vector $r$.

A magnitude of $m$ can be formed by calculating the square root of the sum of the squares of the six numbers. This magnitude $m$ is an indication of the strength of the magnetic field generated by the appliance. If $m$ is given for an appliance, it can be compared with an $m$ from another appliance. This allows an appliance to be characterized relative to a list of appliances. Those with high values of $m$ will have strong magnetic fields and those with low values of $m$ will have weak magnetic fields.

An estimate of the maximum magnetic field that may be present at a given distance from an appliance may be made assuming that $m$ and $r$ are in the same direction. This reduces the equation to

$$
B=\frac{2 \mu_{0} m}{4 \pi r^{3}}
$$

where $m$ is the magnitude of the magnetic moment in ampere-meter ${ }^{2}, r$ is the magnitude of the distance from the moment coordinates to the coordinates where the approximation is desired and $\mu_{0}$ is $4 \pi(10)^{-7}$ Tesla-meter per ampere. If $r$ is in meters and $B$ is desired in milligauss, the equation reduces to

$$
B=\frac{2 m}{r^{3}} \text {. }
$$

This allows very rapid approximation of the maximum magnetic field that can be expected from the appliance. If the value of $m$ is 0.750 ampere-meter ${ }^{2}$ as was found for a blender tested by the appliance test set, the expected $B$ at 60 centimeters 12 feet) is 


$$
B=\frac{20.75}{0.6^{3}}
$$

or $B$ is about 7 milligauss. The moment $m$ for the third harmonic is 0.21 amperemeter ${ }^{2}$. At 60 centimeters the maximum $B$ is calculated as approximately 2 milligauss. The estimations of the moment $m$ at 1 meter is particularly easy as it is simply the moment times two in milligauss.

The moment concept is particularly useful when the effects of an appliance are to be included in other types of measurements. The moment provides the levels of the magnetic field which would add to the ambient fields without the appliance. The moment allows a reasonably accurate magnetic field map of the appliance if the moment components including direction are used. The map will be accurate for distances from the appliance greater than three times the spherical radius of the appliance. The appliance can be assumed to be contained inside a sphere which just encloses the appliance. The radius of this sphere defines the distances where the moment is most useful. Estimation of this distance can be found by measuring a diagonal from the center of the appliance to the farthest corner from the center. As the appliance is approached, the moment will not necessarily provide the maximum field approximation since the moment is assumed to be a point and the actual field may be generated by coils the size of the appliance. Also the presence of magnetic materials in the appliance will shape the fields.

\subsubsection{Electric Field Spatial Data Reduction}

Electric fields are effectively terminated by even poorly conductive surfaces. As stated above, while the human body is a poor conductor, the body is highly conductive relative to air. In air, the electric field is effectively confined between conductive surfaces. The amplitude and direction is directly controlled by the physical locations and shapes of the surfaces and on the voltage potential difference between surfaces. Therefore, electric fields may easily be modified by human presence during measurement.

For many sources, electric field measurements may not be required. The safety codes for most electric appliances utilizing more than 50 volts rms require some type of shielding. The electric fields from these devices are generally very low because the contactable surfaces which are metallic are grounded. These surfaces form shields. There are exceptions to this requirement. There are appliances normally labeled "double-insulated" where the shield is a mechanical, insulating shield which keeps the user from touching the 120 volt circuits. These are found primarily in hand tools and outdoor equipment. Measuring the fields in these circumstances requires that the field 
changes which result from the human operator interaction may be required as part of the measurements.

Quantifying electric fields is difficult around sources since nearby materials that are even slightly conductive will perturb the field. Such objects may either shield the electric field or cause intensification of fields at corners or edge points. The human body will greatly distort a field if the sensor is close to the body.

The electric field quantification problem is compounded by the existence of a measurement probe. Most probes are metallic and measure displacement current in between two plates. These plates perturb the field where they are placed. If fiber optics is used to bring the measurement signal from the probe held in space by insulated supports, the perturbance has been shown to be negligible only if the volume radius of about three probe diameters is free space. If the probe measurement signal is made by recording the displacement current to ground, the field is actually changed by the probe unless the probe is a small flat plate placed on the surface of a ground plane.

Since it is difficult to estimate the electric field strength around objects, except for the simplest geometries, no easy technique exists for reducing the spatial $n$. Even if a tedious mapping of the electric field around a source were performed for the device in a "typical" environment, any change in the environment near the device or approach by an operator may completely change the electric field. For this reason, complete spatial mapping is of little use for most appliance or apparatus sources.

There are standards for measuring electric field strength near sources such as a video display terminal. These standards use the current displacement measurement by setting probes which are spheres of metal connected to a ground plane. The probe is expected to modify the electric field similar to, but not precisely as, a human operator would modify the field. The current measured is then related to the current the human operator may experience should the operator be grounded. These measurements require the distortion of the field and are not truly a measurement of the spatial electric field strength. However, they suggest that only a single spatial point measurement may be as useful in assessing electric field exposure as more complex spatial arrays.

\subsection{Minimum Data Set}

All of the above discussion has focused on reducing the infinite number of samples required to describe electric and magnetic fields through all time and space. The following summarizes the basis for accepting a tractable number of temporal and spatial samples. 
- By definition of the RAPID mission, the upper frequency limit is at the start of radio frequencies, $300 \mathrm{kHz}$. By Nyquist criteria, this set a maximum sample rate of $600 \mathrm{kHz}$.

- Biological-EMF literature to date has focused on the ELF band. Very little data on biological findings exist in the VLF and LF bands. Therefore, a pragmatic approach suggests limiting sampling rates to $6 \mathrm{kHz}$.

- Snapshot techniques allow most sources to be characterized by sampling only in small time windows. Knowledge of the source's steady-state operating . characteristics helps select the time interval between the time windows. This method of sampling greatly reduces the size of the data set.

- Transient characteristics of a source may be recorded with high speed sampling techniques but require triggering logic to initiate a sample window.

- Spatial reduction is best achieved by making use of mathematical models of field sources within an appliance or electrical apparatus. The number of spatial samples required is then set by the degree to which the specific device fields fit those predicted by simple models.

A few past and present environmental quantification studies have used or are using many of these temporal and spatial sampling techniques to develop a data set to quantify environmental electric and magnetic fields. There is a large industrysponsored study on-going which has significantly expanded the parameter set being measured over that of previous studies ${ }^{9}$. This expanded data set is given in Table 2.1. One objective of this study is to look for specific magnetic field parameters that might better correlate to the epidemiological findings than the wire code or simple spot measurements of the $60 \mathrm{~Hz}$ magnitude of the magnetic field. Unfortunately, this study will not produce results until late in 1997. There also was a subset of the 1000 Home Study which reported a data set similar to that suggested above ${ }^{10,11}$.

The recently completed FRA studies all report a data set using most of the suggested techniques ${ }^{12,13,14,15,16}$. The parameters that were selected for these studies were selected to demonstrate known or suspected electric and magnetic characteristics that would be missed or smothered by the simple root-mean-square (rms) measurement which usually constrains $n_{t}$ to one value representing a 40 to $1000 \mathrm{~Hz}$ bandwidth. The FRA studies recorded waveforms which covered nearly the entire ELF bandwidth of $3 \mathrm{~Hz}$ to $3 \mathrm{kHz}$. These studies also recorded dc and frequencies above the ELF band in the VLF band $(3 \mathrm{kHz}$ to $30 \mathrm{k} \mathrm{Hz})$. A recently completed appliance and small motor study collected waveform data in the $60 \mathrm{~Hz}$ to $3 \mathrm{kHz}$ band and rms data in the VLF and LF bands ${ }^{8}$. 
All of these studies demonstrated the feasibility of developing a data base using the suggested sampling techniques.

Table 2.1

Magnetic Field Parameters Recorded in the EPRI Wire Code Study

\begin{tabular}{|c|c|c|c|c|c|c|c|c|}
\hline Frequency & $\begin{array}{l}\text { Major } \\
\text { Axis }\end{array}$ & Resultant & $\begin{array}{ll}\ddots & \\
\text { Polarization } \\
\end{array}$ & $\begin{array}{l}\text { Vertical } \\
\text { Component }\end{array}$ & $\begin{array}{c}\because \\
\text { Horizontal } \\
\text { Component }\end{array}$ & $\begin{array}{l}\therefore \\
\mathrm{THD} \\
\therefore \quad\end{array}$ & $\begin{array}{c}\text { AC } \\
\text { Component } \\
\text { Parallel to } \\
\text { DC }\end{array}$ & $\begin{array}{c}\text { AC } \\
\text { Component } \\
\text { Perpendîcular } \\
\text { to DC } \\
\end{array}$ \\
\hline$O$ or DC & & $\checkmark$ & & $\checkmark$ & $\checkmark$ & & & \\
\hline $15 \mathrm{~Hz}$ & $v$ & $\checkmark$ & $\checkmark$ & $\checkmark$ & $\checkmark$ & & & \\
\hline $30 \mathrm{~Hz}$ & $\checkmark$ & $\checkmark$ & $v^{\prime}$ & $\checkmark$ & $\checkmark$ & & & \\
\hline $45 \mathrm{~Hz}$ & $\checkmark$ & $v$ & $r$ & $\checkmark$ & $\checkmark$ & & & \\
\hline $60 \mathrm{~Hz}$ & $\checkmark$ & $\checkmark$ & $v$ & $\checkmark$ & $\checkmark$ & & & \\
\hline $120 \mathrm{~Hz}$ & $\checkmark$ & $\checkmark$ & $\checkmark$ & $\checkmark$ & $\checkmark$ & & & \\
\hline $180 \mathrm{~Hz}$ & $\checkmark$ & $\checkmark$ & $\checkmark$ & $\checkmark$ & $\checkmark$ & & & \\
\hline $240 \mathrm{~Hz}$ & $\checkmark$ & $\checkmark$ & $\checkmark$ & $\checkmark$ & $\checkmark$ & & & \\
\hline $300 \mathrm{~Hz}$ & $v$ & $\checkmark$ & $\checkmark$ & $\checkmark$ & $\checkmark$ & & & \\
\hline $360 \mathrm{~Hz}$ & $\checkmark$ & $\checkmark$ & $v$ & $\checkmark$ & $\checkmark$ & & & \\
\hline : & $r$ & $\checkmark$ & $\checkmark$ & $\checkmark$ & $\checkmark$ & & & \\
\hline$:$ & $v$ & $\checkmark$ & $\checkmark$ & $\checkmark$ & $\checkmark$ & & & \\
\hline : & $\sim$ & $\checkmark$ & $\checkmark$ & $\nu$ & $\checkmark$ & & & \\
\hline : & $\nu$ & $\checkmark$ & $v$ & $\checkmark$ & $\checkmark$ & & & \\
\hline : & $\checkmark$ & $\checkmark$ & $\checkmark$ & $\checkmark$ & $\checkmark$ & & & \\
\hline $2400 \mathrm{~Hz}$ & $v$ & $\checkmark$ & $\checkmark$ & $\checkmark$ & $\checkmark$ & & & \\
\hline $\begin{array}{l}\text { Bandwidth } \\
15-2400\end{array}$ & $v$ & $\checkmark$ & & $\checkmark$ & $\checkmark$ & $\checkmark$ & $\checkmark$ & $\checkmark$ \\
\hline $\begin{array}{l}\text { Bandwidth } \\
45-795\end{array}$ & $\checkmark$ & $\checkmark$ & & $\boldsymbol{v}$ & $r$ & $\checkmark$ & $\checkmark$ & $\sim$ \\
\hline
\end{tabular}




\subsection{FIELD QUANTIFICATION MEASURES}

The preceding section discussed the scientific means for quantifying electric and magnetic fields in time and space. This section will explore existing techniques and suggest specific parameter measures that will quantify electric and magnetic field attributes for use in health assessment studies.

A specific measure is, by definition, a method to perform spatial and temporal reductions of measurements in a consistent and defined manner. A specific measure, also by definition, forces a loss of data. The specific measure provides a "characteristic" of the data that has been agreed upon by the community as useful. Several specific measures may be selected to "characterize" the data. Specific measures do not characterize the data completely, i.e. the original data cannot be, in general, reconstructed or even accurately estimated from the specific measure.

Specific measures fall into general categories. These category definitions are not explicit because a specific measure may fall into two or three categories, but breaking the specific measures into related groups or field attributes often clarifies the characteristic that results. The attributes include

1. Intensity - Intensity or magnitude is the most commonly thought of attribute of electric and magnetic fields. How inclusive the value is depends on how the specific measure is applied.

2. Frequency - Frequency is possibly the second most important attribute of electric and magnetic fields, yet many specific measures obscure or eliminate most of the frequency characteristics.

3. Intermittency - Intermittency determination requires repeated specific measures over a defined time period, such as an appliance duty cycle.

4. Transients - Transients are non-periodic changes in magnitude that may be related to intermittency but which are usually characterized by damped frequency greater than the base frequency of the source and/or exponential growth and decay of intensity at the base frequency of the source.

5. Spatial Attenuation - Attenuation is a function of source geometry. Background fields from other sources make this a difficult attribute to determine in-situ.

6. Polarization - Polarization is a spatial characteristic of the vector intensity as a function of time within the periodicity of the specific frequencies at any given point in space. 
It is necessary to review the possible specific measures to ensure understanding of what attributes are being determined. Then, when combined with a grouping of biological hypothesis, it will be possible to establish levels of source quantification based on the measurement equipment used.

\subsection{The Wave Form Capture}

The wave form capture of temporal data at a spatial location is not a specific measure but is a summary measure. The wave form normally requires a significant data storage allocation and, as a whole, is difficult to utilize as a "characteristic". For digital waveform capture, fundamental steps in data reduction of $n_{t}$ will necessarily have been applied.

The value of wave capture is that the losses of data are minimal compared to the loss of data from most other commonly used specific measures. Most other specific measures can be calcủlated from stored wave capture data. Observation of "wave forms" from wave capture data can be used to verify the assumption of steady-state source operation. If wave capture systems can be used to trigger on transients, some transient information may become available for observation that would be lost to specific measures.

The temporal waveform data is quite often transferred into frequency data which creates an output that has the same number of data points output as the waveform input. There is no actual reduction of data. Thus, the frequency response analysis simply transfers the data from the temporal domain to the frequency domain. In the frequency domain, the patterns are sometimes easier to identify and mathematical manipulations may be made which are extremely difficult in the time domain. There are several methods of converting temporal data to frequency data but the most popular (because of the simplicity and speed of calculation) is the Fast Fourier Transform or FFT. This algorithm was developed by J. Cooley and J. Tukey and published in April of $1965^{17}$. Almost all spread sheets and plotting packages now include some form of the FFT. Not all FFT calculators present the output data in the same form. Often, the data is not adjusted by a factor of 2 nor averaged over the number of samples. The FFT algorithm equations are

$$
B_{h, j}^{\theta}=\frac{2}{N} \sum_{k=0}^{\frac{N}{2}-1} b_{j, 2 k} e^{\frac{2 \pi i k h}{(N / 2)}}
$$




$$
B_{h, j}^{0}=\frac{2}{N} \sum_{k=0}^{\frac{N}{2}-1} b_{j, 2 k+1} e^{\frac{2 \pi i k h}{(N / 2)}}
$$

where $B$ is the FFT coefficients of the maximum magnetic flux density at each harmonic. The superscript e represents the even coefficients related to the cosine and o represents the odd coefficients related to the sine. The temporal samples begin with sample $\mathrm{O}$ and continue to $\mathrm{N}-1$ samples. The summing index $\mathrm{k}$ selects the samples in an even, odd ordering. The first sample is summed by the even series and then the next sample is summed by the odd series. The $h$ is the "harmonic number" beginning with zero and ending with $\mathrm{N} / 2-1$ or the harmonic just below the Nyquist frequency. The $I$ in the equations is the imaginary $I$ which is often expressed as $j$ in electrical equations. The subscript $j$ indicates the $X, Y$, or $Z$ axis measurement for a three axis transducer.

The coefficients are the magnitudes of the cosine and sine terms at each frequency. Thus the phase as well as magnitude at each frequency is provided. The limitation of the FFT algorithm is that the number of points, $N$, must be a power of 2 . Basic to the FFT is the concept of "steady-state" and period $\mathrm{Nf}_{\mathrm{s}}$ should be selected to allow the principal frequencies expected to have complete cycles.

\subsection{Field Orientation Measures}

\subsubsection{The Resultant}

The most common orientation specific measure is the "resultant". This algorithm combines the data from the $x, y$, and $z$ axis as

$$
B_{R}=\sqrt{B_{x}^{2}+B_{y}^{2}+B_{z}^{2}}
$$

where $B_{R}$ is the resultant magnitude of the magnetic field density and $B_{k} B_{y}$ and $B$ are the magnetic field density from the $X, Y$, and $Z$ axis at a single point in time. The resultant is not an rms measurement but is an instantaneous measurement for every point in time that the data is collected. The data that is lost by this specific measure is the orientation angles of the field in reference to a spatial coordinate system. The temporal information is not lost for the amplitude. However, the resultant is always positive and a "resultant wave form" has little meaning. The rms resultant is discussed in the rms descriptions below. 


\subsubsection{The Spatial Components}

Spatial components are a specific measure that reduce time information while retaining orientation information. The spatial components require analysis at specific frequencies. The components are related to the positioning and magnitude of the orientation vector representing the field at a specific frequency. The governing equation is

$$
\frac{d \sqrt{B_{h, X}^{2} \cos ^{2}\left(h \omega t+\theta_{h, X}\right)+B_{h, Y}^{2} \cos ^{2}\left(h \omega t+\theta_{h, Y}\right)+B_{h, Z}^{2} \cos ^{2}\left(h \omega t+\theta_{h, Z}\right)}}{d t}=0
$$

where $X, Y$ and $Z$ are the axes and $h$ is the harmonic number of the selected frequency. The $B$ magnitudes and angles are taken from the FFT and placed in the equation for the desired harmonic number. The equation is then solved for $t$. Two values of $t$ result. The values are then substituted into the square root expression and the largest number is assigned the "maximum spatial component". The smaller number is assigned the "minimum spatial component". The point in time is then converted to an electrical.angle or time delay from the start of the sampling (sample 0 of the FFT). These angles in degrees are assigned as the "maximum spatial phase" and the "minimum spatial phase". This angle is in degrees of the harmonic waveform selected.

The "polarization" is then calculated for the selected harmonic number by dividing the "minimum spatial component" amplitude by the "maximum spatial component" amplitude. The ratio is a summary measure from 0 to 1 . A measure of 0 indicates that the magnetic field is linearly polarized, i.e. the field amplitude passes through zero (at the selected frequency neglecting $d c$ and other frequency components). A measure of 1 indicates the field is circularly polarized, i.e. the field does not change amplitude but rotates in a circular pattern. Various ratios between 0 and 1 indicate various ratios of an elliptical pattern.

These spatial components provide a method for characterizing the spin of the magnetic field flux density. Polarization is always present when around three-phase (or any phase number above 1) equipment and when near to any motor. The fact that the motor turns is a demonstration that the magnetic field flux density near the rotor of the motor has polarization. 


\subsection{Temporal Measures}

\subsubsection{The RMS Calculation}

The most common specific measure made is the rms measure. This measure was originally developed so that power ratings could be compared between Edison's dc power system and the ac power systems that were being installed by Westinghouse. The basis of comparison was heat in a resistor. The heat from a resistor is the current squared time the resistance. In dc, this is simply $l^{2} R$. When ac is present, the current is alternating and simply squaring the current produced an alternation at twice the frequency which was essentially maximum and minimum power. The average of this squaring was taken to remove the effect of the double frequency component. Finally, the square root was taken so that an ac current in amperes could be compared to the dc current in amperes. This historical note directs the attention of the rms measure toward power relationships. If the time over which the measurement is made is considered, the power relationship becomes an energy relationship. Thus, the rms measure describes the energy that has been transferred over a period of time.

The rms measure characterizes the relative "energy" the field over time to which the measuring transducer was "exposed". RMS has been considered as a way to compare the time averages of "exposure" of an electric or magnetic field without apparent regard for specific frequencies. The rms specific measure does use temporal information in that the sensor will always have a defined bandwidth. Also, the rms circuit will always have time constant, i.e., time period over which the rms signal is derived. However, the output signal blends these factors into a single characterization measure. Time characteristics are removed, except recording the time the characterization was made.

For the $n$ sample data recording approach, the magnitude is characterized by

$$
B_{R M S}=\sqrt{\frac{\sum_{k=0}^{N-1} B_{k}^{2}}{N}}
$$

where $k$ is the data sample number and $N$ is the number of data samples taken over the measurement period $\mathrm{Nf}_{s}$. This formulation is one of many that represent the rms summary measure. The $B_{k}$ can be a value recorded on a specific $X, Y$, or $Z$ axis or it may be a $B_{r}$ resultant value at a time point. 
This rms measure includes $\mathrm{dc}$ and ac contributions to the field. Although the rms measure can be applied to any type of signal over a period of time, the normal use of the rms is for "steady-state" conditions. The rms is good only for the frequencies up to the Nyquist frequency and any anti-aliasing filtering that is applied will modify the rms level.

The results of the rms algorithm for each axis can be combined by the resultant Equation 3.3 to form the resultant rms. This does form an "energy" related resultant from the axes.

\subsubsection{The DC Calculation}

The dc calculation is a special case of the rms calculation. The equation is

$$
B_{0}=\frac{1}{N} \sum_{k=0}^{N-1} B_{k}
$$

where $k$ is the sample number of $\mathrm{N}$ samples of measurements. This equation is a simple average of the data and may be applied to measurements in the $X, Y$ and $Z$ axis. It cannot be applied directly to the resultant since the resultant is a squared specific measure and the sign information has been eliminated. For the resultant $d c$ measure, each axis dc must be calculated and then combined as

$$
B_{0, R}=\sqrt{B_{0, X}^{2}+B_{0, Y}^{2}+B_{0, Z}^{2}} .
$$

This specific measure effectively calculates the contribution of all frequencies below $f_{s} / 2 N$. The earth's field changes are well below $f / 2 N$ limitation for most measurements and is well represented by this calculation. For frequencies that are between $\mathrm{f}_{s} / 2 \mathrm{~N}$ and $\mathrm{f}_{s} / 2 \mathrm{~N}$ this calculation is modified by the phase of the frequency when the measurements were made. This summary measure is also responsive to the phase of any frequency that does not have integral multiples of its cycle within the measurement period. The extent of the effect decreases as the frequency increases. Depending on phasing, the frequency $\mathrm{f}_{\mathrm{s}} / 2 \mathrm{~N}$ modifies the number anywhere between zero and the average of one half the cycle of the $f_{s} / 2 \mathrm{~N}$ wave. A frequency that lies half way between the fundamental $\mathrm{f}_{\mathrm{s}} / \mathrm{N}$ and $2 \mathrm{f} / \mathrm{N}$ will modify the dc calculation between 0 and the half cycle average of that specific frequency divided by 3 . 


\subsubsection{The AC RMS Specific Measure}

The rms most frequently produced in specific measures of magnetic flux density do not include the dc calculation. This is usually because the earth's magnetic field is a quasi-dc field (in the ULF range) and is not desired to be a part of the ac field calculation. In the wave capture system, the algorithm used first calculates the dc level of the $X, Y$, and $Z$ axis over the snapshot duration of $\mathrm{Nf}_{s}$ as three separate numbers. These levels are then subtracted from the wave capture data point by point so that the result has no dc average component. Then the rms measure is applied. The equation is

$$
B_{R M S}=\sqrt{\frac{\sum_{k=0}^{N-1}\left(B_{k}-B_{0}\right)^{2}}{N}} .
$$

Each axis can be calculated in this manner. Then the resultant can be calculated as

$$
B_{R R M S}=\sqrt{B_{X R M S}^{2}+B_{Y R M S}^{2}+B_{Z R M S}^{2}} .
$$

\subsubsection{The Analog RMS Specific Measure}

The rms specific measure can be obtained from analog circuits as well as from digital sampling. The output of these analog circuits can then be "sampled" as a specific measure. The rms "chip" utilizes the equation

$$
B_{R M S}^{2}=\overline{B_{\text {INPUT }}^{2}}
$$

where $B_{\text {INPUT }}$ is the analog signal of one axis of the transducer after any filtering required for the transducer. The bar over the input term indicates an averaging operation. This average is not the same as the digital averages discussed previously but is an analog filter which has a time constant of about 10 to 20 times longer than the period of the lowest frequency expected. This filter controls the effective snapshot duration time for the measurement. The analog averaging produces an output that is longer in snapshot duration than the low frequency filter suggests. Although the measurement records only power frequency waves in "narrow band". mode or power frequency with low order harmonics in the "wide band" mode, these 
frequency contributions are averaged over the much longer snapshot duration. The low frequency of the measurement is controlled by a high pass filter set below the power frequency placed in series with the transducer signal. The fact that the analog average is much slower than the lowest frequency allowed, the identity

$$
B_{R M S}=\frac{\overline{B_{I N P U T}^{2}}}{B_{R M S}}
$$

is used with the output of the chip effectively dividing the average input squared. This process can be used on a single axis at a time since there is no way in most chips to sum the squared inputs before averaging. The rms can then be calculated as in Equation 3.9 after sampling the three axis rms signals.

\subsection{Specific Measures Related to Force}

Forces that are applied to material that will change position in the presence of a magnetic field require calculations that determine the maximum amplitude, minimum amplitude, torque and spin of the magnetic flux density. In some cases, the alinement with a second magnetic field component such as the dc field must be identified. Specific measures which are used for these concepts include peak, peak-to-peak, perpendicular and parallel. Specific equations for ion resonances are presently under development by Dr. Bowman ${ }^{18}$ and are not listed here.

\subsubsection{Peak and Peak-to-Peak Specific Measures}

The peak value of any signal is simply a scan of the signal for the maximum and minimum value and the reporting of these values.

Peak calculations usually assume that "steady-state" is present. For transient events that occur rarely, the peak measurement may require adjustment to account for the transient response of the anti-aliasing filters if the peak does not have at least two adjacent measurements above half-peak.

The peak algorithm for each axis usually produces the peak readings after the removal of the dc level from that axis. The maximum and minimum are calculated and the largest absolute value is reported. The peak-to-peak specific measure subtracts the minimum from the maximum. If peak-to-peak is requested, the dc need not be subtracted from the data. 


\subsubsection{The Peak Resultant Specific Measure}

The peak resultant specific measure calculates the peak using the instantaneous resultant rather than calculating the peak magnitude for each axis. This peak calculation reflects the actual peak field level at a spatial location over a period of time $\mathrm{N} / \mathrm{f}_{\mathrm{s}}$. The instantaneous resultant is calculated as in Equation 3.3 for each point of the wave capture measurement. The resultant is then scanned as for peak magnitudes to determine the actual peak magnitude. A minimum may also be sought although it will be zero for linearly polarized fields. The dc is included in this calculation since there is no way to remove the $d c$ and still preserve the absolute peak information.

The peak resultant specific measure is different from the spatial components discussed under orientation measures. The spatial components are calculated for a single frequency. Polarization is also calculated for a single frequency. This peak magnitude is calculated from the wave capture measurements and no FFT processing is required.

\subsection{Uncommon Specific Measures}

\subsubsection{Relating $A C$ and DC Fields}

A specific measure to relate the strength of the ac field either parallel or perpendicular to the dc field may be useful in ion-resonance calculations. These calculations require a specific frequency to be chosen for the ac components and an FFT performed for all three axes. The limitations on frequency response that applied to the FFT also apply to these calculations. The limitations on the dc calculations discussed in the rms summary measures applies to the dc utilized here. The dc utilized is the resultant dc that comes from using Equations 3.6 and 3.7.

There are a number of approaches to calculating ac parallel or perpendicular to dc. Each method seeks to resolve the definition of where the ac vector is that is to be compared to the dc. If elliptical polarization is present, the ac vector may be chosen as the semi-major axis of the ellipse. This provides the angle to the dc to the maximum spatial component. Another approach is to utilize the wave capture data from the three axes and on an instantaneous point-by-point basis resolve the axes data into planes that are perpendicular and parallel. The resulting point-by-point data then may be processed for the rms summary measure to produce the exposure parallel or perpendicular to the dc. However, unless the data is dominated by a single frequency, this instantaneous algorithm is not compatible to ion-resonance models which require the ac component to be associated with a specific frequency. 
The recommended algorithm calculates the relative magnitudes of the two quadrature phase components and then the angle by

$$
\begin{aligned}
& |B \| D C|_{R E A L}=\frac{B_{D C} \cdot B_{A C_{R E A L}}}{\left|B_{D C}\right|} \\
& |B \| D C|_{\text {IMAG }}=\frac{B_{D C} \cdot B_{A C_{\text {IMAG }}}}{\left|B_{D C}\right|} \\
& \theta=\arctan \left(\frac{|B \| D C|_{\text {IMAG }}}{|B \| D C|_{R E A L}}\right)
\end{aligned}
$$

where $B_{A C}$ and $B_{D C}$ are three dimensional vectors. The equations to determine the magnitude of the ac field parallel to the dc are

$$
|B \| D C|_{M A X}=\frac{B_{D C} \cdot B_{A C \text { at } \theta}}{\left|B_{D C}\right|}
$$

where $B_{A C}$ is evaluated at the angle $\theta$. The ac field perpendicular to the dc field is the projection of the polarization ellipse onto a plane normal to the dc field vector. The quantity is, in general, another elliptically polarized field. If the rms magnitude of the quantity is desired, the principle of conservation of field energy can be used to compute the rms. Since ac field components must resolve into components which are parallel and perpendicular to the dc field (there are no other alternatives), then

$$
\left|B_{R M S}\right|=\sqrt{|B \| D C|^{2}+|B \perp D C|^{2}} \text {. }
$$

This can be rearranged to give

$$
|B \perp D C|=\sqrt{\left|B_{R M S}\right|^{2}-|B \| D C|^{2}} \text {. }
$$


Note: The presence of dc when characterizing sources is somewhat arbitrary since the measurement of a source may occur at a location in the earth's field that is very different than the earth's field where the source is to be utilized. This will require the adaption of dc measurements on an axis basis so that the earth's field may be separated from the source dc enhancements (i.e. concentrations or reductions from the materials used in the source) and the dc field that the source may generate.

\subsubsection{Coherency and Intermittency}

Coherency and intermittency are extremely difficult to characterize. No specific measure has been developed. The normal measurement processes for most sources utilizes the coherency and, except for transients, avoids intermittency. Coherency requires that the source be steady-state at all frequencies generated so that the generated fields are absolutely continuous. Intermittency requires that the steadystate conditions be interrupted.

A coherency summary measure might indicate the drift from perfect coherency as a function of time. As such, it would measure deviation of waveform as a percentage error per time unit desired. The upper frequency limits of the coherency function may need to be lowered to allow longer snapshot durations and shorter snapshot intervals. Litovitz's ${ }^{19}$ earlier publications suggest snapshot durations of at least 10 seconds. Presently there are no algorithms to create this information since there is no commercially available measuring device that records wave capture for periods of 10 seconds or more. Snapshot techniques with short inter-interval spacing would be a good approximation technique to gather the data. However, the same test used to verify steady-state for a given source would have to be applied to verify the validity of the snapshot technique.

An intermittency measure would be the detection of large changes in coherency. It has been suggested that such a measure would be to simply record the on time and off time of source fields. An intermittency summary measure that reported the ontime to the off-time ratio with an on-off period might provide intermittency characterization. Multiple levels of intermittency might be addressed using multiple limits to the coherency index.

\subsubsection{Measures of Induction}

The induction of voltage and current into any material is a function of the internal and external physical characteristics of the object and the proximity to the field source. The induction requires rate-of-change of fields. Therefore, measurements to determine the available induction source strength should be $\mathrm{dV} / \mathrm{dt}$ or $\mathrm{dB} / \mathrm{dt}$. There has been little development in the methods for measuring the rate-of-change of fields even though. 
most magnetic field transducers utilize coil sensing systems which measure rate-ofchange naturally and must utilize integrators to obtain magnetic flux density.

It would be quite simple to construct an ELF rate-of-change magnetic monitor and use all the specific measures that are already discussed to characterize the rate of change. This solution requires the rate-of-change data be also integrated so that magnetic flux density data would also be available.

\subsubsection{Peak Rate-of-Change of Field}

For the ELF band, the maximum rate-of-change can be calculated from the wave capture measurements. The measurements were taken at an incremental time of $1 / f_{s}$. The slope between each point can be approximated by a straight line between each pair of measurements. The rate-of-change of the straight line approximation can be calculated with normal linear equation forms

$$
\frac{d B}{d t}=\frac{B_{k+1}-B_{k}}{1 / f_{s}}
$$

where $\mathrm{k}$ is the sample number from 0 to $\mathrm{N}-2$. This is a conservative approximation as the higher frequencies nearing the Nyquist frequency could have higher rates-of-change between points than is calculated from straight line equations. This calculation does suffer from system noise. If the analog-to-digital converters and the electronics associated with the anti-aliasing filters have a noise level that is within one-tenth of the level of the signal, the $\mathrm{dB} / \mathrm{dt}$ from the noise may overshadow the $\mathrm{dB} / \mathrm{dt}$ from the signals. The assumption of "steady state" is always made to allow snapshot durations and snapshot intervals to be used. The specific measure then uses this calculation to determine a maximum and minimum $\mathrm{dB} / \mathrm{dt}$. The absolute value of the highest $\mathrm{dB} / \mathrm{dt}$ is reported.

\subsubsection{Harmonics}

Measurements of single frequencies determined by FFT analysis of a waveform have the useful characteristic of quantifying $\mathrm{rms}$, peak, and $\mathrm{dB} / \mathrm{dt}$. The single frequency represents the energy at only one frequency $f_{m}$ and is not a measure of overall energy. For the snapshot, the width of the frequency band for each frequency is $f_{s} / N$ so that frequencies included in the band are $f_{m}-f_{s} / 2 N$ to $f_{m}+f_{s} / 2 N$. The harmonic number $h$ is $f_{m} N / f_{s}$ and the harmonics available are $h=1$ to $N / 2-1$ or one harmonic below the Nyquist harmonic of $\mathrm{N} / 2$. The 0 harmonic or dc is calculated as in Equation 3.6. Equation 3.19 gives the rms value of the harmonic magnitude. If the rms value is. multiplied by the square root of 2 , the peak harmonic magnitude results. If the peak 
harmonic magnitude is multiplied by $2 \pi f_{m}$ the peak harmonic rate-of-change results.

$$
B_{R M S h, j}=\frac{\sqrt{B_{h, j}^{\theta 2}+B_{h, j}^{02}}}{\sqrt{2}}
$$

A corollary summary measure associated with individual harmonic magnitudes is harmonic phase. This summary measure allows the relationship of various harmonics to be compared. The harmonic phase is calculated as

$$
\theta_{h}=\arctan \left(\frac{-B_{h}^{0}}{B_{h}^{e}}\right)
$$

where the angle is in electrical degrees related to the harmonic period. The angle is related to the sinusoid in time as

$$
b_{h}(t)=B_{h} \cos \left(\frac{h 2 \pi f_{s}}{N} t+\theta_{h}\right) .
$$

Although biological studies seldom use the harmonic phase, the measure is very useful in source characterization when modeling is required. The harmonic phase of the power frequency between spatial points is useful in determining the location of the source if the source is hidden. It is also useful in tracking source motion. Normally, the reference angle for such calculations is chosen from a separate signal, usually the voltage on a wall outlet, which is expected to remain constant in phase throughout the series of measurements.

\subsection{Measures for Transient Phenomenon}

There is a large body of literature which describes the various transient response measures for control systems. When a change in a control system is required, such specific measures as rise time, overshoot, settling time, droop, and natural frequency allow control system responses to be compared. The calculation of peak and peak rate-of-change values will provide some indication of the characteristics of the transient. However, the use of rise time, etc. can improve the characterization. Rise. time is the time from 10 percent to 90 percent of the desired change. Overshoot is 
the percentage the signal went over the desired change using the desired change as 100 percent. Settling time is the time from the start of change to when the signal does not leave a specified band about the desired change. The natural frequency is half the inverse of the period between oscillation peaks.

Transient phenomenon may need to be coupled with specific measures such as induction because many transient situations occur during steady-state which produce natural frequencies in the VLF and LF ranges. While the device may be at power frequency steady-state, these higher frequencies are seldom generated in a continuous or steady-state manner. The phenomenon of arcing as found in brushes on motors, or the switching of electronic or manual devices creates short bursts of frequencies in the VLF and LF ranges. These bursts often contain high rates-of-change which would fall into the category of possible induction sources.

One method of obtaining the peak rate-of-change is to use a dB/dt transducer (such as a coil) and a peak detecting analog circuit to capture and display the peak rate-ofchange. This is a transient capture since the peak-detecting analog circuit must be reset either by manual means, by a computer switch, or by a slow analog washout circuit. Thus it displays the peak when it is received. If several peaks are received, another circuit might need to display a rate-of-change intermittency summary measure. Such a measure would indicate how often the transient is measured. The limitations on the intermittency measure include the ability of the circuit to discern another transient and the ability to recognize various levels of $\mathrm{dB} / \mathrm{dt}$ as transients. Such summary measures might be useful in determining the presence of $\mathrm{dB} / \mathrm{dt}$ but they are difficult to use in any type of mode that could map the $\mathrm{dB} / \mathrm{dt}$ around a source.

\subsection{Biological Study Considerations}

It is important that the research community should have at its collective fingertips the specific measures that will most easily reveal possible effects.

The Joint NIOSH/DOE Workshop "EMF Exposure Assessment and Epidemiology: Hypotheses, Metrics and Measurements" (Cincinnati, OH, September 26-28, 1994) examined four groups of possible field interaction mechanisms. These mechanisms were selected by the biological community as those which had reportedly been demonstrated. The purpose and goals of this workshop is reprinted here to indicate the broad intent by the biological community to determine metrics which would be useful to explain biological observations.

Purpose

The workshop participants will consider the importance of biologicallybased exposure metrics in EMF epidemiological studies. The primary aim will be to develop exposure assessment methods and epidemiological studies to test hypotheses that these metrics may be 
associated with disease. The product of the workshop will be a report describing approaches for characterizing EMF exposure in terms of these metrics and designs for epidemiological studies.

Goals

1. To determine the 2-3 most plausible biological hypotheses for how occupational and residential EMF may be causing the reported associations with diseases (leukemia and breast cancer particularly) which could be tested in future NIOSH epidemiological studies.

2. To develop quantitative exposure metrics from these hypotheses and strategies for assessing exposures to these metrics in occupational and residential studies.

3. To propose exposure assessment strategies which will collect the data needed to assess future hypotheses with various study populations.

(Taken from the Workshop Agenda)

Although there was considerable discussion on the priority of the research, no priority order for metrics or the most plausible mechanism was reached. The following four mechanism groups were considered to be viable areas for further research:

\section{Group 1: Resonances \\ Group 2: Coherence and Intermittency \\ Group 3: Induced Currents, Transients and Otherwise \\ Group 4: Magnetic Moment Effects}

There are published effects in each group and such effects must be considered when attempting to determine the summary measures that best describe the magnetic field parameters and attributes believed to elicit effects within a group.

\section{Group 1:}

The Group 1 resonances studies indicate an interaction of ac and dc (static) fields both in parallel and perpendicular relationships. The characteristics of the magnetic field involve primarily the temporal aspects of the field with the biological target considered as a point in space. The suggested mechanisms are based on the "mechanical" orientations and force or torque relationships of the biological material and is observed primarily at frequencies below $300 \mathrm{~Hz}$. However, because of the nonlinearity of the biological and biochemical systems, higher frequency fields at harmonics of the resonant frequency may be important. The relationships between ac temporal changes (frequency components) and dc (or static) levels is important. The do level selects the 
resonant frequency with the direction of the ac field also important. The level of the ac field is also a parameter.

\section{Group 2:}

The Group 2 coherence and intermittency models are similar in that they deal with the temporal stability of the field. Coherence requires that a magnetic field should exhibit repeatable temporal patterns for a period of time at a fixed point in space. The report work focused on sinusoidal wave shapes at utility supply frequencies but could apply equally well at other frequencies. Proponents argue that fields which remain stable in frequency, intensity, orientation, and phase for periods of time, in the order of ten seconds, are detected by biological systems by some kind of integration of their coherent pattern over time. The total exposure at a specific frequency seems to be modified by changes in coherency of that field. The response may be related to forces or torques rather than from exposure alone. Hence, for purposes of measurements, detection of exceptions to the repeatable patterns becomes the principal focus. Intermittency suggests that biological systems respond to changes in field conditions more strongly than constant "coherent" fields. Most studies to date have compared constant field exposure to exposures in which fields were turned on and off frequently. Sensitivity to intermittent changes in field orientation, frequency, or phase has not been examined. Consequently, for measurement purposes, the intermittency models require the same sort of field characterization data as the coherency model. Abrupt changes from switching are by definition transient responses. The transient characteristic may also be biologically significant.

Group 3:

Group 3, induced currents, and voltages suggest effects through the induced electric fields and current densities caused by the rate-of-change of the magnetic field. Although much of the work is concerned with the rapid temporal changes of field, such as transients, the above mentioned parameters, such as coherency and intermittency, could equally be involved. The presence of transient activity would be important for this group since the induced voltages could be much higher for large, high frequency transients.

\section{Group 4:}

The Group 4, magnetic moments, suggests that the magnetic material within the cell may respond given specific ac and/or dc field interactions with magnetic material. This concept utilizes the temporal characteristics at a point in space related to the force or torque of the field. The "mechanical" nature of this 
interaction, i.e. the alinement of molecules, operates at the lower frequencies and has measurement requirements similar to resonance relationships.

\subsection{Specific Measures Conclusion}

Specific measures which define the characteristics of a source may be used as a method of reducing the information that would need to be collected to fully describe the orientation, spatial location, and temporal characteristics of a field generated or modified by a source. As the data is reduced by specific measures, data is lost. The cost of the lost data must be judged on the basis of

1. The ability to restore or approximate the lost data using the specific measures recorded.

2. The documented lack of need for the lost data.

3. The ability to approximate lost data using knowledge of the source construction, source location and, in some cases, the source motion.

Since specific measures are characteristics which are exchanged between users in corollary areas, the measures must be defined and agreed upon by these users. Creating specific measures that are undefined either by usefulness to the general users community or by obscure algorithms may have little value.

All measurement processes are bound by limitations of physical systems such as sampling frequency limits, practical filter characteristics, storage capacities, or calculation efficiencies.

Many sources have unique properties which make the data collection less difficult. If sources are normally operating at a steady state or repeatable load cycle for the duration of the measurement process, it allows :

1. Repeated measurements at different spatial locations to be made.

2. The concept of snapshots to be utilized with snapshot durations and intervals.

3. Repeat measurements to be compared even when the source has be turned off and then back on.

4. The mathematical use of rms, FFT, and other algorithms defined only if steady state conditions exist. 
5. The ability to compare results from one test location to another test location.

Not all sources have this characteristic which is basic to many specific measure algorithms. There are transients which occur and must be measured on a one time basis when there is no possibility to repeat the measurement. Such one time measurement poses special restrictions on the specific measures that can be used to characterize the transient nature of the source.

All specific measures of sources must intrinsically contain

1. Spatial information relative to the source. This may be the distance from center of source or distance from some fixed feature of the source. Normally the physical center of the source is utilized but other centers may be justified as discussed later in protocol. Also included in spatial information is the actual location of the source during measurement and the proximity of this source to other sources.

2. Temporal information relative to the measurement. This should include the time and date as well as coordinating times between measurements.

3. State of source information such as actual load current when available, times of load or cycle changes, and times of on-off operations should also be recorded.

4. Orientation of the transducer If the summary measure records individual axis information from the transducer. This orientation should be relative to the source.

Details on the methods of recording this data along with the specific measures is provided in the protocol discussions. Measurements should not be considered complete without this information.

Table 3.1 provides and alphabetized list of the specific measures along with the applicable paragraph and equation numbers for cross reference. The attributes characterized or at least partially quantified are also correlated with the specific measures. As an example, select ac rms specific measure. This measure description is found in Paragraph 3.3.3 and the Equation is 3.8. Axes data are needed for the algorithm, but FFT calculations are not. The attributes of intensity and attenuation can be defined but other information is required to address the other attributes. 


\begin{tabular}{|c|c|c|c|c|c|c|c|c|c|}
\hline \multicolumn{10}{|c|}{$\begin{array}{l}\text { TABLE } 3.1 \quad \text { ALPHABETICAL LISTING OF SPECIFIC } \\
\text { MEASURES AND RELATED SOURCE FIELD ATTRIBUTES }\end{array}$} \\
\hline \multirow[t]{2}{*}{$\begin{array}{l}\text { Specific } \\
\text { Measure }\end{array}$} & \multicolumn{2}{|c|}{$\begin{array}{l}\text { Applicable } \\
\text { Concepts } \\
\text { and/or } \\
\text { Equations }\end{array}$} & \multicolumn{7}{|c|}{ Field Attributes } \\
\hline & 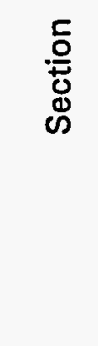 & $\begin{array}{l}\text { 동 } \\
\text { 윰 } \\
\text { 뭄 }\end{array}$ & $\begin{array}{l}\frac{Z}{10} \\
\stackrel{D}{0} \\
\stackrel{ \pm}{E}\end{array}$ & 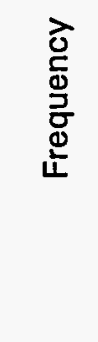 & 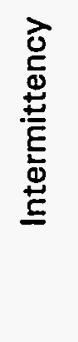 & 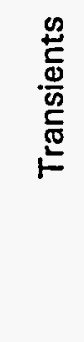 & 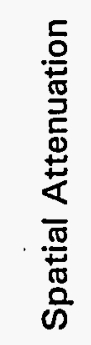 & 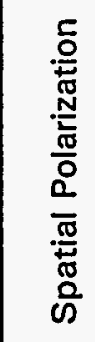 & 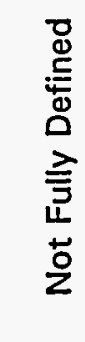 \\
\hline AC-DC Angle & 3.5 .1 & 3.14 & & & & & & & $x$ \\
\hline $\begin{array}{l}\text { AC-DC Parallel } \\
\text { Magnitude }\end{array}$ & 3.5 .1 & 3.15 & $x$ & & & & & & $x$ \\
\hline $\begin{array}{l}\text { AC-DC Perpendicular } \\
\text { Magnitude }\end{array}$ & 3.5 .1 & 3.17 & $x$ & & & & & & $x$ \\
\hline AC RMS & 3.3 .3 & 3.8 . & $x$ & & & & $x$ & & \\
\hline Analog AC RMS & 3.3.4 & 3.11 & $x$ & & & & $x$ & & \\
\hline Coherency Index & 3.5 .2 & & & & & & & & $x$ \\
\hline DC & 3.3.2 & 3.6 & $x$ & $x$ & & & $x$ & & \\
\hline Harmonic Magnitude & 3.5 .5 & 3.19 & $x$ & $x$ & & & $x$ & & \\
\hline Harmonic Phase & 3.5 .5 & 3.20 & & & & & $x$ & & \\
\hline Intermittency Index & 3.5 .2 & & & & & & & & $x$ \\
\hline $\begin{array}{l}\text { Maximum Spatial } \\
\text { Component }\end{array}$ & 3.2 .2 & 3.4 & $x$ & & & & , & $x$ & \\
\hline $\begin{array}{l}\text { Maximum Spatial } \\
\text { Phase }\end{array}$ & 3.2 .2 & 3.4 & $x$ & & & & & $x$ & \\
\hline $\begin{array}{l}\text { Minimum Spatial } \\
\text { Component }\end{array}$ & 3.2 .2 & 3.4 & $x$ & & & & & $x$ & \\
\hline $\begin{array}{l}\text { Minimum Spatial } \\
\text { Phase }\end{array}$ & 3.2 .2 & 3.4 & $x$ & & & & & $x$ & \\
\hline Peak Magnitude & 3.4 .1 & & $x$ & & & & & & \\
\hline Peak Rate-of-Change & 3.5.4 & 3.18 & $x$ & & & $x$ & & & \\
\hline Peak Resultant & 3.4 .2 & & $x$ & & & & & & \\
\hline $\begin{array}{l}\text { Peak-to-Peak } \\
\text { Magnitude }\end{array}$ & 3.4.1 & & $x$ & & & & & & \\
\hline
\end{tabular}




\begin{tabular}{|c|c|c|c|c|c|c|c|c|c|}
\hline \multicolumn{10}{|c|}{$\begin{array}{l}\text { TABLE } 3.1 \text { ALPHABETICAL LISTING OF SPECIFIC } \\
\text { MEASURES AND RELATED SOURCE FIELD ATTRIBUTES }\end{array}$} \\
\hline \multirow[t]{2}{*}{$\begin{array}{l}\text { Specific } \\
\text { Measure }\end{array}$} & \multicolumn{2}{|c|}{$\begin{array}{c}\text { Applicable } \\
\text { Concepts } \\
\text { and/or } \\
\text { Equations }\end{array}$} & \multicolumn{7}{|c|}{ Field Attributes } \\
\hline & 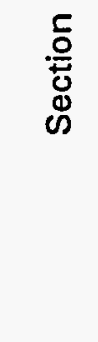 & $\begin{array}{l}\text { 음 } \\
\text { 离 } \\
\text { 号 }\end{array}$ & 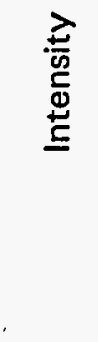 & 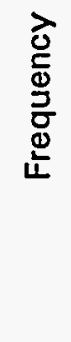 & 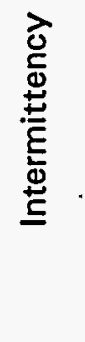 & 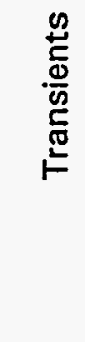 & 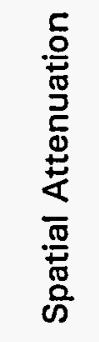 & 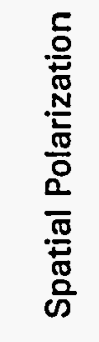 & 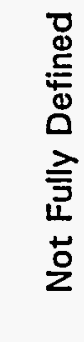 \\
\hline Polarization & 3.2 .2 & 3.4 & & & & & & $x$ & \\
\hline Resultant & 3.2 .1 & 3.3 & $x$ & & & & $x$ & & \\
\hline $\begin{array}{l}\text { Transient Indices of } \\
\text { Rise Time, } \\
\text { Overshoot, Settling } \\
\text { Time, Natural } \\
\text { Frequency. }\end{array}$ & 3.6 & & & & & $x$ & . & & $x$ \\
\hline $\begin{array}{l}\text { Transient Peak Rate- } \\
\text { of-Change }\end{array}$ & 3.6 & & & & & $x$ & & & $x$ \\
\hline
\end{tabular}




\subsection{PRESENTLY AVAILABLE INSTRUMENTATION}

There are many types of magnetic field meters and data recorders. The existing equipment can be grouped into five basic categories, although there is overlap for some equipment. The categories are:

Survey meters, ac and dc.

Broadband or narrowband three-axis recorders.

Single-axis and three-axis wave capture recorders.

Multi-sensor three-axis wave capture recorders.

Special purpose transient capture recorders.

\subsection{Survey Meters}

A meter which produces an rms reading for either one- or three-axis measurements is the easiest system to use in a simple source characterization. Measurements can be taken quickly at key locations to indicate the general field characteristics, providing the various sources of magnetic fields are fairly-well known. These meters rarely measure the $\mathrm{dc}$ levels. The presence of harmonics is usually not determinable, although some meters will indicate either third harmonic or Total Harmonic Distortion (THD). These meters are simple to use, easy to carry, and are rugged. The data taken, however, is subject to transcription error since it usually must be manually recorded.

The normal parameters that an electric and/or magnetic field survey meter can accommodate include:

Analog rms narrowband at power frequency (one or three axis)

Analog rms broadband for the power frequency and power harmonics, for example, up to the 13th harmonic (one or three axes).

Other equipment may function as a survey meter as one of the modes of operation. These include:

Analog rms narrowband or broadband recorders. Wave Capture recorders.

\subsection{Broadband or Narrowband Three-Axis Analog RMS Recorders}

The broadband (an example of $40 \mathrm{~Hz}$ to $800 \mathrm{~Hz}$ ) or narrowband (an example of $40 \mathrm{~Hz}$ to $100 \mathrm{~Hz}$ ) resultant recorders are more complex than survey meters. The recorder normally measures the analog rms levels of the magnetic field density in each of the axes. The resultant is then calculated. Hardware filters provide the band definition and are sometimes selectable through a setup procedure. The use of the recorder 
involves connection to a computer with software to support the up-loading of setup parameters, the down-loading of analog rms axes data and calculated resultants along with time stamps and possibly spatial coordinates. Storage of data is provided by the computer disks and plotting to a printer usually is included in the software. Ranges of magnetic field levels from a low of $0.01 \mu \mathrm{T}$ to as high as $12 \mathrm{mT}$ are examples of commercial offerings. These recorders are economical, small, portable, relatively easy to use, and are rugged. Some of these recorders can also monitor electric fields or ac currents as an auxiliary parameter.

The summary measures that broadband or narrowband recorders can measure include:

Analog rms per axis for a narrow filter band usually set to the power frequency. Analog rms per axis for a broadband filter which includes the power frequency and low-order harmonics.

Time of measurement

Spatial coordinate

Analog rms for the electric field (not necessarily free body)

\subsection{Single-Axis and Three-Axis Wave Capture Recorders}

The few wave capture recorders available greatly vary in size, and complexity. The most common wave capture recorders available presently are portable, carried in a pack, and are easy to use considering the complex measurements that are possible. One variety of wave capture recorder uses a one-axis sensor to determine waveform. This device cannot determine most orientation-related summary measures such as the resultant unless the sensor is rotated to a maximum reading. Polarization can be laboriously determined by rotating the sensor to find the maximum and minimum field magnitudes. In some source measurements the complexity of the field precludes accurate probe rotation to maximums and minimums.

Three-axis recorders have a variety of sensors that can be connected, depending on the instrument and the measurement protocols. A commonly used sensor uses the fluxgate principle, which allows ac rms measurements from $0.01 \mu \mathrm{T}$ to $0.7 \mathrm{mT}$ and dc measurements to $1.0 \mathrm{mT}$. Other sensors do not record dc levels but allow ac rms measurements from $0.001 \mu \mathrm{T}$ to $0.1 \mathrm{mT}$ or $0.01 \mu \mathrm{T}$ to $20 \mathrm{mT}$. The complexity of operation of wave capture recorders is greater than analog rms recorders. The data is normally recorded as a series of samples over a cycle of the power frequency (i.e. 128 samples over $16.67 \mathrm{~ms}$ ). The recording can be extended to allow low frequency effects to be recorded by taking more samples at the same sample frequency (i.e. 2048 samples over $267 \mathrm{~ms}$ ). When the fluxgate is used, the three axes of dc is recorded along with the three axes of ac to allow the relationships between the dc and ac to be calculated. Since the fluxgate measures both dc and ac at the same time with the same sensors, the spatial and temporal relationships are preserved. Such a 
wave capture recorder is normally programmed to allow various sampling frequencies and sample counts to provide recording for either 50 or $60 \mathrm{~Hz}$ or for special low frequency recording. The wave capture recorder is typically broadband across the ELF band (i.e. dc to $3 \mathrm{kHz}$ ) and often includes the capability of operating as a transient recorder as well as a meter. Auxiliary functions include electric field, current, voltage, VLF rms, and LF rms using high frequency sensors.

Wave capture recorders require more understanding of computer usage and of sampling theory than the analog rms recorders. When the recorder is programmed for a specific protocol, the operation is quite simple. Software provides support for setup of sampling parameters, algorithms for a large variety of summary measures and plotting systems. The disadvantages include the cost, management, processing and plotting of the large data sets. As an example of data set size, a recorder set for sampling 128 times during one $60 \mathrm{~Hz}$ cycle, having a snapshot duration of 0.267 seconds, and a snapshot interval of 10 seconds, recording three axis of spatial data will accumulate 737,280 samples in 60 minutes. This translates to roughly 4.4 megabytes of computer storage in binary work format. The waveform capture provides many important relationships of the field measurement which are lost in specific measures, such as analog rms. The increase in data retained may allow subtle biological interactions to be investigated. However, the above example demonstrates that waveform capture has limitations which must be recognized. Determination must be made to ensure that these limitations are consistent with biological response.

The specific measures that can be calculated through FFT processes include a wide variety of possibilities. These calculations depend upon the limitations of sample frequency, snapshot duration and snapshot interval. It should be noted that the only reason the wave capture system inherently limits the characterization of a field is simply because there cannot be an infinite number of samples taken. Wave capture equipment must be utilized with these limitations in mind. The specific measures for source measurements include:

AC rms for each axis over the ELF band.

Harmonic Magnitudes for each axis over the ELF band.

Harmonic Phase for each axis over the ELF band.

DC.

Resultant for AC rms, DC, and Harmonic Magnitudes.

FFT rms.

Polarization.

Maximum Spatial Component.

Maximum Spatial Phase.

Minimum Spatial Component.

Minimum Spatial Phase.

RMS. 
LF RMS.

VLF RMS.

AC-DC Angle.

AC-DC Parallel Magnitude.

AC-DC Perpendicular Magnitude.

Transient wave capture over the ELF band with level triggers.

AC RMS for $E$ using a free body for selected frequency bands.

\subsection{Multi-Sensor Three-Axis Wave Capture Recorders}

The multi-sensor wave capture recorders are an increase in complexity over the single sensor wave capture recorder discussed above. Normally such equipment is so costly that specific designs are provided for specific purposes. Specialty systems, such as the appliance test set with over 40 sensors, provide snapshot data including essentially all of the parameters defined in Section 3.0, except the transient data. The limitations include the inability to measure dc and ac simultaneously if the ac is greater than $0.7 \mathrm{mT}$.

The early part of the EPRI 1000 Home Study used a special system for residential measurements with up to 25 sensors at a $60 \mathrm{~Hz}$ base frequency and a bandwidth to $1.5 \mathrm{kHz}$. This system has no transient capability but can measure currents, voltages and electric fields.

A portable system for measuring up to four Fluxgates simultaneously in transportation systems, such as electric trains and electric vehicles, was developed. The singlesensor wave capture system can be obtained with slave capability to link several systems together.

The value of multiple recordings is to collect coherent temporal data from many spatial locations simultaneously. Although no biological effort has been focused on spatial coherency, subject movement throughout such space may be a variant on the intermittency hypothesis. Although the problem of cost and data volume increase with these more complex systems, the increased volume of data is offset by the value of rapid data assimilation. 


\subsection{Special Purpose Transient Capture Recorders}

There are two types of transient capture recorders. The first type is similar to the analog rms recorder. The equipment captures transient or rare events and calculates specific measures. These specific measures are then displayed or recorded. This equipment is inexpensive, portable and rugged. The transient parameters include the maximum increment of field value relative to the pre-existing field and the maximum time rate of change in the magnetic field. A peak detector with a reset is used to capture the transient. The specific measures include:

Peak Rate-of-Change

Rate-of-Change Intermittency

The second type of rare event or transient capture recorder is far more complex. Presently, there is little in the commercial market prepared for magnetic fields although a broad range of commercial transient recorders and digital storage oscilloscopes are available. Sensors for $30 \mathrm{~Hz}$ to $300 \mathrm{kHz}$ are available. Systems for up to 4 sensors (12 channels) have been connected for measurement up to $1 \mathrm{MHZ}$ sample rates. Another system specifically for magnetic fields to $160 \mathrm{kHz}$ with one sensor (three channels) was assembled and tested with up to 36 trigger parameters available. These systems have been assembled as part of research efforts and they are not generally portable.

There are three primary difficulties with present transient equipment. The high speed waveform capture systems are, in general, not portable. To achieve portability, the sampling rate poses a serious problem. Sampling systems for very high sampling rates require large power pulses to provide the high speed switching necessary. Presently, portable sampling systems have sampling rate limitations of $100 \mathrm{kHz}$, high enough for a single channel to $30 \mathrm{kHz}$ but only good enough for a three-axis sensor above $15 \mathrm{kHz}$. These systems normally do not have the complex trigger systems and suffer from simple trigger protocol. Oscilloscopes have been used for transient observation but often the number of samples that can be recorded for a three-axis sensor is limited. If a $600 \mathrm{kHz}$ sample rate is chosen for a transient ( $300 \mathrm{kHz}$ bandwidth) and there are 4096 sample points available, only $6.8 \mathrm{~ms}$ of waveform is stored. This is less than half of one $60 \mathrm{~Hz}$ cycle.

The second difficulty lies in determining a proper trigger mode. If simple level triggers are used for either $B$ or $\mathrm{dB} / \mathrm{dt}$, the transient will be recorded provided the trigger point is much greater than the normal $B$ or $\mathrm{dB} / \mathrm{dt}$ levels. If the normal levels are near the trigger point, transient after transient will be recorded, overloading the data storage systems. There are trigger protocol that has been developed to reduce this problem but the triggering system must be computer interactive adding a serious complexity to the device. Setting the trigger level poses problems since the level of biological 
activity may be dependent on a biological threshold ${ }^{20}$. This threshold might direct the trigger level settings. As of yet, no commitments to a threshold has been published.

The third difficulty is the latency period present in any transient monitoring system. The data must be transferred to the computers and stored on disk. This time can be (and often is) measured in seconds. The transient capture is usually blind during these transfer periods. The intermittency of the field cannot be well determined because of this transfer time.' Some larger transient recorders allow two or three captures (occasionally more) before the data transfer must occur. However, these systems are usually much more complex.

Another need in transient recorders is the ability to see pre-trigger information. This requires circular memory which is not easily implemented by computer memory systems. Sophisticated transient recorders and oscilloscopes utilize hardware memory especially designed for circular operation. However, these memory systems are often power hungry and are of limited storage capacity.

The summary measures related to transients could be automated from wave shape sample data. Such automation has not been attempted for any past magnetic or electric field studies. There has been some work with Wavelet theory to provide a different type of summary measures for transients.

The summary measures which can come from transient waveform capture include:

Peak Rate-of-Change up to a frequency approaching half the sample frequency. Rate-of-Change Intermittency if rapid triggering is allowed. Wave Capture of the $x, y$, and $z$ axis.

\subsection{Electric Field Recorders}

There are several types of electric field survey meters. These meters are usually selfcontained, battery powered systems with analog or digital meters that can be read at a distance. The survey meter is placed on an insulated rod and positioned so that an analog rms reading can be seen. These meters are considered free body meters since no conductive current path between the meter and any other conductive body exists. The presence of the meter reader must be considered and the meter must be kept reasonably distant from the reader and from nearby electric shields. Normally there is no method available to record the readings other than manually.

There are electric field monitors which measure current from an electrode to ground. In very strong fields with well defined geometry where the distance from the electrode to ground is small compared to the distance from the source to ground (i.e. 
transmission line measurements), these electric field monitors will provide accurate electric field information provided the user is not close to the electrode.

A free body electric field monitor with a fiber-optic link will record electric fields without the difficulty of requiring well defined geometries. The same restrictions which apply to the free body survey meter also apply to the fiber-optic monitor. This monitor can be added to any recording system with auxiliary capability.

When used with a wave capture system, various harmonics of the electric field can be identified. If strong harmonics are found in the electric field, they may increase the field coupling by $\mathrm{dE} / \mathrm{dt}$ and, therefore, the induced current densities.

\subsection{Confounds to Field Measurements}

Loss of data is important to understand and document. In the same way, extraneous data should also be understood and documented. The presence of unrealized or undocumented signals can lead to erroneous conclusions. Such "confounds" must be recognized. Normally, the confounds are specified by the equipment manufacturer but the descriptions may be lost in the "fine print". Manufacturers are challenged to discuss confounds openly in their manuals. Some of the major confounds are discussed here.

1. Temperature: Spurious signal changes from temperature can occur in solid-state equipment such as Hall effect transducers. Since temperature changes are common, spurious signals are usually well controlled over reasonable temperature ranges in most equipment. Very hot (over $45^{\circ} \mathrm{C}$ ) or very cold (below $0^{\circ} \mathrm{C}$ ) may require special designs.

2. Peak Field Level: High peak field levels can damage some equipment creating spurious readings. Magneto-resistive transducers may not work after exposure to $1 \mathrm{mT}$. Floppy disks and "hard disks" in computers which have magnetic media. High fields of 3 to $20 \mathrm{mT}$ have been reported to freeze hard or floppy disk operation and/or change the data on the disk. Arcing from intense electric fields may also corrupt data.

3. Minimum Field Level: Spurious signals from ambient or background fields are always a concern. Elimination of background fields is extremely difficult. Included in this category is the natural electrical noise within the measuring circuits which may provide spurious information at low signal levels.

4. Motion of the Transducer: In magnetic measurements, changes in transducer position from walking, using a profile wheel, or simply holding the transducer in the hand will produce spurious signals within the range of 0.1 to $1 \mu \mathrm{T}$. In 
special circumstances where fields are linearly polarized and field levels are of sufficient magnitude, calculations can remove the effects of transducer motion.

5. Frequency Interactions: Although anti-aliasing filters are commonly used to prevent interaction between frequencies which result in spurious signals, there are interactions that are not able to be controlled in this way. The fluxgatestyle transducer uses high frequency switching, commonly about $30 \mathrm{kHz}$, to provide signal. The user should be aware that both 15 and $30 \mathrm{kHz}$, at about $0.1 \mu \mathrm{T}$, is radiated by the fluxgate for a volume with radius of $10 \mathrm{~cm}$. Also any . $30 \mathrm{kHz}$ magnetic field applied to the fluxgate will appear as a low frequency signal in spite of anti-aliasing filters.

6. High Frequency RF Fields: Many monitors can act as radio receivers for strong RF fields. Although most equipment is heavily shielded, the presence of operational amplifiers, diodes, and capacitors form receiving circuits. Care should be taken to limit mobile radio and mobile telephone use while taking measurements.

\subsection{Conclusion of Measurement Equipment}

The equipment as presently designed may provide most of the specific measure information that is applicable to the biological research efforts. Some of the specific measures are not yet fully designed and the equipment to support these specific measures not yet constructed. Table 4.1 shows the equipment that can support the specific measures that have been discussed. 


\begin{tabular}{|c|c|c|c|c|c|c|c|c|c|c|c|c|c|c|}
\hline \multicolumn{15}{|c|}{$\begin{array}{c}\text { TABLE 4.1 ALPHABETICAL LISTING OF } \\
\text { SOURCE SPECIFIC MEASURES } \\
\text { With Reference to Biological Group and Measurement Equipment }\end{array}$} \\
\hline \multirow[t]{2}{*}{$\begin{array}{l}\text { Specific } \\
\text { Measure }\end{array}$} & \multicolumn{4}{|c|}{$\begin{array}{c}\text { Applicable } \\
\text { Research Areas }\end{array}$} & \multicolumn{10}{|c|}{$\begin{array}{c}\text { Equipment } \\
\text { Available }\end{array}$} \\
\hline & 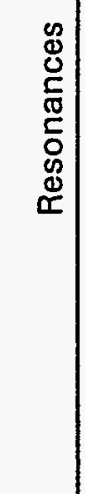 & 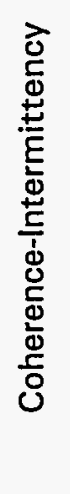 & 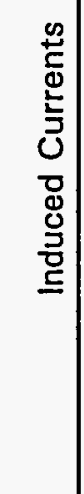 & 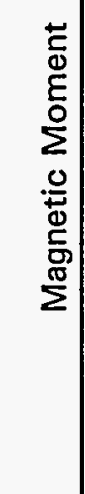 & 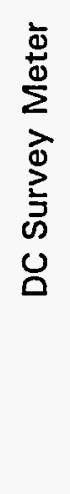 & 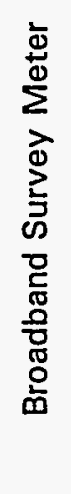 & 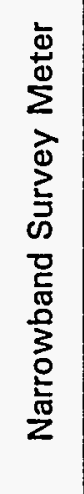 & 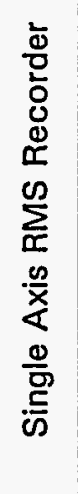 & 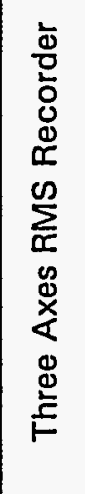 & 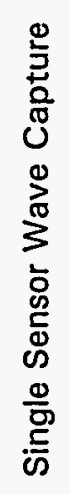 & 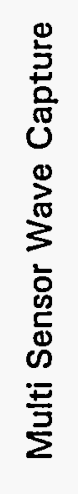 & 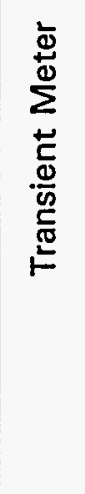 & 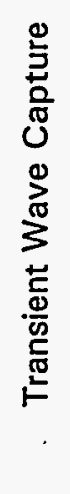 & 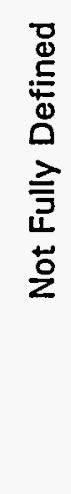 \\
\hline AC-DC Angle & $x$ & & & $x$ & & & & & & $x$ & $x$ & & & \\
\hline $\begin{array}{l}\text { AC-DC Parallel } \\
\text { Magnitude }\end{array}$ & $x$ & & & $x$ & & & & & & $x$ & $x$ & & & \\
\hline $\begin{array}{l}\text { AC-DC Perpendicular } \\
\text { Magnitude }\end{array}$ & $x$ & & & $x$ & & & & & & $x$ & $x$ & & & \\
\hline AC RMS & & $x$ & & & & $x$ & $x$ & $x$ & $x$ & $x$ & $x$ & & & \\
\hline Analog AC RMS & & $x$ & & & & $x$ & $x$ & $x$ & $x$ & & & & & \\
\hline Coherency Index & & $x$ & & & & & & & & & & & & $x$ \\
\hline $\mathrm{DC}$ & $x$ & & & $x$ & $x$ & & & & & $x$ & $x$ & & & \\
\hline Harmonic Magnitude & $x$ & $x$ & $x$ & $x$ & & & $x$ & & & $x$ & $x$ & & & \\
\hline Harmonic Phase & $x$ & $x$ & & $x$ & & & & & & $x$ & $x$ & & & \\
\hline Intermittency Index & & & & & & & & & & & & & & $x$ \\
\hline LF RMS & & & $x$ & & & $x$ & & $x$ & $x$ & $x$ & $x$ & & $x$ & \\
\hline $\begin{array}{l}\text { Maximum Spatial } \\
\text { Component }\end{array}$ & $x$ & $x$ & & $x$ & & & & & & $x$ & $x$ & & & \\
\hline Maximum Spatial Phase & $x$ & $x$ & & $x$ & & & & & & $x$ & $x$ & & & \\
\hline $\begin{array}{l}\text { Minimum Spatial } \\
\text { Component }\end{array}$ & $x$ & $x$ & & $x$ & & & & & & $x$ & $x$ & & & \\
\hline Minimum Spatial Phase & $x$ & $x$ & & $x$ & & & & & & $x$ & $x$ & & & \\
\hline Peak Magnitude & & & $x$ & $x$ & & & & & & $x$ & $x$ & $x$ & $x$ & \\
\hline Peak Rate-of-Change & & & $x$ & & & & & & & $x$ & $x$ & $x$ & $x$ & \\
\hline
\end{tabular}




\begin{tabular}{|c|c|c|c|c|c|c|c|c|c|c|c|c|c|c|}
\hline \multicolumn{15}{|c|}{$\begin{array}{c}\text { TABLE 4.1 ALPHABETICAL LISTING OF } \\
\text { SOURCE SPECIFIC MEASURES } \\
\text { With Reference to Biological Group and Measurement Equipment }\end{array}$} \\
\hline \multirow[t]{2}{*}{$\begin{array}{l}\text { Specific } \\
\text { Measure }\end{array}$} & \multicolumn{4}{|c|}{$\begin{array}{c}\text { Applicable } \\
\text { Research Areas }\end{array}$} & \multicolumn{10}{|c|}{$\begin{array}{c}\text { Equipment } \\
\text { Available }\end{array}$} \\
\hline & 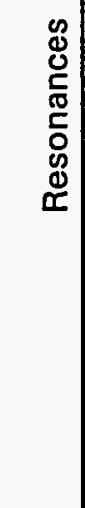 & 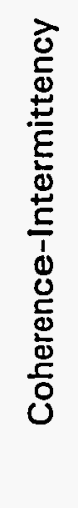 & 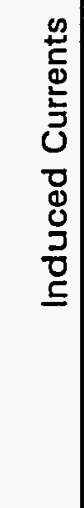 & 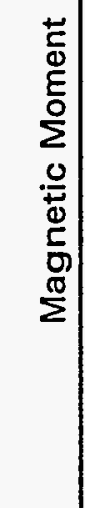 & 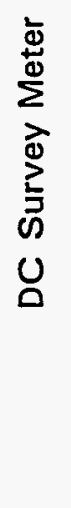 & 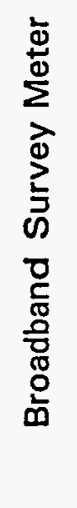 & 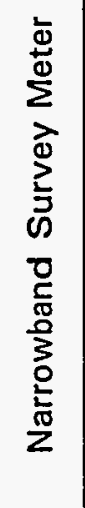 & 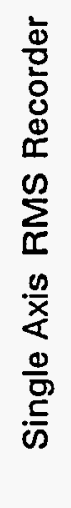 & 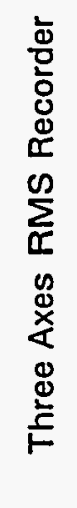 & 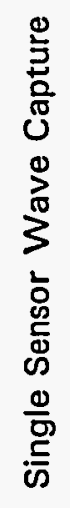 & 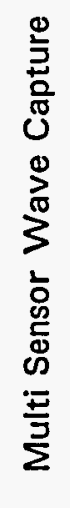 & 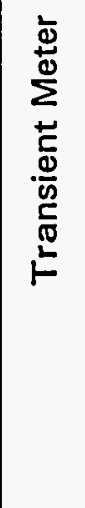 & 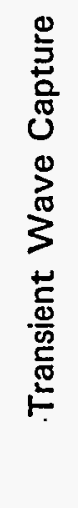 & 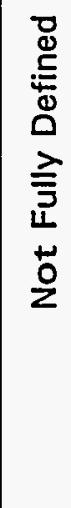 \\
\hline Peak Resultant & & & $x$ & $x$ & & & & & & $x$ & $x$ & & $x$ & \\
\hline Peak-to-Peak Magnitude & & : & $x$ & $x$ & & & & & & $x$ & $x$ & & $x$ & \\
\hline Polarization & $x$ & $x$ & & $x$ & & & & $x$ & & $x$ & $x$ & & & \\
\hline $\begin{array}{l}\text { Rate-of-Change } \\
\text { Intermittency }\end{array}$ & & $x$ & $x$ & & & & & & & & & $x$ & $x$ & \\
\hline Resonance Indices & $x$ & & & $x$ & & & & & & & & & & $x$ \\
\hline Resultant & $x$ & $x$ & & $x$ & & $x$ & $x$ & $x$ & $x$ & $x$ & $x$ & & & \\
\hline $\begin{array}{l}\text { Transient Indices of Rise } \\
\text { Time, Overshoot, } \\
\text { Settling Time, Natural } \\
\text { Frequency. }\end{array}$ & & & $x$ & & & & & & & . & & & $x$ & $x$ \\
\hline $\begin{array}{l}\text { Transient Peak Rate-of- } \\
\text { Change }\end{array}$ & & & $x$ & & & & & & & & & $x$ & $x$ & $x$ \\
\hline VLF RMS & & & $x$ & & & $x$ & & $x$ & $x$ & & & & & \\
\hline
\end{tabular}




\subsection{BIBLIOGRAPHY}

1. IEEE Standard Dictionary of Electrical and Electronic Terms, ANSI/IEEE Std 1001992, Fifth Edition. IEEE, New York, NY. 1992.

2. IEEE Standard for Safety Levels with Respect to Human Exposure to Radio Frequency Electromagnetic Fields, $3 \mathrm{kHz}$ to $300 \mathrm{GHz}$, IEEE C95.1-1991 (Revision of ANSI C95.1-1982). IEEE, New York, NY. 1991.

3. IEEE Guide for the Measurement of DC Electric-Field Strength and lon Related Quantities, IEEE Std 1227-1990. IEEE, New York, NY. 1990.

4. Electromagnetic Fields: Biological Interactions and Mechanisms (Advances in Chemistry Series 250). Edited by Martin Blank. American Chemical Society, Washington, DC. 1995.

5. Biological Effects of Electromagnetic Fields, 2nd Edition. Edited by Charles Polk and Elliot Postow. CRC Press. 1996.

6. Modulation Theory, Chapter 4. H.S. Black. D. Van Nostrand Company, Inc., Princeton, NJ. 1953.

7. IEEE Recommended Practice and Requirements for Harmonic Control in Electric Power Systems, IEEE Recommended Practice 519-1992. IEEE, New York, NY. 1992.

8. Magnetic Field Measurements of Appliances, Draft Report. Submitted to the Electric Power Research Institute. October 1993.

9. Association of Wire Code Configuration with Long-Term Average $60 \mathrm{~Hz}$ Magnetic Fields and Exposures. Electric Power Research Institute Contract. Contractor T. Dan Bracken, Inc. Period of Performance 1992 to 1997.

10. Survey of Residential Magnetic Field Sources (1000 Home Study). EPRI Final Report, TR-102759. Project 3335-02. September 1993.

11. Measurement of Power System Magnetic Fields by Waveform Capture. EPRI Report TR-100061. February 1992.

12. Safety of High Speed Magnetic Levitation Transportation Systems: Magnetic Field Testing of the TRO7 Maglev Vehicle and System, Volume I - Analysis, Volume II - Appendices. Electric Research and Management, Inc. Prepared for U.S. Department of Transportation, Federal Railroad Administration 
(USDOT/FRA). Report No. DOT/FRA/ORD-92/09.I and DOT/FRA/ORD-92/09.II. Available from NTIS, Springfield, VA. April 1993.

13. Safety of High Speed Guided Ground Transportation Systems: Magnetic and Electric Field Testing of the Amtrak Northeast Corridor and New Jersey Transit North Jersey Coast Line Rail Systems, Volume I - Analysis, Volume II Appendices. Electric Research and Management, Inc. Prepared for USDOT/FRA. Report No. DOT/FRA/ORD-93/01.I and DOT/FRA/ORD-93/01.II. Available from NTIS, Springfield, VA. April 1993.

14. Safety of High Speed Guided Ground Transportation Systems: Magnetic and Electric Field Testing of the French Train a Grande Vitesse (TGV) Rail System, Volume 1 - Analysis, Volume II - Appendices. Electric Research and Management, Inc. Prepared for USDOT/FRA. Report No. DOT/FRA/ORD93/03.1 and DOT/FRA/ORD-93/03.II. Available from NTIS, Springfield, VA: May 1993.

15. Safety of High Speed Guided Ground Transportation Systems: Magnetic and Electric Field Testing of the Washington Metropolitan Area Transit Authority (WMATA) Metrorail System, Volume I - Analysis, Volume II - Appendices. Electric Research and Management, Inc. Prepared for USDOT/FRA. Report No. DOT/FRA/ORD-93/04.I and DOT/FRA/ORD-93/04.II. Available from NTIS, Springfield, VA. June 1993.

16. Safety of High Speed Guided Ground Transportation Systems: Magnetic and Electric Field Testing of the Massachusetts Bay Transportation Authority (MBTA) Urban Transit System, Volume I - Analysis, Volume II - Appendices. Electric Research and Management, Inc. Prepared for USDOT/FRA. Report No. DOT/FRA/ORD-93/05.I and DOT/FRA/ORD-93/05.II. Available from NTIS, Springfield, VA. June 1993.

17. An Algorithm for the Machine Calculation of Complex Fourier Series. J.W. Cooley and J.W. Tukey. Mathematics of Computation, Vol. 19, pp. 297-301. April 1965.

18. Hypothesis: The Risk of Childhood Leukemia is Related to Combinations of Power-Frequency and Static Magnetic Fields. J.D. Bowman, D.C. Thomas, S.J. London, and J.M. Peters. Bioelectromagnetics 16:48-59. 1995.

19. Superimposing Spatially Coherent Electromagnetic Noise Inhibits Field-Induced Abnormalities in Developing Chick Embryos. T.A. Litovitz, C.J. Montrose, P. Doinov, K.M. Brown, and M. Barber. Bioelectromagnetics 15:105-113. 1994: 
20. Residential Magnetic Field Transient (RMFT): Induced Signals Versus Thermal Noise. A. Sastre, R. Kavet, J.L. Guttman, and J.C. Weaver. Lecture Presented at the NIOSH/DOE Workshop, Cincinnati, OH. September 26-28, 1994.

\section{OTHER RELEVANT REFERENCES IN ALPHABETICAL ORDER}

ACGIH (1995-96). Threshold limit values (TLVs) for chemical substances and physical agents and biological exposure indices (BELs). ACGIH Worldwide. pp 116-117.

Blackman CF, Blanchard JP, Benane SG, House DE (1994): Empirical test of an ion parametric resonance model for magnetic field interactions with PC-12 cells. Bioelectromagnetics 15:239-260.

Blanchard JP, Blackman CF (1994): Clarification and application of an ion parametric resonance model for magnetic field interactions with biological systems. Bioelectromagnetics 15:217-238.

Bowman JD (Ed) (1992): Manual for Assessing EM Field Exposures. NIOSH CAN 266 Electromanual, September 30, 1992.

EPRI Final Report. Handbook of Shielding Principles for Power System Magnetic Fields. Volume $1:$ Introduction and Application. EPRI TR-103630-VI. Project 3335-02. April 1994.

IEEE Field Effects Design Guide. IEEE PES TF 15.04.06.03 Draft 1.1, Greg Rauch editor, July 1994.

Gold S, Goodman R, Shirley-Henderson A (1994): Exposure of simian virus-40transformed human cells to magnetic fields results in increased levels of tantigen mrna and protein. Bioelectromagnetics 15:329-336.

Grant G, Cadossi R, Steinberg G (1994): Protection Against Focal Cerebral Ischemia Following Exposure to a pulsed electromagnetic field. Bioelectromagnetics 15:205-216.

Grota LJ, Reiter RJ, Keng P, Michaelson S (1994): Electric field exposure alters serum melatonin but not pineal melatonin syntheses in male rats. Bioelectromagnetics 15:427-437. 
Hart FX (1994): Spreadsheet method for calculating the induced currents in bonefracture healing by a low-frequency magnetic field. Bioelectromagnetics 15:465-482.

Kaune WT, Darby SD, Gardner SN, Hrubec A, Iriye RN, Linet MS (1994): Development of a protocol for assessing time-weighted-average exposures of young children to power-frequency magnetic fields. Bioelectromagnetics 15:3351.

Kaune WT, Savitz DA (1994): Simplification of the Wertheimer-Leeper wire code. Bioelectromagnetics 15:275-282.

Lanzerotti LJ, Maclennan CG, Fraser-Smith AC (1988?): Background Magnetic Spectra: $\sim 10^{-5}$ to $\sim 10^{5} \mathrm{~Hz}$.

Maureen P, Hammond SK, Abkollahzadeh S (1994): Power frequency magnetic field exposures among nurses in a neonatal intensive care unit and a normal newborn nursery. Bioelectromagnetics 15:519-530.

Miller, D.A. (Date unknown): Electric and magnetic fields produced by commercial power systems. Low-Frequency Magnetic and Electric Fields, Volume and publisher unknown, pp 62-70.

Moss CE, Booher D (1994): Assessment of Nonionizing Radiation Exposure at a Steel Foundry. Appl Occup Environ Hyg. 9(7), July 1994, pp 465-471.

Nordenson I, Mild KH, Andersson G, Sandstrom M (1994): Chromosomal aberrations in human amniotic cells after intermittent exposure to fifty hertz magnetic fields: Bioelectromagnetics 15:293-301.

Polk C (1994): Effects of extremely-low-frequency magnetic fields on biological magnetite. Bioelectromagnetics 15:261-270.

Robertson DC, Camps OI, Mayer JS, Gish WB (1995). Wavelets and Electromagnetic Power System Transients. Presented at the IEEE PES Summer Power Meeting.

Savitz DA, Ananth CV (1994): Residential magnetic fields, wire codes, and pregnancy outcome. Bioelectromagnetics 15:271-274.

Savitz DA, Ohya T, Loomis DP, Russell S. Sr., Bracken TD, Howard RL (1994): Correlations among indices of electric and magnetic field exposure in electric utility workers. Bioelectromagnetics 15:193-204. 
Valberg PA (1995): Designing EMF experiments: What's required to characterize "exposure"? Bioelectromagnetics (in press).

Valberg PA (1994): Systematic Characterization of Laboratory EMF Exposures. DOE/EPRI Contractors Review Poster P-39, Albuquerque, NM. November 1994.

Wertheimer N, Leeper E (1979): Electrical wiring configurations and childhood cancer. American Journal of Epidemiology, Volume 109, pp. 273-284.

Wilson BW, Hansen NH, Davis KC (1994): Magnetic-field flux density and spectral characteristics of motor-driven personal appliances. Bioelectromagnetics 15:439-446. 


\title{
EMF RAPID PROGRAM ENGINEERING PROJECTS Project 1 DEVELOPMENT OF RECOMMENDATIONS FOR GUIDELINES FOR FIELD SOURCE MEASUREMENT
}

Task B: Identification of Field Sources

\author{
Submitted by: \\ Electric Research and Management, Inc. \\ PO Box 165 \\ State College, PA 16804 \\ Submitted to: \\ Lockheed Martin Energy Research, Inc. \\ PO Box 2008 \\ Bethel Valley Road, \\ Oak Ridge, TN 37831-6070
}

October 2, 1997 


\section{TABLE OF CONTENTS}

\section{PAGE}

1.0 INTRODUCTION ........................... 1

1.1 Arbitrary Assignment of Codes . . . . . . . . . . . 1

1.2 Use of Movable Property System from GSA . . . . . . . . . 2

1.3 Use of Export Control Classification Number (ECCN) . . . . . . . 3

1.4 The Standard Industrial Classification System (SIC) . . . . . . . 3

1.5 Using the Source Name as a Classification System .... . . . 4

2.0 PROPOSED CLASSIFICATION SYSTEM $\ldots \ldots \ldots \ldots \ldots \ldots \ldots \ldots$

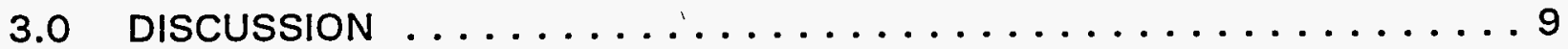

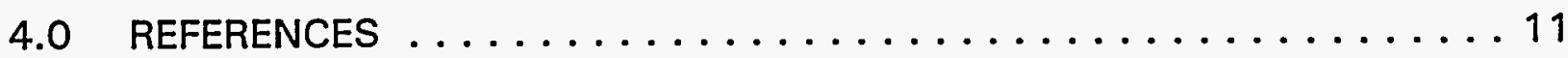

\section{LIST OF TABLES}

Table 2.1 Time Change Codes for Point Sources $\ldots \ldots \ldots \ldots \ldots \ldots$

Table 2.2 Codes that Rate the Attenuation and Polarization to be Expected . . 8

Table 3.1 Appliances and Codes in Alphabetical Order of the

Appliance Name . . . . . . . . . . . . . . . 10 


\title{
EMF RAPID PROGRAM ENGINEERING PROJECTS Project 1 \\ DEVELOPMENT OF RECOMMENDATIONS FOR GUIDELINES FOR FIELD SOURCE MEASUREMENT
}

\author{
Task B: Identification of Field Sources
}

\subsection{INTRODUCTION}

The identification of field sources is a necessary part of survey work in electric and magnetic field measurement. A systematic method of identifying sources during surveys so that they easily could be correlated with measurements made in other surveys or in laboratory tests could simplify survey protocols. Any attempt to codify sources should give some indication of the expected nature of the magnetic field attributes associated with the source. The most often cited source code is the Wertheimer-Leeper Wire Code [Wertheimer and Leeper, 1979, 1982]. However, this code only gives the expected magnitude attribute of a highly specialized subset of source utility power lines. To date, nothing has been reported which attempts to classify apparatus sources.

\subsection{Arbitrary Assignment of Codes}

The concept of identification and classification can take many forms including random or arbitrary assignment of a code, assignment of a code used by others, or assignment of a code that is based on source characteristics. The random or pseudo-random code assignment has been used for electronic components for decades. These numbers are assigned semi-conductors as a way of registering the semi-conductor characteristics. Although there are groupings in the assignment system, generally, the numbers do not directly relate to the characteristic. It is difficult to relate two diodes, a $1 \mathrm{~N} 4001$ and a $1 \mathrm{~N} 4007$, by using the numbers only (they are the same diode except for the maximum peak reverse voltage of 50 and 1000 volts, respectively).

Such a system would require a central clearing house for registry. Each source would be described and characterized. The source would be assigned a number and then a catalog of numbers would be issued. The advantages would include complete documentation of sources as the numbers are assigned. Ultimately, these numbers could be related to other classification systems, such as the bar codes used in point of sale systems. The disadvantage is the immense overhead of documentation and the possibility of multiple assignments. The keeping of the records could become a more complex task than the measurements themselves. 


\subsection{Use of Movable Property System from GSA}

A second method is to piggy-back on the assignment of codes by others. Several codes have been suggested. The Movable Property System that was developed by the General Services Administration (US Government) has been researched for source identification. This system has been in use for decades. The original concept was to number the movable property within the government inventory into classes using a four digit number with possible subclassifications. The system was patterned after the standard Dewey decimal system used in libraries. General equipment classifications were established and specific equipment classified with a specific number. As an example, analyzers were assigned the number 6625 . The subclassifications include:

$\begin{array}{ll}6625 & \text { Voltmeter, pocket } \\ 6625 & \text { Power factor meter } \\ 6625.01 & \text { Ammeter, pocket } \\ 6625.02 & \text { Ammeter, portable } \\ 6625.03 & \text { Analyzer, atomic absorption } \\ 6625.04 & \text { Analyzer electric/electronic (includes tube checker) } \\ 6625.05 & \text { Analyzer, sound and vibration } \\ 6625.06 & \text { Battery Testing Outfit } \\ 6.5 & \\ 6625.35 & \text { Voltmeter, other }\end{array}$

The list continues but the important characteristics are shown in this short example. Characterization is approximate. The above meters may not be substantially alike. For example, an atomic absorption analyzer has very little in common with a power factor meter. Further, many dissimilar meters, such as a pocket voltmeter and a power factor meter, are given identical numbers. It would be possible to find field sources within this list and utilize these numbers. Advantages include the fact that the list is compiled and maintained by the US Government. The list would be available to every interested person. The list would always be retroactive and cover a multitude of sources. The disadvantages include the fact that some modifications would be required to care for obviously different field sources, such as Fixture, Light Portable (florescent or incandescent) 6210.01. There is no classification for permanent light fixtures. Changes in the list would be difficult to track since the US Government would not include these changes in their main lists. Finally, the lists tend to group items together but subgroups are arbitrarily assigned. Such assignments would require the list be updated periodically. 


\subsection{Use of Export Control Classification Number (ECCN)}

The Export Control Classification Number used in the Commerce Control List is provided by the U.S. Bureau of Export Administration. This list has, as a subdivision, Equipment, Assembles and Components. The list, taken from the October 1994 printing, has both materials and equipment intermixed. A search for simple appliances might include a vacuum sweeper. The list provides

$\begin{array}{lr}\text { Substrates } & \text { 3B01A } \\ \text { Supercomputers } & \text { Cat. 4 } \\ \text { Superconductive composite conductors } & \text { 1CO5A } \\ \text { Superconductive solenoids or electromagnets } & \text { 3A01A } \\ \text { Switching devices } & \text { 3A43B } \\ \text { Switching equipment, tèlecommunications } & 5 \mathrm{A03A} \\ \text { Syntactic foam for underwater use } & 8 \mathrm{CO} 1 \mathrm{~A} \\ & 1 \mathrm{CO} 4 \mathrm{~A} \\ \text { Uranium titanium alloys or tungsten alloys } & \text { 3E02A } \\ \text { Vacuum microelectronics } & 1 \mathrm{~B} 50 \mathrm{~A} \\ \text { Vacuum or controlled environment furnaces } & 1 \mathrm{~B} 16 \mathrm{~A} \\ \text { Valves } & \end{array}$

The vacuum sweeper is not found in the list under $s$ or $v$. The list also uses duplicate numbers repeatedly such as $5 \mathrm{AO} 2 \mathrm{~A}$ for underwater communications systems, transportation equipment, transmitter - amplifiers, and transducers as just a few examples. This code is obviously designed to single out equipment and technology which is important to our country's defense system and should be exported only with appropriate permissions and taxes. The code does not fulfill the need for an equipment code for field sources.

\subsection{The Standard Industrial Classification System (SIC)}

During the early investigations of this project, the Standard Industrial Classification system (SIC) was considered as a starting point for field source classifications. The various industries which used field sources were identified in this code using four numbers. The code is excellent for describing the environment in which sources exist. The probability of a source that would be a part of a given industry was discussed as a basis for categorizing sources. The SIC was used to try to determine the most probable industrial classification for a given source. There were obvious matches in this code. Large power generators were associated with a Generation Station 4912 with high probability although they are also associated with industrial sites as well for backup generation and for co-generation. A traction motor could be associated with high probability under the rail classifications of 4010,4020 , and 4030 but also could 
be included under Automobile 4500 in electric-powered cars. The use of traction motors could be subcategorized under Light Rail 4030 , Railroad Train 4010 or Subway system 4020 as essentially all locomotive power is related to the traction motor. There are special sources which are found only in one industry to the exclusion of all others. An MRI is obviously only in Hospitals 8060 or Doctor's Offices 8010. However the list of the specialized sources is short.

Approaching the concept from the possibility of finding a Fluorescent Light fixture in a specific industry or even to describe the industry having the greatest possibility of having a Fluorescent Light is impossible. The Fluorescent Light is essentially in every home, office, shop, or industrial complex. There are few industrial classifications which do not involve Fluorescent Lights. The list might include the Automobile 4119, the Underground Distribution line 4918, the Underground Transmission Line 4916, and Highway \& Street Construction Sites 1610. Even the automobile can have a battery powered Fluorescent Light. The Microwave Oven is another pervasive source. They are principally found in the Home Group 8810, 8811, 8812, 8813, 8814, and 8360. However, they are also found in most break or lunch rooms of any office, store, factory, or school to name only a few. The oven is found in many motel rooms, hotel hallways, and many restaurant facilities. To assign the Microwave Oven to a specific SIC with a probability that it would mostly be found at that SIC has no common sense reasoning. The assignment would be arbitrary. As a last example of this process, the refrigerator, and its immediate sister, the carbonated-beverage vending machine (essentially identical design and construction in relation to magnetic fields), can be found virtually in every part of the environment. They are found in the marble halls of court houses to the restrooms at a highway rest stop, at every hotel and motel, in every home, in every break or lunch room, and even in automobiles and airplanes. Again, assigining a SIC to the refrigerator would be extremely arbitrary.

Most field sources provide assistance to daily living. These assistances can be roughly grouped in categories of environmental control, food preparation, paper-work assistance, transportation, body hygiene, and entertainment. They are all centered around supporting people and not necessarily supporting an industry. The special machinery found in industry that can be typically found only in one class of industry is rare. Categorization according to industry does not fulfill any of the goals of intuitive development of classification.

\subsection{Using the Source Name as a Classification System}

The most obvious method of classification is to simply name the field source. A name with 50 alphanumeric characters would essentially classify all of the sources. The advantage is that the name would facilitate simple key word searches in any data bases of source characteristics. The list would be very easy to use and generate new 
source classifications as they were discovered in survey work. There is the disadvantage that many names may represent the same source. For example, the vacuum sweeper is also referred to as an electric broom, carpet vacuum, vacuum cleaner, etc. Ideally, the list would then require cross referencing by a central classification administrator to ensure that all appliances are listed in the correct order. Practically, the user would assume the responsibility of cross referencing just as we do in a department store when a young sales clerk looks at you blankly when you ask for an electric broom.

It is then only necessary to generate a classification code to append to the name that represents the field source as a classification of field characteristics that might be expected. Sufficient gradations of the characteristics should allow most common sources to be generally quantified and would allow similar equipment to have similar numbers. The advantages include the fact that the list would require little changing once it was started. The source could be classified in the field as the sources are encountered or a query could be placed with the data base to see if the source has been previously quantified. Although it is possible that the same source may be given different numbers, the numbers should be similar in most cases. This type of system is offered as a classification system for field sources for the EMF RAPID Program Engineering Projects. 


\subsection{PROPOSED CLASSIFICATION SYSTEM}

The proposed classification system is restricted to a general quantification of the magnetic field attributes of a source. The electric field is usually so small and almost always dependent on the environment of a source that trying to code the electric field attributes would have little or no usefulness.

Since, in general, the magnetic fields around sources are highly complex, any coding system can only hope to identify the salient attributes of the magnetic field created by the source. Also, to have any chance of being accepted, the code must be intuitive so that only very simple rules will make it possible to uniformly apply or interpret the code. Technicians that are in the process of quantifying the magnetic fields around an appliance or piece of electrical apparatus will want to quickly and correctly code the device. Later, researchers encountering the device code will want to easily apply the code to their specific needs.

The proposed classification system is based on identifying the source by its common name followed by alphanumeric descriptors for a total of 50 characters followed by a four digit code box, for example: UPS-brandxx50kVA208V [4.3] [8].

The first digit of the code box grades the range of magnetic field magnitudes that may be expected at a point $30 \mathrm{~cm}(1 \mathrm{ft})$ in front of the surface of the device. Deciding on how to determine the center front of any appliance is described in Task $C$. The value of the digit is the power of 10 which, when used in the formula $\quad 1 \leq .1 \mathrm{mG} \times 10^{\prime}$ would produce the nearest measure of the magnetic field's resultant magnitude. In the above example, the 4 indicates a magnitude greater than $100 \mathrm{mG}$ and less than $1000 \mathrm{mG}$.

The second digit in the box is a measure of the frequency of the most dominant magnitude. Again, the digits are powers of 10. This time the formula is frequency in

$H z \leq 10^{k_{2}}$, where $k_{2}$ 's value produces the nearest measure of the frequency of the most dominant source. In the above example, the 2 indicates the frequency of the dominant frequency is greater than $10 \mathrm{~Hz}$ and less than $100 \mathrm{~Hz}$.

The third digit in the box is a measure of how intermittent the point source may be. A power of 10 is employed based on an annual cycle being represented by $10^{\circ}$. Since we keep time in years, months, weeks, days, hours, minutes, seconds, and the inverse of cycles per second, a simple time formula is less useful than a tabulation such as given in Table 2.1. In the above example, the third digit in the box, 3 , indicates the point source changes steady state slightly less frequently than every eight hours. 
The fourth digit in the box is a measure of two different attributes, spatial attenuation and polarization. Table 2.2 gives the codes for these attributes. Thus, the final digit of 8 in the example indicates a source that has a dipole characteristic and has a field that is elliptically polarized. For this work, an axial ratio (minor axis over the major axis) greater than .2 is classified as elliptically polarized.

There are many ways this code system can be utilized by the research community. For example, during site surveys, if the appliance or electrical apparatus has been previously classified and logged in the data base, then the survey protocol can make use of the classification. Before measurements are attempted, the maximum magnitude, principal frequency, number of samples over time, expected attenuation away from the appliance, and polarization are all known from the code. Simple measurements can be taken to verify the classification is correct. If the classification is confirmed and the data base is completed, then the source location can be noted and the area measurements continued without further specific attention to the source's existence.

In epidemiological studies, this code could significantly increase the power of the study to quantify exposure levels. Knowing the order of magnitude of the fields near the appliance and its principal frequency will greatly improve the quantification of dose. Should intermittency be part of the study protocol, estimates can be drawn from a knowledge of the codes and work patterns. 
Table 2.1

Time Change Codes for Point Sources

\begin{tabular}{||c|l|l||}
\hline $\begin{array}{c}\text { Power of } \\
10\end{array}$ & $\begin{array}{c}\text { A Change in Steady- } \\
\text { State Occurs Less } \\
\text { Frequently Than Once } \\
\text { Every ... }\end{array}$ & \multicolumn{1}{||}{ Simplifying Interpretation of Time Spans } \\
\hline \hline 0 & Year & Sample at Least Yearly \\
\hline 1 & 1.2 Months & Sample at Least Monthly \\
\hline 2 & .52 Weeks & Sample at Twice a Week \\
\hline 3 & 8.76 Hours & Sample at Least Every 8 Hours \\
\hline 4 & .876 Hours & Sample at Least Every 50 Minutes \\
\hline 5 & 5.256 Minutes & Sample at Least Every 5 Minutes \\
\hline 6 & .5256 Minutes & Sample at Least Every 30 Seconds \\
\hline 7 & 3.1536 Seconds & Sample at Least Every 3 Seconds \\
\hline 8 & .31536 Seconds & Sample at Least Every .3 Seconds \\
\hline 9 & 31.536 Milliseconds & On 60 Hz Power System, Sample Every Cycle \\
\hline
\end{tabular}

Table 2.2

Codes that Rate the Attenuation and Polarization to be Expected

\begin{tabular}{|c|c|c|c|c|c|c|c|c|c|c|}
\hline & \multicolumn{10}{|c|}{ CODE \# } \\
\hline & 0 & 1 & 2 & 3 & 4 & 5 & 6 & 7 & 8 & 9 \\
\hline Unknown & $x$ & & & & & $x$ & & & & \\
\hline $1 / r$ & & $x$ & & & & & $\mathrm{x}$ & & & \\
\hline $1 / r^{2}$ & & & $x$ & & & & & $x$ & & \\
\hline $1 / r^{3}$ & & & & $x$ & & & & & $x$ & \\
\hline Greater Than $1 / r^{3}$ & & & & & $x$ & & & & & $x$ \\
\hline Linear & $x$ & $x$ & $x$ & $x$ & $x$ & & & & & \\
\hline Elliptical & & & & & & $x$ & $\mathrm{x}$ & $x$ & $\mathrm{x}$ & $\mathrm{x}$ \\
\hline
\end{tabular}




\subsection{DISCUSSION}

As discussed in the introduction, a fixed code system where coding is selected and assigned specifically to a device is not advantageous since the paperwork and coordination would require more effort than the process would be worth. Classification of sources is primarily to aid in cross referencing and correlation of data. The need for assigning a specific code to each source is not a primary objective.

Table 3.1 lists some of the appliances that were tested while developing the protocols of Task C. The proposed four digit code was readily determined from the test data. The alphanumeric name is most of the identifying data that would be routinely logged for any device under test. As can be seen from a review of Task $C$ data, most of the salient attributes are indicated by this simple code.

These codes may be assigned at the time of measurements without reference to lengthy lists. There is a possibility that the same device will be assigned two different numbers by different researchers or that two different devices will have the same number. However, these duplications will indicate that approximately the same field characteristics are present in both devices. 


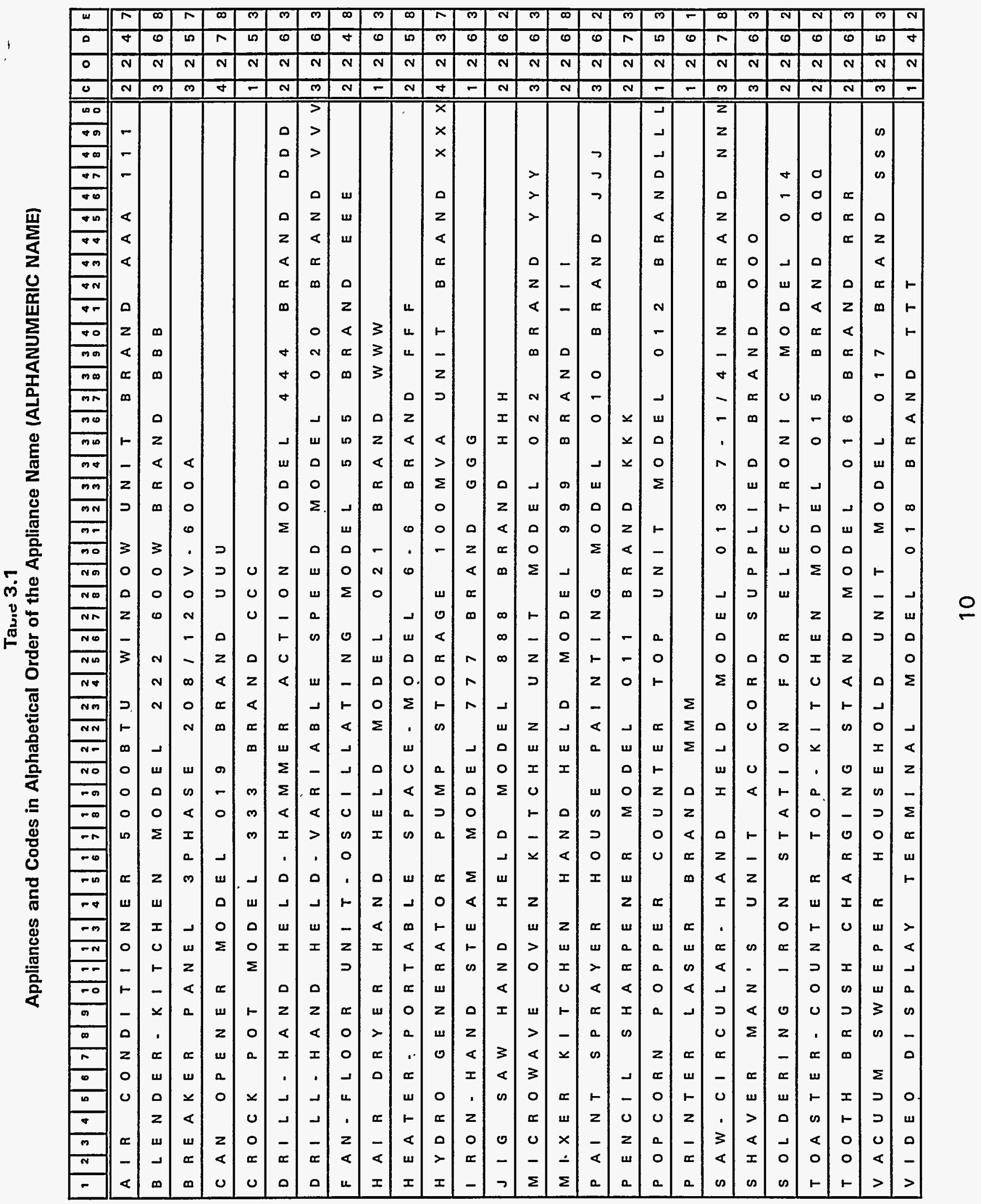




\subsection{REFERENCES}

Wertheimer N, Leeper E (1979): Electrical wiring configurations and childhood cancer. Am J Epidemiol 109:273-284.

Wertheimer N, Leeper E (1982): Adult cancer related to electrical wires near the home. Int J Epidemiol 11:345-355. 


\title{
EMF RAPID PROGRAM ENGINEERING PROJECTS \\ Project 1 \\ DEVELOPMENT OF RECOMMENDATIONS FOR GUIDELINES FOR FIELD SOURCE MEASUREMENT
}

\author{
Task C: Development of Protocol
}

\author{
Submitted by: \\ Electric Research and Management, Inc. \\ PO Box 165 \\ State College, PA 16804 \\ Submitted to: \\ Lockheed Martin Energy Research, Inc. \\ PO Box 2008 \\ Bethel Valley Road, \\ Oak Ridge, TN 37831-6070
}

October 2, 1997 


\section{TABLE OF CONTENTS}

PAGE

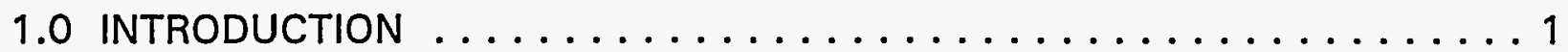

1.1 Review of Guideline Objectives $\ldots \ldots \ldots \ldots \ldots \ldots \ldots \ldots$

2.0 IN-SITU PROTOCOL . ....................... 4

2.1 A Recommended Protocol Guideline for In-Situ Source

Characterization .................... 7

3.0 APPLIANCE TESTING PROTOCOL FOR MEASUREMENTS AT A

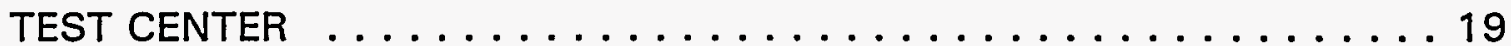

3.1 The Pilot Study . . . . . . . . . . . . . . . 19

3.2 Test Center General Protocol . . . . . . . . . . . . . . 21

3.2.1 Location of the Center of the Appliance ........... 23

3.2.2 Coordinate Systems and Sensor Positioning . . . . . . 24

3.2.3 Sensor Position at the Appliance Surface . . . . . . . 25

3.2.4 The Location of the Distant Sensors . . . . . . . . . 26

3.3 Test Center Production Test Protocol . . . . . . . . . . . 27

3.4 Test Experience . . . . . . . . . . . . . . . . 27

3.5 Prototype Testing Protocol $\ldots \ldots \ldots \ldots \ldots \ldots \ldots \ldots \ldots$

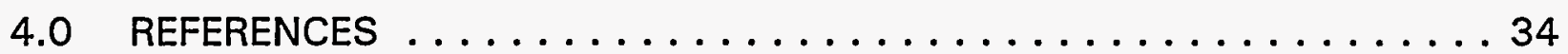




\title{
EMF RAPID PROGRAM ENGINEERING PROJECTS \\ Project 1 \\ DEVELOPMENT OF RECOMMENDATIONS FOR GUIDELINES FOR \\ FIELD SOURCE MEASUREMENT
}

\author{
Task C \\ Development of Measurement Guidelines
}

\subsection{INTRODUCTION}

Before proceeding to discuss guidelines, the definition of protocol as used in this report must be given to allow the reader to understand the nature of the guidelines. Formerly, a protocol is a step by step procedure prepared to lead a person through a measurement sequence. Ideally, the protocol should cover every detail of the sequence and every adjustment of the measurement system. However, this dictionary definition of protocol, "a statement of an experiment in the most fundamental terms without interpretation", can only be practically applied for a narrowly defined range of appliances in a controlled test environment.

Practically, the person, or persons, implementing the protocol must understand the basis of the measurement process. Therefore, the detailed step-by-step procedure must be augmented by comments on the necessity of the measurement step or the rational for the choice of a specific parameter. This augmented step-by-step procedure is used in the appendices of this guideline discussion. The augmented step-by-step procedures allow the reader to be reminded of all the details as they are encountered. Therefore, augmenting comments or "interpretations" allow the user to develop a protocol for their own situation which addresses the measurement concerns presented by the appliance under test and its environment.

The following discussion presents "protocol" concepts but only develops augmented step-by-step procedures for example situations. Through this mechanism, the reader unfamiliar with measurement processes can observe the tedious, but often extremely important, details of a specific protocol so that the general procedures, lists of concerns and selection of parameters can be demonstrated in a specific manner.

Task $A$ of this project demonstrated the necessity for measuring both the temporal and spatial characteristics for an electric or magnetic field. The review of measurement theory and specific measures suggested that waveform capture is the most efficient means to capture all electric and magnetic field attributes. Therefore, the waveform capture approach will be the basis for the following discussion of protocol and the development of guidelines. The waveform capture electric and magnetic field 
monitoring system was chosen to make the guidelines as general as possible. The use of equipment with reduced sophistication may be utilized if the user is mindful of the possible reduction in parameters and attributes that can be quantified. The discussions in Task A provide the basis for determining when the selected measurement systems, which give specific measures that are a subset of waveform capture, might still apply to that specific protocol. Generating augmented step-by-step procedures for every type of measurement system would increase the volume of protocol material without significantly increasing the value of the material. Therefore, the more sophisticated waveform capture equipment is discussed with the expectation that the user choosing to make less sophisticated measurements will eliminate the specific steps in the protocol that are not possible as shown in Task $A$.

\subsection{Review of Guideline Objectives}

Measurement protocol provides a focus on several areas of the measurement process which is required to:

1. Standardize the measurement so that repeatable and comparable results are obtained. from the same or different research teams.

2. Document the procedure to provide permanent records of what was measured.

3. Provide a reasoning process for each step so that the meaning of the step is not lost in future research efforts.

The areas covered by the guidelines include:

1. Preparation: Documentation of the measurement team members, the measurement equipment to be used, the calibrations that apply, the setup details, and the times and dates of the measurement.

2. Environment: Documentation of the environment and/or facilities detail.

3. Location: Documentation of the measurement locations, usually in the form of measurements from a reference or coordinate location.

4. Activity: Documentation of the human activity within the measurement area or over the measurement time.

5. Measurement: Method of taking the measurements, including the order of measurements, the type of calculations required, and the instruments used for each measurement set. 
6. Checking: Check points to ensure that the data collection is making sense.

7. Analysis: Method of analysis and storage of data, including processing into a standard data base. 


\subsection{IN-SITU PROTOCOL}

In-Situ testing is the most frequent measurement situation. Some of the special problems include:

1. The inability to readily repeat measurements. Often, there is only one opportunity to obtain data. Repeated entry into industrial complexes is usually difficult to justify. Special facilities such as nuclear plants or refineries may require special training before measurements may be made. Special dress, i.e. fire retardant safety clothing, hard hats, hearing protection or eye protection, may be needed. Repeated entry into residential or commercial locations where there is some discomfort because the presence of the measurement team is not desirable.

2. The inability to access areas of interest. Industrial complexes are designed to produce a product. Access to the back of machinery, storage areas, electrical vaults, dense piping areas and around control racks may be unavailable.

3. The inability to measure all phases of machine operation. Operation of a specific machine may require the operator to have unencumbered space. Placing cables on the floor or staffs around the operator may unacceptably impede the operator. Also, many machines within a plant do not operate all the time. Setting up for measurements and then waiting for the machine to operate may not be practical.

4. The inability to access operating information such as currents or voltages. Since industrial equipment is installed with more strict electrical codes, access to wiring is often very limited. Simply unplugging an appliance and installing a current measuring loop may not be possible. Installing any measuring sensors in the wiring or control circuits may require an electrician.

5. The inability to locate power outlets for battery chargers for long term monitoring. Industrial complexes seldom provide frequent $A C$ outlets. Outlets that exist may be a significant distance from the monitoring site, often requiring the use of extension cords across walkways and aisles. Safety should always be of first importance and extra time to tape cables and provide signs indicating the presence of extension cords, monitoring sensors and data loggers must be a part of the protocol.

6. The inability to read meters on the monitoring equipment. Often batterypowered equipment uses $L C D$ displays. The poor lighting in industrial locations makes such displays almost impossible to read. Using a flashlight to see the displays often becomes cumbersome. 
Most of these difficulties can be reduced or eliminated by developing a protocol for a specific in-situ situation. This planning should include:

1. Coordinate the timing of the measurement with the local facilities manager. Obtain an agreement on what apparatus will be operational or made available for controlled operation. Give the facilities manager a preliminary test protocol to review. Request a statement on safety rules and any mandatory safety training. Revise protocol to tailor to site specific requirements.

2. Request drawing or sketches of apparatus to be tested and the location in the building. Request floor plans for the apparatus area, including the floor above and the floor below. Look for potentially interfering apparatus fields and/or large building steel structures. From this data, devise maximum reasonable profile paths from the apparatus out to a distance of at least 3 meters.

3. If practical, visit the site to observe operation of the apparatus to be tested. Where permissible, interview operation personnel to determine set-up space, safe access space during operation.

4. During visit, or through requested apparatus data, determine equipment ratings, wiring layouts, and any possible points for attaching clamp-on amp meters or clip-on voltmeters.

5. Make-up any extension cords required or have backup batteries pre-configured to accommodate any required long term measurements.

6. If lighting may be a problem, configure field recorders so that the data can be captured without viewing at all locations.

Although many in-situ tests may be performed in a general office environment, some source characterizations require measurements to be made in extreme environments. These unfavorable environments may include temperature, noise, illumination, other sources of electric and magnetic fields, voltage potentials, and moisture such as fog,. Each of these environments must be considered in the development of a protocol.

Most commercial monitors will function between 0 and 50 degrees Celsius ( 32 and 122 degrees Fahrenheit). Many measurement systems will operate between -5 and 60 degrees Celsius (23 and 140 degrees Fahrenheit). Beyond these temperature extremes, the monitoring equipment should be protected by heating or cooling systems as needed. Displays are often affected by temperature and may be unreadable at the extreme temperatures. Data storage on floppy disks may also be jeopardized. Discussions with the monitoring equipment manufacturer before exposure to extreme temperatures is advised. 
Although audio noise and illumination levels are not normally associated with measurements of fields, they play an important role in the measurement protocol in insitu situations. Audio disturbances such as children playing, presses operating, compressors cycling or any other noise that is not steady in nature will distract the measurement-taking process. The notes tend to be more cryptic from the measurer since the noise must be shut out of the thinking process and the urgency of finishing becomes paramount. Further, lapses in protocol where coordinates are missed enter into the data taking process. It is useful to have ear plugs available when there is expected to be random background noise. In like manner, illumination is equally distracting to the data gathering process. If the light is too low to see displays, the need for a flashlight may also require an additional person to hold the light. Although there is seldom too bright of light, the flashing of light from signals, welding, or strobe lights is extremely distracting. There is little that can be done to hide the measurer from such flashing light and its presence may be corrected by a protocol that takes data at a slower rate.

Although the process of measuring electric and magnetic fields is not usually considered to be affected by extreme fields, the presence of extreme fields may be important. If measurements of magnetic field are taken in a place where very high electric fields exist, then the protocol should call for shielding of the monitors and sensors. Note that if high electric fields exist in the vicinity of a source but are not created by the source, unless you are expert in electric field analysis, do not attempt electric field measurements. High electric fields may cause corona on sharp corners of the sensor electronics and erroneous readings may develop. The protocol must include shielding considerations in any fields above 25 kilovolts per meter. In a similar manner, measurements taken in high magnetic fields requires special considerations. Some types of magnetic sensors and some electronic components will malfunction in high fields. As an example, magneto-resistive sensors will often be damaged in fields above 0.01 Tesla. The manufacturers specifications and warnings must be considered when measurements are taken near large magnetic structures such as hospital MRI units. Sensors that measure only AC magnetic fields, i.e. coil-type sensors, may be used in the presence of high static fields but the effects of sensor motion such as hand shaking will be greatly magnified. The protocol should require calculations to remove motion effects in such circumstances. Finally, measurement equipment may be adversely affected by radio-frequency interference. This interference can come from simple sources such as a local two-way radio that may be used by equipment operators. The protocol should address the need for shielding if the measurement is not certified as insensitive to radio-frequency interference.

Finally, the problem of moisture should be considered. Most commercial monitoring equipment does not work well when moisture condenses inside the equipment. When the equipment is cold and moved into a warm room, condensation takes place. Measurements can be in error from the changes in impedances around operational 
amplifiers and some logic systems will simply stop working. Floppy disks may also be damaged from surface water. The protocol should warn about using equipment when cold environments are expected.

\subsection{A Recommended Protocol Guideline for In-Situ Source Characterization}

This recommended guideline describes the measurement of a source in an office environment although it is applicable to any source characterization. There are two basic conditions that dictate the protocol. If the source has already been coded, then a significantly reduced protocol may be used with only a few measurements to adjust the already available source data. The data from the previous characterization should be studied ahead of measurement time. Four selected measurements are required to determine if the existing database is adequate. If these measurements show that the source is not similar to the previous data, then a full characterization should be made.

Since it cannot be known whether the existing data are adequate until the measurement team is on site, the full measurement protocol must be prepared. Ideally, three people are present for this protocol. The protocol can be executed by two people but the process will be slower.

\section{A. Equipment required for the protocol:}

1. Magnetic wave capture system with a staff to hold four sensors. The data recording system should include provisions for coordinate entry. The magnetic sensors should include DC, ELF wave capture $13 \mathrm{~Hz}$ to 3 $\mathrm{kHz})$, and RMS data for the VLF and LF bands $(3-30 \mathrm{kHz}$ and $30-300$ $\mathrm{kHz})$. A second two sensor system should be available to be used as a magnitude change detector, transient recorder and/or coherency monitor.

2. Electric field sensor with an ELF bandwidth, if electric fields are to be characterized. A monitor which simultaneously records both electric and magnetic fields should be used. A free-body sensor should be used with fiber-optic connections.

3. A video camera and several cassettes of video tape to record the monitoring process. A photographic camera may be used if desired but the frame numbers must carefully be recorded in the log.

4. A staff for the magnetic sensor. Suggested sensor position heights in the staff are $10 \mathrm{~cm}$ for near-floor measurements, $60 \mathrm{~cm}, 110 \mathrm{~cm}$ and $160 \mathrm{~cm}$. If the physical sphere of the source is larger than 6 meters in diameter, a profile wheel is recommended to speed the measurements. The profile wheel should have assemblies to hold the sensors at the same 
levels as the staff. A non-conducting staff for the electric field sensor should be available if profiles of the electric field are to be part of the data set.

5. Measurement equipment including a non-metallic measuring tape long enough to measure four times the physical sphere diameter. Usually a 30 meter or 100 foot tape is used. A small, retractable tape measure (may be metallic since it is not normally used during the actual measurements) should be available to make spot measurements and height measurements as needed. A second non-metallic tape is useful for allowing a grid to be easily approximated.

6. A data log with a supply of pens or pencils. This log should be on a convenient writing surface such as a clipboard. The person recording into the log should also wear a wrist watch that is synchronized to the recorder. Entries should frequently contain the time.

7. A clamp-on current meter or monitor that may be used to measure source current. A meter that can be recorded along with the field data is advantageous. A loop that can be placed in series with the power cord is very useful.

8. A small tool box containing emergency repair tools, duct tape for fastening measuring tapes to the floor, electrician tape to hold cables to staff or hand as needed, and a good multimeter always lends to professional performance.

9. A portable computer if the magnetic field recorder requires configuration or down-loading during or after the measurement process. A printer is usually not necessary.

B. Inspection of the site.

The site should be inspected upon arrival.

1. Meet with the facilities manager and discuss the measurement procedure. Spend a few minutes discussing the process and show some of the measurement equipment. Ask where unneeded equipment may be stashed during measurement process. Answer questions as they are asked. Offer a copy of the written protocol so that the procedure may be more easily observed. Ask the obvious things about needed safety equipment such as hard hats, ear plugs, and safety glasses. Also ask if raised voice levels required to overcome background noise while reading 
coordinates in to the camera microphone will be disturbing. If it is, be prepared to take frequent shots of the log showing the present sample number or coordinate.

2. Look at the sources to be measured. Make a quick sketch of the source. Observe and log specific metallic materials that may be near or around the source. Place major dimensions on the sketch using the retractable tape measure. Sketch the location of walls, desks, and doors. Locate appliances that may be also producing magnetic fields and move the offending appliances if possible. If not possible, then sketch the location of these extra appliances. Look for the little things. A floor heater, a floor fan, a nearby florescent light, a nearby computer, or a telephone can add confusion to the data if the presence and location are not noted in the log. Also look at areas that are on the other side of the wall from the source in adjoining areas such as hallways, offices, or closets. The measurements may be enhanced by using these locations exterior to the immediate office. Do not forget the floor below and the floor above when the source has a physical sphere diameter of more than 1.5 meters. If sources with the same magnetic field level within $30 \mathrm{~cm}$ of their surface are located within 3 to 6 meters $^{1}$ of the apparatus to be measured, the protocol should be expanded at this stage to include these exterior locations. This also may be a time to reduce the protocol. Desks and tables may impede the taking of data on the expected coordinates. Careful elimination of coordinates and modifying profile paths may allow sufficient data to be taken without requiring the movement of tables.

3. Examine the method of powering the source in question. In most of the situations, the power supply will be obvious. However, there are occasions where cords or outlet boxes may be hidden. There are also cases where the wiring to the outlets passes through the floor just below the location of the measurements. The current in this wiring may affect the measurements. Note these details on the log. If it is possible to obtain current to the source, use the clamp-on current meter. Ask before the source is unplugged and replugged into a measurement current loop. If the source utilizes a circuit that is higher than 115 volts or greater than 15 amperes, the power may not be supplied through a plug that is compatible with the current loop. Ask if an electrician is available to take off the cover of the supply panel. Then currents to the source can be

The 3 to 6 meter range is dependent on both the physical size of the source of interest and the interfering source. A rule of thumb is that the measurement radii (from the surface) should be 3 times the diameter of the source. 
measured by clamping around individual wires. Let the electrician do the clamping unless the measurement team is intimately familiar with 240 and 480 volt service panels. Accidental contact with voltage in these panels can be lethal.

4. Draw on the sketches the proposed coordinate grid.

(NOTE: Selection of Cartesian coordinates, cylindrical coordinates, or spherical coordinates is determined by the location of the appliance. For most locations, there may not be access to the top, back, or bottom of an appliance. This is especially true if the appliance is permanently installed. Cartesian coordinates will be more easily used in the measurement process in most industrial sites. Preparation will include a staff with four sensors at fixed separation distances. Dietrich, et al (1993) found that a staff with sensors positioned at $10,60,110$, and $160 \mathrm{~cm}$ was convenient to use. One end of such a staff can be positioned in the horizontal plane with the aid of non-metallic measuring tape to reasonably precise coordinates. The separation distances in the staff, which give the vertical coordinates, and the distances on the measuring tape should be in the same units.

If electric field quantification is to be part of the protocol, then a single sensor staff which can fix the sensor's position at a height from 1 to 2 meters should be available. Remember that electric fields are likely to be effected by most objects or structures. Therefore, selecting more than a few grid points that are in open approach positions in the measurement grid may be counterproductive.

Selection of the grid size for data taking. If possible take data at regular grid intervals. This aids in most calculation and plotting packages. The grid size will directly depend on the size of the area around the appliance and the appliance itself. Around office appliances, a grid size of $30 \mathrm{~cm}$ is suggested. In a large manufacturing facility or a power plant a grid of 1 meter would be suggested. When making measurements of distributed sources such as power cables or distribution lines, 3 meters should be used. Some circumstances may require the measurements be made at irregular intervals to avoid obstacles. 
The use of the staff held in a horizontal position allows data to be obtained in relation to the vertical location of the appliance at fixed distances from the appliance surface. The vertical distance should be recorded.

The intersecting points should be decided and the elimination of hidden or inaccessible points should be discussed. A final grid should be agreed upon. Do not forget to select an "ambient" coordinate to be taken away from the source using a single monitor that will use a sensor placed at 1 $\mathrm{m}$ height $(110 \mathrm{~cm}$ if the Dietrich heights are used in the protocol). Locate a place for the second single monitor about $30 \mathrm{~cm}$ from the source and at a height consistent with one of the mid-heights to be used in the protocol.

C. Assemble the measurement equipment and make measurements.

1. Select file names that can be interpreted later. Simply using File01 is easy but conveys little after a day or two passes. Even if the recording is done manually, a title on the log sheet is very useful. Be sure the video-camera records this title. A shot at the log will suffice. Also arrange a series of code words or letters for problems that arise during measurement. A code such as $T 3,7,1$ to indicate that Two points were taken at coordinate $3,7,1$ instead of the desired single point will facilitate logging of the error.

2. Take four measurements close to the appliance front center, sides and back to ensure the sensor range is acceptable. If overload occurs, change the grid to eliminate all points that are close enough to cause overload. Take four more measurements on a profile from the front center position along a grid line selected above. Take two electric field readings if the approach to the appliance is free of objects. One should be at $30 \mathrm{~cm}$ in front of the appliance at heights from 1 to 2 meters. The other should be at least 3 meters from the appliance.

Use as long a snapshot duration as possible to detect low frequency activity or to separate out off-power frequencies. Use a high frequency sensor to detect activity in the VLF and LF bands. It is suggested that a snapshot duration of 0.2667 seconds be used with sample frequency of $7680 \mathrm{Hertz}$ be used with wave capture equipment in an office with a 60 Hertz power frequency. The spatial orientation of the sensor is very helpful information for source characterization. Log the sensor orientation as well as coordinate location. Take a measurement at the location of the first and second single monitor. If possible, set the 
second single monitor to record transient events as well. Adjust trigger levels using the results from this measurement. The suggested trigger level is suggested to be two times the measured level at this location. Allow these monitors to record at intervals of about 10 seconds or less if the source is known or suspected of having more rapid cycle time. This will allow changes in the source to be recorded.

3. Leave the measurement area to go to the location of the portable computer or another quiet place. If this appliance has been previously coded and has a data file, compare the measurements just taken with the on file readings. If they are consistent within $\pm 10 \%$, no further measurements on this source is justified. If the measured values do not agree with the on file data or no or limited data exists, then proceed.

Take the time to think about the readings of step 2 and the results of the room examination. Take the time to look at the wave forms. Locate low frequency activity if it exists. Locate off-power frequency activity. Locate predominate harmonics. Select a snapshot duration and sample frequency that allows the activity present to be viewed. If no significant activity is seen below the power frequency then select a snapshot duration four times longer than one power-frequency cycle. For a 60 Hertz office, use 0.0667 seconds and a sample frequency of 7680 Hertz. The capture of the 15 and 30 Hertz frequency bands will allow the removal of motion effects from an unsteady hand or a bouncy profiler wheel.

4. Return to the measurement area and begin the measurements starting at a selected grid point and record the sensor location including height. Simple approximations require at least 4 points each in at least 4 directions within 180 degrees and at 4 heights, as shown in Figure 2.1. This will allow the approximate determination of the plane of the field generation and the general attenuation characteristics. If the orientation of the field producing structure inside the source is known, then two points anywhere on the plane of maximum field will determine approximate attenuation. Progress through the grid selected without deviation from the plan discussed just before measurements start. Take only one reading at each point. 


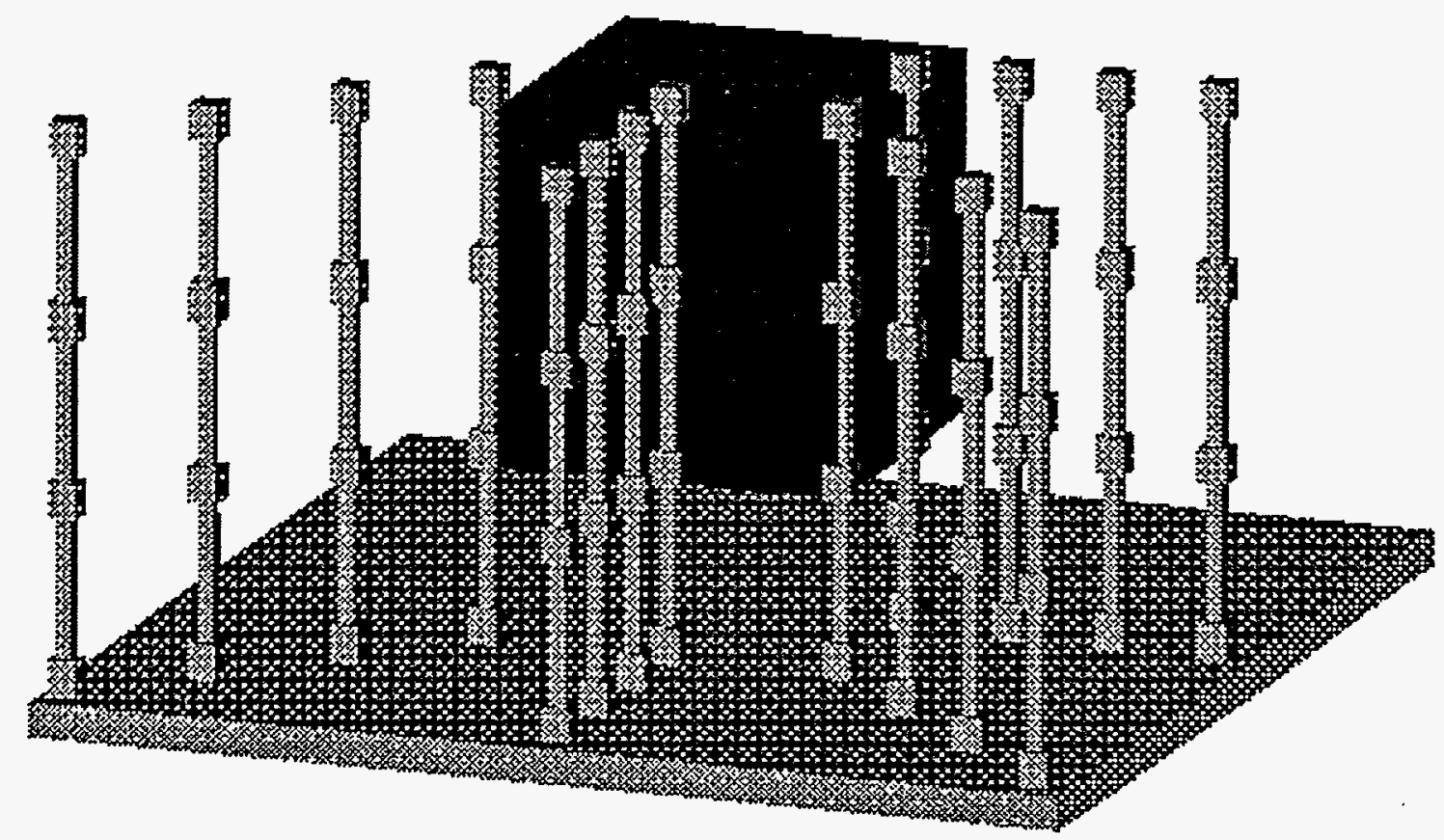

Figure 2.1 An Example of Sensor Positions Selected for an Appliance That is a 1 meter Cube, 0.5 meter From the Floor.

NOTE: Common protocols call for one to three reading per coordinate. There are other protocols that require as many as ten readings. Most protocols call for the averaging of the several readings into a single reading. If the sampling system is not synchronized with any of the field events within the measurement grid, then all samples are truly random in nature. It would be superior to take all samples simultaneously and then there would be no question that all the data was intimately linked for that random point in time. Such sampling requires a large array of sensors and is not practical for most in-situ measurements.

The philosophy of multiple readings is centered on the "noise" or perturbation of the field in the environment and from the appliance that is being measured in-situ. A part of the consideration in setting up a protocol must include the "noise" in the measurements. These result from calculation variations and variations in the spatial position of the sensor such as a shaking hand or a wiggling staff. To reduce the spatial position noise, taking consecutive cycles will reduce the effect of shaking or. wiggling. Further, if wave-capture is used with a snapshot 
duration related to a subharmonic of the power frequency (i.e. snapshot duration of 0.0667 seconds for a minimum frequency of 15 Hertz for a 60 Hertz power frequency), techniques exist to remove the shaking or wiggling. The resultant FFT for the 60 Hertz signal will effectively average four continuous cycles of data as a part of an RMS calculation. Other specific measures will benefit from this four cycle averaging in a similar way.

If the sensor motion from shaking or wiggling is set aside, there is little basis for taking more than one sample of the field at a particular coordinate. Unless significant changes in the environment or in-situ appliance fields is known to be faster than the shortest snapshot interval available, the taking of multiple samples at each coordinate does little to improve accuracy. The relation between samples from different coordinate points is no better than if a single sample were taken since the time involved to move to the new coordinate is much greater than the time between samples at the same coordinate. The "subtle" variations of fields from the environment or the appliance will no better be represented by multiple measurements at a coordinate.

The use of a single stationary sensor located at the outer edge of coordinate measurements is very useful in determining the "subtle" variations in the fields during coordinate sampling. This "monitor" sensor will indicate significant changes in background field activity. A stationary monitor placed close to the appliance will display load changes for many types of appliances. If the general physical arrangement of the area measured does not change, data taken throughout the area can be modified by the level changes from these monitor sensors provided some type of model can be developed for the appliance.

If minor deviations occur, such as an extra sample is taken, be sure to note these exceptions on the log and view the log with the camera. As each measurement is taken, enter the coordinate number.

When measuring large sources such as power generation facilities, the profile wheel is useful. The profile wheel removes the tedium of moving and setting the staff. However, it adds the problem of ensuring the distances remain as recorded. Rough surfaces, sliding surfaces, profiles that are not in straight lines and excessive movement or vibration of the sensor are all circumstances which make data interpretation more difficult. When a profile wheel is used, the video camera should be 
focused not only on the wheel but on the area around the measurements. Simply focusing on the wheel overlooks the possibility of identifying sources that surround the area of the profile.

5. Monitor the location, sensor orientation and the sample numbers or coordinate numbers with the video camera. Be sure the camera views the area around the sensors. Unusual readings may often be explained by an "invisible" source that was overlooked during the initial site examination.

NOTE: Make record keeping. an integral part of the protocol. Photographs of the appliance and video recordings of the measurements as they are made along with sample numbers and coordinates being put on the audio part of the video recording will be invaluable in preparing reports. Write everything down and make sure that there is essentially a duplicate of all information. It is recommended that three people are a part of the protocol. One person moves the staff and takes the actual field data. This person is concerned about the quality of measurement, the direction of the sensors, the location of the coordinates and the operation of the monitors. The second person writes all the coordinate data and sample data down. For measurement devices that log the data with sample numbers, actual field readings need not be recorded. Sketches of the area around the appliance is very useful. The second person should measure the main dimensions of the appliance so that photographs can be used to scale further measurements if needed. The second person also should be concerned about moving into work areas, being polite to the people around the area, and answering questions. The third person should be video taping the measurements and providing good overall views of the appliance, views of appliances near by (in case there are fields from those appliances) and records of the placement of the staff. The first and last measurement must be carefully recorded for time and date so the tape can be viewed later. The audio part of the recording should include the sample numbers and coordinates. If the noise level is high, the third person should repeat the numbers into the camera microphone. If necessary, the camera should be modified to allow the third person to speak into a microphone located very near the person's mouth if extremely high noise is anticipated. A video camera with low lux capability should be chosen so that pictures of relatively dark environments can be recorded. The use of a photographic camera can be used in the same manner as a video camera. 
6. Request people to move from their workplace only when absolutely necessary.

NOTE: Obtain as much data as possible with the minimum impact on the operations in the environment by utilizing monitoring equipment that will record as much data as possible. An office normally cannot simply shutdown and be vacated while measurements are made. Be mindful of desks and workstations so that the employees can continue their work with a minimum of interference. Do not interfere with the work going on next to the appliance that is being measured. This may be obvious to most people but the desire to obtain data often overshadows consideration of the client's economics.

Make the protocol anticipate and adapt. There should be room for changes in the protocol when some part of the appliance is not available to measure. Allow for limited measurements. Anticipate the possibility that near-by appliances may produce fields that obscure the fields from the appliance measured. Such situations as nearby breaker panels, hidden power feeders in a wall, appliances in the next room, the room above or the room below will influence readings and create errors in the data. Look for such influences in the data by noticing that the fields are not attenuating as the measurements are farther from the appliance. As an example, the back of a large power source is against a wall and there is no access to that area. That fact may not have been known when the protocol was prepared. The protocol should be adjusted to make other measurements such as along the wall at the sides of the power supply.

7. When the measurements from the chosen grid are completed, retire from the area where you can examine the data to be sure it has not been corrupted and is consistent with expectations. Think about the measurements taken and review the consequences of grid points that had to be skipped. If all agree that a few more points should be taken, then repeat only these points. Log each point as an exception. If more than a few need to be taken, review the problem with the facilities manager to request permission to repeat the measurements. 
D. Before leaving the site, review the results of a few random measurements.

1. Review a few selected wave forms to ensure that no silly mistake like failing to get a sensor completely plugged in has occurred. If the results are not satisfactory, consider repeating the measurements. Request permission before repeating measurements.

2. If there is to be no further measurements, bring back the first and second single monitors. Log the time the monitors took the last reading before being moved.

3. Rèmove the tape measures, any duct tape and any marking tape from the floor. Pick up any messes and retrieve any measurement equipment including pencils that were left as the measurements were made.

4. Be sure that the data is stored on disks and on the hard drive of the portable computer. The best situation is to have the data on two separate media. At this time put any additional comments on the log so that the last thoughts or observations are remembered.

5. Pack the measurement equipment, thank the facilities manager, and leave promptly. The longer that you are there, the longer the office is disrupted.

E. Reduce the data.

1. Calculate the data needed for the immediate application.

2. Place the summary measures data and the nature of the test into the general data base for access by others.

3. Place the logs, video tapes and archived original data along with all archived calculation results into a file that may be accessed by request.

4. When the data is reduced, the appliance should be coded for future use, the required results calculated and a complete file generated for both the electronic files and for a hard copy file. The raw data should be kept so that at a later date, specific questions may be answered or specific summary measures may be calculated without having to return to the site. 
These protocol steps are common to any in-situ measurement of a magnetic field source. They are made more difficult because the time for measurements are usually limited, the access is usually limited and the temptation to wait until returning to the office to look at the data is intense. There is a feeling that all that is done will be remembered. However, experience has shown that very little is remembered. Ninety percent of the data collection is recording where, when and how the data was collected. The use of sketches and cameras assists in this process. The temptation to send a single person on an in-situ measurement should be avoided unless the measurements have been made several times before in similar circumstances. In that situation, the notes and the video recordings should not be eliminated even though the video will not show the actual measurements. It normally takes one person about three or four times as long to make measurements as it takes three people.

A sample augmented step-by-step protocol is provided in Appendix A. 


\subsection{APPLIANCE TESTING PROTOCOL FOR MEASUREMENTS AT A TEST CENTER}

An electric and magnetic field test center has: extensive measurement apparatus, some knowledge of the appliance characteristics from previous testing of similar appliances, and that there is the ability to repeat tests. The nature and complexity of the protocol may be determined by considering the following:

1. If the appliance type and style have been tested before or if an extensive set of measurements have already been taken on similar appliances, a reduced protocol may be utilized to modify the previous measurements.

2. If the appliance has not been measured before, the known characteristics concerning the operation of the appliance should be documented. Each of these known characteristics may be used to determine the need for a pilot study. If transient fields are known or suspected to exist, then transient measurements should be included in the protocol. If transient fields are not known to exist, the pilot study may include a transient trigger test to determine if transients exist. Similarly, characteristics, such as polarization, dc field modification, harmonics, intermittency and coherency, should be considered for the pilot study.

3. If the electric and magnetic characteristics sought are prespecified, the protocol may be selected to highlight these characteristics. However, the requirements of the characterization should not overly influence the pilot study protocol or the measurement protocol. The tendency to limit the measurements to only those that are needed for the immediate requirements should be avoided. A more general quantification allows other characterizations to be determined with the data at a later date. Any narrowing of the focus should be made after the pilot study.

A sample augmented step-by-step protocol is provided in Appendix B.

\subsection{The Pilot Study}

The pilot study forms an integral part of the full characterization and the data become part of the characterization record. Often the pilot study produces significant findings that are needed for the verification of some characteristic, or the lack of some characteristic, in the future use of the data. The questions which should be included in the study are:

1. What are the maximum and minimum field levels at the power frequency?

2. What are the harmonics present and what levels are the harmonics? 
3. Are there frequencies that are not harmonics or are below the power frequency?

4. Are there sub-radio frequency components in the VLF or LF ranges?

5. Is the dc or static field modified by the appliance?

6. What are the operating cycles of the appliance?

7. Are there transients present when the cycles change or at any other times?

8. What coding, as specified in Task B, should the appliance be given?

The pilot study protocol should include:

1. Operating the appliance through all of the expected cycles to determine the equipment intermittency and loading. The power sources needed, the support required and other special requirements should be addressed at this time. The special characteristics of the appliance are determined. These characteristics include heating (can sensors be used near the heat source) rotation, torques on the appliance supports, acceleration, current and voltage requirements, sizes of the appliance that require special supports, or an appliance that may require opening, closing or changing of shape during operation. Measurements of fields should not be a part of this step. Specific functional characteristics of the equipment should be extracted from the equipment instruction book. A review of the instruction book will ensure there are no unusual circumstances that can occur during operation.

2. Operating the equipment while taking steady state field readings using a wave capture system with $3 \mathrm{~Hz}$ to $3 \mathrm{kHz}$ (ELF) bandwidth. At this point, it can be determined if special loads must be applied to the appliance to maximize the fields. The frequency range $(3 \mathrm{~Hz}$ to $3 \mathrm{kHz})$ indicates the presence of frequencies below the power frequency and creates a fine frequency resolution to detect frequencies that are not power system harmonics. If the high ranges are not needed, the final study protocol should reflect this in the type of sensors used. A series of readings around the perimeter of the equipment near to the equipment surface will usually locate the largest fields.

3. Operating the equipment while taking both $d c$ and sub-radio frequency field measurements ( LF - Low Frequency, between $30 \mathrm{kHz}$ and $300 \mathrm{kHz}$ and VLF Very Low Frequency, between $3 \mathrm{kHz}$ and $30 \mathrm{kHz}$ ). The dc readings will indicate if the equipment produces dc fields or greatly distorts the earth's dc field. The sub-radio frequency levels will determine if such recording should be included in the final study protocol. 
4. Operating the equipment while setting a transient capture based on trigger levels taken from the steady state data. The transient trigger of 1.2 to 1.5 times the steady state level is suggested. Triggering on level changes will allow recording of turn-on transients for most iron-core appliances such as transformers and motors. The turn-off transient usually cannot be detected with level triggers. Rate triggers which sense $\mathrm{dB} / \mathrm{dt}$ can be used with advantage since switching actions often have a very short burst of sub-radio frequency which has substantial $\mathrm{dB} / \mathrm{dt}$. Setting the rate level is more difficult since simple signal noise can be mis-interpreted. Rates of 4 times the normal power frequency rate will work for many applications. The normal power frequency rate is the field amplitude times $2 \pi f$ where $f$ is the power frequency. This rate will tend to ignore the noise and the power system harmonics but will pick up the rapid flux changes for start-up or shut-down of an appliance.

5. Analyzing the pilot study data to determine the type of sensors, the expected maximum levels, the transient trigger levels, the location of dc sensors, the minimum frequencies for recording, the intervals for snapshots, and the LF-VLF recording requirements.

The pilot study should include a series of sketches describing the location of the sensors for the various data snapshots. A three-dimensional drawing package aids in this process since the coordinates of the sensor can be accurately entered and data, including the orientation of the sensor, type of sensor, and reading level, can be attached to the point using the annotation features. A video recording is also very useful to show the process so that little details are not forgotten during the analysis.

\subsection{Test Center General Protocol}

The protocol should develop information to make a complete characterization of the fields as specified in Task $A$. The test center protocol utilizes structures with mounted sensors to allow maximum characterization in the minimum amount of time. The sensors can be located where necessary but a distance between sensors that increases geometrically rather than arithmetically will allow easier analysis of field attenuation. As discussed in Task A, attenuation data is very useful in constructing simple mathematical models of the field production by the appliance. A spherical coordinate system allows the attenuation factors to be analyzed directly while reducing the amount of data taken. 
The final protocol should include:

1. Creation of a sketch of the appliance sufficient to create three-dimensional outline figures for use in displaying field levels in the report. The location of the "center" of the appliance should be included. A discussion of the method of locating the center of the appliance is included in a separate section below.

2. Preparation of a set of photographs to document the details of the device, including a photograph of the nameplate (if any).

3. The size, construction or wiring, voltage rating, current or power rating, and any other data which may be related to the field sources within the appliance as given in the instruction book.

4. Collection of field data on spherical coordinates to completely surround the appliance. This collection should be done for each major operational cycle or each significant load situation for the appliance. There should be some measurements that are always repeated in the same place. This may conveniently be the top or bottom measurement. In this manner, changes in operating level may be detected. The recommended maximum angles between collection positions should be no more than 30 degrees.

5. Sub-radio frequency data should be recorded with the coordinates precisely recorded unless such data is a part of the sensor structure data collection. The measurement of the LF and VLF data, as a function of distance from the appliance, is very useful to determine the LF and VLF source location. It is recommended that the first three sensors at all angles that are radial from the appliance record the .LF and VLF levels.

6. Transient data should be taken from the first three radial sensors at all angles similar to the sub-radio frequency. Triggering of the recording of all sensors should be controlled at a single point using a sub-radio frequency sensor with $\mathrm{dB} / \mathrm{dt}$ capability on all three axes. This point should be nearest the appliance where the pilot study found the largest or most time variable magnetic fields. The pre-test data should have indicated the location of the most intense field activity. As the appliance is rotated, the transient should be repeated. The transient will not be repeatable and the data will not directly be comparable, but the attenuation of the fields may be predicted by such data.

7. Analysis of the acquired data includes verification that no recording failures occurred. Failures of sensor data from improper gains will result in overloads or low readings. If only one or two overload measurements are found, the data may be replaced by the maximum field level the sensor can sense. This will 
distort the plotting somewhat but will maintain the grid so that large deviations do not take place (as would occur if the data were ignored). If more than a two overloads are found, the test should be repeated with the gains adjusted. Since the test set sensors are positioned in a geometric pattern, checking for low readings is accomplished by observing that as the measurements proceed radially away from the appliance, the fields will drop by approximately a power of 10 (a divider of 5 to 30 ) from one sensor to the next. If the field does not drop by a power of 10 or drops by a power of 100 , then the data should be adjusted by a power of 10 to provide a reasonable reading. Since the test set does not have the problem of external appliances to distort the field, it can be assumed that the gain was simply mis-adjusted by a power of 10 . The presence of LF and VLF fields, transient fields and significant harmonics should be noted on all studies although detailed analysis of this data may be postponed.

8. All data and analysis should be put into the same folder as the pictures, sketches and instruction book information. This data should be in electronic form as much as possible.

9. Summary data should be entered into the general data base.

10. A report should be prepared for each appliance so that it may be filed separately in an appliance folder.

11. The appliance should be coded for retrieval using the source classification system.

\subsubsection{Location of the Center of the Appliance}

Selection of a "center" for measurements in the spherical coordinate system is a complex issue. The physical "center", i.e. the bisection of all three axes of the appliance, is an obvious choice. However, practical considerations must be weighed when such a selection is made. If the physical center is easy to locate because the device is rectangular on all sides and the appliance has homogeneous density, then the physical center should be used. Other factors may enter into the decision. Since the semi-spherical array of sensors of the test set should have access to the bottom of the appliance at the angle of 60 degrees from the horizontal, the appliance must be balanced on a rather small pivot point. Such a point requires that the weight of the appliance be "centered". A microwave oven is an example of this problem. The oven center is located in the space within the cooking cavity. The weight is concentrated on one side. To simply set the oven on the test stand at the physical center 
would require extra-ordinary supports. Therefore, shifting the center to the center of gravity would be acceptable.

\subsubsection{Coordinate Systems and Sensor Positioning}

For the laboratory test fixture, the use of spherical coordinates and geometric ratios of distance provide an improvement in data reduction for the purposes of modeling or detailed study. The attenuation coefficients can readily be calculated for essentially any direction from the appliance being tested. Unfortunately it is more difficult to display the data in spherical coordinates since the profiles are radial and most presentations are presented in rectangular format.

The tendency is to analyze data in profiles at the foot level, the torso level or the head level since body movement is an important dosimetry consideration. As a person moves toward the source, the question of the exposure to the head requires a profile at head height. The radial data can be converted to such profiles but the mathematical process is more difficult. However, if a model is developed then the model can be computed to any profile desired. It would seem more general to make a good model and then develop profiles (that can be verified at the spherical measurement points) rather than develop the profiles and attempt to create the model from the more difficult Cartesian coordinates.

There is always the issue of taking so many points of data. The test fixture used to develop and test the recommended guidelines for source measurement protocol makes three-axis magnetic measurements at 560 points ( 35 sensors at 22.5 degree rotation locations). As discussed in Task A only two or three points need be measured to model a point source. Indeed, if the point source is a simple magnetic dipole or a simple point of charge and the surrounding environment is non-magnetic or insulating, then linear conditions exist. For this process to work effectively, the source geometry must be very well known, the dipole length and spatial orientation must be known and measurements near the dipole (less than three or four lengths of the dipole) may indicate dipole strength but not attenuation. However, if the magnetic dipole geometry is this well known, a single measurement at a known orientation can be used along with a model to rapidly obtain the entire field structure and profiles at any point, any line or any surface.

When the geometry becomes more complex, such as in a motor structure, and there is ferris material for magnetic fields or semiconducting material for electric fields, the source may not be clearly defined. The different size dipoles or charge plates that form harmonics or sub-radio frequency signals may change the attenuation at each frequency. The model cannot be developed from a few 
points. However, with the large number of data points, patterns can be recognized as to location and size of the various dipoles.

While spherical coordinates will generally produce the most information for modeling with the minimum of data, cylindrical coordinates are useful for some appliances. If the appliance is elongated and the current is actually running through the equipment, such as in a breaker box or conduit, the use of cylindrical coordinates is superior.

The use of Cartesian coordinates normally produces excessive data in the laboratory environment. It has the advantage of being used directly for plotting from most 3-D plotting packages with good results.

\subsubsection{Sensor Position at the Appliance Surface}

For magnetic field measurements, locating the first sensor so that it touches the appliance surface would give the most complete data set. However, repositioning of the sensor arrays for small appliances often takes considerable time for each measurement. The analysis and plotting of the data is much less complicated by having the sensors fixed throughout the measurements as the table with the appliance is turned around. This would require that the sensors be positioned outside the "physical sphere" which the appliance will occupy when it is turned in a circle. In some cases, this sphere would be so large as to allow only remote field measurements.

The determination of the distance from an appliance where the assumptions for a remote field are not always obvious. Although some appliances may have a motor that is nearly the size of the case, i.e. a pencil sharpener or a drill, many appliances have a case with a much smaller motor or coil located inside. The size of the motor or coil will approximate the size of the magnetic dipole for estimation purposes when locating sensors. Literature provides the guidance that a distance two or three object dimensions away will allow the source to be approximated as a dipole and the remote field calculations using the inverse of the cube of the distance are appropriate (EPRI, 1994, pp 2-15 to 2-17). This implies that a pencil sharpener that is approximately a $10 \mathrm{~cm}$ cube will have measurements that approach the remote field characteristics at $30 \mathrm{~cm}$.

It is recommended that for an appliance which has a "physical sphere" of about $30 \mathrm{~cm}$. radius, the measurements should be made at a fixed position with the first sensor located on the physical sphere, i.e. the sensors just barely touch as the appliance is rotated on the test platform. In addition, at least one measurement is made with all sensors positioned as close to the appliance as possible. For appliances which have a physical sphere greater than $30 \mathrm{~cm}$, the 
first sensors should be placed as close to the appliance surface as possible for each measurement. If the fixed positions are used, documentation as to the reason for such selection should be included.

The fact that the appliance must sit on some type of platform limits the access of the lower first sensors. This platform must be sturdy enough to accept the weight of the appliance without tilting or bending as the appliance is rotated. The sensors below the horizontal may not be able to be positioned at the appliance surface. It is recommended that these lower sensors be positioned as close to the appliance as possible allowing that the platform is between the sensor and the appliance. The platform must be considered when determining the physical sphere of the appliance.

\subsubsection{The Location of the Distant Sensors}

The "first" sensors located at the "surface" (or physical sphere) of the appliance should have a fixed relationship with the second and third sensor. Recommended distances are $10 \mathrm{~cm}$ between the first and second sensor and $30 \mathrm{~cm}$ between the first and third sensor.

The fourth sensor should be located $100 \mathrm{~cm}$ from the first sensor. For smaller appliances with a physical sphere of $30 \mathrm{~cm}$ in radius, the fourth sensor may be permanently located $100 \mathrm{~cm}$ from the center of the test unit. This allows the use of shorter movable spars for the sensors. For such small appliances, the use of a fifth sensor at $300 \mathrm{~cm}$ from the first sensor is usually not needed as the attenuation can usually be determined using the $100 \mathrm{~cm}$ data with the assumption that the remote field attenuation of the inverse of the distance cubed is valid.

For appliances with a physical sphere larger than $30 \mathrm{~cm}$ radius, the fourth sensor should be linked to the movement of the first sensor so that there is a fixed distance of $100 \mathrm{~cm}$ between the first and fourth sensor for measurements around that appliance. Exceptions due to unusual shapes or other obvious characteristics should be documented. For these larger appliances, the fifth sensor should be located at a fixed distance of $300 \mathrm{~cm}$ from the center of the test appliance. For this $300 \mathrm{~cm}$ sensor location, the assumption of a remote field measurement will be valid for appliances having a physical sphere radius of $70 \mathrm{~cm}$.

\subsection{Test Center Production Test Protocol}

When the same type and general shape of equipment is to be studied, a determination of the variability from previous characterizations may be all that is needed. 
Such a protocol might be used to collect information to bracket the fields to be expected from a class of equipment such as home refrigerators. This protocol should include:

1. Documentation of sizes and shapes with any unique changes noted, i.e. half height, double width, etc.

2. Documentation of ratings or loads with any unique cycling noted.

3. Documentation of the waveforms of the fields at six locations around the appliance. These should be at the center of each side and top and bottom of the appliance. These measurements should be taken at the surface of the appliance and at $30 \mathrm{~cm}$ from the equipment. Waveform data is especially useful for inter-class comparison since the waveforms will indicate changes in the field generation that will be obscured in simple measures using harmonic spectrum or rms levels.

4. Reporting any changes or exceptions for the appliance.

If the changes or exceptions are considered significant, a pilot study should be initiated as though the appliance was of a new type and a final study made to completely characterize the appliance.

\subsection{Test Experience}

The use of the pre-test protocol was developed as appliance testing progressed. Pretests take time, however it was shown after several tests that the pre-test time saved time on the final testing. Practical problems, including the ability to mount the appliance appropriately and the ability to obtain a continuous load for the appliance, were encountered and resolved during this prototype testing phase. The pencil sharpener was an obvious case of having to reload the appliance at frequent intervals. The toaster proved difficult to monitor at the top since the top sensors became very warm. The appliances that have data available (as shown in Appendix $\mathrm{C}$ ) include:

$\begin{array}{lll}\text { Crock Pot } & \text { CRT Monitor-VGA } & \text { Fan - Oscillating } \\ \text { Space Heater } & \text { Pencil Sharpener } & \text { Popcorn Popper - Hot Air } \\ \text { Printer - Laser } & \text { Electric Shaver } & \text { Toaster } \\ \text { Hair Dryer } & \text { Can Opener } & \text { Microwave Oven } \\ \text { Vacuum - upright } & \text { Hand Drill } & \text { Mixer } \\ \text { Blender } & \text { Skil Saw } & \text { Soldering Iron } \\ \text { Jig Saw } & \text { Hammer Drill } & \text { Iron } \\ \text { Air Conditioner-window } & \end{array}$




\subsection{Prototype Testing Protocol}

This prototype protocol for the electric and magnetic field characterization of a new type of appliance that has not been tested before was developed as the appliance testing progressed. No single appliance has all steps listed, although each step was used on at least one appliance.

1. Examine the appliance. Determine:

A. What physical load is required for correct operation.

(i.e. liquid for blender, sprayer, cooker; pencils for pencil sharpener; wood for drill or saw, carpet for sweeper, bread for toaster, etc.)

B. What special considerations are required for putting the appliance on the test set.

(i.e. a bag to catch spray, weight and balance of a printer or microwave oven, computer interface and extension cables for printer or CRT, etc.)

C. What special considerations are required for powering the appliance.

(i.e. a switch to turn the toaster off between measurements to keep the sensors from excessive heat, three phase voltage requirements, 240 volt operation, etc.)

D. Record the nameplate data. Include manufacturer, model and serial number, voltage rating, current or power rating, etc.)

E. Make a sketch of the outline of the appliance and general sizes so that a rough outline can be overlaid on the magnetic field plots.

F. Determine the classification code for the appliance.

G. Determine if the test set environment is suitable for the appliance. To eliminate fields around the test set, no heating, air-conditioning, lighting or other source of electric and magnetic fields are allowed. The computer must be at least 6 meters from the test set and on the opposite side of the appliance from the sensors. The computer should be operating during all pre-test measurements.

2. Mount the appliance on the test table.

A. Determine the physical "sphere" for the sensors by spinning the appliance on the table and adjusting the sensors so they just barely touch the appliance somewhere in the circle. The table legs or supports should be 
included in the alinement of the sensors. Make notes of reasons for offsetting the appliance such as weight balance.

B. Measure the distance from the sensing center of the first level of sensors to the center of the appliance. Record these measurements for entry into the probe position file.

C. Locate power cord to the appliance so that it drops from the appliance directly to the turntable and then out from the turntable at the 12 degree location (between the 0 and 22.5 degree measurement points). Plug the appliance into a shielded power cable with tightly twisted wire and run the shielded cable away from the sensors to the current and voltage monitors. Be sure any unpolarized plugs are correctly inserted to achieve the effect of plug polarization. Do not put loops in the appliance power cable unless the appliance is normally operated with the loops in place. Describe any variations of this connection process.

D. Photograph the appliance on the table with the sensors positioned.

3. Conduct a pre-test test of the appliance.

A. Determine the possible cycles the appliance may have.

(i.e. wash and spin on a washing machine, charge and run on an electric toothbrush, etc.)

B. For each identified cycle, monitor the following data:

1. With a static sensor monitor with a snapshot duration of 0.2667 seconds (minimum frequency of $3.75 \mathrm{Hertz}$ ) and a sample frequency of $7680.5 \mathrm{Hertz},(3 \mathrm{kHz}$ anti-aliasing filters) take 6 coordinates around the six axes centered on the physical sphere of the appliance. If the static sensor overloads, back the sensor away from the appliance until readings return to range. Record the distance required. (These readings allow the data to be augmented with close-in readings and indicates the influence of the appliance on the static dc field.)

2. Using the static sensor, take two readings at a coordinate in front of the appliance at 1 meter from the surface. Record the coordinate. One reading should be taken with the appliance on and one with the appliance off. (These readings will provide the basis for background and de levels and modification of the dc levels with the appliance operating.) 
3. Using the static sensor, take a reading at the coordinate on the surface of the appliance which produces the highest RMS field levels. Record the coordinate. (This is used to scale the levels of the sensor readings on the test set.) If the static sensor overloads, then substitute a high field ac sensor.

4. Set the AC field sensor near the highest field area, record the coordinate, and turn on transient capture. Set the capture level to 2 times the AC flux level. Turn on and off the appliance to trigger transient capture during transition between cycles or from off to on. (The transient capture may be limited to the $3 \mathrm{kHz}$ bandwidth of the Fluxgate unless there is reason to expect more than $0.1 \mu \mathrm{T}$ above $3 \mathrm{kHz}$. Then the high- frequency transient three-axis capture system should be used to capture up to $100 \mathrm{kHz}$ for short bursts.)

5. Using a free-body electric field probe, record the electric field at a distance of $10 \mathrm{~cm}$ from the surface of the appliance on the six appliance axes using the same snapshot duration and sample frequency as for the magnetic probes.

6. Scan the appliance for the highest level of electric field no closer than $10 \mathrm{~cm}$ to the appliance. Record the coordinate. Turn off the appliance and take a measurement at the same coordinate. This is used to verify background and estimate the electric field characteristics.

C. Review the waveforms captured and determine the harmonic levels, the low frequency components, the off-power-frequency components or other anomalies of the field. Set the sample frequency and snapshot duration of the test set based on the lowest or off-power frequencies found. The snapshot duration must be set so the off-power frequencies appear in separate FFT frequencies.

4. Sample the fields with the test set.

A. Energize the appliance for the selected cycle and scan all sensors with the computer to ensure that no sensors are malfunctioning. (During the setup of the appliance, cables may be bumped and a sensor may be operating incorrectly.) Set all sensors to the ranges dictated by the pretest. Set the computer sample frequency and snapshot duration. Select the sensors on the 1 meter and 3 meter distances as appropriate for the appliance size. Larger appliances may require 3 meter 
measurements. The sensors are at "physical sphere" surface, $10 \mathrm{~cm}$ from surface, $30 \mathrm{~cm}$ from surface, 1 meter from surface or from coordinate center, and 3 meters from coordinate center. Sensors are 0-2 G AC, 0-20 G AC, or 0-200 G AC peak for the first three levels. RMS readings of 3 to $30 \mathrm{kHz}$ and 30 to $300 \mathrm{kHz}$ are recorded. The fourth and fifth level use 0-1 G AC sensors with a $10 \mathrm{~Hz}$ to $3 \mathrm{kHz}$ bandwidth. Current and voltage readings on the power cord are also monitored.

B. With appliance operating at the 0 degree position of the turntable, measure a sample with the test set reading all sensors.

C. If required, turn the appliance off. Turn the turntable 22.5 degrees. Turn the appliance back on if necessary and measure all sensors for a sample. Repeat this process for each 22.5 degree rotation for 16 rotations.

D. Turn appliance off and measure a sample from all sensors. This is used to determine the sensor operation as well as background levels.

E. Repeat this process for each identified cycle of the appliance.

F. Transfer all data to floppy disks for later processing. Do not erase the data until a copy exists on a hard drive in the office.

5. Remove the appliance.

A. Dismount the appliance. Remove any marks or tape placed on the appliance to indicate centers of the physical sphere.

B. Return the appliance to the owner with any promise documentation.

6. Reduce the data.

A. Run the calculations to create required specific measures. Simply running all calculations may be wasteful of effort. The pre-test data must be examined to determine when the signal levels in each group of calculations is significant enough to require the calculation to be made. These calculations include:

1. RMS calculation at power system frequency from test set data. (Provides information on the fundamental energy relationships within the appliance and serves as a basis for modeling of many appliances that operate principally on power frequency circuits. 
2. RMS calculation at power system harmonics from test set data. This is done when the harmonic levels exceed 5 percent of the fundamental. This determination is made primarily from the pretest information.

3. Using the pre-test data, calculation of the dc levels for analysis of the dc field modifications. Unless otherwise specified, deviations of more than 10 percent from the local earth field may justify calculations and documentation.

4. Using pretest data, calculation of low frequency or "off-frequency" field RMS levels by harmonic. Presence of off-frequency of 5 percent or greater of the fundamental or maximum harmonic should be calculated and documented.

5. Examination of the transient data waveforms and record the extent of transient levels, rise times, and decay times in terms of frequency envelopes.

6. Calculation of the $3-30 \mathrm{kHz}$ and $30-300 \mathrm{kHz}$ RMS data if activity above $0.1 \mu \mathrm{T}$ at $3-30 \mathrm{kHz}$ and/or $0.02 \mu \mathrm{T}$ for $30-300 \mathrm{kHz}$ exists.

7. Calculation of the electric field data over the ELF frequencies that are of interest for the magnetic field data.

B. Assemble a descriptive file indicating the nature of the appliance and the nature of the test. Add the specific measures as ASCll data for submission to the general data base.

C. Prepare the magnetic field contour plots (or other types of plots of interest) for each of the pertinent harmonics including sub-power frequencies and off-power frequencies as needed. (The fundamental and harmonic plots may not be similar since the sources of the field can be different inside the appliance.)

D. Place the waveform data onto an archive device for long term storage. Assemble a file folder for storage of plots, ASCII printouts and pictures. 


\subsection{REFERENCES}

Dietrich, F.M., Robertson, D.C. and Steiner, G.A. (1993). Magnetic and electric field testing of the Amtrak Northeast Corridor, and New Jersey Transit/ North Jersey Coast Line rail systems. U.S. Department of Transportation DOT-VNTSC-FRA93-4.2, April 1993.

EPRI, (1994). Handbook of shielding principles for power system magnetic fields. EPRI TR-103630-V1, Final Report, April 1994.

ERM (1993). The MF3D modeling program: User's manual. Version 2.0, Electric Research and Management, Inc. State College, PA: April 1993.

IEEE, (1994). IEEE Field Effects Design Guide. IEEE PES TF 15.04.06.03 Draft 1.1, Greg Rauch editor, July 1994.

Miller, D.A. (1972). Electric and magnetic fields produced by commercial power systems. Chapter IV, Low-Frequency Magnetic and Electric Fields, (We need to call IITRI to find out the exact reference). 


\title{
EMF RAPID PROGRAM ENGINEERING PROJECTS Project 1 DEVELOPMENT OF RECOMMENDATIONS FOR GUIDELINES FOR FIELD SOURCE MEASUREMENT
}

\author{
Appendices
}

\author{
Submitted by: \\ Electric Research and Management, Inc. \\ PO Box 165 \\ State College, PA 16804 \\ Submitted to: \\ Lockheed Martin Energy Research, Inc. \\ PO Box 2008 \\ Bethel Valley Road, \\ Oak Ridge, TN 37831-6070
}

March 11, 1997 


\section{TABLE OF CONTENTS}

PAGE

Appendix A An Example Protocol for In-Situ Appliance Measurements . . . . A-1

In-Situ Test Set Protocol for an Uninterruptible Power Supply . . . . . . A-1

Appendix B An Example Protocol for Appliance Testing . . . . . . . . . . B-1

Appliance Test Set Protocol for a Toaster . . . . . . . . . . . . B-1

Appendix C Appliance Test Data .................. C-1

Introduction $\ldots \ldots \ldots \ldots \ldots \ldots \ldots \ldots \ldots \ldots \ldots \ldots \ldots \ldots$

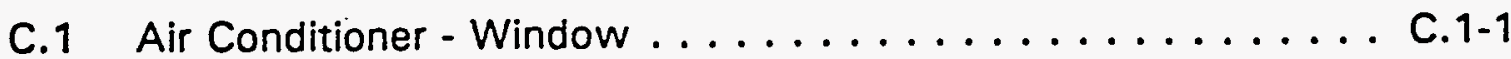

C.2 Blender .........................

C.3 Crock Pot ....................... C.3-1

C.4 Drill - Hand Hammer . . . . . . . . . . . . . . C.4-1

C.5 Fan-Oscillating ..................... C.5-1

C.6 Heater - Portable Space . . . . . . . . . . . . . C.6-1

C.7 Iron-Steam . . . . . . . . . . . . . . . . C.1

C.8 Jig Saw - Hand . . . . . . . . . . . . . . . C.8-1

C.9 Mixer - Hand Kitchen . . . . . . . . . . . . . . . C.9-1

C.10 Paint Sprayer . . . . . . . . . . . . . . . . C.10-1

C.11 Pencil Sharpener .......................11-1

C.12 Popcorn Popper ..................... C.12-1

C.13 Laser Printer . . . . . . . . . . . . . . . . . C.13-1

C.14 Saw - Skil Hand . . . . . . . . . . . . . . . . C.14-1

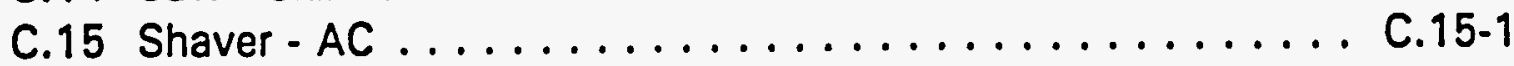

C.16 Soldering Iron Station $\ldots \ldots \ldots \ldots \ldots \ldots \ldots \ldots \ldots$ C.16-1

C.17 Toaster ......................... C.17-1

C.18 Tooth Brush - Electric . . . . . . . . . . . . . . . C.18-1

C.19 Vacuum Sweeper - Upright . . . . . . . . . . . . C.19-1

C.20 Video Display Terminal . . . . . . . . . . . . . C.20-1 
PAGE

Appendix D In-Situ Test Data . . . . . . . . . . . . . . D-1

Introduction $\ldots \ldots \ldots \ldots \ldots \ldots \ldots \ldots \ldots \ldots \ldots \ldots \ldots \ldots \ldots \ldots \ldots$

D.1 The Uninterruptible Power Supply . . . . . . . . . . D-1

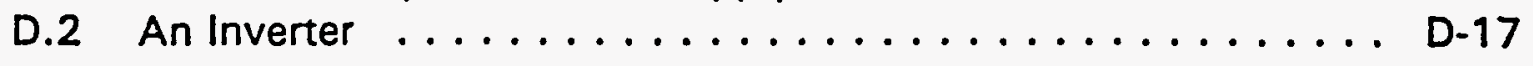


The in-situ protocol discussion provides the basis for the development of an augmented step-by-step procedure. An example procedure is given in this appendix to highlight the concepts discussed. The situation chosen was a measurement in a computer complex where graphical editing and printing work was in progress. The work could not be interrupted. Furniture could not be moved. The appliance to be measured was a three-phase, uninterruptible power supply (UPS). The supply was approximately $80 \mathrm{~cm}$ wide by $80 \mathrm{~cm}$ deep by $180 \mathrm{~cm}$ high. The unit sat on a raised floor typical of large computer rooms. The unit was against a wall and the other side of the wall was accessible as a hallway. The protocol was developed without having seen the site before. Verbal descriptions of the size and location of the unit along with a general description of the room layout formed the basis of the protocol. Instructions were given that no photographic or video equipment was allowed for security purposes and that the test crew should be as quiet as possible. The objective was to determine the level of magnetic field inside the computer room created by the UPS so that magnetic field management techniques might be applied.

\section{In-Situ Test Set Protocol for an Uninterruptible Power Supply}

Purpose: To measure and quantify the steady state magnetic fields around an appliance using Cartesian coordinates.

Items not Covered in this Protocol: Waveform or RMS data above $3 \mathrm{kHz}$ and electric fields are not addressed in this protocol.

Overview: The room is entered and quickly sketched. Locations for measurements are chosen and the grid is established on a grid based on the floor tiles. Waveforms for the ELF band $(3 \mathrm{~Hz}-3 \mathrm{kHz})$ are recorded. Data is developed for the RMS resultant of the power frequency and for harmonics to allow contour plotting. The data is quickly reviewed and a few added data points are taken. The room is exited and the data is processed at a later time.

Personnel Required: Two people are required. One will operate the wave capture system and the other will maintain a log. 
1. Equipment Required.

A wave capture system with four transducers on a staff with spacings of 10 $\mathrm{cm}, 60 \mathrm{~cm}, 110 \mathrm{~cm}$, and $160 \mathrm{~cm}$ from the floor.

- A two-transducer wave capture system with sensor locations of $110 \mathrm{~cm}$ from the floor.

Six static magnetic monitors $(0$ to 5 Gauss peak, DC to $3 \mathrm{kHz}$ ). Have available four high flux density monitors 10 to 200 Gauss peak, $3 \mathrm{~Hz}$ to $3 \mathrm{kHz}$ ).

ㅁ At least 10 blank formatted floppy disks, 3.5 inch High Density. Apply blank labels to allow the disks to be marked with their contents.

- A laptop computer to allow the waveforms to be viewed.

- A ruler and a tape measure marked in centimeters and meters for measurement.

ㅁ Masking tape to mark the floor at critical locations.

2. Test Set Operation Check:

Note the last time the wave capture system was calibrated.

Before arriving at the site, power up all the wave capture system with the three axis magnetic field verification coil. Verify that all three axes of all sensors are working properly.

3. Identification of the appliance:

- Upon arriving at the site, make a file folder for the appliance. Mark the name of the appliance on the folder - use the full name to designate the appliance along with its location.

․ Generate a log sheet for the appliance putting the full name of the appliance on the log sheet. Also mark your name, the date, and the time on the sheet. Record the name plate data on the log sheet.

Draw a sketch of the appliance providing approximate measurements in $\mathrm{cm}$. Show on the sketch of the power cables as they enter the appliance.

Draw the location of tables, chairs, and computer equipment on the sketch with approximate distances in $\mathrm{cm}$.

4. Pretest the appliance.

ㅁ Select four grid point locations for the pretest measurements. Three points should be near the appliance and the fourth near the position of the computer. Verify the appliance is operating in the normal mode.

Use a base FFT frequency of 3 Hertz with a sample frequency of $6144 \mathrm{Hertz}$ and 2048 samples. This will allow the determination of frequencies below 
power frequency and frequencies that may not be harmonics of the power frequency.

Name the computer data file with the three letters indicating the type of appliance, one letter for the location code and two numbers for the order of the files. The naming convention is totally arbitrary but the selections can help when many appliances and sites are to be reviewed. An example is UPSNOO. The UPS stands for Uninterruptible Power Supply, the $\mathrm{N}$ for the New York office location and 00 for the pretest file.

Take the data at the four locations with the four level staff.

5. Examine the Pretest data.

Transfer the wave capture file to a floppy disk. Do not erase the files from the static memory. Label the disk as the wave capture disk and mark the appliance file name and date on the disk.

口 Place the floppy disk into disk $A$ on the laptop. Start the plotting program. Observe the waveforms of the three axes of each of the four measurements at each of the four levels.

- If there are any overloaded signals (above 5 Gauss peak), note the overload and adjust the grid pattern to use the High Flux Density sensors in the overload region. If there is a question about the areas of the overload, the pretest may be run again with points in the vicinity of the high flux levels.

Using the FFT selection, determine the predominate harmonics. If there is no activity below the power frequency, then set the wave capture systems for a base frequency of $60 \mathrm{~Hz}$ with a $7680 \mathrm{Hertz}$ sampling frequency and 128 points within the sample. If there are frequencies below the power frequency or frequencies that are not a harmonic of the power frequency, select the appropriate sampling frequency and number of sample points.

Exit the plotting program. Remove the floppy disk from the laptop and place it in the folder.

6. Test the appliance.

Set the two-sensor wave capture system in the room with the first sensor located about 3 to 4 meters from the appliance. Be sure it is not near a computer terminal or printer. Verify from the readings being taken as the staff is moved around that the field measured is approximately a minimum in the area to remove the influence of power cables under the raised floor. This transducer is to observe the background fields in the room as the measurements progress. Set to record data at 10 second intervals.

Record the location of the first sensor based on the physical center of the appliance. 
Locate the second sensor of the two-sensor wave capture system at about 30 $\mathrm{cm}$ from the appliance surface. Enable the transient capture system and record data regularly at 10 second intervals. This will provide data on possible load changes of the appliance.

Place masking tape marks on the floor to define the desired grids. Each point need not be marked but the starting point and the final point of each profile line is useful.

․ Measure the four vertical levels at each grid crossing. Record the coordinates as the measurement is made. Record the sample number in the log. If video or photographic cameras were allowed, the measurement process and the room layout would be recorded during the measurement process. In this specific case, these types of recording are not allowed.

7. Check the data taken for validity and leave the site

Download the data from the four-sensor wave capture system onto floppy disks. Label the disks.

a Quickly look at the data waveforms with the plotter program on the laptop to verify that the signals were present and that overloads did not occur. If there were problems, repeat the data measurements. Remember to unplug the battery charger during any measurements.

口 Download the data from the two-sensor wave capture system onto floppy disks. Label the disks.

Quickly look at the data waveforms with the plotter program on the laptop to verify the signals were present.

Repack the wave capture systems and the laptop computer.

Remove the masking tape from the floor and retrieve any pencils and paper that may have been laid down in the room.

Be sure the logs and sketches are in the appliance file folder.

Thank the host or hostess and leave the site.

8. Prepare basic calculations of the data.

This section of the protocol is to be done after the site visit.

Verify that the files are present on the laptop computer. If they are absent, load the files from the floppy disks.

Prepare the data using the analysis programs. The data will be analyzed for the summary measures selected. An ASCII file will result with the summary measures listed along with coordinates.

Load the ASCIl files into a commercial spreadsheet package to adjust the levels based on the data from the two-sensor wave capture system. Export corrected ASCII files. 
- Load the ASCII files into the commercial plotting package and generate contour plots for the four vertical levels.

9. Reporting and Archiving.

口 Sign off on the log sheets with your name. If more than one person participated, sign all names. Date the signatures.

口 Note that calibration dates for the test set and for wave capture system were checked before their use.

- Verify that the folder contains data disks marked with the appliance names and dates, log sheets, and the contour plots.

Write a test report to the customer.

- Archive the data on a magnetic tape backup system. 
A-6 


\section{Appendix B An Example Protocol for Appliance Testing}

An actual protocol applied to a specific appliance is presented as an example of exercising the recommended guidelines. A toaster was chosen as an extremely simple appliance that falls into a category of the most simple of devices. It is a loop of wire run in a zig-zag manner to produce heat. The appliance could be characterized by one well chosen measurement. However, the simplicity of the toaster allows attention to be focused on the protocol rather than on the complexity of the appliance. This protocol may be used for measuring fields from much more complex sources.

\section{Appliance Test Set Protocol for a TOASTEB}

Purpose: To measure and quantify the steady state magnetic fields around an appliance using spherical coordinates and geometric distances as assembled on the appliance test set. This data may be used for modeling the appliance.

Items not Covered in this Protocol: Transient magnetic fields are not-addressed. Waveform data above $3 \mathrm{kHz}$ is not recorded (the RMS resultant is recorded). Electric fields are not recorded since the appliance is shielded. DC data is not taken for all sensors.

Overview: The test set is powered up and checked for consistency of operation. The appliance is examined and mounted on the test set. A pre-check test is performed to determine the highest fields around the appliance. The test set is adjusted to accommodate these fields. The appliance test sequence is conducted. The waveforms for the ELF band $(3 \mathrm{~Hz}-3 \mathrm{kHz})$ are recorded. The RMS resultant for the VLF $(3-30 \mathrm{kHz})$ and LF $(30-300 \mathrm{kHz})$ bands are recorded. The current and voltage waveforms are recorded. Nine measurements are taken near the appliance to assess the impact of the appliance on the DC (static) magnetic field. Data is developed for the RMS resultant of the power frequency and for harmonics to allow contour plotting. The data is stored and the appliance removed from the test set. Calculations are made to develop contour plots of eight vertical slices and one horizontal slice for the fundamental and each significant harmonic. Data is then archived.

Personnel Required: One person experienced in the operation of the test set is required for this test. 
1. Equipment Required.

- A loaf of bread to act as a load to the IOASTER.

The TOASTER. Select a residential toaster from a local supplier based on the mission of the test series.

- At least 4 blank formatted floppy disks, 3.5 inch High Density. Only 2 will be used to archive the data but have spares available. Apply blank labels to allow the disks to be marked with their contents.

- A laptop computer to allow the waveforms to be viewed.

口 A ruler and a tape measure marked in centimeters and meters for measurement.

\section{Test Set Operation Check:}

Note the last time the test set was calibrated. Recalibrate the test set every 6 months using the appliance test set calibration protocol.

$\square \quad$ Power up the Test Set computer.

$\square \quad$ Place the 200 Gauss calibration coil on the Test Set table and plug it into the shielded appliance power cable.

$\square \quad$ Set the dial on the variable transformer to the second notch.

$\square \quad$ Turn on the calibration coil using the appliance switch.

$\square$ Return to the computer and Select the CLBOO1 site file using the Site File menu.

Select the Probe Setup menu.

$\square \quad$ Cycle through the probes using the F10 key and enter the probe ID. Verify that each probe is operating by observing a waveform.

$\square \quad$ Turn off the calibration coil using the appliance switch.

$\square$ Disconnect the calibration coil and remove the coil from the table.

3. Identification of the appliance:

ㅁ Make a file folder for the appliance. Mark the name of the appliance on the folder - use the full name to designate the appliance.

Generate a log sheet for the appliance putting the full name of the appliance on the log sheet. Also mark your name, the date, and the time on the sheet. Use a separate sheet for all cycles of the appliance. Note that the IOASTEB has only one cycle.

ㅁ Record the name plate data on the log sheet.

$\square$ Draw a sketch of the appliance providing approximate measurements in $\mathrm{cm}$.

․ Measure the appliance and adjust the table height to bring the appliance horizontal physical center into the spar 4 axis (horizontal spar).

Place the appliance on the Test Set table.

Adjust the Turntable to 0 degrees and leave table unlocked. 
Adjust spar 1 (vertical top) until probe 11 is in contact with the top of the appliance.

Note: For the Toaster, move the probe 11 so that it is $2.5 \mathrm{~cm}$ from the top of the toaster to avoid excessive heat from the toasting process.

Adjust spar 7 (vertical bottom) until probe 71 is in contact with the bottom of the table.

Move spars 2, 3, 4, 5 and 6 until the probes 21, 31, 41, 51, and 61 just touch the appliance or the table as the appliance is rotated through 337.5 degrees. Note: For the Toaster, move probe 21,31 , and 41 so that they are $2.5 \mathrm{~cm}$ from the surface of the appliance to avoid excessive heat from the toasting process.

- Record in the log the distances from the center of the appliance (also now the center point of the test set) to the midpoint of the first sensor on each spar (the center of the cube transducer).

ㅁ Route the power cord straight down from the table at the location where the cord exits the appliance. Connect it to the shielded power cable of the test set located at 12 degrees on the 1 meter rod.

- Turn the appliance on and off to test the power connections. Lock the turntable at 0 degrees.

Photograph the appliance on the test bed at 0 degrees position on the turn table.

Assign the first three numbers of the classification code and put it on the log sheet - 101_for the toaster..

Assign three letters and three numbers for the file names - TSTO01 for the toaster.

4. Pretest the appliance.

Repeat this section of the protyocol for each cycle. of the appliance. Sections 4,5 and 6 should be done in order for each cycle of the appliance. Note the Toaster has only one cycle.

Use the wave capture system with a static field sensor to measure the magnetic fields.

Set the sample frequency to $7680 \mathrm{~Hz}$ and the sample count to 2048 .

Use the Spot-Measurement mode.

Set the file name to the same three letters and three numbers as for the test set, i.e. TSTO01.

Load slices of bread.

Turn on the appliance.

Take 6 measurements at the center of each of the six faces of the appliance. Note: for the IOASTEB do not keep the Static field transducer in the hot areas. Measure at least $2 \mathrm{~cm}$ from the hot faces. If the transducer overloads, take 
readings as close to the faces as possible and record the distances. The order is TOP, BOTTOM, 0 degree turntable mark, 90 degree mark, 180 degree mark, and 270 degree mark. Record the orientation of the transducer for each measurement. Have the $Z$ axis pointing toward the appliance and the $X$ axis pointing to the operator's left.

- Scan the appliance for the highest RMS Resultant reading. Record this reading as measurement 7. Draw the location of this reading on the sketches of the appliance. If the transducer overloads, locate the point where the highest fields that the transducer can measure and take sample 7 . Have the $Z$ axis pointing toward the appliance and the $X$ axis pointing to the operator's left.

- Take a sample 1 meter from the appliance on the horizontal axis at 0 location using the transducer (near probe 44 but stay $30 \mathrm{~cm}$ to the right of the probe). This is measurement 8 . Have the $Z$ axis pointing toward the appliance and the $X$ axis pointing to the operator's left.

․ Turn off the appliance.

Take a sample at the same location 1 meter from the appliance near probe 44 . This is measurement 9.

If there were overloads on the transducer, repeat the measurements 1 through 7 using a high field sensor.

Turn on the appliance.

Name the file with the three letters, the letter $C$ and the last two numbers, i.e. TSTC01

Get as close to the six surfaces as possible with measurements 1 through 6 and then locate the maximum field as measurement 7 . Log the location of measurement 7. Use the same axis orientations as for the static field transducer.

Turn off the appliance.

5. Examine the Pretest data.

Repeat this section of the protocol for each cycle.

- Transfer all wave capture files to a floppy disk. Do not erase the files from the static memory. Label the disk as the wave capture disk marking the appliance file name and date on the disk.

Power up the laptop computer either on battery or on the charger. (Do not have the charger plugged in during appliance testing.)

- Place the floppy disk into disk $A$ on the laptop. Start the plotting program. Select any printer, and load the data file, i.e. A:ITST001 lor A:ITSTC01 if the Static field transducer overloaded).

Observe the waveforms of the three axes of each of the first six measurements of the static field transducer (or high field sensor if the static field transducer 
overloaded). Select to remove DC but do not select to remove Motion. Select the next waveform using Enter.

Determine and record the highest harmonic present using the $F$ selection on the waveform plots. Return to the waveforms using $W$.

If the waveforms show sub-power harmonics or off-power harmonics greater than 10 percent of the highest harmonic present, calculate the sample frequency and snapshot duration. For the TOASTER set the sample frequency to $60 \mathrm{~Hz}$ base with 128 samples on the analog hardware entry.

Determine the maximum field expected using measurement 7 . Use a time plot and calculate the RMS Resultant.

- Exit the plotting program with ALT-X. Turn off the laptop and unplug the battery charger if it was used. Remove the floppy disk and place it in the folder.

Set the range switches on probes $11,21,31,4151,61$, and 71 (all must be set alike) to accommodate this highest reading. If the reading is below $100 \mu \mathrm{T}$, use the lowest scale ( $2 \mathrm{G}$ range). If the reading is between 100 and $1000 \mu \mathrm{T}$, use the middle scale $(20 \mathrm{G}$ range). If the reading is above $1000 \mu \mathrm{T}$, use the high scale $(200 \mathrm{G}$ range). Note the range selection on the log.

Set the range switches on probes $12,22,32,42,52,62$, and 72 (all must be set alike) to one range lower than the probes $11 . .71$ above. Note the range selection on the log.

- Set the range switches on probes $13,23,33,43,53,63$, and 73 (all must be set alike) to the lowest range ( $2 \mathrm{G}$ range). Note the range selection on the $\log$.

Go through all probes and set appropriate levels. The first sensor on each spar should be set to accommodate the largest field measured. Address all probes which are at first level, $11,21,31,41,51,61$, and 71 .

The second sensor on each spar should be set to 0.1 times the largest field measured. Since the sensors on a single spar are located geometrically, the second sensor should have an amplitude reduction in field level of about 10 for most appliances. Address all probes which are at second level, 12, 22, 32, $42,52,62$, and 72 .

The third sensor on each spar should be set to 0.01 times the largest.field measured. The reduction of 100 is derived from the geometric spacing of the sensors. Address all probes which are at the third level, $13,23,33,43,53$, 63 , and 73 .

The fourth sensor on each spar should be set to 0.001 times the largest field measured. Address all probes which are at the fourth level, 14, 24, 34, 44, 54,64 , and 74.

Address the current and voltage probes 84 and 85 to be sure they respond. 
6. Test the appliance.

Repeat this section of the protocol for each cycle of the appliance.

Load bread into TOASTER as needed.

Turn on appliance. Note that the toaster must be turned off after every reading and turned on before the next reading to limit heat on the transducers.

Take one reading of all probes in the record mode at turntable angle 0 degrees.

Take one reading of all probes for each turntable angle 22.5, 45, 67.5, 90, $112.5,135,157.5,180,202.5,225,247.5,270,292.5,315$, and 337.5. This is a total of 16 measurement samples (each sample has 30 transducer outputs at 3 axis each for ELF data and 21 transducer RMS outputs for VLF and LF data).

$\square \quad$ Turn the appliance off.

Take one sample of all probes with the appliance off. This is the 17th sample. Note in the log any errors in procedure such as taking two samples instead of one at one of the angles.

7. Remove the appliance from the test set.

Verify the power is off to the appliance.

Remove the appliance plug from the shielded power cable.

Remove the appliance from the test set table.

Transfer all Test Set Computer files to floppy disks. This includes the .SF2 file, the .LOG file and the .WFM file. Do not erase the files from the fixed disks. Label the disk as the Test Set Disk and mark the appliance file name and date on the disk.

Take the floppy disks to the appliance processing computer in the office.

Load the floppy disk data from both disks into the IDATA directory on the appliance processing computer.

When the floppy disk data has been transferred successfully to the appliance processing computer, erase the files from the test set computer and wave capture system. Do not erase the floppy disks. The data is now stored on the appliance processing computer and the floppy disks.

Return the appliance to the supplier or owner.

Place the two floppy disks in the file folder for the appliance.

Send the photographs out for processing.

Place the returned photographs in the file folder for the appliance.

Place the completed file folder into the appliance file drawer under the heading NEW MEASUREMENTS. 
8. Prepare basic calculations of the data.

This section of the protocol may be done at a later time. Performing this section of the protocol for several appliances would be an efficient use of time.

Remove the file folder for the appliance from the appliance file drawer from under the heading NEW MEASUREMENTS.

Verify that the files are present on the appliance computer. If they are absent, load the files from the floppy disks. Indicate on the computer log that data files were not present.

Run the A.BAT file followed by the six letter-numbers of the appliance, i.e. A TST001.

Use the ESC key twice. Use the cursor to select Conversion and then Convert. Use the Enter when Convert is highlighted. Use Enter again to select the 1st DAC board. Type A, and use Enter twice more to answer the two questions to the affirmative. The calculations will take about 10 minutes to obtain all the FFT and Time course data for the 1st DAC board.

When the Calculations Finished message appears, use ESC. Then select the 2nd DAC board with the cursor. Use Enter, type $A$ and use Enter twice more to begin the second set of calculations. The calculations will take about 20 minutes.

When the Calculations Finished message appears, use ESC five times to obtain the Exit Wave-A question. Answer the question by typing $Y$.

Use Enter at the Pause question on the screen. The Wave-A program will load and calculate all the $60 \mathrm{~Hz}$ RMS resultant levels for all transducers. Each transducer data will be placed in a separate file and a single beep on the computer will acknowledge that the file has been written. Thirty files will be written. The time required is about 10 minutes.

When the DOS Pause appears on the screen, use Enter to return to DOS. Change directories to the IDATA directory. Several files should now be present in that directory. They include .TT1, .TT2, .CT1, .DT1, DT2 and .TXT files. There should be 41 files with the first three letters and three numbers of the appliance.

- Copy the GENERAL.PRB file to a file name with the three letters and three numbers, i.e. COPY GENERAL.PRB TST001.PRB.

口 Open this file with an editor, i.e. EDIT TSTO01.PRB. Edit the distances from the center of the appliance to the center of the first probe on each spar using the data from the log sheet. Also edit any changes in the sample number at the various turntable angles as noted on the log sheet. Change any scaling as noted on the log sheet. Close the editor with ALT F and then $X$.

Run B.BAT with the file name of the appliance, i.e. B TSTO01. The program will read 28 text files and generate 11 files. These are the profile data sets for plotting magnetic profiles at eight vertical cuts $1.000, .023, .045, .067, .090$, 
$.113, .135$, and .157$)$ and one horizontal cut $(.00 \mathrm{H})$ through the test set. The file .COR contains the three-dimensional plot coordinates and values. The .COX contains the error file.

Open the .COX file with the editor, i.e. EDIT TST001.COX. Examine the file for probes that were removed from the calculation. If there were more than eight probe entries in this file, the appliance should be retested. Check carefully the maximum field and the settings of the probe ranges. Increase the range by one step for the probes that are noted in this file.

Note from the log the harmonic levels. If there were harmonic levels above 10 percent of the $60 \mathrm{~Hz}$ (power frequency), then process each harmonic. The IOASTEB had no harmonics other than $60 \mathrm{~Hz}$ fundamental.

- Use a commercial plotting program to plot the contours. Import each of the nine slice files for each harmonic. Make a plot of each slice.

This completes the calculation process for contour plotting.

9. Reporting and Archiving.

Sign off on the log sheets with your name. If more than one person participated, sign all names. Date the signatures.

Note that calibration dates for the test set and for wave capture system were checked before their use.

Verify that the folder contains 2 data disks marked with the appliance names and dates, one or more photographs, a log sheet or sheets, and at least nine contour plots.

Write a test report using EDIT with a file name of the first three letters and the three numbers followed by .RPT, i.e. EDIT TST001.RPT.

Write the report in ASCII format without a word processor. State the appliance, the appliance classification code, the appliance name plate, the date, the test person's name, and any special circumstances of the test, i.e. the load. Describe the dimensions and the location of the center of the appliance. State the voltage and current from the 30.TXT and 29.TXT files. Add statements about the purpose and limitations of the data. Add the pretyped section on location of further detailed data so that those having further interest can locate the original data. State the highest RMS resultant level detected and the location of that level. Load the $.00 \mathrm{H}$ slice file as an example of the activity on the horizontal plane for the $60 \mathrm{~Hz}$ fundamental. Include the. $\mathrm{OOH}$ slice files for any significant harmonics. Exit from EDIT with ALT F and $X$. Copy all files from the IDATA directory for each specific appliance onto four blank floppy disks. Label the disks with appliance name and date. Place these floppy disks into the folder.

Append the files from the IDATA directory for each specific appliance onto the master appliance archive tape. 


\section{Appendix C Appliance Test Data}

\section{Introduction}

The data presented in this appendix are sub-groups of the data recorded while the protocol presented in Task $C$ was developed. As the protocol development progressed, all the steps of the protocol were applied. However, not any one appliance had all steps applied. Some parts of the protocol had minimum testing as a result of equipment limitations.

The data taken demonstrates many of the characteristics that were found in the magnetic fields surrounding the appliances. Although the most intense magnetic field levels were recorded for each appliance, the focus of the measurements was not on a single magnetic measure. Rather, specific measures using harmonics and peaks were sought in an attempt to better characterize each appliance.

Three measurement systems were used. These systems had the following parameters:

System A. Wave-capture system with a three-axis magnetic field transducer measuring up to 5.7 Gauss peak with a frequency response of dc to $3 \mathrm{kHz}$. A base frequency of 3.75 Hertz was used with 2048 sample points taken which results in a sampling frequency per channel of 7680 Hertz. The resolution for the dc signal processing using FFT techniques was 0.6 milligauss and for the ac signal processing 0.015 milligauss.

System B. Wave-capture system with a three-axis magnetic field transducer measuring up to 200 Gauss peak with a frequency response of $5 \mathrm{Hertz}$ to $3 \mathrm{kHz}$. A base frequency of 60 Hertz was used with 128 samples for a sampling frequency per channel of $7680 \mathrm{Hertz}$. The resolution for the ac signal processing on the 20 Gauss range was 2.4 milligauss and on the 200 Gauss range was 24 milligauss.

System C. A multi-transducer wave-capture system with:

1. 42 three-axis ac transducers with ranges of 2, 20, and 200 Gauss peak. These transducers had a frequency bandwidth of $24 \mathrm{Hertz}$ to $300 \mathrm{kHz}$. There were filtering systems which separated the signals into $24 \mathrm{Hertz}$ to $3 \mathrm{kHz}$ which was recorded as three waveforms per transducer. Three $\mathrm{kHz}$ to $30 \mathrm{kHz}$ and $30 \mathrm{kHz}$ to $300 \mathrm{kHz}$ ranges which recorded as RMS resultant signals. The base frequency for the wave capture was 60 Hertz using 128 sample points for a sampling frequency of 7680 Hertz. 
2. 7 three-axis ac transducers with ranges of $1 \mathrm{mG}, 10 \mathrm{mG}, 100 \mathrm{mG}$ and 1000 mG RMS. These transducers had a frequency.bandwidth of $20 \mathrm{Hertz}$ to $3 \mathrm{kHz}$. The wave capture system had a base frequency of 60 Hertz using 128 points for a sampling frequency of 7680 Hertz per channel.

3. 2 special transducers to record ac current for a maximum of 20 Amperes RMS and ac voltage for a maximum of 150 Volts RMS. The frequency response was $20 \mathrm{Hertz}$ to $3 \mathrm{kHz}$. The wave capture had a base frequency of $60 \mathrm{Hertz}$ using 128 points for a sampling frequency of 7680 Hertz per channel.

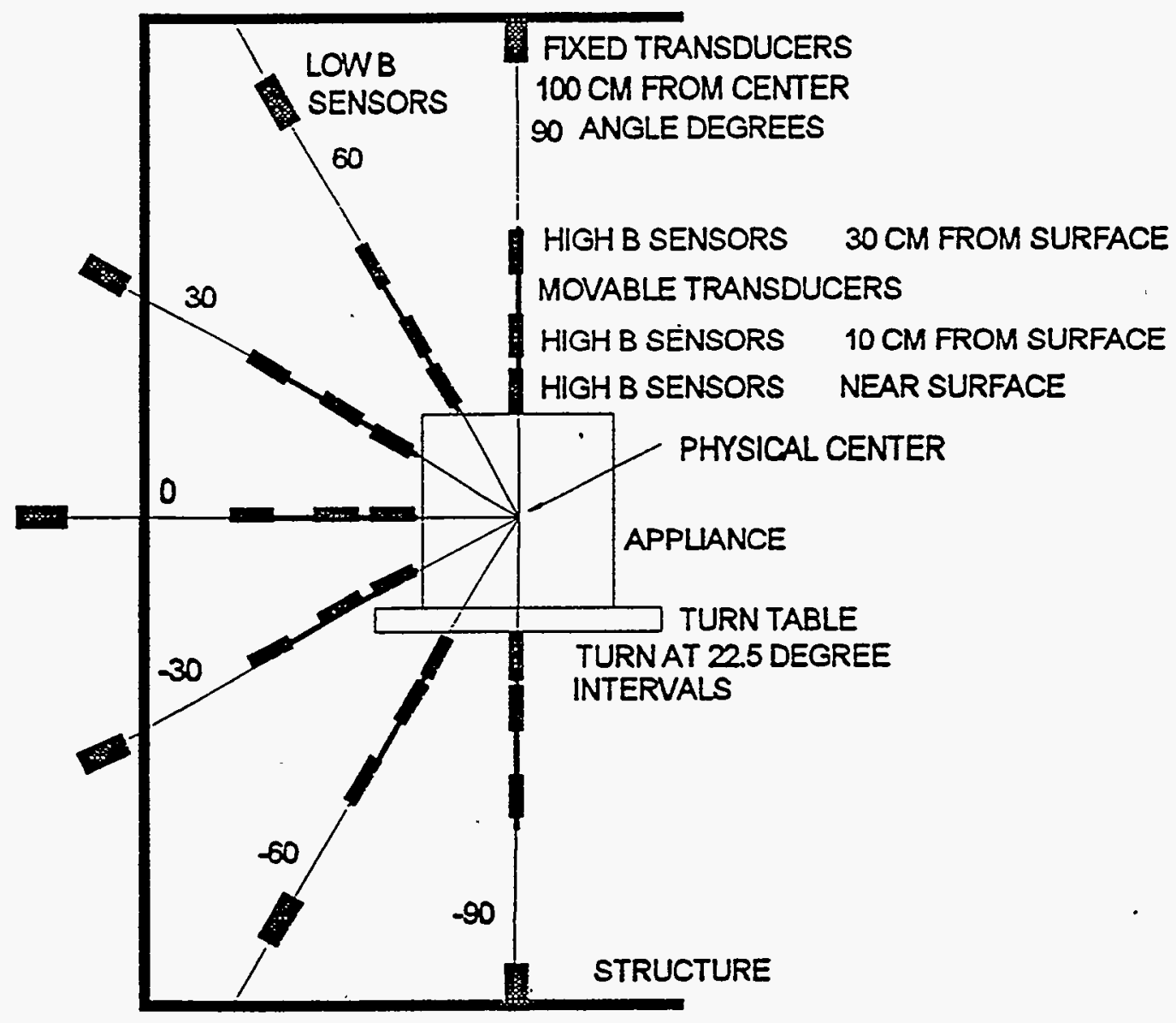

Figure C.1 A Simplified Diagram of the Appliance Test Set 


\section{C.1 Air Conditioner - Window}

Appliance Tested: Window Air Conditioner

Manufacturer: AAA

Model: 111

Rating: $5000 \mathrm{BTU} / \mathrm{Hr}$

Rated current: 6.2 Amperes

Appliance Code: 2247

Conditions for Testing: The air conditioner was operating with the fan and compressor running.

Measured voltage and current: 117.0 Volts and 5.39 Amperes.

Magnetic Field Summary:

$60 \mathrm{~Hz}$ RMS resultant level at $30 \mathrm{~cm}$ from front surface: $1.1 \mathrm{mG}$

RMS resultant low surface level: $3.6 \mathrm{mG}$ on right side

RMS resultant high surface level: $98 \mathrm{mG}$ on left side

Highest recorded surface levels:

60 Hertz RMS resultant: $97 \mathrm{mG}$

180 Hertz RMS resultant: $1.99 \mathrm{mG}$

300 Hertz RMS resultant: $0.91 \mathrm{mG}$

420 Hertz RMS resultant: $0.41 \mathrm{mG}$

For the appliance test set, the front of the unit was located at 180 degrees on the test table.

Summary of characteristics: The magnetic fields were primarily $60 \mathrm{Hertz}$ and fell rapidly from the appliance. 
Distance Horizontal Vertical $60 \mathrm{~Hz}$ meters angle deg angle deg mGauss

\begin{tabular}{|r|r|r|r|}
\hline 0.203 & 0.0 & 90 & 12.6 \\
\hline 0.303 & 0.0 & 90 & 5.0 \\
\hline 0.503 & 0.0 & 90 & 1.2 \\
\hline 1.000 & 0.0 & 90 & 0.2 \\
\hline 0.229 & 0.0 & 60 & 4.0 \\
\hline 0.229 & -22.5 & 60 & 3.0 \\
\hline 0.229 & -45.0 & 60 & 3.4 \\
\hline 0.229 & -67.5 & 60 & 4.1 \\
\hline 0.229 & -90.0 & 60 & 3.5 \\
\hline 0.229 & -112.5 & 60 & 2.7 \\
\hline 0.229 & -135.0 & 60 & 3.1 \\
\hline 0.229 & -157.5 & 60 & 3.7 \\
\hline 0.229 & 180.0 & 60 & 4.6 \\
\hline 0.229 & 157.5 & 60 & 6.3 \\
\hline 0.229 & 135.0 & 60 & 9.1 \\
\hline 0.229 & 112.5 & 60 & 13.0 \\
\hline 0.229 & 90.0 & 60 & 14.5 \\
\hline 0.229 & 67.5 & 60 & 13.0 \\
\hline 0.229 & 45.0 & 60 & 9.5 \\
\hline 0.229 & 22.5 & 60 & 6.3 \\
\hline 0.329 & 0.0 & 60 & 4.2 \\
\hline 0.329 & -22.5 & 60 & 4.0 \\
\hline 0.329 & -45.0 & 60 & 3.7 \\
\hline 0.329 & -67.5 & 60 & 3.3 \\
\hline 0.329 & -90.0 & 60 & 2.8 \\
\hline 0.329 & -112.5 & 60 & 2.5 \\
\hline 0.329 & -135.0 & 60 & 2.5 \\
\hline 0.329 & -157.5 & 60 & 2.6 \\
\hline 0.329 & 180.0 & 60 & 2.9 \\
\hline 0.329 & 157.5 & 60 & 3.5 \\
\hline
\end{tabular}

Table C.1 Air Conditioner $60 \mathrm{~Hz}$ RMS Resultant Fields

Distance Horizontal Vertical $60 \mathrm{~Hz}$ meters angle deg angle deg mGauss

\begin{tabular}{|r|r|r|r|}
\hline 0.329 & 135.0 & 60 & 4.5 \\
\hline 0.329 & 112.5 & 60 & 5.3 \\
\hline 0.329 & 90.0 & 60 & 5.6 \\
\hline 0.329 & 67.5 & 60 & 5.5 \\
\hline 0.329 & 45.0 & 60 & 5.0 \\
\hline 0.329 & 22.5 & 60 & 4.6 \\
\hline 0.529 & 0.0 & 60 & 1.3 \\
\hline 0.529 & -22.5 & 60 & 1.2 \\
\hline 0.529 & -45.0 & 60 & 1.1 \\
\hline 0.529 & -67.5 & 60 & 1.0 \\
\hline 0.529 & -90.0 & 60 & 1.0 \\
\hline 0.529 & -112.5 & 60 & 0.9 \\
\hline 0.529 & -135.0 & 60 & 0.9 \\
\hline 0.529 & -157.5 & 60 & 0.8 \\
\hline 0.529 & 180.0 & 60 & 0.9 \\
\hline 0.529 & 157.5 & 60 & 1.1 \\
\hline 0.529 & 135.0 & 60 & 1.3 \\
\hline 0.529 & 112.5 & 60 & 1.4 \\
\hline 0.529 & 90.0 & 60 & 1.5 \\
\hline 0.529 & 67.5 & 60 & 1.5 \\
\hline 0.529 & 45.0 & 60 & 1.4 \\
\hline 0.529 & 22.5 & 60 & 1.4 \\
\hline 1.000 & 0.0 & 60 & 0.3 \\
\hline 1.000 & -22.5 & 60 & 0.2 \\
\hline 1.000 & -45.0 & 60 & 0.2 \\
\hline 1.000 & -67.5 & 60 & 0.2 \\
\hline 1.000 & -90.0 & 60 & 0.2 \\
\hline 1.000 & -112.5 & 60 & 0.2 \\
\hline 1.000 & -135.0 & 60 & 0.2 \\
\hline 1.000 & -157.5 & 60 & 0.2 \\
\hline & & & \\
\hline
\end{tabular}

C. $1-2$ 
Distance Horizontal Vertical $60 \mathrm{~Hz}$ meters angle deg angle deg mGauss

\begin{tabular}{|r|r|r|r|}
\hline 1.000 & 180.0 & 60 & 0.2 \\
\hline 1.000 & 157.5 & 60 & 0.3 \\
\hline 1.000 & 135.0 & 60 & 0.3 \\
\hline 1.000 & 112.5 & 60 & 0.3 \\
\hline 1.000 & 90.0 & 60 & 0.3 \\
\hline 1.000 & 67.5 & 60 & 0.3 \\
\hline 1.000 & 45.0 & 60 & 0.3 \\
\hline 1.000 & 22.5 & 60 & 0.3 \\
\hline 0.305 & 0.0 & 30 & 6.3 \\
\hline 0.305 & -22.5 & 30 & 5.7 \\
\hline 0.305 & -45.0 & 30 & 5.4 \\
\hline 0.305 & -67.5 & 30 & 3.9 \\
\hline 0.305 & -90.0 & 30 & 2.4 \\
\hline 0.305 & -112.5 & 30 & 2.7 \\
\hline 0.305 & -135.0 & 30 & 4.0 \\
\hline 0.305 & -157.5 & 30 & 2.9 \\
\hline 0.305 & 180.0 & 30 & 3.4 \\
\hline 0.305 & 157.5 & 30 & 4.8 \\
\hline 0.305 & 135.0 & 30 & 9.5 \\
\hline 0.305 & 112.5 & 30 & 13.3 \\
\hline 0.305 & 90.0 & 30 & 17.0 \\
\hline 0.305 & 67.5 & 30 & 16.2 \\
\hline 0.305 & 45.0 & 30 & 17.2 \\
\hline 0.305 & 22.5 & 30 & 8.7 \\
\hline 0.405 & 0.0 & 30 & 3.1 \\
\hline 0.405 & -22.5 & 30 & 2.7 \\
\hline 0.405 & -45.0 & 30 & 2.4 \\
\hline 0.405 & -67.5 & 30 & 2.1 \\
\hline 0.405 & -90.0 & 30 & 1.7 \\
\hline 0.405 & -112.5 & 30 & 1.6 \\
\hline
\end{tabular}

Table C.1 Air Conditioner $60 \mathrm{~Hz}$ RMS Resultant Fields

Dislance Horizontal Vertical $60 \mathrm{~Hz}$

meters angle deg angle deg $m$ Gauss

\begin{tabular}{|c|c|c|c|}
\hline 0.405 & -135.0 & 30 & 1.8 \\
\hline 0.405 & -157.5 & 30 & 1.8 \\
\hline 0.405 & 180.0 & 30 & 2.1 \\
\hline 0.405 & 157.5 & 30 & 2.8 \\
\hline 0.405 & 135.0 & 30 & 4.2 \\
\hline 0.405 & 112.5 & 30 & 5.5 \\
\hline 0.405 & 90.0 & 30 & 6.4 \\
\hline 0.405 & 67.5 & 30 & 6.4 \\
\hline 0.405 & 45.0 & 30 & 5.6 \\
\hline 0.405 & 22.5 & 30 & 4.1 \\
\hline 0.605 & 0.0 & 30 & 1.1 \\
\hline 0.605 & -22.5 & 30 & 1.1 \\
\hline 0.605 & -45.0 & 30 & 0.9 \\
\hline 0.605 & -67.5 & 30 & 1.0 \\
\hline 0.605 & .90 .0 & 30 & 0.8 \\
\hline 0.605 & -112.5 & 30 & 0.8 \\
\hline 0.605 & -135.0 & 30 & 0.6 \\
\hline 0.605 & -157.5 & 30 & 0.6 \\
\hline 0.605 & 180.0 & 30 & 0.6 \\
\hline 0.605 & 157.5 & 30 & 0.7 \\
\hline 0.605 & 135.0 & 30 & 1.1 \\
\hline 0.605 & 112.5 & 30 & 1.4 \\
\hline 0.605 & 90.0 & 30 & 1.7 \\
\hline 0.605 & 67.5 & 30 & 1.8 \\
\hline 0.605 & 45.0 & 30 & 1.6 \\
\hline 0.605 & 22.5 & 30 & 1.5 \\
\hline 1.305 & 0.0 & 30 & 0.1 \\
\hline 1.305 & -22.5 & 30 & 0.1 \\
\hline 1.305 & -45.0 & 30 & 0.1 \\
\hline 1.305 & -67.5 & 30 & 0.1 \\
\hline
\end{tabular}


Distance Horizontal Vertical $60 \mathrm{~Hz}$ meters angle deg angle deg mGauss

\begin{tabular}{|r|r|r|r|}
\hline 1.305 & -90.0 & 30 & 0.1 \\
\hline 1.305 & -112.5 & 30 & 0.1 \\
\hline 1.305 & -135.0 & 30 & 0.1 \\
\hline 1.305 & -157.5 & 30 & 0.1 \\
\hline 1.305 & 180.0 & 30 & 0.2 \\
\hline 1.305 & 157.5 & 30 & 0.2 \\
\hline 1.305 & 135.0 & 30 & 0.2 \\
\hline 1.305 & 112.5 & 30 & 0.2 \\
\hline 1.305 & 90.0 & 30 & 0.2 \\
\hline 1.305 & 67.5 & 30 & 0.2 \\
\hline 1.305 & 45.0 & 30 & 0.2 \\
\hline 1.305 & 22.5 & 30 & 0.2 \\
\hline 0.267 & 0.0 & 0 & 8.1 \\
\hline 0.267 & -22.5 & 0 & 6.7 \\
\hline 0.267 & -45.0 & 0 & 5.3 \\
\hline 0.267 & -67.5 & 0 & 4.0 \\
\hline 0.267 & -90.0 & 0 & 2.5 \\
\hline 0.267 & -112.5 & 0 & 2.2 \\
\hline 0.267 & -135.0 & 0 & 4.3 \\
\hline 0.267 & -157.5 & 0 & 3.0 \\
\hline 0.267 & 180.0 & 0 & 3.6 \\
\hline 0.267 & 157.5 & 0 & 5.5 \\
\hline 0.267 & 135.0 & 0 & 12.5 \\
\hline 0.267 & 112.5 & 0 & 19.7 \\
\hline 0.267 & 90.0 & 0 & 22.4 \\
\hline 0.267 & 67.5 & -0 & 21.7 \\
\hline 0.267 & 45.0 & 0 & 20.5 \\
\hline 0.267 & 22.5 & 0 & 13.2 \\
\hline 0.367 & 0.0 & 0 & 3.8 \\
\hline 0.367 & -22.5 & 0 & 3.2 \\
\hline
\end{tabular}

Table C.1 Air Conditioner

\section{$60 \mathrm{~Hz}$ RMS Resultant Fields}

Distance Horizontal Vertical $60 \mathrm{~Hz}$

meters angle deg angle deg mGauss

\begin{tabular}{|c|c|c|c|}
\hline 0.367 & -45.0 & 0 & 2.8 \\
\hline 0.367 & -67.5 & 0 & 2.2 \\
\hline 0.367 & -90.0 & 0 & 1.8 \\
\hline 0.367 & -112.5 & 0 & 1.6 \\
\hline 0.367 & -135.0 & 0 & 1.9 \\
\hline 0.367 & -157.5 & 0 & 1.8 \\
\hline 0.367 & 180.0 & 0 & 2.0 \\
\hline 0.367 & 157.5 & 0 & 2.9 \\
\hline 0.367 & 135.0 & 0 & 4.8 \\
\hline 0.367 & 112.5 & 0 & 7.5 \\
\hline 0.367 & 90.0 & 0 & 10.1 \\
\hline 0.367 & 67.5 & 0 & 10.6 \\
\hline 0.367 & 45.0 & 0 & 8.9 \\
\hline 0.367 & 22.5 & 0 & 5.6 \\
\hline 0.567 & 0.0 & 0 & 1.2 \\
\hline 0.567 & -22.5 & 0 & 1.0 \\
\hline 0.567 & -45.0 & 0 & 1.0 \\
\hline 0.567 & -67.5 & 0 & 0.8 \\
\hline 0.567 & -90.0 & 0 & 0.7 \\
\hline 0.567 & -112.5 & 0 & 0.7 \\
\hline 0.567 & -135.0 & 0 & 0.7 \\
\hline 0.567 & -157.5 & 0 & 0.7 \\
\hline 0.567 & 180.0 & 0 & 0.8 \\
\hline 0.567 & 157.5 & 0 & 1.1 \\
\hline 0.567 & 135.0 & 0 & 1.4 \\
\hline 0.567 & 112.5 & 0 & 2.0 \\
\hline 0.567 & 90.0 & 0 & 2.4 \\
\hline 0.567 & 67.5 & 0 & 2.4 \\
\hline 0.567 & 45.0 & 0 & 2.1 \\
\hline 0.567 & 22.5 & 0 & 1.5 \\
\hline
\end{tabular}


Distance Horizontal Vertical $60 \mathrm{~Hz}$ meters angle deg angle deg mGauss

\begin{tabular}{|c|r|r|r|}
\hline 1.267 & 0.0 & 0 & 0.1 \\
\hline 1.267 & -22.5 & 0 & 0.1 \\
\hline 1.267 & -45.0 & 0 & 0.1 \\
\hline 1.267 & -67.5 & 0 & 0.1 \\
\hline 1.267 & -90.0 & 0 & 0.1 \\
\hline 1.267 & -112.5 & 0 & 0.1 \\
\hline 1.267 & -135.0 & 0 & 0.1 \\
\hline 1.267 & -157.5 & 0 & 0.1 \\
\hline 1.267 & 180.0 & 0 & 0.1 \\
\hline 1.267 & 157.5 & 0 & 0.2 \\
\hline 1.267 & 135.0 & 0 & 0.2 \\
\hline 1.267 & 112.5 & 0 & 0.2 \\
\hline 1.267 & 90.0 & 0 & 0.2 \\
\hline 1.267 & 67.5 & 0 & 0.2 \\
\hline 1.267 & 45.0 & 0 & 0.2 \\
\hline 1.267 & 22.5 & 0 & 0.2 \\
\hline 0.330 & 0.0 & -30 & 4.4 \\
\hline 0.330 & -22.5 & -30 & 3.7 \\
\hline 0.330 & -45.0 & -30 & 3.9 \\
\hline 0.330 & -67.5 & -30 & 3.3 \\
\hline 0.330 & -90.0 & -30 & 2.7 \\
\hline 0.330 & -112.5 & -30 & 2.6 \\
\hline 0.330 & -135.0 & -30 & 3.0 \\
\hline 0.330 & -157.5 & -30 & 2.4 \\
\hline 0.330 & 180.0 & -30 & 3.2 \\
\hline 0.330 & 157.5 & -30 & 7.0 \\
\hline 0.330 & 135.0 & -30 & 9.9 \\
\hline 0.330 & 112.5 & -30 & 10.9 \\
\hline 0.330 & 90.0 & -30 & 11.8 \\
\hline 0.330 & 67.5 & -30 & 11.9 \\
\hline
\end{tabular}

Table C.1 Air Conditioner

$60 \mathrm{~Hz}$ RMS Resultant Fields

Distance Horizontal Vertical $60 \mathrm{~Hz}$

meters angle deg angle deg mGauss

\begin{tabular}{|r|r|r|r|}
\hline 0.330 & 45.0 & -30 & 11.2 \\
\hline 0.330 & 22.5 & -30 & 6.5 \\
\hline 0.430 & 0.0 & -30 & 2.4 \\
\hline 0.430 & -22.5 & -30 & 2.0 \\
\hline 0.430 & -45.0 & -30 & 1.9 \\
\hline 0.430 & -67.5 & -30 & 1.7 \\
\hline 0.430 & -90.0 & -30 & 1.5 \\
\hline 0.430 & -112.5 & -30 & 1.5 \\
\hline 0.430 & -135.0 & -30 & 1.5 \\
\hline 0.430 & -157.5 & -30 & 1.5 \\
\hline 0.430 & 180.0 & -30 & 1.8 \\
\hline 0.430 & 157.5 & -30 & 2.7 \\
\hline 0.430 & 135.0 & -30 & 5.1 \\
\hline 0.430 & 112.5 & -30 & 4.7 \\
\hline 0.430 & 90.0 & -30 & 5.3 \\
\hline 0.430 & 67.5 & -30 & 5.3 \\
\hline 0.430 & 45.0 & -30 & 4.6 \\
\hline 0.430 & 22.5 & -30 & 3.3 \\
\hline 0.630 & 0.0 & -30 & 0.8 \\
\hline 0.630 & -22.5 & -30 & 0.8 \\
\hline 0.630 & -45.0 & -30 & 0.7 \\
\hline 0.630 & -67.5 & -30 & 0.6 \\
\hline 0.630 & -90.0 & -30 & 0.6 \\
\hline 0.630 & -112.5 & -30 & 0.6 \\
\hline 0.630 & -135.0 & -30 & 0.7 \\
\hline 0.630 & -157.5 & -30 & 0.7 \\
\hline 0.630 & 180.0 & -30 & 0.8 \\
\hline 0.630 & 157.5 & -30 & 0.9 \\
\hline 0.630 & 135.0 & -30 & 1.7 \\
\hline 0.630 & 112.5 & -30 & 1.5 \\
\hline
\end{tabular}


Distance Horizontal Vertical $60 \mathrm{~Hz}$ meters angle deg angle deg mGauss

\begin{tabular}{|c|c|c|c|}
\hline 0.630 & 90.0 & -30 & 1.7 \\
\hline 0.630 & 67.5 & .30 & 1.6 \\
\hline 0.630 & 45.0 & .30 & 1.4 \\
\hline 0.630 & 22.5 & .30 & 1.1 \\
\hline 1.330 & 0.0 & .30 & 0.1 \\
\hline 1.330 & -22.5 & -30 & 0.1 \\
\hline 1.330 & -45.0 & .30 & 0.1 \\
\hline 1.330 & -67.5 & -30 & 0.1 \\
\hline 1.330 & -90.0 & -30 & 0.1 \\
\hline 1.330 & -112.5 & -30 & 0.1 \\
\hline 1.330 & -135.0 & .30 & 0.1 \\
\hline 1.330 & -157.5 & -30 & 0.1 \\
\hline 1.330 & 180.0 & -30 & 0.1 \\
\hline 1.330 & 157.5 & -30 & 0.1 \\
\hline 1.330 & 135.0 & .30 & 0.1 \\
\hline 1.330 & $\$ 12.5$ & -30 & 0.1 \\
\hline 1.330 & 90.0 & -30 & 0.1 \\
\hline 1.330 & 67.5 & -30 & 0.1 \\
\hline 1.330 & 45.0 & -30 & 0.1 \\
\hline 1.330 & 22.5 & -30 & 0.1 \\
\hline 0.279 & 0.0 & -60 & 7.9 \\
\hline 0.279 & -22.5 & -60 & 6.6 \\
\hline 0.279 & -45.0 & -60 & 5.4 \\
\hline 0.279 & -67.5 & -60 & 4.7 \\
\hline 0.279 & -90.0 & -60 & 4.1 \\
\hline 0.279 & -112.5 & -60 & 4.0 \\
\hline 0.279 & -135.0 & -60 & 4.3 \\
\hline 0.279 & -157.5 & -60 & 4.8 \\
\hline 0.279 & 180.0 & -60 & 5.6 \\
\hline 0.279 & 157.5 & -60 & 9.1 \\
\hline
\end{tabular}

Table C.1 Air Conditioner

$60 \mathrm{~Hz}$ RMS Resultant Fields

Distance Horizontal Vertical $60 \mathrm{~Hz}$

meters angle deg angle deg mGauss

\begin{tabular}{|l|r|r|r|}
\hline 0.279 & 135.0 & -60 & 14.5 \\
\hline 0.279 & 112.5 & -60 & 13.2 \\
\hline 0.279 & 90.0 & -60 & 14.2 \\
\hline 0.279 & 67.5 & -60 & 14.0 \\
\hline 0.279 & 45.0 & -60 & 12.2 \\
\hline 0.279 & 22.5 & -60 & 9.9 \\
\hline 0.379 & 0.0 & -60 & 2.9 \\
\hline 0.379 & -22.5 & -60 & 2.5 \\
\hline 0.379 & -45.0 & -60 & 2.2 \\
\hline 0.379 & -67.5 & -60 & 2.1 \\
\hline 0.379 & -90.0 & -60 & 2.0 \\
\hline 0.379 & -112.5 & -60 & 1.9 \\
\hline 0.379 & -135.0 & -60 & 1.9 \\
\hline 0.379 & -157.5 & -60 & 2.1 \\
\hline 0.379 & 180.0 & -60 & 2.4 \\
\hline 0.379 & 157.5 & -60 & 7.6 \\
\hline 0.379 & 135.0 & -60 & 6.8 \\
\hline 0.379 & 112.5 & -60 & 4.1 \\
\hline 0.379 & 90.0 & -60 & 5.0 \\
\hline 0.379 & 67.5 & -60 & 5.1 \\
\hline 0.379 & 45.0 & -60 & 4.7 \\
\hline 0.379 & 22.5 & -60 & 3.7 \\
\hline 0.579 & 0.0 & -60 & 0.9 \\
\hline 0.579 & -22.5 & -60 & 0.9 \\
\hline 0.579 & -45.0 & -60 & 0.8 \\
\hline 0.579 & -67.5 & -60 & 0.8 \\
\hline 0.579 & -90.0 & -60 & 0.8 \\
\hline 0.579 & -112.5 & -60 & 0.8 \\
\hline 0.579 & -135.0 & -60 & 0.9 \\
\hline 0.579 & -157.5 & -60 & 0.9 \\
\hline
\end{tabular}


Distance Horizontal Vertical $60 \mathrm{~Hz}$

meters angle deg angle deg $m$ Gauss

\begin{tabular}{|l|r|r|r|}
\hline 0.579 & 180.0 & -60 & 1.0 \\
\hline 0.579 & 157.5 & -60 & 2.8 \\
\hline 0.579 & 135.0 & -60 & 2.1 \\
\hline 0.579 & 112.5 & -60 & 3.5 \\
\hline 0.579 & 90.0 & -60 & 3.1 \\
\hline 0.579 & 67.5 & -60 & 2.3 \\
\hline 0.579 & 45.0 & -60 & 1.8 \\
\hline 0.579 & 22.5 & -60 & 1.3 \\
\hline 1.000 & 0.0 & -60 & 0.3 \\
\hline 1.000 & -22.5 & -60 & 0.2 \\
\hline 1.000 & -45.0 & -60 & 0.2 \\
\hline 1.000 & -67.5 & -60 & 0.2 \\
\hline 1.000 & -90.0 & -60 & 0.2 \\
\hline 1.000 & -112.5 & -60 & 0.2 \\
\hline
\end{tabular}

Table C.1 Air Conditioner

$60 \mathrm{~Hz}$ RMS Resultant Fields

Distance Horizontal Vertical $60 \mathrm{~Hz}$ melers angle deg angle deg mGauss

\begin{tabular}{|l|r|r|r|}
\hline 1.000 & -135.0 & -60 & 0.2 \\
\hline 1.000 & -157.5 & -60 & 0.2 \\
\hline 1.000 & 180.0 & -60 & 0.2 \\
\hline 1.000 & 157.5 & -60 & 0.3 \\
\hline 1.000 & 135.0 & -60 & 1.6 \\
\hline 1.000 & 112.5 & -60 & 0.4 \\
\hline 1.000 & 90.0 & -60 & 0.3 \\
\hline 1.000 & 67.5 & -60 & 0.3 \\
\hline 1.000 & 45.0 & -60 & 0.2 \\
\hline 1.000 & 22.5 & -60 & 0.3 \\
\hline 0.203 & 0.0 & -90 & 6.4 \\
\hline 0.303 & 0.0 & -90 & 3.7 \\
\hline 0.503 & 0.0 & -90 & 1.7 \\
\hline
\end{tabular}




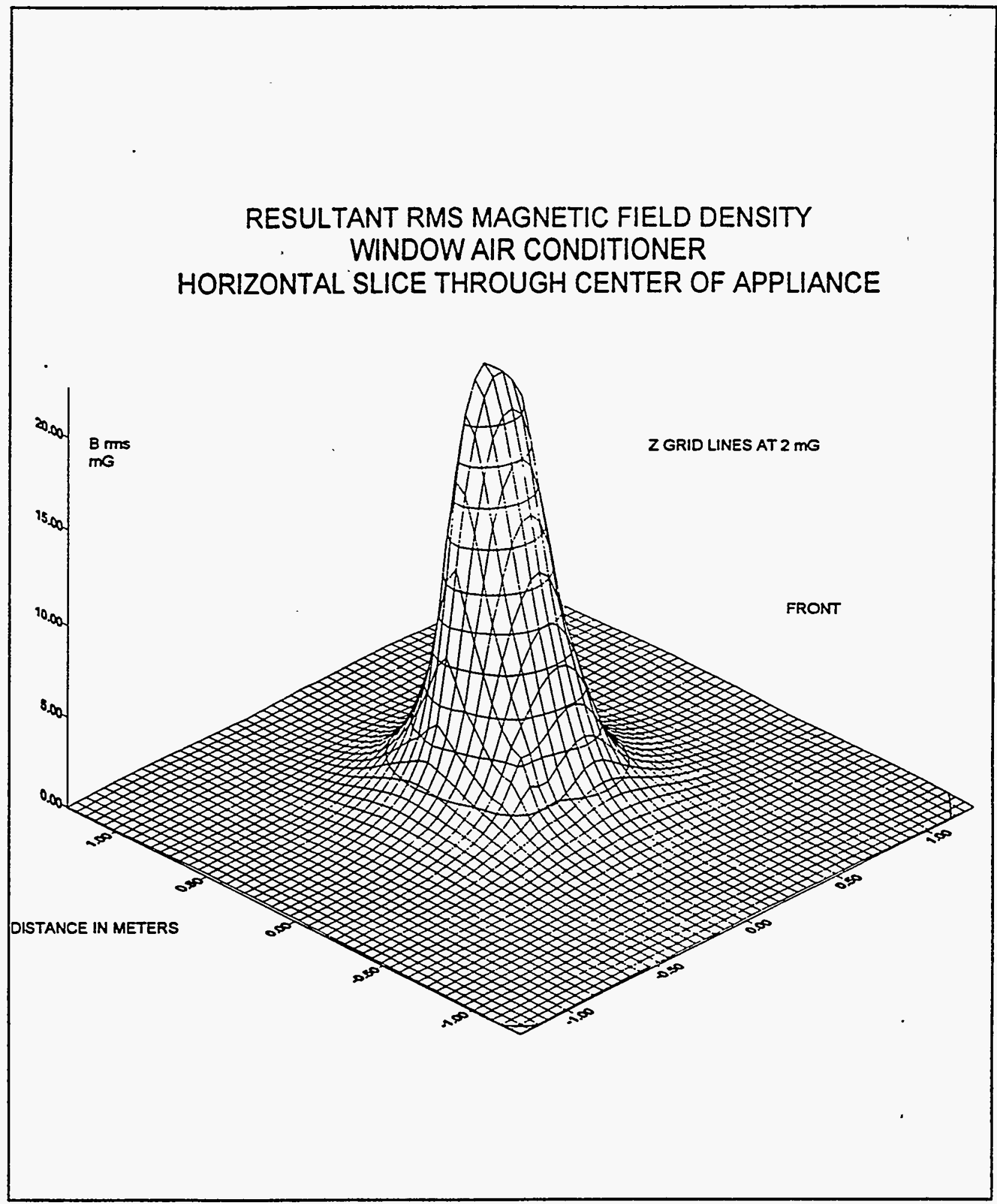

Figure C.1.1 Fundamental Resultant RMS Magnetic Field Density - Window Air Conditioner. 


\section{C.2 Blender}

Appliance Tested: Kitchen Blender

Manufacturer: BBB

Model: 222

Rated power: 600 Watts

Appliance Code: 3263

Conditions for Testing: The blender was half-full of water and was operating on the highest speed selection.

Measured voltage and current: 116.7 Volts and 2.66 Amperes.

Magnetic Field Summary:

$60 \mathrm{~Hz}$ RMS resultant level at $30 \mathrm{~cm}$ from front surface: $18.6 \mathrm{mG}$

RMS resultant low surface level: $177 \mathrm{mG}$ at bottom

RMS resultant high surface level: $3210 \mathrm{mG}$ at rear

Highest recorded surface levels:

60 Hertz RMS resultant: $7913 \mathrm{mG}$

180 Hertz RMS resultant: $1825 \mathrm{mG}$

300 Hertz RMS resultant: $468 \mathrm{mG}$

420 Hertz RMS resultant: $27 \mathrm{mG}$

For the appliance test set, the front of the unit was located at 180 degrees on the test table.

Summary of characteristics: The blender has a significant field near the device but the field falls off rapidly. There are strong harmonics in the field. 
Table C.2 Blender

$60 \mathrm{~Hz}, 180 \mathrm{~Hz}$, and $300 \mathrm{~Hz}$ RMS Resultant Fields

Distance Horizontal Vertical $60 \mathrm{~Hz} \quad 180 \mathrm{~Hz} \quad 300 \mathrm{~Hz}$ meters Angle deg Angle deg mGauss mGauss mGauss

$\begin{array}{llll} & 180 \mathrm{~Hz} & 300 \mathrm{~Hz}\end{array}$

\begin{tabular}{|r|r|r|r|r|r|}
\hline 0.318 & 0.0 & 90 & 266.0 & 73.8 & 20.0 \\
\hline 0.418 & 0.0 & 90 & 13.0 & 4.0 & 1.0 \\
\hline 0.618 & 0.0 & 90 & 3.5 & 1.0 & 0.3 \\
\hline 1.000 & 0.0 & 90 & 1.0 & 0.3 & 0.1 \\
\hline 0.254 & 0.0 & 60 & 573.0 & 158.5 & 40.1 \\
\hline 0.254 & -22.5 & 60 & 609.4 & 166.7 & 42.6 \\
\hline 0.254 & -45.0 & 60 & 574.9 & 154.1 & 37.6 \\
\hline 0.254 & -67.5 & 60 & 503.7 & 134.0 & 33.4 \\
\hline 0.254 & -90.0 & 60 & 453.4 & 130.3 & 36.2 \\
\hline 0.254 & -112.5 & 60 & 441.6 & 130.6 & 37.3 \\
\hline 0.254 & -135.0 & 60 & 486.0 & 145.8 & 42.7 \\
\hline 0.254 & -157.5 & 60 & 543.2 & 156.1 & 41.6 \\
\hline 0.254 & 180.0 & 60 & 549.3 & 158.6 & 45.6 \\
\hline 0.254 & 157.5 & 60 & 527.1 & 143.7 & 39.5 \\
\hline 0.254 & 135.0 & 60 & 516.8 & 138.1 & 36.7 \\
\hline 0.254 & 112.5 & 60 & 460.2 & 120.6 & 35.4 \\
\hline 0.254 & 90.0 & 60 & 438.4 & 117.4 & 33.0 \\
\hline 0.254 & 67.5 & 60 & 405.9 & 107.9 & 27.3 \\
\hline 0.254 & 45.0 & 60 & 420.8 & 118.7 & 33.3 \\
\hline 0.254 & 22.5 & 60 & 478.3 & 138.5 & 38.0 \\
\hline 0.354 & 0.0 & 60 & 21.2 & 5.8 & 1.4 \\
\hline 0.354 & -22.5 & 60 & 22.5 & 6.0 & 1.4 \\
\hline 0.354 & -45.0 & 60 & 21.3 & 5.7 & 1.4 \\
\hline 0.354 & -67.5 & 60 & 18.8 & 5.0 & 1.2 \\
\hline 0.354 & -90.0 & 60 & 17.1 & 4.8 & 1.2 \\
\hline 0.354 & -112.5 & 60 & 16.8 & 5.0 & 1.4 \\
\hline 0.354 & -135.0 & 60 & 18.5 & 5.6 & 1.6 \\
\hline 0.354 & -157.5 & 60 & 20.9 & 6.1 & 1.7 \\
\hline 0.354 & 180.0 & 60 & 21.6 & 6.3 & 1.8 \\
\hline 0.354 & 157.5 & 60 & 21.0 & 5.8 & 1.6 \\
\hline
\end{tabular}

\begin{tabular}{|l|r|r|r|r|r|}
\hline 0.354 & 135.0 & 60 & 20.7 & 5.6 & 1.5 \\
\hline 0.354 & 112.5 & 60 & 18.3 & 4.9 & 1.5 \\
\hline 0.354 & 90.0 & 60 & 17.2 & 4.8 & 1.4 \\
\hline 0.354 & 67.5 & 60 & 15.7 & 4.3 & 1.1 \\
\hline 0.354 & 45.0 & 60 & 15.9 & 4.6 & 1.2 \\
\hline 0.354 & 22.5 & 60 & 17.9 & 5.1 & 1.4 \\
\hline 0.554 & 0.0 & 60 & 5.5 & 1.5 & 0.4 \\
\hline 0.554 & -22.5 & 60 & 5.8 & 1.6 & 0.4 \\
\hline 0.554 & -45.0 & 60 & 5.5 & 1.4 & 0.4 \\
\hline 0.554 & -67.5 & 60 & 5.0 & 1.3 & 0.3 \\
\hline 0.554 & -90.0 & 60 & 4.6 & 1.3 & 0.3 \\
\hline 0.554 & -112.5 & 60 & 4.5 & 1.3 & 0.4 \\
\hline 0.554 & -135.0 & 60 & 5.0 & 1.5 & 0.4 \\
\hline 0.554 & -157.5 & 60 & 5.8 & 1.7 & 0.5 \\
\hline 0.554 & 180.0 & 60 & 6.1 & 1.8 & 0.5 \\
\hline 0.554 & 157.5 & 60 & 5.9 & 1.7 & 0.5 \\
\hline 0.554 & 135.0 & 60 & 5.9 & 1.6 & 0.4 \\
\hline 0.554 & 112.5 & 60 & 5.2 & 1.4 & 0.4 \\
\hline 0.554 & 90.0 & 60 & 4.8 & 1.3 & 0.4 \\
\hline 0.554 & 67.5 & 60 & 4.3 & 1.2 & 0.3 \\
\hline 0.554 & 45.0 & 60 & 4.3 & 1.2 & 0.3 \\
\hline 0.554 & 22.5 & 60 & 4.7 & 1.3 & 0.4 \\
\hline 1.000 & 0.0 & 60 & 0.9 & 0.2 & 0.1 \\
\hline 9.000 & -22.5 & 60 & 1.0 & 0.2 & 0.1 \\
\hline 1.000 & -45.0 & 60 & 0.9 & 0.2 & 0.1 \\
\hline 1.000 & -67.5 & 60 & 0.8 & 0.2 & 0.1 \\
\hline 1.000 & -90.0 & 60 & 0.7 & 0.2 & 0.1 \\
\hline 1.000 & -112.5 & 60 & 0.8 & 0.2 & 0.1 \\
\hline 1.000 & -135.0 & 60 & 0.9 & 0.3 & 0.1 \\
\hline 1.000 & -157.5 & 60 & 1.1 & 0.3 & 0.1 \\
\hline
\end{tabular}

C. $2-2$ 
Table C.2 Blender

$60 \mathrm{~Hz}, 180 \mathrm{~Hz}$, and $300 \mathrm{~Hz}$ RMS Resultant Fields

Distance Horizontal Vertical $60 \mathrm{~Hz} \quad 180 \mathrm{~Hz} \quad 300 \mathrm{~Hz}$ meters Angle deg Angle deg mGauss mGauss mGauss

\begin{tabular}{|c|c|c|c|c|c|}
\hline 1.000 & 180.0 & 60 & 1.3 & 0.4 & 0.1 \\
\hline 1.000 & 157.5 & 60 & 1.2 & 0.3 & 0.1 \\
\hline 1.000 & 135.0 & 60 & 1.2 & 0.3 & 0.1 \\
\hline 1.000 & 112.5 & 60 & 1.1 & 0.3 & 0.1 \\
\hline 1.000 & 90.0 & 60 & 0.9 & 0.3 & 0.1 \\
\hline 1.000 & 67.5 & 60 & 0.8 & 0.2 & 0.0 \\
\hline 1.000 & 45.0 & 60 & 0.7 & 0.2 & 0.1 \\
\hline 1.000 & 22.5 & 60 & 0.8 & 0.2 & 0.1 \\
\hline 0.140 & 0.0 & 30 & 5288.4 & 1274.1 & 306.0 \\
\hline 0.140 & -22.5 & 30 & 5927.8 & 1410.4 & 324.3 \\
\hline 0.140 & -45.0 & 30 & 5143.6 & 1304.0 & 327.8 \\
\hline 0.140 & -67.5 & 30 & 3428.4 & 916.5 & 292.0 \\
\hline 0.140 & -90.0 & 30 & 2310.2 & 729.0 & 239.2 \\
\hline 0.140 & -112.5 & 30 & 2376.1 & 780.6 & 237.4 \\
\hline 0.140 & -135.0 & 30 & 2546.7 & 732.6 & 204.3 \\
\hline 0.140 & -157.5 & 30 & 2755.1 & 775.0 & 203.3 \\
\hline 0.140 & 180.0 & 30 & 2554.2 & 707.9 & 201.8 \\
\hline 0.140 & 157.5 & 30 & 2300.3 & 618.1 & 173.0 \\
\hline 0.140 & 135.0 & 30 & 2088.1 & 527.3 & 127.1 \\
\hline 0.140 & 112.5 & 30 & 1813.6 & 446.9 & 116.2 \\
\hline 0.140 & 90.0 & 30 & 1656.1 & 428.4 & 144.4 \\
\hline 0.140 & 67.5 & 30 & 1610.1 & 505.6 & 113.6 \\
\hline 0.140 & 45.0 & 30 & 2268.0 & 632.0 & 141.9 \\
\hline 0.140 & 22.5 & 30 & 3602.2 & 913.0 & 207.5 \\
\hline 0.240 & 0.0 & 30 & 93.2 & 23.5 & 5.8 \\
\hline 0.240 & -22.5 & 30 & 102.9 & 25.6 & 6.1 \\
\hline 0.240 & -45.0 & 30 & 99.1 & 24.7 & 5.7 \\
\hline 0.240 & -67.5 & 30 & 83.3 & 21.9 & 6.0 \\
\hline 0.240 & -90.0 & 30 & 63.1 & 17.2 & 5.1 \\
\hline 0.240 & -912.5 & 30 & 55.6 & 16.9 & 4.8 \\
\hline
\end{tabular}

Distance Horizontal Vertical $60 \mathrm{~Hz} \quad 180 \mathrm{~Hz} \quad 300 \mathrm{~Hz}$

meters Angle deg Angle de $\mathrm{g}$ mGauss mGauss mGauss

\begin{tabular}{|r|r|r|r|r|r|}
\hline 0.240 & -135.0 & 30 & 55.8 & 16.0 & 4.5 \\
\hline 0.240 & -157.5 & 30 & 64.3 & 18.4 & 4.8 \\
\hline 0.240 & 180.0 & 30 & 63.6 & 18.0 & 5.1 \\
\hline 0.240 & 157.5 & 30 & 60.8 & 16.7 & 4.8 \\
\hline 0.240 & 135.0 & 30 & 58.4 & 15.4 & 3.9 \\
\hline 0.240 & 112.5 & 30 & 52.8 & 13.6 & 3.6 \\
\hline 0.240 & 90.0 & 30 & 50.1 & 13.0 & 3.5 \\
\hline 0.240 & 67.5 & 30 & 49.9 & 13.0 & 3.6 \\
\hline 0.240 & 45.0 & 30 & 56.9 & 15.0 & 3.8 \\
\hline 0.240 & 22.5 & 30 & 72.1 & 18.6 & 4.6 \\
\hline 0.440 & 0.0 & 30 & 15.5 & 4.0 & 0.9 \\
\hline 0.440 & -22.5 & 30 & 16.9 & 4.1 & 0.9 \\
\hline 0.440 & -45.0 & 30 & 15.7 & 3.8 & 0.9 \\
\hline 0.440 & -67.5 & 30 & 12.7 & 3.2 & 0.9 \\
\hline 0.440 & -90.0 & 30 & 9.3 & 2.5 & 0.8 \\
\hline 0.440 & -112.5 & 30 & 8.5 & 2.7 & 0.9 \\
\hline 0.440 & -135.0 & 30 & 9.7 & 2.9 & 0.9 \\
\hline 0.440 & -157.5 & 30 & 12.1 & 3.6 & 1.1 \\
\hline 0.440 & 180.0 & 30 & 12.8 & 3.7 & 1.1 \\
\hline 0.440 & 157.5 & 30 & 12.6 & 3.5 & 1.0 \\
\hline 0.440 & 135.0 & 30 & 11.9 & 3.1 & 0.9 \\
\hline 0.440 & 112.5 & 30 & 10.3 & 2.6 & 0.7 \\
\hline 0.440 & 90.0 & 30 & 8.8 & 2.2 & 0.6 \\
\hline 0.440 & 67.5 & 30 & 7.9 & 2.1 & 0.5 \\
\hline 0.440 & 45.0 & 30 & 8.8 & 2.4 & 0.6 \\
\hline 0.440 & 22.5 & 30 & 11.8 & 3.1 & 0.7 \\
\hline 1.140 & 0.0 & 30 & 1.2 & 0.3 & 0.1 \\
\hline 1.140 & -22.5 & 30 & 1.3 & 0.3 & 0.1 \\
\hline 1.140 & -45.0 & 30 & 1.3 & 0.3 & 0.1 \\
\hline 1.140 & -67.5 & 30 & 1.0 & 0.2 & 0.1 \\
\hline
\end{tabular}


Table C. 2 Blender

$60 \mathrm{~Hz}, 180 \mathrm{~Hz}$, and $300 \mathrm{~Hz}$ RMS Resultant Fields

Distance Horizontal Vertical $60 \mathrm{~Hz} \quad 180 \mathrm{~Hz} \quad 300 \mathrm{~Hz}$

meters Angle deg Angle deg mGauss mGauss mGauss

Distance Horizontal Vertical $60 \mathrm{~Hz} \quad 180 \mathrm{~Hz} \quad 300 \mathrm{~Hz}$

\begin{tabular}{|r|r|r|r|r|r|}
\hline 1.140 & -90.0 & 30 & 0.7 & 0.2 & 0.0 \\
\hline 1.140 & -112.5 & 30 & 0.6 & 0.2 & 0.1 \\
\hline 1.140 & -135.0 & 30 & 0.9 & 0.3 & 0.1 \\
\hline 1.140 & -157.5 & 30 & 1.2 & 0.3 & 0.1 \\
\hline 1.140 & 180.0 & 30 & 1.3 & 0.4 & 0.1 \\
\hline 1.140 & 157.5 & 30 & 1.4 & 0.4 & 0.1 \\
\hline 1.140 & 135.0 & 30 & 1.3 & 0.3 & 0.1 \\
\hline 1.140 & 112.5 & 30 & 1.1 & 0.3 & 0.1 \\
\hline 1.140 & 90.0 & 30 & 0.8 & 0.2 & 0.1 \\
\hline 1.140 & 67.5 & 30 & 0.6 & 0.2 & 0.0 \\
\hline 1.140 & 45.0 & 30 & 0.6 & 0.2 & 0.1 \\
\hline 1.140 & 22.5 & 30 & 0.9 & 0.2 & 0.1 \\
\hline 0.127 & 0.0 & 0 & 7100.5 & 1635.7 & 387.0 \\
\hline 0.127 & -22.5 & 0 & 7913.4 & 1825.2 & 468.1 \\
\hline 0.127 & -45.0 & 0 & 6751.9 & 1740.2 & 446.2 \\
\hline 0.127 & -67.5 & 0 & 4116.3 & 1158.7 & 358.9 \\
\hline 0.127 & -90.0 & 0 & 2655.6 & 805.8 & 259.0 \\
\hline 0.127 & -112.5 & 0 & 2581.7 & 818.7 & 248.8 \\
\hline 0.127 & -135.0 & 0 & 2678.8 & 766.3 & 201.9 \\
\hline 0.127 & -157.5 & 0 & 2781.2 & 785.7 & 210.4 \\
\hline 0.127 & 180.0 & 0 & 2554.5 & 689.8 & 188.8 \\
\hline 0.127 & 157.5 & 0 & 2275.4 & 565.1 & 131.5 \\
\hline 0.127 & 135.0 & 0 & 2027.4 & 531.9 & 144.8 \\
\hline 0.127 & 112.5 & 0 & 1790.9 & 458.8 & 138.3 \\
\hline 0.127 & 90.0 & 0 & 1546.5 & 453.4 & 120.0 \\
\hline 0.127 & 67.5 & 0 & 1754.4 & 509.3 & 107.9 \\
\hline 0.127 & 45.0 & 0 & 2729.7 & 703.2 & 172.3 \\
\hline 0.127 & 22.5 & 0 & 4641.5 & 1141.9 & 281.2 \\
\hline 0.227 & 0.0 & 0 & 122.3 & 31.2 & 7.7 \\
\hline 0.227 & -22.5 & 0 & 133.4 & 32.8 & 8.4 \\
\hline
\end{tabular}

meters Angle deg Angle de $\mathrm{g}$ mGauss mGauss mGauss

\begin{tabular}{|c|c|c|c|c|c|}
\hline 0.227 & -45.0 & 0 & 123.7 & 30.6 & 7.6 \\
\hline 0.227 & -67.5 & 0 & 90.5 & 23.6 & 6.9 \\
\hline 0.227 & -90.0 & 0 & 58.6 & 16.1 & 4.7 \\
\hline 0.227 & -112.5 & 0 & 52.0 & 16.7 & 4.9 \\
\hline 0.227 & -135.0 & 0 & 59.0 & 17.4 & 4.6 \\
\hline 0.227 & -157.5 & 0 & 68.6 & 20.0 & 5.3 \\
\hline 0.227 & 180.0 & 0 & 67.6 & 18.6 & 5.1 \\
\hline 0.227 & 157.5 & 0 & 63.8 & 16.5 & 3.9 \\
\hline 0.227 & 135.0 & 0 & 58.2 & 15.4 & 4.1 \\
\hline 0.227 & 112.5 & 0 & 50.6 & 12.7 & 3.4 \\
\hline 0.227 & 90.0 & 0 & 42.2 & 10.6 & 2.7 \\
\hline 0.227 & 67.5 & 0 & 42.1 & 11.5 & 2.9 \\
\hline 0.227 & 45.0 & 0 & 55.6 & 15.3 & 3.8 \\
\hline 0.227 & 22.5 & 0 & 85.1 & 23.5 & 6.1 \\
\hline 0.427 & 0.0 & 0 & 18.6 & 5.0 & 1.3 \\
\hline 0.427 & -22.5 & 0 & 19.9 & 5.1 & 1.4 \\
\hline 0.427 & -45.0 & 0 & 19.0 & 4.7 & 1.2 \\
\hline 0.427 & -67.5 & 0 & 15.2 & 3.9 & 1.1 \\
\hline 0.427 & -90.0 & 0 & 10.4 & 2.7 & 0.8 \\
\hline 0.427 & -112.5 & 0 & 8.7 & 2.7 & 0.8 \\
\hline 0.427 & -135.0 & 0 & 10.0 & 3.0 & 0.8 \\
\hline 0.427 & -157.5 & 0 & 12.6 & 3.8 & 1.0 \\
\hline 0.427 & 180.0 & 0 & 13.2 & 3.7 & 1.0 \\
\hline 0.427 & 157.5 & 0 & 13.1 & 3.4 & 0.8 \\
\hline 0.427 & 135.0 & 0 & 12.3 & 3.3 & 0.8 \\
\hline 0.427 & 112.5 & 0 & 10.6 & 2.6 & 0.7 \\
\hline 0.427 & 90.0 & 0 & 8.5 & 2.1 & 0.5 \\
\hline 0.427 & 67.5 & 0 & 7.8 & 2.2 & 0.5 \\
\hline 0.427 & 45.0 & 0 & 9.4 & 2.7 & 0.7 \\
\hline 0.427 & 22.5 & 0 & 13.6 & 3.9 & 1.1 \\
\hline
\end{tabular}

C. $2-4$ 
Table C. 2 Blender

$60 \mathrm{~Hz}, 180 \mathrm{~Hz}$, and $300 \mathrm{~Hz}$ RMS Resultant Fields

Distance Horizontal Vertical $60 \mathrm{~Hz} \quad 180 \mathrm{~Hz} \quad 300 \mathrm{~Hz}$ meters Angle deg Angle deg mGauss mGauss mGauss

Distance Horizontal Vertical $60 \mathrm{~Hz} \quad 180 \mathrm{~Hz} \quad 300 \mathrm{~Hz}$

meters Angle deg Angle de $\mathrm{g}$ mGauss mGauss mGauss

\begin{tabular}{|c|c|c|c|c|c|}
\hline 9.127 & 0.0 & 0 & 1.2 & 0.3 & 0.1 \\
\hline 1.127 & -22.5 & 0 & 1.3 & 0.3 & 0.1 \\
\hline 1.127 & -45.0 & 0 & 1.3 & 0.3 & 0.1 \\
\hline 1.127 & -67.5 & 0 & 1.0 & 0.2 & 0.1 \\
\hline 1.127 & -90.0 & 0 & 0.7 & 0.2 & 0.0 \\
\hline 1.127 & -112.5 & 0 & 0.5 & 0.1 & 0.0 \\
\hline 1.127 & -135.0 & 0 & 0.6 & 0.2 & 0.0 \\
\hline 9.127 & -157.5 & 0 & 0.9 & 0.3 & 0.1 \\
\hline 1.127 & 180.0 & 0 & 1.0 & 0.3 & 0.1 \\
\hline 1.127 & 157.5 & 0 & 1.0 & 0.3 & 0.1 \\
\hline 1.127 & 135.0 & 0 & 1.0 & 0.3 & 0.1 \\
\hline 1.127 & 112.5 & 0 & 0.9 & 0.2 & 0.1 \\
\hline 1.127 & 90.0 & 0 & 0.6 & 0.1 & 0.0 \\
\hline 1.127 & 67.5 & 0 & 0.5 & 0.1 & 0.0 \\
\hline 1.127 & 45.0 & 0 & 0.6 & 0.2 & 0.0 \\
\hline 1.127 & 22.5 & 0 & 0.9 & 0.2 & 0.1 \\
\hline 0.279 & 0.0 & -30 & 499.7 & 137.7 & 37.1 \\
\hline 0.279 & -22.5 & -30 & 547.3 & 148.0 & 38.5 \\
\hline 0.279 & -45.0 & -30 & 515.0 & 137.9 & 35.3 \\
\hline 0.279 & -67.5 & -30 & 415.2 & 113.9 & 32.8 \\
\hline 0.279 & -90.0 & -30 & 306.0 & 87.6 & 23.8 \\
\hline 0.279 & -112.5 & -30 & 255.5 & 78.7 & 23.0 \\
\hline 0.279 & -135.0 & -30 & 269.9 & 82.6 & 21.8 \\
\hline 0.279 & -157.5 & -30 & 304.6 & 88.8 & 24.4 \\
\hline 0.279 & 180.0 & -30 & 311.6 & 84.8 & 20.9 \\
\hline 0.279 & 157.5 & -30 & 302.4 & 81.7 & 23.0 \\
\hline 0.279 & 135.0 & -30 & 281.1 & 69.5 & 16.8 \\
\hline 0.279 & 112.5 & -30 & 243.7 & 61.4 & 16.4 \\
\hline 0.279 & 90.0 & -30 & 212.5 & 54.2 & 14.2 \\
\hline 0.279 & 67.5 & -30 & 215.2 & 59.9 & 16.2 \\
\hline
\end{tabular}

\begin{tabular}{|r|r|r|r|r|r|}
\hline 0.279 & 45.0 & -30 & 290.9 & 88.4 & 24.6 \\
\hline 0.279 & 22.5 & -30 & 373.3 & 101.5 & 25.1 \\
\hline 0.379 & 0.0 & -30 & 21.1 & 5.9 & 1.6 \\
\hline 0.379 & -22.5 & -30 & 23.0 & 6.3 & 1.6 \\
\hline 0.379 & -45.0 & -30 & 21.6 & 5.7 & 1.4 \\
\hline 0.379 & -67.5 & -30 & 17.4 & 4.7 & 1.3 \\
\hline 0.379 & -90.0 & -30 & 12.8 & 3.6 & 1.0 \\
\hline 0.379 & -112.5 & -30 & 10.5 & 3.2 & 0.9 \\
\hline 0.379 & -135.0 & -30 & 11.4 & 3.5 & 0.9 \\
\hline 0.379 & -157.5 & -30 & 13.4 & 3.9 & 1.0 \\
\hline 0.379 & 180.0 & -30 & 14.2 & 3.8 & 0.9 \\
\hline 0.379 & 157.5 & -30 & 14.0 & 3.8 & 1.1 \\
\hline 0.379 & 135.0 & -30 & 13.2 & 3.2 & 0.7 \\
\hline 0.379 & 112.5 & -30 & 11.3 & 2.8 & 0.7 \\
\hline 0.379 & 90.0 & -30 & 9.5 & 2.4 & 0.6 \\
\hline 0.379 & 67.5 & -30 & 9.0 & 2.5 & 0.7 \\
\hline 0.379 & 45.0 & -30 & 12.0 & 3.7 & 1.1 \\
\hline 0.379 & 22.5 & -30 & 15.8 & 4.3 & 1.1 \\
\hline 0.579 & 0.0 & -30 & 6.6 & 1.9 & 0.5 \\
\hline 0.579 & -22.5 & -30 & 7.1 & 2.0 & 0.6 \\
\hline 0.579 & -45.0 & -30 & 6.8 & 1.8 & 0.5 \\
\hline 0.579 & -67.5 & -30 & 5.6 & 1.5 & 0.4 \\
\hline 0.579 & -90.0 & -30 & 4.1 & 1.1 & 0.3 \\
\hline 0.579 & -112.5 & -30 & 3.4 & 1.0 & 0.3 \\
\hline 0.579 & -135.0 & -30 & 3.7 & 1.1 & 0.3 \\
\hline 0.579 & -157.5 & -30 & 4.6 & 1.3 & 0.3 \\
\hline 0.579 & 180.0 & -30 & 4.9 & 1.3 & 0.3 \\
\hline 0.579 & 157.5 & -30 & 4.9 & 1.3 & 0.3 \\
\hline 0.579 & 135.0 & -30 & 4.7 & 1.1 & 0.2 \\
\hline 0.579 & 112.5 & -30 & 4.0 & 1.0 & 0.2 \\
\hline
\end{tabular}

C.2-5 
Table C.2 Blender

$60 \mathrm{~Hz}, 180 \mathrm{~Hz}$, and $300 \mathrm{~Hz}$ RMS Resultant Fields

Distance Horizontal Vertical $60 \mathrm{~Hz} \quad 180 \mathrm{~Hz} \quad 300 \mathrm{~Hz}$ meters Angle deg Angle deg mGauss mGauss mGauss

Distance Horizontal Vertical $60 \mathrm{~Hz} \quad 180 \mathrm{~Hz} \quad 300 \mathrm{~Hz}$

\begin{tabular}{|c|c|c|c|c|c|}
\hline 0.579 & 90.0 & -30 & 3.3 & 0.8 & 0.2 \\
\hline 0.579 & 67.5 & -30 & 3.0 & 0.9 & 0.2 \\
\hline 0.579 & 45.0 & -30 & 3.8 & 1.2 & 0.4 \\
\hline 0.579 & 22.5 & -30 & 5.0 & 1.4 & 0.4 \\
\hline 1.279 & 0.0 & -30 & 0.8 & 0.2 & 0.0 \\
\hline 1.279 & -22.5 & -30 & 0.8 & 0.2 & 0.1 \\
\hline 1.279 & -45.0 & -30 & 0.8 & 0.2 & 0.0 \\
\hline 1.279 & -67.5 & -30 & 0.7 & 0.2 & 0.0 \\
\hline 1.279 & -90.0 & -30 & 0.5 & 0.1 & 0.0 \\
\hline 1.279 & -112.5 & .30 & 0.3 & 0.1 & 0.0 \\
\hline 1.279 & -135.0 & -30 & 0.3 & 0.1 & 0.0 \\
\hline 1.279 & -157.5 & .30 & 0.5 & 0.1 & 0.0 \\
\hline 1.279 & 180.0 & -30 & 0.5 & 0.1 & 0.0 \\
\hline 1.279 & 157.5 & -30 & 0.6 & 0.1 & 0.0 \\
\hline 1.279 & 135.0 & -30 & 0.6 & 0.1 & 0.0 \\
\hline 1.279 & $\$ 12.5$ & .30 & 0.5 & 0.1 & 0.0 \\
\hline 1.279 & 90.0 & .30 & 0.4 & 0.1 & 0.0 \\
\hline 1.279 & 67.5 & .30 & 0.3 & 0.1 & 0.0 \\
\hline 1.279 & 45.0 & .30 & 0.4 & 0.1 & 0.0 \\
\hline 1.279 & 22.5 & -30 & 0.6 & 0.2 & 0.0 \\
\hline 0.330 & 0.0 & -60 & 225.0 & 72.0 & 21.0 \\
\hline 0.330 & -22.5 & -60 & 238.6 & 76.7 & 21.7 \\
\hline 0.330 & -45.0 & -60 & 224.2 & 67.1 & 17.7 \\
\hline 0.330 & -67.5 & -60 & 208.0 & 63.9 & 17.6 \\
\hline 0.330 & -90.0 & -60 & 175.1 & 55.7 & 15.4 \\
\hline 0.330 & -112.5 & -60 & $\{51.3$ & 46.6 & 13.4 \\
\hline 0.330 & -135.0 & -60 & 146.2 & 45.1 & 11.3 \\
\hline 0.330 & -157.5 & -60 & 153.8 & 45.3 & 12.1 \\
\hline 0.330 & 180.0 & -60 & 161.2 & 47.9 & 12.9 \\
\hline 0.330 & 157.5 & -60 & 156.9 & 43.4 & 11.4 \\
\hline
\end{tabular}

\begin{tabular}{|c|c|c|c|c|c|}
\hline 0.330 & 135.0 & -60 & 153.8 & 41.7 & 12.0 \\
\hline 0.330 & 112.5 & -60 & 141.5 & 37.4 & 10.5 \\
\hline 0.330 & 90.0 & -60 & 139.3 & 37.7 & 9.9 \\
\hline 0.330 & 67.5 & -60 & 121.0 & 46.6 & 14.4 \\
\hline 0.330 & 45.0 & -60 & 213.7 & 56.1 & 15.5 \\
\hline 0.330 & 22.5 & -60 & 188.6 & 56.6 & 13.8 \\
\hline 0.430 & 0.0 & -60 & 11.0 & 3.4 & 1.0 \\
\hline 0.430 & -22.5 & -60 & 11.4 & 3.5 & 1.0 \\
\hline 0.430 & -45.0 & -60 & 10.7 & 3.1 & 0.9 \\
\hline 0.430 & -67.5 & -60 & 9.9 & 3.0 & 0.8 \\
\hline 0.430 & -90.0 & -60 & 8.3 & 2.6 & 0.7 \\
\hline 0.430 & -112.5 & -60 & 7.2 & 2.2 & 0.6 \\
\hline 0.430 & -135.0 & -60 & 7.1 & 2.2 & 0.6 \\
\hline 0.430 & -157.5 & -60 & 7.6 & 2.2 & 0.6 \\
\hline 0.430 & 180.0 & -60 & 8.0 & 2.3 & 0.6 \\
\hline 0.430 & 157.5 & -60 & 7.9 & 2.1 & 0.5 \\
\hline 0.430 & 135.0 & -60 & 7.8 & 2.1 & 0.5 \\
\hline 0.430 & 112.5 & -60 & 7.2 & 1.9 & 0.5 \\
\hline 0.430 & 90.0 & -60 & 7.1 & 1.9 & 0.5 \\
\hline 0.430 & 67.5 & -60 & 7.1 & 2.2 & 0.7 \\
\hline 0.430 & 45.0 & -60 & 6.7 & 2.3 & 0.8 \\
\hline 0.430 & 22.5 & -60 & 9.5 & 2.7 & 0.7 \\
\hline 0.630 & 0.0 & -60 & 3.9 & 1.2 & 0.3 \\
\hline 0.630 & -22.5 & -60 & 4.0 & 1.2 & 0.3 \\
\hline 0.630 & -45.0 & -60 & 3.7 & 1.1 & 0.2 \\
\hline 0.630 & -67.5 & -60 & 3.5 & 1.0 & 0.2 \\
\hline 0.630 & -90.0 & -60 & 3.0 & 0.9 & 0.2 \\
\hline 0.630 & -112.5 & -60 & 2.5 & 0.8 & 0.2 \\
\hline 0.630 & -135.0 & -60 & 2.5 & 0.8 & 0.2 \\
\hline 0.630 & -157.5 & -60 & 2.6 & 0.8 & 0.2 \\
\hline
\end{tabular}


Table C.2 Blender

$60 \mathrm{~Hz}, 180 \mathrm{~Hz}$, and $300 \mathrm{~Hz}$ RMS Resultant Fields

Distance Horizontal Vertical $60 \mathrm{~Hz} 180 \mathrm{~Hz} 300 \mathrm{~Hz}$

meters Angle deg Angle deg mGauss mGauss mGauss

Distance Horizontal Vertical $60 \mathrm{~Hz} \quad 180 \mathrm{~Hz} \quad 300 \mathrm{~Hz}$

meters Angle deg Angle de $g$ mGauss mGauss mGauss

\begin{tabular}{|r|r|r|r|r|r|}
\hline 0.630 & 180.0 & -60 & 2.7 & 0.8 & 0.2 \\
\hline 0.630 & 157.5 & -60 & 2.7 & 0.8 & 0.2 \\
\hline 0.630 & 135.0 & -60 & 2.7 & 0.8 & 0.2 \\
\hline 0.630 & 112.5 & -60 & 2.5 & 0.7 & 0.2 \\
\hline 0.630 & 90.0 & -60 & 2.5 & 0.7 & 0.2 \\
\hline 0.630 & 67.5 & -60 & 2.5 & 0.8 & 0.2 \\
\hline 0.630 & 45.0 & -60 & 2.0 & 0.8 & 0.3 \\
\hline 0.630 & 22.5 & -60 & 3.3 & 0.9 & 0.2 \\
\hline 1.000 & 0.0 & -60 & 0.8 & 0.3 & 0.1 \\
\hline 1.000 & -22.5 & -60 & 0.9 & 0.3 & 0.1 \\
\hline 1.000 & -45.0 & -60 & 0.9 & 0.3 & 0.1 \\
\hline 1.000 & -67.5 & -60 & 0.8 & 0.2 & 0.1 \\
\hline 1.000 & -90.0 & -60 & 0.7 & 0.2 & 0.0 \\
\hline 1.000 & -112.5 & -60 & 0.5 & 0.2 & 0.0 \\
\hline
\end{tabular}

\begin{tabular}{|r|r|r|r|r|r|}
\hline 1.000 & -135.0 & -60 & 0.5 & 0.1 & 0.0 \\
\hline 1.000 & -157.5 & -60 & 0.5 & 0.1 & 0.0 \\
\hline 1.000 & 180.0 & -60 & 0.6 & 0.1 & 0.1 \\
\hline 1.000 & 157.5 & -60 & 0.6 & 0.1 & 0.0 \\
\hline 1.000 & 135.0 & -60 & 0.6 & 0.1 & 0.0 \\
\hline 1.000 & 112.5 & -60 & 0.5 & 0.1 & 0.0 \\
\hline 1.000 & 90.0 & -60 & 0.5 & 0.1 & 0.0 \\
\hline 1.000 & 67.5 & -60 & 0.5 & 0.2 & 0.0 \\
\hline 1.000 & 45.0 & -60 & 0.5 & 0.2 & 0.1 \\
\hline 1.000 & 22.5 & -60 & 0.7 & 0.2 & 0.0 \\
\hline 0.114 & 0.0 & -90 & 1671.4 & 662.8 & 220.8 \\
\hline 0.414 & 0.0 & -90 & 8.7 & 2.8 & 0.7 \\
\hline
\end{tabular}




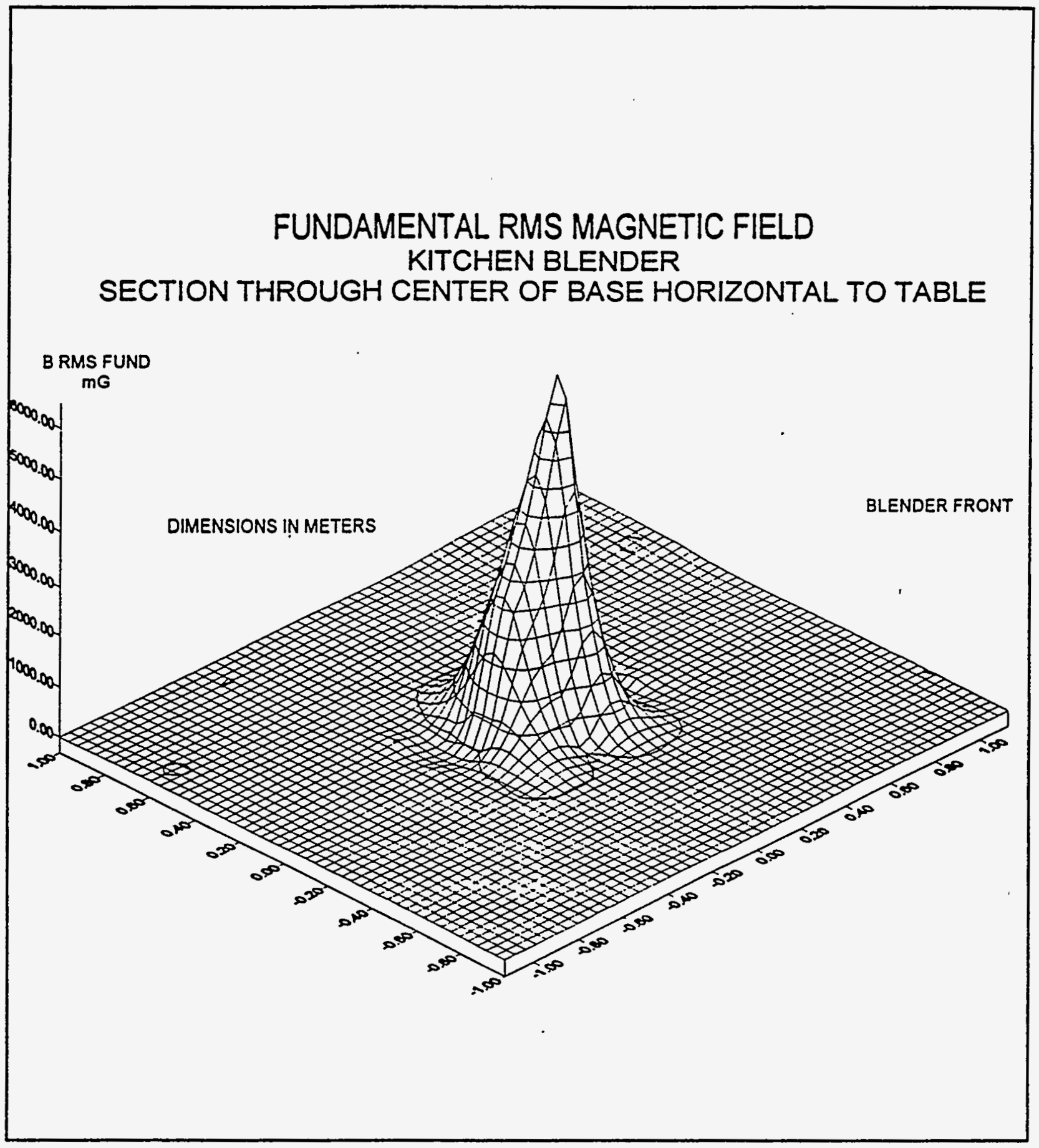

Figure C.2.1 Fundamental RMS Magnetic Field - Kitchen Blender. 


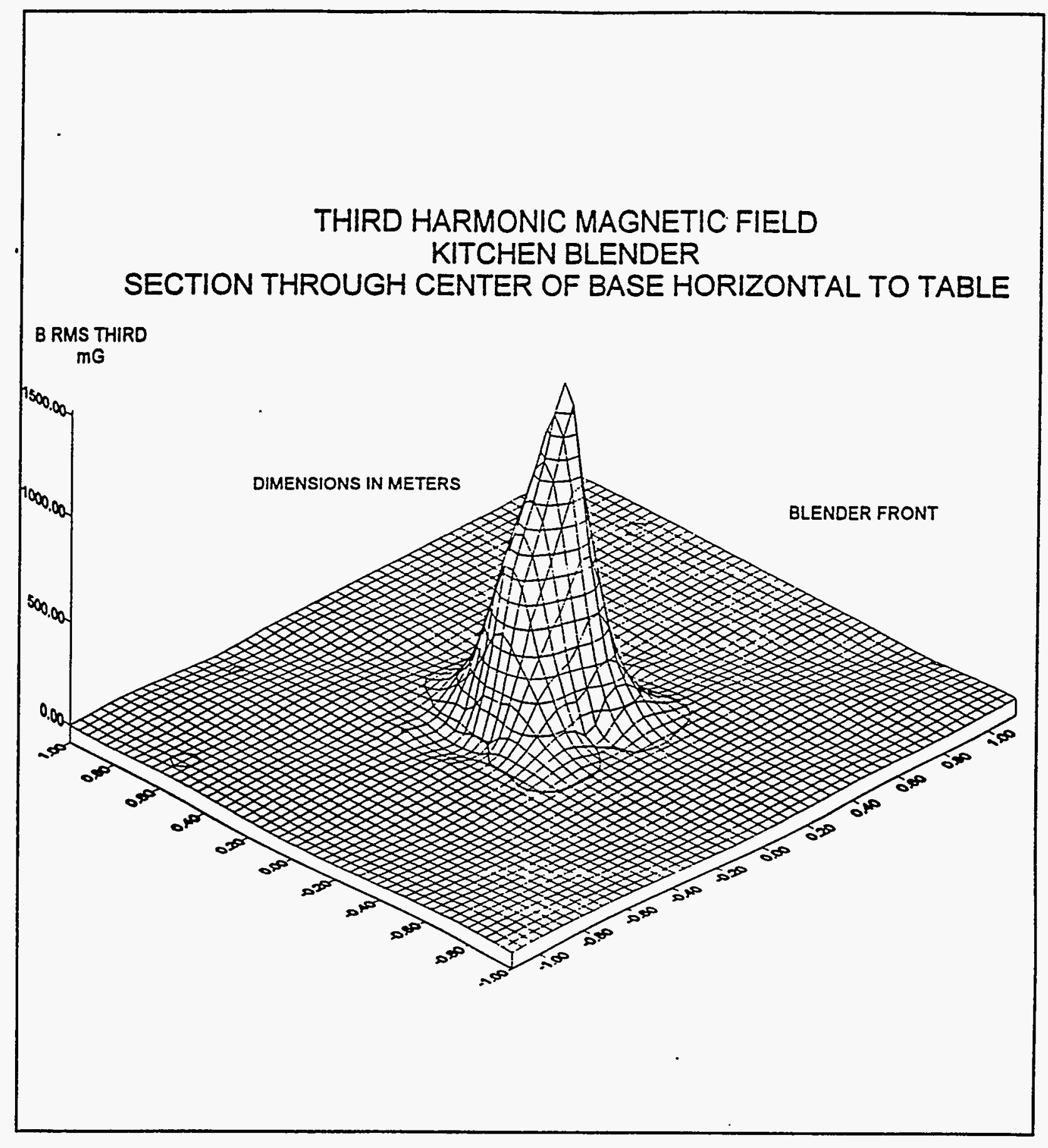

Figure C.2.2 Third Harmonic Magnetic Field - Kitchen Blender. 


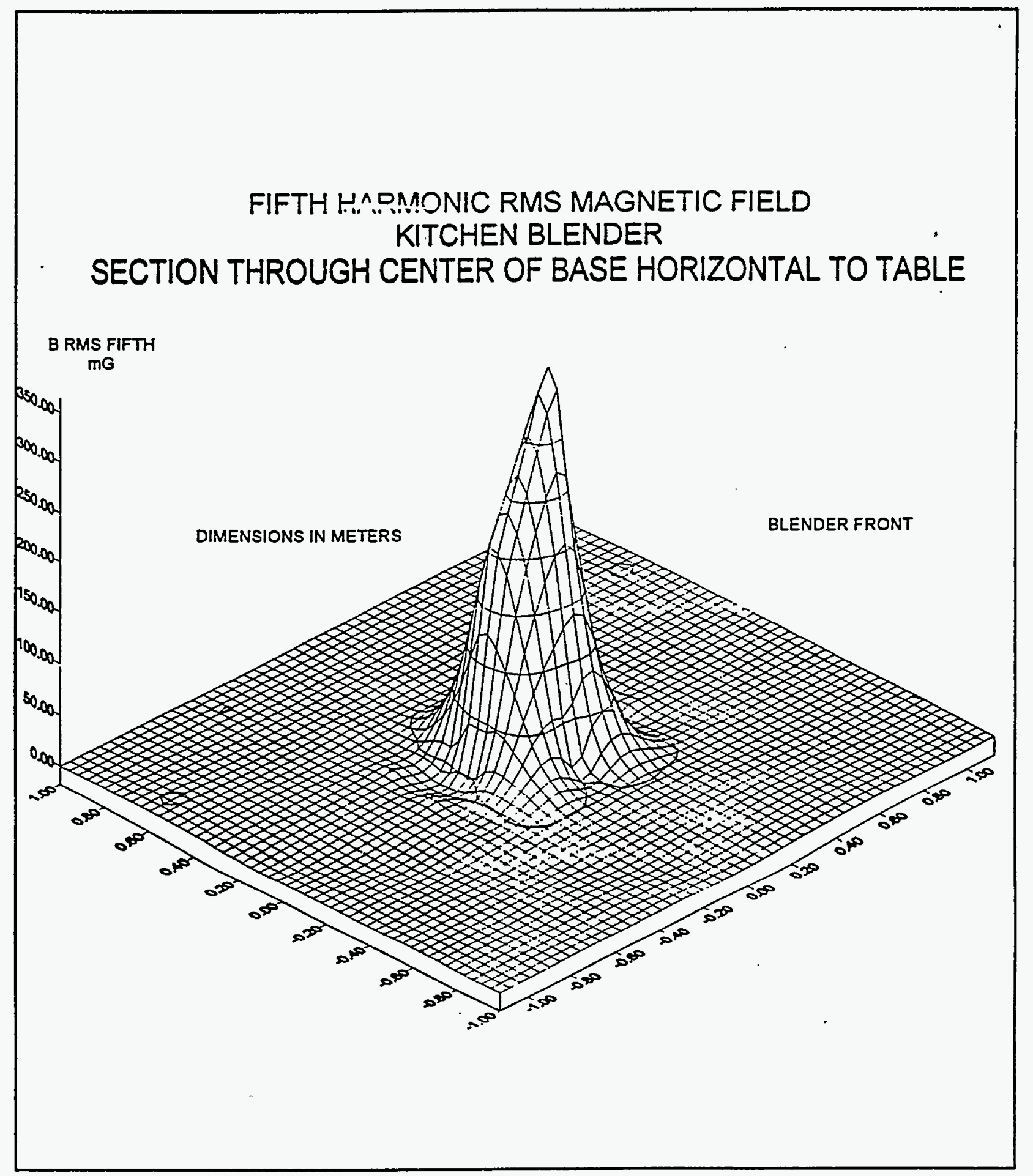

Figure C.2.3 Fifth Harmonic RMS Magnetic Field - Kitchen Blender. 


\section{C.3 Crock Pot}

Appliance Tested: Crock Pot for kitchen use

Manufacturer: CCC

- Model: 333

Appliance Code: 1253

Conditions for Testing: The crock pot had water covering the bottom and the heat was turned to HIGH heat.

Measured voltage and current: 119.3 Volts and 1.77 Amperes.

Magnetic Field Summary:

$60 \mathrm{~Hz}$ RMS resultant level at $30 \mathrm{~cm}$ from front surface: $0.7 \mathrm{mG}$

RMS resultant low surface level: $15.7 \mathrm{mG}$

RMS resultant high surface level: $41.3 \mathrm{mG}$ at rear

Highest recorded surface levels:

60 Hertz RMS resultant: $41.3 \mathrm{mG}$

180 Hertz RMS resultant: $0.28 \mathrm{mG}$

300 Hertz RMS resultant: $0.18 \mathrm{mG}$

420 Hertz RMS resultant: $0.04 \mathrm{mG}$

For the appliance test set, the front of the unit was located at 0 degrees on the test table.

Summary of characteristics: The magnetic fields were primarily $60 \mathrm{Hertz}$ and decayed in the pattern of a solenoid. There were essentially no harmonics from this primarily resistive device. 
Distance Horizontal Vertical $60 \mathrm{~Hz}$ meters Angle deg Angle deg mGauss

\begin{tabular}{|r|r|r|r|}
\hline 0.152 & 0.0 & 90 & 9.8 \\
\hline 0.252 & 0.0 & 90 & 4.0 \\
\hline 0.452 & 0.0 & 90 & 0.1 \\
\hline 1.000 & -90.0 & 90 & 1.1 \\
\hline 0.178 & 0.0 & 60 & 9.1 \\
\hline 0.178 & -22.5 & 60 & 9.3 \\
\hline 0.178 & -45.0 & 60 & 9.2 \\
\hline 0.178 & -67.5 & 60 & 8.8 \\
\hline 0.178 & -90.0 & 60 & 8.3 \\
\hline 0.178 & -112.5 & 60 & 7.7 \\
\hline 0.178 & -135.0 & 60 & 7.3 \\
\hline 0.178 & -157.5 & 60 & 6.9 \\
\hline 0.178 & 180.0 & 60 & 6.6 \\
\hline 0.178 & 157.5 & 60 & 6.6 \\
\hline 0.178 & 135.0 & 60 & 6.7 \\
\hline 0.178 & 112.5 & 60 & 7.0 \\
\hline 0.178 & 90.0 & 60 & 7.5 \\
\hline 0.178 & 67.5 & 60 & 8.0 \\
\hline 0.178 & 45.0 & 60 & 8.4 \\
\hline 0.178 & 22.5 & 60 & 8.9 \\
\hline 0.278 & 0.0 & 60 & 2.5 \\
\hline 0.278 & -22.5 & 60 & 2.5 \\
\hline 0.278 & -45.0 & 60 & 2.5 \\
\hline 0.278 & -67.5 & 60 & 2.4 \\
\hline 0.278 & -90.0 & 60 & 2.3 \\
\hline 0.278 & -112.5 & 60 & 2.3 \\
\hline 0.278 & -135.0 & 60 & 2.9 \\
\hline 0.278 & -157.5 & 60 & 2.1 \\
\hline 0.278 & 180.0 & 60 & 2.0 \\
\hline 0.278 & 157.5 & 60 & 2.0 \\
\hline
\end{tabular}

Table C.3 Crock Pot

$60 \mathrm{~Hz}$ RMS Resultant Fields

Distance Horizontal Vertical $60 \mathrm{~Hz}$

meters Angle deg Angle deg mGauss

\begin{tabular}{|c|c|c|c|}
\hline 0.278 & 135.0 & 60 & 2.0 \\
\hline 0.278 & 112.5 & 60 & 2.1 \\
\hline 0.278 & 90.0 & 60 & 2.2 \\
\hline 0.278 & 67.5 & 60 & 2.3 \\
\hline 0.278 & 45.0 & 60 & 2.4 \\
\hline 0.278 & 22.5 & 60 & 2.4 \\
\hline 0.478 & 0.0 & 60 & 0.5 \\
\hline 0.478 & -22.5 & 60 & 0.5 \\
\hline 0.478 & -45.0 & 60 & 0.5 \\
\hline 0.478 & -67.5 & 60 & 0.5 \\
\hline 0.478 & -90.0 & 60 & 0.5 \\
\hline 0.478 & -112.5 & 60 & 0.5 \\
\hline 0.478 & -135.0 & 60 & 0.5 \\
\hline 0.478 & -157.5 & 60 & 0.5 \\
\hline 0.478 & 180.0 & 60 & 0.5 \\
\hline 0.478 & 157.5 & 60 & 0.5 \\
\hline 0.478 & 135.0 & 60 & 0.4 \\
\hline 0.478 & 112.5 & 60 & 0.5 \\
\hline 0.478 & 90.0 & 60 & 0.5 \\
\hline 0.478 & 67.5 & 60 & 0.5 \\
\hline 0.478 & 45.0 & 60 & 0.5 \\
\hline 0.478 & 22.5 & 60 & 0.5 \\
\hline 1.000 & 0.0 & 60 & 0.1 \\
\hline 1.000 & -22.5 & 60 & 0.1 \\
\hline 1.000 & -45.0 & 60 & 0.1 \\
\hline 1.000 & -67.5 & 60 & 0.1 \\
\hline 1.000 & -90.0 & 60 & 0.1 \\
\hline 1.000 & -112.5 & 60 & 0.1 \\
\hline 1.000 & -135.0 & 60 & 0.1 \\
\hline 1.000 & -157.5 & 60 & 0.1 \\
\hline
\end{tabular}


Distance Horizontal Vertical $60 \mathrm{~Hz}$ meters Angle deg Angle deg mGauss

\begin{tabular}{|c|c|c|c|}
\hline 1.000 & 180.0 & 60 & 0.1 \\
\hline 1.000 & 157.5 & 60 & 0.1 \\
\hline 1.000 & 135.0 & 60 & 0.1 \\
\hline 1.000 & 112.5 & 60 & 0.1 \\
\hline 1.000 & 90.0 & 60 & 0.1 \\
\hline 1.000 & 67.5 & 60 & 0.1 \\
\hline 1.000 & 45.0 & 60 & 0.1 \\
\hline 1.000 & 22.5 & 60 & 0.1 \\
\hline 0.2229 & 0.0 & 30 & 4.8 \\
\hline 0.229 & -22.5 & 30 & 4.9 \\
\hline 0.229 & -45.0 & 30 & 4.8 \\
\hline 0.229 & -67.5 & 30 & 4.6 \\
\hline 0.229 & -90.0 & 30 & 4.3 \\
\hline 0.229 & -112.5 & 30 & 3.9 \\
\hline 0.229 & -135.0 & 30 & 3.6 \\
\hline 0.222 & -157.5 & 30 & 3.4 \\
\hline 0.229 & 180.0 & 30 & 3.2 \\
\hline 0.2229 & 157.5 & 30 & 3.2 \\
\hline 0.229 & 135.0 & 30 & 3.3 \\
\hline 0.229 & 112.5 & 30 & 3.5 \\
\hline 0.2229 & 90.0 & 30 & 3.7 \\
\hline 0.2229 & 67.5 & 30 & 4.0 \\
\hline 0.229 & 45.0 & 30 & 4.2 \\
\hline 0.229 & 22.5 & 30 & 4.5 \\
\hline 0.329 & 0.0 & 30 & 1.4 \\
\hline 0.329 & -22.5 & 30 & 1.4 \\
\hline 0.329 & -45.0 & 30 & 1.4 \\
\hline 0.329 & -67.5 & 30 & 1.4 \\
\hline 0.329 & .90 .0 & 30 & 1.4 \\
\hline 0.329 & -112.5 & 30 & 1.3 \\
\hline
\end{tabular}

Table C.3 Crock Pot

$60 \mathrm{~Hz}$ RMS Resultant Fields

Distance Horizontal Vertical $60 \mathrm{~Hz}$

meters Angle deg Angle deg mGauss

\begin{tabular}{|r|r|r|r|}
\hline 0.329 & -135.0 & 30 & 1.2 \\
\hline 0.329 & -157.5 & 30 & 1.2 \\
\hline 0.329 & 180.0 & 30 & 1.1 \\
\hline 0.329 & 157.5 & 30 & 1.1 \\
\hline 0.329 & 135.0 & 30 & 1.2 \\
\hline 0.329 & 112.5 & 30 & 1.2 \\
\hline 0.329 & -90.0 & 30 & 1.2 \\
\hline 0.329 & 67.5 & 30 & 1.3 \\
\hline 0.329 & 45.0 & 30 & 1.3 \\
\hline 0.329 & 22.5 & 30 & 1.4 \\
\hline 0.529 & 0.0 & 30 & 0.4 \\
\hline 0.529 & -22.5 & 30 & 0.4 \\
\hline 0.529 & -45.0 & 30 & 0.4 \\
\hline 0.529 & -57.5 & 30 & 0.4 \\
\hline 0.529 & -90.0 & 30 & 0.3 \\
\hline 0.529 & -112.5 & 30 & 0.4 \\
\hline 0.529 & -135.0 & 30 & 0.4 \\
\hline 0.529 & -157.5 & 30 & 0.3 \\
\hline 0.529 & 180.0 & 30 & 0.3 \\
\hline 0.529 & 157.5 & 30 & 0.4 \\
\hline 0.529 & 135.0 & 30 & 0.4 \\
\hline 0.529 & 112.5 & 30 & 0.4 \\
\hline 0.529 & 90.0 & 30 & 0.3 \\
\hline 0.529 & 67.5 & 30 & 0.3 \\
\hline 0.529 & 45.0 & 30 & 0.3 \\
\hline 0.529 & 22.5 & 30 & 0.3 \\
\hline 1.229 & 0.0 & 30 & 0.2 \\
\hline 1.229 & -22.5 & 30 & 0.3 \\
\hline 1.229 & -45.0 & 30 & 0.2 \\
\hline 1.229 & -67.5 & 30 & 0.3 \\
\hline
\end{tabular}


Table C.3 Crock Pot

$60 \mathrm{~Hz}$ RMS Resultant Fields

Distance Horizontal Vertical $60 \mathrm{~Hz}$

meters Angle deg Angle deg mGauss

Distance Horizontal Vertical $60 \mathrm{~Hz}$

meters Angle deg Angle deg mGauss

\begin{tabular}{|r|r|r|r|}
\hline 1.229 & -90.0 & 30 & 0.3 \\
\hline 1.229 & -112.5 & 30 & 0.3 \\
\hline 1.229 & -135.0 & 30 & 0.3 \\
\hline 1.229 & -157.5 & 30 & 0.2 \\
\hline 1.229 & 180.0 & 30 & 0.2 \\
\hline 1.229 & 157.5 & 30 & 0.3 \\
\hline 1.229 & 135.0 & 30 & 0.2 \\
\hline 1.229 & 112.5 & 30 & 0.2 \\
\hline 1.229 & 90.0 & 30 & 0.2 \\
\hline 1.229 & 67.5 & 30 & 0.3 \\
\hline 1.229 & 45.0 & 30 & 0.1 \\
\hline 1.229 & 22.5 & 30 & 0.2 \\
\hline 0.178 & 0.0 & 0 & 6.4 \\
\hline 0.178 & -22.5 & 0 & 6.7 \\
\hline 0.178 & -45.0 & 0 & 6.6 \\
\hline 0.178 & -67.5 & 0 & 6.1 \\
\hline 0.178 & -90.0 & 0 & 5.5 \\
\hline 0.178 & -112.5 & 0 & 4.9 \\
\hline 0.178 & -135.0 & 0 & 4.4 \\
\hline 0.178 & -157.5 & 0 & 4.0 \\
\hline 0.178 & 180.0 & 0 & 3.8 \\
\hline 0.178 & 157.5 & 0 & 3.7 \\
\hline 0.178 & 135.0 & 0 & 3.9 \\
\hline 0.178 & 112.5 & 0 & 4.1 \\
\hline 0.178 & 90.0 & 0 & 4.4 \\
\hline 0.178 & 67.5 & 0 & 4.8 \\
\hline 0.178 & 45.0 & 0 & 5.2 \\
\hline 0.178 & 22.5 & 0 & 5.9 \\
\hline 0.278 & 0.0 & 0 & 1.7 \\
\hline 0.278 & -22.5 & 0 & 1.7 \\
\hline
\end{tabular}

\begin{tabular}{|c|c|c|c|}
\hline 0.278 & -45.0 & 0 & 1.6 \\
\hline 0.278 & -67.5 & 0 & 1.6 \\
\hline 0.278 & -90.0 & 0 & 1.5 \\
\hline 0.278 & -112.5 & 0 & 1.4 \\
\hline 0.278 & -135.0 & 0 & 1.3 \\
\hline 0.278 & -157.5 & 0 & 1.2 \\
\hline 0.278 & 180.0 & 0 & 1.1 \\
\hline 0.278 & 157.5 & 0 & 1.1 \\
\hline 0.278 & 135.0 & 0 & 1.2 \\
\hline 0.278 & 112.5 & 0 & 1.2 \\
\hline 0.278 & 90.0 & 0 & 1.3 \\
\hline 0.278 & 67.5 & 0 & 1.4 \\
\hline 0.278 & 45.0 & 0 & 1.5 \\
\hline 0.278 & 22.5 & 0 & 1.5 \\
\hline 0.478 & 0.0 & 0 & 0.4 \\
\hline 0.478 & -22.5 & 0 & 0.4 \\
\hline 0.478 & -45.0 & 0 & 0.3 \\
\hline 0.478 & -67.5 & 0 & 0.4 \\
\hline 0.478 & -90.0 & 0 & 0.4 \\
\hline 0.478 & -112.5 & 0 & 0.3 \\
\hline 0.478 & -135.0 & 0 & 0.4 \\
\hline 0.478 & -157.5 & 0 & 0.3 \\
\hline 0.478 & 180.0 & 0 & 0.3 \\
\hline 0.478 & 157.5 & 0 & 0.3 \\
\hline 0.478 & 135.0 & 0 & 0.3 \\
\hline 0.478 & 112.5 & 0 & 0.3 \\
\hline 0.478 & 90.0 & 0 & 0.3 \\
\hline 0.478 & 67.5 & 0 & 0.4 \\
\hline 0.478 & 45.0 & 0 & 0.3 \\
\hline 0.478 & 22.5 & 0 & 0.4 \\
\hline
\end{tabular}

C.3-4 
Distance Horizontal Vertical $60 \mathrm{~Hz}$ meters Angle deg Angle deg mGauss

\begin{tabular}{|c|c|c|c|}
\hline 0.279 & 0.0 & 30 & 2.7 \\
\hline 0.279 & -22.5 & 30 & 2.5 \\
\hline 0.279 & -45.0 & 30 & 2.4 \\
\hline 0.279 & -67.5 & -30 & 2.3 \\
\hline 0.279 & .90 .0 & .30 & 2.2 \\
\hline 0.279 & -112.5 & .30 & 2.1 \\
\hline 0.279 & -135.0 & -30 & 2.0 \\
\hline 0.279 & -157.5 & -30 & 1.8 \\
\hline 0.279 & 180.0 & .30 & 1.9 \\
\hline 0.279 & 157.5 & -30 & 1.4 \\
\hline 0.279 & 135.0 & -30 & 1.6 \\
\hline 0.279 & 112.5 & .30 & 1.7 \\
\hline 0.279 & 90.0 & -30 & 1.7 \\
\hline 0.279 & 67.5 & -30 & 2.0 \\
\hline 0.279 & 45.0 & .30 & 2.0 \\
\hline 0.279 & 22.5 & .30 & 1.9 \\
\hline 0.379 & 0.0 & .30 & 1.0 \\
\hline 0.379 & -22.5 & -30 & 1.0 \\
\hline 0.379 & -45.0 & -30 & 0.9 \\
\hline 0.379 & -67.5 & .30 & 0.9 \\
\hline 0.379 & -90.0 & .30 & 0.9 \\
\hline 0.379 & -112.5 & -30 & 0.8 \\
\hline 0.379 & -135.0 & -30 & 0.7 \\
\hline 0.379 & -157.5 & -30 & 0.7 \\
\hline 0.379 & 180.0 & -30 & 0.7 \\
\hline 0.379 & 157.5 & -30 & 0.7 \\
\hline 0.379 & 135.0 & -30 & 0.7 \\
\hline 0.379 & 112.5 & -30 & 0.7 \\
\hline 0.379 & 90.0 & .30 & 0.7 \\
\hline 0.379 & 67.5 & -30 & 1.0 \\
\hline
\end{tabular}

Table C.3 Crock Pot

$60 \mathrm{~Hz}$ RMS Resultant Fields

Distance Horizontal Vertical $60 \mathrm{~Hz}$

meters Angle deg Angle deg mGauss

\begin{tabular}{|r|r|r|r|}
\hline 0.379 & 45.0 & -30 & 0.8 \\
\hline 0.379 & 22.5 & -30 & 1.0 \\
\hline 0.579 & 0.0 & -30 & 0.3 \\
\hline 0.579 & -22.5 & -30 & 0.3 \\
\hline 0.579 & -45.0 & -30 & 0.3 \\
\hline 0.579 & -67.5 & -30 & 0.3 \\
\hline 0.579 & -90.0 & -30 & 0.2 \\
\hline 0.579 & -112.5 & -30 & 0.3 \\
\hline 0.579 & -135.0 & -30 & 0.2 \\
\hline 0.579 & -157.5 & -30 & 0.2 \\
\hline 0.579 & 180.0 & -30 & 0.2 \\
\hline 0.579 & 157.5 & -30 & 0.2 \\
\hline 0.579 & 135.0 & -30 & 0.2 \\
\hline 0.579 & 112.5 & -30 & 0.2 \\
\hline 0.579 & 90.0 & -30 & 0.2 \\
\hline 0.579 & 67.5 & -30 & 0.2 \\
\hline 0.579 & 45.0 & -30 & 0.3 \\
\hline 0.579 & 22.5 & -30 & 0.3 \\
\hline 0.229 & 0.0 & -60 & 1.7 \\
\hline 0.229 & -45.0 & -60 & 1.4 \\
\hline 0.229 & -67.5 & -60 & 1.4 \\
\hline 0.229 & -90.0 & -60 & 1.3 \\
\hline 0.229 & -112.5 & -60 & 1.4 \\
\hline 0.229 & -135.0 & -60 & 1.6 \\
\hline 0.229 & -157.5 & -60 & 1.3 \\
\hline 0.229 & 180.0 & -60 & 1.2 \\
\hline 0.229 & 157.5 & -60 & 1.1 \\
\hline 0.229 & 112.5 & -60 & 1.2 \\
\hline 0.229 & 90.0 & -60 & 1.8 \\
\hline 0.229 & 67.5 & -60 & 2.8 \\
\hline
\end{tabular}


Distance Horizontal Vertical $60 \mathrm{~Hz}$ meters Angle deg Angle deg mGauss

\begin{tabular}{|r|r|r|r|}
\hline 0.229 & 45.0 & -60 & 4.5 \\
\hline 0.229 & 22.5 & -60 & 7.0 \\
\hline 0.329 & 0.0 & -60 & 0.6 \\
\hline 0.329 & -22.5 & -60 & 0.6 \\
\hline 0.329 & -45.0 & -60 & 0.6 \\
\hline 0.329 & -67.5 & -60 & 0.6 \\
\hline 0.329 & -90.0 & -60 & 0.6 \\
\hline 0.329 & -112.5 & -60 & 0.6 \\
\hline 0.329 & -135.0 & -60 & 0.6 \\
\hline 0.329 & -157.5 & -60 & 0.6 \\
\hline 0.329 & 180.0 & -60 & 0.6 \\
\hline 0.329 & 157.5 & -60 & 0.5 \\
\hline 0.329 & 135.0 & -60 & 0.5 \\
\hline 0.329 & 112.5 & -60 & 0.6 \\
\hline 0.329 & 90.0 & -60 & 0.6 \\
\hline 0.329 & 67.5 & -60 & 0.9 \\
\hline 0.329 & 45.0 & -60 & 1.5 \\
\hline 0.329 & 22.5 & -60 & 1.8 \\
\hline
\end{tabular}

Table C.3 Crock Pot

$60 \mathrm{~Hz}$ RMS Resultant Fields

Distance Horizontal Vertical $60 \mathrm{~Hz}$

meters Angle deg Angle deg mGauss

\begin{tabular}{|r|r|r|r|}
\hline 0.529 & 0.0 & -60 & 0.3 \\
\hline 0.529 & -22.5 & -60 & 0.3 \\
\hline 0.529 & -45.0 & -60 & 0.2 \\
\hline 0.529 & -67.5 & -60 & 0.2 \\
\hline 0.529 & -90.0 & -60 & 0.2 \\
\hline 0.529 & -112.5 & -60 & 0.2 \\
\hline 0.529 & -135.0 & -60 & 0.2 \\
\hline 0.529 & -157.5 & -60 & 0.2 \\
\hline 0.529 & 180.0 & $-\infty 0$ & 0.1 \\
\hline 0.529 & 112.5 & -60 & 0.2 \\
\hline 0.529 & 90.0 & -60 & 0.2 \\
\hline 0.529 & 67.5 & -60 & 0.2 \\
\hline 0.529 & 45.0 & -60 & 0.6 \\
\hline 0.529 & 22.5 & -60 & 0.9 \\
\hline 0.203 & 0.0 & -90 & 5.8 \\
\hline 0.303 & 0.0 & -90 & 2.0 \\
\hline
\end{tabular}




\section{RESULTANT RMS MAGNETIC FIELD DENSITY CROCK POT HORIZONTAL SLICE THROUGH CENTER OF APPLIANCE}

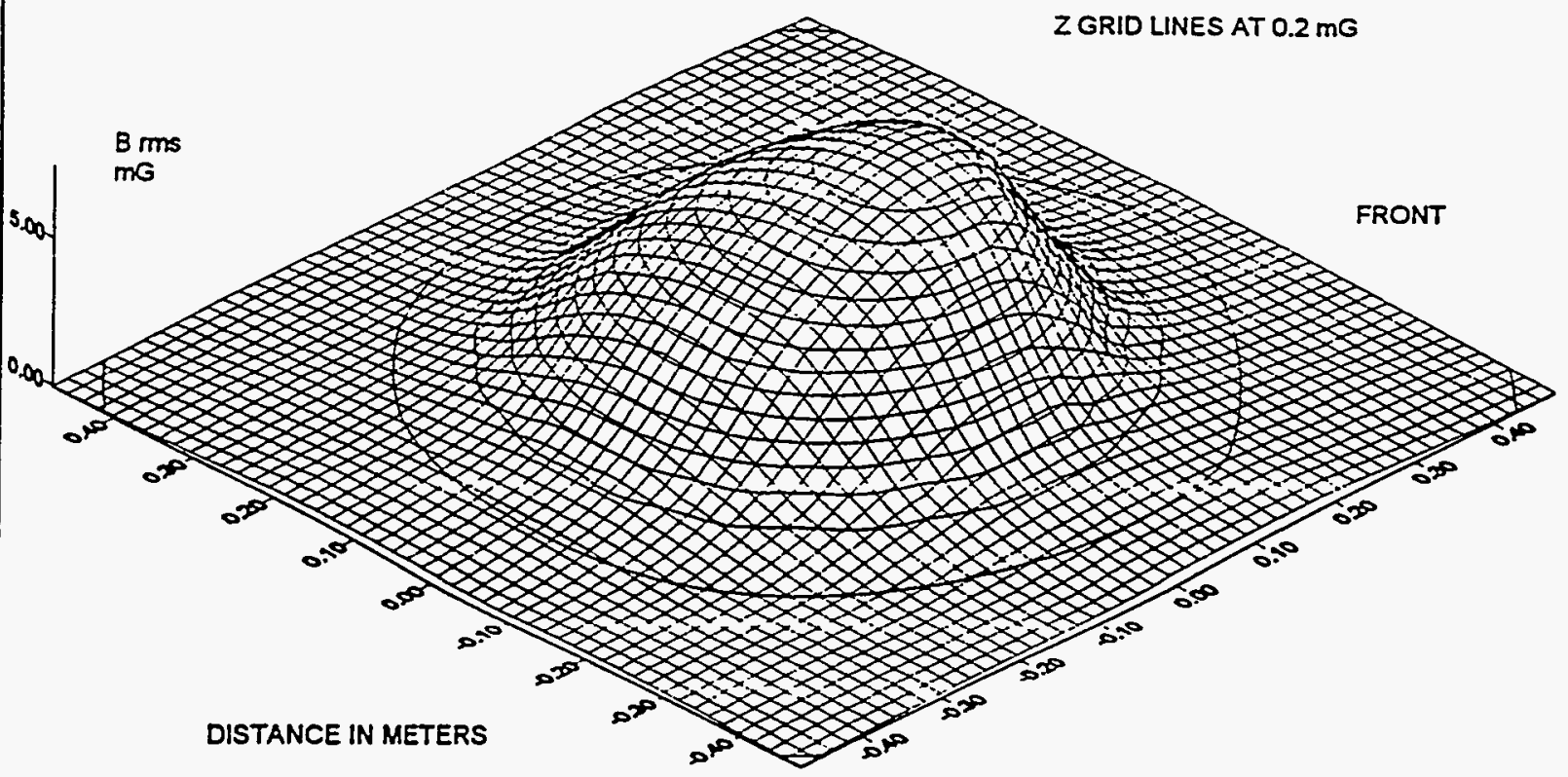

Figure C.3.1 Fundamental Resultant RMS Magnetic Field Density - Crock Pot.

C.3-7 


\section{C.4 Drill - Hand Hammer}

Appliance Tested: A three-eights inch variable speed hand drill with the option of selecting "hammer" operation for certain types of materials such as concrete. Manufacturer: DDD

Model: 444

Rated current: 3.5 Amperes

Appliance Code: 2263

Conditions for Testing: A block of wood was held tight to the chuck since the hammer effect is not present until there is load.

Measured voltage and current: 120.8 Volts and 1.65 Amperes.

Magnetic Field Summary:

$60 \mathrm{~Hz}$ RMS resultant level at $30 \mathrm{~cm}$ from front surface: $7.0 \mathrm{mG}$

RMS resultant low surface level: $410 \mathrm{mG}$

RMS resultant high surface level: $6672 \mathrm{mG}$ at the triggering

Highest recorded surface levels:

60 Hertz RMS resultant: $6103 \mathrm{mG}$

180 Hertz RMS resultant: $2547 \mathrm{mG}$

300 Hertz RMS resultant: $472 \mathrm{mG}$

420 Hertz RMS resultant: $155 \mathrm{mG}$

Maximum Total Harmonic Distortion: 58 percent

Maximum recorded RMS resultant in the $30-300 \mathrm{kHz}$ range: $11 \mathrm{mG}$

Maximum recorded RMS resultant in the $3-30 \mathrm{kHz}$ range: $10 \mathrm{mG}$

For the appliance test set, the handle of the unit was located at 0 degrees on the test table. The unit was laying on its side.

Summary of characteristics: The magnetic fields were rich in harmonics and were relatively strong. The unit had a field at $300 \mathrm{~Hz}$ that was one-fourth the ACGIH limit of 2 Gauss. 
Table C.4.1 Drill - Hand Hammer

$60 \mathrm{~Hz}$ RMS Resultant Fields

Distance Horizontal Vertical $60 \mathrm{~Hz}$ meters angle deg angle deg mGauss

\begin{tabular}{|c|c|c|c|}
\hline 0.038 & 0.0 & 90 & 4055.5 \\
\hline 0.338 & 0.0 & 90 & 190.0 \\
\hline 1.000 & 0.0 & $\infty 0$ & 1.0 \\
\hline 0.076 & 0.0 & 60 & 436.1 \\
\hline 0.076 & -22.5 & 60 & 396.9 \\
\hline 0.076 & -45.0 & 60 & 376.7 \\
\hline 0.076 & -67.5 & 60 & 448.8 \\
\hline 0.076 & -90.0 & 60 & 336.0 \\
\hline 0.076 & -112.5 & 60 & 290.7 \\
\hline 0.076 & -135.0 & 60 & 306.5 \\
\hline 0.076 & -157.5 & 60 & 292.9 \\
\hline 0.076 & 180.0 & 60 & 279.8 \\
\hline 0.076 & 157.5 & 60 & 264.5 \\
\hline 0.076 & $135: 0$ & 60 & 338.0 \\
\hline 0.076 & 112.5 & 60 & 438.4 \\
\hline 0.076 & 90.0 & 60 & 434.8 \\
\hline 0.076 & 67.5 & 60 & 448.3 \\
\hline 0.076 & 45.0 & 60 & 483.9 \\
\hline 0.076 & 22.5 & 60 & 436.2 \\
\hline 0.176 & 0.0 & ${ }^{4} 60$ & 69.8 \\
\hline 0.176 & -22.5 & 60 & 66.1 \\
\hline 0.176 & -45.0 & 60 & 66.0 \\
\hline 0.176 & -67.5 & 60 & 82.7 \\
\hline 0.176 & .90 .0 & 60 & 76.5 \\
\hline 0.176 & -112.5 & 60 & 77.7 \\
\hline 0.176 & -135.0 & 60 & 81.7 \\
\hline 0.176 & -157.5 & 60 & 92.8 \\
\hline 0.176 & 180.0 & 60 & 101.2 \\
\hline 0.176 & 157.5 & 60 & 103.4 \\
\hline 0.176 & 135.0 & 60 & 97.4 \\
\hline
\end{tabular}

Distance Horizontal Vertical $60 \mathrm{~Hz}$

meters angle deg angle deg mGauss

\begin{tabular}{|r|r|r|r|}
\hline 0.176 & 112.5 & 60 & 92.5 \\
\hline 0.176 & 90.0 & 60 & 76.9 \\
\hline 0.176 & 67.5 & 60 & 78.9 \\
\hline 0.176 & 45.0 & 60 & 76.7 \\
\hline 0.176 & 22.5 & 60 & 69.7 \\
\hline 0.376 & 0.0 & 60 & 9.6 \\
\hline 0.376 & -22.5 & 60 & 9.3 \\
\hline 0.376 & -45.0 & 60 & 9.4 \\
\hline 0.376 & -67.5 & 60 & 11.6 \\
\hline 0.376 & -90.0 & 60 & 11.1 \\
\hline 0.376 & -112.5 & 60 & 11.5 \\
\hline 0.376 & -135.0 & 60 & 11.9 \\
\hline 0.376 & -157.5 & 60 & 13.1 \\
\hline 0.376 & 180.0 & 60 & 13.9 \\
\hline 0.376 & 157.5 & 60 & 13.8 \\
\hline 0.376 & 135.0 & 60 & 12.8 \\
\hline 0.376 & 112.5 & 60 & 12.4 \\
\hline 0.376 & 90.0 & 60 & 10.6 \\
\hline 0.376 & 67.5 & 60 & 10.4 \\
\hline 0.376 & 45.0 & 60 & 10.0 \\
\hline 0.376 & 22.5 & 60 & 9.2 \\
\hline 1.000 & 0.0 & 60 & 0.8 \\
\hline 1.000 & -22.5 & 60 & 0.7 \\
\hline 1.000 & -45.0 & 60 & 0.8 \\
\hline 1.000 & -67.5 & 60 & 0.9 \\
\hline 1.000 & -90.0 & 60 & 0.9 \\
\hline 1.000 & -112.5 & 60 & 1.0 \\
\hline 1.000 & -135.0 & 60 & 0.9 \\
\hline 1.000 & -157.5 & 60 & 0.9 \\
\hline 1.000 & 180.0 & 60 & 1.0 \\
\hline
\end{tabular}

C. $4-2$ 
Table C.4.1 Drill - Hand Hammer

$60 \mathrm{~Hz}$ RMS Resultant Fields

Distance Horizontal Vertical $60 \mathrm{~Hz}$

Distance Horizontal Vertical $60 \mathrm{~Hz}$

meters angle deg angle deg mGauss

meters angle deg angle deg mGauss

\begin{tabular}{|c|c|c|c|}
\hline 1.000 & 157.5 & 60 & 1.0 \\
\hline 1.000 & 135.0 & 60 & 0.9 \\
\hline 1.000 & 1125 & 60 & 0.9 \\
\hline 1.000 & 90.0 & 60 & 0.9 \\
\hline 1.000 & 67.5 & 60 & 0.7 \\
\hline 1.000 & 45.0 & 60 & 0.8 \\
\hline 1.000 & 22.5 & 60 & 0.7 \\
\hline 0.102 & 0.0 & 30 & 221.6 \\
\hline 0.102 & -22.5 & 30 & 195.0 \\
\hline 0.102 & -45.0 & 30 & 191.5 \\
\hline 0.102 & -67.5 & 30 & 254.4 \\
\hline 0.102 & -90.0 & 30 & 228.5 \\
\hline 0.102 & -112.5 & 30 & 243.9 \\
\hline 0.102 & -135.0 & 30 & 287.0 \\
\hline 0.102 & -157.5 & 30 & 344.1 \\
\hline 0.102 & 180.0 & 30 & 383.8 \\
\hline 0.102 & 157.5 & 30 & 445.4 \\
\hline 0.102 & 135.0 & 30 & 394.6 \\
\hline 0.102 & 112.5 & 30 & 330.2 \\
\hline 0.102 & 90.0 & 30 & 240.5 \\
\hline 0.102 & 67.5 & 30 & 275.9 \\
\hline 0.102 & 45.0 & 30 & 292.2 \\
\hline 0.102 & 22.5 & 30 & 246.7 \\
\hline 0.202 & 0.0 & 30 & 36.4 \\
\hline 0.202 & -22.5 & 30 & 33.2 \\
\hline 0.202 & -45.0 & 30 & 33.8 \\
\hline 0.202 & -67.5 & 30 & 42.4 \\
\hline 0.202 & -90.0 & 30 & 40.2 \\
\hline 0.202 & -112.5 & 30 & 44.2 \\
\hline 0.202 & -135.0 & 30 & 48.9 \\
\hline
\end{tabular}

\begin{tabular}{|c|c|c|c|}
\hline 0.202 & -157.5 & 30 & 56.1 \\
\hline 0.202 & 180.0 & 30 & 60.4 \\
\hline 0.202 & 157.5 & 30 & 67.5 \\
\hline 0.202 & 135.0 & 30 & 62.8 \\
\hline 0.202 & 112.5 & 30 & 57.9 \\
\hline 0.202 & 90.0 & 30 & 45.4 . \\
\hline 0.202 & 67.5 & 30 & 44.6 \\
\hline 0.202 & 45.0 & 30 & 45.9 \\
\hline 0.202 & 22.5 & 30 & 39.3 \\
\hline 0.402 & 0.0 & 30 & 5.8 \\
\hline 0.402 & -22.5 & 30 & 5.4 \\
\hline 0.402 & -45.0 & 30 & 5.6 \\
\hline 0.402 & -67.5 & 30 & 6.9 \\
\hline 0.402 & -90.0 & 30 & - 6.8 \\
\hline 0.402 & -112.5 & 30 & 7.7 \\
\hline 0.402 & -135.0 & 30 & 8.4 \\
\hline 0.402 & -157.5 & 30 & 9.3 \\
\hline 0.402 & 180.0 & 30 & 9.8 \\
\hline 0.402 & 157.5 & 30 & 10.5 \\
\hline 0.402 & 135.0 & 30 & 9.4 \\
\hline 0.402 & 112.5 & 30 & 8.5 \\
\hline 0.402 & 90.0 & 30 & 6.7 \\
\hline 0.402 & 67.5 & 30 & 6.4 \\
\hline 0.402 & 45.0 & 30 & 6.5 \\
\hline 0.402 & 22.5 & 30 & 5.8 \\
\hline 1.102 & 0.0 & 30 & 0.4 \\
\hline 1.102 & -22.5 & 30 & 0.3 \\
\hline 1.102 & -45.0 & 30 & 0.4 \\
\hline 1.102 & -67.5 & 30 & 0.5 \\
\hline 1.102 & -90.0 & 30 & 0.6 \\
\hline
\end{tabular}

C. $4-3$ 
Table C.4.1 Drill - Hand Hammer

$60 \mathrm{~Hz}$ RMS Resultant Fields

Distance Horizontal Vertical $60 \mathrm{~Hz}$

Distance Horizontal Vertical $60 \mathrm{~Hz}$

meters angle deg angle deg mGauss

meters angle deg angle deg mGauss

\begin{tabular}{|r|r|r|r|}
\hline 1.102 & -112.5 & 30 & 0.7 \\
\hline 1.102 & -135.0 & 30 & 0.8 \\
\hline 1.102 & -157.5 & 30 & 0.8 \\
\hline 1.102 & 180.0 & 30 & 0.9 \\
\hline 1.102 & 157.5 & 30 & 0.9 \\
\hline 1.102 & 135.0 & 30 & 0.8 \\
\hline 1.102 & 112.5 & 30 & 0.7 \\
\hline 1.102 & 90.0 & 30 & 0.5 \\
\hline 1.102 & 67.5 & 30 & 0.5 \\
\hline 1.102 & 45.0 & 30 & 0.4 \\
\hline 1.102 & 22.5 & 30 & 0.3 \\
\hline 0.165 & 0.0 & 0 & 85.5 \\
\hline 0.165 & -22.5 & 0 & 70.7 \\
\hline 0.165 & -45.0 & 0 & 64.6 \\
\hline 0.165 & -67.5 & 0 & 72.8 \\
\hline 0.165 & -90.0 & 0 & 61.3 \\
\hline 0.165 & -112.5 & 0 & 61.8 \\
\hline 0.165 & -135.0 & 0 & 75.1 \\
\hline 0.165 & -157.5 & 0 & 85.8 \\
\hline 0.165 & 180.0 & 0 & 105.0 \\
\hline 0.165 & 157.5 & 0 & 109.3 \\
\hline 0.165 & 135.0 & 0 & 99.9 \\
\hline 0.165 & 112.5 & 0 & 89.6 \\
\hline 0.165 & 90.0 & 0 & 74.0 \\
\hline 0.165 & 67.5 & 0 & 82.6 \\
\hline 0.165 & 45.0 & 0 & 101.2 \\
\hline 0.165 & 22.5 & 0 & 95.3 \\
\hline 0.265 & 0.0 & 0 & 22.6 \\
\hline 0.265 & -22.5 & 0 & 19.3 \\
\hline 0.265 & -45.0 & 0 & 18.3 \\
\hline 1.0 & & & \\
\hline & 30 & & \\
\hline
\end{tabular}

\begin{tabular}{|c|c|c|c|}
\hline 0.265 & -67.5 & 0 & 19.6 \\
\hline 0.265 & -90.0 & 0 & 16.8 \\
\hline 0.265 & -112.5 & 0 & 17.1 \\
\hline 0.265 & -135.0 & 0 & 20.1 \\
\hline 0.265 & -157.5 & 0 & 22.4 \\
\hline 0.265 & 180.0 & 0 & 26.9 \\
\hline 0.265 & 157.5 & 0 & 27.5 \\
\hline 0.265 & 135.0 & 0 & 24.5 \\
\hline 0.265 & 112.5 & 0 & 22.6 \\
\hline 0.265 & 90.0 & 0 & 18.8 \\
\hline 0.265 & 67.5 & 0 & 20.0 \\
\hline 0.265 & 45.0 & 0 & 24.2 \\
\hline 0.265 & 22.5 & 0 & 23.7 \\
\hline 0.465 & 0.0 & 0 & 4.9 \\
\hline 0.465 & -22.5 & 0 & 4.3 \\
\hline 0.465 & -45.0 & 0 & 4.9 \\
\hline 0.465 & -67.5 & 0 & 4.3 \\
\hline 0.465 & -90.0 & 0 & 3.7 \\
\hline 0.465 & -112.5 & 0 & 3.8 \\
\hline 0.465 & -135.0 & 0 & 4.3 \\
\hline 0.465 & -157.5 & 0 & 4.7 \\
\hline 0.465 & 180.0 & 0 & 5.6 \\
\hline 0.465 & 157.5 & 0 & 5.6 \\
\hline 0.465 & 135.0 & 0 & 5.0 \\
\hline 0.465 & 112.5 & 0 & 4.6 \\
\hline 0.465 & 90.0 & 0 & 3.9 \\
\hline 0.465 & 67.5 & 0 & 4.0 \\
\hline 0.465 & 45.0 & 0 & 4.8 \\
\hline 0.465 & 22.5 & 0 & 4.8 \\
\hline 1.165 & 0.0 & 0 & 0.4 \\
\hline
\end{tabular}


Table C.4.1 Drill - Hand Hammer $60 \mathrm{~Hz}$ RMS Resultant Fields

Distance Horizontal Vertical $60 \mathrm{~Hz}$ meters angle deg angle deg mGauss

\begin{tabular}{|r|r|r|r|}
\hline 1.165 & -22.5 & 0 & 0.3 \\
\hline 1.165 & -45.0 & 0 & 0.3 \\
\hline 1.165 & -67.5 & 0 & 0.3 \\
\hline 1.165 & -90.0 & 0 & 0.2 \\
\hline 1.165 & -112.5 & 0 & 0.3 \\
\hline 1.165 & -135.0 & 0 & 0.3 \\
\hline 1.165 & -157.5 & 0 & 0.3 \\
\hline 1.165 & 180.0 & 0 & 0.4 \\
\hline 1.165 & 157.5 & 0 & 0.4 \\
\hline 1.165 & 135.0 & 0 & 0.3 \\
\hline 1.165 & 112.5 & .0 & 0.3 \\
\hline 1.165 & 90.0 & 0 & .0 .2 \\
\hline 1.165 & 67.5 & 0 & 0.2 \\
\hline 1.165 & 45.0 & 0 & 0.3 \\
\hline 1.165 & 22.5 & 0 & 0.3 \\
\hline 0.241 & 0.0 & -30 & 46.3 \\
\hline 0.241 & -22.5 & -30 & 39.4 \\
\hline 0.241 & -45.0 & -30 & 41.3 \\
\hline 0.241 & -67.5 & -30 & 40.7 \\
\hline 0.241 & -90.0 & -30 & 27.9 \\
\hline 0.241 & -112.5 & -30 & 25.1 \\
\hline 0.241 & -135.0 & -30 & 23.1 \\
\hline 0.241 & -157.5 & -30 & 24.9 \\
\hline 0.241 & 180.0 & -30 & 27.1 \\
\hline 0.241 & 157.5 & -30 & 27.8 \\
\hline 0.241 & 135.0 & -30 & 29.5 \\
\hline 0.241 & 112.5 & -30 & 33.9 \\
\hline 0.241 & 90.0 & -30 & 35.2 \\
\hline 0.241 & 67.5 & -30 & 40.9 \\
\hline 0.241 & 45.0 & -30 & 51.9 \\
\hline
\end{tabular}

Distance Horizontal Vertical $60 \mathrm{~Hz}$

meters angle deg angle deg mGauss

\begin{tabular}{|r|r|r|r|}
\hline 0.241 & 22.5 & -30 & 49.4 \\
\hline 0.341 & 0.0 & -30 & 16.0 \\
\hline 0.341 & -22.5 & -30 & 14.0 \\
\hline 0.341 & -45.0 & -30 & 14.4 \\
\hline 0.341 & -67.5 & -30 & 14.3 \\
\hline 0.341 & -90.0 & -30 & 10.0 \\
\hline 0.341 & -112.5 & -30 & 9.1 \\
\hline 0.341 & -135.0 & -30 & 8.5 \\
\hline 0.341 & -157.5 & -30 & 8.9 \\
\hline 0.341 & 180.0 & -30 & 9.6 \\
\hline 0.341 & 157.5 & -30 & 9.8 \\
\hline 0.341 & 135.0 & -30 & 10.1 \\
\hline 0.341 & 112.5 & -30 & 11.5 \\
\hline 0.341 & 90.0 & -30 & 12.0 \\
\hline 0.341 & 67.5 & -30 & 13.5 \\
\hline 0.341 & 45.0 & -30 & 17.1 \\
\hline 0.341 & 22.5 & -30 & 16.7 \\
\hline 0.541 & 0.0 & -30 & 4.6 \\
\hline 0.541 & -22.5 & -30 & 4.1 \\
\hline 0.541 & -45.0 & -30 & 4.2 \\
\hline 0.541 & -67.5 & -30 & 4.1 \\
\hline 0.541 & -90.0 & -30 & 2.9 \\
\hline 0.541 & -112.5 & -30 & 2.6 \\
\hline 0.541 & -135.0 & -30 & 2.4 \\
\hline 0.541 & -157.5 & -30 & 2.5 \\
\hline 0.541 & 180.0 & -30 & 2.6 \\
\hline 0.541 & 157.5 & -30 & 2.7 \\
\hline 0.541 & 135.0 & -30 & 2.7 \\
\hline 0.541 & 112.5 & -30 & 3.1 \\
\hline 0.541 & 90.0 & -30 & 3.3 \\
\hline
\end{tabular}


Table C.4.1 Drill - Hand Hammer

Distance Horizontal Vertical $60 \mathrm{~Hz}$ meters angle deg angle deg mGauss

\begin{tabular}{|c|c|c|c|}
\hline 0.541 & 67.5 & -30 & 3.7 \\
\hline 0.541 & 45.0 & -30 & 4.7 \\
\hline 0.541 & 22.5 & -30 & 4.6 \\
\hline 1.241 & 0.0 & -30 & 0.5 \\
\hline 1.241 & -22.5 & -30 & 0.4 \\
\hline 1.241 & -45.0 & -30 & 0.4 \\
\hline 1.241 & -67.5 & -30 & 0.4 \\
\hline 1.241 & -90.0 & -30 & 0.3 \\
\hline 1.241 & -112.5 & -30 & 0.2 \\
\hline 1.241 & -135.0 & .30 & 0.2 \\
\hline 1.241 & -157.5 & -30 & 0.2 \\
\hline 1.241 & 180.0 & -30 & 0.2 \\
\hline 1.241 & 157.5 & -30 & 0.2 \\
\hline 1.241 & 112.5 & -30 & 0.2 \\
\hline 1.241 & 90.0 & -30 & 0.3 \\
\hline 1.241 & 67.5 & -30 & 0.3 \\
\hline 1.241 & 45.0 & -30 & 0.5 \\
\hline 1.241 & 22.5 & -30 & 0.4 \\
\hline 0.356 & 0.0 & -60 & 99.0 \\
\hline 0.356 & -22.5 & -60 & 17.7 \\
\hline 0.356 & -45.0 & -60 & 17.9 \\
\hline 0.356 & -67.5 & -60 & 18.2 \\
\hline 0.356 & .90 .0 & -60 & 14.0 \\
\hline 0.356 & -112.5 & -60 & 12.8 \\
\hline 0.356 & -135.0 & -60 & 11.7 \\
\hline 0.356 & -157.5 & -60 & 11.9 \\
\hline 0.356 & 180.0 & -60 & 11.0 \\
\hline 0.356 & 157.5 & -60 & 12.2 \\
\hline 0.356 & 135.0 & -60 & 13.3 \\
\hline 0.356 & 112.5 & -60 & 15.8 \\
\hline
\end{tabular}

\section{$60 \mathrm{~Hz}$ RMS Resultant Fields}

Distance Horizontal Vertical $60 \mathrm{~Hz}$

meters angle deg angle deg mGauss

\begin{tabular}{|c|c|c|c|}
\hline 0.356 & 90.0 & -60 & 15.4 \\
\hline 0.356 & 67.5 & -60 & 17.3 \\
\hline 0.355 & 45.0 & $-\infty$ & 19.4 \\
\hline 0.356 & 22.5 & $-\infty 0$ & 20.0 \\
\hline 0.456 & 0.0 & -60 & 8.3 \\
\hline 0.456 & -22.5 & -60 & 7.5 \\
\hline 0.456 & -45.0 & $-\infty 0$ & 7.5 \\
\hline 0.456 & -67.5 & -60 & 8.0 \\
\hline 0.456 & -90.0 & $-\infty$ & 5.8 \\
\hline 0.456 & -112.5 & -60 & 5.8 \\
\hline 0.456 & -135.0 & -60 & 5.2 \\
\hline 0.456 & -157.5 & -60 & 5.3 \\
\hline 0.456 & 180.0 & -60 & 5.2 \\
\hline 0.456 & 157.5 & -60 & 5.5 \\
\hline 0.456 & 135.0 & -60 & 5.5 \\
\hline 0.456 & 112.5 & -60 & 6.6 \\
\hline 0.456 & 90.0 & -60 & 6.4 \\
\hline 0.456 & 67.5 & -60 & 7.3 \\
\hline 0.456 & 45.0 & -60 & 8.0 \\
\hline 0.456 & 22.5 & .60 & 8.3 \\
\hline 0.656 & 0.0 & -60 & 3.0 \\
\hline 0.656 & -22.5 & -60 & 2.8 \\
\hline 0.656 & -45.0 & -60 & 2.8 \\
\hline 0.656 & -67.5 & -60 & 3.0 \\
\hline 0.656 & .90 .0 & -60 & 2.2 \\
\hline 0.656 & -112.5 & -60 & 2.2 \\
\hline 0.656 & -135.0 & -60 & 2.0 \\
\hline 0.656 & -157.5 & -60 & 2.0 \\
\hline 0.656 & 180.0 & .60 & 1.9 \\
\hline 0.656 & 157.5 & -60 & 2.0 \\
\hline
\end{tabular}


Distance Horizontal Vertical $60 \mathrm{~Hz}$ meters angle deg angle deg mGauss

\begin{tabular}{|r|r|r|r|}
\hline 0.656 & 135.0 & -60 & 2.0 \\
\hline 0.656 & 112.5 & -60 & 2.4 \\
\hline 0.656 & 90.0 & -60 & 2.3 \\
\hline 0.656 & 67.5 & -60 & 2.6 \\
\hline 0.656 & 45.0 & -60 & 2.8 \\
\hline 0.656 & 22.5 & -60 & 3.0 \\
\hline 1.000 & 0.0 & -60 & 0.7 \\
\hline 1.000 & -22.5 & -60 & 0.6 \\
\hline 1.000 & -45.0 & -60 & 0.7 \\
\hline 1.000 & -67.5 & -60 & 0.8 \\
\hline 1.000 & -90.0 & -60 & 0.6 \\
\hline 1.000 & -112.5 & -60 & 0.5 \\
\hline 1.000 & -135.0 & -60 & 0.5 \\
\hline 1.000 & -157.5 & -60 & 0.5 \\
\hline
\end{tabular}

\section{Table C.4.1 Drill - Hand Hammer} $60 \mathrm{~Hz}$ RMS Resultant Fields

Distance Horizontal Vertical $60 \mathrm{~Hz}$ meters angle deg angle deg mGauss

\begin{tabular}{|r|r|r|r|}
\hline 1.000 & 180.0 & -60 & 0.4 \\
\hline 1.000 & 157.5 & -60 & 0.5 \\
\hline 1.000 & 135.0 & -60 & 0.5 \\
\hline 1.000 & 112.5 & -60 & 0.6 \\
\hline 1.000 & 90.0 & -60 & 0.5 \\
\hline 1.000 & 67.5 & -60 & 0.7 \\
\hline 1.000 & 45.0 & -60 & 0.8 \\
\hline 1.000 & 22.5 & -60 & 0.7 \\
\hline 0.076 & 0.0 & -90 & 1610.3 \\
\hline 0.176 & 0.0 & -90 & 139.2 \\
\hline 0.376 & 0.0 & -90 & 15.6 \\
\hline 0.376 & 22.5 & -90 & 13.4 \\
\hline
\end{tabular}




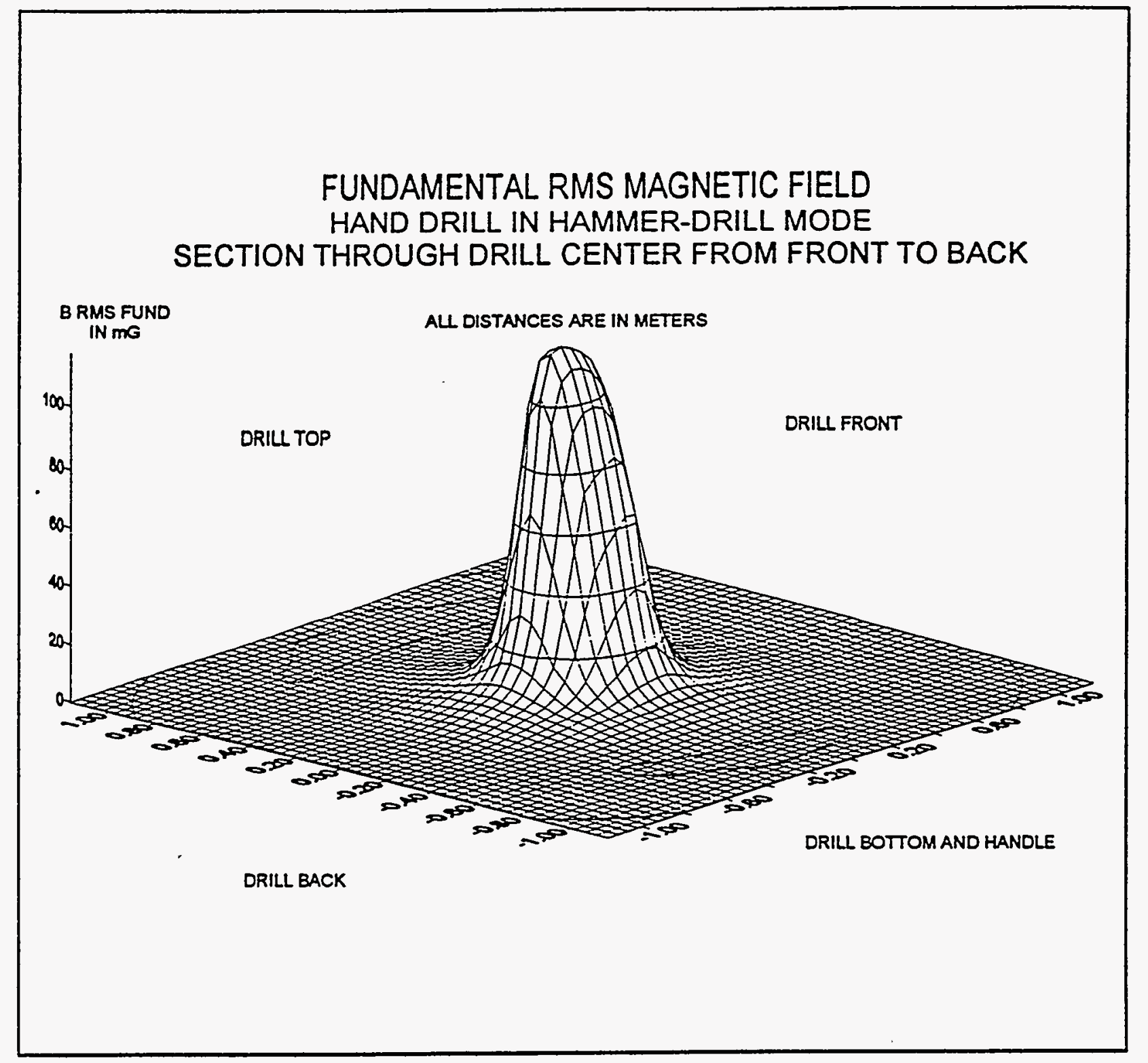

Figure C.4.1 Fundamental RMS Magnetic Field - Hand Drill in Hammer-Drill Mode. 


\section{C.5 Fan - Oscillating}

Appliance Tested: An oscillating table fan was selected.

Manufacturer: EEE

Model: 555

Rated current: 0.32 Amperes

Appliance Code: 2248

Conditions for Testing: The fan was running at high speed with the oscillation feature disengaged.

Measured voltage and current: 119.5 Volts and 0.28 Amperes.

Magnetic Field Summary:

$60 \mathrm{~Hz}$ RMS resultant level at $30 \mathrm{~cm}$ from front surface: $1.1 \mathrm{mG}$

RMS resultant low surface level: $15.8 \mathrm{mG}$ in front

RMS resultant high surface level: $622 \mathrm{mG}$

Highest recorded surface levels:

60 Hertz RMS resultant: $606 \mathrm{mG}$ 180 Hertz RMS resultant: $100 \mathrm{mG}$ 300 Hertz RMS resultant: $30.9 \mathrm{mG}$

420 Hertz RMS resultant: $17.5 \mathrm{mG}$

For the appliance test set, the front of the unit was located at 180 degrees on the test table.

Summary of characteristics: The magnetic fields were moderate with strong harmonic content. 
Distance Horizontal Vertical $60 \mathrm{~Hz}$ meters angle deg angle deg mGauss

\begin{tabular}{|c|c|c|c|}
\hline 0.076 & 0.0 & 90 & 112.3 \\
\hline 0.176 & 0.0 & 90 & 4.0 \\
\hline 0.376 & 0.0 & 90 & 0.2 \\
\hline 1.000 & 0.0 & 90 & 0.2 \\
\hline 0.127 & 0.0 & 60 & 7.2 \\
\hline 0.127 & -22.5 & 60 & 9.1 \\
\hline 0.127 & -45.0 & 60 & 12.6 \\
\hline 0.127 & -67.5 & 60 & 15.5 \\
\hline 0.127 & -90.0 & 60 & 16.6 \\
\hline 0.127 & -112.5 & 60 & 15.3 \\
\hline 0.127 & -135.0 & 60 & 11.8 \\
\hline 0.127 & -157.5 & 60 & 9.3 \\
\hline 0.127 & 180.0 & 60 & 7.0 \\
\hline 0.127 & 157.5 & 60 & 8.1 \\
\hline 0.127 & 135.0 & 60 & 9.9 \\
\hline 0.127 & 112.5 & 60 & 9.5 \\
\hline 0.127 & 90.0 & 60 & 10.3 \\
\hline 0.127 & 67.5 & 60 & 11.3 \\
\hline 0.127 & 45.0 & 60 & 8.7 \\
\hline 0.127 & 22.5 & 60 & 8.0 \\
\hline 0.227 & 0.0 & 60 & 2.5 \\
\hline 0.227 & -22.5 & 60 & 3.2 \\
\hline 0.227 & -45.0 & 60 & 4.0 \\
\hline 0.227 & -67.5 & 60 & 4.4 \\
\hline 0.227 & .90 .0 & 60 & 4.6 \\
\hline 0.227 & -112.5 & 60 & 4.3 \\
\hline 0.227 & -135.0 & 60 & 3.4 \\
\hline 0.227 & -157.5 & 60 & 2.9 \\
\hline 0.227 & 180.0 & 60 & 2.5 \\
\hline 0.227 & 157.5 & 60 & 2.8 \\
\hline
\end{tabular}

Table C.5 Fan - Oscillating

$60 \mathrm{~Hz}$ RMS Resultant Fields

Distance Horizontal Vertical $60 \mathrm{~Hz}$

meters angle deg angle deg mGauss

\begin{tabular}{|r|r|r|r|}
\hline 0.227 & 135.0 & 60 & 3.2 \\
\hline 0.227 & 112.5 & 60 & 3.1 \\
\hline 0.227 & 90.0 & 60 & 3.3 \\
\hline 0.227 & 67.5 & 60 & 3.7 \\
\hline 0.227 & 45.0 & 60 & 2.8 \\
\hline 0.227 & 22.5 & 60 & 2.7 \\
\hline 0.427 & 0.0 & 60 & 0.5 \\
\hline 0.427 & -22.5 & 60 & 0.6 \\
\hline 0.427 & -45.0 & 60 & 0.7 \\
\hline 0.427 & -67.5 & 60 & 0.7 \\
\hline 0.427 & -90.0 & 60 & 0.8 \\
\hline 0.427 & -112.5 & 60 & 0.8 \\
\hline 0.427 & -135.0 & 60 & 0.6 \\
\hline 0.427 & -157.5 & 60 & 0.6 \\
\hline 0.427 & 180.0 & 60 & 0.6 \\
\hline 0.427 & 157.5 & 60 & 0.6 \\
\hline 0.427 & 135.0 & 60 & 0.8 \\
\hline 0.427 & 112.5 & 60 & 0.7 \\
\hline 0.427 & 90.0 & 60 & 0.7 \\
\hline 0.427 & 67.5 & 60 & 0.8 \\
\hline 0.427 & 45.0 & 60 & 0.6 \\
\hline 0.427 & 22.5 & 60 & 0.6 \\
\hline 1.000 & 0.0 & 60 & 0.1 \\
\hline 1.000 & -22.5 & 60 & 0.1 \\
\hline 1.000 & -45.0 & 60 & 0.1 \\
\hline 1.000 & -67.5 & 60 & 0.1 \\
\hline 1.000 & -90.0 & 60 & 0.2 \\
\hline 1.000 & -112.5 & 60 & 0.1 \\
\hline 1.000 & -135.0 & 60 & 0.1 \\
\hline 1.000 & -157.5 & 60 & 0.1 \\
\hline
\end{tabular}


Distance Horizontal Vertical $60 \mathrm{~Hz}$ meters angle deg angle deg mGauss

\begin{tabular}{|r|r|r|r|}
\hline 1.000 & 180.0 & 60 & 0.1 \\
\hline 1.000 & 157.5 & 60 & 0.2 \\
\hline 1.000 & 135.0 & 60 & 0.2 \\
\hline 1.000 & 112.5 & 60 & 0.2 \\
\hline 1.000 & 90.0 & 60 & 0.2 \\
\hline 1.000 & 67.5 & 60 & 0.2 \\
\hline 1.000 & 45.0 & 60 & 0.2 \\
\hline 1.000 & 22.5 & 60 & 0.2 \\
\hline 0.254 & 0.0 & 30 & 5.9 \\
\hline 0.254 & -22.5 & 30 & 8.9 \\
\hline 0.254 & -45.0 & 30 & 13.7 \\
\hline 0.254 & -67.5 & 30 & 17.4 \\
\hline 0.254 & -90.0 & 30 & 18.9 \\
\hline 0.254 & -112.5 & 30 & 16.0 \\
\hline 0.254 & -135.0 & 30 & 9.3 \\
\hline 0.254 & -157.5 & 30 & 6.5 \\
\hline 0.254 & 180.0 & 30 & 6.4 \\
\hline 0.254 & 157.5 & 30 & 8.4 \\
\hline 0.254 & 135.0 & 30 & 10.0 \\
\hline 0.254 & 112.5 & 30 & 9.6 \\
\hline 0.254 & 90.0 & 30 & 10.3 \\
\hline 0.254 & 67.5 & 30 & 10.6 \\
\hline 0.254 & 45.0 & 30 & 8.3 \\
\hline 0.254 & 22.5 & 30 & 6.8 \\
\hline 0.354 & 0.0 & 30 & 2.5 \\
\hline 0.354 & -22.5 & 30 & 3.0 \\
\hline 0.354 & -45.0 & 30 & 3.8 \\
\hline 0.354 & -67.5 & 30 & 4.5 \\
\hline 0.354 & -90.0 & 30 & 5.1 \\
\hline 0.354 & -112.5 & 30 & 5.0 \\
\hline
\end{tabular}

Table C.5 Fan - Oscillating $60 \mathrm{~Hz}$ RMS Resultant Fields

Distance Horizontal Vertical $60 \mathrm{~Hz}$ meters angle deg angle deg mGauss

\begin{tabular}{|r|r|r|r|}
\hline 0.354 & -135.0 & 30 & 3.4 \\
\hline 0.354 & -157.5 & 30 & 2.7 \\
\hline 0.354 & 180.0 & 30 & 2.5 \\
\hline 0.354 & 157.5 & 30 & 2.9 \\
\hline 0.354 & 135.0 & 30 & 3.3 \\
\hline 0.354 & 112.5 & 30 & 3.3 \\
\hline 0.354 & 90.0 & 30 & 3.6 \\
\hline 0.354 & 67.5 & 30 & 3.8 \\
\hline 0.354 & 45.0 & 30 & 3.2 \\
\hline 0.354 & 22.5 & 30 & 2.7 \\
\hline 0.554 & 0.0 & 30 & 0.3 \\
\hline 0.554 & -45.0 & 30 & 0.2 \\
\hline 0.554 & -67.5 & 30 & 0.3 \\
\hline 0.554 & -90.0 & 30 & 0.4 \\
\hline 0.554 & -135.0 & 30 & 0.6 \\
\hline 0.554 & 135.0 & 30 & 0.5 \\
\hline 0.554 & 112.5 & 30 & 0.3 \\
\hline 0.554 & 90.0 & 30 & 0.3 \\
\hline 0.554 & 67.5 & 30 & 0.4 \\
\hline 0.554 & 45.0 & 30 & 0.4 \\
\hline 1.254 & 0.0 & 30 & 0.1 \\
\hline 1.254 & -22.5 & 30 & 0.1 \\
\hline 1.254 & -45.0 & 30 & 0.1 \\
\hline 1.254 & -67.5 & 30 & 0.1 \\
\hline 1.254 & -90.0 & 30 & 0.1 \\
\hline 1.254 & -112.5 & 30 & 0.1 \\
\hline 1.254 & -135.0 & 30 & 0.1 \\
\hline 1.254 & -157.5 & 30 & 0.1 \\
\hline 1.254 & 180.0 & 30 & 0.1 \\
\hline 1.254 & 157.5 & 30 & 0.1 \\
\hline
\end{tabular}


Distance Horizontal Vertical $60 \mathrm{~Hz}$ meters angle deg angle deg mGauss

\begin{tabular}{|c|c|c|c|}
\hline 1.254 & 135.0 & 30 & 0.1 \\
\hline 1.254 & 112.5 & 30 & 0.2 \\
\hline 1.254 & 90.0 & 30 & 0.2 \\
\hline 1.254 & 67.5 & 30 & 0.2 \\
\hline 1.254 & 45.0 & 30 & 0.2 \\
\hline 1.254 & 22.5 & 30 & 0.1 \\
\hline 0.254 & 0.0 & 0 & 4.3 \\
\hline 0.254 & -22.5 & 0 & 5.8 \\
\hline 0.254 & -45.0 & 0 & 8.4 \\
\hline 0.254 & -67.5 & 0 & 11.3 \\
\hline 0.254 & -90.0 & 0 & 12.6 \\
\hline 0.254 & -112.5 & 0 & 11.5 \\
\hline 0.254 & -135.0 & 0 & 6.4 \\
\hline 0.254 & -157.5 & 0 & 4.9 \\
\hline 0.254 & 180.0 & 0 & 5.3 \\
\hline 0.254 & 157.5 & 0 & 6.5 \\
\hline 0.254 & 135.0 & 0 & 7.2 \\
\hline 0.254 & 112.5 & 0 & 6.9 \\
\hline 0.254 & 90.0 & 0 & 7.4 \\
\hline 0.254 & 67.5 & 0 & 7.1 \\
\hline 0.254 & 45.0 & 0 & 6.2 \\
\hline 0.254 & 22.5 & 0 & 5.0 \\
\hline 0.354 & 0.0 & 0 & 1.7 \\
\hline 0.354 & -22.5 & 0 & 1.9 \\
\hline 0.354 & -45.0 & 0 & 2.7 \\
\hline 0.354 & -67.5 & 0 & 3.4 \\
\hline 0.354 & -90.0 & 0 & 3.9 \\
\hline 0.354 & -112.5 & 0 & 3.7 \\
\hline 0.354 & -135.0 & 0 & 2.3 \\
\hline 0.354 & -157.5 & 0 & 1.8 \\
\hline
\end{tabular}

Table C.5 Fan - Oscillating $60 \mathrm{~Hz}$ RMS Resultant Fields

Distance Horizontal Vertical $60 \mathrm{~Hz}$ meters angle deg angle deg mGauss

\begin{tabular}{|c|c|c|c|}
\hline 0.354 & 180.0 & 0 & 1.9 \\
\hline 0.354 & 157.5 & 0 & 2.5 \\
\hline 0.354 & 135.0 & 0 & 2.8 \\
\hline 0.354 & 112.5 & 0 & 2.8 \\
\hline 0.354 & 90.0 & 0 & 3.0 \\
\hline 0.354 & 67.5 & 0 & 2.9 \\
\hline 0.354 & 45.0 & 0 & 2.6 \\
\hline 0.354 & 22.5 & 0 & 2.0 \\
\hline 0.554 & 0.0 & 0 & 0.4 \\
\hline 0.554 & -22.5 & 0 & 0.6 \\
\hline 0.554 & -45.0 & 0 & 0.7 \\
\hline 0.554 & -67.5 & 0 & 0.8 \\
\hline 0.554 & -90.0 & 0 & 0.9 \\
\hline 0.554 & -112.5 & 0 & 1.0 \\
\hline 0.554 & -135.0 & 0 & 0.6 \\
\hline 0.554 & -157.5 & 0 & 0.6 \\
\hline 0.554 & 180.0 & 0 & 0.5 \\
\hline 0.554 & 157.5 & 0 & 0.7 \\
\hline 0.554 & 135.0 & 0 & 0.7 \\
\hline 0.554 & 112.5 & 0 & 0.7 \\
\hline 0.554 & 90.0 & 0 & 0.9 \\
\hline 0.554 & 67.5. & 0 & 0.7 \\
\hline 0.554 & 45.0 & 0 & 0.6 \\
\hline 0.554 & 22.5 & 0 & 0.5 \\
\hline 1.254 & 0.0 & 0 & 0.1 \\
\hline 1.254 & -22.5 & 0 & 0.1 \\
\hline 1.254 & -45.0 & 0 & 0.1 \\
\hline 1.254 & -67.5 & 0 & 0.1 \\
\hline 1.254 & -90.0 & 0 & 0.1 \\
\hline 1.254 & -112.5 & 0 & 0.1 \\
\hline & & & \\
\hline 0.5 & 0 & 0 & 0 \\
\hline
\end{tabular}


Distance Horizontal Vertical $60 \mathrm{~Hz}$ meters angle deg angle deg mGauss

\begin{tabular}{|r|r|r|r|}
\hline 1.254 & -135.0 & 0 & 0.1 \\
\hline 1.254 & -157.5 & 0 & 0.1 \\
\hline 1.254 & 180.0 & 0 & 0.1 \\
\hline 1.254 & 157.5 & 0 & 0.1 \\
\hline 1.254 & 135.0 & 0 & 0.1 \\
\hline 1.254 & 112.5 & 0 & 0.1 \\
\hline 1.254 & 90.0 & 0 & 0.1 \\
\hline 1.254 & 67.5 & 0 & 0.1 \\
\hline 1.254 & 45.0 & 0 & 0.1 \\
\hline 1.254 & 22.5 & 0 & 0.1 \\
\hline 0.457 & 0.0 & -30 & 1.9 \\
\hline 0.457 & -22.5 & -30 & 2.3 \\
\hline 0.457 & -45.0 & -30 & 2.8 \\
\hline 0.457 & -67.5 & -30 & 3.3 \\
\hline 0.457 & -90.0 & -30 & 3.8 \\
\hline 0.457 & -112.5 & -30 & 3.7 \\
\hline 0.457 & -135.0 & -30 & 2.6 \\
\hline 0.457 & -157.5 & -30 & 2.6 \\
\hline 0.457 & 180.0 & -30 & 2.8 \\
\hline 0.457 & 157.5 & -30 & 3.3 \\
\hline 0.457 & 135.0 & -30 & 3.1 \\
\hline 0.457 & 112.5 & -30 & 2.8 \\
\hline 0.457 & 90.0 & -30 & 2.7 \\
\hline 0.457 & 67.5 & -30 & 2.6 \\
\hline 0.457 & 45.0 & -30 & 2.8 \\
\hline 0.457 & 22.5 & -30 & 2.1 \\
\hline 0.557 & 0.0 & -30 & 1.0 \\
\hline 0.557 & -22.5 & -30 & 1.0 \\
\hline 0.557 & -45.0 & -30 & 1.1 \\
\hline 0.557 & -67.5 & -30 & 1.4 \\
\hline & & & \\
\hline 1.20 & 0 & 0 & 0 \\
\hline
\end{tabular}

Table C.5 Fan - Oscillating $60 \mathrm{~Hz}$ RMS Resultant Fields

Distance Horizontal Vertical $60 \mathrm{~Hz}$ meters angle deg angle deg mGauss

\begin{tabular}{|c|c|c|c|}
\hline 0.557 & -90.0 & -30 & 1.5 \\
\hline 0.557 & -112.5 & -30 & 1.5 \\
\hline 0.557 & -135.0 & -30 & 1.1 \\
\hline 0.557 & -157.5 & -30 & 1.1 \\
\hline 0.557 & 180.0 & -30 & 1.3 \\
\hline 0.557 & 157.5 & -30 & 1.6 \\
\hline 0.557 & 135.0 & -30 & 1.6 \\
\hline 0.557 & 112.5 & -30 & 1.5 \\
\hline 0.557 & 90.0 & -30 & 1.6 \\
\hline 0.557 & 67.5 & -30 & 1.4 \\
\hline 0.557 & 45.0 & -30 & 1.5 \\
\hline 0.557 & 22.5 & -30 & 1.2 \\
\hline 0.757 & 0.0 & -30 & 0.4 \\
\hline 0.757 & -22.5 & -30 & 0.4 \\
\hline 0.757 & -45.0 & -30 & 0.5 \\
\hline 0.757 & -67.5 & -30 & 0.6 \\
\hline 0.757 & -90.0 & -30 & 0.5 \\
\hline 0.757 & -112.5 & -30 & 0.6 \\
\hline 0.757 & -135.0 & -30 & 0.4 \\
\hline 0.757 & -157.5 & -30 & 0.4 \\
\hline 0.757 & 180.0 & -30 & 0.3 \\
\hline 0.757 & 157.5 & -30 & 0.5 \\
\hline 0.757 & 135.0 & -30 & 0.4 \\
\hline 0.757 & 112.5 & -30 & 0.4 \\
\hline 0.757 & 90.0 & -30 & 0.5 \\
\hline 0.757 & 67.5 & -30 & 0.4 \\
\hline 0.757 & 45.0 & -30 & 0.4 \\
\hline 0.757 & 22.5 & -30 & 0.3 \\
\hline 1.457 & 0.0 & -30 & 0.1 \\
\hline 1.457 & -22.5 & -30 & 0.1 \\
\hline & & & \\
\hline 0.50 & -30 & -30 & \\
\hline
\end{tabular}


Distance Horizontal Vertical $60 \mathrm{~Hz}$ meters angle deg angle deg mGauss

\begin{tabular}{|l|r|r|r|}
\hline 1.457 & -45.0 & -30 & 0.1 \\
\hline 1.457 & -67.5 & -30 & 0.1 \\
\hline 1.457 & -90.0 & -30 & 0.1 \\
\hline 1.457 & -112.5 & -30 & 0.1 \\
\hline 1.457 & -135.0 & -30 & 0.1 \\
\hline 1.457 & -157.5 & -30 & 0.1 \\
\hline 1.457 & 180.0 & -30 & 0.1 \\
\hline 1.457 & 157.5 & -30 & 0.1 \\
\hline 1.457 & 135.0 & -30 & 0.1 \\
\hline 1.457 & 112.5 & -30 & 0.1 \\
\hline 1.457 & 90.0 & -30 & 0.1 \\
\hline 1.457 & 67.5 & -30 & 0.1 \\
\hline 1.457 & 45.0 & -30 & 0.1 \\
\hline 1.457 & 22.5 & -30 & 0.1 \\
\hline 0.483 & 0.0 & -60 & 0.8 \\
\hline 0.483 & -22.5 & -60 & 1.0 \\
\hline 0.483 & -45.0 & -60 & 1.1 \\
\hline 0.483 & -67.5 & -60 & 1.0 \\
\hline 0.483 & -90.0 & -60 & 1.0 \\
\hline 0.483 & -112.5 & -60 & 1.1 \\
\hline 0.483 & -135.0 & -60 & 0.8 \\
\hline 0.483 & -157.5 & -60 & 1.0 \\
\hline 0.483 & 180.0 & -60 & 1.0 \\
\hline 0.483 & 157.5 & -60 & 1.2 \\
\hline 0.483 & 135.0 & -60 & 1.0 \\
\hline 0.483 & 112.5 & -60 & 1.0 \\
\hline 0.483 & 90.0 & -60 & 1.1 \\
\hline 0.483 & 67.5 & -60 & 0.8 \\
\hline 0.483 & 45.0 & -60 & 1.1 \\
\hline 0.483 & 22.5 & -60 & 2.2 \\
\hline
\end{tabular}

Table C.5 Fan - Oscillating

\section{$60 \mathrm{~Hz}$ RMS Resultant Fields}

Distance Horizontal Vertical $60 \mathrm{~Hz}$

meters angle deg angle deg mGauss

\begin{tabular}{|r|r|r|r|}
\hline 0.583 & 0.0 & -60 & 0.5 \\
\hline 0.583 & -22.5 & -60 & 0.5 \\
\hline 0.583 & -45.0 & -60 & 0.5 \\
\hline 0.583 & -67.5 & -60 & 0.5 \\
\hline 0.583 & -90.0 & -60 & 0.4 \\
\hline 0.583 & -112.5 & -60 & 0.5 \\
\hline 0.583 & -135.0 & -60 & 0.5 \\
\hline 0.583 & -157.5 & -60 & 0.6 \\
\hline 0.583 & 180.0 & -60 & 0.7 \\
\hline 0.583 & 157.5 & -60 & 0.7 \\
\hline 0.583 & 135.0 & -60 & 0.7 \\
\hline 0.583 & 112.5 & -60 & 0.7 \\
\hline 0.583 & 90.0 & -60 & 0.7 \\
\hline 0.583 & 67.5 & -60 & 0.7 \\
\hline 0.583 & 45.0 & -60 & 0.8 \\
\hline 0.583 & 22.5 & -60 & 1.0 \\
\hline 0.783 & 0.0 & -60 & 0.2 \\
\hline 0.783 & -22.5 & -60 & 0.3 \\
\hline 0.783 & -45.0 & -60 & 0.2 \\
\hline 0.783 & -90.0 & -60 & 0.3 \\
\hline 0.783 & -135.0 & -60 & 0.3 \\
\hline 0.783 & 180.0 & -60 & 0.3 \\
\hline 0.783 & 157.5 & -60 & 0.3 \\
\hline 0.783 & 135.0 & -60 & 0.3 \\
\hline 0.783 & 112.5 & -60 & 0.3 \\
\hline 0.783 & 90.0 & -60 & 0.3 \\
\hline 0.783 & 67.5 & -60 & 0.3 \\
\hline 0.783 & 45.0 & -60 & 0.3 \\
\hline 0.783 & 22.5 & -60 & 0.3 \\
\hline 1.000 & 0.0 & -60 & 0.1 \\
\hline
\end{tabular}


Table C.5 Fan - Oscillating

$60 \mathrm{~Hz}$ RMS Resultant Fields

Distance Horizontal Vertical $60 \mathrm{~Hz}$

Distance Horizontal Vertical $60 \mathrm{~Hz}$

meters angle deg angle deg mGauss

meters angle deg angle deg mGauss

\begin{tabular}{|l|r|r|r|}
\hline 1.000 & -22.5 & -60 & 0.1 \\
\hline 1.000 & -45.0 & -60 & 0.1 \\
\hline 1.000 & -67.5 & -60 & 0.1 \\
\hline 1.000 & -90.0 & -60 & 0.1 \\
\hline 1.000 & -112.5 & -60 & 0.1 \\
\hline 1.000 & -135.0 & -60 & 0.1 \\
\hline 1.000 & -157.5 & -60 & 0.1 \\
\hline 1.000 & 180.0 & -60 & 0.1 \\
\hline 1.000 & 157.5 & -60 & 0.1 \\
\hline
\end{tabular}

\begin{tabular}{|l|l|l|l|}
\hline 1.000 & 135.0 & -60 & 0.1 \\
\hline 1.000 & 112.5 & -60 & 0.1 \\
\hline 1.000 & 90.0 & -60 & 0.1 \\
\hline 1.000 & 67.5 & -60 & 0.1 \\
\hline 1.000 & 45.0 & -60 & 0.1 \\
\hline 1.000 & 22.5 & -60 & 0.1 \\
\hline 0.432 & 0.0 & -90 & 1.1 \\
\hline 0.532 & 0.0 & -90 & 0.7 \\
\hline
\end{tabular}




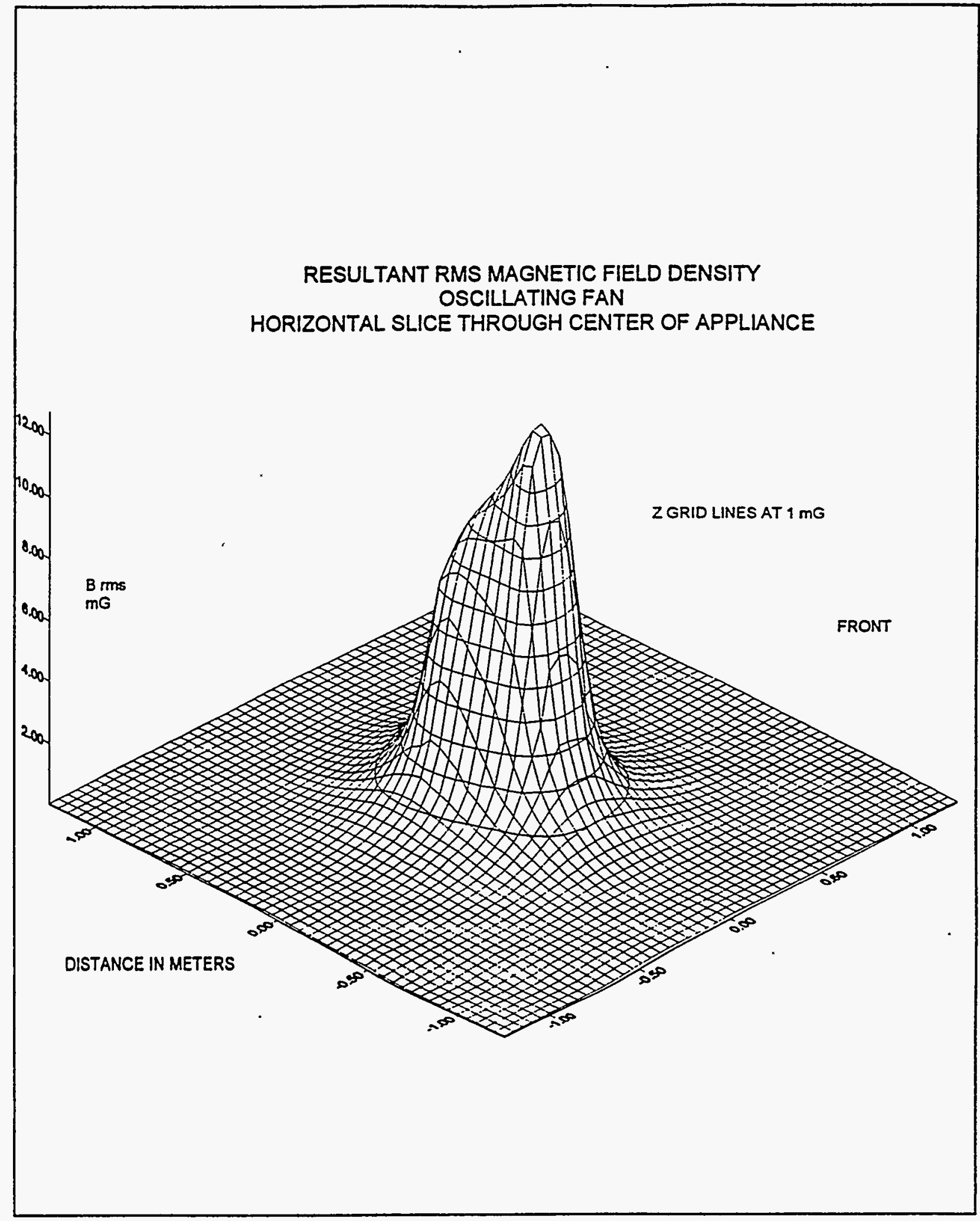

Figure C.5.1 Fundamental Resultant RMS Magnetic Field Density - Oscillating Fan. C. $5-8$ 


\section{C.6 Heater - Portable Space}

Appliance Tested: A small portable electric space heater with a fan. Manufacturer: FFF

Model: 6-6

Rated current: 12.5 Amperes

Appliance Code: 2258

Conditions for Testing: The heater element and fan were operating at the HIGH heat selection.

Measured voltage and current: 110.1 Volts and 10.4 Amperes.

Magnetic Field Summary:

$60 \mathrm{~Hz}$ RMS resultant level at $30 \mathrm{~cm}$ from front surface: $2.2 \mathrm{mG}$

RMS resultant low surface level: $31.3 \mathrm{mG}$ on bottom

RMS resultant high surface level: $156 \mathrm{mG}$ at rear

Highest recorded surface levels:

60 Hertz RMS resultant: $156 \mathrm{mG}$

180 Hertz RMS resultant: $39.7 \mathrm{mG}$

300 Hertz RMS resultant: $10.8 \mathrm{mG}$

420 Hertz RMS resultant: $1.41 \mathrm{mG}$

For the appliance test set, the front of the unit was located at 0 degrees on the test table.

Summary of characteristics: The heater had little magnetic field even though the current was relatively high. The higher harmonics were generated by the fan motor. 
Table C. 6 Heater - Portable Space

$60 \mathrm{~Hz}$ RMS Resultant Fields

Distance Horizontal Vortical $60 \mathrm{~Hz}$ metere ongle dog angle dog mGause

\begin{tabular}{|r|r|r|r|}
\hline 0.127 & 0.0 & 90 & 48.3 \\
\hline 0.227 & 0.0 & 90 & 10.0 \\
\hline 0.427 & 0.0 & 90 & 1.1 \\
\hline 1.000 & 0.0 & 90 & 0.1 \\
\hline 0.127 & 0.0 & 60 & 34.8 \\
\hline 0.127 & -22.5 & 60 & 31.1 \\
\hline 0.127 & -45.0 & 60 & 29.4 \\
\hline 0.127 & -67.5 & 60 & 28.5 \\
\hline 0.127 & -90.0 & 60 & 28.0 \\
\hline 0.127 & -112.5 & 60 & 25.0 \\
\hline 0.127 & -135.0 & 60 & 21.7 \\
\hline 0.127 & -157.5 & 60 & 20.3 \\
\hline 0.127 & 180.0 & 60 & 21.3 \\
\hline 0.127 & 157.5 & 60 & 24.1 \\
\hline 0.127 & 135.0 & 60 & 26.9 \\
\hline 0.127 & 112.5 & 60 & 24.6 \\
\hline 0.127 & 90.0 & 60 & 36.9 \\
\hline 0.127 & 67.5 & 60 & 44.9 \\
\hline 0.127 & 45.0 & 60 & 44.1 \\
\hline 0.127 & 22.5 & 60 & 40.5 \\
\hline 0.227 & 0.0 & 60 & 7.8 \\
\hline 0.227 & -22.5 & 60 & 7.1 \\
\hline 0.227 & -45.0 & 60 & 6.2 \\
\hline 0.227 & -67.5 & 60 & 5.6 \\
\hline 0.227 & -90.0 & 60 & 5.4 \\
\hline 0.227 & -112.5 & 60 & 5.4 \\
\hline 0.227 & -135.0 & 60 & 5.5 \\
\hline 0.227 & -157.5 & 60 & 5.7 \\
\hline 0.227 & 180.0 & 60 & 5.9 \\
\hline 0.227 & 157.5 & 60 & 6.1 \\
\hline
\end{tabular}

Distance Horizontal Vertical $60 \mathrm{~Hz}$

meters angle deg angle deg mGause

\begin{tabular}{|r|r|r|r|}
\hline 0.227 & 135.0 & 60 & 6.1 \\
\hline 0.227 & 112.5 & 60 & 6.1 \\
\hline 0.227 & 90.0 & 60 & 6.3 \\
\hline 0.227 & 67.5 & 60 & 7.0 \\
\hline 0.227 & 45.0 & 60 & 8.0 \\
\hline 0.227 & 22.5 & 60 & 8.2 \\
\hline 0.427 & 0.0 & 60 & 1.3 \\
\hline 0.427 & -22.5 & 60 & 1.2 \\
\hline 0.427 & -45.0 & 60 & 1.1 \\
\hline 0.427 & -67.5 & 60 & 1.1 \\
\hline 0.427 & -90.0 & 60 & 1.0 \\
\hline 0.427 & -112.5 & 60 & 1.0 \\
\hline 0.427 & -135.0 & 60 & 1.0 \\
\hline 0.427 & -157.5 & 60 & 1.0 \\
\hline 0.427 & 180.0 & 60 & 1.1 \\
\hline 0.427 & 157.5 & 60 & 1.1 \\
\hline 0.427 & 135.0 & 60 & 1.1 \\
\hline 0.427 & 112.5 & 60 & 1.1 \\
\hline 0.427 & 90.0 & 60 & 1.0 \\
\hline 0.427 & 67.5 & 60 & 1.1 \\
\hline 0.427 & 45.0 & 60 & 1.2 \\
\hline 0.427 & 22.5 & 60 & 1.2 \\
\hline 1.000 & 0.0 & 60 & 0.1 \\
\hline 1.000 & -22.5 & 60 & 0.1 \\
\hline 1.000 & -45.0 & 60 & 0.1 \\
\hline 1.000 & -67.5 & 60 & 0.1 \\
\hline 1.000 & -90.0 & 60 & 0.1 \\
\hline 1.000 & -112.5 & 60 & 0.1 \\
\hline 1.000 & -135.0 & 60 & 0.1 \\
\hline 1.000 & -157.5 & 60 & 0.1 \\
\hline
\end{tabular}


Table C. 6 Heater - Portable Space $60 \mathrm{~Hz}$ RMS Resultant Fields

Distance Horizontal Vertical $60 \mathrm{~Hz}$ meters angle deg angle deg mGauss

Distance Horizontal Vertical $60 \mathrm{~Hz}$ meters angle deg angle deg mGauss

\begin{tabular}{|c|c|c|c|}
\hline 1.000 & 180.0 & 60 & 0.1 \\
\hline 1.000 & 157.5 & 60 & 0.1 \\
\hline 1.000 & 135.0 & 60 & 0.1 \\
\hline 1.000 & 112.5 & 60 & 0.1 \\
\hline 1.000 & 90.0 & 60 & 0.1 \\
\hline 1.000 & 67.5 & 60 & 0.1 \\
\hline 1.000 & 45.0 & 60 & 0.1 \\
\hline 1.000 & 22.5 & 60 & 0.1 \\
\hline 0.140 & 0.0 & 30 & 33.0 \\
\hline 0.140 & -22.5 & 30 & 27.9 \\
\hline 0.140 & -45.0 & 30 & 21.6 \\
\hline 0.140 & -67.5 & 30 & 17.2 \\
\hline 0.140 & -90.0 & 30 & 16.2 \\
\hline 0.140 & -112.5 & 30 & 14.7 \\
\hline 0.140 & -135.0 & 30 & 13.1 \\
\hline 0.140 & -157.5 & 30 & 13.6 \\
\hline 0.140 & 180.0 & 30 & 16.4 \\
\hline 0.140 & 157.5 & 30 & 20.8 \\
\hline 0.140 & 135.0 & 30 & 26.1 \\
\hline 0.140 & 112.5 & 30 & 28.4 \\
\hline 0.140 & 90.0 & 30 & 26.5 \\
\hline 0.140 & 67.5 & 30 & 30.3 \\
\hline 0.140 & 45.0 & 30 & 37.0 \\
\hline 0.140 & 22.5 & 30 & 36.4 \\
\hline 0.240 & 0.0 & 30 & 7.7 \\
\hline 0.240 & -22.5 & 30 & 7.1 \\
\hline 0.240 & -45.0 & 30 & 6.1 \\
\hline 0.240 & -67.5 & 30 & 5.1 \\
\hline 0.240 & -90.0 & 30 & 4.3 \\
\hline 0.240 & -112.5 & 30 & 4.0 \\
\hline
\end{tabular}

\begin{tabular}{|c|c|c|c|}
\hline 0.240 & -135.0 & 30 & 4.1 \\
\hline 0.240 & -157.5 & 30 & 4.4 \\
\hline 0.240 & 180.0 & 30 & 5.0 \\
\hline 0.240 & 157.5 & 30 & 5.6 \\
\hline 0.240 & 135.0 & 30 & 6.0 \\
\hline 0.240 & 112.5 & 30 & 6.1 \\
\hline 0.240 & 90.0 & 30 & 6.1 \\
\hline 0.240 & 67.5 & 30 & 6.4 \\
\hline 0.240 & 45.0 & 30 & 7.3 \\
\hline 0.240 & 22.5 & 30 & 7.9 \\
\hline 0.440 & 0.0 & 30 & 1.6 \\
\hline 0.440 & -22.5 & 30 & 1.4 \\
\hline 0.440 & -45.0 & 30 & 1.2 \\
\hline 0.440 & -67.5 & 30 & 1.1 \\
\hline 0.440 & -90.0 & 30 & 0.9 \\
\hline 0.440 & -112.5 & 30 & 0.8 \\
\hline 0.440 & -135.0 & 30 & 0.9 \\
\hline 0.440 & -157.5 & 30 & 1.0 \\
\hline 0.440 & 180.0 & 30 & 1.1 \\
\hline 0.440 & 157.5 & 30 & 1.3 \\
\hline 0.440 & 135.0 & 30 & 1.2 \\
\hline 0.440 & 112.5 & 30 & 1.1 \\
\hline 0.440 & 90.0 & 30 & 1.1 \\
\hline 0.440 & 67.5 & 30 & 1.0 \\
\hline 0.440 & 45.0 & 30 & 1.2 \\
\hline 0.440 & 22.5 & 30 & 1.4 \\
\hline 1.140 & 0.0 & 30 & 0.1 \\
\hline 1.140 & -22.5 & 30 & 0.1 \\
\hline 1.140 & -45.0 & 30 & 0.1 \\
\hline 1.140 & -67.5 & 30 & 0.1 \\
\hline
\end{tabular}

C. 6-3 
Table C. 6 Heater - Portable Space

\section{$60 \mathrm{~Hz}$ RMS Resultant Fields}

Distance Horizontal Vertical $60 \mathrm{~Hz}$

metere angle deg angle deg mGause

\begin{tabular}{|r|r|r|r|}
\hline 1.140 & -90.0 & 30 & 0.1 \\
\hline 1.140 & -112.5 & 30 & 0.1 \\
\hline 1.140 & -135.0 & 30 & 0.1 \\
\hline 1.140 & -157.5 & 30 & 0.1 \\
\hline 1.140 & 180.0 & 30 & 0.1 \\
\hline 1.140 & 157.5 & 30 & 0.1 \\
\hline 1.140 & 135.0 & 30 & 0.1 \\
\hline 1.140 & 112.5 & 30 & 0.1 \\
\hline 1.140 & 90.0 & 30 & 0.1 \\
\hline 1.140 & 67.5 & 30 & 0.1 \\
\hline 1.140 & 45.0 & 30 & 0.1 \\
\hline 1.140 & 22.5 & 30 & 0.1 \\
\hline 0.114 & 0.0 & 0 & 35.5 \\
\hline 0.114 & -22.5 & 0 & 32.7 \\
\hline 0.114 & -45.0 & 0 & 25.2 \\
\hline 0.114 & -67.5 & 0 & 18.0 \\
\hline 0.114 & -90.0 & 0 & 14.0 \\
\hline 0.114 & -112.5 & 0 & 12.0 \\
\hline 0.114 & -135.0 & 0 & 11.1 \\
\hline 0.114 & -157.5 & 0 & 12.4 \\
\hline 0.114 & 180.0 & 0 & 16.1 \\
\hline 0.114 & 157.5 & 0 & 21.6 \\
\hline 0.114 & 135.0 & 0 & 28.5 \\
\hline 0.114 & 112.5 & 0 & 32.2 \\
\hline 0.114 & 90.0 & 0 & 28.6 \\
\hline 0.114 & 67.5 & 0 & 28.6 \\
\hline 0.114 & 45.0 & 0 & 35.5 \\
\hline 0.114 & 22.5 & 0 & 37.9 \\
\hline 0.214 & 0.0 & 0 & 8.6 \\
\hline 0.22 .5 & 0 & 8.1 \\
\hline 1.14 & & & \\
\hline & 30 & & 0 \\
\hline
\end{tabular}

Distance Horizontal Vertical $60 \mathrm{~Hz}$

meters angle dog angle deg mGauss

\begin{tabular}{|c|c|c|c|}
\hline 0.214 & -45.0 & 0 & 6.7 \\
\hline 0.214 & -67.5 & 0 & 5.1 \\
\hline 0.214 & -90.0 & 0 & 3.9 \\
\hline 0.214 & -112.5 & 0 & 3.5 \\
\hline 0.214 & -135.0 & 0 & 3.6 \\
\hline 0.214 & -157.5 & 0 & 4.2 \\
\hline 0.214 & 180.0 & 0 & 5.2 \\
\hline 0.214 & 157.5 & 0 & 6.3 \\
\hline 0.214 & 135.0 & 0 & 7.1 \\
\hline 0.214 & 112.5 & 0 & 6.9 \\
\hline 0.214 & 90.0 & 0 & 5.8 \\
\hline 0.214 & 67.5 & 0 & 5.4 \\
\hline 0.214 & 45.0 & 0 & 7.1 \\
\hline 0.214 & 22.5 & 0 & 8.9 \\
\hline 0.414 & 0.0 & 0 & 1.6 \\
\hline 0.414 & -22.5 & 0 & 1.6 \\
\hline 0.414 & -45.0 & 0 & 1.3 \\
\hline 0.414 & -67.5 & 0 & 1.1 \\
\hline 0.414 & -90.0 & 0 & 0.8 \\
\hline 0.414 & -112.5 & 0 & 0.7 \\
\hline 0.414 & -135.0 & 0 & 0.8 \\
\hline 0.414 & -157.5 & 0 & 1.0 \\
\hline 0.414 & 180.0 & 0 & 1.2 \\
\hline 0.414 & 157.5 & 0 & 1.4 \\
\hline 0.414 & 135.0 & 0 & 1.4 \\
\hline 0.414 & 112.5 & 0 & 1.3 \\
\hline 0.414 & 90.0 & 0 & 1.1 \\
\hline 0.414 & 67.5 & 0 & 0.7 \\
\hline 0.414 & 45.0 & 0 & 0.9 \\
\hline 0.414 & 22.5 & 0 & 1.4 \\
\hline
\end{tabular}


Table C.6 Heater - Portable Space $60 \mathrm{~Hz}$ RMS Resultant Fields

Distance Horizontal Vertical $60 \mathrm{~Hz}$ meters angle deg angle deg mGauss

\begin{tabular}{|r|r|r|r|}
\hline 1.114 & 0.0 & 0 & 0.1 \\
\hline 1.114 & -22.5 & 0 & 0.1 \\
\hline 1.114 & -45.0 & 0 & 0.1 \\
\hline 1.114 & -67.5 & 0 & 0.1 \\
\hline 1.114 & -90.0 & 0 & 0.1 \\
\hline 1.114 & -112.5 & 0 & 0.1 \\
\hline 1.114 & -135.0 & 0 & 0.1 \\
\hline 1.114 & -157.5 & 0 & 0.1 \\
\hline 1.114 & 180.0 & 0 & 0.1 \\
\hline 1.114 & 157.5 & 0 & 0.1 \\
\hline 1.114 & 135.0 & 0 & 0.1 \\
\hline 1.114 & 112.5 & 0 & 0.1 \\
\hline 1.114 & 90.0 & 0 & 0.1 \\
\hline 1.114 & 67.5 & 0 & 0.1 \\
\hline 1.114 & 45.0 & 0 & 0.1 \\
\hline 1.114 & 22.5 & 0 & 0.1 \\
\hline 0.279 & 0.0 & -30 & 4.1 \\
\hline 0.279 & -22.5 & -30 & 4.0 \\
\hline 0.279 & -45.0 & -30 & 3.6 \\
\hline 0.279 & -67.5 & -30 & 3.1 \\
\hline 0.279 & -90.0 & -30 & 2.6 \\
\hline 0.279 & -112.5 & -30 & 2.2 \\
\hline 0.279 & -135.0 & -30 & 2.5 \\
\hline 0.279 & -157.5 & -30 & 2.7 \\
\hline 0.279 & 180.0 & -30 & 3.0 \\
\hline 0.279 & 157.5 & -30 & 3.4 \\
\hline 0.279 & 135.0 & -30 & 3.7 \\
\hline 0.279 & 112.5 & -30 & 3.1 \\
\hline 0.279 & 90.0 & -30 & 3.9 \\
\hline 0.279 & 67.5 & -30 & 4.1 \\
\hline
\end{tabular}

Distance Horizontal Vertical $60 \mathrm{~Hz}$ meters angle deg ongle deg mGauss

\begin{tabular}{|r|r|r|r|}
\hline 0.279 & 45.0 & -30 & 22.0 \\
\hline 0.279 & 22.5 & -30 & 28.6 \\
\hline 0.379 & 0.0 & -30 & 2.0 \\
\hline 0.379 & -22.5 & -30 & 1.9 \\
\hline 0.379 & -45.0 & -30 & 1.8 \\
\hline 0.379 & -67.5 & -30 & 1.5 \\
\hline 0.379 & -90.0 & -30 & 1.3 \\
\hline 0.379 & -112.5 & -30 & 1.2 \\
\hline 0.379 & -135.0 & -30 & 1.2 \\
\hline 0.379 & -157.5 & -30 & 1.4 \\
\hline 0.379 & 180.0 & -30 & 1.5 \\
\hline 0.379 & 157.5 & -30 & 1.7 \\
\hline 0.379 & 135.0 & -30 & 1.7 \\
\hline 0.379 & 112.5 & -30 & 1.6 \\
\hline 0.379 & 90.0 & -30 & 2.0 \\
\hline 0.379 & 67.5 & -30 & 1.2 \\
\hline 0.379 & 45.0 & -30 & 2.9 \\
\hline 0.379 & 22.5 & -30 & 5.9 \\
\hline 0.579 & 0.0 & -30 & 0.7 \\
\hline 0.579 & -22.5 & -30 & 0.7 \\
\hline 0.579 & -45.0 & -30 & 0.6 \\
\hline 0.579 & -67.5 & -30 & 0.5 \\
\hline 0.579 & -90.0 & -30 & 0.4 \\
\hline 0.579 & -112.5 & -30 & 0.4 \\
\hline 0.579 & -135.0 & -30 & 0.5 \\
\hline 0.579 & -157.5 & -30 & 0.5 \\
\hline 0.579 & 180.0 & -30 & 0.5 \\
\hline 0.579 & 157.5 & -30 & 0.6 \\
\hline 0.579 & 135.0 & -30 & 0.6 \\
\hline 0.579 & 112.5 & -30 & 0.6 \\
\hline
\end{tabular}


Table C.6 Heater - Portable Space $60 \mathrm{~Hz}$ RMS Resultant Fields

Distance Horizontal Vertical $60 \mathrm{~Hz}$ moters angle deg angle deg mGause

\begin{tabular}{|c|c|c|c|}
\hline 0.579 & 90.0 & .30 & 0.7 \\
\hline 0.579 & 67.5 & -30 & 0.4 \\
\hline 0.579 & 45.0 & -30 & 0.5 \\
\hline 0.579 & 22.5 & -30 & 0.9 \\
\hline 1.279 & 0.0 & 30 & 0.1 \\
\hline 1.279 & -22.5 & -30 & 0.1 \\
\hline 1.279 & -45.0 & -30 & 0.1 \\
\hline 1.279 & -67.5 & .30 & 0.1 \\
\hline 1.279 & .90 .0 & -30 & 0.1 \\
\hline 1.279 & -112.5 & .30 & 0.1 \\
\hline 1.279 & -135.0 & -30 & 0.1 \\
\hline 1.279 & -157.5 & -30 & 0.1 \\
\hline 1.279 & 180.0 & -30 & 0.1 \\
\hline 1.279 & 157.5 & -30 & 0.1 \\
\hline 1.279 & 135.0 & -30 & 0.1 \\
\hline 1.279 & 112.5 & -30 & 0.1 \\
\hline 1.279 & 90.0 & -30 & 0.1 \\
\hline 1.279 & 67.5 & -30 & 0.1 \\
\hline 1.279 & 45.0 & -30 & 0.1 \\
\hline 1.279 & 22.5 & -30 & 0.1 \\
\hline 0.356 & 0.0 & -60 & 2.3 \\
\hline 0.356 & -22.5 & -60 & 2.2 \\
\hline 0.356 & -45.0 & -60 & 2.1 \\
\hline 0.356 & -67.5 & -60 & 2.0 \\
\hline 0.356 & -90.0 & -60 & 1.9 \\
\hline 0.356 & -112.5 & -60 & 1.7 \\
\hline 0.356 & -135.0 & .60 & 1.8 \\
\hline 0.356 & -157.5 & -60 & 1.9 \\
\hline 0.356 & 180.0 & -60 & 2.0 \\
\hline 0.356 & 157.5 & -60 & 2.2 \\
\hline
\end{tabular}

Distance Horizontal Vertical $60 \mathrm{~Hz}$

meters angle deg angle deg mGauss

\begin{tabular}{|l|r|r|r|}
\hline 0.356 & 135.0 & -60 & 2.4 \\
\hline 0.356 & 112.5 & -60 & 3.0 \\
\hline 0.356 & 90.0 & -60 & 6.7 \\
\hline 0.356 & 67.5 & -60 & 9.8 \\
\hline 0.356 & 45.0 & -60 & 16.5 \\
\hline 0.356 & 22.5 & -60 & 26.8 \\
\hline 0.456 & 0.0 & -60 & 1.6 \\
\hline 0.456 & -22.5 & -60 & 1.3 \\
\hline 0.456 & -45.0 & -60 & 1.2 \\
\hline 0.456 & -67.5 & -60 & 1.1 \\
\hline 0.456 & -90.0 & -60 & 1.1 \\
\hline 0.456 & -112.5 & -60 & 1.0 \\
\hline 0.456 & -135.0 & -60 & 1.1 \\
\hline 0.456 & -157.5 & -60 & 1.2 \\
\hline 0.456 & 180.0 & -60 & 1.2 \\
\hline 0.456 & 157.5 & -60 & 1.3 \\
\hline 0.456 & 135.0 & -60 & 1.4 \\
\hline 0.456 & 112.5 & -60 & 1.8 \\
\hline 0.456 & 90.0 & -60 & 3.9 \\
\hline 0.456 & 67.5 & -60 & 3.6 \\
\hline 0.456 & 45.0 & -60 & 6.6 \\
\hline 0.456 & 22.5 & -60 & 23.6 \\
\hline 0.656 & 0.0 & -60 & 1.0 \\
\hline 0.656 & -22.5 & -60 & 0.6 \\
\hline 0.656 & -45.0 & -60 & 0.5 \\
\hline 0.656 & -67.5 & -60 & 0.5 \\
\hline 0.656 & -90.0 & -60 & 0.4 \\
\hline 0.656 & -112.5 & -60 & 0.4 \\
\hline 0.656 & -135.0 & -60 & 0.4 \\
\hline 0.656 & -157.5 & -60 & 0.4 \\
\hline
\end{tabular}


HIletaili. Hoilzontal Vartical $60 \mathrm{~Hz}$ inelane Hoise deo angle deg mGause

\begin{tabular}{|c|c|c|c|}
\hline onsin & 1800 & -60 & 0.5 \\
\hline 11 and & 157.5 & -60 & 0.4 \\
\hline o nins & 135.0 & $-\infty 0$ & 0.5 \\
\hline $0 n \infty 6$ & 112.5 & -60 & 0.8 \\
\hline 0 and & $\infty 0.0$ & -60 & 1.4 \\
\hline lnan & 67.5 & .60 & 1.4 \\
\hline (I) & 45.0 & -60 & 1.7 \\
\hline n nas & 22.5 & -60 & 6.0 \\
\hline $1(k x)$ & 0.0 & -60 & 0.2 \\
\hline $1(x)$ & .22 .5 & -60 & 0.1 \\
\hline$f(x)$ & -45.0 & -60 & 0.1 \\
\hline$f(x)$ & .67 .5 & -60 & 0.2 \\
\hline 1000 & .90 .0 & -60 & 0.1 \\
\hline 1000 & -112.5 & -60 & 0.1 \\
\hline
\end{tabular}

Distance Horizontal Vertical $60 \mathrm{~Hz}$ meters angle deg angle deg mGausa

\begin{tabular}{|r|r|r|r|}
\hline 1.000 & -135.0 & -60 & 0.1 \\
\hline 1.000 & -157.5 & -60 & 0.1 \\
\hline 1.000 & 180.0 & -60 & 0.1 \\
\hline 1.000 & 157.5 & -60 & 0.1 \\
\hline 1.000 & 135.0 & -60 & 0.1 \\
\hline 1.000 & 112.5 & -60 & 0.2 \\
\hline 1.000 & 90.0 & -60 & 0.2 \\
\hline 1.000 & 67.5 & -60 & 0.2 \\
\hline 1.000 & 45.0 & -60 & 0.4 \\
\hline 1.000 & 22.5 & -60 & 1.8 \\
\hline 0.152 & 0.0 & -90 & 26.0 \\
\hline 0.252 & 0.0 & -90 & 5.9 \\
\hline 0.452 & 0.0 & -90 & 1.8 \\
\hline
\end{tabular}




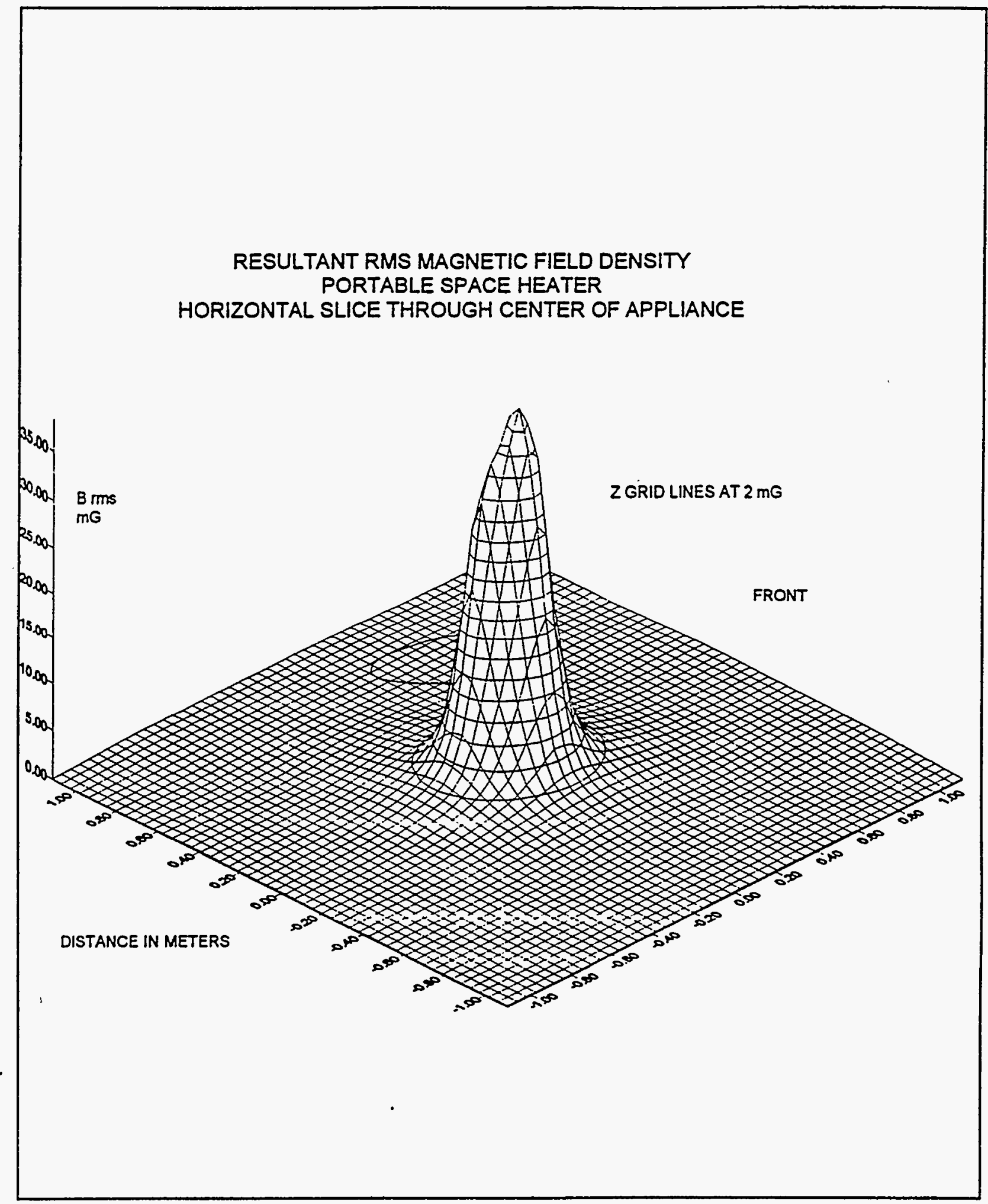

Figure C.6.1 Fundamental Resultant RMS Magnetic Field Density - Portable Space Heater.

C.6-8 


\section{C.7 Iron - Steam}

Appliance Tested: A steam iron to use on clothing.

Manufacturer: GGG

Model: 777

Rated Power: 1100 Watts

Appliance Code: 1263

Conditions for Testing: The iron was set to maximum heat.

Measured voltage and current: 112.9 Volts and 8.05 Amperes.

Magnetic Field Summary:

$60 \mathrm{~Hz}$ RMS resultant level at $30 \mathrm{~cm}$ from front surface: $0.8 \mathrm{mG}$

RMS resultant low surface level: $23 \mathrm{mG}$ on bottom

RMS resultant high surface level: $166 \mathrm{mG}$ at the rear of handle

Highest recorded surface levels:

60 Hertz RMS resultant: $166 \mathrm{mG}$

180 Hertz RMS resultant: $23.9 \mathrm{mG}$

300 Hertz RMS resultant: $5.9 \mathrm{mG}$

420 Hertz RMS resultant: $3.2 \mathrm{mG}$

For the appliance test set, the front of the unit was located at -90 degrees on the test table.

Summary of characteristics: The magnetic fields were moderate. 
Table C.7 Iron - Steam

Distence Horizontal Vertical $60 \mathrm{~Hz}$ metere angle deg angle deg mGause

\begin{tabular}{|c|c|c|c|}
\hline 0.076 & 0.0 & 90 & 31.1 \\
\hline 0.176 & 0.0 & 90 & 7.0 \\
\hline 0.376 & 0.0 & 90 & 1.0 \\
\hline 1.000 & 0.0 & 90 & 0.2 \\
\hline 0.127 & 0.0 & 60 & 7.8 \\
\hline 0.127 & -22.5 & 60 & 8.3 \\
\hline 0.127 & -45.0 & 60 & 8.5 \\
\hline 0.127 & -67.5 & 60 & 8.1 \\
\hline 0.127 & -112.5 & 60 & 6.5 \\
\hline 0.127 & -135.0 & 60 & 5.9 \\
\hline 0.127 & -157.5 & 60 & 5.1 \\
\hline 0.127 & 180.0 & 60 & 4.1 \\
\hline 0.127 & 157.5 & 60 & 2.8 \\
\hline 0.127 & 135.0 & 60 & 3.3 \\
\hline 0.127 & 90.0 & 60 & 31.5 \\
\hline 0.127 & 67.5 & 60 & 7.1 \\
\hline 0.127 & 45.0 & 60 & 6.5 \\
\hline 0.127 & 22.5 & 60 & 7.7 \\
\hline 0.227 & 0.0 & 60 & 3.4 \\
\hline 0.227 & -22.5 & 60 & 3.4 \\
\hline 0.227 & -45.0 & 60 & 3.3 \\
\hline 0.227 & -67.5 & 60 & 3.3 \\
\hline 0.227 & -112.5 & 60 & 2.7 \\
\hline 0.227 & -135.0 & 60 & 3.4 \\
\hline 0.227 & -157.5 & 60 & 3.7 \\
\hline 0.227 & 180.0 & 60 & 4.2 \\
\hline 0.227 & 157.5 & 60 & 4.3 \\
\hline 0.227 & 135.0 & 60 & 4.4 \\
\hline 0.227 & 90.0 & 60 & 4.0 \\
\hline 0.227 & 67.5 & 60 & 3.9 \\
\hline
\end{tabular}

\section{$60 \mathrm{~Hz}$ RMS Resultant Fields}

Distance Horizonsal Vertical $60 \mathrm{~Hz}$

moters angle deg angle deg mGause

\begin{tabular}{|r|r|r|r|}
\hline 0.227 & 45.0 & 60 & 4.2 \\
\hline 0.227 & 22.5 & 60 & 4.0 \\
\hline 0.427 & 0.0 & 60 & 0.6 \\
\hline 0.427 & -22.5 & 60 & 0.6 \\
\hline 0.427 & -45.0 & 60 & 0.7 \\
\hline 0.427 & -67.5 & 60 & 0.7 \\
\hline 0.427 & -112.5 & 60 & 0.5 \\
\hline 0.427 & -135.0 & 60 & 0.7 \\
\hline 0.427 & -157.5 & 60 & 0.8 \\
\hline 0.427 & 180.0 & 60 & 0.8 \\
\hline 0.427 & 157.5 & 60 & 0.8 \\
\hline 0.427 & 135.0 & 60 & 0.8 \\
\hline 0.427 & 90.0 & 60 & 0.8 \\
\hline 0.427 & 67.5 & 60 & 0.7 \\
\hline 0.427 & 45.0 & 60 & 0.7 \\
\hline 0.427 & 22.5 & 60 & 0.7 \\
\hline 1.000 & 0.0 & 60 & 0.1 \\
\hline 1.000 & -22.5 & 60 & 0.1 \\
\hline 1.000 & -45.0 & 60 & 0.1 \\
\hline 1.000 & -67.5 & 60 & 0.1 \\
\hline 1.000 & -90.0 & 60 & 0.1 \\
\hline 1.000 & -112.5 & 60 & 0.1 \\
\hline 1.000 & -135.0 & 60 & 0.1 \\
\hline 1.000 & -157.5 & 60 & 0.2 \\
\hline 1.000 & .180 .0 & 60 & 0.2 \\
\hline 1.000 & 157.5 & 60 & 0.2 \\
\hline 1.000 & 135.0 & 60 & 0.2 \\
\hline 1.000 & 90.0 & 60 & 0.2 \\
\hline 1.000 & 67.5 & 60 & 0.2 \\
\hline 1.000 & 45.0 & 60 & 0.1 \\
\hline
\end{tabular}


Table C.7 Iron - Steam

Distence Horizontal Vertical $60 \mathrm{~Hz}$ meters angle deg angle deg mGauss

\begin{tabular}{|r|r|r|r|}
\hline 1.000 & 22.5 & 60 & 0.1 \\
\hline 0.178 & 0.0 & 30 & 6.1 \\
\hline 0.178 & -22.5 & 30 & 5.2 \\
\hline 0.178 & -45.0 & 30 & 4.8 \\
\hline 0.178 & -67.5 & 30 & 4.5 \\
\hline 0.178 & -112.5 & 30 & 3.8 \\
\hline 0.178 & -135.0 & 30 & 5.2 \\
\hline 0.178 & -157.5 & 30 & 6.5 \\
\hline 0.178 & 180.0 & 30 & 8.0 \\
\hline 0.178 & 157.5 & 30 & 9.3 \\
\hline 0.178 & 135.0 & 30 & 10.6 \\
\hline 0.178 & 90.0 & 30 & 18.6 \\
\hline 0.178 & 67.5 & 30 & 15.9 \\
\hline 0.178 & 45.0 & 30 & 10.4 \\
\hline 0.178 & 22.5 & 30 & 8.3 \\
\hline 0.278 & 0.0 & 30 & 1.8 \\
\hline 0.278 & -22.5 & 30 & 1.7 \\
\hline 0.278 & -45.0 & 30 & 1.7 \\
\hline 0.278 & -57.5 & 30 & 1.5 \\
\hline 0.278 & -112.5 & 30 & 1.3 \\
\hline 0.278 & -135.0 & 30 & 1.8 \\
\hline 0.278 & -157.5 & 30 & 2.2 \\
\hline 0.278 & 180.0 & 30 & 2.5 \\
\hline 0.278 & 157.5 & 30 & 2.7 \\
\hline 0.278 & 135.0 & 30 & 2.9 \\
\hline 0.278 & 90.0 & 30 & 3.0 \\
\hline 0.278 & 67.5 & 30 & 2.8 \\
\hline 0.278 & 45.0 & 30 & 2.7 \\
\hline 0.278 & 22.5 & 30 & 2.4 \\
\hline 0.478 & 0.0 & 30 & 0.6 \\
\hline
\end{tabular}

\section{$60 \mathrm{~Hz}$ RMS Resultant Fields}

Distance Horizontal Vortical $60 \mathrm{~Hz}$

meters angle deg anglo deg mGauss

\begin{tabular}{|l|r|r|r|}
\hline 0.478 & -22.5 & 30 & 0.8 \\
\hline 0.478 & -45.0 & 30 & 0.7 \\
\hline 0.478 & -67.5 & 30 & 0.7 \\
\hline 0.478 & -90.0 & 30 & 0.7 \\
\hline 0.478 & -112.5 & 30 & 0.5 \\
\hline 0.478 & -135.0 & 30 & 0.7 \\
\hline 0.478 & -157.5 & 30 & 0.7 \\
\hline 0.478 & 180.0 & 30 & 0.9 \\
\hline 0.478 & 157.5 & 30 & 0.7 \\
\hline 0.478 & 135.0 & 30 & 0.7 \\
\hline 0.478 & 112.5 & 30 & 0.7 \\
\hline 0.478 & 90.0 & 30 & 0.8 \\
\hline 0.478 & 67.5 & 30 & 0.7 \\
\hline 0.478 & 45.0 & 30 & 1.0 \\
\hline 0.478 & 22.5 & 30 & 0.8 \\
\hline 1.178 & 0.0 & 30 & 0.1 \\
\hline 1.178 & -22.5 & 30 & 0.1 \\
\hline 1.178 & -45.0 & 30 & 0.1 \\
\hline 1.178 & -67.5 & 30 & 0.1 \\
\hline 1.178 & -90.0 & 30 & 0.1 \\
\hline 1.178 & -112.5 & 30 & 0.1 \\
\hline 1.178 & -135.0 & 30 & 0.1 \\
\hline 1.178 & -157.5 & 30 & 0.1 \\
\hline 1.178 & 180.0 & 30 & 0.1 \\
\hline 1.178 & 157.5 & 30 & 0.1 \\
\hline 1.178 & 135.0 & 30 & 0.1 \\
\hline 1.178 & 112.5 & 30 & 0.1 \\
\hline 1.178 & 90.0 & 30 & 0.1 \\
\hline 1.178 & 67.5 & 30 & 0.1 \\
\hline 1.178 & 45.0 & 30 & 0.1 \\
\hline
\end{tabular}


Distance Horizontal Vortical $60 \mathrm{~Hz}$ metere angle deg angle deg mGauss

\begin{tabular}{|c|c|c|c|}
\hline 1.178 & 22.5 & 30 & 0.1 \\
\hline 0.127 & 0.0 & 0 & 14.9 \\
\hline 0.127 & -22.5 & 0 & 13.4 \\
\hline 0.127 & -45.0 & 0 & 13.9 \\
\hline 0.127 & -67.5 & 0 & 14.5 \\
\hline 0.127 & -112.5 & 0 & 11.7 \\
\hline 0.127 & -135.0 & 0 & 10.3 \\
\hline 0.127 & -157.5 & 0 & 11.6 \\
\hline 0.127 & 180.0 & 0 & 14.4 \\
\hline 0.127 & 157.5 & 0 & 18.1 \\
\hline 0.127 & 135.0 & 0 & 22.0 \\
\hline 0.127 & 90.0 & 0 & 29.3 \\
\hline 0.127 & 67.5 & 0 & 39.8 \\
\hline 0.127 & 45.0 & 0 & 24.4 \\
\hline 0.127 & 22.5 & 0 & 19.1 \\
\hline 0.227 & 0.0 & 0 & 2.9 \\
\hline 0.227 & -22.5 & 0 & 2.9 \\
\hline 0.227 & -45.0 & 0 & 3.2 \\
\hline 0.227 & -67.5 & 0 & 3.3 \\
\hline 0.227 & -112.5 & 0 & 2.5 \\
\hline 0.227 & -135.0 & 0 & 2.6 \\
\hline 0.227 & -157.5 & 0 & 2.9 \\
\hline 0.227 & 180.0 & 0 & 3.5 \\
\hline 0.227 & 157.5 & 0 & 4.1 \\
\hline 0.227 & 135.0 & 0 & 4.5 \\
\hline 0.227 & 90.0 & 0 & 5.7 \\
\hline 0.227 & 67.5 & 0 & 4.9 \\
\hline 0.227 & 45.0 & 0 & 4.0 \\
\hline 0.227 & 22.5 & 0 & 3.6 \\
\hline 0.427 & 0.0 & 0 & 0.6 \\
\hline
\end{tabular}

Table C.7 Iron - Steam

\section{$60 \mathrm{~Hz}$ RMS Resultant Fields}

Distance Horizontal Vertical $60 \mathrm{~Hz}$ meters angle deg angle deg mGauss

\begin{tabular}{|c|c|c|c|}
\hline 0.427 & -22.5 & 0 & 0.6 \\
\hline 0.427 & -45.0 & 0 & 0.6 \\
\hline 0.427 & -67.5 & 0 & 0.6 \\
\hline 0.427 & -112.5 & 0 & 0.5 \\
\hline 0.427 & -135.0 & 0 & 0.6 \\
\hline 0.427 & -157.5 & 0 & 0.6 \\
\hline 0.427 & 180.0 & 0 & 0.7 \\
\hline 0.427 & 157.5 & 0 & 0.8 \\
\hline 0.427 & 135.0 & 0 & 0.8 \\
\hline 0.427 & 90.0 & 0 & 0.8 \\
\hline 0.427 & 67.5 & 0 & 0.7 \\
\hline 0.427 & 45.0 & 0 & 0.7 \\
\hline 0.427 & 22.5 & 0 & 0.7 \\
\hline 1.127 & 0.0 & 0 & 0.1 \\
\hline 1.127 & -22.5 & 0 & 0.1 \\
\hline 1.127 & -45.0 & 0 & 0.1 \\
\hline 1.127 & -67.5 & 0 & 0.0 \\
\hline 1.127 & -90.0 & 0 & 0.1 \\
\hline 1.127 & -112.5 & 0 & 0.0 \\
\hline 1.127 & -135.0 & 0 & 0.1 \\
\hline 1.127 & -157.5 & 0 & 0.9 \\
\hline 1.127 & 180.0 & 0 & 0.1 \\
\hline 1.127 & 157.5 & 0 & 0.1 \\
\hline 1.127 & 135.0 & 0 & 0.1 \\
\hline 1.127 & 90.0 & 0 & 0.1 \\
\hline 1.127 & 67.5 & 0 & 0.1 \\
\hline 1.127 & 45.0 & 0 & 0.1 \\
\hline 1.127 & 22.5 & 0 & 0.0 \\
\hline 0.318 & 0.0 & -30 & 1.3 \\
\hline 0.318 & -22.5 & -30 & 1.7 \\
\hline
\end{tabular}


Distance Horizontal Vortical $60 \mathrm{~Hz}$

moters angle deg anglo deg mGause

\begin{tabular}{|c|c|c|c|}
\hline 0.318 & -45.0 & -30 & 2.9 \\
\hline 0.318 & -67.5 & -30 & 2.2 \\
\hline 0.318 & -90.0 & -30 & 1.5 \\
\hline 0.318 & -112.5 & -30 & 1.5 \\
\hline 0.318 & -135.0 & -30 & 1.4 \\
\hline 0.318 & -157.5 & -30 & 1.5 \\
\hline 0.318 & 180.0 & -30 & 1.5 \\
\hline 0.318 & 157.5 & -30 & 1.4 \\
\hline 0.318 & 135.0 & -30 & 1.0 \\
\hline 0.318 & 90.0 & -30 & 2.4 \\
\hline 0.318 & 67.5 & -30 & 0.9 \\
\hline 0.318 & 45.0 & .30 & 1.0 \\
\hline 0.318 & 22.5 & -30 & 1.2 \\
\hline 0.418 & 0.0 & -30 & 0.7 \\
\hline 0.418 & -22.5 & -30 & 0.8 \\
\hline 0.418 & -45.0 & -30 & 1.0 \\
\hline 0.418 & .67 .5 & -30 & 1.0 \\
\hline 0.418 & -112.5 & -30 & 0.8 \\
\hline 0.418 & -135.0 & -30 & 0.9 \\
\hline 0.418 & -157.5 & -30 & 0.8 \\
\hline 0.418 & 180.0 & -30 & 0.8 \\
\hline 0.418 & 157.5 & -30 & 0.7 \\
\hline 0.418 & 135.0 & -30 & 0.6 \\
\hline 0.418 & $\$ 12.5$ & -30 & 0.6 \\
\hline 0.418 & 90.0 & .30 & 0.9 \\
\hline 0.418 & 67.5 & -30 & 0.5 \\
\hline 0.418 & 45.0 & .30 & 0.4 \\
\hline 0.418 & 22.5 & -30 & 0.6 \\
\hline 0.618 & 0.0 & -30 & 0.3 \\
\hline 0.618 & -22.5 & -30 & 0.3 \\
\hline
\end{tabular}

Table C.7 Iron - Steam

$60 \mathrm{~Hz}$ RMS Resultant Fields

Distance Horizontal Vertical $60 \mathrm{~Hz}$

meters angle deg ongle deg mGause

\begin{tabular}{|c|c|c|c|}
\hline 0.618 & -45.0 & -30 & 0.3 \\
\hline 0.618 & -67.5 & -30 & 0.3 \\
\hline 0.618 & -90.0 & .30 & 0.3 \\
\hline 0.618 & -112.5 & -30 & 0.3 \\
\hline 0.618 & -135.0 & -30 & 0.3 \\
\hline 0.618 & -157.5 & -30 & 0.4 \\
\hline 0.618 & 180.0 & -30 & 0.4 \\
\hline 0.618 & 157.5 & -30 & 0.4 \\
\hline 0.618 & 135.0 & -30 & 0.4 \\
\hline 0.618 & 112.5 & -30 & 0.2 \\
\hline 0.618 & 90.0 & -30 & 0.4 \\
\hline 0.618 & 22.5 & .30 & 0.3 \\
\hline 1.318 & 0.0 & .30 & 0.1 \\
\hline 1.318 & -22.5 & .30 & 0.1 \\
\hline 1.318 & -45.0 & -30 & 0.1 \\
\hline 1.318 & -67.5 & -30 & 0.1 \\
\hline 1.318 & .90 .0 & -30 & 0.1 \\
\hline 1.318 & -112.5 & .30 & 0.1 \\
\hline 1.318 & -135.0 & -30 & 0.1 \\
\hline 1.318 & -157.5 & -30 & 0.1 \\
\hline 1.318 & 180.0 & -30 & 0.1 \\
\hline 1.318 & 157.5 & -30 & 0.1 \\
\hline 1.318 & 135.0 & -30 & 0.0 \\
\hline 1.318 & 90.0 & -30 & 0.1 \\
\hline 1.318 & 67.5 & -30 & 0.0 \\
\hline 1.318 & 45.0 & -30 & 0.1 \\
\hline 1.398 & 22.5 & -30 & 0.1 \\
\hline 0.318 & 0.0 & -60 & 2.5 \\
\hline 0.318 & -22.5 & .60 & 2.9 \\
\hline 0.318 & -45.0 & .60 & 3.3 \\
\hline
\end{tabular}


Table C.7 Iron - Steam

Distance Horizonsal Vortical $60 \mathrm{~Hz}$ meters angle dog angle dog mGause

\begin{tabular}{|c|c|c|c|}
\hline 0.318 & -67.5 & -60 & 3.4 \\
\hline 0,318 & .112 .5 & -60 & 2.7 \\
\hline 0.318 & -135.0 & -60 & 2.9 \\
\hline 0.318 & -157.5 & -60 & 2.3 \\
\hline 0.318 & 180.0 & -60 & 2.2 \\
\hline 0.318 & 157.5 & -60 & 1.9 \\
\hline 0.318 & 135.0 & $-\infty 0$ & 1.7 \\
\hline 0.318 & 90.0 & -60 & 8.3 \\
\hline 0.318 & 67.5 & -60 & 10.5 \\
\hline 0.318 & 45.0 & -60 & 7.0 \\
\hline 0.318 & 22.5 & -60 & 3.8 \\
\hline 0.418 & 0.0 & -60 & 1.1 \\
\hline 0.418 & -22.5 & -60 & 1.2 \\
\hline 0.418 & -45.0 & -60 & 1.4 \\
\hline 0.418 & -67.5 & -60 & 1.4 \\
\hline 0.418 & -112.5 & -60 & 1.1 \\
\hline 0.418 & -135.0 & -60 & 1.2 \\
\hline 0.418 & -157.5 & -60 & 1.2 \\
\hline 0.418 & 180.0 & -60 & 1.1 \\
\hline 0.418 & 157.5 & -60 & 1.0 \\
\hline 0.418 & 135.0 & -60 & 0.8 \\
\hline 0.418 & 90.0 & -60 & 1.6 \\
\hline 0.418 & 67.5 & -60 & 2.6 \\
\hline 0.418 & 45.0 & -60 & 1.6 \\
\hline 0.418 & 22.5 & -60 & 1.3 \\
\hline 0.618 & 0.0 & -60 & 0.5 \\
\hline 0.618 & .22 .5 & -60 & 0.6 \\
\hline 0.618 & -45.0 & -60 & 0.6 \\
\hline 0.618 & -67.5 & -60 & 0.6 \\
\hline 0.618 & -112.5 & -60 & 0.6 \\
\hline
\end{tabular}

$60 \mathrm{~Hz}$ RMS Resultant Fields

Distance Horizontal Vertical $60 \mathrm{~Hz}$

meters angle deg angle deg mGauss

\begin{tabular}{|c|c|c|c|}
\hline 0.618 & -135.0 & -60 & 0.6 \\
\hline 0.618 & -157.5 & -60 & 0.6 \\
\hline 0.618 & 180.0 & -60 & 0.6 \\
\hline 0.618 & 157.5 & -60 & 0.5 \\
\hline 0.618 & 135.0 & -60 & 0.5 \\
\hline 0.618 & 90.0 & -60 & 0.7 \\
\hline 0.618 & 67.5 & -60 & 1.1 \\
\hline 0.618 & 45.0 & -60 & 0.9 \\
\hline 0.618 & 22.5 & -60 & 0.7 \\
\hline 1.000 & 0.0 & -60 & 0.1 \\
\hline 1.000 & -22.5 & -60 & 0.1 \\
\hline 1.000 & -45.0 & -60 & 0.1 \\
\hline 1.000 & -67.5 & -60 & 0.1 \\
\hline 1.000 & -90.0 & -60 & 0.1 \\
\hline 1.000 & -112.5 & -60 & 0.1 \\
\hline 1.000 & -135.0 & -60 & 0.1 \\
\hline 1.000 & -157.5 & -60 & 0.1 \\
\hline 1.000 & 180.0 & -60 & 0.1 \\
\hline 1.000 & 157.5 & -60 & 0.1 \\
\hline 1.000 & 135.0 & -60 & 0.1 \\
\hline 1.000 & 112.5 & -60 & 0.1 \\
\hline 1.000 & 90.0 & -60 & 0.1 \\
\hline 1.000 & 67.5 & -60 & 0.1 \\
\hline 1.000 & 45.0 & -60 & 0.1 \\
\hline 1.000 & 22.5 & -60 & 0.1 \\
\hline 0.152 & 0.0 & -90 & 22.0 \\
\hline 0.252 & 0.0 & -90 & 5.3 \\
\hline 0.452 & 0.0 & -90 & 1.2 \\
\hline
\end{tabular}




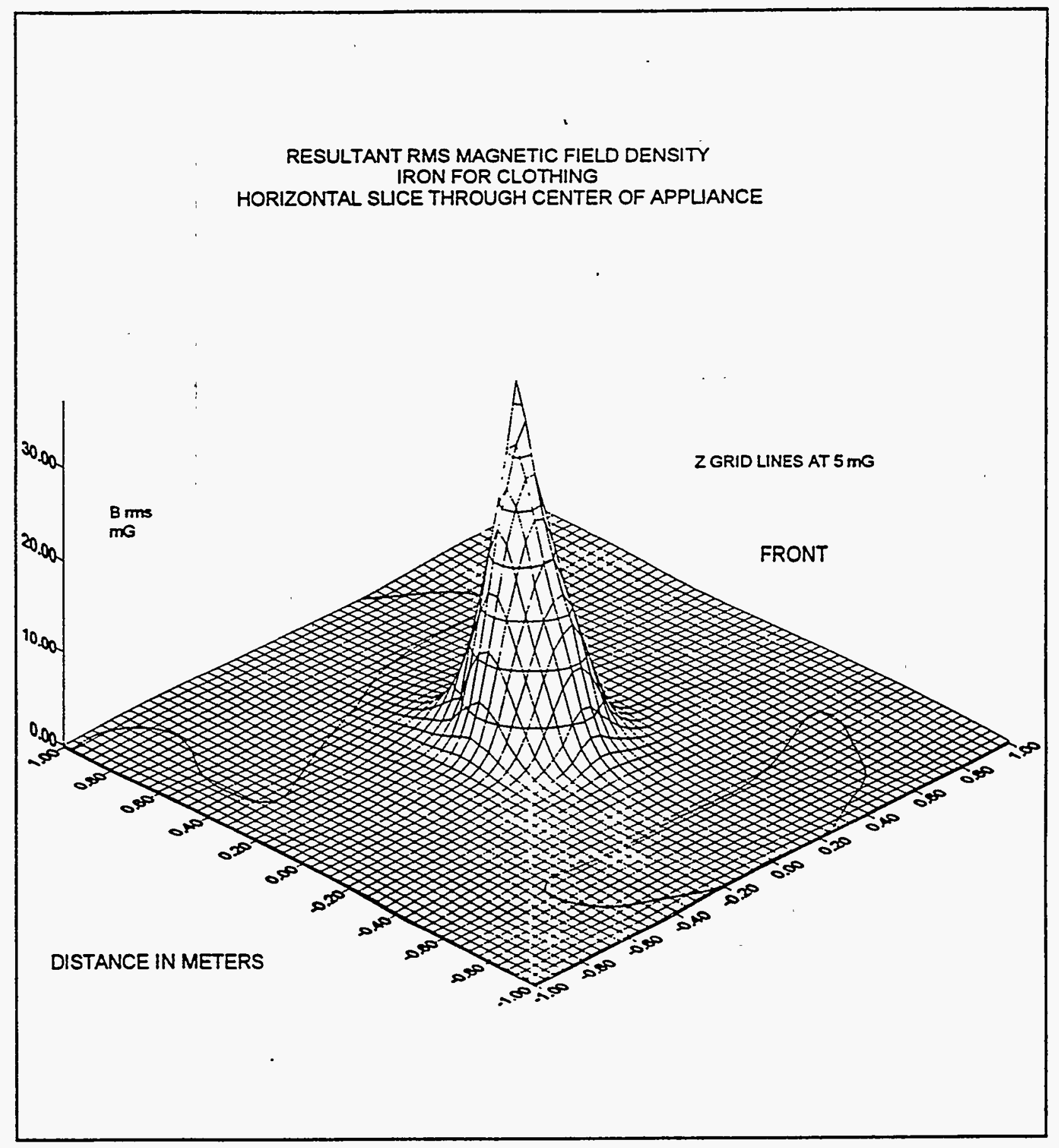

Figure C.7.1 Fundamental Resultant RMS Magnetic Field Density - Iron for Clothing. 


\section{C.8 Jig Saw - Hand}

Appliance Tested: A hand jig saw with variable speed control Manufacturer: $\mathrm{HHH}$

Model: 888

Rated current: 2.5 Amperes

Appliance Code: 2262

Conditions for Testing: The trigger was locked on at high speed but there was no material being cut by the blade

Measured voltage and current: 120.0 Volts and 5.34 Amperes.

Magnetic Field Summary:

$60 \mathrm{~Hz}$ RMS resultant level at $30 \mathrm{~cm}$ from front surface: $3.2 \mathrm{mG}$

RMS resultant low surface level: $107 \mathrm{mG}$

RMS resultant high surface level: $1796 \mathrm{mG}$

Highest recorded surface levels:

60 Hertz RMS resultant: $1536 \mathrm{mG}$

180 Hertz RMS resultant: $870 \mathrm{mG}$

300 Hertz RMS resultant: $313 \mathrm{mG}$

420 Hertz RMS resultant: $50.7 \mathrm{mG}$

Peak resultant: $5700 \mathrm{mG}$

Maximum recorded Total Harmonic Distortion: 76 percent

Maximum recorded RMS resultant in the $3-30 \mathrm{kHz}$ range: $12.2 \mathrm{mG}$

For the appliance test set, the front of the unit was located at 90 degrees on the test table. The unit was standing up without a blade.

Summary of characteristics: The magnetic fields were rich in harmonics and were relatively strong close to the surface. 
Distance Horizontal Vertical $60 \mathrm{~Hz}$ meters angle deg angle deg mGauss

\begin{tabular}{|r|r|r|r|}
\hline 0.114 & 0.0 & 90 & 135.4 \\
\hline 0.214 & 0.0 & 90 & 27.0 \\
\hline 0.414 & 0.0 & 90 & 3.5 \\
\hline 1.000 & 0.0 & 90 & 0.4 \\
\hline 0.114 & 0.0 & 60 & 167.5 \\
\hline 0.114 & -22.5 & 60 & 134.7 \\
\hline 0.114 & -45.0 & 60 & 92.4 \\
\hline 0.114 & -67.5 & 60 & 58.1 \\
\hline 0.114 & -90.0 & 60 & 49.8 \\
\hline 0.194 & -112.5 & 60 & 33.5 \\
\hline 0.114 & -135.0 & 60 & 23.7 \\
\hline 0.114 & -157.5 & 60 & 23.1 \\
\hline 0.114 & 180.0 & 60 & 29.0 \\
\hline 0.114 & 157.5 & 60 & 37.4 \\
\hline 0.114 & 135.0 & 60 & 45.6 \\
\hline 0.114 & 112.5 & 60 & 53.3 \\
\hline 0.114 & 90.0 & 60 & 57.6 \\
\hline 0.114 & 67.5 & 60 & 58.8 \\
\hline 0.114 & 45.0 & 60 & 39.5 \\
\hline 0.114 & 22.5 & 60 & 18.6 \\
\hline 0.214 & 0.0 & 60 & 31.9 \\
\hline 0.214 & -22.5 & 60 & 28.0 \\
\hline 0.214 & -45.0 & 60 & 22.2 \\
\hline 0.214 & -67.5 & 60 & 15.8 \\
\hline 0.214 & -90.0 & 60 & 13.7 \\
\hline 0.214 & -112.5 & 60 & 12.4 \\
\hline 0.214 & -135.0 & 60 & 11.5 \\
\hline 0.214 & -157.5 & 60 & 11.9 \\
\hline 0.214 & 180.0 & 60 & 12.0 \\
\hline 0.214 & 157.5 & 60 & 12.1 \\
\hline
\end{tabular}

Table C.8 Jig Saw - Hand

$60 \mathrm{~Hz}$ RMS Resultant Fields

Distance Horizontal Vertical $60 \mathrm{~Hz}$

meters angle deg angle deg mGauss

\begin{tabular}{|r|r|r|r|}
\hline 0.214 & 135.0 & 60 & 12.3 \\
\hline 0.214 & 112.5 & 60 & 14.2 \\
\hline 0.214 & 90.0 & 60 & 17.2 \\
\hline 0.214 & 67.5 & 60 & 22.9 \\
\hline 0.214 & 45.0 & 60 & 27.6 \\
\hline 0.214 & 22.5 & 60 & 30.7 \\
\hline 0.414 & 0.0 & 60 & 4.8 \\
\hline 0.414 & -22.5 & 60 & 4.4 \\
\hline 0.414 & -45.0 & 60 & 4.0 \\
\hline 0.414 & -67.5 & 60 & 3.8 \\
\hline 0.414 & -90.0 & 60 & 2.6 \\
\hline 0.414 & -112.5 & 60 & 2.4 \\
\hline 0.414 & -135.0 & 60 & 2.2 \\
\hline 0.414 & -157.5 & 60 & 2.2 \\
\hline 0.414 & 180.0 & 60 & 2.2 \\
\hline 0.414 & 157.5 & 60 & 2.2 \\
\hline 0.414 & 135.0 & 60 & 2.3 \\
\hline 0.414 & 112.5 & 60 & 2.6 \\
\hline 0.414 & 90.0 & 60 & 3.0 \\
\hline 0.414 & 67.5 & 60 & 3.7 \\
\hline 0.414 & 45.0 & 60 & 4.3 \\
\hline 0.414 & 22.5 & 60 & 4.7 \\
\hline 1.000 & 0.0 & 60 & 0.5 \\
\hline 1.000 & -22.5 & 60 & 0.3 \\
\hline 1.000 & -45.0 & 60 & 0.4 \\
\hline 1.000 & -67.5 & 60 & 0.3 \\
\hline 1.000 & -90.0 & 60 & 0.2 \\
\hline 1.000 & -112.5 & 60 & 0.3 \\
\hline 1.000 & -135.0 & 60 & 0.3 \\
\hline 1.000 & -157.5 & 60 & 0.3 \\
\hline
\end{tabular}


Distance Horizontal Vertical $60 \mathrm{~Hz}$ meters angle deg angle deg mGauss

\begin{tabular}{|r|r|r|r|}
\hline 1.000 & 180.0 & 60 & 0.3 \\
\hline 1.000 & 135.0 & 60 & 0.2 \\
\hline 1.000 & 112.5 & 60 & 0.3 \\
\hline 1.000 & 90.0 & 60 & 0.3 \\
\hline 1.000 & 67.5 & 60 & 0.4 \\
\hline 1.000 & 45.0 & 60 & 0.3 \\
\hline 1.000 & 22.5 & 60 & 0.3 \\
\hline 0.165 & 0.0 & 30 & 82.6 \\
\hline 0.165 & -22.5 & 30 & 64.7 \\
\hline 0.165 & -45.0 & 30 & 41.7 \\
\hline 0.165 & -67.5 & 30 & 25.6 \\
\hline 0.165 & -90.0 & 30 & 23.5 \\
\hline 0.165 & -112.5 & 30 & 23.2 \\
\hline 0.165 & -135.0 & 30 & 25.3 \\
\hline 0.165 & -157.5 & 30 & 26.6 \\
\hline 0.165 & 180.0 & 30 & 26.8 \\
\hline 0.165 & 157.5 & 30 & 24.3 \\
\hline 0.165 & 135.0 & 30 & 22.0 \\
\hline 0.165 & 112.5 & 30 & 22.1 \\
\hline 0.165 & 90.0 & 30 & 28.5 \\
\hline 0.165 & 67.5 & 30 & 47.3 \\
\hline 0.165 & 45.0 & 30 & 67.0 \\
\hline 0.165 & 22.5 & 30 & 81.1 \\
\hline 0.265 & 0.0 & 30 & 20.6 \\
\hline 0.265 & -22.5 & 30 & 18.5 \\
\hline 0.265 & -45.0 & 30 & 14.6 \\
\hline 0.265 & -67.5 & 30 & 10.3 \\
\hline 0.265 & -90.0 & 30 & 8.7 \\
\hline 0.265 & -112.5 & 30 & 7.7 \\
\hline 0.265 & -135.0 & 30 & 7.9 \\
\hline
\end{tabular}

Table C.8 Jig Saw - Hand $60 \mathrm{~Hz}$ RMS Resultant Fields

Distance Horizontal Vertical $60 \mathrm{~Hz}$

meters angle deg angle deg mGauss

\begin{tabular}{|r|r|r|r|}
\hline 0.265 & -157.5 & 30 & 8.2 \\
\hline 0.265 & 180.0 & 30 & 8.3 \\
\hline 0.265 & 157.5 & 30 & 7.8 \\
\hline 0.265 & 135.0 & 30 & 7.8 \\
\hline 0.265 & 112.5 & 30 & 8.0 \\
\hline 0.265 & 90.0 & 30 & 9.7 \\
\hline 0.265 & 67.5 & 30 & 13.7 \\
\hline 0.265 & 45.0 & 30 & 17.1 \\
\hline 0.265 & 22.5 & 30 & 19.6 \\
\hline 0.465 & 0.0 & 30 & 4.0 \\
\hline 0.465 & -22.5 & 30 & 3.6 \\
\hline 0.465 & -45.0 & 30 & 2.7 \\
\hline 0.465 & -67.5 & 30 & 2.0 \\
\hline 0.465 & -90.0 & 30 & 1.7 \\
\hline 0.465 & -112.5 & 30 & 1.7 \\
\hline 0.465 & -135.0 & 30 & 2.0 \\
\hline 0.465 & -157.5 & 30 & 2.1 \\
\hline 0.465 & 180.0 & 30 & 2.1 \\
\hline 0.465 & 157.5 & 30 & 1.9 \\
\hline 0.465 & 135.0 & 30 & 1.8 \\
\hline 0.465 & 112.5 & 30 & 1.7 \\
\hline 0.465 & 90.0 & 30 & 2.0 \\
\hline 0.465 & 67.5 & 30 & 2.8 \\
\hline 0.465 & 45.0 & 30 & 3.5 \\
\hline 0.465 & 22.5 & 30 & 4.0 \\
\hline 1.165 & 0.0 & 30 & 0.4 \\
\hline 1.165 & -22.5 & 30 & 0.4 \\
\hline 1.165 & -45.0 & 30 & 0.3 \\
\hline 1.165 & -67.5 & 30 & 0.2 \\
\hline 1.165 & -90.0 & 30 & 0.2 \\
\hline
\end{tabular}


Distance Horizontal Vertical $60 \mathrm{~Hz}$ meters angle deg angle deg mGauss

\begin{tabular}{|r|r|r|r|}
\hline 1.165 & -112.5 & 30 & 0.2 \\
\hline 1.165 & -135.0 & 30 & 0.3 \\
\hline 1.165 & -157.5 & 30 & 0.3 \\
\hline 1.165 & 180.0 & 30 & 0.3 \\
\hline 1.165 & 157.5 & 30 & 0.2 \\
\hline 1.165 & 135.0 & 30 & 0.2 \\
\hline 1.165 & 112.5 & 30 & 0.2 \\
\hline 1.165 & 90.0 & 30 & 0.2 \\
\hline 1.165 & 67.5 & 30 & 0.3 \\
\hline 1.165 & 45.0 & 30 & 0.3 \\
\hline 1.165 & 22.5 & 30 & 0.4 \\
\hline 0.127 & 0.0 & 0 & 142.0 \\
\hline 0.127 & -22.5 & 0 & 106.3 \\
\hline 0.127 & -45.0 & 0 & 63.3 \\
\hline 0.127 & -67.5 & 0 & 42.7 \\
\hline 0.127 & -90.0 & 0 & 40.4 \\
\hline 0.127 & -112.5 & 0 & 40.0 \\
\hline 0.127 & -135.0 & 0 & 42.0 \\
\hline 0.127 & -157.5 & 0 & 43.9 \\
\hline 0.127 & 180.0 & 0 & 43.8 \\
\hline 0.127 & 157.5 & 0 & 40.7 \\
\hline 0.127 & 135.0 & 0 & 36.6 \\
\hline 0.127 & 112.5 & 0 & 31.4 \\
\hline 0.127 & 90.0 & 0 & 31.6 \\
\hline 0.127 & 67.5 & 0 & 52.1 \\
\hline 0.127 & 45.0 & 0 & 87.1 \\
\hline 0.127 & 22.5 & 0 & 127.6 \\
\hline 0.227 & 0.0 & 0 & 28.3 \\
\hline 0.227 & -22.5 & 0 & 23.5 \\
\hline 0.227 & -45.0 & 0 & 16.1 \\
\hline
\end{tabular}

Table C.8 Jig Saw - Hand

\section{$60 \mathrm{~Hz}$ RMS Resultant Fields}

Distance Horizontal Vertical $60 \mathrm{~Hz}$

meters angle deg angle deg mGauss

\begin{tabular}{|r|r|r|r|}
\hline 0.227 & -67.5 & 0 & 10.9 \\
\hline 0.227 & -90.0 & 0 & 11.0 \\
\hline 0.227 & -112.5 & 0 & 19.5 \\
\hline 0.227 & -135.0 & 0 & 12.6 \\
\hline 0.227 & -157.5 & 0 & 13.5 \\
\hline 0.227 & 180.0 & 0 & 13.4 \\
\hline 0.227 & 157.5 & 0 & 12.5 \\
\hline 0.227 & 135.0 & 0 & 11.2 \\
\hline 0.227 & 112.5 & 0 & 9.2 \\
\hline 0.227 & 90.0 & 0 & 9.6 \\
\hline 0.227 & 67.5 & 0 & 13.5 \\
\hline 0.227 & 45.0 & 0 & 20.2 \\
\hline 0.227 & 22.5 & 0 & 26.3 \\
\hline 0.427 & 0.0 & 0 & 4.5 \\
\hline 0.427 & -22.5 & 0 & 3.9 \\
\hline 0.427 & -45.0 & 0 & 2.9 \\
\hline 0.427 & -67.5 & 0 & 2.1 \\
\hline 0.427 & -90.0 & 0 & 2.2 \\
\hline 0.427 & -112.5 & 0 & 2.4 \\
\hline 0.427 & -135.0 & 0 & 2.7 \\
\hline 0.427 & -157.5 & 0 & 3.0 \\
\hline 0.427 & 180.0 & 0 & 3.0 \\
\hline 0.427 & 157.5 & 0 & 2.8 \\
\hline 0.427 & 135.0 & 0 & 2.5 \\
\hline 0.427 & 112.5 & 0 & 1.9 \\
\hline 0.427 & 90.0 & 0 & 2.0 \\
\hline 0.427 & 67.5 & 0 & 2.6 \\
\hline 0.427 & 45.0 & 0 & 3.6 \\
\hline 0.427 & 22.5 & 0 & 4.3 \\
\hline 1.127 & 0.0 & 0 & 0.3 \\
\hline
\end{tabular}


Distance Horizontal Vertical $60 \mathrm{~Hz}$ meters angle deg angle deg mGauss

\begin{tabular}{|c|r|r|r|}
\hline 1.127 & -22.5 & 0 & 0.3 \\
\hline 1.127 & -45.0 & 0 & 0.2 \\
\hline 1.127 & -67.5 & 0 & 0.2 \\
\hline 1.127 & -90.0 & 0 & 0.2 \\
\hline 1.127 & -112.5 & 0 & 0.2 \\
\hline 1.127 & -135.0 & 0 & 0.3 \\
\hline 1.127 & -157.5 & 0 & 0.2 \\
\hline 1.127 & 180.0 & 0 & 0.2 \\
\hline 1.127 & 157.5 & 0 & 0.2 \\
\hline 1.127 & 135.0 & 0 & 0.2 \\
\hline 1.127 & 112.5 & 0 & 0.2 \\
\hline 1.127 & 90.0 & 0 & 0.2 \\
\hline 1.127 & 67.5 & 0 & 0.2 \\
\hline 1.127 & 45.0 & 0 & 0.3 \\
\hline 1.127 & 22.5 & 0 & 0.3 \\
\hline 0.279 & 0.0 & -30 & 10.4 \\
\hline 0.279 & -22.5 & -30 & 9.2 \\
\hline 0.279 & -45.0 & -30 & 7.5 \\
\hline 0.279 & -67.5 & -30 & 7.1 \\
\hline 0.279 & -90.0 & -30 & 8.4 \\
\hline 0.279 & -112.5 & -30 & 8.9 \\
\hline 0.279 & -135.0 & -30 & 8.9 \\
\hline 0.279 & -157.5 & -30 & 9.4 \\
\hline 0.279 & 180.0 & -30 & 9.7 \\
\hline 0.279 & 157.5 & -30 & 9.2 \\
\hline 0.279 & 135.0 & -30 & 8.3 \\
\hline 0.279 & 112.5 & -30 & 7.2 \\
\hline 0.279 & 90.0 & -30 & 6.3 \\
\hline 0.279 & 67.5 & -30 & 6.9 \\
\hline 0.279 & 45.0 & -30 & 8.0 \\
\hline
\end{tabular}

Table C.8 Jig Saw - Hand

$60 \mathrm{~Hz}$ RMS Resultant Fields

Distance Horizontal Vertical $60 \mathrm{~Hz}$

meters angle deg angle deg mGauss

\begin{tabular}{|c|c|c|c|}
\hline 0.279 & 22.5 & -30 & 9.8 \\
\hline 0.379 & 0.0 & -30 & 4.3 \\
\hline 0.379 & -22.5 & -30 & 3.9 \\
\hline 0.379 & -45.0 & -30 & 3.1 \\
\hline 0.379 & -67.5 & -30 & 2.9 \\
\hline 0.379 & -90.0 & -30 & 3.6 \\
\hline 0.379 & -112.5 & -30 & 4.1 \\
\hline 0.379 & -135.0 & -30 & 4.2 \\
\hline 0.379 & -157.5 & -30 & 4.5 \\
\hline 0.379 & 180.0 & -30 & 4.7 \\
\hline 0.379 & 157.5 & -30 & 4.4 \\
\hline 0.379 & 135.0 & -30 & 3.9 \\
\hline 0.379 & 112.5 & -30 & 3.3 \\
\hline 0.379 & 90.0 & -30 & 2.8 \\
\hline 0.379 & 67.5 & -30 & 3.0 \\
\hline 0.379 & 45.0 & -30 & 3.4 \\
\hline 0.379 & 22.5 & -30 & 4.1 \\
\hline 0.579 & 0.0 & -30 & 1.4 \\
\hline 0.579 & -22.5 & -30 & 1.2 \\
\hline 0.579 & -45.0 & -30 & 0.9 \\
\hline 0.579 & -67.5 & -30 & 0.9 \\
\hline 0.579 & -90.0 & -30 & 1.2 \\
\hline 0.579 & -112.5 & -30 & 1.4 \\
\hline 0.579 & -135.0 & -30 & 1.4 \\
\hline 0.579 & -157.5 & -30 & 1.6 \\
\hline 0.579 & 180.0 & -30 & 1.6 \\
\hline 0.579 & 157.5 & -30 & 1.5 \\
\hline 0.579 & 135.0 & -30 & 1.3 \\
\hline 0.579 & 112.5 & -30 & 1.1 \\
\hline 0.579 & 90.0 & -30 & 0.9 \\
\hline
\end{tabular}


Distance Horizontal Vertical $60 \mathrm{~Hz}$ meters angle deg angle deg mGauss

\begin{tabular}{|r|r|r|r|}
\hline 0.579 & 67.5 & -30 & 1.0 \\
\hline 0.579 & 45.0 & -30 & 1.2 \\
\hline 0.579 & 22.5 & -30 & 1.3 \\
\hline 1.279 & 0.0 & -30 & 0.3 \\
\hline 1.279 & -22.5 & -30 & 0.2 \\
\hline 1.279 & -45.0 & -30 & 0.1 \\
\hline 1.279 & -67.5 & -30 & 0.1 \\
\hline 1.279 & -90.0 & -30 & 0.1 \\
\hline 1.279 & -112.5 & -30 & 0.1 \\
\hline 1.279 & -135.0 & -30 & 0.2 \\
\hline 1.279 & -157.5 & -30 & 0.2 \\
\hline 1.279 & 180.0 & -30 & 0.1 \\
\hline 1.279 & 157.5 & -30 & 0.2 \\
\hline 1.279 & 112.5 & -30 & 0.1 \\
\hline 1.279 & 90.0 & -30 & 0.1 \\
\hline 1.279 & 67.5 & -30 & 0.2 \\
\hline 1.279 & 45.0 & -30 & 0.2 \\
\hline 1.279 & 22.5 & -30 & 0.2 \\
\hline 0.330 & 0.0 & -60 & 4.1 \\
\hline 0.330 & -22.5 & -60 & 4.4 \\
\hline 0.330 & -45.0 & -60 & 4.4 \\
\hline 0.330 & -67.5 & -60 & 4.6 \\
\hline 0.330 & -90.0 & -60 & 5.3 \\
\hline 0.330 & -112.5 & -60 & 5.5 \\
\hline 0.330 & -135.0 & -60 & 5.9 \\
\hline 0.330 & -157.5 & -60 & 6.4 \\
\hline 0.330 & 180.0 & -60 & 6.0 \\
\hline 0.330 & 157.5 & -60 & 6.1 \\
\hline 0.330 & 135.0 & -60 & 5.3 \\
\hline 0.330 & 112.5 & -60 & 4.9 \\
\hline
\end{tabular}

Table C.8 Jig Saw - Hand $60 \mathrm{~Hz}$ RMS Resultant Fields

Distance Horizontal Vertical $60 \mathrm{~Hz}$ meters angle deg angle deg mGauss

\begin{tabular}{|c|c|c|c|}
\hline 0.330 & 90.0 & -60 & 5.9 \\
\hline 0.330 & 67.5 & -60 & 4.4 \\
\hline 0.330 & 45.0 & -60 & 4.3 \\
\hline 0.330 & 22.5 & -60 & 4.4 \\
\hline 0.430 & 0.0 & -60 & 1.9 \\
\hline 0.430 & -22.5 & -60 & 2.0 \\
\hline 0.430 & -45.0 & -60 & 2.0 \\
\hline 0.430 & -67.5 & -60 & 2.2 \\
\hline 0.430 & -90.0 & -60 & 2.6 \\
\hline 0.430 & -112.5 & -60 & 2.7 \\
\hline 0.430 & -135.0 & -60 & 3.0 \\
\hline 0.430 & -157.5 & -60 & 3.2 \\
\hline 0.430 & 180.0 & -60 & 3.2 \\
\hline 0.430 & 157.5 & -60 & 3.2 \\
\hline 0.430 & 135.0 & -60 & 2.7 \\
\hline 0.430 & 112.5 & -60 & 2.5 \\
\hline 0.430 & 90.0 & -60 & 2.9 \\
\hline 0.430 & 67.5 & -60 & 2.0 \\
\hline 0.430 & 45.0 & -60 & 2.0 \\
\hline 0.430 & 22.5 & -60 & 2.0 \\
\hline 0.630 & 0.0 & -60 & 0.7 \\
\hline 0.630 & -22.5 & -60 & 0.7 \\
\hline 0.630 & -45.0 & -60 & 0.7 \\
\hline 0.630 & -67.5 & -60 & 0.7 \\
\hline 0.630 & -90.0 & -60 & 0.9 \\
\hline 0.630 & -112.5 & -60 & 0.9 \\
\hline 0.630 & -135.0 & -60 & 1.0 \\
\hline 0.630 & -157.5 & -60 & 1.1 \\
\hline 0.630 & 180.0 & -60 & 1.1 \\
\hline 0.630 & 157.5 & -60 & 1.1 \\
\hline
\end{tabular}


Table C.8 Jig Saw - Hand $60 \mathrm{~Hz}$ RMS Resultant Fields

Distance Horizontal Vertical $60 \mathrm{~Hz}$ meters angle deg angle deg mGauss

\begin{tabular}{|r|r|r|r|}
\hline 0.630 & 135.0 & -60 & 1.0 \\
\hline 0.630 & 112.5 & -60 & 0.9 \\
\hline 0.630 & 90.0 & -60 & 0.7 \\
\hline 0.630 & 67.5 & -60 & 0.7 \\
\hline 0.630 & 45.0 & -60 & 0.7 \\
\hline 0.630 & 22.5 & -60 & 0.7 \\
\hline 1.000 & 0.0 & -60 & 0.2 \\
\hline 1.000 & -22.5 & -60 & 0.2 \\
\hline 1.000 & -45.0 & -60 & 0.2 \\
\hline 1.000 & -67.5 & -60 & 0.1 \\
\hline 1.000 & -112.5 & -60 & 0.3 \\
\hline 1.000 & -135.0 & -60 & 0.2 \\
\hline
\end{tabular}

Distance Horizontal Vertical $60 \mathrm{~Hz}$ meters angle deg angle deg mGauss

\begin{tabular}{|r|r|r|r|}
\hline 1.000 & -157.5 & -60 & 0.2 \\
\hline 1.000 & 180.0 & -60 & 0.2 \\
\hline 1.000 & 157.5 & -60 & 0.2 \\
\hline 1.000 & 135.0 & -60 & 0.2 \\
\hline 1.000 & 112.5 & -60 & 0.2 \\
\hline 1.000 & 67.5 & -60 & 0.2 \\
\hline 1.000 & 45.0 & -60 & 0.3 \\
\hline 1.000 & 22.5 & -60 & 0.4 \\
\hline 0.114 & 0.0 & -90 & 73.0 \\
\hline 0.214 & 0.0 & -90 & 17.2 \\
\hline 0.414 & 0.0 & -90 & 3.1 \\
\hline
\end{tabular}




\section{RESULTANT RMS MAGNETIC FIELD DENSITY HAND JIG SAW \\ HORIZONTAL SLICE THROUGH CENTER OF APPLIANCE}

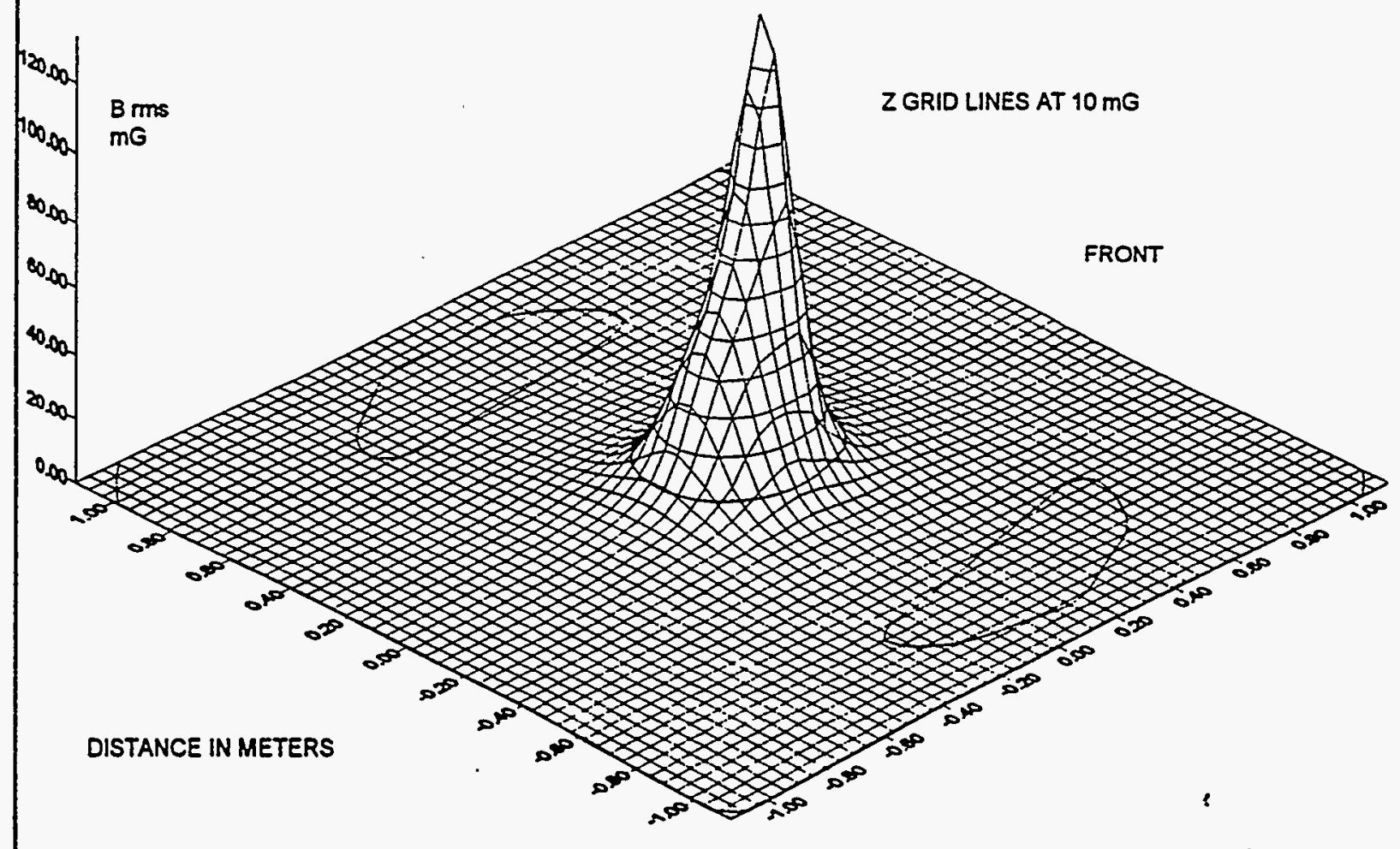

Figure C.8.1 Fundamental Resultant RMS Magnetic Field Density - Hand Jig Saw. 


\section{C.9 Mixer - Hand Kitchen}

Appliance Tested: A hand mixer as used in the kitchen for food preparation Manufacturer: III

Model: 999

Rated power: 160 Watts

Appliance Code: 2268

Conditions for Testing: The mixer was running at the highest speed with no load on the beaters.

Measured voltage and current: 121.5 Volts and 0.67 Amperes.

Magnetic Field Summary:

$60 \mathrm{~Hz}$ RMS resultant level at $30 \mathrm{~cm}$ from front surface: $4.0 \mathrm{mG}$

RMS resultant low surface level: $128 \mathrm{mG}$

RMS resultant high surface level: $1463 \mathrm{mG}$

Highest recorded surface levels:

60 Hertz RMS resultant: $1458 \mathrm{mG}$

180 Hertz RMS resultant: $75.7 \mathrm{mG}$

300 Hertz RMS resultant: $13.9 \mathrm{mG}$

420 Hertz RMS resultant: $12.8 \mathrm{mG}$

2220 Hertz RMS resultant: $6 \mathrm{mG}$

Maximum Total Harmonic Distortion: 8.8 percent

Maximum recorded RMS resultant in the $30-300 \mathrm{kHz}$ range: $2.2 \mathrm{mG}$

Maximum recorded RMS resultant in the $3-30 \mathrm{kHz}$ range: $7.3 \mathrm{mG}$

For the appliance test set, the front of the unit was located at -90 degrees on the test table. The unit was laying on its bottom without beaters.

Summary of characteristics: The magnetic fields were rich in harmonics and were relatively strong. The presence of a significant field level centered at 2220 Hertz spread over a band of 120 Hertz was unusual. There were high frequency components caused by brush arcing. 
Table C.9 Mixer - Hand Kitchen $60 \mathrm{~Hz}$ RMS Resultant Fields

Distance Horizontal Vertical $60 \mathrm{~Hz}$ meters angle deg angle deg mGauss

\begin{tabular}{|c|c|c|c|}
\hline 0.114 & 0.0 & 90 & 127.3 \\
\hline 0.214 & 0.0 & 90 & 17.0 \\
\hline 0.414 & 0.0 & 90 & 2.1 \\
\hline 1.000 & -45.0 & 90 & 0.7 \\
\hline 0.194 & 0.0 & 60 & 100.3 \\
\hline 0.114 & -22.5 & 60 & 97.0 \\
\hline 0.914 & -45.0 & 60 & 104.9 \\
\hline 0.114 & -67.5 & 60 & 102.1 \\
\hline 0.114 & -90.0 & 60 & 83.8 \\
\hline 0.194 & -112.5 & 60 & 62.1 \\
\hline 0.914 & -135.0 & 60 & 46.2 \\
\hline 0.114 & -157.5 & 60 & 38.8 \\
\hline 0.114 & 180.0 & 60 & 39.9 \\
\hline 0.114 & 157.5 & 60 & 46.5 \\
\hline 0.114 & 135.0 & 60 & 57.8 \\
\hline 0.114 & 112.5 & 60 & 71.1 \\
\hline 0.114 & 90.0 & 60 & 88.9 \\
\hline 0.114 & 67.5 & 60 & 105.6 \\
\hline 0.114 & 45.0 & 60 & 121.0 \\
\hline 0.114 & 22.5 & $\infty$ & 115.3 \\
\hline 0.214 & 0.0 & 60 & 16.3 \\
\hline 0.214 & -22.5 & 60 & 15.3 \\
\hline 0.214 & -45.0 & 60 & 16.0 \\
\hline 0.214 & -67.5 & 60 & 16.6 \\
\hline 0.214 & .90 .0 & 60 & 16.1 \\
\hline 0.214 & -112.5 & 60 & 13.2 \\
\hline 0.214 & -135.0 & 60 & 10.6 \\
\hline 0.214 & -157.5 & 60 & 8.9 \\
\hline 0.214 & 180.0 & 60 & 8.4 \\
\hline 0.214 & 157.5 & 60 & 9.3 \\
\hline
\end{tabular}

Distance Horizontal Vertical $60 \mathrm{~Hz}$

meters angle deg angle deg mGauss

\begin{tabular}{|r|r|r|r|}
\hline 0.214 & 135.0 & 60 & 10.9 \\
\hline 0.214 & 112.5 & 60 & 12.6 \\
\hline 0.214 & 90.0 & 60 & 14.2 \\
\hline 0.214 & 67.5 & 60 & 15.9 \\
\hline 0.214 & 45.0 & 60 & 17.9 \\
\hline 0.214 & 22.5 & 60 & 17.8 \\
\hline 0.414 & 0.0 & 60 & 2.2 \\
\hline 0.414 & -22.5 & 60 & 2.1 \\
\hline 0.414 & -45.0 & 60 & 2.1 \\
\hline 0.414 & -67.5 & 60 & 2.3 \\
\hline 0.414 & -90.0 & 60 & 2.4 \\
\hline 0.414 & -112.5 & 60 & 2.1 \\
\hline 0.414 & -135.0 & 60 & 1.8 \\
\hline 0.414 & -157.5 & 60 & 1.6 \\
\hline 0.414 & 180.0 & 60 & 1.5 \\
\hline 0.414 & 157.5 & 60 & 1.5 \\
\hline 0.414 & 135.0 & 60 & 1.7 \\
\hline 0.414 & 112.5 & 60 & 1.9 \\
\hline 0.414 & 90.0 & 60 & 2.0 \\
\hline 0.414 & 67.5 & 60 & 2.1 \\
\hline 0.414 & 45.0 & 60 & 2.3 \\
\hline 0.414 & 22.5 & 60 & 2.3 \\
\hline 1.000 & 0.0 & 60 & 0.4 \\
\hline 1.000 & -45.0 & 60 & 0.2 \\
\hline 1.000 & -67.5 & 60 & 0.2 \\
\hline 1.000 & -90.0 & 60 & 0.3 \\
\hline 1.000 & -112.5 & 60 & 0.3 \\
\hline 1.000 & -157.5 & 60 & 0.1 \\
\hline 1.000 & 180.0 & 60 & 0.2 \\
\hline 1.000 & 157.5 & 60 & 0.4 \\
\hline
\end{tabular}


Table C.9 Mixer - Hand Kitchen

$60 \mathrm{~Hz}$ RMS Resultant Fields

Distance Horizontal Vertical $60 \mathrm{~Hz}$

meters angle deg angle deg mGauss

\begin{tabular}{|r|r|r|r|}
\hline 1.000 & 90.0 & 60 & 0.2 \\
\hline 1.000 & 45.0 & 60 & 0.4 \\
\hline 1.000 & 22.5 & 60 & 0.3 \\
\hline 0.127 & 0.0 & 30 & 88.3 \\
\hline 0.127 & -22.5 & 30 & 78.5 \\
\hline 0.127 & -45.0 & 30 & 97.5 \\
\hline 0.127 & -67.5 & 30 & 103.1 \\
\hline 0.127 & -90.0 & 30 & 84.1 \\
\hline 0.127 & -112.5 & 30 & 56.5 \\
\hline 0.127 & -135.0 & 30 & 36.8 \\
\hline 0.127 & -157.5 & 30 & 24.9 \\
\hline 0.127 & 180.0 & 30 & 24.2 \\
\hline 0.127 & 157.5 & 30 & 30.6 \\
\hline 0.127 & 135.0 & 30 & 43.5 \\
\hline 0.127 & 112.5 & 30 & 62.3 \\
\hline 0.127 & 90.0 & 30 & 77.9 \\
\hline 0.127 & 67.5 & 30 & 85.6 \\
\hline 0.127 & 45.0 & 30 & 110.1 \\
\hline 0.127 & 22.5 & 30 & 115.9 \\
\hline 0.227 & 0.0 & 30 & 17.8 \\
\hline 0.227 & -22.5 & 30 & 16.8 \\
\hline 0.227 & -45.0 & 30 & 17.2 \\
\hline 0.227 & -67.5 & 30 & 17.6 \\
\hline 0.227 & -90.0 & 30 & 16.7 \\
\hline 0.227 & -112.5 & 30 & 13.7 \\
\hline 0.227 & -135.0 & 30 & 10.6 \\
\hline 0.227 & -157.5 & 30 & 7.8 \\
\hline 0.227 & 180.0 & 30 & 6.5 \\
\hline 0.227 & 157.5 & 30 & 7.2 \\
\hline 0.227 & 135.0 & 30 & 9.1 \\
\hline
\end{tabular}

Distance Horizontal Vertical $60 \mathrm{~Hz}$ meters angle deg angle deg mGauss

\begin{tabular}{|r|r|r|r|}
\hline 0.227 & 112.5 & 30 & 11.1 \\
\hline 0.227 & 90.0 & 30 & 12.9 \\
\hline 0.227 & 67.5 & 30 & 15.1 \\
\hline 0.227 & 45.0 & 30 & 17.5 \\
\hline 0.227 & 22.5 & 30 & 18.6 \\
\hline 0.427 & 0.0 & 30 & 2.8 \\
\hline 0.427 & -22.5 & 30 & 2.9 \\
\hline 0.427 & -45.0 & 30 & 2.8 \\
\hline 0.427 & -67.5 & 30 & 3.0 \\
\hline 0.427 & -112.5 & 30 & 2.7 \\
\hline 0.427 & -135.0 & 30 & 1.9 \\
\hline 0.427 & -157.5 & 30 & 1.3 \\
\hline 0.427 & 180.0 & 30 & 1.0 \\
\hline 0.427 & 157.5 & 30 & 1.0 \\
\hline 0.427 & 135.0 & 30 & 1.4 \\
\hline 0.427 & 112.5 & 30 & 1.6 \\
\hline 0.427 & 90.0 & 30 & 1.7 \\
\hline 0.427 & 67.5 & 30 & 1.9 \\
\hline 0.427 & 45.0 & 30 & 2.3 \\
\hline 0.427 & 22.5 & 30 & 2.7 \\
\hline 1.127 & 0.0 & 30 & 0.3 \\
\hline 1.127 & -22.5 & 30 & 0.2 \\
\hline 1.127 & -45.0 & 30 & 0.1 \\
\hline 1.127 & -67.5 & 30 & 0.2 \\
\hline 1.127 & -90.0 & 30 & 0.2 \\
\hline 1.127 & -112.5 & 30 & 0.2 \\
\hline 1.127 & -135.0 & 30 & 0.1 \\
\hline 1.127 & -157.5 & 30 & 0.1 \\
\hline 1.127 & 180.0 & 30 & 0.2 \\
\hline 1.127 & 157.5 & 30 & 0.2 \\
\hline
\end{tabular}


Table C.9 Mixer - Hand Kitchen

$60 \mathrm{~Hz}$ RMS Resultant Fields

Distance Horizontal Vertical $60 \mathrm{~Hz}$ meters angle deg angle deg mGauss

\begin{tabular}{|c|c|c|c|}
\hline 1.127 & 135.0 & 30 & 0.2 \\
\hline 1.127 & 112.5 & 30 & 0.2 \\
\hline 1.127 & 90.0 & 30 & 0.3 \\
\hline 1.127 & 67.5 & 30 & 0.3 \\
\hline 1.127 & 45.0 & 30 & 0.3 \\
\hline 1.127 & 22.5 & 30 & 0.2 \\
\hline 0.127 & 0.0 & 0 & 86.0 \\
\hline 0.127 & -22.5 & 0 & 73.8 \\
\hline 0.127 & -45.0 & 0 & 90.8 \\
\hline 0.127 & -67.5 & 0 & 95.4 \\
\hline 0.127 & -90.0 & 0 & 75.9 \\
\hline 0.127 & -112.5 & 0 & 49.8. \\
\hline 0.127 & -135.0 & 0 & 31.3 \\
\hline 0.127 & -157.5 & 0 & 20.2 \\
\hline 0.127 & 180.0 & 0 & 18.8 \\
\hline 0.127 & 157.5 & 0 & 26.3 \\
\hline 0.127 & 135.0 & 0 & 45.6 \\
\hline 0.127 & 112.5 & 0 & 76.5 \\
\hline 0.127 & 90.0 & 0 & 99.2 \\
\hline 0.127 & 67.5 & 0 & 90.9 \\
\hline 0.127 & 45.0 & 0 & 101.7 \\
\hline 0.127 & 22.5 & 0 & 110.8 \\
\hline 0.227 & 0.0 & 0 & 18.0 \\
\hline 0.227 & -22.5 & 0 & 15.1 \\
\hline 0.227 & -45.0 & 0 & 16.8 \\
\hline 0.227 & -67.5 & 0 & 18.9 \\
\hline 0.227 & -90.0 & 0 & 17.4 \\
\hline 0.227 & -112.5 & 0 & 13.4 \\
\hline 0.227 & -135.0 & 0 & 9.1 \\
\hline 0.227 & -157.5 & 0 & 5.8 \\
\hline
\end{tabular}

Distance Horizontal Vertical $60 \mathrm{~Hz}$ meters angle deg angle deg mGauss

\begin{tabular}{|c|c|c|c|}
\hline 0.227 & 180.0 & 0 & 4.8 \\
\hline 0.227 & 157.5 & 0 & 6.4 \\
\hline 0.227 & 135.0 & 0 & 9.5 \\
\hline 0.227 & 112.5 & 0 & 13.1 \\
\hline 0.227 & 90.0 & 0 & 14.8 \\
\hline 0.227 & 67.5 & 0 & 14.0 \\
\hline 0.227 & 45.0 & 0 & 16.6 \\
\hline 0.227 & 22.5 & 0 & 19.9 \\
\hline 0.427 & 0.0 & 0 & 2.9 \\
\hline 0.427 & -22.5 & 0 & 2.6 \\
\hline 0.427 & -45.0 & 0 & 2.7 \\
\hline 0.427 & -67.5 & 0 & 2.9 \\
\hline 0.427 & -90.0 & 0 & 2.9 \\
\hline 0.427 & -112.5 & 0 & 2.5 \\
\hline 0.427 & -135.0 & 0 & 1.9 \\
\hline 0.427 & -157.5 & 0 & 1.3 \\
\hline 0.427 & 180.0 & 0 & 1.1 \\
\hline 0.427 & 157.5 & 0 & 1.5 \\
\hline 0.427 & 135.0 & 0 & 1.7 \\
\hline 0.427 & 112.5 & 0 & 2.0 \\
\hline 0.427 & 90.0 & 0 & 2.1 \\
\hline 0.427 & 67.5 & 0 & 2.0 \\
\hline 0.427 & 45.0 & 0 & 2.4 \\
\hline 0.427 & 22.5 & 0 & 3.0 \\
\hline 1.127 & 0.0 & 0 & 0.2 \\
\hline 1.127 & -22.5 & 0 & 0.2 \\
\hline 1.127 & -45.0 & 0 & 0.2 \\
\hline 1.127 & -67.5 & 0 & 0.2 \\
\hline 1.127 & -90.0 & 0 & 0.2 \\
\hline 1.127 & -112.5 & 0 & 0.2 \\
\hline
\end{tabular}


Table C.9 Mixer - Hand Kitchen

Distance Horizontal Vertical $60 \mathrm{~Hz}$

meters angle deg angle deg mGauss

\begin{tabular}{|r|r|r|r|}
\hline 1.127 & -135.0 & 0 & 0.2 \\
\hline 1.127 & -157.5 & 0 & 0.2 \\
\hline 1.127 & 157.5 & 0 & 0.1 \\
\hline 1.127 & 135.0 & 0 & 0.2 \\
\hline 1.127 & 112.5 & 0 & 0.2 \\
\hline 1.127 & 90.0 & 0 & 0.2 \\
\hline 1.127 & 45.0 & 0 & 0.2 \\
\hline 1.127 & 22.5 & 0 & 0.2 \\
\hline 0.267 & 0.0 & -30 & 10.8 \\
\hline 0.267 & -22.5 & -30 & 9.6 \\
\hline 0.267 & -45.0 & -30 & 9.4 \\
\hline 0.267 & -67.5 & -30 & 9.7 \\
\hline 0.267 & -90.0 & -30 & 9.3 \\
\hline 0.267 & -112.5 & -30 & 7.7 \\
\hline 0.267 & -135.0 & -30 & 5.6 \\
\hline 0.267 & -157.5 & -30 & 4.0 \\
\hline 0.267 & 180.0 & -30 & 2.8 \\
\hline 0.267 & 157.5 & -30 & 3.9 \\
\hline 0.267 & 135.0 & -30 & 5.2 \\
\hline 0.267 & 112.5 & -30 & 6.8 \\
\hline 0.267 & 90.0 & -30 & 7.8 \\
\hline 0.267 & 67.5 & -30 & 7.7 \\
\hline 0.267 & 45.0 & -30 & 8.9 \\
\hline 0.267 & 22.5 & -30 & 11.0 \\
\hline 0.367 & 0.0 & -30 & 4.1 \\
\hline 0.367 & -22.5 & -30 & 3.7 \\
\hline 0.367 & -45.0 & -30 & 3.7 \\
\hline 0.367 & -67.5 & -30 & 3.9 \\
\hline 0.367 & -90.0 & -30 & 3.8 \\
\hline-112.5 & -30 & 3.3 \\
\hline
\end{tabular}

\section{$60 \mathrm{~Hz}$ RMS Resultant Fields}

Distance Horizontal Vertical $60 \mathrm{~Hz}$

meters angle deg angle deg mGauss

\begin{tabular}{|r|r|r|r|}
\hline 0.367 & -135.0 & -30 & 2.5 \\
\hline 0.367 & -157.5 & -30 & 1.8 \\
\hline 0.367 & 180.0 & -30 & 1.4 \\
\hline 0.367 & 157.5 & -30 & 1.6 \\
\hline 0.367 & 135.0 & -30 & 2.1 \\
\hline 0.367 & 112.5 & -30 & 2.4 \\
\hline 0.367 & 90.0 & -30 & 2.6 \\
\hline 0.367 & 67.5 & -30 & 2.6 \\
\hline 0.367 & 45.0 & -30 & 3.3 \\
\hline 0.367 & 22.5 & -30 & 4.0 \\
\hline 0.567 & 0.0 & -30 & 1.2 \\
\hline 0.567 & -22.5 & -30 & 1.0 \\
\hline 0.567 & -45.0 & -30 & 0.9 \\
\hline 0.567 & -67.5 & -30 & 0.9 \\
\hline 0.567 & -90.0 & -30 & 0.7 \\
\hline 0.567 & -112.5 & -30 & 0.9 \\
\hline 0.567 & -135.0 & -30 & 0.7 \\
\hline 0.567 & -157.5 & -30 & 0.6 \\
\hline 0.567 & 180.0 & -30 & 0.6 \\
\hline 0.567 & 157.5 & -30 & 0.8 \\
\hline 0.567 & 135.0 & -30 & 0.8 \\
\hline 0.567 & 112.5 & -30 & 0.9 \\
\hline 0.567 & 90.0 & -30 & 0.9 \\
\hline 0.567 & 67.5 & -30 & 1.0 \\
\hline 0.567 & 45.0 & -30 & 1.1 \\
\hline 0.567 & 22.5 & -30 & 1.3 \\
\hline 1.267 & 0.0 & -30 & 0.2 \\
\hline 1.267 & -45.0 & -30 & 0.1 \\
\hline 1.267 & -90.0 & -30 & 0.2 \\
\hline 1.267 & -112.5 & -30 & 0.1 \\
\hline
\end{tabular}


Table C.9 Mixer - Hand Kitchen

$60 \mathrm{~Hz}$ RMS Resultant Fields

Distance Horizontal Vertical $60 \mathrm{~Hz}$ meters angle deg angle deg mGauss

\begin{tabular}{|c|c|c|c|}
\hline 1.267 & -135.0 & -30 & 0.2 \\
\hline 1.267 & -157.5 & -30 & 0.2 \\
\hline 1.267 & 180.0 & -30 & 0.1 \\
\hline 1.257 & 157.5 & -30 & 0.1 \\
\hline 1.267 & 135.0 & -30 & 0.1 \\
\hline 1.267 & 112.5 & -30 & 0.1 \\
\hline 1.267 & 90.0 & -30 & 0.2 \\
\hline 1.267 & 45.0 & -30 & 0.2 \\
\hline 0.318 & 0.0 & -60 & 5.5 \\
\hline 0.318 & -22.5 & -60 & 5.3 \\
\hline 0.318 & -45.0 & -60 & 5.2 \\
\hline 0.318 & -67.5 & -60 & 5.0 \\
\hline 0.318 & -90.0 & -60 & 5.3 \\
\hline 0.318 & -112.5 & -60 & 4.2 \\
\hline 0.318 & -135.0 & -60 & 3.6 \\
\hline 0.318 & -157.5 & -60 & 2.8 \\
\hline 0.318 & 180.0 & -60 & 2.2 \\
\hline 0.318 & 157.5 & -60 & 2.2 \\
\hline 0.318 & 135.0 & -60 & 2.1 \\
\hline 0.318 & 112.5 & -60 & 5.3 \\
\hline 0.318 & 90.0 & -60 & 3.0 \\
\hline 0.318 & 67.5 & -60 & 3.5 \\
\hline 0.318 & 45.0 & -60 & 4.5 \\
\hline 0.318 & 22.5 & -60 & 5.1 \\
\hline 0.418 & 0.0 & -60 & 2.4 \\
\hline 0.418 & -22.5 & -60 & 2.4 \\
\hline 0.418 & -45.0 & -60 & 2.4 \\
\hline 0.418 & -67.5 & -60 & 2.3 \\
\hline 0.418 & -90.0 & -60 & 2.1 \\
\hline 0.418 & -112.5 & -60 & 1.9 \\
\hline
\end{tabular}

Distance Horizontal Vertical $60 \mathrm{~Hz}$ meters angle deg angle deg mGauss

\begin{tabular}{|r|r|r|r|}
\hline 0.418 & -135.0 & -60 & 1.6 \\
\hline 0.418 & -157.5 & -60 & 1.2 \\
\hline 0.418 & 180.0 & -60 & 1.0 \\
\hline 0.418 & 157.5 & -60 & 0.8 \\
\hline 0.418 & 135.0 & -60 & 1.0 \\
\hline 0.418 & 112.5 & -60 & 1.5 \\
\hline 0.418 & 90.0 & -60 & 1.3 \\
\hline 0.418 & 67.5 & -60 & 1.4 \\
\hline 0.418 & 45.0 & -60 & 1.9 \\
\hline 0.418 & 22.5 & -60 & 2.2 \\
\hline 0.618 & 0.0 & -60 & 0.7 \\
\hline 0.618 & -22.5 & -60 & 0.8 \\
\hline 0.618 & -45.0 & -60 & 0.9 \\
\hline 0.618 & -67.5 & -60 & 0.9 \\
\hline 0.618 & -90.0 & -60 & 1.1 \\
\hline 0.618 & -112.5 & -60 & 0.9 \\
\hline 0.618 & -135.0 & -60 & 0.9 \\
\hline 0.618 & -157.5 & -60 & 0.7 \\
\hline 0.618 & 180.0 & -60 & 0.6 \\
\hline 0.618 & 157.5 & -60 & 0.5 \\
\hline 0.618 & 135.0 & -60 & 0.5 \\
\hline 0.618 & 112.5 & -60 & 0.5 \\
\hline 0.618 & 90.0 & -60 & 0.5 \\
\hline 0.618 & 67.5 & -60 & 0.4 \\
\hline 0.618 & 45.0 & -60 & 0.5 \\
\hline 0.618 & 22.5 & -60 & 0.5 \\
\hline 1.000 & 0.0 & -60 & 0.2 \\
\hline 1.000 & -22.5 & -60 & 0.2 \\
\hline 1.000 & -45.0 & -60 & 0.2 \\
\hline 1.000 & -112.5 & -60 & 0.3 \\
\hline
\end{tabular}


Table C.9 Mixer - Hand Kitchen

$60 \mathrm{~Hz}$ RMS Resultant Fields

Distance Horizontal Vertical $60 \mathrm{~Hz}$

Distance Horizontal Vertical $60 \mathrm{~Hz}$

meters angle deg angle deg mGauss

\begin{tabular}{|c|c|c|c|}
\hline 1.000 & -135.0 & -60 & 0.2 \\
\hline 1.000 & -157.5 & -60 & 0.2 \\
\hline 1.000 & 135.0 & -60 & 0.2 \\
\hline 1.000 & 45.0 & -60 & 0.3 \\
\hline
\end{tabular}

meters angle deg angle deg mGauss

\begin{tabular}{|c|c|c|c|}
\hline 0.102 & 0.0 & -90 & 93.4 \\
\hline 0.202 & 0.0 & -90 & 14.7 \\
\hline 0.402 & 0.0 & -90 & 2.1 \\
\hline
\end{tabular}




\section{RESULTANT RMS MAGNETIC FIELD DENSITY KITCHEN MIXER \\ HORIZONTAL SLICE THROUGH CENTER OF APPLIANCE}

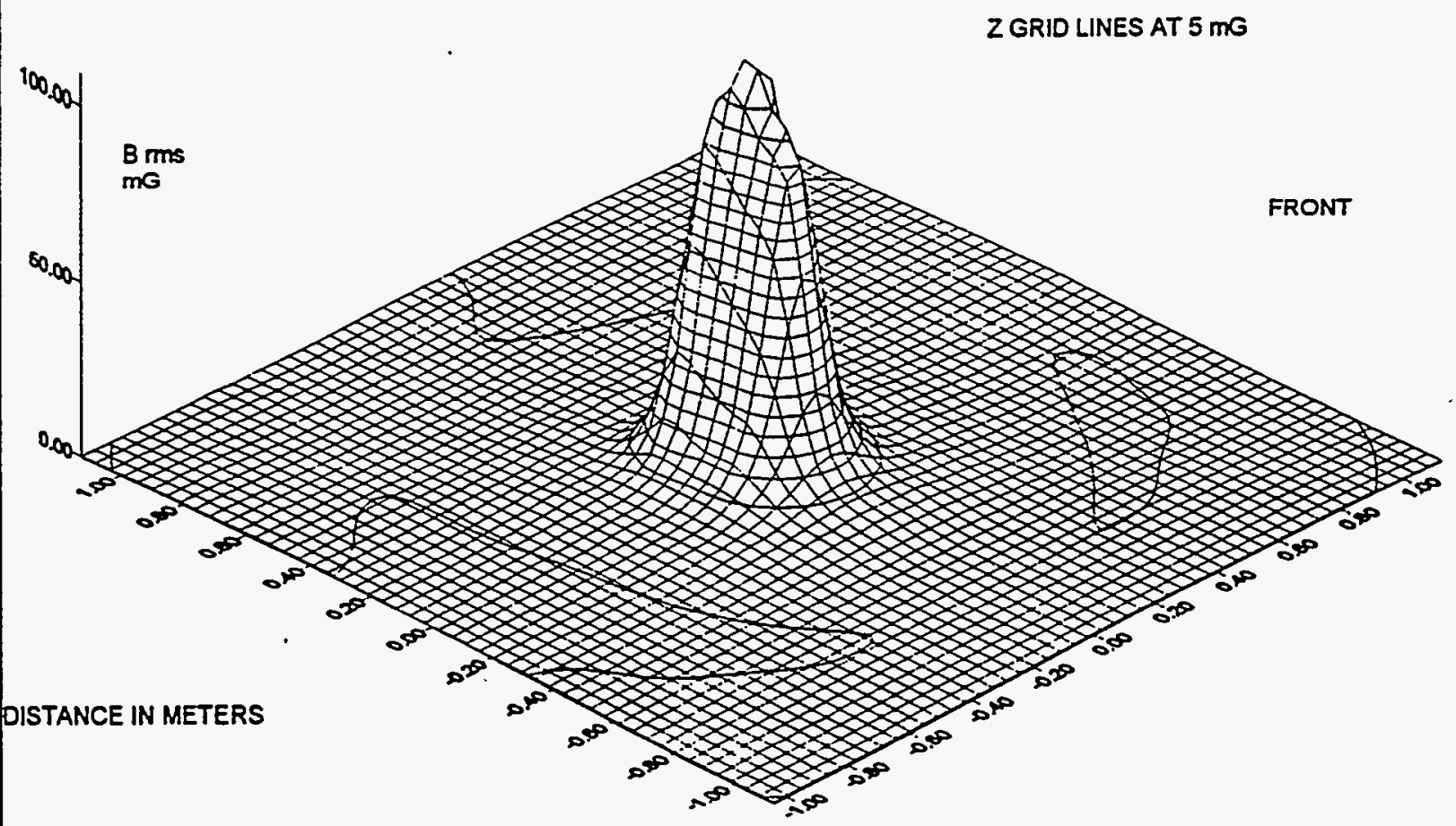

Figure C.9.1 Fundamental Resultant RMS Magnetic Field Density - Kitchen Mixer. 


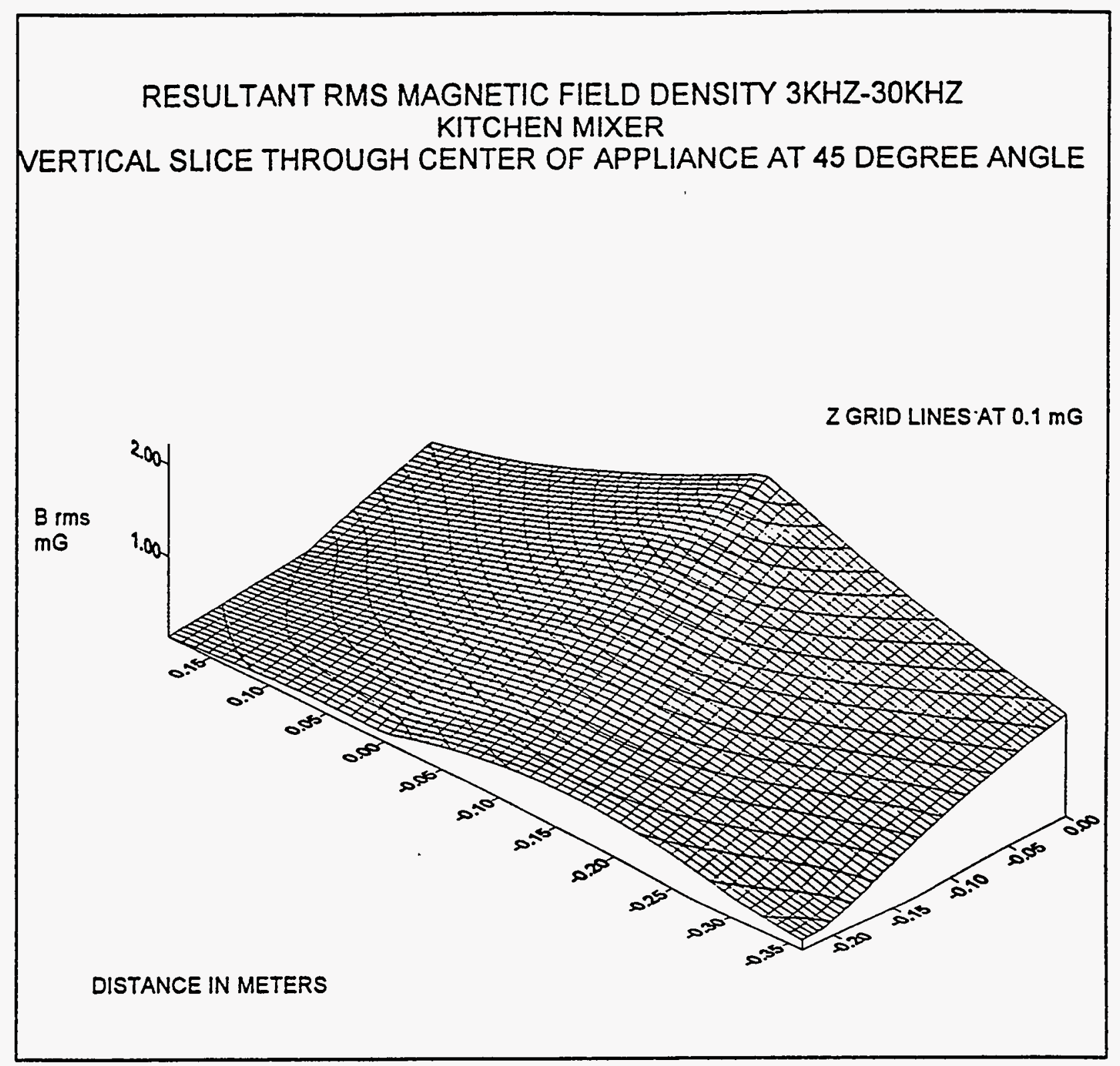

Figure C.9.2 Resultant RMS Magnetic Field Density 3 kHz-30 kHz - Kitchen Mixer. 


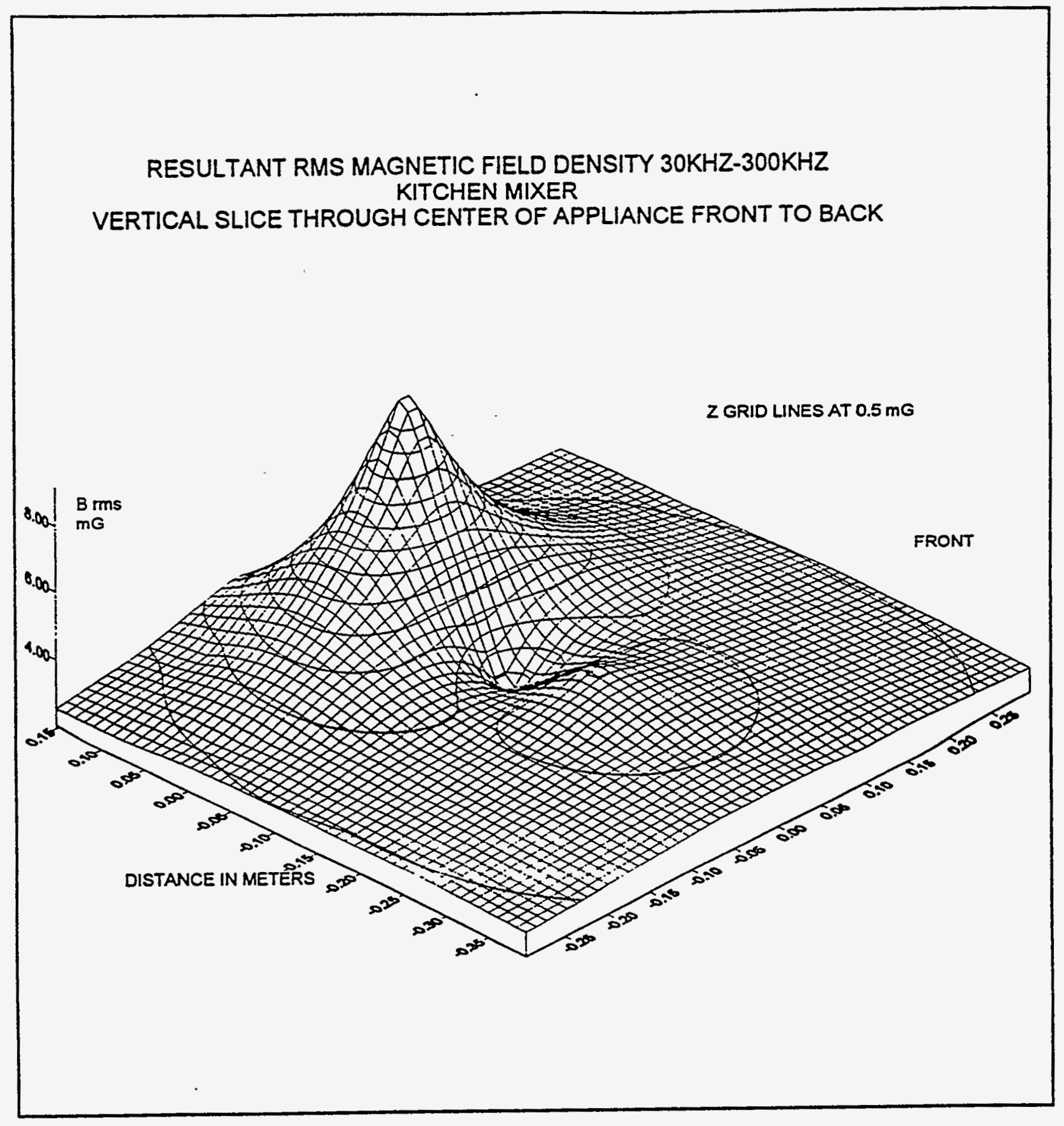

Figure C.9.3 Resultant RMS Magnetic Field Density $30 \mathrm{kHz}-300 \mathrm{kHz}$ - Kitchen Mixer. 


\section{C.10 Paint Sprayer}

Appliance Tested: A power paint sprayer used for household painting Manufacturer: JJJ

Model: 010

Rated current: 2.0 Amperes

Appliance Code: 3262

Conditions for Testing: The sprayer was operating at full spray with water.

Measured voltage and current: 121.2 Volts and 1.74 Amperes.

Magnetic Field Summary:

$60 \mathrm{~Hz}$ RMS resultant level at $30 \mathrm{~cm}$ from front surface: $61.5 \mathrm{mG}$

RMS resultant low surface level: $2545 \mathrm{mG}$ to the left

RMS resultant high surface level: $21,846 \mathrm{mG}$ to the right

Highest recorded surface levels:

60 Hertz RMS resultant: $21,659 \mathrm{mG}$

180 Hertz RMS resultant: $2643 \mathrm{mG}$

300 Hertz RMS resultant: $965 \mathrm{mG}$

420 Hertz RMS resultant: $411 \mathrm{mG}$

Maximum Total Harmonic Distortion: 37 percent

For the appliance test set, the front of the unit was located at 90 degrees on the test table.

Summary of characteristics: The magnetic fields were rich in harmonics and were very strong. The unit had a field at $300 \mathrm{~Hz}$ that was nearly one-half the ACGIH limit of 2 Gauss. 
Table C.10 Paint Sprayer

$60 \mathrm{~Hz}$ RMS Resultant Fields

Distance Horizontal Vertical $60 \mathrm{~Hz}$ meters angle deg angle deg mGauss

Distance Horizontal Vertical $60 \mathrm{~Hz}$ meters angle deg angle deg mGauss

\begin{tabular}{|c|c|c|c|}
\hline 0.038 & 0.0 & 90 & 10411.9 \\
\hline 0.138 & 0.0 & 90 & 776.0 \\
\hline 0.338 & 0.0 & 90 & 62.6 \\
\hline 1.000 & 0.0 & 90 & 4.0 \\
\hline 0.051 & 0.0 & 60 & 2043.2 \\
\hline 0.051 & -22.5 & 60 & 4247.2 \\
\hline 0.051 & -45.0 & 60 & 7491.9 \\
\hline 0.051 & -67.5 & 60 & 7959.1 \\
\hline 0.051 & -90.0 & 60 & 7932.6 \\
\hline 0.051 & -112.5 & 60 & 6157.9 \\
\hline 0.051 & -135.0 & 60 & 3389.1 \\
\hline 0.051 & -157.5 & 60 & 1829.9 \\
\hline 0.051 & 180.0 & 60 & 1170.2 \\
\hline 0.051 & 157.5 & 60 & 901.9 \\
\hline 0.051 & 135.0 & 60 & 819.6 \\
\hline 0.051 & 112.5 & 60 & 789.9 \\
\hline 0.051 & 90.0 & 60 & 782.7 \\
\hline 0.051 & 67.5 & 60 & 807.2 \\
\hline 0.051 & 45.0 & 60 & 869.8 \\
\hline 0.051 & 22.5 & 60 & 949.5 \\
\hline 0.151 & 0.0 & 60 & 479.8 \\
\hline 0.151 & -22.5 & 60 & 508.2 \\
\hline 0.151 & -45.0 & 60 & 574.0 \\
\hline 0.151 & -67.5 & 60 & 594.2 \\
\hline 0.151 & -90.0 & 60 & 597.6 \\
\hline 0.151 & -112.5 & 60 & 532.1 \\
\hline 0.151 & -135.0 & 60 & 431.2 \\
\hline 0.151 & -157.5 & 60 & 372.5 \\
\hline 0.151 & 180.0 & 60 & 356.8 \\
\hline 0.151 & 157.5 & 60 & 351.6 \\
\hline
\end{tabular}

\begin{tabular}{|c|c|c|c|}
\hline 0.151 & 135.0 & 60 & 358.9 \\
\hline 0.151 & 112.5 & 60 & 368.2 \\
\hline 0.151 & 90.0 & 60 & 375.5 \\
\hline 0.151 & 67.5 & 60 & 385.4 \\
\hline 0.151 & 45.0 & 60 & 399.7 \\
\hline 0.151 & 22.5 & 60 & 410.9 \\
\hline 0.351 & 0.0 & 60 & 53.7 \\
\hline 0.351 & -22.5 & 60 & 52.9 \\
\hline 0.351 & -45.0 & 60 & 57.0 \\
\hline 0.351 & -67.5 & 60 & 60.1 \\
\hline 0.351 & -90.0 & 60 & 60.9 \\
\hline 0.351 & -112.5 & 60 & 56.9 \\
\hline 0.351 & -135.0 & 60 & 49.8 \\
\hline 0.351 & -157.5 & 60 & 45.8 \\
\hline 0.351 & 180.0 & 60 & 45.5 \\
\hline 0.351 & 157.5 & 60 & 49.6 \\
\hline 0.351 & 135.0 & 60 & 54.1 \\
\hline 0.351 & 112.5 & 60 & 57.1 \\
\hline 0.351 & 90.0 & 60 & 58.6 \\
\hline 0.351 & 67.5 & 60 & 58.8 \\
\hline 0.351 & 45.0 & 60 & 57.5 \\
\hline 0.351 & 22.5 & 60 & 54.2 \\
\hline 1.000 & 0.0 & 60 & 3.4 \\
\hline 1.000 & -22.5 & 60 & 3.0 \\
\hline 1.000 & -45.0 & 60 & 3.1 \\
\hline 1.000 & -67.5 & 60 & 3.3 \\
\hline 1.000 & .90 .0 & 60 & 3.5 \\
\hline 1.000 & -112.5 & 60 & 3.3 \\
\hline 1.000 & -135.0 & 60 & 2.9 \\
\hline 1.000 & -157.5 & 60 & 2.7 \\
\hline
\end{tabular}


Table C.10 Paint Sprayer

$60 \mathrm{~Hz}$ RMS Resultant Fields

Distance Horizontal Vertical $60 \mathrm{~Hz}$ meters angle deg angle deg mGauss

Distance Horizontal Vertical $60 \mathrm{~Hz}$

meters angle deg angle deg mGauss

\begin{tabular}{|c|c|c|c|}
\hline 1.000 & 180.0 & 60 & 3.0 \\
\hline 1.000 & 157.5 & 60 & 3.6 \\
\hline 1.000 & 135.0 & 60 & 4.2 \\
\hline 1.000 & 112.5 & 60 & 4.7 \\
\hline 1.000 & 90.0 & 60 & 4.9 \\
\hline 1.000 & 67.5 & 60 & 4.9 \\
\hline 1.000 & 45.0 & 60 & 4.6 \\
\hline 1.000 & 22.5 & 60 & 4.1 \\
\hline 0.102 & 0.0 & 30 & 1555.3 \\
\hline 0.102 & -22.5 & 30 & 2178.6 \\
\hline 0.102 & -45.0 & 30 & 3916.6 \\
\hline 0.102 & -67.5 & 30 & 5478.3 \\
\hline 0.102 & -90.0 & 30 & 5303.5 \\
\hline 0.102 & -112.5 & 30 & 2939.4 \\
\hline 0.102 & -135.0 & 30 & 1447.9 \\
\hline 0.102 & -157.5 & 30 & 1125.6 \\
\hline 0.102 & 180.0 & 30 & 999.5 \\
\hline 0.102 & 157.5 & 30 & 917.0 \\
\hline 0.102 & 135.0 & 30 & 910.1 \\
\hline 0.102 & 112.5 & 30 & 931.4 \\
\hline 0.102 & 90.0 & 30 & 952.6 \\
\hline 0.102 & 67.5 & 30 & 1021.3 \\
\hline 0.102 & 45.0 & 30 & 1133.0 \\
\hline 0.102 & 22.5 & $30^{\circ}$ & 1280.9 \\
\hline 0.202 & 0.0 & 30 & 295.8 \\
\hline 0.202 & -22.5 & 30 & 364.9 \\
\hline 0.202 & -45.0 & 30 & 477.7 \\
\hline 0.202 & -67.5 & 30 & 569.5 \\
\hline 0.202 & -90.0 & 30 & 588.0 \\
\hline 0.202 & -112.5 & 30 & 487.0 \\
\hline
\end{tabular}

\begin{tabular}{|c|c|c|c|}
\hline 0.202 & -135.0 & 30 & 341.6 \\
\hline 0.202 & -157.5 & 30 & 242.0 \\
\hline 0.202 & 180.0 & 30 & 205.3 \\
\hline 0.202 & 157.5 & 30 & 199.6 \\
\hline 0.202 & 135.0 & 30 & 206.8 \\
\hline 0.202 & 112.5 & 30 & 217.1 \\
\hline 0.202 & 90.0 & 30 & 225.7 \\
\hline 0.202 & 67.5 & 30 & 233.4 \\
\hline 0.202 & 45.0 & 30 & 243.7 \\
\hline 0.202 & 22.5 & 30 & 253.3 \\
\hline 0.402 & 0.0 & 30 & 38.0 \\
\hline 0.402 & -22.5 & 30 & 43.1 \\
\hline 0.402 & -45.0 & 30 & 57.8 \\
\hline 0.402 & -67.5 & 30 & 72.1 \\
\hline 0.402 & .90 .0 & 30 & 76.1 \\
\hline 0.402 & -112.5 & 30 & 66.4 \\
\hline 0.402 & -135.0 & 30 & 48.9 \\
\hline 0.402 & -157.5 & 30 & 35.4 \\
\hline 0.402 & 180.0 & 30 & 32.9 \\
\hline 0.402 & 157.5 & 30 & 36.8 \\
\hline 0.402 & 135.0 & 30 & 41.5 \\
\hline 0.402 & 112.5 & 30 & 45.0 \\
\hline 0.402 & 90.0 & 30 & 47.6 \\
\hline 0.402 & 67.5 & 30 & 48.0 \\
\hline 0.402 & 45.0 & 30 & 46.3 \\
\hline 0.402 & 22.5 & 30 & 42.2 \\
\hline 1.102 & 0.0 & 30 & 2.7 \\
\hline 1.102 & -22.5 & 30 & 2.7 \\
\hline 1.102 & -45.0 & 30 & 3.8 \\
\hline 1.102 & -67.5 & 30 & 5.0 \\
\hline
\end{tabular}

C. $10-3$ 
Table C.10 Paint Sprayer

$60 \mathrm{~Hz}$ RMS Resultant Fields

Distance Horizontal Vertical $60 \mathrm{~Hz}$ meters angle deg angle deg mGauss

\begin{tabular}{|c|c|c|c|}
\hline 1.102 & -90.0 & 30 & 5.4 \\
\hline 1.102 & -112.5 & 30 & 5.0 \\
\hline 1.102 & -135.0 & 30 & 3.9 \\
\hline 1.102 & -157.5 & 30 & 2.7 \\
\hline 1.102 & 180.0 & 30 & 2.4 \\
\hline 1.102 & 157.5 & 30 & 3.2 \\
\hline 1.102 & 135.0 & 30 & 4.2 \\
\hline 1.102 & 112.5 & 30 & 4.9 \\
\hline 1.102 & 90.0 & 30 & 5.3 \\
\hline 1.102 & 67.5 & 30 & 5.2 \\
\hline 1.102 & 45.0 & 30 & 4.8 \\
\hline 1.102 & 22.5 & 30 & 3.9 \\
\hline 0.089 & 0.0 & 0 & 2248.5 \\
\hline 0.089 & -22.5 & 0 & 3855.0 \\
\hline 0.089 & -45.0 & 0 & 9068.9 \\
\hline 0.089 & -67.5 & 0 & 13054.7 \\
\hline 0.089 & .90 .0 & 0 & 13073.3 \\
\hline 0.089 & -112.5 & 0 & 6446.4 \\
\hline 0.089 & -135.0 & 0 & 2559.7 \\
\hline 0.089 & -157.5 & 0 & 1314.9 \\
\hline 0.089 & 180.0 & 0 & 1105.2 \\
\hline 0.089 & 157.5 & 0 & 972.2 \\
\hline 0.089 & 135.0 & 0 & 951.3 \\
\hline 0.089 & 112.5 & 0 & 970.7 \\
\hline 0.089 & 90.0 & 0 & 1000.8 \\
\hline 0.089 & 67.5 & 0 & 1059.7 \\
\hline 0.089 & 45.0 & 0 & 1179.3 \\
\hline 0.089 & 22.5 & 0 & 1364.9 \\
\hline 0.189 & 0.0 & 0 & 314.3 \\
\hline 0.189 & -22.5 & 0 & 435.2 \\
\hline
\end{tabular}

Distance Horizontal Vertical $60 \mathrm{~Hz}$

meters angle deg angle deg mGauss

\begin{tabular}{|c|c|c|c|}
\hline 0.189 & -45.0 & 0 & 748.0 \\
\hline 0.189 & -67.5 & 0 & 997.2 \\
\hline 0.189 & -90.0 & 0 & 1037.7 \\
\hline 0.189 & -112.5 & 0 & 798.2 \\
\hline 0.189 & -135.0 & 0 & 472.6 \\
\hline 0.189 & -157.5 & 0 & 270.8 \\
\hline 0.189 & 180.0 & 0 & 212.5 \\
\hline 0.189 & 157.5 & 0 & 291.7 \\
\hline 0.189 & 135.0 & 0 & 224.4 \\
\hline 0.189 & 112.5 & 0 & 238.6 \\
\hline 0.189 & 90.0 & 0 & 248.7 \\
\hline 0.189 & 67.5 & 0 & 256.8 \\
\hline 0.189 & 45.0 & 0 & 265.2 \\
\hline 0.189 & 22.5 & 0 & 269.5 \\
\hline 0.389 & 0.0 & 0 & 42.5 \\
\hline 0.389 & -22.5 & 0 & 52.7 \\
\hline 0.389 & -45.0 & 0 & 79.0 \\
\hline 0.389 & -67.5 & 0 & 101.7 \\
\hline 0.389 & -90.0 & 0 & 107.6 \\
\hline 0.389 & -112.5 & 0 & 94.9 \\
\hline 0.389 & -135.0 & 0 & 68.5 \\
\hline 0.389 & -157.5 & 0 & 44.6 \\
\hline 0.389 & 180.0 & 0 & 34.1 \\
\hline 0.389 & 157.5 & 0 & 36.7 \\
\hline 0.389 & 135.0 & 0 & 42.2 \\
\hline 0.389 & 912.5 & 0 & 47.1 \\
\hline 0.389 & 90.0 & 0 & 49.9 \\
\hline 0.389 & 67.5 & 0 & 50.5 \\
\hline 0.389 & 45.0 & 0 & 49.0 \\
\hline 0.389 & 22.5 & 0 & 44.9 \\
\hline
\end{tabular}


Table C.10 Paint Sprayer

$60 \mathrm{~Hz}$ RMS Resultant Fields

Distance Horizontal Vertical $60 \mathrm{~Hz}$ meters angle deg angle deg mGauss

Distance Horizontal Vertical $60 \mathrm{~Hz}$

meters angle deg angle deg mGauss

\begin{tabular}{|c|c|c|c|}
\hline 1.089 & 0.0 & 0 & 2.2 \\
\hline 1.089 & -22.5 & 0 & 2.5 \\
\hline 1.089 & -45.0 & 0 & 3.8 \\
\hline 1.089 & -67.5 & 0 & 5.1 \\
\hline 1.089 & -90.0 & 0 & 5.6 \\
\hline 1.089 & -112.5 & 0 & 5.2 \\
\hline 1.089 & -135.0 & 0 & 4.1 \\
\hline 1.089 & -157.5 & 0 & 2.7 \\
\hline 1.089 & 180.0 & 0 & 1.9 \\
\hline 1.089 & 157.5 & 0 & 2.2 \\
\hline 1.089 & 135.0 & 0 & 3.0 \\
\hline 1.089 & 112.5 & 0 & 3.7 \\
\hline 1.089 & 90.0 & 0 & 4.1 \\
\hline 1.089 & 67.5 & 0 & 4.1 \\
\hline 1.089 & 45.0 & $b$ & 3.8 \\
\hline 1.089 & 22.5 & 0 & 3.1 \\
\hline 0.305 & 0.0 & -30 & 101.3 \\
\hline 0.305 & -22.5 & .30 & 135.2 \\
\hline 0.305 & -45.0 & -30 & 193.4 \\
\hline 0.305 & -67.5 & -30 & 248.9 \\
\hline 0.305 & .90 .0 & .30 & 254.6 \\
\hline 0.305 & -112.5 & -30 & 223.0 \\
\hline 0.305 & -135.0 & .30 & 160.0 \\
\hline 0.305 & -157.5 & 30 & 106.9 \\
\hline 0.305 & 180.0 & -30 & 82.0 \\
\hline 0.305 & 157.5 & -30 & 80.3 \\
\hline 0.305 & 135.0 & .30 & 87.2 \\
\hline 0.305 & 112.5 & -30 & 93.7 \\
\hline 0.305 & 90.0 & .30 & 99.1 \\
\hline 0.305 & 67.5 & -30 & 100.1 \\
\hline
\end{tabular}

\begin{tabular}{|c|c|c|c|}
\hline 0.305 & 45.0 & -30 & 98.5 \\
\hline 0.305 & 22.5 & -30 & 94.5 \\
\hline 0.405 & 0.0 & -30 & 38.6 \\
\hline 0.405 & -22.5 & .30 & 50.1 \\
\hline 0.405 & -45.0 & -30 & 71.0 \\
\hline 0.405 & -67.5 & .30 & 90.7 \\
\hline 0.405 & -90.0 & -30 & 97.1 \\
\hline 0.405 & -112.5 & -30 & 84.9 \\
\hline 0.405 & -135.0 & -30 & 63.5 \\
\hline 0.405 & -157.5 & -30 & 43.2 \\
\hline 0.405 & 180.0 & -30 & 33.4 \\
\hline 0.405 & 157.5 & -30 & 34.2 \\
\hline 0.405 & 135.0 & -30 & 38.9 \\
\hline 0.405 & 112.5 & .30 & 42.2 \\
\hline 0.405 & 90.0 & -30 & 44.8 \\
\hline 0.405 & 67.5 & -30 & 44.8 \\
\hline 0.405 & 45.0 & -30 & 43.0 \\
\hline 0.405 & 22.5 & .30 & 39.0 \\
\hline 0.605 & 0.0 & .30 & 12.2 \\
\hline 0.605 & -22.5 & -30 & 15.5 \\
\hline 0.605 & -45.0 & -30 & 21.6 \\
\hline 0.605 & -67.5 & -30 & 27.1 \\
\hline 0.605 & .90 .0 & -30 & 29.1 \\
\hline 0.605 & -112.5 & -30 & 26.2 \\
\hline 0.605 & -135.0 & -30 & 20.4 \\
\hline 0.605 & -157.5 & .30 & 14.3 \\
\hline 0.605 & 180.0 & .30 & 11.2 \\
\hline 0.605 & 157.5 & -30 & 11.6 \\
\hline 0.605 & 135.0 & .30 & 13.7 \\
\hline 0.605 & 112.5 & -30 & 15.4 \\
\hline
\end{tabular}

C. $10-5$ 
Table C.10 Paint Sprayer

$60 \mathrm{~Hz}$ RMS Resultant Fields

Distance Morizontal Vertical $60 \mathrm{~Hz}$ meters angle deg angle deg maauss

\begin{tabular}{|c|c|c|c|}
\hline 0.605 & 90.0 & -30 & 16.3 \\
\hline 0.605 & 67.5 & -30 & 16.1 \\
\hline 0.605 & 45.0 & 30 & 14.9 \\
\hline 0.605 & 22.5 & -30 & 13.0 \\
\hline 1.305 & 0.0 & .30 & 1.2 \\
\hline 1.305 & .22 .5 & .30 & 1.5 \\
\hline 1.305 & -45.0 & -30 & 2.2 \\
\hline 1.305 & -67.5 & -30 & 2.8 \\
\hline 1.305 & -90.0 & -30 & 3.1 \\
\hline 1.305 & -112.5 & -30 & 3.0 \\
\hline 1.305 & -135.0 & -30 & 2.4 \\
\hline 1.305 & -157.5 & .30 & 1.7 \\
\hline 1.305 & 180.0 & -30 & 1.2 \\
\hline 1.305 & 157.5 & -30 & 1.2 \\
\hline 1.305 & 135.0 & -30 & 1.6 \\
\hline 1.305 & 112.5 & .30 & 1.9 \\
\hline 1.305 & 90.0 & -30 & 2.1 \\
\hline 1.305 & 67.5 & -30 & 2.1 \\
\hline 1.305 & 45.0 & -30 & 1.9 \\
\hline 1.305 & 22.5 & -30 & 1.5 \\
\hline 0.356 & 0.0 & -60 & 74.4 \\
\hline 0.356 & -22.5 & -60 & 88.2 \\
\hline 0.356 & -45.0 & -60 & 109.5 \\
\hline 0.356 & -67.5 & -60 & 128.3 \\
\hline 0.356 & .90 .0 & -60 & 132.4 \\
\hline 0.356 & -112.5 & -60 & 119.9 \\
\hline 0.356 & -135.0 & -60 & 101.3 \\
\hline 0.356 & -157.5 & -60 & 78.7 \\
\hline 0.356 & 980.0 & -60 & 64.5 \\
\hline 0.356 & 157.5 & -60 & 59.3 \\
\hline
\end{tabular}

Distance Horizontal Vertical $60 \mathrm{~Hz}$ meters angle deg angle deg mGauss

\begin{tabular}{|c|c|c|c|}
\hline 0.356 & 135.0 & -60 & 60.7 \\
\hline 0.356 & 112.5 & -60 & 58.8 \\
\hline 0.356 & 90.0 & -60 & 65.1 \\
\hline 0.356 & 67.5 & -60 & 66.9 \\
\hline 0.356 & 45.0 & -60 & 65.8 \\
\hline 0.356 & 22.5 & -60 & 66.0 \\
\hline 0.456 & 0.0 & -60 & 31.6 \\
\hline 0.456 & -22.5 & -60 & 36.7 \\
\hline 0.456 & -45.0 & -60 & 44.2 \\
\hline 0.456 & -67.5 & -60 & 51.1 \\
\hline 0.456 & -90.0 & -60 & 53.0 \\
\hline 0.456 & -112.5 & .60 & 49.2 \\
\hline 0.456 & -135.0 & -60 & 42.4 \\
\hline 0.456 & -157.5 & -60 & 34.0 \\
\hline 0.456 & 180.0 & -60 & 28.9 \\
\hline 0.456 & 157.5 & -60 & 27.6 \\
\hline 0.456 & 135.0 & -60 & 29.2 \\
\hline 0.456 & 112.5 & -60 & 29.3 \\
\hline 0.456 & 90.0 & -60 & 31.0 \\
\hline 0.456 & 67.5 & -60 & 31.2 \\
\hline 0.456 & 45.0 & -60 & 30.5 \\
\hline 0.456 & 22.5 & -60 & 29.6 \\
\hline 0.656 & 0.0 & -60 & 10.5 \\
\hline 0.656 & -22.5 & -60 & 12.1 \\
\hline 0.656 & -45.0 & -60 & 14.4 \\
\hline 0.656 & -67.5 & -60 & 16.3 \\
\hline 0.656 & -90.0 & -60 & 16.8 \\
\hline 0.656 & -112.5 & -60 & 15.8 \\
\hline 0.656 & -135.0 & -60 & 14.1 \\
\hline 0.656 & -157.5 & -60 & 11.7 \\
\hline
\end{tabular}


Table C.10 Paint Sprayer

$60 \mathrm{~Hz}$ RMS Resultant Fields

Distance Horizontal Vertical $60 \mathrm{~Hz}$

meters angle deg angle deg mGauss

\begin{tabular}{|r|r|r|r|}
\hline 0.656 & 180.0 & -60 & 10.0 \\
\hline 0.656 & 157.5 & -60 & 9.4 \\
\hline 0.656 & 135.0 & -60 & 9.7 \\
\hline 0.656 & 112.5 & -60 & 10.1 \\
\hline 0.656 & 90.0 & -60 & 10.5 \\
\hline 0.656 & 67.5 & -60 & 10.4 \\
\hline 0.656 & 45.0 & -60 & 10.1 \\
\hline 0.656 & 22.5 & -60 & 9.8 \\
\hline 1.000 & 0.0 & -60 & 1.9 \\
\hline 1.000 & -22.5 & -60 & 2.2 \\
\hline 1.000 & -45.0 & -60 & 2.8 \\
\hline 1.000 & -67.5 & -60 & 3.3 \\
\hline 1.000 & -90.0 & -60 & 3.5 \\
\hline 1.000 & -112.5 & -60 & 3.4 \\
\hline
\end{tabular}

Distance Horizontal Vertical $60 \mathrm{~Hz}$

meters angle deg angle deg mGauss

\begin{tabular}{|r|r|r|r|}
\hline 1.000 & -135.0 & -60 & 3.0 \\
\hline 1.000 & -157.5 & -60 & 2.4 \\
\hline 1.000 & 180.0 & -60 & 1.9 \\
\hline 1.000 & 157.5 & -60 & 1.7 \\
\hline 1.000 & 135.0 & -60 & 1.9 \\
\hline 1.000 & 112.5 & -60 & 21 \\
\hline 1.000 & 90.0 & -60 & 2.3 \\
\hline 1.000 & 67.5 & -60 & 2.3 \\
\hline 1.000 & 45.0 & -60 & 2.1 \\
\hline 1.000 & 22.5 & -60 & 1.9 \\
\hline 0.229 & 0.0 & -90 & 289.4 \\
\hline 0.329 & 0.0 & -90 & 86.1 \\
\hline 0.529 & 0.0 & -90 & 20.9 \\
\hline
\end{tabular}




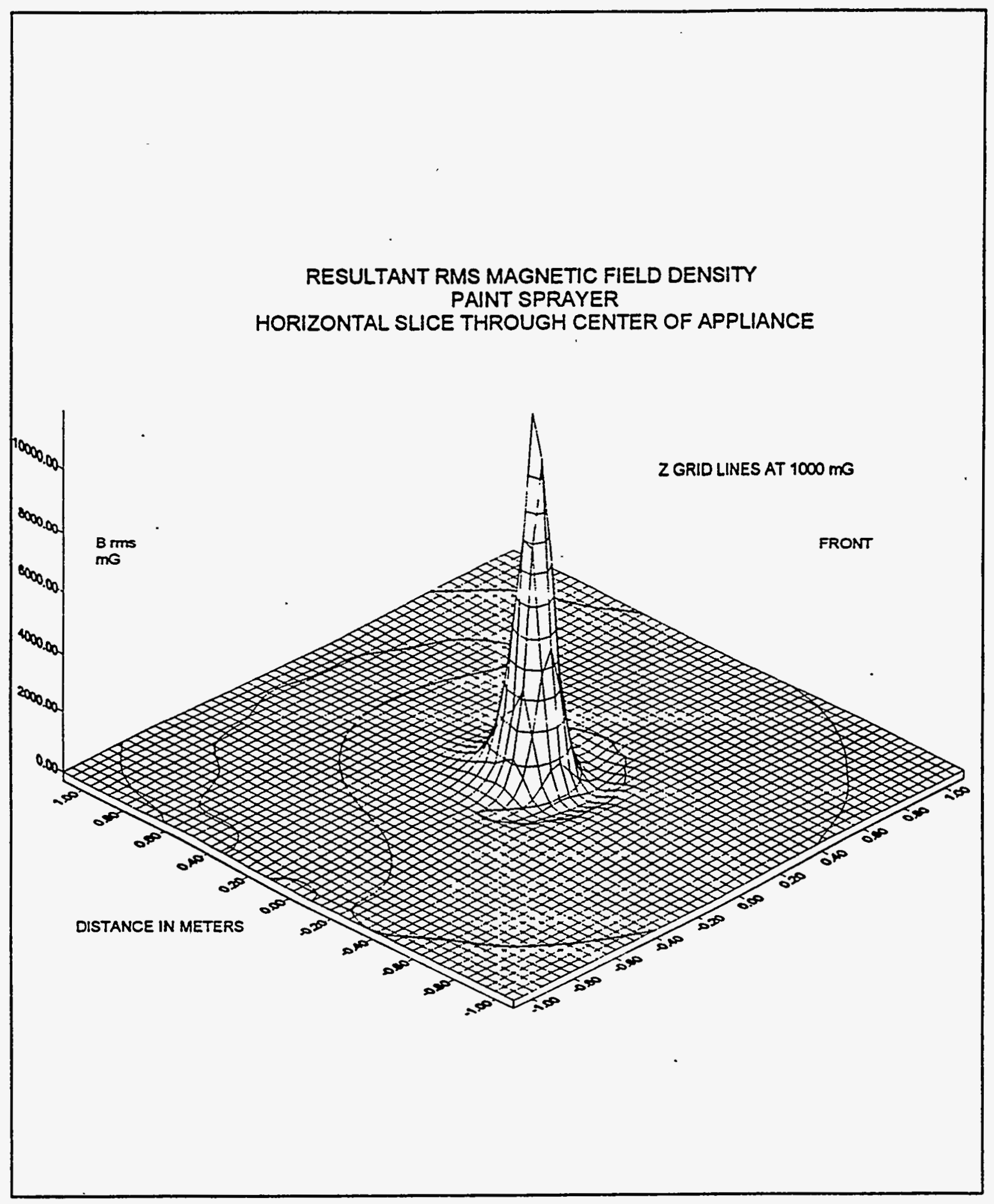

Figure C.10.1 Fundamental Resultant RMS Magnetic Field Density $3 \mathrm{~Hz}-3 \mathrm{kHz}$ - Paint Sprayer.

C.10-8 


\section{C.11 Pencil Sharpener}

Appliance Tested: A pencil sharpener as found on a desk in an office environment Manufacturer: KKK

Model: 011

Rated current: 1.2 Amperes

Appliance Code: 2273

Conditions for Testing: The unit was running with a pencil

Measured voltage and current: 119.6 Volts and 5.34 Amperes.

Magnetic Field Summary:

$60 \mathrm{~Hz}$ RMS resultant level at $30 \mathrm{~cm}$ from front surface: $9.5 \mathrm{mG}$

RMS resultant low surface level: $623 \mathrm{mG}$

RMS resultant high surface level: $5510 \mathrm{mG}$

Highest recorded surface levels:

60 Hertz RMS resultant: $4900 \mathrm{mG}$

180 Hertz RMS resultant: $2410 \mathrm{mG}$

300 Hertz RMS resultant: $706 \mathrm{mG}$

420 Hertz RMS resultant: $24 \mathrm{mG}$

Maximum Total Harmonic Distortion: 66 percent

For the appliance test set, the front of the unit was located at 0 degrees on the test table.

Summary of characteristics: The magnetic fields were rich in harmonics and were relatively strong. 
Table C.11 Pencil Sharpener

$60 \mathrm{~Hz}, 180 \mathrm{~Hz}, 300 \mathrm{~Hz}$ and $420 \mathrm{~Hz}$ RMS Resultant Fields

Distance Horizontal Vertical $60 \mathrm{~Hz} 180 \mathrm{~Hz} 300 \mathrm{~Hz} 420 \mathrm{~Hz}$ Distance Horizontal Vertical $60 \mathrm{~Hz} \quad 180 \mathrm{~Hz} \quad 300 \mathrm{~Hz} 420 \mathrm{~Hz}$ moters angle deg angle deg mGauss mGauss mGauss mGauss meters angle deg angle deg mGauss mGauss mGauss mGauss

\begin{tabular}{|c|c|c|c|c|c|c|c|c|c|c|c|c|c|}
\hline 0.051 & 0.0 & 90 & 3001.4 & 1621.0 & 503.9 & 59.6 & 0.176 & 90.0 & 60 & 111.8 & 63.4 & 19.1 & 1.8 \\
\hline 0.151 & 0.0 & 90 & 218.0 & 126.0 & 39.0 & 4.0 & 0.176 & 67.5 & 60 & 116.0 & 66.8 & 20.2 & 2.1 \\
\hline 0.351 & 0.0 & 90 & 20.0 & 12.1 & 3.8 & 0.4 & 0.176 & 45.0 & 60 & 112.5 & 66.0 & 20.2 & 2.1 \\
\hline 1.000 & 0.0 & 90 & 1.4 & 0.7 & 0.2 & 0.0 & 0.176 & 22.5 & 60 & 109.3 & 66.4 & 20.1 & 2.3 \\
\hline 0.076 & 0.0 & 60 & 623.4 & 381.0 & 117.3 & 14.9 & 0.376 & 0.0 & 60 & 13.9 & 8.6 & 2.7 & 0.3 \\
\hline 0.076 & -22.5 & 60 & 561.9 & 349.0 & 108.1 & 13.3 & 0.376 & -22.5 & 60 & 13.4 & 8.5 & 2.7 & 0.3 \\
\hline 0.076 & -45.0 & 60 & 513.1 & 318.2 & 99.1 & 12.0 & 0.376 & .45 .0 & 60 & 12.8 & 8.4 & 2.6 & 0.3 \\
\hline 0.076 & .67 .5 & 60 & 446.5 & 276.5 & 86.9 & 11.6 & 0.376 & .67 .5 & 60 & 12.4 & 8.1 & 2.5 & 0.3 \\
\hline 0.076 & -90.0 & 60 & 382.6 & 238.9 & 74.1 & 9.7 & 0.376 & .90 .0 & 60 & 11.9 & 7.8 & 2.4 & 0.3 \\
\hline 0.076 & -112.5 & 6 & 340.4 & 213.0 & 65.0 & 8.5 & 0.376 & -112.5 & 60 & 11.7 & 7.6 & 2.4 & 0.3 \\
\hline 0.076 & -135.0 & 60 & 328.3 & 203.4 & 62.2 & 7.4 & 0.376 & -135.0 & 60 & 11.6 & 7.5 & 2.3 & 0.3 \\
\hline 0.076 & -157.5 & 60 & 346.1 & 208.4 & 63.4 & 6.9 & 0.376 & -157.5 & 60 & 12.3 & 7.7 & 2.4 & 0.3 \\
\hline 0.076 & i80.0 & 60 & 379.0 & 219.3 & 66.3 & 6.7 & 0.376 & 180.0 & 60 & 12.6 & 7.7 & 2.4 & 0.3 \\
\hline 0.076 & 157.5 & 60 & 441.2 & 245.4 & 74.3 & 7.7 & 0.376 & 157.5 & 60 & 13.2 & 7.8 & 2.4 & 0.3 \\
\hline 0.076 & 135.0 & 60 & 536.4 & 288.3 & 86.1 & 8.4 & 0.376 & 135.0 & 60 & 13.9 & 8.1 & 2.5 & 0.2 \\
\hline 0.076 & 112.5 & 60 & 683.9 & 361.9 & 108.1 & 10.4 & 0.376 & 112.5 & 60 & 14.9 & 8.6 & 2.6 & 0.2 \\
\hline 0.076 & 90.0 & 60 & 798.6 & 421.3 & 124.8 & 12.3 & 0.376 & 90.0 & 60 & 15.2 & 8.8 & 2.6 & 0.3 \\
\hline 0.076 & 67.5 & 60 & 866.3 & 467.7 & 140.2 & 14.9 & 0.376 & 67.5 & 60 & 15.4 & 9.0 & 2.7 & 0.3 \\
\hline 0.076 & 45.0 & 60 & 799.1 & 444.4 & 131.8 & 13.9 & 0.376 & 45.0 & 60 & 15.0 & 8.9 & 2.7 & 0.3 \\
\hline 0.076 & 22.5 & 60 & 747.0 & 437.9 & 132.8 & 16.6 & 0.376 & 22.5 & 60 & 14.8 & 9.0 & 2.7 & 0.3 \\
\hline 0.176 & 0.0 & 60 & 99.3 & 61.9 & 19.5 & 2.2 & 1.000 & 0.0 & 60 & 1.2 & 0.7 & 0.2 & 0.0 \\
\hline 0.176 & -22.5 & 60 & 92.6 & 59.2 & 18.6 & 2.2 & 1.000 & -22.5 & 60 & 1.2 & 0.7 & 0.2 & 0.0 \\
\hline 0.176 & .45 .0 & 60 & 86.9 & 56.6 & 17.7 & 1.9 & 1.000 & .45 .0 & 60 & 1.2 & 0.7 & 0.2 & 0.0 \\
\hline 0.176 & .67 .5 & 60 & 81.0 & 52.8 & 16.8 & 2.1 & 1.000 & .67 .5 & 60 & 1.1 & 0.7 & 0.2 & 0.0 \\
\hline 0.176 & -90.0 & 60 & 75.2 & 49.2 & 15.4 & 1.9 & 1.000 & -90.0 & 60 & 1.1 & 0.7 & 0.2 & 0.0 \\
\hline 0.176 & -112.5 & $\infty$ & 71.9 & 46.5 & 14.5 & 1.6 & 1.000 & -112.5 & 60 & 1.1 & 0.7 & 0.2 & 0.0 \\
\hline 0.176 & -135.0 & 60 & 71.1 & 45.3 & 14.2 & 1.6 & 1.000 & -135.0 & 60 & 1.1 & 0.6 & 0.2 & 0.0 \\
\hline 0.176 & -157.5 & 60 & 75.2 & 46.5 & 14.5 & 1.5 & 1.000 & .157 .5 & 60 & 1.2 & 0.7 & 0.2 & 0.0 \\
\hline 0.176 & 180.0 & 60 & 78.8 & 47.3 & 14.7 & 1.5 & 1.000 & 180.0 & 60 & 1.2 & 0.7 & 0.2 & 0.0 \\
\hline 0.176 & 157.5 & 60 & 85.1 & 49.8 & 15.4 & 1.5 & 1.000 & 157.5 & 60 & 1.2 & 0.7 & 0.2 & 0.0 \\
\hline 0.176 & 135.0 & 60 & 93.4 & 53.7 & 16.2 & 1.7 & 1.000 & 135.0 & 60 & 1.3 & 0.7 & 0.2 & 0.0 \\
\hline 0.176 & 112.5 & 60 & 105.2 & 59.7 & 17.9 & 1.7 & 1.000 & 112.5 & 60 & 1.3 & 0.7 & 0.2 & 0.0 \\
\hline
\end{tabular}


Table C.11 Pencil Sharpener

$60 \mathrm{~Hz}, 180 \mathrm{~Hz}, 300 \mathrm{~Hz}$ and $420 \mathrm{~Hz}$ RMS Resultant Fields

Distanee Horizontal Vertical $60 \mathrm{~Hz} \quad 180 \mathrm{~Hz} \quad 300 \mathrm{~Hz} \quad 420 \mathrm{~Hz}$ Distence Horizontal Vertical $60 \mathrm{~Hz} \quad 180 \mathrm{~Hz} 300 \mathrm{~Hz} 420 \mathrm{~Hz}$ maters angle deg engle deg mQause mGausz mGauss mGauss meters engle deg angle deg mGauss mGauss mGanss mGauss

\begin{tabular}{|c|c|c|c|c|c|c|c|c|c|c|c|c|c|}
\hline 1.000 & 80.0 & 60 & 1.3 & 0.7 & 0.2 & 0.0 & 0.227 & 90.0 & 30 & 52.0 & 28.6 & 8.6 & 0.9 \\
\hline 1.000 & 67.5 & 60 & 1.3 & 0.7 & 0.2 & 0.0 & 0.227 & 67.5 & 30 & 55.0 & 30.5 & 9.2 & 0.8 \\
\hline 1.000 & 45.0 & 60 & 1.3 & 0.7 & 0.2 & 0.0 & 0.227 & 45.0 & 30 & 53.0 & 30.3 & 9.2 & 1.0 \\
\hline 1.000 & 22.5 & $\infty$ & 1.3 & 0.7 & 0.2 & 0.0 & 0.227 & 22.5 & 30 & 50.7 & 30.1 & 9.0 & 1.1 \\
\hline 0.127 & 0.0 & 30 & 202.2 & 123.5 & 39.0 & 5.3 & 0.427 & 0.0 & 30) & 7.7 & 4.9 & 1.5 & 0.2 \\
\hline 0.127 & .22 .5 & 30 & 189.7 & 194.0 & 35.9 & 5.0 & 0.427 & -22.5 & 30 & 7.2 & 4.8 & 1.5 & 0.2 \\
\hline 0.127 & -45.0 & 30 & 181.3 & 106.1 & 33.3 & 4.0 & 0.427 & -45.0 & 30 & 6.8 & 4.6 & 1.4 & 0.2 \\
\hline 0.127 & -67.5 & 30 & 165.1 & 95.0 & 30.4 & 4.5 & 0.427 & .67 .5 & 30 & 6.5 & 4.4 & 1.4 & 0.2 \\
\hline 0.127 & -90.0 & 30 & 146.7 & 83.8 & 26.8 & 3.2 & 0.427 & -90.0 & 30 & 6.1 & 4.2 & 1.3 & 0.2 \\
\hline 0.127 & -112.5 & 30 & 129.9 & 75.6 & 23.7 & 2.9 & 0.427 & -112.5 & 30 & 5.9 & 4.1 & 1.2 & 0.2 \\
\hline 0.127 & -135.0 & 30 & 122.1 & 72.1 & 22.5 & 2.5 & 0.427 & -135.0 & 30 & 5.9 & 4.0 & 1.2 & 0.2 \\
\hline 0.127 & -157.5 & 30 & 121.8 & 72.2 & 22.4 & 2.8 & 0.427 & -157.5 & 30 & 6.2 & 4.1 & 1.2 & 0.2 \\
\hline 0.127 & 180.0 & 30 & 128.3 & 74.4 & 23.2 & 2.9 & 0.427 & 180.0 & 30 & 6.5 & 4.2 & 1.2 & 0.2 \\
\hline 0.127 & 157.5 & 30 & 146.6 & 82.0 & 25.4 & 3.0 & 0.427 & 157.5 & 30 & 7.1 & 4.3 & 1.2 & 0.1 \\
\hline 0.127 & 135.0 & 30 & 177.1 & 95.4 & 29.1 & 2.8 & 0.427 & 135.0 & 30 & 8.0 & 4.5 & 1.3 & 0.1 \\
\hline 0.127 & 112.5 & 30 & 219.2 & 115.0 & 34.8 & 4.1 & 0.427 & 112.5 & 30 & 8.6 & 4.9 & 1.4 & 0.2 \\
\hline 0.127 & 90.0 & 30 & 252.8 & 132.9 & 40.0 & 4.2 & 0.427 & 90.0 & 30 & 9.1 & 5.1 & 1.5 & 0.1 \\
\hline 0.127 & 67.5 & 30 & 270.0 & 145.0 & 43.5 & 4.6 & 0.427 & 67.5 & 30 & 9.2 & 5.3 & 1.5 & 0.2 \\
\hline 0.127 & 45.0 & 30 & 248.3 & 140.2 & 42.8 & 4.6 & 0.427 & 45.0 & 30 & 8.9 & 5.2 & 1.5 & 0.2 \\
\hline 0.127 & 22.5 & 30 & 227.9 & 137.3 & 41.9 & 4.7 & 0.427 & 22.5 & 30 & 8.5 & 6.2 & 1.5 & 0.2 \\
\hline 0.227 & 0.0 & 30 & 45.6 & 28.2 & 8.9 & 1.1 & 1.127 & 0.0 & 30 & 0.7 & 0.4 & 0.1 & 0.0 \\
\hline 0.227 & -22.5 & 30 & 41.5 & 26.6 & 8.4 & 1.0 & 1.127 & -22.5 & 30 & 0.6 & 0.4 & 0.1 & 0.0 \\
\hline 0.227 & -45.0 & 30 & 38.4 & 25.1 & 7.9 & 0.9 & 1.127 & -45.0 & 30 & 0.5 & 0.4 & 0.1 & 0.0 \\
\hline 0.227 & .67 .5 & 30 & 35.4 & 23.3 & 7.5 & 1.0 & 1.127 & .67 .5 & 30 & 0.5 & 0.4 & 0.1 & 0.0 \\
\hline 0.227 & -90.0 & 30 & 32.5 & 29.4 & 6.7 & 0.9 & 1.127 & -90.0 & 30 & 0.5 & 0.3 & 0.1 & 0.0 \\
\hline 0.227 & -912.5 & 30 & 30.5 & 20.0 & 6.2 & 0.8 & 1.127 & -112.5 & 30 & 0.5 & 0.3 & 0.1 & 0.0 \\
\hline 0.227 & -135.0 & 30 & 29.8 & 19.4 & 6.1 & 0.8 & 1.127 & .135 .0 & 30 & 0.5 & 0.3 & 0.1 & 0.0 \\
\hline 0.227 & .157 .5 & 30 & 31.0 & 19.7 & 6.1 & 0.7 & 1.127 & -157.5 & 30 & 0.6 & 0.3 & 0.1 & 0.0 \\
\hline 0.227 & 180.0 & 30 & 32.7 & 20.0 & 6.2 & 0.7 & 1.127 & 180.0 & 30 & 0.6 & 0.4 & 0.1 & 0.0 \\
\hline 0.227 & 157.5 & 30 & 36.1 & 21.2 & 6.5 & 0.7 & 1.127 & 157.5 & 30 & 0.7 & 0.4 & 0.1 & 0.0 \\
\hline 0.227 & 135.0 & 30 & 41.4 & 23.3 & 7.1 & 0.7 & 1.127 & 135.0 & 30 & 0.8 & 0.4 & 0.1 & 0.0 \\
\hline 0.227 & 112.5 & 30 & 47.5 & 26.2 & 8.0 & 0.8 & 1.127 & 112.5 & 30 & 0.8 & 0.4 & 0.1 & 0.0 \\
\hline
\end{tabular}


Table C.11 Pencil Sharpener

$60 \mathrm{~Hz}, 180 \mathrm{~Hz}, 300 \mathrm{~Hz}$ and $420 \mathrm{~Hz}$ RMS Resultant Fields

Dlstance Horizontal Vertical $60 \mathrm{~Hz} 180 \mathrm{~Hz} 300 \mathrm{~Hz} \quad 420 \mathrm{~Hz}$ Distunce Horizontel Vertical $60 \mathrm{~Hz} \quad 180 \mathrm{~Hz} 300 \mathrm{~Hz} \quad 420 \mathrm{~Hz}$ meters angle deg angle deg mGauss mGauss mGauss moeús:

maters engle deg angle deg moauss moauss moauss mGauss

\begin{tabular}{|c|c|c|c|c|c|c|c|c|c|c|c|c|c|}
\hline 1.127 & 90.0 & 30 & 0.9 & 0.4 & 0.1 & 0.0 & 0.252 & 90.0 & 0 & 27.3 & 15.8 & 4.9 & 0.6 \\
\hline 1.127 & 67.5 & 30 & 0.8 & 0.4 & 0.1 & 0.0 & 0.252 & 67.5 & o & 28.4 & 16.7 & 5.2 & 0.6 \\
\hline 1.127 & 45.0 & 30 & 0.8 & 0.4 & 0.1 & 0.0 & 0.252 & 45.0 & 0 & 27.7 & 16.7 & 5.2 & 0.7 \\
\hline 1.127 & 22.5 & 30 & 0.8 & 0.4 & 0.1 & 0.0 & 0.252 & 22.5 & o & 27.4 & 16.9 & 5.2 & 0.6 \\
\hline 0.552 & 0.0 & 0 & 112.6 & 67.1 & 21.1 & 2.7 & 0.452 & 0.0 & 0 & 5.2 & 3.1 & 1.0 & 0.1 \\
\hline 0.152 & -22.5 & 0 & 111.3 & 64.6 & 19.5 & 0.0 & 0.452 & -22.5 & 0 & 5.2 & 3.1 & 1.0 & 0.1 \\
\hline 0.152 & -45.0 & of & 109.3 & 61.0 & 18.6 & 1.8 & 0.452 & .45 .0 & 0 & 5.3 & 3.1 & 1.0 & 0.1 \\
\hline 0.152 & .67 .5 & 0 & 101.7 & 55.9 & 16.7 & 2.4 & 0.452 & -67.5 & 0 & 5.3 & 3.0 & 0.9 & 0.1 \\
\hline 0.152 & -90.0 & 0 & 91.2 & 50.7 & 15.3 & 1.7 & 0.452 & .90 .0 & 0 & 5.1 & 2.9 & 0.9 & 0.1 \\
\hline 0.152 & -112.5 & 0 & 81.1 & 46.2 & 13.8 & 1.8 & 0.452 & -112.5 & 0 & 4.8 & 2.8 & 0.9 & 0.1 \\
\hline 0.152 & -135.0 & o & 74.9 & 43.6 & 13.7 & 1.8 & 0.452 & -135.0 & 0 & 4.5 & 2.7 & 0.8 & 0.1 \\
\hline 0.152 & -157.5 & 0 & 72.8 & 43.8 & 13.1 & 0.0 & 0.452 & -157.5 & 0 & 4.5 & 2.7 & 0.8 & 0.1 \\
\hline 0.152 & 180.0 & 0 & 72.5 & 44.4 & 12.9 & 1.8 & 0.452 & 180.0 & 0 & 4.4 & 2.7 & 0.8 & 0.1 \\
\hline 0.152 & 157.5 & 0 & 77.9 & 46.8 & 14.8 & 1.8 & 0.452 & 157.5 & o & 4.5 & 2.7 & 0.8 & 0.1 \\
\hline 0.152 & 135.0 & 0 & 89.9 & 53.0 & 16.1 & 2.4 & 0.452 & 135.0 & 0 & 4.8 & 2.8 & 0.9 & 0.1 \\
\hline 0.152 & 112.5 & 0 & 106.0 & 61.1 & 18.6 & 2.4 & 0.452 & 112.5 & 0 & 5.1 & 3.0 & 0.9 & 0.1 \\
\hline 0.152 & 90.0 & 0 & 117.5 & 67.4 & 20.2 & 0.0 & 0.452 & 90.0 & 0 & 5.3 & 3.1 & 0.9 & 0.1 \\
\hline 0.152 & 67.5 & 0 & 124.4 & 72.7 & 22.7 & 3.1 & 0.452 & 67.5 & o & 5.5 & 3.2 & 1.0 & 0.1 \\
\hline 0.152 & 45.0 & 0 & 119.9 & 71.7 & 22.3 & 2.8 & 0.452 & 45.0 & 0 & 5.3 & 3.2 & 1.0 & 0.1 \\
\hline 0.152 & 22.5 & 0 & 116.5 & 71.9 & 22.0 & 2.7 & 0.452 & 22.5 & 0 & 5.3 & 3.2 & 1.0 & 0.1 \\
\hline 0.252 & 0.0 & 0 & 26.4 & 16.1 & 5.1 & 0.6 & 1.152 & 0.0 & 0 & 0.3 & 0.2 & 0.0 & 0.0 \\
\hline 0.252 & -22.5 & 0 & 26.5 & 15.8 & 4.9 & 0.5 & 1.152 & .22 .5 & 0 & 0.3 & 0.2 & 0.0 & 0.0 \\
\hline 0.252 & .45 .0 & 0 & 26.4 & 15.3 & 4.7 & 0.6 & 1.152 & .45 .0 & 0 & 0.3 & 0.2 & 0.0 & 0.0 \\
\hline 0.252 & -67.5 & o & 25.5 & 14.5 & 4.5 & 0.5 & 1.152 & .67 .5 & o & 0.3 & 0.2 & 0.0 & 0.0 \\
\hline 0.252 & .90 .0 & o) & 23.8 & 13.5 & 4.1 & 0.5 & 1.152 & -90.0 & o) & 0.3 & 0.2 & 0.0 & 0.0 \\
\hline 0.252 & -112.5 & of & 21.9 & 12.6 & 3.9 & 0.4 & 1.152 & -112.5 & 0 & 0.3 & 0.2 & 0.0 & 0.0 \\
\hline 0.252 & -135.0 & o) & 20.6 & 12.2 & 3.7 & 0.4 & 1.152 & -135.0 & 0 & 0.3 & 0.2 & 0.0 & 0.0 \\
\hline 0.252 & -157.5 & o) & 20.2 & 12.3 & 3.7 & 0.4 & 1.152 & -157.5 & 이 & 0.3 & 0.2 & 0.0 & 0.0 \\
\hline 0.252 & 180.0 & of & 19.9 & 12.2 & 3.7 & 0.4 & 1.152 & 180.0 & o & 0.2 & 0.2 & 0.0 & 0.0 \\
\hline 0.252 & 157.5 & 0 & 20.8 & 12.6 & 3.9 & 0.4 & 1.152 & 157.5 & 0 & 0.2 & 0.2 & 0.0 & 0.0 \\
\hline 0.252 & 135.0 & of & 22.8 & 13.5 & 4.2 & 0.5 & 1.152 & 135.0 & -0 & 0.3 & 0.2 & 0.0 & 0.0 \\
\hline 0.252 & 112.5 & o & 25.5 & 14.9 & 4.5 & 0.5 & 1.152 & 112.5 & 0 & 0.3 & 0.2 & 0.0 & 0.0 \\
\hline
\end{tabular}

C.11-4 
Table C.11 Pencil Sharpener

$60 \mathrm{~Hz}, 180 \mathrm{~Hz}, 300 \mathrm{~Hz}$ and $420 \mathrm{~Hz}$ RMS Resultant Fields

Dietence Horizontal Vertlcal $60 \mathrm{~Hz} \quad 180 \mathrm{~Hz} \quad 300 \mathrm{~Hz} \quad 420 \mathrm{~Hz}$ Distance Horizontel Vertical $60 \mathrm{~Hz} \quad 180 \mathrm{~Hz} \quad 300 \mathrm{~Hz} \quad 120 \mathrm{~Hz}$ meters angle degangle deg mGauss mGauss mGauss mGauss maters angle deg angle deg mGauss mGauss mQsuss mGauss

\begin{tabular}{|c|c|c|c|c|c|c|}
\hline 1.152 & 90.0 & 0 & 0.3 & 0.2 & 0.0 & 0.0 \\
\hline 1.152 & 67.5 & 0 & 0.3 & 0.2 & 0.0 & 0.0 \\
\hline 1,152 & 45.0 & 0 & 0.3 & 0.2 & 0.0 & 0.0 \\
\hline 1.152 & 22.5 & ol & 0.3 & 0.2 & 0.0 & 0.0 \\
\hline 0.254 & 0.0 & .30 & 35.0 & 19.9 & 6.2 & 1.7 \\
\hline 0.254 & -22.5 & .30 & 36.4 & 20.0 & 6.1 & 0.9 \\
\hline 0.254 & .45 .0 & .30 & 37.1 & 19.9 & 6.0 & 1.2 \\
\hline 0.254 & .67 .5 & .30 & 36.2 & 18.5 & 5.8 & 0.0 \\
\hline 0.254 & .90 .0 & -30 & 34.3 & 17.9 & 5.5 & 1.0 \\
\hline 0.254 & -112.5 & -30 & 31.4 & 16.6 & 4.8 & 1.2 \\
\hline 0.254 & $-135,0$ & .30 & 29.0 & 15.8 & 4.8 & 1.0 \\
\hline 0.254 & -157.5 & -30 & 27.3 & 16.0 & 4.2 & 0.7 \\
\hline 0.254 & 180.0 & .30 & 24.8 & 15.9 & 4.3 & 1.0 \\
\hline 0.254 & 157.5 & -30 & 23.8 & 15.9 & 4.8 & 0.0 \\
\hline 0.254 & 135.0 & -30 & 24.1 & 16.1 & 5.1 & 1.4 \\
\hline 0.254 & 112.5 & .30 & 25.3 & 17.7 & 5.7 & 1.3 \\
\hline 0.254 & 90.0 & -30 & 27.8 & 18.2 & 5.2 & 1.6 \\
\hline 0.254 & 67.5 & -30 & 29.1 & 20.4 & 6.1 & 0.0 \\
\hline 0.254 & 45.0 & -30 & 30.8 & 20.0 & 6.0 & 0.0 \\
\hline 0.254 & 22.5 & .30 & 33.3 & 20.8 & 6.7 & 0.0 \\
\hline 0.354 & 0.0 & -30 & 12.6 & 7.7 & 2.4 & 0.3 \\
\hline 0.354 & -22.5 & -30 & 13.4 & 7.7| & 2.4 & 0.2 \\
\hline 0.354 & .45 .0 & -30 & 13.6 & 7.6 & 2.3 & 0.2 \\
\hline 0.354 & .67.5 & -30 & 13.7 & 7.5 & 2.3 & 0.2 \\
\hline 0,354 & -90.0 & .30 & 13.0 & 7.1 & 2.1 & 0.2 \\
\hline 0.354 & .112 .5 & .30 & 12.3 & 6.8 & 2.1 & 0.2 \\
\hline 0.354 & -135.0 & -30 & 11.5 & 6.5 & 2.0 & 0.2 \\
\hline 0.354 & -157.5 & -30 & 10.9 & 6.5 & 2.0 & 0.2 \\
\hline 0.354 & 180.0 & .30 & 10.2 & 6.3 & 1.9 & 0.2 \\
\hline 0.354 & 157.5 & .30 & 9.7 & 6.3 & 2.0 & 0.3 \\
\hline 0.354 & 135.0 & .30 & 9.7 & 6.5 & 2.0 & 0.3 \\
\hline 0.354 & 112.5 & -30 & 10.1 & 6.9 & 2.2 & 0.3 \\
\hline
\end{tabular}

\begin{tabular}{|r|r|r|r|r|r|r|}
\hline 0.354 & 90.0 & -30 & 10.4 & 7.1 & 2.2 & 0.3 \\
\hline 0.354 & 67.5 & -30 & 11.0 & 7.4 & 2.3 & 0.3 \\
\hline 0.354 & 45.0 & -30 & 11.3 & 7.5 & 2.3 & 0.3 \\
\hline 0.354 & 22.5 & -30 & 12.1 & 7.8 & 2.4 & 0.3 \\
\hline 0.554 & 0.0 & -30 & 3.8 & 2.3 & 0.7 & 0.1 \\
\hline 0.554 & -22.5 & -30 & 4.1 & 2.3 & 0.7 & 0.1 \\
\hline 0.554 & -45.0 & -30 & 4.2 & 2.3 & 0.7 & 0.1 \\
\hline 0.554 & -67.5 & -30 & 4.2 & 2.3 & 0.7 & 0.1 \\
\hline 0.554 & -90.0 & -30 & 4.1 & 2.2 & 0.7 & 0.1 \\
\hline 0.554 & -112.5 & -30 & 4.0 & 2.1 & 0.7 & 0.1 \\
\hline 0.554 & -1350 & -30 & 3.7 & 2.1 & 0.6 & 0.1 \\
\hline 0.554 & -157.5 & -30 & 3.5 & 2.1 & 0.6 & 0.1 \\
\hline 0.554 & 180.0 & -30 & 3.2 & 2.0 & 0.6 & 0.1 \\
\hline 0.554 & 157.5 & -30 & 3.1 & 2.0 & 0.6 & 0.1 \\
\hline 0.554 & 135.0 & -30 & 3.0 & 2.0 & 0.6 & 0.1 \\
\hline 0.554 & 112.5 & -30 & 3.1 & 2.1 & 0.7 & 0.1 \\
\hline 0.554 & 90.0 & -30 & 3.1 & 2.1 & 0.7 & 0.1 \\
\hline 0.554 & 67.5 & -30 & 3.2 & 2.2 & 0.7 & 0.1 \\
\hline 0.554 & 45.0 & -30 & 3.3 & 2.2 & 0.7 & 0.1 \\
\hline 0.554 & 22.5 & -30 & 3.6 & 2.3 & 0.7 & 0.1 \\
\hline 1.254 & 0.0 & -30 & 0.4 & 0.2 & 0.1 & 0.0 \\
\hline 1.254 & -22.5 & -30 & 0.4 & 0.2 & 0.1 & 0.0 \\
\hline 1.254 & -45.0 & -30 & 0.4 & 0.2 & 0.1 & 0.0 \\
\hline 1.254 & -67.5 & -30 & 0.5 & 0.2 & 0.1 & 0.0 \\
\hline 1.254 & -90.0 & -30 & 0.5 & 0.2 & 0.1 & 0.0 \\
\hline 1.254 & -112.5 & -30 & 0.4 & 0.2 & 0.1 & 0.0 \\
\hline 1.254 & -135.0 & -30 & 0.4 & 0.2 & 0.1 & 0.0 \\
\hline 1.254 & -157.5 & -30 & 0.4 & 0.2 & 0.1 & 0.0 \\
\hline 1.254 & 180.0 & -30 & 0.3 & 0.2 & 0.1 & 0.0 \\
\hline 1.254 & 157.5 & -30 & 0.3 & 0.2 & 0.1 & 0.0 \\
\hline 1.254 & 135.0 & -30 & 0.3 & 0.2 & 0.1 & 0.0 \\
\hline 1.254 & 112.5 & -30 & 0.3 & 0.2 & 0.1 & 0.0 \\
\hline
\end{tabular}


Table C.11 Pencil Sharpener

$60 \mathrm{~Hz}, 180 \mathrm{~Hz}, 300 \mathrm{~Hz}$ and $420 \mathrm{~Hz}$ RMS Resultant Fields

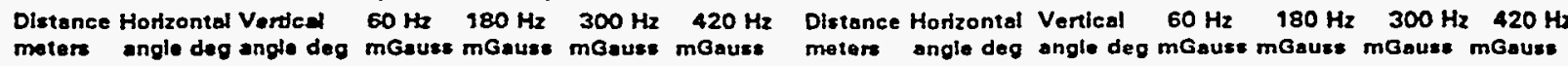

\begin{tabular}{|r|r|r|r|r|r|r|}
\hline 1.254 & 90.0 & -30 & 0.3 & 0.2 & 0.1 & 0.0 \\
\hline 1.254 & 67.5 & -30 & 0.3 & 0.2 & 0.1 & 0.0 \\
\hline 1.254 & 45.0 & -30 & 0.3 & 0.2 & 0.1 & 0.0 \\
\hline 1.254 & 22.5 & -30 & 0.3 & 0.2 & 0.1 & 0.0 \\
\hline 0.432 & 0.0 & -60 & 14.6 & 6.4 & 2.9 & 0.0 \\
\hline 0.432 & -22.5 & -60 & 14.9 & 6.5 & 3.1 & 0.7 \\
\hline 0.432 & -45.0 & -60 & 15.2 & 6.5 & 2.5 & 0.6 \\
\hline 0.432 & -67.5 & -60 & 15.2 & 6.3 & 2.8 & 0.6 \\
\hline 0.432 & -90.0 & -60 & 15.3 & 6.9 & 3.0 & 0.9 \\
\hline 0.432 & -112.5 & -60 & 14.7 & 6.0 & 2.8 & 1.0 \\
\hline 0.432 & -135.0 & -60 & 13.6 & 6.4 & 2.7 & 1.2 \\
\hline 0.432 & -157.5 & -60 & 13.6 & 6.2 & 2.8 & 0.4 \\
\hline 0.432 & 180.0 & -60 & 12.9 & 6.0 & 2.2 & 0.0 \\
\hline 0.432 & 157.5 & -60 & 12.5 & 5.6 & 2.2 & 0.6 \\
\hline 0.432 & 135.0 & -60 & 11.4 & 6.4 & 2.2 & 0.0 \\
\hline 0.432 & 112.5 & -60 & 12.6 & 6.0 & 2.4 & 0.0 \\
\hline 0.432 & 90.0 & -60 & 12.0 & 6.1 & 2.5 & 0.7 \\
\hline 0.432 & 67.5 & -60 & 13.1 & 6.3 & 2.4 & 0.7 \\
\hline 0.432 & 45.0 & -60 & 13.3 & 6.4 & 3.0 & 0.8 \\
\hline 0.432 & 22.5 & -60 & 13.3 & 6.9 & 2.8 & 0.0 \\
\hline 0.532 & 0.0 & -60 & 6.3 & 3.7 & 1.2 & 0.1 \\
\hline 0.532 & -22.5 & -60 & 6.5 & 3.7 & 1.2 & 0.1 \\
\hline 0.532 & -45.0 & -60 & 6.5 & 3.7 & 1.2 & 0.1 \\
\hline 0.532 & -67.5 & -60 & 6.6 & 3.7 & 1.2 & 0.1 \\
\hline 0.532 & -90.0 & -60 & 6.4 & 3.6 & 1.1 & 0.1 \\
\hline 0.532 & -112.5 & -60 & 6.3 & 3.6 & 1.1 & 0.1 \\
\hline 0.532 & -135.0 & -60 & 6.0 & 3.5 & 1.1 & 0.1 \\
\hline 0.532 & -157.5 & -60 & 5.9 & 3.5 & 1.1 & 0.1 \\
\hline 0.532 & 180.0 & -60 & 5.6 & 3.4 & 1.1 & 0.1 \\
\hline 0.532 & 157.5 & -60 & 5.4 & 3.3 & 1.1 & 0.1 \\
\hline 0.532 & 135.0 & -60 & 5.4 & 3.4 & 1.1 & 0.1 \\
\hline 0.532 & 112.5 & -60 & 5.5 & 3.5 & 1.1 & 0.1 \\
\hline
\end{tabular}

\begin{tabular}{|r|r|r|r|r|r|r|}
\hline 0.532 & 90.0 & -60 & 5.5 & 3.5 & 1.1 & 0.2 \\
\hline 0.532 & 67.5 & -60 & 5.6 & 3.6 & 1.2 & 0.2 \\
\hline 0.532 & 45.0 & -60 & 5.7 & 3.6 & 1.2 & 0.2 \\
\hline 0.532 & 22.5 & -60 & 6.0 & 3.7 & 1.2 & 0.2 \\
\hline 0.732 & 0.0 & -60 & 2.5 & 1.4 & 0.5 & 0.1 \\
\hline 0.732 & -22.5 & -60 & 2.6 & 1.4 & 0.5 & 0.0 \\
\hline 0.732 & -45.0 & -60 & 2.6 & 1.5 & 0.5 & 0.1 \\
\hline 0.732 & -67.5 & -60 & 2.6 & 1.5 & 0.5 & 0.0 \\
\hline 0.732 & -90.0 & -60 & 2.7 & 1.4 & 0.5 & 0.0 \\
\hline 0.732 & -112.5 & -60 & 2.6 & 1.4 & 0.5 & 0.0 \\
\hline 0.732 & -135.0 & -60 & 2.5 & 1.4 & 0.4 & 0.0 \\
\hline 0.732 & -157.5 & -60 & 2.5 & 1.4 & 0.5 & 0.0 \\
\hline 0.732 & 180.0 & -60 & 2.3 & 1.3 & 0.4 & 0.0 \\
\hline 0.732 & 157.5 & .60 & 2.3 & 1.3 & 0.4 & 0.1 \\
\hline 0.732 & 135.0 & -60 & 2.2 & 1.3 & 0.4 & 0.0 \\
\hline 0.732 & 112.5 & -60 & 2.2 & 1.4 & 0.5 & 0.1 \\
\hline 0.732 & 90.0 & -60 & 2.2 & 1.4 & 0.4 & 0.1 \\
\hline 0.732 & 67.5 & -60 & 2.2 & 1.4 & 0.5 & 0.1 \\
\hline 0.732 & 45.0 & -60 & 2.4 & 1.4 & 0.5 & 0.1 \\
\hline 0.732 & 22.5 & -60 & 2.4 & 1.4 & 0.5 & 0.1 \\
\hline 1.000 & 0.0 & -60 & 0.8 & 0.4 & 0.1 & 0.0 \\
\hline 1.000 & -22.5 & -60 & 0.8 & 0.4 & 0.1 & 0.0 \\
\hline 1.000 & -45.0 & -60 & 0.9 & 0.4 & 0.1 & 0.0 \\
\hline 1.000 & -67.5 & -60 & 0.9 & 0.4 & 0.1 & 0.0 \\
\hline 1.000 & -90.0 & -60 & 0.9 & 0.4 & 0.1 & 0.0 \\
\hline 1.000 & -112.5 & -60 & 0.8 & 0.4 & 0.1 & 0.0 \\
\hline 1.000 & -135.0 & -60 & 0.8 & 0.4 & 0.1 & 0.0 \\
\hline 1.000 & -157.5 & -60 & 0.8 & 0.4 & 0.1 & 0.0 \\
\hline 1.000 & 180.0 & -60 & 0.8 & 0.4 & 0.1 & 0.0 \\
\hline 1.000 & 157.5 & -60 & 0.7 & 0.4 & 0.1 & 0.0 \\
\hline 1.000 & 135.0 & -60 & 0.7 & 0.4 & 0.1 & 0.0 \\
\hline 112.5 & -60 & 0.7 & 0.4 & 0.1 & 0.0 \\
\hline
\end{tabular}


Table C.11 Pencil Sharpener

$60 \mathrm{~Hz}, 180 \mathrm{~Hz}, 300 \mathrm{~Hz}$ and $420 \mathrm{~Hz}$ RMS Resultant Fields

Distance Horizonted Vertled $60 \mathrm{~Hz} \quad 180 \mathrm{~Hz} \quad 300 \mathrm{~Hz} \quad 420 \mathrm{~Hz}$ Distence Horizontal Vertical $60 \mathrm{~Hz} \quad 180 \mathrm{~Hz} \quad 300 \mathrm{~Hz} \quad 420 \mathrm{~Hz}$

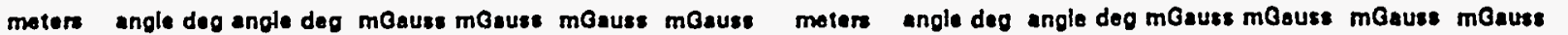

\begin{tabular}{|l|l|l|l|l|l|l|}
\hline 1.000 & 90.0 & -60 & 0.7 & 0.4 & 0.1 & 0.0 \\
\hline 1.000 & 67.5 & -60 & 0.7 & 0.4 & 0.1 & 0.0 \\
\hline 1.000 & 45.0 & -60 & 0.7 & 0.4 & 0.1 & 0.0 \\
\hline 1.000 & 22.5 & -60 & 0.8 & 0.4 & 0.1 & 0.0 \\
\hline
\end{tabular}

\begin{tabular}{|r|r|r|r|r|r|r|}
\hline 0.076 & 0.0 & -90 & 1100.0 & 576.4 & 181.5 & 19.6 \\
\hline 0.176 & 0.0 & -90 & 129.4 & 76.0 & 24.1 & 2.7 \\
\hline 0.376 & 0.0 & -90 & 16.7 & 9.9 & 3.2 & 0.4 \\
\hline
\end{tabular}

C.11-7 


\section{RESULTANT RMS MAGNETIC FIELD DENSITY \\ PENCIL SHARPENER \\ HORIZONTAL SLICE THROUGH CENTER OF APPLIANCE}

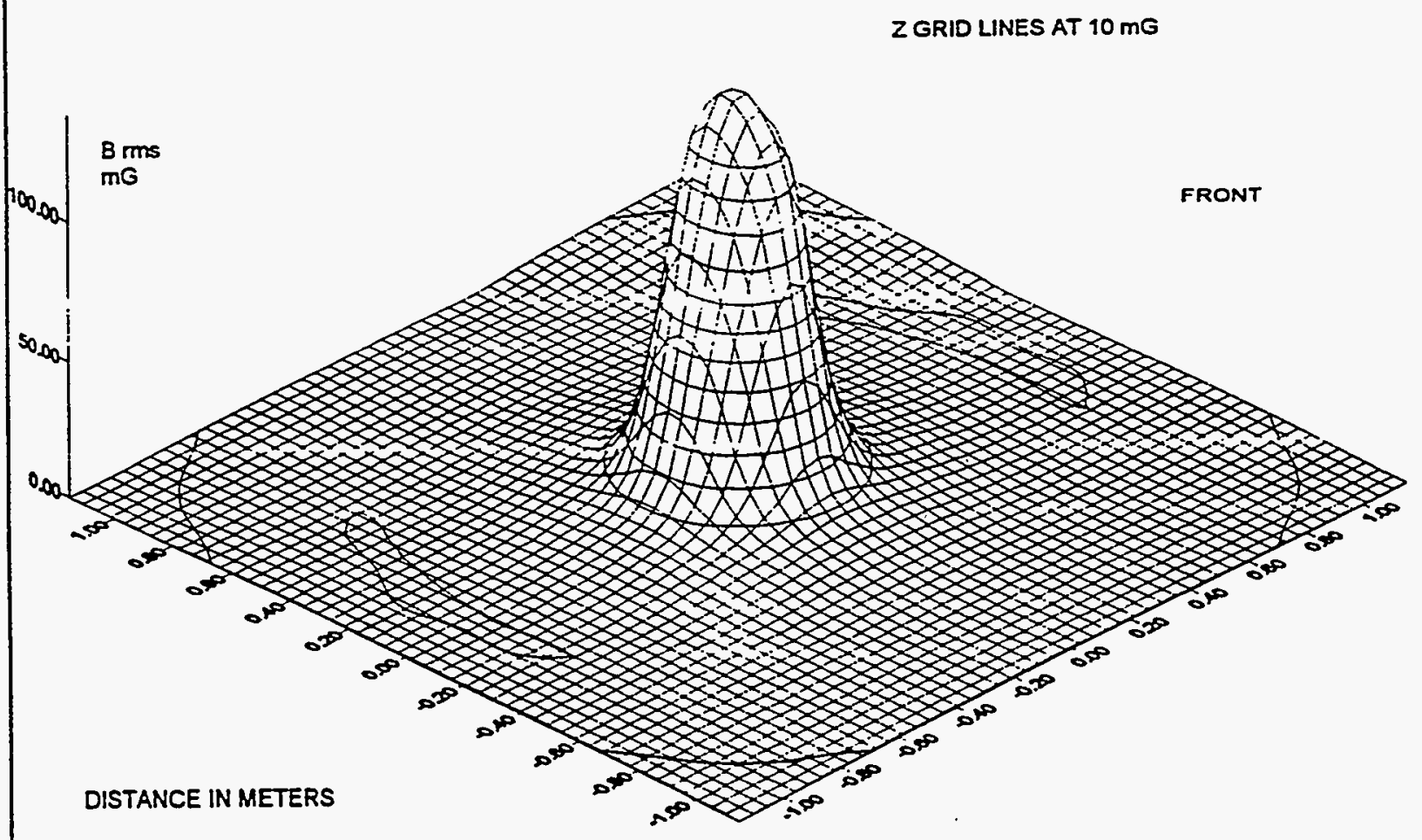

Figure C.11.1 Fundamental Resultant RMS Magnetic Field Density - Pencil Sharpener.

C.11-8 
THIRD HARMONIC RESULTANT RMS MAGNETIC FIELD DENSITY PENCIL SHARPENER

HORIZONTAL SLICE THROUGH CENTER OF APPLIANCE

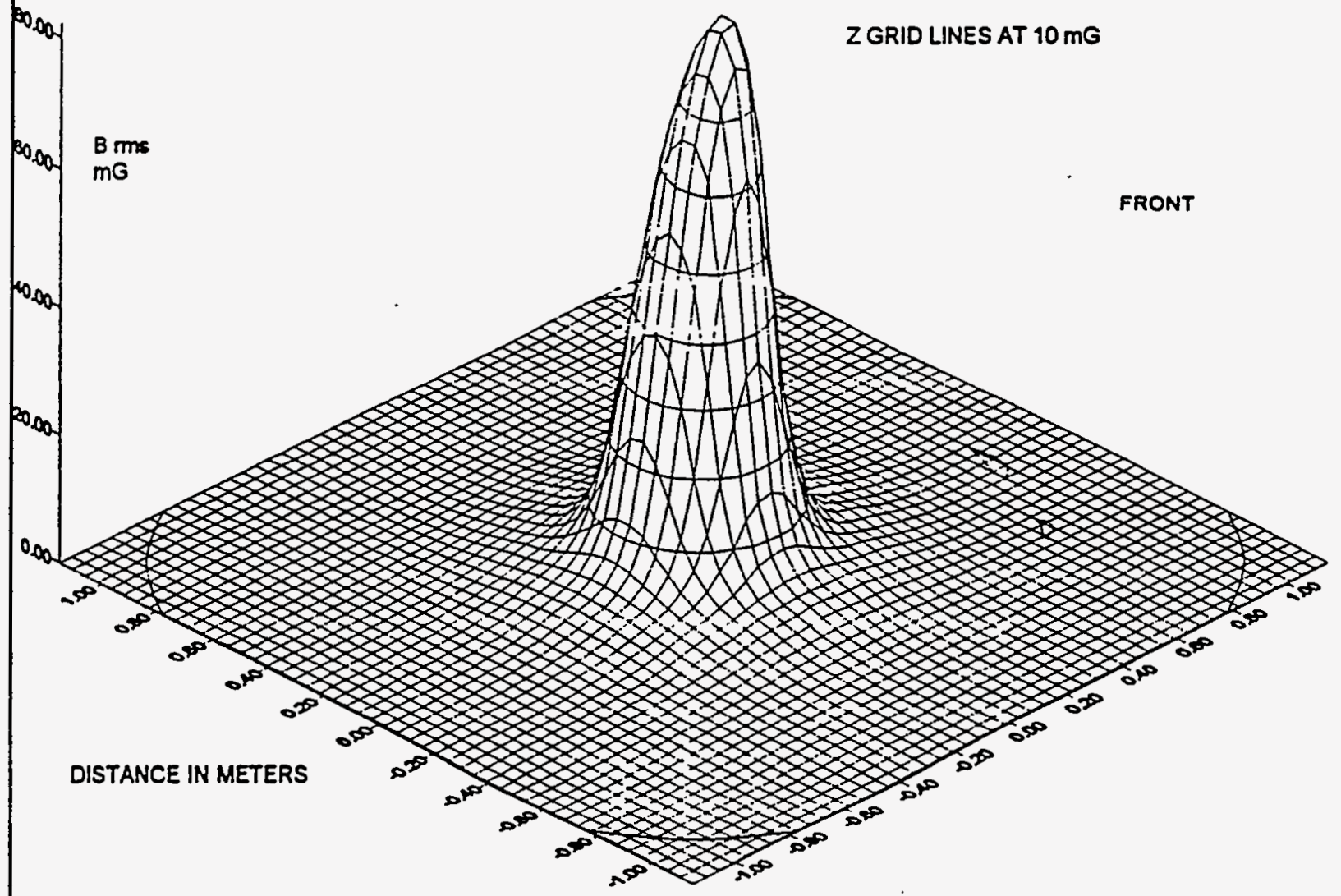

Figure C.11.2 Third Harmonic Resultant RMS Magnetic Field Density - Pencil Sharpener.

C.11-9 


\title{
FIFTH HARMONIC RESULTANT RMS MAGNETIC FIELD DENSITY PENCIL SHARPENER \\ HORIZONTAL SLICE THROUGH CENTER OF APPLIANCE
}

\author{
Z GRID LINES AT 2 mG
}

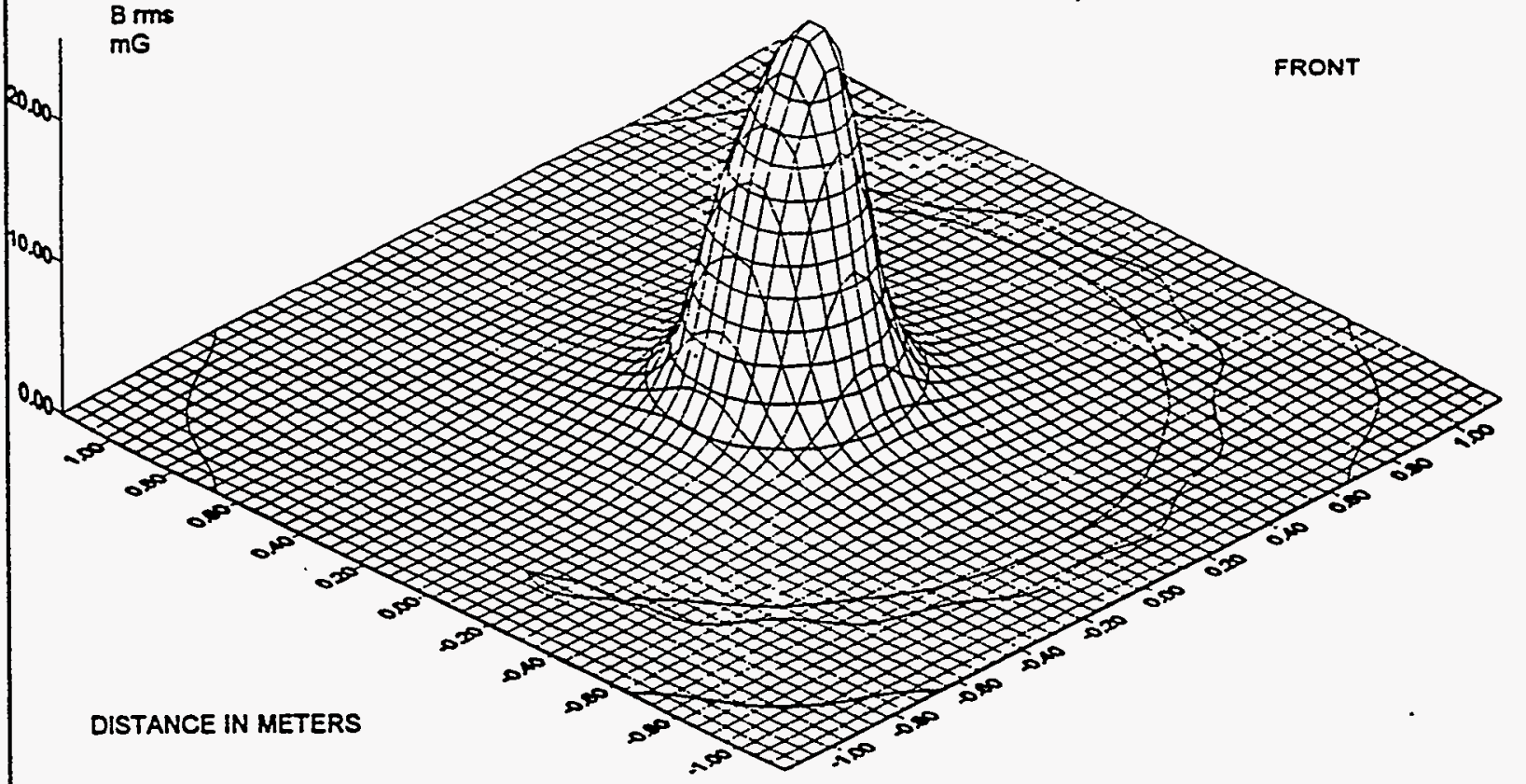

Figure C.11.3 Fifth Harmonic Resultant RMS Magnetic Field Density - Pencil Sharpener. 


\section{SEVENTH HARMONIC RESULTANT RMS MAGNETIC FIELD DENSITY PENCIL SHARPENER \\ HORIZONTAL SLICE THROUGH CENTER OF APPLIANCE}

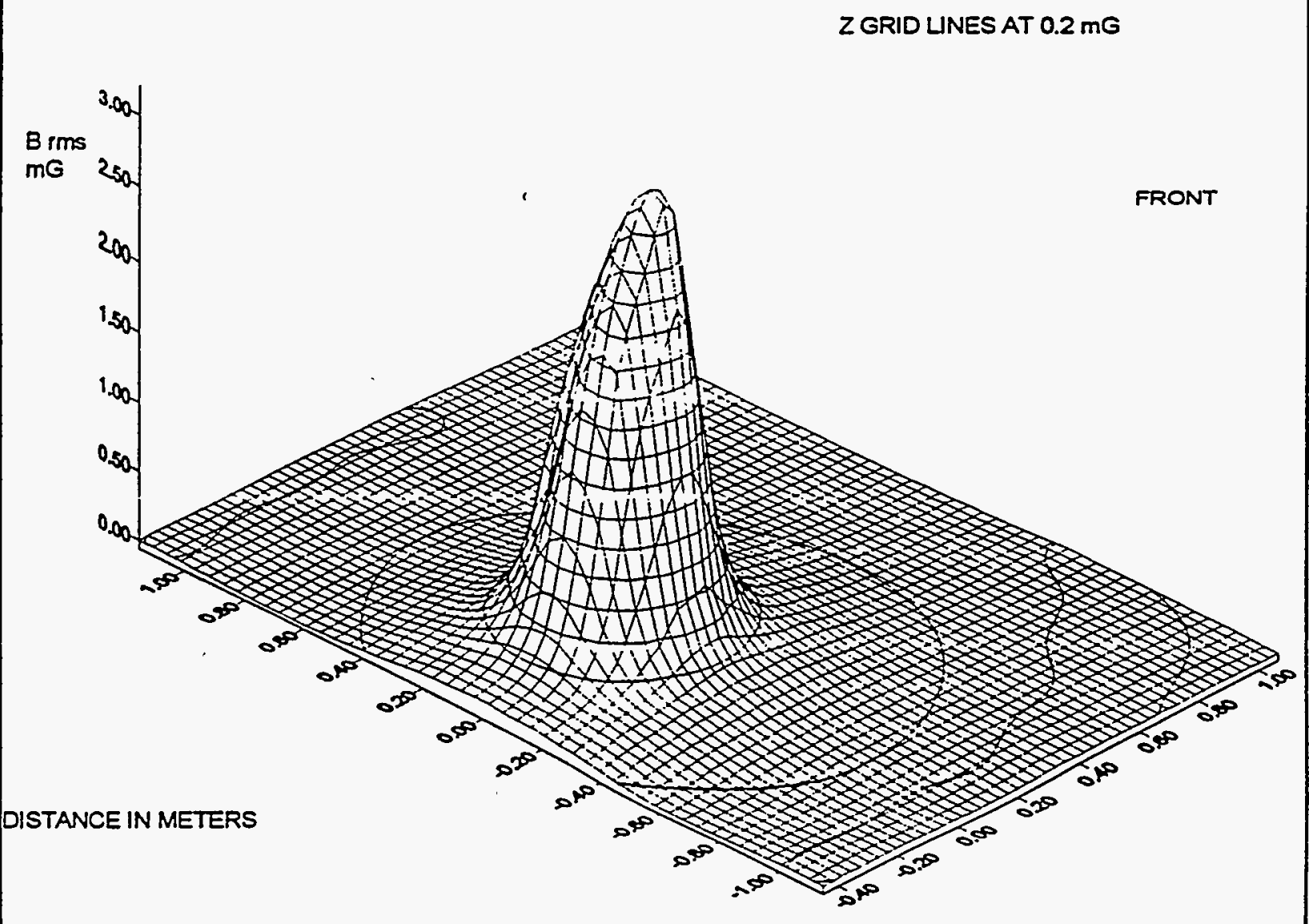

Figure C.11.4 Seventh Harmonic Resultant RMS Magnetic Field Density - Pencil Sharpener. 


\section{C.12 Popcorn Popper}

Appliance Tested: A hot air popcorn popper

Manufacturer: $\mathrm{LLL}$

Model: 012

Rated power: 140 Watts

Appliance Code: 1253

Conditions for Testing: The unit was running with no popcorn. The unit was turned off periodically to cool so the transducers were not damaged.

Measured voltage and current: 112.2 Volts and 10.56 Amperes.

Magnetic Field Summary:

$60 \mathrm{~Hz}$ RMS resultant level at $30 \mathrm{~cm}$ from front surface: $0.2 \mathrm{mG}$

RMS resultant low surface level: $11.2 \mathrm{mG}$

RMS resultant high surface level: $257 \mathrm{mG}$

Highest recorded surface levels:

60 Hertz RMS resultant: $255 \mathrm{mG}$

120 Hertz RMS resultant: $124.9 \mathrm{mG}$

180 Hertz RMS resultant: $2.4 \mathrm{mG}$

240 Hertz RMS resultant: $21.7 \mathrm{mG}$

300 Hertz RMS resultant: $2.7 \mathrm{mG}$

360 Hertz RMS resultant: $8.45 \mathrm{mG}$

420 Hertz RMS resultant: $1.1 \mathrm{mG}$

Maximum Total Harmonic Distortion: 52 percent

For the appliance test set, the front of the unit was located at 180 degrees on the test table.

Summary of characteristics: The magnetic fields were rich in even as well as odd harmonics. 
Table C.12 Popcorn Popper

Distance Horizontal Vertical $60 \mathrm{~Hz}$ $60 \mathrm{~Hz}$ RMS Resultant Fields

Distance Horizontal Vertical $60 \mathrm{~Hz}$ meters angle deg angle deg mGauss

\begin{tabular}{|r|r|r|r|}
\hline 0.229 & 0.0 & 90 & 5.0 \\
\hline 0.329 & 0.0 & 90 & 3.0 \\
\hline 0.529 & 0.0 & 90 & 0.1 \\
\hline 1.000 & 0.0 & 90 & 3.8 \\
\hline 0.229 & 0.0 & 60 & 5.1 \\
\hline 0.229 & -22.5 & 60 & 5.1 \\
\hline 0.229 & -45.0 & 60 & 4.8 \\
\hline 0.229 & -67.5 & 60 & 4.5 \\
\hline 0.229 & -90.0 & 60 & 4.0 \\
\hline 0.229 & -112.5 & 60 & 3.7 \\
\hline 0.229 & -135.0 & 60 & 3.4 \\
\hline 0.229 & -157.5 & 60 & 3.2 \\
\hline 0.229 & 180.0 & 60 & 3.0 \\
\hline 0.229 & 157.5 & 60 & 2.9 \\
\hline 0.229 & 135.0 & 60 & 3.0 \\
\hline 0.229 & 112.5 & 60 & 3.2 \\
\hline 0.229 & 90.0 & 60 & 3.5 \\
\hline 0.229 & 67.5 & 60 & 3.9 \\
\hline 0.229 & 45.0 & 60 & 4.2 \\
\hline 0.229 & 22.5 & 60 & 4.7 \\
\hline 0.329 & 0.0 & 60 & 1.4 \\
\hline 0.329 & -22.5 & 60 & 1.4 \\
\hline 0.329 & -45.0 & 60 & 1.4 \\
\hline 0.329 & -67.5 & 60 & 1.4 \\
\hline 0.329 & -90.0 & 60 & 1.4 \\
\hline 0.329 & -112.5 & 60 & 1.3 \\
\hline 0.329 & -135.0 & 60 & 1.3 \\
\hline 0.329 & -157.5 & 60 & 1.2 \\
\hline 0.329 & 180.0 & 60 & 1.2 \\
\hline 0.329 & 157.5 & 60 & 1.1 \\
\hline
\end{tabular}

\begin{tabular}{|c|c|c|c|}
\hline 0.329 & 135.0 & 60 & 1.1 \\
\hline 0.329 & 112.5 & 60 & 1.1 \\
\hline 0.329 & 90.0 & 60 & 1.1 \\
\hline 0.329 & 67.5 & 60 & 1.2 \\
\hline 0.329 & 45.0 & 60 & 1.2 \\
\hline 0.329 & 22.5 & 60 & 1.2 \\
\hline 0.529 & 0.0 & 60 & 0.3 \\
\hline 0.529 & -22.5 & 60 & 0.3 \\
\hline 0.529 & -45.0 & 60 & 0.3 \\
\hline 0.529 & -67.5 & 60 & 0.3 \\
\hline 0.529 & -90.0 & 60 & 0.4 \\
\hline 0.529 & -112.5 & 60 & 0.3 \\
\hline 0.529 & -135.0 & 60 & 0.3 \\
\hline 0.529 & -157.5 & 60 & 0.3 \\
\hline 0.529 & 180.0 & 60 & 0.3 \\
\hline 0.529 & 157.5 & 60 & 0.3 \\
\hline 0.529 & 135.0 & 60 & 0.3 \\
\hline 0.529 & 112.5 & 60 & 0.3 \\
\hline 0.529 & 90.0 & 60 & 0.3 \\
\hline 0.529 & 67.5 & 60 & 0.3 \\
\hline 0.529 & 45.0 & 60 & 0.3 \\
\hline 0.529 & 22.5 & 60 & 0.3 \\
\hline 1.000 & 0.0 & 60 & 0.1 \\
\hline 1.000 & -22.5 & 60 & 0.1 \\
\hline 1.000 & -45.0 & 60 & 0.1 \\
\hline 1.000 & -67.5 & 60 & 0.1 \\
\hline 1.000 & -90.0 & 60 & 0.1 \\
\hline 1.000 & -112.5 & 60 & 0.1 \\
\hline 1.000 & -135.0 & 60 & 0.1 \\
\hline 1.000 & -157.5 & 60 & 0.1 \\
\hline
\end{tabular}


Table C.12 Popcorn Popper

Distance Horizontal Vertical $60 \mathrm{~Hz}$ meters angle deg angle deg mGauss

\section{$60 \mathrm{~Hz}$ RMS Resultant Fields}

Distance Horizontal Vertical $60 \mathrm{~Hz}$

meters angle deg angle deg mGauss

\begin{tabular}{|c|c|c|c|}
\hline 1.000 & 180.0 & 60 & 0.1 \\
\hline 1.000 & 157.5 & 60 & 0.1 \\
\hline 1.000 & 135.0 & 60 & 0.1 \\
\hline 1.000 & 112.5 & 60 & 0.1 \\
\hline 1.000 & 90.0 & $\infty$ & 0.1 \\
\hline 1.000 & 67.5 & 60 & 0.1 \\
\hline 1.000 & 45.0 & 60 & 0.1 \\
\hline 1.000 & 22.5 & 60 & 0.1 \\
\hline 0.229 & 0.0 & 30 & 3.2 \\
\hline 0.229 & -22.5 & 30 & 3.2 \\
\hline 0.229 & -45.0 & 30 & 3.0 \\
\hline 0.229 & -57.5 & 30 & 2.8 \\
\hline 0.229 & -90.0 & 30 & 2.5 \\
\hline 0.2229 & -112.5 & 30 & 2.3 \\
\hline 0.2229 & -135.0 & 30 & 2.1 \\
\hline 0.229 & -157.5 & 30 & 1.9 \\
\hline 0.229 & 180.0 & 30 & 1.8 \\
\hline 0.229 & 157.5 & 30 & 1.7 \\
\hline 0.229 & 135.0 & 30 & 1.7 \\
\hline 0.229 & 112.5 & 30 & 1.8 \\
\hline 0.229 & $\infty 0.0$ & 30 & 2.0 \\
\hline 0.229 & 67.5 & 30 & 2.2 \\
\hline 0.229 & 45.0 & 30 & 2.6 \\
\hline 0.229 & 22.5 & 30 & 2.9 \\
\hline 0.329 & 0.0 & 30 & 1.0 \\
\hline 0.329 & .22 .5 & 30 & 1.0 \\
\hline 0.329 & -45.0 & 30 & 1.0 \\
\hline 0.329 & -67.5 & 30 & 1.0 \\
\hline 0.329 & -90.0 & 30 & 1.0 \\
\hline 0.329 & -112.5 & 30 & 1.0 \\
\hline
\end{tabular}

\begin{tabular}{|c|c|c|c|}
\hline 0.329 & -135.0 & 30 & 0.9 \\
\hline 0.329 & -157.5 & 30 & 0.8 \\
\hline 0.329 & 180.0 & 30 & 0.8 \\
\hline 0.329 & 157.5 & 30 & 0.8 \\
\hline 0.329 & 135.0 & 30 & 0.8 \\
\hline 0.329 & 112.5 & 30 & 0.7 \\
\hline 0.329 & 90.0 & 30 & 0.8 \\
\hline 0.329 & 67.5 & 30 & 0.9 \\
\hline 0.329 & 45.0 & 30 & 0.9 \\
\hline 0.329 & 22.5 & 30 & 0.9 \\
\hline 0.529 & 0.0 & 30 & 0.4 \\
\hline 0.529 & -22.5 & 30 & 0.3 \\
\hline 0.529 & -45.0 & 30 & 0.3 \\
\hline 0.529 & -67.5 & 30 & 0.3 \\
\hline 0.529 & -90.0 & 30 & 0.3 \\
\hline 0.529 & -112.5 & 30 & 0.3 \\
\hline 0.529 & -135.0 & 30 & 0.3 \\
\hline 0.529 & -157.5 & 30 & 0.4 \\
\hline 0.529 & 180.0 & 30 & 0.3 \\
\hline 0.529 & 157.5 & 30 & 0.3 \\
\hline 0.529 & 135.0 & 30 & 0.2 \\
\hline 0.529 & 112.5 & 30 & 0.3 \\
\hline 0.529 & 90.0 & 30 & 0.3 \\
\hline 0.529 & 67.5 & 30 & 0.3 \\
\hline 0.529 & 45.0 & 30 & 0.3 \\
\hline 0.529 & 22.5 & 30 & 0.3 \\
\hline 1.229 & 0.0 & 30 & 0.2 \\
\hline 1.229 & -22.5 & 30 & 0.2 \\
\hline 1.229 & -45.0 & 30 & 0.1 \\
\hline 1.229 & -90.0 & 30 & 0.0 \\
\hline
\end{tabular}


Table C.12 Popcorn Popper

Distance Horizontal Vertical $60 \mathrm{~Hz}$ meters angle deg angle deg mGauss

\begin{tabular}{|c|c|c|c|}
\hline 1.229 & -112.5 & 30 & 0.0 \\
\hline 1.229 & -135.0 & 30 & 0.1 \\
\hline 1.220 & -157.5 & 30 & 0.1 \\
\hline 1.229 & 67.5 & 30 & 0.0 \\
\hline 1.229 & 45.0 & 30 & 0.1 \\
\hline 0.127 & 0.0 & 0 & 16.7 \\
\hline 0.127 & -22.5 & io & 96.4 \\
\hline 0.127 & -45.0 & 0 & 11.5 \\
\hline 0.127 & -67.5 & 0 & 8.0 \\
\hline 0.127 & .90 .0 & 0 & 6.2 \\
\hline 0.127 & -112.5 & 0 & 5.1 \\
\hline 0.127 & '-135.0 & 0 & 4.3 \\
\hline 0.127 & -157.5 & 0 & 3.7 \\
\hline 0.127 & 180.0 & 0 & 3.3 \\
\hline 0.127 & 157.5 & 0 & 3.3 \\
\hline 0.127 & 135.0 & 0 & 3.4 \\
\hline 0.127 & 112.5 & 0 & 3.9 \\
\hline 0.127 & 90.0 & 0 & 4.8 \\
\hline 0.127 & 67.5 & 0 & 6.2 \\
\hline 0.127 & 45.0 & 0 & 8.5 \\
\hline 0.127 & 22.5 & 0 & 12.1 \\
\hline 0.227 & 0.0 & 0 & 4.1 \\
\hline 0.227 & -22.5 & 0 & 3.8 \\
\hline 0.227 & -45.0 & 0 & 2.7 \\
\hline 0.227 & -67.5 & 0 & 2.0 \\
\hline 0.227 & -90.0 & 0 & 1.8 \\
\hline 0.227 & -112.5 & 0 & 1.6 \\
\hline 0.227 & -135.0 & 0 & 1.4 \\
\hline 0.227 & -157.5 & 0 & 1.2 \\
\hline 0.227 & 180.0 & 0 & 1.1 \\
\hline
\end{tabular}

$60 \mathrm{~Hz}$ RMS Resultant Fields

Distance Horizontal Vertical $60 \mathrm{~Hz}$

meters angle deg angle deg mGauss

\begin{tabular}{|c|r|r|r|}
\hline 0.227 & 157.5 & 0 & 1.1 \\
\hline 0.227 & 135.0 & 0 & 1.0 \\
\hline 0.227 & 112.5 & 0 & 1.1 \\
\hline 0.227 & 90.0 & 0 & 1.3 \\
\hline 0.227 & 67.5 & 0 & 1.7 \\
\hline 0.227 & 45.0 & 0 & 2.4 \\
\hline 0.227 & 22.5 & 0 & 3.1 \\
\hline 0.427 & 0.0 & 0 & 0.8 \\
\hline 0.427 & -22.5 & 0 & 0.7 \\
\hline 0.427 & -45.0 & 0 & 0.5 \\
\hline 0.427 & -67.5 & 0 & 0.5 \\
\hline 0.427 & -90.0 & 0 & 0.4 \\
\hline 0.427 & -112.5 & 0 & 0.4 \\
\hline 0.427 & -135.0 & 0 & 0.4 \\
\hline 0.427 & -157.5 & 0 & 0.3 \\
\hline 0.427 & 180.0 & 0 & 0.3 \\
\hline 0.427 & 157.5 & 0 & 0.3 \\
\hline 0.427 & 135.0 & 0 & 0.3 \\
\hline 0.427 & 112.5 & 0 & 0.3 \\
\hline 0.427 & 90.0 & 0 & 0.4 \\
\hline 0.427 & 67.5 & 0 & 0.4 \\
\hline 0.427 & 45.0 & 0 & 0.7 \\
\hline 0.427 & 22.5 & 0 & 0.7 \\
\hline 0.279 & 0.0 & -30 & 4.1 \\
\hline 0.279 & -22.5 & -30 & 5.4 \\
\hline 0.279 & -45.0 & -30 & 3.5 \\
\hline 0.279 & -67.5 & -30 & 1.8 \\
\hline 0.279 & -90.0 & -30 & 1.4 \\
\hline 0.279 & -112.5 & -30 & 1.1 \\
\hline 0.279 & -135.0 & -30 & 1.0 \\
\hline
\end{tabular}


Table C.12 Popcorn Popper

$60 \mathrm{~Hz}$ RMS Resultant Fields

Distance Horizontal Vertical $60 \mathrm{~Hz}$ meters angle deg angle deg mGauss

\begin{tabular}{|l|r|r|r|}
\hline 0.279 & 180.0 & -30 & 0.7 \\
\hline 0.279 & 157.5 & -30 & 0.8 \\
\hline 0.279 & 135.0 & 30 & 0.7 \\
\hline 0.279 & 112.5 & -30 & 0.9 \\
\hline 0.279 & 90.0 & -30 & 1.4 \\
\hline 0.279 & 67.5 & -30 & 2.5 \\
\hline 0.279 & 45.0 & -30 & 4.1 \\
\hline 0.279 & 22.5 & -30 & 12.7 \\
\hline 0.379 & 0.0 & -30 & 3.5 \\
\hline 0.379 & -22.5 & -30 & 1.8 \\
\hline 0.379 & -45.0 & -30 & 1.2 \\
\hline 0.379 & -67.5 & -30 & 0.8 \\
\hline 0.379 & -90.0 & -30 & 0.6 \\
\hline 0.379 & -112.5 & -30 & 0.5 \\
\hline 0.379 & -135.0 & -30 & 0.5 \\
\hline 0.379 & -157.5 & -30 & 0.5 \\
\hline 0.379 & 180.0 & -30 & 0.4 \\
\hline 0.379 & 157.5 & -30 & 0.4 \\
\hline 0.379 & 135.0 & -30 & 0.4 \\
\hline 0.379 & 112.5 & -30 & 0.5 \\
\hline 0.379 & 90.0 & -30 & 0.7 \\
\hline 0.379 & 67.5 & -30 & 1.2 \\
\hline 0.379 & 45.0 & -30 & 2.1 \\
\hline 0.379 & 22.5 & -30 & 3.7 \\
\hline 0.579 & 0.0 & -30 & 1.1 \\
\hline 0.579 & -22.5 & -30 & 0.7 \\
\hline 0.579 & -45.0 & -30 & 0.3 \\
\hline 0.579 & -67.5 & -30 & 0.3 \\
\hline 0.579 & -90.0 & -30 & 0.2 \\
\hline 0.579 & -112.5 & -30 & 0.2 \\
\hline
\end{tabular}

Distance Horizontal Vertical $60 \mathrm{~Hz}$ meters angle deg angle deg mGauss

\begin{tabular}{|c|c|c|c|}
\hline 0.579 & -135.0 & .30 & 0.2 \\
\hline 0.579 & -157.5 & -30 & 0.2 \\
\hline 0.579 & 180.0 & -30 & 0.2 \\
\hline 0.579 & 157.5 & -30 & 0.1 \\
\hline 0.579 & 135.0 & -30 & 0.1 \\
\hline 0.579 & 112.5 & .30 & 0.2 \\
\hline 0.579 & 90.0 & -30 & 0.4 \\
\hline 0.579 & 67.5 & -30 & 0.5 \\
\hline 0.579 & 45.0 & -30 & 0.9 \\
\hline 0.579 & 22.5 & -30 & 0.9 \\
\hline 0.305 & 0.0 & -60 & 29.9 \\
\hline 0.305 & -22.5 & .60 & 3.9 \\
\hline 0.305 & -45.0 & -60 & 2.0 \\
\hline 0.305 & -67.5 & -60 & 0.9 \\
\hline 0.305 & -90.0 & -60 & 0.7 \\
\hline 0.305 & -112.5 & -60 & 0.6 \\
\hline 0.305 & -135.0 & -60 & 0.5 \\
\hline 0.305 & -157.5 & -60 & 0.5 \\
\hline 0.305 & 180.0 & -60 & 0.5 \\
\hline 0.305 & 157.5 & -60 & 0.5 \\
\hline 0.305 & 135.0 & -60 & 0.6 \\
\hline 0.305 & 112.5 & -60 & 0.8 \\
\hline 0.305 & 90.0 & -60 & 1.2 \\
\hline 0.305 & 67.5 & -60 & 1.9 \\
\hline 0.305 & 45.0 & -60 & 4.1 \\
\hline 0.305 & 22.5 & -60 & 54.4 \\
\hline 0.405 & 0.0 & -60 & 12.4 \\
\hline 0.405 & -22.5 & -60 & 3.4 \\
\hline 0.405 & -45.0 & -60 & 1.3 \\
\hline 0.405 & .67 .5 & -60 & 0.7 \\
\hline
\end{tabular}


Table C.12 Popcorn Popper

Distance Horizontal Vertical $60 \mathrm{~Hz}$ meters angle deg angle deg mGauss

\begin{tabular}{|r|r|r|r|}
\hline 0.405 & -90.0 & -60 & 0.5 \\
\hline 0.405 & -112.5 & -60 & 0.4 \\
\hline 0.405 & -135.0 & -60 & 0.4 \\
\hline 0.405 & -157.5 & -60 & 0.4 \\
\hline 0.405 & 180.0 & -60 & 0.4 \\
\hline 0.405 & 157.5 & -60 & 0.4 \\
\hline 0.405 & 135.0 & -60 & 0.5 \\
\hline 0.405 & 112.5 & -60 & 0.6 \\
\hline 0.405 & 90.0 & -60 & 0.9 \\
\hline 0.405 & 67.5 & -60 & 1.7 \\
\hline 0.405 & 45.0 & -60 & 4.1 \\
\hline 0.405 & 22.5 & -60 & 15.0 \\
\hline 0.605 & 0.0 & -60 & 4.8 \\
\hline 0.605 & -22.5 & -60 & 1.5 \\
\hline 0.605 & -45.0 & -60 & 0.6 \\
\hline 0.605 & -67.5 & -60 & 0.4 \\
\hline
\end{tabular}

$60 \mathrm{~Hz}$ RMS Resultant Fields

Distance Horizontal Vertical $60 \mathrm{~Hz}$

meters angle deg angle deg mGauss

\begin{tabular}{|r|r|r|r|}
\hline 0.605 & -90.0 & -60 & 0.3 \\
\hline 0.605 & -112.5 & -60 & 0.2 \\
\hline 0.605 & -135.0 & -60 & 0.2 \\
\hline 0.605 & -157.5 & -60 & 0.2 \\
\hline 0.605 & 180.0 & -60 & 0.2 \\
\hline 0.605 & 157.5 & -60 & 0.2 \\
\hline 0.605 & 135.0 & -60 & 0.3 \\
\hline 0.605 & 112.5 & -60 & 0.3 \\
\hline 0.605 & 90.0 & -60 & 0.5 \\
\hline 0.605 & 67.5 & -60 & 1.0 \\
\hline 0.605 & 45.0 & -60 & 2.9 \\
\hline 0.605 & 22.5 & -60 & 4.6 \\
\hline 0.229 & 0.0 & -90 & 4.1 \\
\hline 0.329 & 0.0 & -90 & 0.7 \\
\hline
\end{tabular}




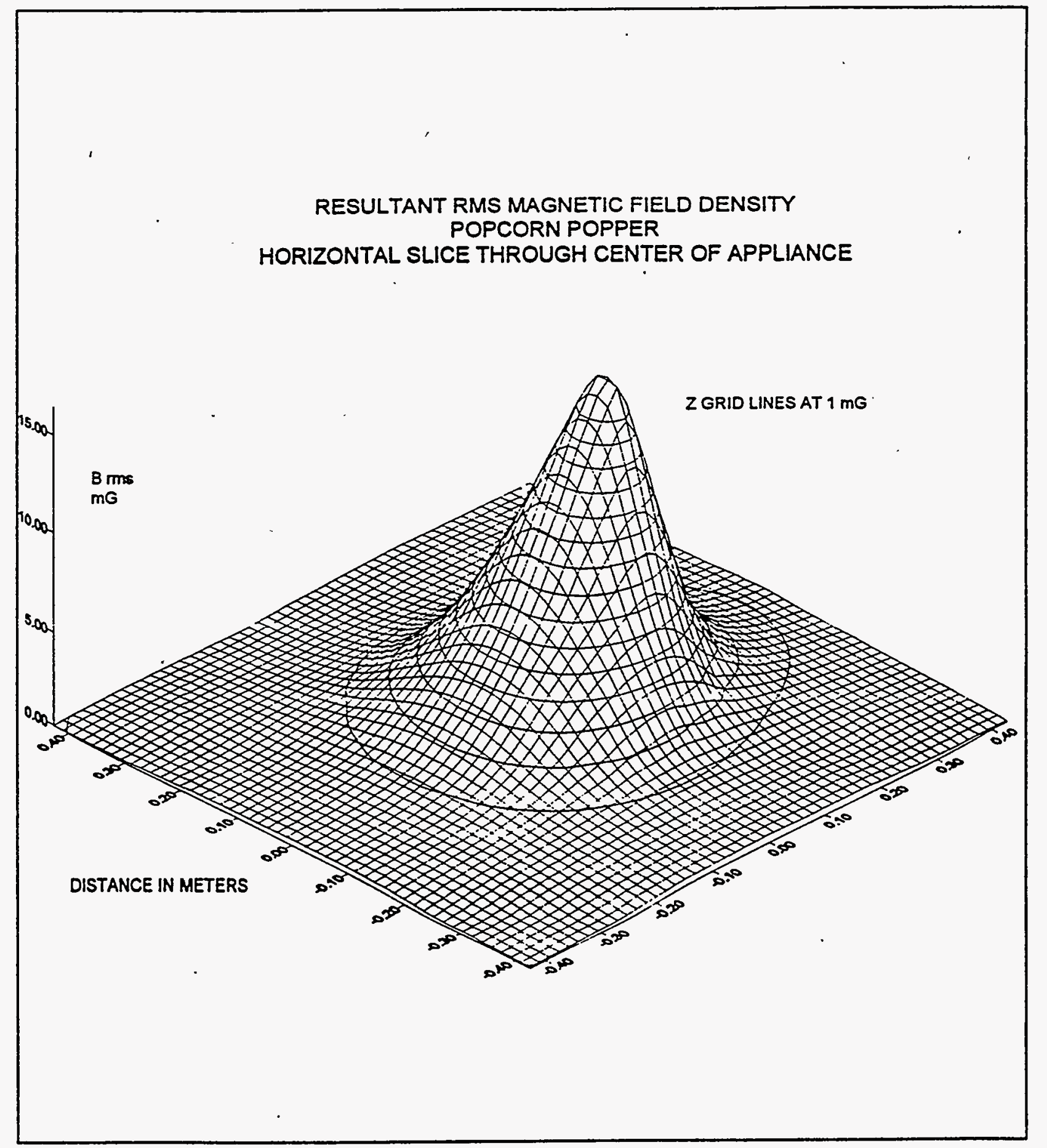

Figure C.12.1 Fundamental Resultant RMS Magnetic Field Density - Popcorn Popper. 


\section{C.13 Laser Printer}

Appliance Tested: A laser printer as used in the home and office environments. Manufacturer: MMM

Appliance Code: 1261

Conditions for Testing: The printer was connected to a laptop computer with a 50 foot parallel port cable and was printing documents.

Measured voltage and current: 119.1 Volts and 1.5 Amperes.

Magnetic Field Summary:

$60 \mathrm{~Hz}$ RMS resultant level at $30 \mathrm{~cm}$ from front surface: $0.2 \mathrm{mG}$

RMS resultant low surface level: $1.18 \mathrm{mG}$

RMS resultant high surface level: $83.5 \mathrm{mG}$

Highest recorded surface levels:

60 Hertz RMS resultant: $82.7 \mathrm{mG}$

180 Hertz RMS resultant: $7.61 \mathrm{mG}$

300 Hertz RMS resultant: $5.22 \mathrm{mG}$

420 Hertz RMS resultant: $3.82 \mathrm{mG}$

$1822 \mathrm{~Hz}$ RMS resultant: $0.92 \mathrm{mG}$

Maximum Total Harmonic Distortion: 14 percent

For the appliance test set, the front of the unit was located at 0 degrees on the test table.

Summary of characteristics: The magnetic fields were moderate but contained many off-power-frequency components. 
Distance Horizontal Vertical $60 \mathrm{~Hz}$ meters angle deg angle deg mGauss

\begin{tabular}{|c|c|c|c|}
\hline 0.152 & 0.0 & 90 & 0.5 \\
\hline 0.252 & 0.0 & 90 & 0.3 \\
\hline 0.452 & 0.0 & 90 & 0.2 \\
\hline 1.000 & 0.0 & 90 & 0.1 \\
\hline 0.203 & 0.0 & 60 & 0.5 \\
\hline 0.203 & -22.5 & 60 & 0.8 \\
\hline 0.203 & -45.0 & 60 & 0.6 \\
\hline 0.203 & -112.5 & 60 & 0.4 \\
\hline 0.203 & 157.5 & 60 & 1.0 \\
\hline 0.203 & 135.0 & 60 & 0.7 \\
\hline 0.203 & 112.5 & 60 & 0.4 \\
\hline 0.203 & 90.0 & 60 & 0.3 \\
\hline 0.303 & -22.5 & 60 & 0.4 \\
\hline 0.303 & -45.0 & 60 & 0.5 \\
\hline 0.303 & -112.5 & 60 & 0.3 \\
\hline 0.303 & 180.0 & 60 & 0.8 \\
\hline 0.303 & 112.5 & 60 & 0.4 \\
\hline 0.503 & 0.0 & 60 & 0.2 \\
\hline 0.503 & -22.5 & 60 & 0.2 \\
\hline 0.503 & 45.0 & 60 & 0.2 \\
\hline 0.503 & 157.5 & 60 & 0.1 \\
\hline 0.503 & 135.0 & 60 & 0.1 \\
\hline 0.503 & 112.5 & 60 & 0.1 \\
\hline 1.000 & -22.5 & 60 & 6.4 \\
\hline 1.000 & -45.0 & 60 & 6.4 \\
\hline 1.000 & -67.5 & 60 & 6.5 \\
\hline 1.000 & -90.0 & 60 & 6.5 \\
\hline 1.000 & -112.5 & 60 & 6.4 \\
\hline 1.000 & -135.0 & 60 & 6.5 \\
\hline 1.000 & -157.5 & 60 & 6.5 \\
\hline
\end{tabular}

Table C.13 Laser Printer

$60 \mathrm{~Hz}$ RMS Resultant Fields

Distance Horizontal Vertical $60 \mathrm{~Hz}$ meters angle deg angle deg mGauss

\begin{tabular}{|r|r|r|r|}
\hline 1.000 & 180.0 & 60 & 6.5 \\
\hline 1.000 & 157.5 & 60 & 6.4 \\
\hline 1.000 & 135.0 & 60 & 6.4 \\
\hline 1.000 & 112.5 & 60 & 6.4 \\
\hline 1.000 & 90.0 & 60 & 6.4 \\
\hline 1.000 & 67.5 & 60 & 6.5 \\
\hline 1.000 & 45.0 & 60 & 6.5 \\
\hline 1.000 & 22.5 & 60 & 6.5 \\
\hline 0.203 & -22.5 & 30 & 0.6 \\
\hline 0.203 & -45.0 & 30 & 1.2 \\
\hline 0.203 & -112.5 & 30 & 0.3 \\
\hline 0.203 & 157.5 & 30 & 0.3 \\
\hline 0.203 & 135.0 & 30 & 0.3 \\
\hline 0.203 & 112.5 & 30 & 0.2 \\
\hline 0.203 & 90.0 & 30 & 0.2 \\
\hline 0.303 & 0.0 & 30 & 0.5 \\
\hline 0.303 & -22.5 & 30 & 0.5 \\
\hline 0.303 & -45.0 & 30 & .0 .7 \\
\hline 0.303 & -112.5 & 30 & 0.4 \\
\hline 0.303 & -135.0 & 30 & 0.5 \\
\hline 0.303 & 180.0 & 30 & 0.5 \\
\hline 0.303 & 157.5 & 30 & 0.4 \\
\hline 0.303 & 135.0 & 30 & 0.4 \\
\hline 0.303 & 112.5 & 30 & 0.4 \\
\hline 0.303 & 90.0 & 30 & 0.4 \\
\hline 0.503 & 0.0 & 30 & 0.4 \\
\hline 0.503 & -22.5 & 30 & 0.3 \\
\hline 0.503 & -67.5 & 30 & 0.4 \\
\hline 0.503 & -90.0 & 30 & 0.3 \\
\hline 0.503 & -112.5 & 30 & 0.3 \\
\hline
\end{tabular}


Table C.13 Laser Printer

Distance Horizontal Vertical $60 \mathrm{~Hz}$ meters angle deg angle deg mGauss

\begin{tabular}{|c|c|c|c|}
\hline 0.503 & -135.0 & 30 & 0.4 \\
\hline 0.503 & -157.5 & 30 & 0.4 \\
\hline 0.503 & 180.0 & 30 & 0.4 \\
\hline 0.503 & 157.5 & 30 & 0.3 \\
\hline 0.503 & 135.0 & 30 & 0.3 \\
\hline 0.503 & 112.5 & 30 & 0.3 \\
\hline 0.503 & 90.0 & 30 & 0.3 \\
\hline 0.503 & 67.5 & 30 & 0.3 \\
\hline 0.503 & 45.0 & 30 & 0.4 \\
\hline 0.503 & 22.5 & 30 & 0.4 \\
\hline 1.203 & 0.0 & 30 & 0.0 \\
\hline 1.203 & -22.5 & 30 & 0.0 \\
\hline 1.203 & -45.0 & 30 & 0.0 \\
\hline 1.203 & -67.5 & 30 & 0.0 \\
\hline 1.203 & -90.0 & 30 & 0.0 \\
\hline 1.203 & -135.0 & 30 & 0.0 \\
\hline 1.203 & 157.5 & 30 & 0.0 \\
\hline 1.203 & 112.5 & 30 & 0.1 \\
\hline 1.203 & 45.0 & 30 & 0.1 \\
\hline 1.203 & 22.5 & 30 & 0.0 \\
\hline 0.178 & 0.0 & 0 & 0.5 \\
\hline 0.178 & -22.5 & 0 & 1.0 \\
\hline 0.178 & -45.0 & 0 & 1.5 \\
\hline 0.178 & -90.0 & 0 & 0.5 \\
\hline 0.178 & -112.5 & 0 & 0.4 \\
\hline 0.178 & 180.0 & 0 & 0.5 \\
\hline 0.178 & 157.5 & 0 & 0.4 \\
\hline 0.178 & 135.0 & 0 & 0.4 \\
\hline 0.178 & 112.5 & 0 & 0.4 \\
\hline 0.178 & 90.0 & 0 & 0.4 \\
\hline 0.5 & & & 30 \\
\hline
\end{tabular}

$60 \mathrm{~Hz}$ RMS Resultant Fields

Distance Horizontal Vertical $60 \mathrm{~Hz}$

meters angle deg angle deg mGauss

\begin{tabular}{|c|c|c|c|}
\hline 0.278 & 0.0 & 0 & 0.4 \\
\hline 0.278 & -22.5 & 0 & 0.5 \\
\hline 0.278 & -45.0 & 0 & 0.6 \\
\hline 0.278 & .90 .0 & 0 & 0.4 \\
\hline 0.278 & -112.5 & 0 & 0.4 \\
\hline 0.278 & -157.5 & 0 & 0.4 \\
\hline 0.278 & 180.0 & 0 & 0.5 \\
\hline 0.278 & 157.5 & 0 & 0.5 \\
\hline 0.278 & 135.0 & 0 & 0.5 \\
\hline 0.278 & 112.5 & 0 & 0.5 \\
\hline 0.278 & 90.0 & 0 & 0.4 \\
\hline 0.478 & -22.5 & 0 & 0.1 \\
\hline 0.478 & -45.0 & 0 & 0.2 \\
\hline 0.478 & -90.0 & 0 & 0.2 \\
\hline 0.478 & -112.5 & 0 & 0.1 \\
\hline 0.478 & -135.0 & 0 & 0.2 \\
\hline 0.478 & -157.5 & 0 & 0.2 \\
\hline 0.478 & 157.5 & 0 & 0.2 \\
\hline 0.478 & 112.5 & 0 & 0.1 \\
\hline 0.279 & 0.0 & -30 & 0.8 \\
\hline 0.279 & -45.0 & -30 & 2.2 \\
\hline 0.279 & .90 .0 & -30 & 0.7 \\
\hline 0.279 & -112.5 & .30 & 0.5 \\
\hline 0.279 & -157.5 & .30 & 0.6 \\
\hline 0.279 & 180.0 & .30 & 0.7 \\
\hline 0.279 & 112.5 & -30 & 0.5 \\
\hline 0.379 & 0.0 & -30 & 0.4 \\
\hline 0.379 & -22.5 & .30 & 0.5 \\
\hline 0.379 & -45.0 & -30 & 0.7 \\
\hline 0.379 & -67.5 & -30 & 0.8 \\
\hline
\end{tabular}


Distance Horizontal Vertical $60 \mathrm{~Hz}$ meters angle deg angle deg mGauss

\begin{tabular}{|l|r|r|r|}
\hline 0.379 & -90.0 & -30 & 0.4 \\
\hline 0.379 & -112.5 & -30 & 0.3 \\
\hline 0.379 & -157.5 & -30 & 0.3 \\
\hline 0.379 & 180.0 & -30 & 0.4 \\
\hline 0.379 & 157.5 & -30 & 0.4 \\
\hline 0.379 & 135.0 & -30 & 0.4 \\
\hline 0.379 & 112.5 & -30 & 0.4 \\
\hline 0.379 & 90.0 & -30 & 0.4 \\
\hline 0.579 & 0.0 & -30 & 0.2 \\
\hline 0.579 & -22.5 & -30 & 0.1 \\
\hline 0.579 & -45.0 & -30 & 0.2 \\
\hline 0.579 & -67.5 & -30 & 0.2 \\
\hline 0.579 & -90.0 & -30 & 0.2 \\
\hline 0.579 & -135.0 & -30 & 0.2 \\
\hline 0.579 & -157.5 & -30 & 0.1 \\
\hline 0.579 & 180.0 & -30 & 0.3 \\
\hline 0.579 & 112.5 & -30 & 0.2 \\
\hline 0.305 & 0.0 & -60 & 0.7 \\
\hline 0.305 & -22.5 & -60 & 0.6 \\
\hline 0.305 & -45.0 & -60 & 0.8 \\
\hline 0.305 & -57.5 & -60 & 0.7 \\
\hline 0.305 & -90.0 & -60 & 0.7 \\
\hline 0.305 & -112.5 & -60 & 0.5 \\
\hline 0.305 & -135.0 & -60 & 0.6 \\
\hline 0.305 & -157.5 & -60 & 0.5 \\
\hline 0.305 & 180.0 & -60 & 0.7 \\
\hline 0.305 & 157.5 & -60 & 0.8 \\
\hline 0.305 & 135.0 & -60 & 0.7 \\
\hline 0.305 & 112.5 & -60 & 0.7 \\
\hline
\end{tabular}

Table C.13 Laser Printer $60 \mathrm{~Hz}$ RMS Resultant Fields

Distance Horizontal Vertical $60 \mathrm{~Hz}$ meters angle deg angle deg mGauss

\begin{tabular}{|c|c|c|c|}
\hline 0.305 & 90.0 & -60 & 0.7 \\
\hline 0.405 & 0.0 & .60 & 0.3 \\
\hline 0.405 & -22.5 & -60 & 0.3 \\
\hline 0.405 & -45.0 & -60 & 0.4 \\
\hline 0.405 & -67.5 & -60 & 0.3 \\
\hline 0.405 & 90.0 & -60 & 0.3 \\
\hline 0.405 & -112.5 & -60 & 0.3 \\
\hline 0.405 & -135.0 & -60 & 0.4 \\
\hline 0.405 & -157.5 & .60 & 0.3 \\
\hline 0.405 & 180.0 & -60 & 0.3 \\
\hline 0.405 & 157.5 & -60 & 0.4 \\
\hline 0.405 & 135.0 & .60 & 0.3 \\
\hline 0.405 & 112.5 & .60 & 0.4 \\
\hline 0.405 & 90.0 & -60 & 0.4 \\
\hline 0.605 & 0.0 & -60 & 0.2 \\
\hline 0.605 & -22.5 & -60 & 0.2 \\
\hline 0.605 & .90 .0 & -60 & 0.2 \\
\hline 0.605 & -112.5 & -60 & 0.2 \\
\hline 0.605 & -135.0 & -60 & 0.3 \\
\hline 0.605 & -157.5 & -60 & 0.2 \\
\hline 0.605 & 180.0 & -60 & 0.2 \\
\hline 0.605 & 157.5 & -60 & 0.2 \\
\hline 0.605 & 1355.0 & -60 & 0.2 \\
\hline 0.605 & 90.0 & -60 & 0.2 \\
\hline 0.605 & 67.5 & .60 & 0.2 \\
\hline 0.605 & 45.0 & -60 & 0.2 \\
\hline 0.152 & -22.5 & .90 & 0.4 \\
\hline 0.252 & 0.0 & .90 & 0.4 \\
\hline
\end{tabular}




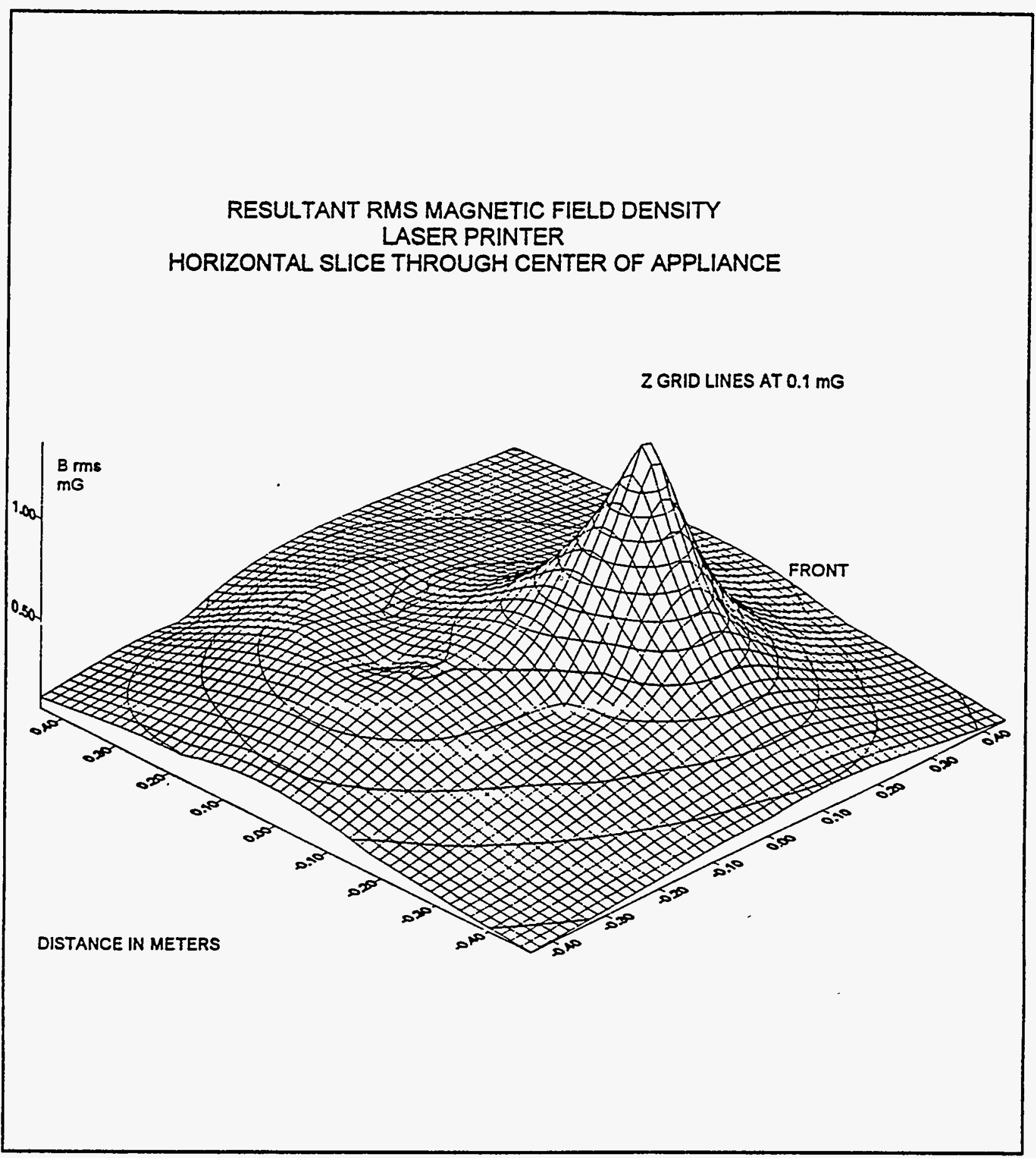

Figure C.13.1 Fundamental Resultant RMS Magnetic Field Density - Laser Printer.

C.13-5 


\section{C.14 Saw - Skil Hand}

Appliance Tested: A portable circular saw for cutting wood.

Manufacturer: NNN

Model: 013

Rated current: 13 Amperes

Appliance Code: 3278

Conditions for Testing: The unit was not loaded.

Measured voltage and current: 115.1 Volts and 4.94 Amperes.

Magnetic Field Summary:

$60 \mathrm{~Hz}$ RMS resultant level at $30 \mathrm{~cm}$ from front surface: $13.2 \mathrm{mG}$

RMS resultant low surface level: $253 \mathrm{mG}$

RMS resultant high surface level: $3222 \mathrm{mG}$ in back

Highest recorded surface levels:

60 Hertz RMS resultant: $2560 \mathrm{mG}$

180 Hertz RMS resultant: $1826 \mathrm{mG}$

300 Hertz RMS resultant: $650 \mathrm{mG}$

420 Hertz RMS resultant: $99.7 \mathrm{mG}$

For the appliance test set, the motor of the unit was located at 0 degrees on the test table. The saw was laid on its back with the blade in a vertical position and the front of the saw toward the top of the test set.

Summary of characteristics: The magnetic fields were rich in harmonics and were relatively strong. 
Table C.14 Saw - Skil Hand

Distance Horizontal Vertical $60 \mathrm{~Hz}$ meters angle deg angle deg mGauss

\begin{tabular}{|r|r|r|r|}
\hline 0.140 & 0.0 & 90 & 474.3 \\
\hline 0.240 & 0.0 & 90 & 115.0 \\
\hline 0.440 & 0.0 & 90 & 19.2 \\
\hline 1.000 & 0.0 & 90 & 1.2 \\
\hline 0.114 & 0.0 & 60 & 699.7 \\
\hline 0.114 & -22.5 & 60 & 670.5 \\
\hline 0.114 & -45.0 & 60 & 549.2 \\
\hline 0.114 & -67.5 & 60 & 415.5 \\
\hline 0.114 & -90.0 & 60 & 296.8 \\
\hline 0.114 & -112.5 & 60 & 226.1 \\
\hline 0.114 & -135.0 & 60 & 186.8 \\
\hline 0.114 & -157.5 & 60 & 179.4 \\
\hline 0.114 & 180.0 & 60 & 184.9 \\
\hline 0.114 & 157.5 & 60 & 208.7 \\
\hline 0.114 & 135.0 & 60 & 245.2 \\
\hline 0.114 & 112.5 & 60 & 296.3 \\
\hline 0.114 & 90.0 & 60 & 362.9 \\
\hline 0.114 & 67.5 & 60 & 448.8 \\
\hline 0.114 & 45.0 & 60 & 540.9 \\
\hline 0.114 & 22.5 & 60 & 630.9 \\
\hline 0.214 & 0.0 & 60 & 135.6 \\
\hline 0.214 & -22.5 & 60 & 122.9 \\
\hline 0.214 & -45.0 & 60 & 107.1 \\
\hline 0.214 & -67.5 & 60 & 92.6 \\
\hline 0.214 & -90.0 & 60 & 79.2 \\
\hline 0.214 & -112.5 & 60 & 68.8 \\
\hline 0.214 & -135.0 & 60 & 61.6 \\
\hline 0.214 & -157.5 & 60 & 61.3 \\
\hline 0.214 & 180.0 & 60 & 64.3 \\
\hline 0.214 & 157.5 & 60 & 72.1 \\
\hline
\end{tabular}

$60 \mathrm{~Hz}$ RMS Resultant Fields

Distance Horizontal Vertical $60 \mathrm{~Hz}$

meters angle deg angle deg mGauss

\begin{tabular}{|c|c|c|c|}
\hline 0.214 & 135.0 & 60 & 82.8 \\
\hline 0.214 & 112.5 & 60 & 95.0 \\
\hline 0.214 & 90.0 & 60 & 108.0 \\
\hline 0.214 & 67.5 & 60 & 121.9 \\
\hline 0.214 & 45.0 & 60 & 130.8 \\
\hline 0.214 & 22.5 & 60 & 135.5 \\
\hline 0.414 & 0.0 & 60 & 22.1 \\
\hline 0.414 & -22.5 & 60 & 19.8 \\
\hline 0.414 & -45.0 & 60 & 17.4 \\
\hline 0.414 & -67.5 & 60 & 15.8 \\
\hline 0.414 & -90.0 & 60 & 14.6 \\
\hline 0.414 & -112.5 & 60 & 13.8 \\
\hline 0.414 & -135.0 & 60 & 13.2 \\
\hline 0.414 & -157.5 & 60 & 13.7 \\
\hline 0.414 & 180.0 & 60 & 14.6 \\
\hline 0.414 & 157.5 & 60 & 16.5 \\
\hline 0.414 & 135.0 & 60 & 18.6 \\
\hline 0.414 & 112.5 & 60 & 20.7 \\
\hline 0.414 & 90.0 & 60 & 22.3 \\
\hline 0.414 & 67.5 & 60 & 23.3 \\
\hline 0.414 & 45.0 & 60 & 23.5 \\
\hline 0.414 & 22.5 & 60 & 23.1 \\
\hline 1.000 & 0.0 & 60 & 1.3 \\
\hline 1.000 & -22.5 & 60 & 1.3 \\
\hline 1.000 & -45.0 & 60 & 1.3 \\
\hline 1.000 & -67.5 & 60 & 1.2 \\
\hline 1.000 & -90.0 & 60 & 1.1 \\
\hline 1.000 & -112.5 & 60 & 1.0 \\
\hline 1.000 & -135.0 & 60 & 1.1 \\
\hline 1.000 & -157.5 & 60 & 1.2 \\
\hline
\end{tabular}


Distance Horizontal Vertical $60 \mathrm{~Hz}$ meters angle deg angle deg mGauss

\begin{tabular}{|r|r|r|r|}
\hline 1.140 & -90.0 & 30 & 1.1 \\
\hline 1.140 & -112.5 & 30 & 1.0 \\
\hline 1.140 & -135.0 & 30 & 0.8 \\
\hline 1.140 & -157.5 & 30 & 0.8 \\
\hline 1.140 & 180.0 & 30 & 1.0 \\
\hline 1.140 & 157.5 & 30 & 1.1 \\
\hline 1.140 & 135.0 & 30 & 1.2 \\
\hline 1.140 & 112.5 & 30 & 1.3 \\
\hline 1.140 & 90.0 & 30 & 1.3 \\
\hline 1.140 & 67.5 & 30 & 1.4 \\
\hline 1.140 & 45.0 & 30 & 1.4 \\
\hline 1.140 & 22.5 & 30 & 1.5 \\
\hline 0.089 & 0.0 & 0 & 1014.5 \\
\hline 0.089 & -22.5 & 0 & 1053.8 \\
\hline 0.089 & -45.0 & 0 & 1096.3 \\
\hline 0.089 & -67.5 & 0 & 827.2 \\
\hline 0.089 & -90.0 & 0 & 394.4 \\
\hline 0.089 & -112.5 & 0 & 198.5 \\
\hline 0.089 & -135.0 & 0 & 124.7 \\
\hline 0.089 & -157.5 & 0 & 95.8 \\
\hline 0.089 & 180.0 & 0 & 92.0 \\
\hline 0.089 & 157.5 & 0 & 102.3 \\
\hline 0.089 & 135.0 & 0 & 126.8 \\
\hline 0.089 & 112.5 & 0 & 159.2 \\
\hline 0.089 & 90.0 & 0 & 204.8 \\
\hline 0.089 & 67.5 & 0 & 255.6 \\
\hline 0.089 & 45.0 & 0 & 345.7 \\
\hline 0.089 & 22.5 & 0 & 433.7 \\
\hline 0.189 & 0.0 & 0 & 132.3 \\
\hline 0.22 .5 & 0 & 158.8 \\
\hline 1.199 & & \\
\hline
\end{tabular}

Table C.14 Saw - Skil Hand $60 \mathrm{~Hz}$ RMS Resultant Fields

Distance Horizontal Vertical $60 \mathrm{~Hz}$

meters angle deg angle deg mGauss

\begin{tabular}{|c|c|c|c|}
\hline 0.189 & -45.0 & 0 & 191.5 \\
\hline 0.189 & -67.5 & 0 & 177.7 \\
\hline 0.189 & .90 .0 & 0 & 124.8 \\
\hline 0.189 & -112.5 & 0 & 71.4 \\
\hline 0.189 & -135.0 & 0 & 46.9 \\
\hline 0.189 & -157.5 & 0 & 35.9 \\
\hline 0.189 & 180.0 & 0 & 32.4 \\
\hline 0.189 & 157.5 & 0 & 35.7 \\
\hline 0.189 & 135.0 & 0 & 43.1 \\
\hline 0.189 & 112.5 & 0 & 53.0 \\
\hline 0.189 & 90.0 & 0 & 63.9 \\
\hline 0.189 & 67.5 & 0 & 75.5 \\
\hline 0.189 & 45.0 & 0 & 89.4 \\
\hline 0.189 & 22.5 & 0 & 95.7 \\
\hline 0.389 & 0.0 & 0 & 18.5 \\
\hline 0.389 & -22.5 & 0 & 21.3 \\
\hline 0.389 & -45.0 & 0 & 26.4 \\
\hline 0.389 & -67.5 & 0 & 27.7 \\
\hline 0.389 & .90 .0 & 0 & 23.9 \\
\hline 0.389 & -112.5 & 0 & 16.6 \\
\hline 0.389 & -135.0 & 0 & 12.0 \\
\hline 0.389 & -157.5 & 0 & 9.2 \\
\hline 0.389 & 180.0 & 0 & 8.1 \\
\hline 0.389 & 157.5 & 0 & 8.9 \\
\hline 0.389 & 135.0 & 0 & 10.8 \\
\hline 0.389 & 112.5 & 0 & 12.9 \\
\hline 0.389 & 90.0 & 0 & 15.0 \\
\hline 0.389 & 67.5 & 0 & 16.3 \\
\hline 0.389 & 45.0 & 0 & 17.2 \\
\hline 0.389 & 22.5 & 0 & 16.5 \\
\hline
\end{tabular}


Distance Horizontal Vertical $60 \mathrm{~Hz}$ meters angle deg angle deg mGauss

\begin{tabular}{|c|c|c|c|}
\hline 1.089 & 0.0 & 0 & 0.9 \\
\hline 1.089 & -22.5 & 0 & 1.0 \\
\hline 1.089 & -45.0 & 0 & 1.2 \\
\hline 1.089 & -67.5 & 0 & 1.3 \\
\hline 1.089 & -90.0 & 0 & 1.3 \\
\hline 1.089 & -112.5 & 0 & 1.2 \\
\hline 1.089 & -135.0 & 0 & 1.0 \\
\hline 1.089 & -157.5 & 0 & 0.8 \\
\hline 1.089 & 180.0 & 0 & 0.7 \\
\hline 1.089 & 157.5 & 0 & 0.7 \\
\hline 1.089 & 135.0 & 0 & 1.0 \\
\hline 1.089 & 112.5 & 0 & 1.1 \\
\hline 1.089 & 90.0 & 0 & 1.2 \\
\hline 1.089 & 67.5 & 0 & 1.2 \\
\hline 1.089 & 45.0 & 0 & 1.2 \\
\hline 1.089 & 22.5 & 0 & 1.1 \\
\hline 0.267 & 0.0 & -30 & 65.0 \\
\hline 0.267 & -22.5 & -30 & 86.5 \\
\hline 0.267 & -45.0 & -30 & 100.9 \\
\hline 0.267 & -67.5 & -30 & 97.6 \\
\hline 0.267 & -90.0 & -30 & 81.4 \\
\hline 0.267 & -112.5 & -30 & 60.5 \\
\hline 0.267 & -135.0 & -30 & 43.0 \\
\hline 0.267 & -157.5 & -30 & 32.2 \\
\hline 0.267 & 180.0 & -30 & 26.3 \\
\hline 0.267 & 157.5 & -30 & 24.2 \\
\hline 0.267 & 135.0 & -30 & 25.4 \\
\hline 0.267 & 112.5 & -30 & 28.8 \\
\hline 0.267 & 90.0 & -30 & 32.7 \\
\hline 0.267 & 67.5 & -30 & 37.2 \\
\hline & & & \\
\hline 1.25 & 0 & 0 & 0 \\
\hline
\end{tabular}

Table C.14 Saw - Skil Hand

\section{$60 \mathrm{~Hz}$ RMS Resultant Fields}

Distance Horizontal Vertical $60 \mathrm{~Hz}$ meters angle deg angle deg mGauss

\begin{tabular}{|l|r|r|r|}
\hline 0.267 & 45.0 & -30 & 42.5 \\
\hline 0.267 & 22.5 & -30 & 49.9 \\
\hline 0.367 & 0.0 & -30 & 25.5 \\
\hline 0.367 & -22.5 & -30 & 33.6 \\
\hline 0.367 & -45.0 & -30 & 40.1 \\
\hline 0.367 & -67.5 & -30 & 40.7 \\
\hline 0.367 & -90.0 & -30 & 35.8 \\
\hline 0.367 & -112.5 & -30 & 28.2 \\
\hline 0.367 & -135.0 & -30 & 20.8 \\
\hline 0.367 & -157.5 & -30 & 15.6 \\
\hline 0.367 & 180.0 & -30 & 12.8 \\
\hline 0.367 & 157.5 & -30 & 11.4 \\
\hline 0.367 & 135.0 & -30 & 12.0 \\
\hline 0.367 & 112.5 & -30 & 13.5 \\
\hline 0.367 & 90.0 & -30 & 15.1 \\
\hline 0.367 & 67.5 & -30 & 16.6 \\
\hline 0.367 & 45.0 & -30 & 18.0 \\
\hline 0.367 & 22.5 & -30 & 20.0 \\
\hline 0.567 & 0.0 & -30 & 7.6 \\
\hline 0.567 & -22.5 & -30 & 10.0 \\
\hline 0.567 & -45.0 & -30 & 12.0 \\
\hline 0.567 & -67.5 & -30 & 12.6 \\
\hline 0.567 & -90.0 & -30 & 11.8 \\
\hline 0.567 & -112.5 & -30 & 9.9 \\
\hline 0.567 & -135.0 & -30 & 7.7 \\
\hline 0.567 & -157.5 & -30 & 5.8 \\
\hline 0.567 & 180.0 & -30 & 4.6 \\
\hline 0.567 & 157.5 & -30 & 4.1 \\
\hline 0.567 & 135.0 & -30 & 4.2 \\
\hline 0.567 & 112.5 & -30 & 4.7 \\
\hline
\end{tabular}

C. 14-5 
Distance Horizontal Vertical $60 \mathrm{~Hz}$ meters angle deg angle deg mGauss

\begin{tabular}{|r|r|r|r|}
\hline 0.567 & 90.0 & -30 & 5.2 \\
\hline 0.567 & 67.5 & -30 & 5.4 \\
\hline 0.567 & 45.0 & -30 & 5.7 \\
\hline 0.567 & 22.5 & -30 & 6.0 \\
\hline 1.267 & 0.0 & -30 & 0.7 \\
\hline 1.267 & -22.5 & -30 & 0.8 \\
\hline 1.267 & -45.0 & -30 & 1.0 \\
\hline 1.267 & -67.5 & -30 & 1.1 \\
\hline 1.267 & -90.0 & -30 & 1.0 \\
\hline 1.267 & -112.5 & -30 & 1.0 \\
\hline 1.267 & -135.0 & -30 & 0.9 \\
\hline 1.267 & -157.5 & -30 & 0.8 \\
\hline 1.267 & 180.0 & -30 & 0.6 \\
\hline 1.267 & 157.5 & -30 & 0.5 \\
\hline 1.267 & 135.0 & -30 & 0.5 \\
\hline 1.267 & 112.5 & -30 & 0.6 \\
\hline 1.267 & 90.0 & -30 & 0.6 \\
\hline 1.267 & 67.5 & -30 & 0.7 \\
\hline 1.267 & 45.0 & -30 & 0.6 \\
\hline 1.267 & 22.5 & -30 & 0.6 \\
\hline 0.343 & 0.0 & -60 & 41.4 \\
\hline 0.343 & -22.5 & -60 & 48.5 \\
\hline 0.343 & -45.0 & -60 & 51.9 \\
\hline 0.343 & -67.5 & -60 & 52.1 \\
\hline 0.343 & -90.0 & -60 & 48.2 \\
\hline 0.343 & -112.5 & -60 & 41.0 \\
\hline 0.343 & -135.0 & -60 & 33.8 \\
\hline 0.343 & -157.5 & -60 & 27.1 \\
\hline 0.343 & 180.0 & -60 & 23.7 \\
\hline 0.343 & 157.5 & -60 & 21.3 \\
\hline
\end{tabular}

Table C.14 Saw - Skil Hand

\section{$60 \mathrm{~Hz}$ RMS Resultant Fields}

Distance Horizontal Vertical $60 \mathrm{~Hz}$

meters angie deg angle deg mGauss

\begin{tabular}{|c|c|c|c|}
\hline 0.343 & 135.0 & -60 & 20.4 \\
\hline 0.343 & 112.5 & -60 & 20.3 \\
\hline 0.343 & 90.0 & -60 & 21.5 \\
\hline 0.343 & 67.5 & -60 & 36.2 \\
\hline 0.343 & 45.0 & -60 & 27.4 \\
\hline 0.343 & 22.5 & -60 & 33.6 \\
\hline 0.443 & 0.0 & -60 & 19.0 \\
\hline 0.443 & -22.5 & -60 & 22.3 \\
\hline 0.443 & -45.0 & -60 & 24.0 \\
\hline 0.443 & -67.5 & -60 & 24.5 \\
\hline 0.443 & .90 .0 & -60 & 23.2 \\
\hline 0.443 & -112.5 & -60 & 20.3 \\
\hline 0.443 & -135.0 & .60 & 17.2 \\
\hline 0.443 & -157.5 & -60 & 14.1 \\
\hline 0.443 & 180.0 & -60 & 12.4 \\
\hline 0.443 & 157.5 & -60 & 11.1 \\
\hline 0.443 & 135.0 & -60 & 10.5 \\
\hline 0.443 & 112.5 & -60 & 10.2 \\
\hline 0.443 & 90.0 & -60 & 10.7 \\
\hline 0.443 & 67.5 & -60 & 11.4 \\
\hline 0.443 & 45.0 & -60 & 13.2 \\
\hline 0.443 & 22.5 & -60 & 15.8 \\
\hline 0.643 & 0.0 & -60 & 6.5 \\
\hline 0.643 & -22.5 & -60 & 7.4 \\
\hline 0.643 & -45.0 & -60 & 8.0 \\
\hline 0.643 & -67.5 & -60 & 8.3 \\
\hline 0.643 & -90.0 & -60 & 8.0 \\
\hline 0.643 & -112.5 & -60 & 7.3 \\
\hline 0.643 & -1350 & -60 & 6.4 \\
\hline 0.643 & -157.5 & -60 & 5.4 \\
\hline
\end{tabular}


Table C.14 Saw - Skil Hand

$60 \mathrm{~Hz}$ RMS Resultant Fields

Distance Horizontal Vertical $60 \mathrm{~Hz}$ meters angle deg angle deg mGauss

\begin{tabular}{|l|r|r|r|}
\hline 0.643 & 180.0 & -60 & 4.8 \\
\hline 0.643 & 157.5 & -60 & 4.2 \\
\hline 0.643 & 135.0 & -60 & 4.0 \\
\hline 0.643 & 112.5 & -60 & 3.8 \\
\hline 0.643 & 90.0 & -60 & 4.0 \\
\hline 0.643 & 67.5 & -60 & 4.2 \\
\hline 0.643 & 45.0 & -60 & 4.6 \\
\hline 0.643 & 22.5 & -60 & 5.4 \\
\hline 1.000 & 0.0 & -60 & 1.0 \\
\hline 1.000 & -22.5 & -60 & 1.0 \\
\hline 1.000 & -45.0 & -60 & 1.0 \\
\hline 1.000 & -67.5 & -60 & 1.1 \\
\hline 1.000 & -90.0 & -60 & 1.2 \\
\hline 1.000 & -112.5 & -60 & 1.1 \\
\hline
\end{tabular}

Distance Horizontal Vertical $60 \mathrm{~Hz}$

meters angle deg angle deg mGauss

\begin{tabular}{|r|r|r|r|}
\hline 1.000 & -135.0 & -60 & 1.1 \\
\hline 1.000 & -157.5 & -60 & 1.1 \\
\hline 1.000 & 180.0 & -60 & 1.0 \\
\hline 1.000 & 157.5 & $-\infty$ & 0.9 \\
\hline 1.000 & 135.0 & -60 & 0.8 \\
\hline 1.000 & 112.5 & -60 & 0.8 \\
\hline 1.000 & 90.0 & -60 & 0.8 \\
\hline 1.000 & 67.5 & -60 & 0.8 \\
\hline 1.000 & 45.0 & -60 & 1.2 \\
\hline 1.000 & 22.5 & -60 & 1.2 \\
\hline 0.191 & 0.0 & -90 & 206.9 \\
\hline 0.291 & 0.0 & -90 & 66.1 \\
\hline 0.491 & 0.0 & -90 & 14.9 \\
\hline & & & \\
\hline
\end{tabular}




\section{RESULTANT RMS MAGNETIC FIELD DENSITY SKIL SAW \\ HORIZONTAL SLICE THROUGH CENTER OF APPLIANCE}

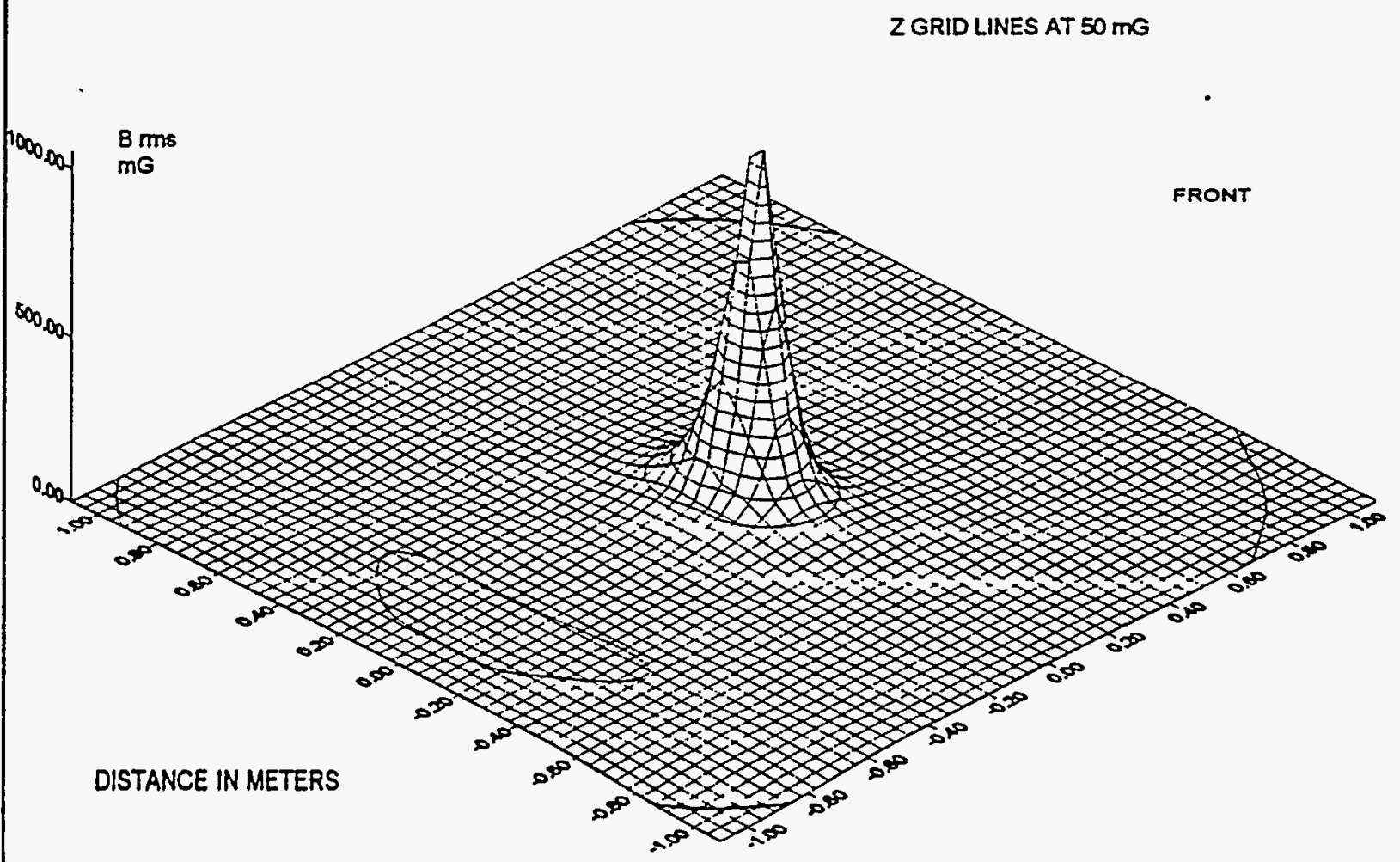

Figure C.14.1 Fundamental Resultant RMS Magnetic Field Density - Skil Saw.

\section{C.14-8}




\section{RESULTANT RMS MAGNETIC FIELD DENSITY 3KHZ-30KHZ SKIL SAW \\ HORIZONTAL SLICE THROUGH CENTER OF APPLIANCE}

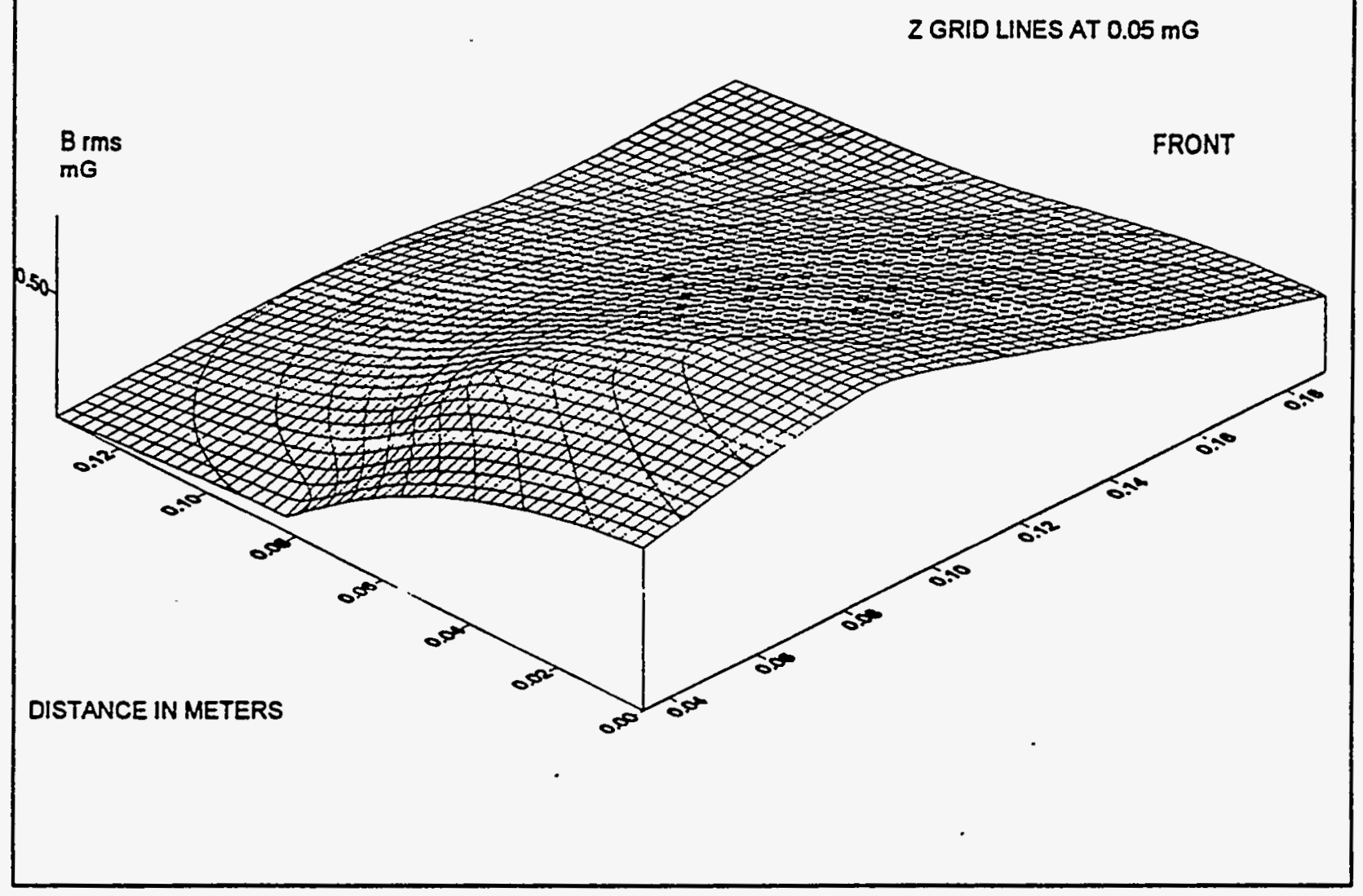

Figure C.14.2 Resultant RMS Magnetic Field Density $3 \mathrm{kHz}-30 \mathrm{kHz}$ - Skil Saw. 


\section{C.15 Shaver - AC}

Appliance Tested: A shaver used for men having an ac cord. Manufacturer: 000

Appliance Code: 3263

Conditions for Testing: The unit was running with no load.

Measured voltage and current: 120.8 Volts and 0.15 Amperes.

Magnetic Field Summary:

$60 \mathrm{~Hz}$ RMS resultant level at $30 \mathrm{~cm}$ from front surface: $23.9 \mathrm{mG}$ RMS resultant low surface level: $940 \mathrm{mG}$ right side

RMS resultant high surface level: $11,777 \mathrm{mG}$ front

Highest recorded surface levels:

60 Hertz RMS resultant: $11,471 \mathrm{mG}$

180 Hertz RMS resultant: $2637 \mathrm{mG}$

300 Hertz RMS resultant: $348 \mathrm{mG}$

420 Hertz RMS resultant: $199 \mathrm{mG}$

Maximum Total Harmonic Distortion: 34 percent

For the appliance test set, the front of the unit was located at 0 degrees on the test table. The unit was laid on the table.

Summary of characteristics: The magnetic fields were rich in harmonics and were relatively strong. 
Table C.15 Shaver-AC $60 \mathrm{~Hz}$ RMS Resultant Fields

Distance Horizontal Vertical $60 \mathrm{~Hz}$ meters angle deg angle deg mGauss

\begin{tabular}{|c|c|c|c|}
\hline 0.025 & 0.0 & 90 & 5087.7 \\
\hline 0.125 & 0.0 & 90 & 234.0 \\
\hline 0.325 & 0.0 & 90 & 17.2 \\
\hline 1.000 & 0.0 & 90 & 0.9 \\
\hline 0.051 & 0.0 & 60 & 1957.1 \\
\hline 0.051 & -22.5 & 60 & 1985.3 \\
\hline 0.051 & -45.0 & 60 & 1844.3 \\
\hline 0.051 & -67.5 & 60 & 1587.9 \\
\hline 0.051 & -90.0 & 60 & 1646.9 \\
\hline 0.051 & -112.5 & 60 & 1781.7 \\
\hline 0.051 & -135.0 & 60 & 2382.8 \\
\hline 0.051 & -157.5 & 60 & 2474.4 \\
\hline 0.051 & 180.0 & 60 & 2561.1 \\
\hline 0.051 & 157.5 & 60 & 2357.0 \\
\hline 0.051 & 135.0 & 60 & 1952.7 \\
\hline 0.051 & 112.5 & 60 & 1652.3 \\
\hline 0.051 & 90.0 & 60 & 1532.8 \\
\hline 0.051 & 67.5 & 60 & 1621.2 \\
\hline 0.051 & 45.0 & 60 & 1895.8 \\
\hline 0.051 & 22.5 & 60 & 2045.4 \\
\hline 0.151 & 0.0 & 60 & 166.6 \\
\hline 0.151 & -22.5 & 60 & 164.7 \\
\hline 0.159 & -45.0 & 60 & 152.8 \\
\hline 0.151 & -67.5 & 60 & 133.3 \\
\hline 0.151 & -90.0 & 60 & 132.5 \\
\hline 0.151 & -112.5 & 60 & 139.6 \\
\hline 0.151 & -135.0 & 60 & 166.0 \\
\hline 0.151 & -157.5 & 60 & 177.5 \\
\hline 0.151 & 180.0 & 60 & 183.5 \\
\hline 0.151 & 157.5 & 60 & 174.6 \\
\hline
\end{tabular}

Distance Horizontal Vertical $60 \mathrm{~Hz}$ meters angle deg angle deg mGauss

\begin{tabular}{|c|c|c|c|}
\hline 0.151 & 135.0 & 60 & 150.3 \\
\hline 0.151 & 112.5 & 60 & 130.2 \\
\hline 0.151 & 90.0 & 60 & 125.7 \\
\hline 0.151 & 67.5 & 60 & 131.7 \\
\hline 0.951 & 45.0 & 60 & 150.7 \\
\hline 0.151 & 22.5 & 60 & 165.0 \\
\hline 0.351 & 0.0 & 60 & 17.9 \\
\hline 0.351 & -22.5 & 60 & 17.5 \\
\hline 0.351 & -45.0 & 60 & 16.3 \\
\hline 0.351 & -67.5 & 60 & 14.3 \\
\hline 0.351 & -90.0 & 60 & 14.0 \\
\hline 0.351 & -112.5 & 60 & 14.6 \\
\hline 0.351 & -135.0 & 60 & 16.7 \\
\hline 0.351 & -157.5 & 60 & 18.0 \\
\hline 0.351 & 180.0 & 60 & 18.8 \\
\hline 0.351 & 157.5 & 60 & 18.0 \\
\hline 0.351 & 135.0 & 60 & 15.8 \\
\hline 0.351 & 112.5 & 60 & 13.8 \\
\hline 0.351 & 90.0 & 60 & 13.6 \\
\hline 0.351 & 67.5 & 60 & 14.2 \\
\hline 0.351 & 45.0 & 60 & 15.9 \\
\hline 0.351 & 22.5 & 60 & 17.5 \\
\hline 1.000 & 0.0 & 60 & 1.0 \\
\hline 1.000 & -22.5 & 60 & 1.0 \\
\hline 1.000 & -45.0 & 60 & 0.9 \\
\hline 1.000 & -67.5 & 60 & 0.8 \\
\hline 1.000 & -90.0 & 60 & 0.8 \\
\hline 1.000 & -112.5 & 60 & 0.8 \\
\hline 1.000 & -135.0 & 60 & 1.0 \\
\hline 1.000 & -157.5 & 60 & 1.1 \\
\hline
\end{tabular}


Distance Horizontal Vertical $60 \mathrm{~Hz}$ meters angle deg angle deg mGauss

\begin{tabular}{|r|r|r|r|}
\hline 1.000 & 180.0 & 60 & 1.2 \\
\hline 1.000 & 157.5 & 60 & 1.2 \\
\hline 1.000 & 135.0 & 60 & 1.0 \\
\hline 1.000 & 112.5 & 60 & 0.7 \\
\hline 1.000 & 90.0 & 60 & 0.7 \\
\hline 1.000 & 67.5 & 60 & 0.7 \\
\hline 1.000 & 45.0 & 60 & 0.9 \\
\hline 1.000 & 22.5 & 60 & 1.0 \\
\hline 0.076 & 0.0 & 30 & 1462.0 \\
\hline 0.076 & -22.5 & 30 & 1457.8 \\
\hline 0.076 & -45.0 & 30 & 1236.3 \\
\hline 0.076 & -67.5 & 30 & 869.8 \\
\hline 0.076 & -90.0 & 30 & 909.1 \\
\hline 0.076 & -112.5 & 30 & 1165.6 \\
\hline 0.076 & -135.0 & 30 & 1978.5 \\
\hline 0.076 & -157.5 & 30 & 2057.9 \\
\hline 0.076 & 180.0 & 30 & 2141.9 \\
\hline 0.076 & 157.5 & 30 & 1818.4 \\
\hline 0.076 & 135.0 & 30 & 1179.9 \\
\hline 0.076 & 112.5 & 30 & 867.4 \\
\hline 0.076 & 90.0 & 30 & 869.8 \\
\hline 0.076 & 67.5 & 30 & 1091.4 \\
\hline 0.076 & 45.0 & 30 & 1465.0 \\
\hline 0.076 & 22.5 & 30 & 1620.5 \\
\hline 0.176 & 0.0 & 30 & 157.0 \\
\hline 0.176 & -22.5 & 30 & 158.6 \\
\hline 0.176 & -45.0 & 30 & 146.2 \\
\hline 0.176 & -67.5 & 30 & 121.9 \\
\hline 0.176 & -90.0 & 30 & 121.8 \\
\hline 0.112 .5 & 30 & 132.5 \\
\hline 0.0 & & \\
\hline 0.0 & & & \\
\hline
\end{tabular}

Table C.15 Shaver-AC

\section{$60 \mathrm{~Hz}$ RMS Resultant Fields}

Distance Horizontal Vertical $60 \mathrm{~Hz}$

meters angle deg angle deg mGauss

\begin{tabular}{|r|r|r|r|}
\hline 0.176 & -135.0 & 30 & 170.8 \\
\hline 0.176 & -157.5 & 30 & 180.7 \\
\hline 0.176 & 180.0 & 30 & 187.8 \\
\hline 0.176 & 157.5 & 30 & 173.3 \\
\hline 0.176 & 135.0 & 30 & 140.5 \\
\hline 0.176 & 112.5 & 30 & 119.3 \\
\hline 0.176 & 90.0 & 30 & 114.1 \\
\hline 0.176 & 67.5 & 30 & 124.0 \\
\hline 0.176 & 450 & 30 & 146.0 \\
\hline 0.176 & 22.5 & 30 & 161.7 \\
\hline 0.376 & 0.0 & 30 & 21.2 \\
\hline 0.376 & -22.5 & 30 & 20.5 \\
\hline 0.376 & -45.0 & 30 & 17.6 \\
\hline 0.376 & -67.5 & 30 & 13.0 \\
\hline 0.376 & -90.0 & 30 & 12.2 \\
\hline 0.376 & -112.5 & 30 & 13.9 \\
\hline 0.376 & -135.0 & 30 & 18.8 \\
\hline 0.376 & -157.5 & 30 & 21.8 \\
\hline 0.376 & 180.0 & 30 & 22.8 \\
\hline 0.376 & 157.5 & 30 & 20.9 \\
\hline 0.376 & 135.0 & 30 & 16.0 \\
\hline 0.376 & 112.5 & 30 & 12.4 \\
\hline 0.376 & 90.0 & 30 & 12.2 \\
\hline 0.376 & 67.5 & 30 & 14.5 \\
\hline 0.376 & 45.0 & 30 & 18.5 \\
\hline 0.376 & 22.5 & 30 & 21.2 \\
\hline 1.076 & 0.0 & 30 & 1.5 \\
\hline 1.076 & -22.5 & 30 & 1.5 \\
\hline 1.076 & -45.0 & 30 & 1.2 \\
\hline 1.076 & -67.5 & 30 & 0.8 \\
\hline
\end{tabular}


Distance Horizontal Vertical $60 \mathrm{~Hz}$ meters angle deg angle deg mGauss

\begin{tabular}{|r|r|r|r|}
\hline 1.076 & -90.0 & 30 & 0.7 \\
\hline 1.076 & -112.5 & 30 & 0.9 \\
\hline 1.076 & -135.0 & 30 & 1.3 \\
\hline 1.076 & -157.5 & 30 & 1.6 \\
\hline 1.076 & 180.0 & 30 & 1.6 \\
\hline 1.076 & 157.5 & 30 & 1.6 \\
\hline 1.076 & 135.0 & 30 & 1.2 \\
\hline 1.076 & 112.5 & 30 & 0.7 \\
\hline 1.076 & 90.0 & 30 & 0.7 \\
\hline 1.076 & 67.5 & 30 & 0.8 \\
\hline 1.076 & 45.0 & 30 & 1.2 \\
\hline 1.076 & 22.5 & 30 & 1.5 \\
\hline 0.089 & 0.0 & 0 & 899.5 \\
\hline 0.089 & -22.5 & 0 & 925.7 \\
\hline 0.089 & -45.0 & 0 & 807.0 \\
\hline 0.089 & -67.5 & 0 & 562.8 \\
\hline 0.089 & -90.0 & 0 & 542.5 \\
\hline 0.089 & -112.5 & 0 & 646.3 \\
\hline 0.089 & -135.0 & 0 & 1092.5 \\
\hline 0.089 & -157.5 & 0 & 1176.6 \\
\hline 0.089 & 180.0 & 0 & 1244.3 \\
\hline 0.089 & 157.5 & 0 & 1083.9 \\
\hline 0.089 & 135.0 & 0 & 733.5 \\
\hline 0.089 & 112.5 & 0 & 490.4 \\
\hline 0.089 & 90.0 & 0 & 470.1 \\
\hline 0.089 & 67.5 & 0 & 578.4 \\
\hline 0.089 & 45.0 & 0 & 811.0 \\
\hline 0.089 & 22.5 & 0 & 947.2 \\
\hline 0.189 & 0.0 & 0 & 134.8 \\
\hline 0.189 & -22.5 & 0 & 135.3 \\
\hline & & & \\
\hline 1.07 & & & \\
\hline
\end{tabular}

Table C.15 Shaver - AC

$60 \mathrm{~Hz}$ RMS Resultant Fields

Distance Horizontal Vertical $60 \mathrm{~Hz}$ meters angle deg angle deg mGauss

\begin{tabular}{|c|c|c|c|}
\hline 0.189 & -45.0 & 0 & 118.4 \\
\hline 0.189 & -67.5 & 0 & 83.5 \\
\hline 0.189 & -90.0 & 0 & 75.8 \\
\hline 0.189 & -112.5 & 0 & 87.1 \\
\hline 0.189 & -135.0 & 0 & 131.4 \\
\hline 0.189 & -157.5 & 0 & 150.5 \\
\hline 0.189 & 180.0 & 0 & 159.4 \\
\hline 0.189 & 157.5 & 0 & 144.6 \\
\hline 0.189 & 135.0 & 0 & 106.2 \\
\hline 0.189 & 112.5 & 0 & 72.8 \\
\hline 0.189 & 90.0 & 0 & 70.8 \\
\hline 0.189 & 67.5 & 0 & 84.9 \\
\hline 0.189 & 45.0 & 0 & 114.9 \\
\hline 0.189 & 22.5 & 0 & 136.2 \\
\hline 0.389 & 0.0 & 0 & 20.1 \\
\hline 0.389 & -22.5 & 0 & 19.8 \\
\hline 0.389 & -45.0 & 0 & 17.2 \\
\hline 0.389 & -67.5 & 0 & 12.0 \\
\hline 0.389 & -90.0 & 0 & 10.5 \\
\hline 0.389 & -112.5 & 0 & 12.0 \\
\hline 0.389 & -135.0 & 0 & 17.2 \\
\hline 0.389 & -157.5 & 0 & 20.4 \\
\hline 0.389 & 180.0 & 0 & 21.7 \\
\hline 0.389 & 157.5 & 0 & 20.2 \\
\hline 0.389 & 135.0 & 0 & 15.3 \\
\hline 0.389 & 112.5 & 0 & 10.4 \\
\hline 0.389 & 90.0 & 0 & 10.2 \\
\hline 0.389 & 67.5 & 0 & 12.3 \\
\hline 0.389 & 450 & 0 & 16.5 \\
\hline 0.389 & 22.5 & 0 & 19.8 \\
\hline
\end{tabular}

C.15-4 
Distance Horizontal Vertical $60 \mathrm{~Hz}$ meters angle deg angle deg mGauss

\begin{tabular}{|c|c|c|c|}
\hline 1.089 & 0.0 & 0 & 1.3 \\
\hline 1.089 & -22.5 & 0 & 1.3 \\
\hline 1.089 & -45.0 & 0 & 1.1 \\
\hline 1.089 & -67.5 & 0 & 0.7 \\
\hline 1.089 & -90.0 & 0 & 0.5 \\
\hline 1.089 & -112.5 & 0 & 0.6 \\
\hline 1.089 & -135.0 & 0 & 1.0 \\
\hline 1.089 & -157.5 & 0 & 1.2 \\
\hline 1.089 & 180.0 & 0 & 1.3 \\
\hline 1.089 & 157.5 & 0 & 1.3 \\
\hline 1.089 & 135.0 & 0 & 1.0 \\
\hline 1.089 & 112.5 & 0 & 0.5 \\
\hline 1.089 & 90.0 & 0 & 0.5 \\
\hline 1.089 & 67.5 & 0 & 0.7 \\
\hline 1.089 & 45.0 & 0 & 1.0 \\
\hline 1.089 & 22.5 & 0 & 1.3 \\
\hline 0.279 & 0.0 & -30 & 56.5 \\
\hline 0.279 & -22.5 & .30 & 55.7 \\
\hline 0.279 & -45.0 & -30 & 48.7 \\
\hline 0.279 & -67.5 & -30 & 37.4 \\
\hline 0.279 & -90.0 & -30 & 32.8 \\
\hline 0.279 & -112.5 & .30 & 34.7 \\
\hline 0.279 & -135.0 & -30 & 44.5 \\
\hline 0.278 & -157.5 & -30 & 51.5 \\
\hline 0.279 & 180.0 & .30 & 54.2 \\
\hline 0.279 & 157.5 & -30 & 51.4 \\
\hline 0.279 & 135.0 & -30 & 40.8 \\
\hline 0.279 & 112.5 & .30 & 35.5 \\
\hline 0.279 & 90.0 & .30 & 34.8 \\
\hline 0.279 & 67.5 & -30 & 40.1 \\
\hline
\end{tabular}

Table C.15 Shaver-AC

$60 \mathrm{~Hz}$ RMS Resultant Fields

Distance Horizontal Vertical $60 \mathrm{~Hz}$

meters angle deg angle deg mGauss

\begin{tabular}{|c|c|c|c|}
\hline 0.279 & 45.0 & -30 & 50.3 \\
\hline 0.279 & 22.5 & -30 & 57.2 \\
\hline 0.379 & 0.0 & 30 & 20.6 \\
\hline 0.379 & -22.5 & -30 & 20.2 \\
\hline 0.379 & -45.0 & -30 & 17.7 \\
\hline 0.379 & -67.5 & -30 & 13.2 \\
\hline 0.379 & -90.0 & -30 & 11.9 \\
\hline 0.379 & -112.5 & -30 & 13.0 \\
\hline 0.379 & -135.0 & -30 & 17.3 \\
\hline 0.379 & -157.5 & -30 & 20.2 \\
\hline 0.379 & 180.0 & -30 & 21.2 \\
\hline 0.379 & 157.5 & -30 & 19.9 \\
\hline 0.379 & 135.0 & -30 & 15.6 \\
\hline 0.379 & 112.5 & .30 & 11.8 \\
\hline 0.379 & 90.0 & -30 & 11.7 \\
\hline 0.379 & 67.5 & -30 & 13.5 \\
\hline 0.379 & 45.0 & .30 & 17.5 \\
\hline 0.379 & 22.5 & -30 & 20.5 \\
\hline 0.579 & 0.0 & -30 & 6.5 \\
\hline 0.579 & -22.5 & -30 & 6.3 \\
\hline 0.579 & -45.0 & -30 & 5.5 \\
\hline 0.579 & -67.5 & -30 & 4.0 \\
\hline 0.579 & -90.0 & -30 & 3.6 \\
\hline 0.579 & -112.5 & -30 & 4.0 \\
\hline 0.579 & -935.0 & -30 & 5.4 \\
\hline 0.579 & -157.5 & -30 & 6.4 \\
\hline 0.579 & 180.0 & -30 & 6.7 \\
\hline 0.579 & 157.5 & -30 & 6.2 \\
\hline 0.579 & 135.0 & -30 & 4.9 \\
\hline 0.579 & 112.5 & -30 & 3.5 \\
\hline
\end{tabular}


Distance Horizontal Vertical $60 \mathrm{~Hz}$ meters angle deg angle deg mGauss

\begin{tabular}{|c|c|c|c|}
\hline 0.579 & 90.0 & -30 & 3.5 \\
\hline 0.579 & 67.5 & -30 & 4.1 \\
\hline 0.579 & 45.0 & -30 & 5.4 \\
\hline 0.579 & 22.5 & -30 & 6.4 \\
\hline 1.279 & 0.0 & -30 & 0.8 \\
\hline 1.279 & -22.5 & -30 & 0.8 \\
\hline 1.279 & -45.0 & -30 & 0.7 \\
\hline 1.279 & -67.5 & .30 & 0.5 \\
\hline 1.279 & -90.0 & .30 & 0.4 \\
\hline 1.279 & -112.5 & -30 & 0.4 \\
\hline 1.279 & -135.0 & -30 & 0.5 \\
\hline 1.279 & -157.5 & .30 & 0.6 \\
\hline 1.279 & 180.0 & .30 & 0.7 \\
\hline 1.279 & 157.5 & -30 & 0.7 \\
\hline 1.279 & 135.0 & .30 & 0.5 \\
\hline 1.279 & 112.5 & -30 & 0.3 \\
\hline 1.279 & 90.0 & -30 & 0.3 \\
\hline 1.278 & 67.5 & -30 & 0.4 \\
\hline 1.279 & 45.0 & -30 & 0.6 \\
\hline 1.279 & 22.5 & -30 & 0.8 \\
\hline 0.330 & 0.0 & -60 & 29.2 \\
\hline 0.330 & -22.5 & -60 & 27.5 \\
\hline 0.330 & -45.0 & -60 & 24.5 \\
\hline 0.330 & -67.5 & -60 & 21.0 \\
\hline 0.330 & -90.0 & -60 & 17.9 \\
\hline 0.330 & -112.5 & -60 & 16.4 \\
\hline 0.330 & -135.0 & -60 & 15.8 \\
\hline 0.330 & -157.5 & .60 & 16.7 \\
\hline 0.330 & 180.0 & -60 & 17.8 \\
\hline 0.330 & 157.5 & .60 & 18.2 \\
\hline
\end{tabular}

Table C.15 Shaver - AC

$60 \mathrm{~Hz}$ RMS Resultant Fields

Distance Horizontal Vertical $60 \mathrm{~Hz}$

meters angle deg angle deg mGauss

\begin{tabular}{|c|c|c|c|}
\hline 0.330 & 135.0 & -60 & 17.5 \\
\hline 0.330 & 112.5 & -60 & 19.8 \\
\hline 0.330 & 90.0 & -60 & 20.5 \\
\hline 0.330 & 67.5 & -60 & 22.6 \\
\hline 0.330 & 45.0 & -60 & 26.0 \\
\hline 0.330 & 22.5 & -60 & 28.0 \\
\hline 0.430 & 0.0 & -60 & 10.4 \\
\hline 0.430 & -22.5 & -60 & 10.1 \\
\hline 0.430 & -45.0 & -60 & 9.4 \\
\hline 0.430 & -67.5 & -60 & 8.0 \\
\hline 0.430 & -90.0 & -60 & 7.6 \\
\hline 0.430 & -112.5 & -60 & 7.6 \\
\hline 0.430 & -135.0 & -60 & 8.5 \\
\hline 0.430 & -157.5 & -60 & 9.2 \\
\hline 0.430 & 180.0 & -60 & 9.6 \\
\hline 0.430 & 157.5 & -60 & 9.4 \\
\hline 0.430 & 135.0 & -60 & 8.5 \\
\hline 0.430 & 112.5 & -60 & 7.6 \\
\hline 0.430 & 90.0 & -60 & 7.6 \\
\hline 0.430 & 67.5 & -60 & 8.0 \\
\hline 0.430 & 45.0 & -60 & 9.2 \\
\hline 0.430 & 22.5 & -60 & 10.1 \\
\hline 0.630 & 0.0 & -60 & 3.6 \\
\hline 0.630 & -22.5 & -60 & 3.6 \\
\hline 0.630 & -45.0 & -60 & 3.3 \\
\hline 0.630 & -67.5 & .60 & 2.9 \\
\hline 0.630 & -90.0 & -60 & 2.7 \\
\hline 0.630 & -112.5 & .60 & 2.7 \\
\hline 0.630 & -135.0 & .60 & 2.9 \\
\hline 0.630 & -157.5 & -60 & 3.0 \\
\hline
\end{tabular}


Table C.15 Shaver-AC

Distance Horizontal Vertical $60 \mathrm{~Hz}$ $60 \mathrm{~Hz}$ RMS Resultant Fields

Distance Horizontal Vertical $60 \mathrm{~Hz}$ meters angle deg angle deg mGauss

\begin{tabular}{|l|r|r|r|}
\hline 0.630 & 180.0 & -60 & 3.1 \\
\hline 0.630 & 157.5 & -60 & 3.0 \\
\hline 0.630 & 135.0 & -60 & 2.7 \\
\hline 0.630 & 112.5 & -60 & 2.6 \\
\hline 0.630 & 90.0 & -60 & 2.6 \\
\hline 0.630 & 67.5 & -60 & 2.8 \\
\hline 0.630 & 45.0 & -60 & 3.2 \\
\hline 0.630 & 22.5 & -60 & 3.5 \\
\hline 1.000 & 0.0 & -60 & 0.9 \\
\hline 1.000 & -22.5 & -60 & 0.8 \\
\hline 1.000 & -45.0 & -60 & 0.8 \\
\hline 1.000 & -67.5 & -60 & 0.6 \\
\hline 1.000 & -90.0 & -60 & 0.5 \\
\hline 1.000 & -112.5 & -60 & 0.5 \\
\hline
\end{tabular}

\begin{tabular}{|r|r|r|r|}
\hline 1.000 & -135.0 & -60 & 0.6 \\
\hline 1.000 & -157.5 & -60 & 0.7 \\
\hline 1.000 & 180.0 & -60 & 0.7 \\
\hline 1.000 & 157.5 & -60 & 0.7 \\
\hline 1.000 & 135.0 & -60 & 0.6 \\
\hline 1.000 & 112.5 & -60 & 0.5 \\
\hline 1.000 & 90.0 & -60 & 0.5 \\
\hline 1.000 & 67.5 & -60 & 0.6 \\
\hline 1.000 & 45.0 & -60 & 0.7 \\
\hline 1.000 & 22.5 & -60 & 0.8 \\
\hline 0.127 & 0.0 & -90 & 242.5 \\
\hline 0.227 & 0.0 & -90 & 48.9 \\
\hline 0.427 & 0.0 & -90 & 8.8 \\
\hline
\end{tabular}




\section{RESULTANT RMS MAGNETIC FIELD DENSITY ELECTRIC SHAVER \\ HORIZONTAL SLICE THROUGH CENTER OF APPLIANCE}

Z GRID LINES AT $100 \mathrm{mG}$

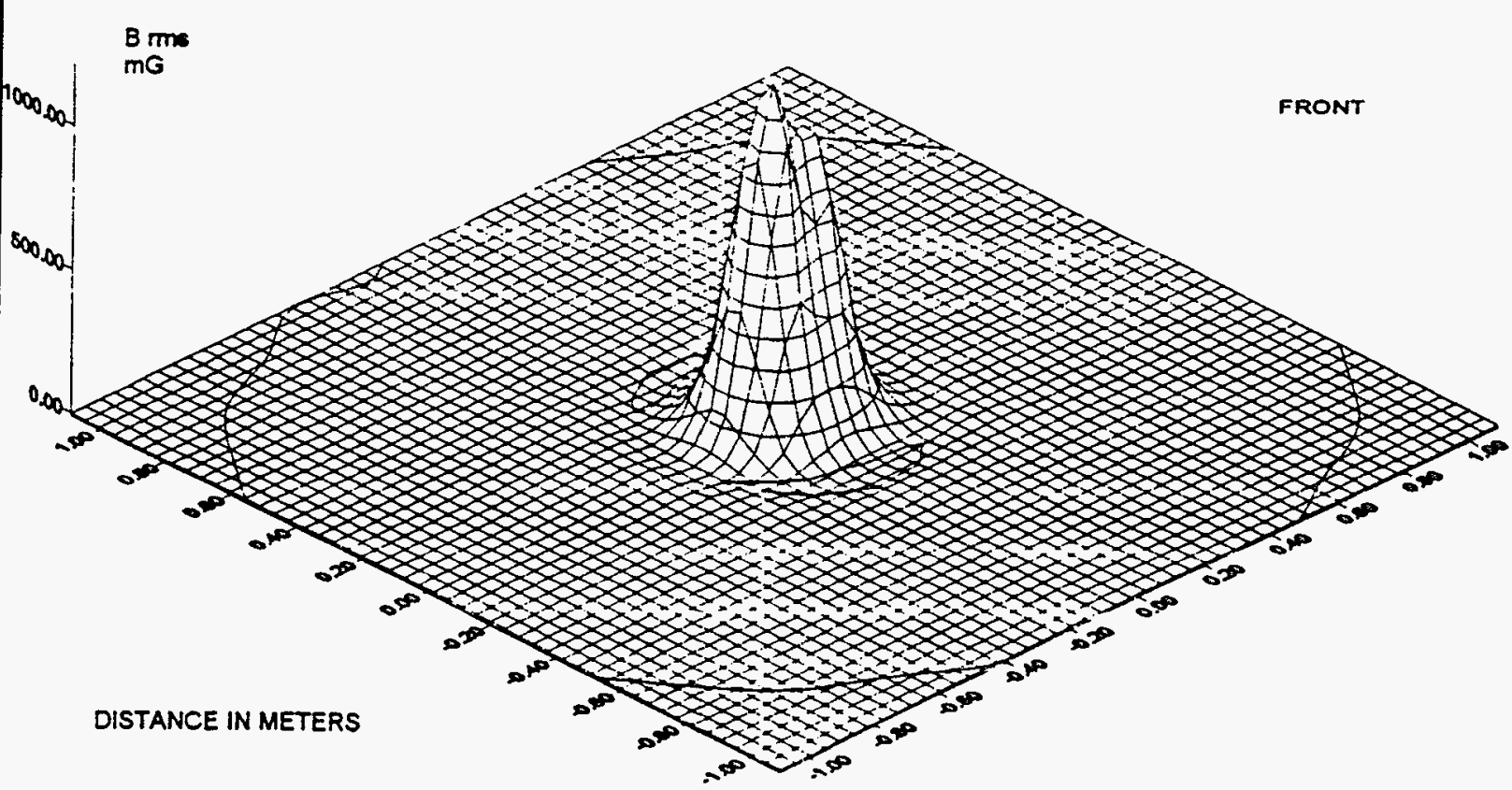

Figure C.15.1 Fundamental Resultant RMS Magnetic Field Density - Electric Shaver.

C. $15-8$ 


\section{C.16 Soldering Iron Station}

Appliance Tested: A soldering iron station with temperature control as used in an electronics assembly area

Manufacturer: PPP

Model: 014

Rated power: 60 Watts

Appliance Code: 2262

Conditions for Testing: The unit was set to 1000 degrees and the soldering iron was placed in cold water.

Measured voltage and current: 119.2 Volts and 0.41 Amperes.

Magnetic Fieid Summary:

$60 \mathrm{~Hz}$ RMS resultant level at $30 \mathrm{~cm}$ from front surface: $2.6 \mathrm{mG}$

RMS resultant low surface level: $139 \mathrm{mG}$ back

RMS resultant high surface level: $2884 \mathrm{mG}$ top

Highest recorded surface levels:

60 Hertz RMS resultant: $2865 \mathrm{mG}$

180 Hertz RMS resultant: $363 \mathrm{mG}$

300 Hertz RMS resultant: $67.9 \mathrm{mG}$

420 Hertz RMS resultant: $27.8 \mathrm{mG}$

Peak field $Y$ axis top: $3702 \mathrm{mG}$

Maximum Total Harmonic Distortion: 43 percent

For the appliance test set, the front of the unit was located at 180 degrees on the test table.

Summary of characteristics: The magnetic fields were strong with mild harmonics.

C.16-1 
Table C.16 Soldering Iron Station $60 \mathrm{~Hz}$ RMS Resultant Fields

Distance Horizontal Vertical $60 \mathrm{~Hz}$

meters angle deg angle deg mGauss

\begin{tabular}{|c|c|c|c|}
\hline 0.064 & 0.0 & $\infty$ & 644.6 \\
\hline 0.164 & 0.0 & 90 & 41.0 \\
\hline 0.364 & 0.0 & 90 & 4.8 \\
\hline 1.000 & 0.0 & 90 & 0.3 \\
\hline 0.140 & 0.0 & 60 & 46.8 \\
\hline 0.140 & -22.5 & 60 & 38.7 \\
\hline 0.140 & -45.0 & 60 & 34.8 \\
\hline 0.140 & -67.5 & 60 & 33.8 \\
\hline 0.140 & -90.0 & 60 & .33 .2 \\
\hline 0.140 & -112.5 & 60 & 31.4 \\
\hline 0.140 & -135.0 & 60 & 29.0 \\
\hline 0.140 & -157.5 & 60 & 26.7 \\
\hline 0.140 & 180.0 & 60 & 25.2 \\
\hline 0.140 & 157.5 & 60 & 24.2 \\
\hline 0.140 & 135.0 & 60 & 24.1 \\
\hline 0.140 & 112.5 & 60 & 26.2 \\
\hline 0.140 & 90.0 & 60 & 30.4 \\
\hline 0.140 & 67.5 & 60 & 38.4 \\
\hline 0.940 & 45.0 & 60 & 48.0 \\
\hline 0.140 & 22.5 & 60 & 52.4 \\
\hline 0.240 & 0.0 & 60 & 14.7 \\
\hline 0.240 & -22.5 & 60 & 15.4 \\
\hline 0.240 & -45.0 & 60 & 16.0 \\
\hline 0.240 & -67.5 & 60 & 15.6 \\
\hline 0.240 & -90.0 & 60 & 14.2 \\
\hline 0.240 & -112.5 & 60 & 12.3 \\
\hline 0.240 & -135.0 & 60 & 10.4 \\
\hline 0.240 & -157.5 & 60 & 9.0 \\
\hline 0.240 & 180.0 & 60 & 8.6 \\
\hline 0.240 & 157.5 & 60 & 9.1 \\
\hline
\end{tabular}

Distance Horizontal Vertical $60 \mathrm{~Hz}$

meters angle deg angle deg mGauss

\begin{tabular}{|r|r|r|r|}
\hline 0.240 & 135.0 & 60 & 10.6 \\
\hline 0.240 & 112.5 & 60 & 12.4 \\
\hline 0.240 & 90.0 & 60 & 13.8 \\
\hline 0.240 & 67.5 & 60 & 14.7 \\
\hline 0.240 & 45.0 & 60 & 14.9 \\
\hline 0.240 & 22.5 & 60 & 14.8 \\
\hline 0.440 & 0.0 & 60 & 2.6 \\
\hline 0.440 & -22.5 & 60 & 2.7 \\
\hline 0.440 & -45.0 & 60 & 3.1 \\
\hline 0.440 & -67.5 & 60 & 3.1 \\
\hline 0.440 & -90.0 & 60 & 3.0 \\
\hline 0.440 & -112.5 & 60 & 2.8 \\
\hline 0.440 & -135.0 & 60 & 2.5 \\
\hline 0.440 & -157.5 & 60 & 2.2 \\
\hline 0.440 & 180.0 & 60 & 2.0 \\
\hline 0.440 & 157.5 & 60 & 2.1 \\
\hline 0.440 & 135.0 & 60 & 2.3 \\
\hline 0.440 & 112.5 & 60 & 2.6 \\
\hline 0.440 & 90.0 & 60 & 2.9 \\
\hline 0.440 & 67.5 & 60 & 3.0 \\
\hline 0.440 & 45.0 & 60 & 2.8 \\
\hline 0.440 & 22.5 & 60 & 2.7 \\
\hline 1.000 & 0.0 & 60 & 0.3 \\
\hline 1.000 & -22.5 & 60 & 0.3 \\
\hline 1.000 & -450 & 60 & 0.3 \\
\hline 1.000 & -67.5 & 60 & 0.3 \\
\hline 1.000 & -90.0 & 60 & 0.3 \\
\hline 1.000 & -112.5 & 60 & 0.3 \\
\hline 1.000 & -135.0 & 60 & 0.3 \\
\hline 1.000 & -157.5 & 60 & 0.2 \\
\hline
\end{tabular}


Table C.16 Soldering Iron Station

\section{$60 \mathrm{~Hz}$ RMS Resultant Fields}

Distance Horizontal Vertical $60 \mathrm{~Hz}$

meters angle deg angle deg mGauss

\begin{tabular}{|r|r|r|r|}
\hline 1.000 & 180.0 & 60 & 0.2 \\
\hline 1.000 & 157.5 & 60 & 0.3 \\
\hline 1.000 & 135.0 & 60 & 0.4 \\
\hline 1.000 & 112.5 & 60 & 0.4 \\
\hline 1.000 & 90.0 & 60 & 0.4 \\
\hline 1.000 & 67.5 & 60 & 0.4 \\
\hline 1.000 & 45.0 & 60 & 0.4 \\
\hline 1.000 & 22.5 & 60 & 0.3 \\
\hline 0.178 & 0.0 & 30 & 40.8 \\
\hline 0.178 & -22.5 & 30 & 45.2 \\
\hline 0.178 & -45.0 & 30 & 48.8 \\
\hline 0.178 & -67.5 & 30 & 45.5 \\
\hline 0.178 & -90.0 & 30 & 37.4 \\
\hline 0.178 & -112.5 & 30 & 28.3 \\
\hline 0.178 & -135.0 & 30 & 21.2 \\
\hline 0.178 & -157.5 & 30 & 16.0 \\
\hline 0.178 & 180.0 & 30 & 15.4 \\
\hline 0.178 & 157.5 & 30 & 18.8 \\
\hline 0.178 & 135.0 & 30 & 25.2 \\
\hline 0.178 & 112.5 & 30 & 32.2 \\
\hline 0.178 & 90.0 & 30 & 39.5 \\
\hline 0.178 & 67.5 & 30 & 45.6 \\
\hline 0.178 & 45.0 & 30 & 47.7 \\
\hline 0.178 & 22.5 & 30 & 43.4 \\
\hline 0.278 & 0.0 & 30 & 12.8 \\
\hline 0.278 & -22.5 & 30 & 13.1 \\
\hline 0.278 & -45.0 & 30 & 13.9 \\
\hline 0.278 & -67.5 & 30 & 13.9 \\
\hline 0.278 & -90.0 & 30 & 12.6 \\
\hline 0.278 & -112.5 & 30 & 10.7 \\
\hline
\end{tabular}

Distance Horizontal Vertical $60 \mathrm{~Hz}$ meters angle deg angle deg mGauss

\begin{tabular}{|r|r|r|r|}
\hline 0.278 & -135.0 & 30 & 8.9 \\
\hline 0.278 & -157.5 & 30 & 7.4 \\
\hline 0.278 & 180.0 & 30 & 6.9 \\
\hline 0.278 & 157.5 & 30 & 7.6 \\
\hline 0.278 & 135.0 & 30 & 9.2 \\
\hline 0.278 & 112.5 & 30 & 11.0 \\
\hline 0.278 & 90.0 & 30 & 12.5 \\
\hline 0.278 & 67.5 & 30 & 13.7 \\
\hline 0.278 & 45.0 & 30 & 14.1 \\
\hline 0.278 & 22.5 & 30 & 13.5 \\
\hline 0.478 & 0.0 & 30 & 2.7 \\
\hline 0.478 & -22.5 & 30 & 3.2 \\
\hline 0.478 & -45.0 & 30 & 3.4 \\
\hline 0.478 & -67.5 & 30 & 3.7 \\
\hline 0.478 & -90.0 & 30 & 3.3 \\
\hline 0.478 & -112.5 & 30 & 2.9 \\
\hline 0.478 & -135.0 & 30 & 2.3 \\
\hline 0.478 & -157.5 & 30 & 1.6 \\
\hline 0.478 & 180.0 & 30 & 1.2 \\
\hline 0.478 & 157.5 & 30 & 1.2 \\
\hline 0.478 & 135.0 & 30 & 1.8 \\
\hline 0.478 & 112.5 & 30 & 2.4 \\
\hline 0.478 & 90.0 & 30 & 2.9 \\
\hline 0.478 & 67.5 & 30 & 3.2 \\
\hline 0.478 & 45.0 & 30 & 3.0 \\
\hline 0.478 & 22.5 & 30 & 2.8 \\
\hline 1.178 & 0.0 & 30 & 0.2 \\
\hline 1.178 & -22.5 & 30 & 0.2 \\
\hline 1.178 & -45.0 & 30 & 0.3 \\
\hline 1.178 & -67.5 & 30 & 0.3 \\
\hline
\end{tabular}


Table C.16 - Soldering Iron Station

\section{$60 \mathrm{~Hz}$ RMS Resultant Fields}

Distance Horizontal Vertical $60 \mathrm{~Hz}$ meters angle deg angle deg mGauss

\begin{tabular}{|c|c|c|c|}
\hline 1.178 & -90.0 & 30 & 0.3 \\
\hline 1.178 & -112.5 & 30 & 0.3 \\
\hline 1.178 & -135.0 & 30 & 0.2 \\
\hline 1.178 & -157.5 & 30 & 0.2 \\
\hline 1.178 & 180.0 & 30 & 0.2 \\
\hline 1.178 & 157.5 & 30 & 0.2 \\
\hline 1.178 & 135.0 & 30 & 0.3 \\
\hline 1.178 & 112.5 & 30 & 0.4 \\
\hline 1.178 & 90.0 & 30 & 0.4 \\
\hline 1.178 & 67.5 & 30 & 0.4 \\
\hline 1.178 & 45.0 & 30 & 0.4 \\
\hline 1.178 & 22.5 & 30 & 0.3 \\
\hline 0.140 & 0.0 & 0 & 66.9 \\
\hline 0.140 & -22.5 & 0 & 70.0 \\
\hline 0.140 & -45.0 & 0 & 79.6 \\
\hline 0.140 & -67.5 & 0 & $\pi .5$ \\
\hline 0.140 & -90.0 & 0 & 60.0 \\
\hline 0.140 & -112.5 & 0 & 45.0 \\
\hline 0.140 & -135.0 & 0 & 30.5 \\
\hline 0.140 & -157.5 & 0 & 21.3 \\
\hline 0.140 & 180.0 & 0 & 18.9 \\
\hline 0.140 & 157.5 & 0 & 23.0 \\
\hline 0.140 & 135.0 & 0 & 31.1 \\
\hline 0.140 & 112.5 & 0 & 42.3 \\
\hline 0.140 & 90.0 & 0 & 55.3 \\
\hline 0.140 & 67.5 & 0 & 70.5 \\
\hline 0.140 & 45.0 & 0 & 83.0 \\
\hline 0.140 & 22.5 & 0 & 80.8 \\
\hline 0.240 & 0.0 & 0 & 15.1 \\
\hline 0.240 & -22.5 & 0 & 16.8 \\
\hline
\end{tabular}

Distance Horizontal Vertical $60 \mathrm{~Hz}$ meters angle deg angle deg mGauss

\begin{tabular}{|c|c|c|c|}
\hline 0.240 & -45.0 & 0 & 20.3 \\
\hline 0.240 & -67.5 & 0 & 21.0 \\
\hline 0.240 & -90.0 & 0 & 18.6 \\
\hline 0.240 & -112.5 & 0 & 14.6 \\
\hline 0.240 & -135.0 & 0 & 10.6 \\
\hline 0.240 & -157.5 & 0 & 7.4 \\
\hline 0.240 & 180.0 & 0 & 6.4 \\
\hline 0.240 & 157.5 & 0 & 7.6 \\
\hline 0.240 & 135.0 & 0 & 10.5 \\
\hline 0.240 & 112.5 & 0 & 13.9 \\
\hline 0.240 & 90.0 & 0 & 17.3 \\
\hline 0.240 & 67.5 & 0 & 19.9 \\
\hline 0.240 & 45.0 & 0 & 21.0 \\
\hline 0.240 & 22.5 & 0 & 18.4 \\
\hline 0.440 & 0.0 & 0 & 2.8 \\
\hline 0.440 & -22.5 & 0 & 3.2 \\
\hline 0.440 & -45.0 & 0 & 4.0 \\
\hline 0.440 & -67.5 & 0 & 4.4 \\
\hline 0.440 & -90.0 & 0 & 4.2 \\
\hline 0.440 & -112.5 & 0 & 3.5 \\
\hline 0.440 & -135.0 & 0 & 2.7 \\
\hline 0.440 & -157.5 & 0 & 1.9 \\
\hline 0.440 & 180.0 & 0 & 1.6 \\
\hline 0.440 & 157.5 & 0 & 1.8 \\
\hline 0.440 & 135.0 & 0 & 2.6 \\
\hline 0.440 & 112.5 & 0 & 3.4 \\
\hline 0.440 & 90.0 & 0 & 4.0 \\
\hline 0.440 & 67.5 & 0 & 4.3 \\
\hline 0.440 & 45.0 & 0 & 4.2 \\
\hline 0.440 & 22.5 & 0 & 3.6 \\
\hline
\end{tabular}


Table C.16 Soldering Iron Station $60 \mathrm{~Hz}$ RMS Resultant Fields

Distance Horizontal Vertical $60 \mathrm{~Hz}$ meters angle deg angle deg mGauss

\begin{tabular}{|r|r|r|r|}
\hline 1.140 & 0.0 & 0 & 0.2 \\
\hline 1.140 & -22.5 & 0 & 0.2 \\
\hline 1.140 & -45.0 & 0 & 0.3 \\
\hline 1.140 & -67.5 & 0 & 0.4 \\
\hline 1.140 & -90.0 & 0 & 0.4 \\
\hline 1.140 & -112.5 & 0 & 0.3 \\
\hline 1.140 & -135.0 & 0 & 0.3 \\
\hline 1.140 & -157.5 & 0 & 0.2 \\
\hline 1.140 & 180.0 & 0 & 0.2 \\
\hline 1.140 & 157.5 & 0 & 0.2 \\
\hline 1.140 & 135.0 & 0 & 0.3 \\
\hline 1.140 & 112.5 & 0 & 0.3 \\
\hline 1.140 & 90.0 & 0 & 0.4 \\
\hline 1.140 & 67.5 & 0 & 0.4 \\
\hline 1.140 & 45.0 & 0 & 0.3 \\
\hline 1.140 & 22.5 & 0 & 0.2 \\
\hline 0.279 & 0.0 & -30 & 11.5 \\
\hline 0.279 & -22.5 & -30 & 12.9 \\
\hline 0.279 & -45.0 & -30 & 14.8 \\
\hline 0.279 & -67.5 & -30 & 14.9 \\
\hline 0.279 & -900 & -30 & 13.5 \\
\hline 0.279 & -112.5 & -30 & 11.0 \\
\hline 0.279 & -135.0 & -30 & 8.4 \\
\hline 0.279 & -157.5 & -30 & 6.7 \\
\hline 0.279 & 180.0 & -30 & 5.6 \\
\hline 0.279 & 157.5 & -30 & 6.7 \\
\hline 0.279 & 135.0 & -30 & 8.8 \\
\hline 0.279 & 112.5 & -30 & 11.0 \\
\hline 0.279 & 90.0 & -30 & 12.9 \\
\hline 0.279 & 67.5 & -30 & 14.3 \\
\hline
\end{tabular}

Distance Horizontal Vertical $60 \mathrm{~Hz}$ meters angle deg angle deg mGauss

\begin{tabular}{|r|r|r|r|}
\hline 0.279 & 45.0 & -30 & 14.2 \\
\hline 0.279 & 22.5 & -30 & 12.8 \\
\hline 0.379 & 0.0 & -30 & 4.4 \\
\hline 0.379 & -22.5 & -30 & 5.2 \\
\hline 0.379 & -45.0 & -30 & 6.2 \\
\hline 0.379 & -67.5 & -30 & 6.6 \\
\hline 0.379 & -90.0 & -30 & 6.2 \\
\hline 0.379 & -112.5 & -30 & 5.2 \\
\hline 0.379 & -135.0 & -30 & 4.1 \\
\hline 0.379 & -157.5 & -30 & 3.1 \\
\hline 0.379 & 180.0 & -30 & 2.7 \\
\hline 0.379 & 157.5 & -30 & 3.1 \\
\hline 0.379 & 135.0 & -30 & 4.0 \\
\hline 0.379 & 112.5 & -30 & 5.0 \\
\hline 0.379 & 90.0 & -30 & 5.8 \\
\hline 0.379 & -67.5 & -30 & 6.2 \\
\hline 0.379 & 45.0 & -30 & 5.9 \\
\hline 0.379 & 22.5 & -30 & 4.9 \\
\hline 0.579 & 0.0 & -30 & 1.2 \\
\hline 0.579 & -22.5 & -30 & 1.4 \\
\hline 0.579 & -450 & -30 & 1.9 \\
\hline 0.579 & -67.5 & -30 & 2.1 \\
\hline 0.579. & -90.0 & -30 & 2.1 \\
\hline 0.579 & -112.5 & -30 & 1.8 \\
\hline 0.579 & -135.0 & -30 & 1.4 \\
\hline 0.579 & -157.5 & -30 & 1.0 \\
\hline 0.579 & 180.0 & -30 & 1.1 \\
\hline 0.579 & 157.5 & -30 & 1.3 \\
\hline 0.579 & 135.0 & -30 & 1.5 \\
\hline 0.579 & 112.5 & -30 & 1.9 \\
\hline
\end{tabular}


Table C.16 Soldering Iron Station $60 \mathrm{~Hz}$ RMS Resultant Fields

Distance Horizontal Vertical $60 \mathrm{~Hz}$ meters angle deg angle deg mGauss

\begin{tabular}{|r|r|r|r|}
\hline 0.579 & 90.0 & -30 & 2.1 \\
\hline 0.579 & 67.5 & -30 & 2.1 \\
\hline 0.579 & 45.0 & -30 & 2.0 \\
\hline 0.579 & 22.5 & -30 & 1.5 \\
\hline 1.279 & 0.0 & -30 & 0.1 \\
\hline 1.279 & -22.5 & -30 & 0.2 \\
\hline 1.279 & -45.0 & -30 & 0.2 \\
\hline 1.279 & -67.5 & -30 & 0.3 \\
\hline 1.279 & -90.0 & -30 & 0.3 \\
\hline 1.279 & -112.5 & -30 & 0.3 \\
\hline 1.279 & -135.0 & -30 & 0.2 \\
\hline 1.279 & -157.5 & -30 & 0.2 \\
\hline 1.279 & 180.0 & -30 & 0.1 \\
\hline 1.279 & 157.5 & -30 & 0.1 \\
\hline 1.279 & 135.0 & -30 & 0.1 \\
\hline 1.279 & 112.5 & -30 & 0.2 \\
\hline 1.279 & 90.0 & -30 & 0.2 \\
\hline 1.279 & 67.5 & -30 & 0.2 \\
\hline 1.279 & 45.0 & -30 & 0.2 \\
\hline 1.279 & 22.5 & -30 & 0.1 \\
\hline 0.267 & 0.0 & -60 & 13.0 \\
\hline 0.267 & -22.5 & -60 & 14.1 \\
\hline 0.267 & -45.0 & -60 & 15.1 \\
\hline 0.267 & -67.5 & -60 & 15.1 \\
\hline 0.267 & -90.0 & -60 & 14.0 \\
\hline 0.267 & -112.5 & -60 & 12.0 \\
\hline 0.267 & -135.0 & -60 & 9.9 \\
\hline 0.267 & -157.5 & -60 & 8.3 \\
\hline 0.267 & 180.0 & -60 & 7.3 \\
\hline 0.267 & 157.5 & -60 & 7.7 \\
\hline
\end{tabular}

Distance Horizontal Vertical $60 \mathrm{~Hz}$ meters angle deg angle deg mGauss

\begin{tabular}{|r|r|r|r|}
\hline 0.267 & 135.0 & -60 & 9.2 \\
\hline 0.267 & 112.5 & -60 & 10.9 \\
\hline 0.267 & 90.0 & -60 & 12.5 \\
\hline 0.267 & 67.5 & -60 & 13.7 \\
\hline 0.267 & 45.0 & -60 & 13.5 \\
\hline 0.267 & 22.5 & -60 & 13.1 \\
\hline 0.367 & 0.0 & -60 & 4.9 \\
\hline 0.367 & -22.5 & -60 & 5.3 \\
\hline 0.367 & -45.0 & -60 & 6.0 \\
\hline 0.367 & -67.5 & -60 & 6.2 \\
\hline 0.367 & -90.0 & -60 & 6.0 \\
\hline 0.367 & -112.5 & -60 & 5.2 \\
\hline 0.367 & -135.0 & -60 & 4.4 \\
\hline 0.367 & -157.5 & -60 & 3.7 \\
\hline 0.367 & 180.0 & -60 & 3.3 \\
\hline 0.367 & 157.5 & -60 & 3.5 \\
\hline 0.367 & 135.0 & -60 & 4.1 \\
\hline 0.367 & 112.5 & -60 & 4.7 \\
\hline 0.367 & 90.0 & -60 & 5.2 \\
\hline 0.367 & 67.5 & -60 & 5.5 \\
\hline 0.367 & 45.0 & -60 & 5.4 \\
\hline 0.367 & 22.5 & -60 & 5.0 \\
\hline 0.567 & 0.0 & -60 & 1.3 \\
\hline 0.567 & -22.5 & -60 & 1.6 \\
\hline 0.567 & -45.0 & -60 & 1.8 \\
\hline 0.567 & -67.5 & -60 & 1.9 \\
\hline 0.567 & -90.0 & -60 & 1.9 \\
\hline 0.567 & -112.5 & -60 & 1.8 \\
\hline 0.567 & -135.0 & -60 & 1.7 \\
\hline 0.567 & -157.5 & -60 & 1.4 \\
\hline
\end{tabular}


Table C.16 Soldering Iron Station $60 \mathrm{~Hz}$ RMS Resultant Fields

Distance Horizontal Vertical $60 \mathrm{~Hz}$ meters angle deg angle deg mGauss

\begin{tabular}{|r|r|r|r|}
\hline 0.567 & 180.0 & -60 & 1.2 \\
\hline 0.567 & 157.5 & -60 & 1.0 \\
\hline 0.567 & 135.0 & -60 & 1.2 \\
\hline 0.567 & 112.5 & -60 & 1.3 \\
\hline 0.567 & 90.0 & -60 & 1.4 \\
\hline 0.567 & 67.5 & -60 & 1.4 \\
\hline 0.567 & 45.0 & -60 & 1.3 \\
\hline 0.567 & 22.5 & -60 & 1.2 \\
\hline 1.000 & 0.0 & -60 & 0.2 \\
\hline 1.000 & -22.5 & -60 & 0.2 \\
\hline 1.000 & -45.0 & -60 & 0.3 \\
\hline 1.000 & -67.5 & -60 & 0.3 \\
\hline 1.000 & -90.0 & -60 & 0.3 \\
\hline 1.000 & -112.5 & -60 & 0.3 \\
\hline
\end{tabular}

Distance Horizontal Vertical $60 \mathrm{~Hz}$ melers angle deg angle deg mGauss

\begin{tabular}{|r|r|r|r|}
\hline 1.000 & -135.0 & -60 & 0.3 \\
\hline 1.000 & -157.5 & -60 & 0.2 \\
\hline 1.000 & 180.0 & -60 & 0.2 \\
\hline 1.000 & 157.5 & -60 & 0.2 \\
\hline 1.000 & 135.0 & -60 & 0.2 \\
\hline 1.000 & 112.5 & -60 & 0.2 \\
\hline 1.000 & 90.0 & -60 & 0.2 \\
\hline 1.000 & 67.5 & -60 & 0.2 \\
\hline 1.000 & 45.0 & -60 & 0.2 \\
\hline 1.000 & 22.5 & -60 & 0.2 \\
\hline 0.127 & 0.0 & -90 & 143.9 \\
\hline 0.227 & 0.0 & -90 & 23.9 \\
\hline 0.427 & 0.0 & -90 & 3.6 \\
\hline
\end{tabular}




\title{
RESULTANT RMS MAGNETIC FIELD DENSITY SOLDERING STATION HORIZONTAL SLICE THROUGH CENTER OF APPLIANCE
}

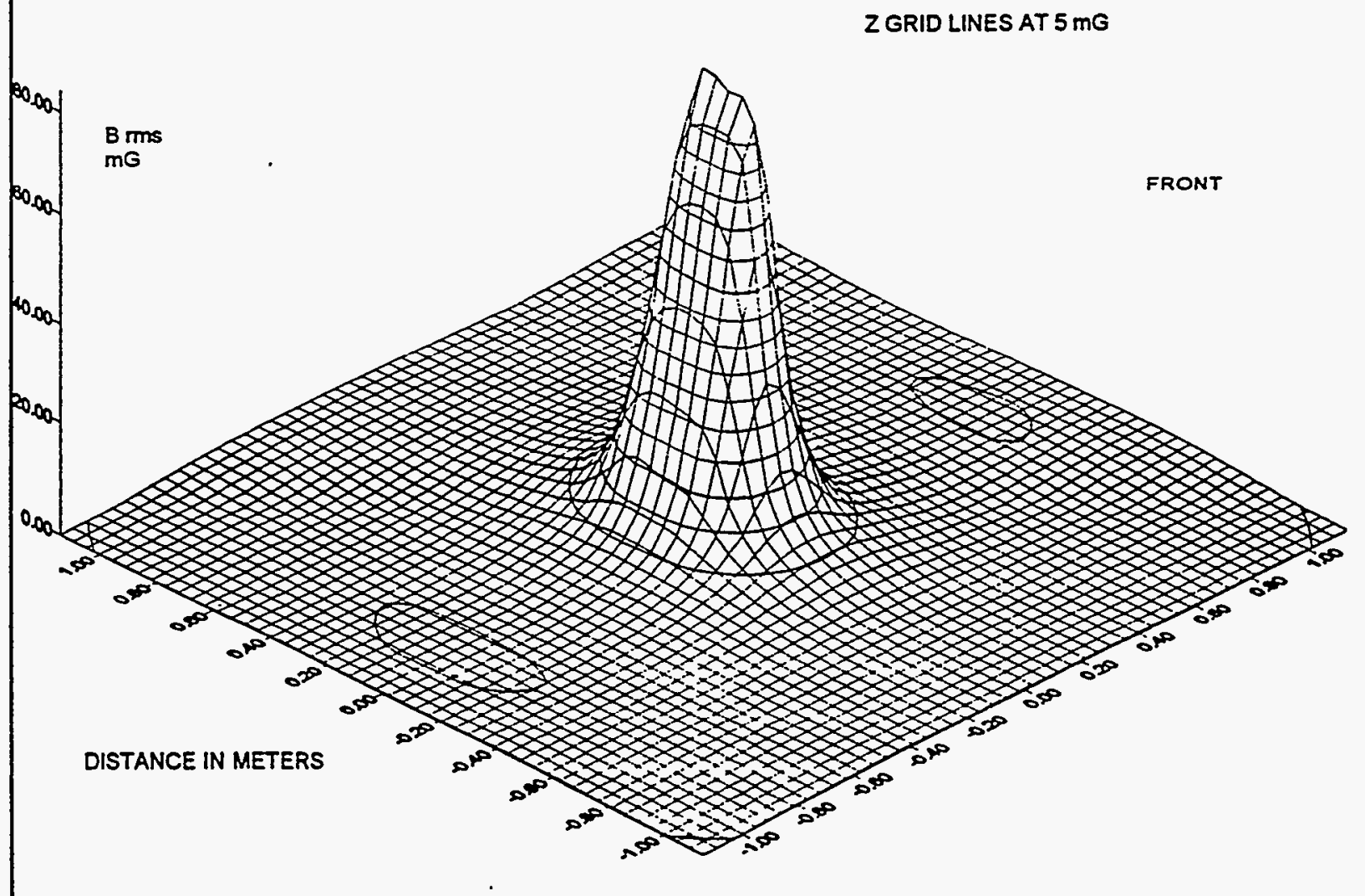

Figure C.16.1 Fundamental Resultant RMS Magnetic Field Density - Soldering Station.

\author{
C.16-8
}




\section{C.17 Toaster}

Appliance Tested: A kitchen toaster

Manufacturer: QQQ

Model: 015

Rated power: 900 Watts

Appliance Code: 2262

Conditions for Testing: The toaster was toasting bread

Measured voltage and current: 112.6 Volts and 6.77 Amperes.

Magnetic Field Summary:

$60 \mathrm{~Hz}$ RMS resultant level at $30 \mathrm{~cm}$ from front surface: $1.1 \mathrm{mG}$

RMS resultant low surface level: $18.1 \mathrm{mG}$ bottom

RMS resultant high surface level: $191 \mathrm{mG}$ rear

Highest recorded surface levels:

60 Hertz RMS resultant: $191 \mathrm{mG}$

180 Hertz RMS resultant: $3.35 \mathrm{mG}$

300 Hertz RMS resultant: $2.57 \mathrm{mG}$

420 Hertz RMS resultant: $0.84 \mathrm{mG}$

Maximum Total Harmonic Distortion: 4.16 percent

For the appliance test set, the front of the unit was located at 180 degrees on the test table.

Summary of characteristics: Low magnetic fields indicate a heating element only. 
Distance Horizontal Vertical $60 \mathrm{~Hz}$ meters angle deg angle deg mGauss

\begin{tabular}{|r|r|r|r|}
\hline 0.203 & 0.0 & 90 & 39.6 \\
\hline 0.303 & 0.0 & 90 & 10.0 \\
\hline 0.503 & 0.0 & 90 & 0.2 \\
\hline 1.000 & 0.0 & 90 & 0.3 \\
\hline 0.203 & 0.0 & 60 & 25.8 \\
\hline 0.203 & -22.5 & 60 & 28.1 \\
\hline 0.203 & -45.0 & 60 & 30.3 \\
\hline 0.203 & -67.5 & 60 & 26.9 \\
\hline 0.203 & -90.0 & 60 & 22.6 \\
\hline 0.203 & -112.5 & 60 & 18.0 \\
\hline 0.203 & -135.0 & 60 & 14.2 \\
\hline 0.203 & -157.5 & 60 & 12.1 \\
\hline 0.203 & 180.0 & 60 & 11.4 \\
\hline 0.203 & 157.5 & 60 & 12.3 \\
\hline 0.203 & 135.0 & 60 & 15.1 \\
\hline 0.203 & 112.5 & 60 & 20.3 \\
\hline 0.203 & 90.0 & 60 & 26.4 \\
\hline 0.203 & 67.5 & 60 & 30.7 \\
\hline 0.203 & 45.0 & 60 & 29.8 \\
\hline 0.203 & 22.5 & 60 & 26.2 \\
\hline 0.303 & 0.0 & 60 & 7.5 \\
\hline 0.303 & -22.5 & 60 & 7.1 \\
\hline 0.303 & -45.0 & 60 & 6.7 \\
\hline 0.303 & -67.5 & 60 & 5.9 \\
\hline 0.303 & -90.0 & 60 & 5.3 \\
\hline 0.303 & -135.0 & 60 & 4.3 \\
\hline 0.303 & -157.5 & 60 & 4.1 \\
\hline 0.303 & 180.0 & 60 & 4.3 \\
\hline 0.303 & 157.5 & 60 & 4.9 \\
\hline 0.303 & 135.0 & 60 & 5.9 \\
\hline
\end{tabular}

Table C.17 Toaster

\section{$60 \mathrm{~Hz}$ RMS Resultant Fields}

Distance Horizontal Vertical $60 \mathrm{~Hz}$

meters angle deg angle deg mGauss

\begin{tabular}{|l|r|r|r|}
\hline 0.303 & 112.5 & 60 & 7.2 \\
\hline 0.303 & 90.0 & 60 & 8.4 \\
\hline 0.303 & 67.5 & 60 & 9.1 \\
\hline 0.303 & 45.0 & 60 & 8.8 \\
\hline 0.303 & 22.5 & 60 & 8.1 \\
\hline 0.503 & 0.0 & 60 & 1.6 \\
\hline 0.503 & -22.5 & 60 & 1.5 \\
\hline 0.503 & -45.0 & 60 & 1.3 \\
\hline 0.503 & -67.5 & 60 & 1.2 \\
\hline 0.503 & -90.0 & 60 & 1.1 \\
\hline 0.503 & -112.5 & 60 & 1.1 \\
\hline 0.503 & -135.0 & 60 & 1.0 \\
\hline 0.503 & -157.5 & 60 & 1.1 \\
\hline 0.503 & 180.0 & 60 & 1.1 \\
\hline 0.503 & 157.5 & 60 & 1.3 \\
\hline 0.503 & 135.0 & 60 & 1.5 \\
\hline 0.503 & 112.5 & 60 & 1.7 \\
\hline 0.503 & 90.0 & 60 & 1.9 \\
\hline 0.503 & 67.5 & 60 & 2.0 \\
\hline 0.503 & 45.0 & 60 & 1.9 \\
\hline 0.503 & 22.5 & 60 & 1.8 \\
\hline 1.000 & 0.0 & 60 & 0.2 \\
\hline 1.000 & -22.5 & 60 & 0.2 \\
\hline 1.000 & -45.0 & 60 & 0.2 \\
\hline 1.000 & -67.5 & 60 & 0.2 \\
\hline 1.000 & -90.0 & 60 & 0.2 \\
\hline 1.000 & -112.5 & 60 & 0.2 \\
\hline 1.000 & -135.0 & 60 & 0.2 \\
\hline 1.000 & -157.5 & 60 & 0.2 \\
\hline 1.000 & 180.0 & 60 & 0.2 \\
\hline
\end{tabular}


Table C.17 Toaster

Distance Horizontal Vertical $60 \mathrm{~Hz}$

$60 \mathrm{~Hz}$ RMS Resultant Fields

meters angle deg angle deg mGauss

Distance Horizontal Vertical $60 \mathrm{~Hz}$

meters angle deg angle deg mGauss

\begin{tabular}{|r|r|r|r|}
\hline 1.000 & 157.5 & 60 & 0.2 \\
\hline 1.000 & 135.0 & 60 & 0.2 \\
\hline 1.000 & 112.5 & 60 & 0.2 \\
\hline 1.000 & 90.0 & 60 & 0.3 \\
\hline 1.000 & 67.5 & 60 & 0.3 \\
\hline 1.000 & 45.0 & 60 & 0.3 \\
\hline 1.000 & 22.5 & 60 & 0.3 \\
\hline 0.203 & 0.0 & 30 & 12.7 \\
\hline 0.203 & -22.5 & 30 & 12.1 \\
\hline 0.203 & -45.0 & 30 & 13.3 \\
\hline 0.203 & -67.5 & 30 & 12.5 \\
\hline 0.203 & -90.0 & 30 & 11.4 \\
\hline 0.203 & -112.5 & 30 & 9.6 \\
\hline 0.203 & -135.0 & 30 & 7.8 \\
\hline 0.203 & -157.5 & 30 & 6.4 \\
\hline 0.203 & 180.0 & 30 & 5.9 \\
\hline 0.203 & 157.5 & 30 & 6.8 \\
\hline 0.203 & 135.0 & 30 & 10.2 \\
\hline 0.203 & 112.5 & 30 & 16.8 \\
\hline 0.203 & 90.0 & 30 & 23.3 \\
\hline 0.203 & 67.5 & 30 & 25.7 \\
\hline 0.203 & 45.0 & 30 & 22.3 \\
\hline 0.203 & 22.5 & 30 & 16.1 \\
\hline 0.303 & 0.0 & 30 & 4.9 \\
\hline 0.303 & -22.5 & 30 & 4.2 \\
\hline 0.303 & -45.0 & 30 & 3.9 \\
\hline 0.303 & -67.5 & 30 & 3.6 \\
\hline 0.303 & -90.0 & 30 & 3.3 \\
\hline 0.303 & -112.5 & 30 & 3.1 \\
\hline 0.303 & -135.0 & 30 & 2.7 \\
\hline
\end{tabular}

\begin{tabular}{|r|r|r|r|}
\hline 0.303 & -157.5 & 30 & 2.5 \\
\hline 0.303 & 180.0 & 30 & 2.6 \\
\hline 0.303 & 157.5 & 30 & 3.1 \\
\hline 0.303 & 135.0 & 30 & 4.0 \\
\hline 0.303 & 112.5 & 30 & 5.4 \\
\hline 0.303 & 90.0 & 30 & 6.7 \\
\hline 0.303 & 67.5 & 30 & 7.3 \\
\hline 0.303 & 45.0 & 30 & 6.9 \\
\hline 0.303 & 22.5 & 30 & 5.8 \\
\hline 0.503 & 0.0 & 30 & 1.6 \\
\hline 0.503 & -22.5 & 30 & 1.5 \\
\hline 0.503 & -45.0 & 30 & 1.1 \\
\hline 0.503 & -67.5 & 30 & 1.2 \\
\hline 0.503 & -90.0 & 30 & 1.0 \\
\hline 0.503 & -112.5 & 30 & 1.4 \\
\hline 0.503 & -135.0 & 30 & 0.9 \\
\hline 0.503 & -157.5 & 30 & 1.1 \\
\hline 0.503 & 180.0 & 30 & 1.1 \\
\hline 0.503 & 157.5 & 30 & 1.1 \\
\hline 0.503 & 135.0 & 30 & 1.3 \\
\hline 0.503 & 112.5 & 30 & 1.7 \\
\hline 0.503 & 90.0 & 30 & 1.9 \\
\hline 0.503 & 67.5 & 30 & 2.0 \\
\hline 0.503 & 45.0 & 30 & 1.8 \\
\hline 0.503 & 22.5 & 30 & 1.5 \\
\hline 1.203 & 0.0 & 30 & 0.2 \\
\hline 1.203 & -22.5 & 30 & 0.1 \\
\hline 1.203 & -45.0 & 30 & 0.1 \\
\hline 1.203 & -67.5 & 30 & 0.1 \\
\hline 1.203 & -90.0 & 30 & 0.1 \\
\hline
\end{tabular}

C.17-3 
Distance Horizontal Vertical $60 \mathrm{~Hz}$ meters angle deg angle deg mGauss

\begin{tabular}{|c|c|c|c|}
\hline 1.203 & -112.5 & 30 & 0.1 \\
\hline 1.203 & -135.0 & 30 & 0.1 \\
\hline 1.203 & -157.5 & 30 & 0.1 \\
\hline 1.203 & 180.0 & 30 & 0.1 \\
\hline 1.203 & 157.5 & 30 & 0.1 \\
\hline 1.203 & 135.0 & 30 & 0.1 \\
\hline 1.203 & 112.5 & 30 & 0.2 \\
\hline 1.203 & 90.0 & 30 & 0.2 \\
\hline 1.203 & 67.5 & 30 & 0.2 \\
\hline 1.203 & 45.0 & 30 & 0.2 \\
\hline 1.203 & 22.5 & 30 & 0.2 \\
\hline 0.178 & 0.0 & 0 & 11.3 \\
\hline 0.178 & -22.5 & 0 & 11.5 \\
\hline 0.178 & -45.0 & 0 & 13.4 \\
\hline 0.178 & -67.5 & 0 & 13.1 \\
\hline 0.178 & -90.0 & 0 & 12.1 \\
\hline 0.178 & -112.5 & 0 & 10.3 \\
\hline 0.178 & -135.0 & 0 & 8.2 \\
\hline 0.178 & -157.5 & 0 & 6.8 \\
\hline 0.178 & 180.0 & 0 & 6.2 \\
\hline 0.178 & 157.5 & 0 & 6.9 \\
\hline 0.178 & 135.0 & 0 & 10.5 \\
\hline 0.178 & 112.5 & 0 & 17.5 \\
\hline 0.178 & 90.0 & 0 & 23.7 \\
\hline 0.178 & 67.5 & 0 & 24.5 \\
\hline 0.178 & 45.0 & 0 & 20.2 \\
\hline 0.178 & 22.5 & 0 & 14.1 \\
\hline 0.278 & 0.0 & 0 & 3.9 \\
\hline 0.278 & -22.5 & 0 & 3.8 \\
\hline 0.278 & -45.0 & 0 & 4.1 \\
\hline
\end{tabular}

Table C.17 Toaster

$60 \mathrm{~Hz}$ RMS Resultant Fields

Distance Horizontal Vertical $60 \mathrm{~Hz}$

meters angle deg angle deg mGauss

\begin{tabular}{|c|c|c|c|}
\hline 0.278 & -67.5 & 0 & 4.2 \\
\hline 0.278 & -90.0 & 0 & 4.1 \\
\hline 0.278 & -112.5 & 0 & 3.7 \\
\hline 0.278 & -135.0 & 0 & 3.2 \\
\hline 0.278 & -157.5 & 0 & 2.8 \\
\hline 0.278 & 180.0 & 0 & 2.5 \\
\hline 0.278 & 157.5 & 0 & 2.7 \\
\hline 0.278 & 135.0 & 0 & 3.6 \\
\hline 0.278 & 112.5 & 0 & 5.3 \\
\hline 0.278 & 90.0 & 0 & 6.8 \\
\hline 0.278 & 67.5 & 0 & 7.0 \\
\hline 0.278 & 45.0 & 0 & 6.2 \\
\hline 0.278 & 22.5 & 0 & 4.7 \\
\hline 0.478 & 0.0 & 0 & 0.9 \\
\hline 0.478 & -22.5 & 0 & 0.9 \\
\hline 0.478 & -45.0 & 0 & 1.0 \\
\hline 0.478 & -67.5 & 0 & 1.0 \\
\hline 0.478 & -90.0 & 0 & 1.0 \\
\hline 0.478 & -135.0 & 0 & 0.9 \\
\hline 0.478 & -157.5 & 0 & 0.8 \\
\hline 0.478 & 180.0 & 0 & 0.7 \\
\hline 0.478 & 157.5 & 0 & 0.8 \\
\hline 0.478 & 135.0 & 0 & 0.9 \\
\hline 0.478 & 112.5 & 0 & 1.3 \\
\hline 0.478 & 90.0 & 0 & 1.4 \\
\hline 0.478 & 67.5 & 0 & 1.5 \\
\hline 0.478 & 45.0 & 0 & 1.3 \\
\hline 0.478 & 22.5 & 0 & 1.0 \\
\hline 1.178 & 0.0 & 0 & 0.1 \\
\hline 1.178 & -22.5 & 0 & 0.1 \\
\hline
\end{tabular}

\section{17-4}


Distance Horizontal Vertical $60 \mathrm{~Hz}$ meters angle deg angle deg mGauss

\begin{tabular}{|r|r|r|r|}
\hline 1.178 & -45.0 & 0 & 0.1 \\
\hline 1.178 & -67.5 & 0 & 0.1 \\
\hline 1.178 & -90.0 & 0 & 0.1 \\
\hline 1.178 & -112.5 & 0 & 0.1 \\
\hline 1.178 & -135.0 & 0 & 0.1 \\
\hline 1.178 & -157.5 & 0 & 0.1 \\
\hline 1.178 & 180.0 & 0 & 0.1 \\
\hline 1.178 & 157.5 & 0 & 0.1 \\
\hline 1.178 & 135.0 & 0 & 0.1 \\
\hline 1.178 & 112.5 & 0 & 0.1 \\
\hline 1.178 & 90.0 & 0 & 0.1 \\
\hline 1.178 & 67.5 & 0 & 0.2 \\
\hline 1.178 & 45.0 & 0 & 0.1 \\
\hline 1.178 & 22.5 & 0 & 0.1 \\
\hline 0.279 & 0.0 & -30 & 11.0 \\
\hline 0.279 & -22.5 & -30 & 11.4 \\
\hline 0.279 & -45.0 & -30 & 12.3 \\
\hline 0.279 & -67.5 & -30 & 12.2 \\
\hline 0.279 & -90.0 & -30 & 11.6 \\
\hline 0.279 & -112.5 & -30 & 11.7 \\
\hline 0.279 & -135.0 & -30 & 9.0 \\
\hline 0.279 & -157.5 & -30 & 8.1 \\
\hline 0.279 & 180.0 & -30 & 7.4 \\
\hline 0.279 & 157.5 & -30 & 7.6 \\
\hline 0.279 & 135.0 & -30 & 8.6 \\
\hline 0.279 & 112.5 & -30 & 11.2 \\
\hline 0.279 & 90.0 & -30 & 13.9 \\
\hline 0.279 & 67.5 & -30 & 21.5 \\
\hline 0.279 & 45.0 & -30 & 30.6 \\
\hline 0.279 & 22.5 & -30 & 13.6 \\
\hline & & & \\
\hline 130 & 0 & 0 & 0 \\
\hline
\end{tabular}

Table C.17 Toaster

$60 \mathrm{~Hz}$ RMS Resultant Fields

Distance Horizontal Vertical $60 \mathrm{~Hz}$

meters angle deg angie deg muauss

\begin{tabular}{|r|r|r|r|}
\hline 0.379 & 0.0 & -30 & 4.2 \\
\hline 0.379 & -22.5 & -30 & 4.3 \\
\hline 0.379 & -45.0 & -30 & 4.6 \\
\hline 0.379 & -67.5 & -30 & 4.6 \\
\hline 0.379 & -90.0 & -30 & 4.6 \\
\hline 0.379 & -112.5 & -30 & 4.3 \\
\hline 0.379 & -135.0 & -30 & 3.9 \\
\hline 0.379 & -157.5 & -30 & 3.4 \\
\hline 0.379 & 1800 & -30 & 3.1 \\
\hline 0.379 & 157.5 & -30 & 2.9 \\
\hline 0.379 & 135.0 & -30 & 2.9 \\
\hline 0.379 & 112.5 & -30 & 3.5 \\
\hline 0.379 & 90.0 & -30 & 4.0 \\
\hline 0.379 & 67.5 & -30 & 4.0 \\
\hline 0.379 & 45.0 & -30 & 5.1 \\
\hline 0.379 & 22.5 & -30 & 4.1 \\
\hline 0.579 & 0.0 & -30 & 1.2 \\
\hline 0.579 & -22.5 & -30 & 1.3 \\
\hline 0.579 & -45.0 & -30 & 1.3 \\
\hline 0.579 & -67.5 & -30 & 1.3 \\
\hline 0.579 & -90.0 & -30 & 1.4 \\
\hline 0.579 & -112.5 & -30 & 1.6 \\
\hline 0.579 & -135.0 & -30 & 1.2 \\
\hline 0.579 & -157.5 & -30 & 1.1 \\
\hline 0.579 & 180.0 & -30 & 0.9 \\
\hline 0.579 & 157.5 & -30 & 0.8 \\
\hline 0.579 & 135.0 & -30 & 0.7 \\
\hline 0.579 & 112.5 & -30 & 0.8 \\
\hline 0.579 & 90.0 & -30 & 0.9 \\
\hline 0.579 & 67.5 & -30 & 0.9 \\
\hline
\end{tabular}

C.17-5 
Distance Horizontal Vertical $60 \mathrm{~Hz}$ meters angle deg angle deg mGauss

\begin{tabular}{|c|c|c|c|}
\hline 0.579 & 45.0 & .30 & 0.8 \\
\hline 0.579 & 22.5 & -30 & 0.7 \\
\hline 1.279 & 0.0 & .30 & 0.1 \\
\hline 1.279 & -22.5 & -30 & 0.1 \\
\hline 1.279 & -45.0 & -30 & 0.1 \\
\hline 1.279 & -67.5 & 30 & 0.1 \\
\hline 1.279 & -90.0 & -30 & 0.1 \\
\hline 1.279 & -112.5 & -30 & 0.1 \\
\hline 1.279 & -135.0 & .30 & 0.1 \\
\hline 1.279 & -157.5 & -30 & 0.1 \\
\hline 1.279 & 180.0 & -30 & 0.1 \\
\hline 1.279 & 157.5 & -30 & 0.1 \\
\hline 1.279 & 135.0 & -30 & 0.1 \\
\hline 1.279 & 112.5 & .30 & 0.1 \\
\hline 1.279 & 90.0 & -30 & 0.1 \\
\hline 1.279 & 67.5 & -30 & 0.1 \\
\hline 1.279 & 45.0 & -30 & 0.1 \\
\hline 1.279 & 22.5 & -30 & 0.1 \\
\hline 0.305 & 0.0 & -60 & 4.1 \\
\hline 0.305 & .22 .5 & -60 & 3.7 \\
\hline 0.305 & -45.0 & -60 & 3.3 \\
\hline 0.305 & -67.5 & -60 & 3.2 \\
\hline 0.305 & -90.0 & -60 & 3.2 \\
\hline 0.305 & -112.5 & -60 & 3.7 \\
\hline 0.305 & -135.0 & -60 & 3.0 \\
\hline 0.305 & -157.5 & -60 & 2.8 \\
\hline 0.305 & 180.0 & -60 & 2.7 \\
\hline 0.305 & 157.5 & -60 & 2.7 \\
\hline 0.305 & 135.0 & -60 & 2.6 \\
\hline 0.305 & 112.5 & -60 & 3.0 \\
\hline
\end{tabular}

Table C.17 Toaster

$60 \mathrm{~Hz}$ RMS Resultant Fields

Distance Horizontal Vertical $60 \mathrm{~Hz}$

meters angle deg angle deg mGauss

\begin{tabular}{|l|r|r|r|}
\hline 0.305 & 90.0 & -60 & 3.7 \\
\hline 0.305 & 67.5 & -60 & 8.4 \\
\hline
\end{tabular}


Distance Horizontal Vertical $60 \mathrm{~Hz}$

meters angle deg angle deg mGauss

\begin{tabular}{|r|r|r|r|}
\hline 0.605 & 112.5 & -60 & 0.6 \\
\hline 0.605 & 90.0 & -60 & 0.7 \\
\hline 0.605 & 67.5 & -60 & 0.8 \\
\hline 0.605 & 45.0 & -60 & 1.6 \\
\hline 0.605 & 22.5 & -60 & 1.1 \\
\hline 1.000 & 0.0 & -60 & 0.4 \\
\hline 1.000 & -22.5 & -60 & 0.3 \\
\hline 1.000 & -45.0 & -60 & 0.2 \\
\hline 1.000 & -67.5 & -60 & 0.2 \\
\hline 1.000 & -90.0 & -60 & 0.2 \\
\hline 1.000 & -112.5 & -60 & 0.2 \\
\hline 1.000 & -135.0 & -60 & 0.2 \\
\hline
\end{tabular}

Table C.17 Toaster

\section{$60 \mathrm{~Hz}$ RMS Resultant Fields}

Distance Horizontal Vertical $60 \mathrm{~Hz}$

meters angle deg angle deg mGauss

\begin{tabular}{|r|r|r|r|}
\hline 1.000 & -157.5 & -60 & 0.2 \\
\hline 1.000 & 180.0 & -60 & 0.2 \\
\hline 1.000 & 157.5 & -60 & 0.1 \\
\hline 1.000 & 135.0 & -60 & 0.1 \\
\hline 1.000 & 112.5 & -60 & 0.1 \\
\hline 1.000 & 90.0 & -60 & 0.1 \\
\hline 1.000 & 67.5 & -60 & 0.1 \\
\hline 1.000 & 45.0 & -60 & 0.2 \\
\hline 1.000 & 22.5 & -60 & 0.2 \\
\hline 0.178 & 0.0 & -90 & 17.8 \\
\hline 0.278 & 0.0 & -90 & 6.2 \\
\hline
\end{tabular}




\section{FUNDAMENTAL RMS MAGNETIC FIELD TWO SLICE BREAD TOASTER HORIZONTAL SECTION THROUGH CENTER OF TOASTER}

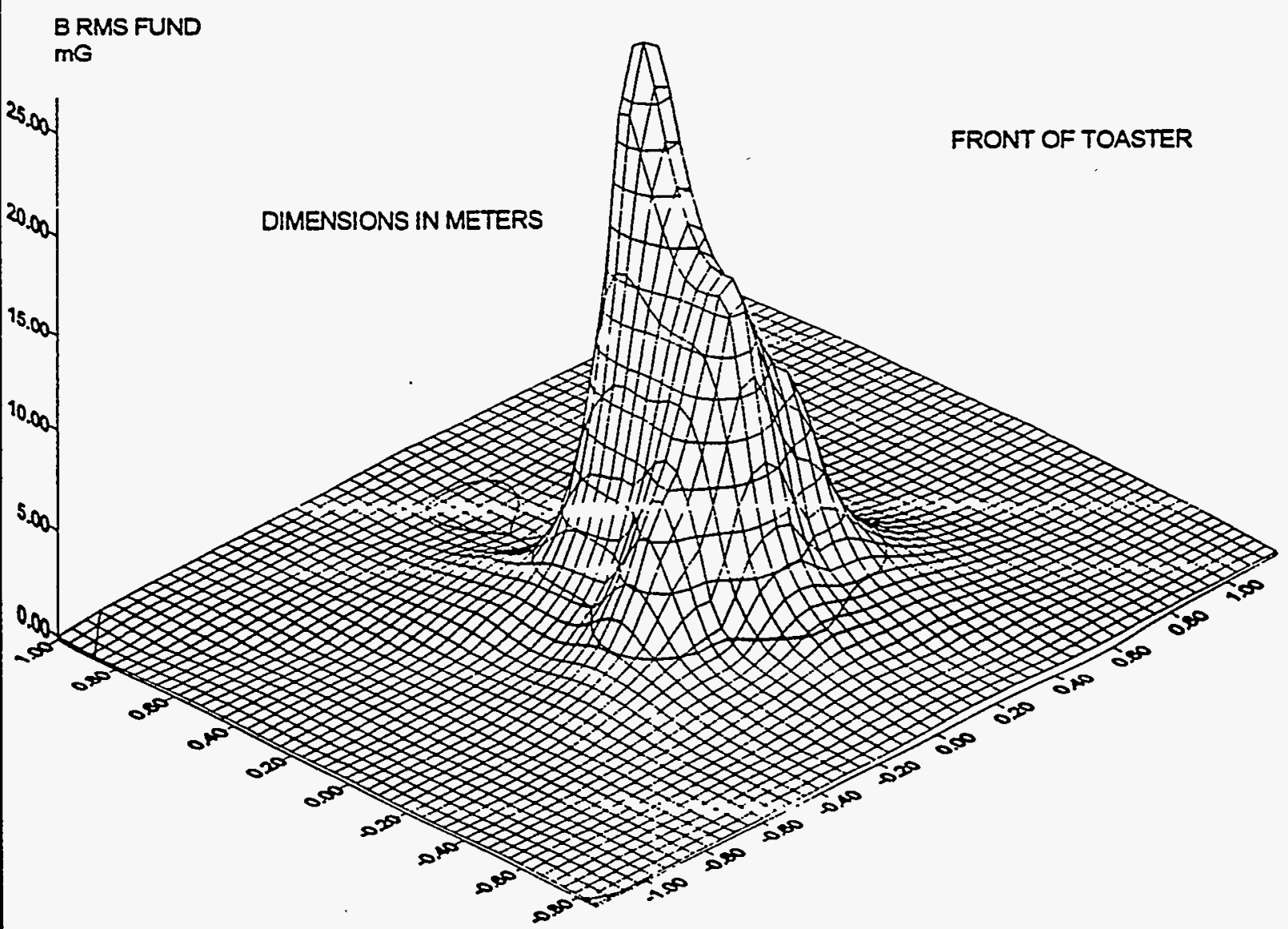

Figure C.17.1 Fundamental RMS Magnetic Field - Two Slice Bread Toaster. 


\section{C.18 Tooth Brush - Electric}

Appliance Tested: An electric tooth brush which charges the batteries in the brush through induction from a stand.

Manufacturer: RRR

Model: 016

Appliance Code: 2263

Conditions for Testing: The unit was charging the tooth brush

Measured voltage and current: 121.7 Volts and 0.024 Amperes.

Magnetic Field Summary:

$60 \mathrm{~Hz}$ RMS resultant level at $30 \mathrm{~cm}$ from front surface: $4.2 \mathrm{mG}$

RMS resultant low surface level: $119 \mathrm{mG}$ bottom

RMS resultant high surface level: $943 \mathrm{mG}$ back

Highest recorded surface levels:

60 Hertz RMS resultant: $939 \mathrm{mG}$

180 Hertz RMS resultant: $33.1 \mathrm{mG}$

300 Hertz RMS resultant: $5.47 \mathrm{mG}$

420 Hertz RMS resultant: $2.23 \mathrm{mG}$

Maximum Total Harmonic Distortion: 8.4 percent

For the appliance test set, the front of the unit was located at 180 degrees on the test table.

Summary of characteristics: The magnetic fields were relatively strong for this small device. 
Table C.18 Tooth Brush - Electric

\section{$60 \mathrm{~Hz}$ RMS Resultant Fields}

Distance Horizontal Vertical $60 \mathrm{~Hz}$ meters angle deg angle deg mGauss

\begin{tabular}{|c|c|c|c|}
\hline 0.178 & 0.0 & 90 & 62.3 \\
\hline 0.278 & 0.0 & 90 & 18.0 \\
\hline 0.478 & 0.0 & 90 & 3.7 \\
\hline 1.000 & 0.0 & 90 & 0.8 \\
\hline 0.102 & 0.0 & 60 & 57.4 \\
\hline 0.102 & -22.5 & 60 & 38.7 \\
\hline 0.102 & -45.0 & 60 & 65.9 \\
\hline 0.102 & -67.5 & 60 & 65.1 \\
\hline 0.102 & -90.0 & 60 & 64.1 \\
\hline 0.102 & -112.5 & 60 & 69.5 \\
\hline 0.102 & -135.0 & 60 & 62.4 \\
\hline 0.102 & -157.5 & 60 & 56.6 \\
\hline 0.102 & 180.0 & 60 & 58.8 \\
\hline 0.902 & 157.5 & 60 & 65.4 \\
\hline 0.102 & 135.0 & 60 & 65.0 \\
\hline 0.102 & 112.5 & 60 & 65.1 \\
\hline 0.102 & 90.0 & 60 & 68.0 \\
\hline 0.102 & 67.5 & 60 & 49.7 \\
\hline 0.102 & 45.0 & 60 & 48.0 \\
\hline 0.102 & 22.5 & 60 & 33.4 \\
\hline 0.202 & 0.0 & 60 & 37.4 \\
\hline 0.202 & -22.5 & 60 & 39.6 \\
\hline 0.202 & -45.0 & 60 & 40.0 \\
\hline 0.202 & -67.5 & 60 & 39.5 \\
\hline 0.202 & -90.0 & 60 & 39.0 \\
\hline 0.202 & -112.5 & 60 & 38.5 \\
\hline 0.202 & -135.0 & 60 & 37.8 \\
\hline 0.202 & -157.5 & 60 & 37.6 \\
\hline 0.202 & 180.0 & 60 & 37.1 \\
\hline 0.202 & 157.5 & 60 & 37.4 \\
\hline
\end{tabular}

Distance Horizontal Vertical $60 \mathrm{~Hz}$ meters angle deg angle deg mGauss

\begin{tabular}{|r|r|r|r|}
\hline 0.202 & 135.0 & 60 & 37.0 \\
\hline 0.202 & 112.5 & 60 & 38.2 \\
\hline 0.202 & 90.0 & 60 & 38.6 \\
\hline 0.202 & 67.5 & 60 & 40.2 \\
\hline 0.202 & 45.0 & 60 & 39.9 \\
\hline 0.202 & 22.5 & 60 & 41.9 \\
\hline 0.402 & 0.0 & 60 & 5.4 \\
\hline 0.402 & -22.5 & 60 & 5.7 \\
\hline 0.402 & -45.0 & 60 & 5.8 \\
\hline 0.402 & -67.5 & 60 & 5.7 \\
\hline 0.402 & -90.0 & 60 & 5.6 \\
\hline 0.402 & -112.5 & 60 & 5.5 \\
\hline 0.402 & -135.0 & 60 & 5.4 \\
\hline 0.402 & -157.5 & 60 & 5.4 \\
\hline 0.402 & 180.0 & 60 & 5.3 \\
\hline 0.402 & 157.5 & 60 & 5.3 \\
\hline 0.402 & 135.0 & 60 & 5.3 \\
\hline 0.402 & 112.5 & 60 & 5.5 \\
\hline 0.402 & 90.0 & 60 & 5.5 \\
\hline 0.402 & 67.5 & 60 & 5.7 \\
\hline 0.402 & 45.0 & 60 & 5.7 \\
\hline 0.402 & 22.5 & 60 & 5.8 \\
\hline 1.000 & 0.0 & 60 & 0.6 \\
\hline 1.000 & -22.5 & 60 & 0.6 \\
\hline 1.000 & -45.0 & 60 & 0.6 \\
\hline 1.000 & -67.5 & 60 & 0.6 \\
\hline 1.000 & -90.0 & 60 & 0.6 \\
\hline 1.000 & -112.5 & 60 & 0.6 \\
\hline 1.000 & -135.0 & 60 & 0.6 \\
\hline 1.000 & -157.5 & 60 & 0.6 \\
\hline
\end{tabular}


Table C.18 Tooth Brush - Electric $60 \mathrm{~Hz}$ RMS Resultant Fields

Distance Horizontal Vertical $60 \mathrm{~Hz}$ meters angle deg angle deg mGauss

\begin{tabular}{|c|c|c|c|}
\hline 1.000 & 180.0 & 60 & 0.6 \\
\hline 1.000 & 157.5 & 60 & 0.6 \\
\hline 1.000 & 135.0 & 60 & 0.6 \\
\hline 1.000 & 112.5 & 60 & 0.6 \\
\hline 1.000 & 90.0 & 60 & 0.6 \\
\hline 1.000 & 67.5 & 60 & 0.6 \\
\hline 1.000 & 45.0 & 60 & 0.6 \\
\hline 1.000 & 22.5 & 60 & 0.6 \\
\hline 0.102 & 0.0 & 30 & 175.6 \\
\hline 0.102 & -22.5 & 30 & 204.7 \\
\hline 0.102 & -45.0 & 30 & 202.7 \\
\hline 0.102 & -67.5 & 30 & 192.9 \\
\hline 0.102 & -90.0 & 30 & 187.7 \\
\hline 0.102 & -112.5 & 30 & 181.1 \\
\hline 0.102 & -135.0 & 30 & $\$ 76.6$ \\
\hline 0.102 & -157.5 & 30 & 180.7 \\
\hline 0.102 & 180.0 & 30 & 177.0 \\
\hline 0.102 & 157.5 & 30 & 187.0 \\
\hline 0.102 & 135.0 & 30 & 177.9 \\
\hline 0.102 & 112.5 & 30 & 198.8 \\
\hline 0.102 & 90.0 & 30 & 204.3 \\
\hline 0.102 & 67.5 & 30 & 224.7 \\
\hline 0.102 & 45.0 & 30 & 214.6 \\
\hline 0.102 & 22.5 & 30 & 237.1 \\
\hline 0.202 & 0.0 & 30 & 27.9 \\
\hline 0.202 & -22.5 & 30 & 30.9 \\
\hline 0.202 & -45.0 & 30 & 31.7 \\
\hline 0.202 & -67.5 & 30 & 30.8 \\
\hline 0.202 & -90.0 & 30 & 30.0 \\
\hline 0.202 & -112.5 & 30 & 29.0 \\
\hline
\end{tabular}

Distance Horizontal Vertical $60 \mathrm{~Hz}$ meters angle deg angle deg mGauss

\begin{tabular}{|r|r|r|r|}
\hline 0.202 & -135.0 & 30 & 28.0 \\
\hline 0.202 & -157.5 & 30 & 27.5 \\
\hline 0.202 & 180.0 & 30 & 26.8 \\
\hline 0.202 & 157.5 & 30 & 27.3 \\
\hline 0.202 & 135.0 & 30 & 26.8 \\
\hline 0.202 & 112.5 & 30 & 28.5 \\
\hline 0.202 & 90.0 & 30 & 29.2 \\
\hline 0.202 & 67.5 & 30 & 31.0 \\
\hline 0.202 & 45.0 & 30 & 30.9 \\
\hline 0.202 & 22.5 & 30 & 32.9 \\
\hline 0.402 & 0.0 & 30 & 3.9 \\
\hline 0.402 & -22.5 & 30 & 4.2 \\
\hline 0.402 & -45.0 & 30 & 4.2 \\
\hline 0.402 & -67.5 & 30 & 4.2 \\
\hline 0.402 & -90.0 & 30 & 4.1 \\
\hline 0.402 & -112.5 & 30 & 3.9 \\
\hline 0.402 & -135.0 & 30 & 3.8 \\
\hline 0.402 & -157.5 & 30 & 3.7 \\
\hline 0.402 & 180.0 & 30 & 3.7 \\
\hline 0.402 & 157.5 & 30 & 3.7 \\
\hline 0.402 & 135.0 & 30 & 3.6 \\
\hline 0.402 & 112.5 & 30 & 3.7 \\
\hline 0.402 & 90.0 & 30 & 3.9 \\
\hline 0.402 & 67.5 & 30 & 4.1 \\
\hline 0.402 & 45.0 & 30 & 4.1 \\
\hline 0.402 & 22.5 & 30 & 4.3 \\
\hline 1.102 & 0.0 & 30 & 0.4 \\
\hline 1.102 & -22.5 & 30 & 0.4 \\
\hline 1.102 & -45.0 & 30 & 0.4 \\
\hline 1.102 & -67.5 & 30 & 0.4 \\
\hline
\end{tabular}


Table C.18 Tooth Brush - Electric $60 \mathrm{~Hz}$ RMS Resultant Fields

Distance Horizontal Vertical $60 \mathrm{~Hz}$ meters angle deg angle deg mGauss

\begin{tabular}{|r|r|r|r|}
\hline 1.102 & -90.0 & 30 & 0.4 \\
\hline 1.102 & -112.5 & 30 & 0.3 \\
\hline 1.102 & -135.0 & 30 & 0.3 \\
\hline 1.102 & -157.5 & 30 & 0.3 \\
\hline 1.102 & 180.0 & 30 & 0.3 \\
\hline 1.102 & 157.5 & 30 & 0.3 \\
\hline 1.102 & 135.0 & 30 & 0.3 \\
\hline 1.102 & 112.5 & 30 & 0.3 \\
\hline 1.102 & 90.0 & 30 & 0.3 \\
\hline 1.102 & 67.5 & 30 & 0.3 \\
\hline 1.102 & 45.0 & 30 & 0.4 \\
\hline 1.102 & 22.5 & 30 & 0.4 \\
\hline 0.089 & 0.0 & 0 & 177.3 \\
\hline 0.089 & -22.5 & 0 & 218.0 \\
\hline 0.089 & -45.0 & 0 & 222.4 \\
\hline 0.089 & -67.5 & 0 & 207.8 \\
\hline 0.089 & -90.0 & 0 & 201.1 \\
\hline 0.089 & -112.5 & 0 & 195.0 \\
\hline 0.089 & -135.0 & 0 & 188.7 \\
\hline 0.089 & -157.5 & 0 & 192.8 \\
\hline 0.089 & 180.0 & 0 & 189.1 \\
\hline 0.089 & 157.5 & 0 & 198.3 \\
\hline 0.089 & 135.0 & 0 & 183.2 \\
\hline 0.089 & 112.5 & 0 & 207.5 \\
\hline 0.089 & 90.0 & 0 & 214.4 \\
\hline 0.089 & 67.5 & 0 & 240.1 \\
\hline 0.089 & 45.0 & 0 & 222.2 \\
\hline 0.089 & 22.5 & 0 & 256.4 \\
\hline 0.189 & 0.0 & 0 & 25.8 \\
\hline 0.189 & -22.5 & 0 & 29.1 \\
\hline
\end{tabular}

Distance Horizontal Vertical $60 \mathrm{~Hz}$

meters angle deg angle deg mGauss

\begin{tabular}{|c|c|c|c|}
\hline 0.189 & -45.0 & 0 & 30.0 \\
\hline 0.189 & -67.5 & 0 & 29.0 \\
\hline 0.189 & -90.0 & 0 & 28.4 \\
\hline 0.189 & -112.5 & 0 & 27.9 \\
\hline 0.189 & -135.0 & 0 & 27.2 \\
\hline 0.189 & -157.5 & 0 & 27.2 \\
\hline 0.189 & 180.0 & 0 & 25.8 \\
\hline 0.189 & 157.5 & 0 & 27.4 \\
\hline 0.189 & 135.0 & 0 & 25.9 \\
\hline 0.189 & 112.5 & 0 & 27.8 \\
\hline 0.189 & 90.0 & 0 & 28.0 \\
\hline 0.189 & 67.5 & 0 & 30.2 \\
\hline 0.189 & 45.0 & 0 & 29.2 \\
\hline 0.189 & 22.5 & 0 & 31.6 \\
\hline 0.389 & 0.0 & 0 & 3.4 \\
\hline 0.389 & -22.5 & 0 & 3.7 \\
\hline 0.389 & -45.0 & 0 & 3.7 \\
\hline 0.389 & -67.5 & 0 & 3.7 \\
\hline 0.389 & -90.0 & 0 & 3.6 \\
\hline 0.389 & -112.5 & 0 & 3.6 \\
\hline 0.389 & -135.0 & 0 & 3.5 \\
\hline 0.389 & -157.5 & 0 & 3.6 \\
\hline 0.389 & 180.0 & 0 & 3.5 \\
\hline 0.389 & 157.5 & 0 & 3.5 \\
\hline 0.389 & 135.0 & 0 & 3.4 \\
\hline 0.389 & 112.5 & 0 & 3.5 \\
\hline 0.389 & 90.0 & 0 & 3.5 \\
\hline 0.389 & 67.5 & 0 & 3.7 \\
\hline 0.389 & 45.0 & 0 & 3.6 \\
\hline 0.389 & 22.5 & 0 & 3.7 \\
\hline
\end{tabular}


Table C.18 Tooth Brush - Electric $60 \mathrm{~Hz}$ RMS Resultant Fields

Distance Horizontal Vertical $60 \mathrm{~Hz}$ meters angle deg angle deg mGauss

\begin{tabular}{|c|c|c|c|}
\hline 1.089 & 0.0 & 0 & 0.1 \\
\hline 9.089 & -22.5 & 0 & 0.1 \\
\hline 1.089 & -45.0 & 0 & 0.1 \\
\hline 1.089 & -67.5 & 0 & 0.1 \\
\hline 1.089 & -90.0 & 0 & 0.1 \\
\hline 1.089 & -112.5 & 0 & 0.1 \\
\hline 1.089 & -135.0 & 0 & 0.1 \\
\hline 1.089 & -157.5 & 0 & 0.1 \\
\hline 1.089 & 980.0 & 0 & 0.1 \\
\hline 1.089 & 157.5 & 0 & 0.1 \\
\hline 1.089 & 135.0 & 0 & 0.1 \\
\hline 1.089 & 112.5 & 0 & 0.1 \\
\hline 1.089 & 90.0 & 0 & 0.1 \\
\hline 1.089 & 67.5 & 0 & 0.1 \\
\hline 1.089 & 45.0 & 0 & 0.1 \\
\hline 1.089 & 22.5 & 0 & 0.1 \\
\hline 0.279 & 0.0 & -30 & 9.9 \\
\hline 0.279 & -22.5 & -30 & 10.7 \\
\hline 0.279 & -45.0 & -30 & 11.2 \\
\hline 0.279 & -67.5 & -30 & 11.0 \\
\hline 0.279 & -90.0 & -30 & 11.3 \\
\hline 0.279 & -112.5 & -30 & 11.6 \\
\hline 0.279 & -135.0 & .30 & 11.6 \\
\hline 0.279 & -157.5 & -30 & 11.8 \\
\hline 0.279 & 180.0 & .30 & 11.7 \\
\hline 0.279 & 157.5 & .30 & 12.1 \\
\hline 0.279 & 135.0 & -30 & 11.2 \\
\hline 0.279 & 112.5 & -30 & 11.8 \\
\hline 0.279 & 90.0 & .30 & 11.6 \\
\hline 0.279 & 67.5 & .30 & 12.2 \\
\hline
\end{tabular}

Distance Horizontal Vertical $60 \mathrm{~Hz}$

meters angle deg angle deg mGauss

\begin{tabular}{|r|r|r|r|}
\hline 0.279 & 45.0 & -30 & 11.3 \\
\hline 0.279 & 22.5 & -30 & 11.9 \\
\hline 0.379 & 0.0 & -30 & 4.1 \\
\hline 0.379 & -22.5 & -30 & 4.4 \\
\hline 0.379 & -45.0 & -30 & 4.5 \\
\hline 0.379 & -57.5 & -30 & 4.5 \\
\hline 0.379 & -90.0 & -30 & 4.6 \\
\hline 0.379 & -112.5 & -30 & 4.7 \\
\hline 0.379 & -135.0 & -30 & 4.7 \\
\hline 0.379 & -157.5 & -30 & 4.9 \\
\hline 0.379 & 180.0 & -30 & 4.9 \\
\hline 0.379 & 157.5 & -30 & 5.0 \\
\hline 0.379 & 135.0 & -30 & 4.7 \\
\hline 0.379 & 112.5 & -30 & 4.8 \\
\hline 0.379 & 90.0 & -30 & 4.7 \\
\hline 0.379 & 67.5 & -30 & 5.0 \\
\hline 0.379 & 45.0 & -30 & 4.6 \\
\hline 0.379 & 22.5 & -30 & 4.8 \\
\hline 0.579 & 0.0 & -30 & 1.4 \\
\hline 0.579 & -22.5 & -30 & 1.4 \\
\hline 0.579 & -45.0 & -30 & 1.4 \\
\hline 0.579 & -67.5 & -30 & 1.4 \\
\hline 0.579 & -90.0 & -30 & 1.4 \\
\hline 0.579 & -112.5 & -30 & 1.5 \\
\hline 0.579 & -135.0 & -30 & 1.5 \\
\hline 0.579 & -157.5 & -30 & 1.6 \\
\hline 0.579 & 180.0 & -30 & 1.5 \\
\hline 0.579 & 157.5 & -30 & 1.6 \\
\hline 0.579 & 135.0 & -30 & 1.5 \\
\hline 0.579 & 112.5 & -30 & 1.5 \\
\hline
\end{tabular}


Table C:18 Tooth Brush - Electric $60 \mathrm{~Hz}$ RMS Resultant Fields

Distance Horizontal Vertical $60 \mathrm{~Hz}$ meters angle deg angle deg mGauss

\begin{tabular}{|r|r|r|r|}
\hline 0.579 & 90.0 & -30 & 1.5 \\
\hline 0.579 & 67.5 & -30 & 1.5 \\
\hline 0.579 & 45.0 & -30 & 1.5 \\
\hline 0.579 & 22.5 & -30 & 1.5 \\
\hline 1.279 & 0.0 & -30 & 0.1 \\
\hline 1.279 & -22.5 & -30 & 0.1 \\
\hline 1.279 & -45.0 & -30 & 0.1 \\
\hline 1.279 & -67.5 & -30 & 0.1 \\
\hline 1.279 & -90.0 & -30 & 0.2 \\
\hline 1.279 & -112.5 & -30 & 0.2 \\
\hline 1.279 & -135.0 & -30 & 0.2 \\
\hline 1.279 & -157.5 & -30 & 0.2 \\
\hline 1.279 & 180.0 & -30 & 0.2 \\
\hline 1.279 & 157.5 & -30 & 0.2 \\
\hline 1.279 & 135.0 & -30 & 0.2 \\
\hline 1.279 & 112.5 & -30 & 0.2 \\
\hline 1.279 & 90.0 & -30 & 0.2 \\
\hline 1.279 & 67.5 & -30 & 0.2 \\
\hline 1.279 & 45.0 & -30 & 0.2 \\
\hline 1.279 & 22.5 & -30 & 0.2 \\
\hline 0.330 & 0.0 & -60 & 8.4 \\
\hline 0.330 & -22.5 & -60 & 8.8 \\
\hline 0.330 & -45.0 & -60 & 9.0 \\
\hline 0.330 & -67.5 & -60 & 8.9 \\
\hline 0.330 & -90.0 & -60 & 9.0 \\
\hline 0.330 & -112.5 & -60 & 9.1 \\
\hline 0.330 & -135.0 & -60 & 9.0 \\
\hline 0.330 & -157.5 & -60 & 8.9 \\
\hline 0.330 & 180.0 & -60 & 9.2 \\
\hline 0.330 & 157.5 & -60 & 9.2 \\
\hline
\end{tabular}

Distance Horizontal Vertical $60 \mathrm{~Hz}$ meters angle deg angle deg mGauss

\begin{tabular}{|c|c|c|c|}
\hline 0.330 & 135.0 & -60 & 9.0 \\
\hline 0.330 & 112.5 & -60 & 9.1 \\
\hline 0.330 & 90.0 & -60 & 9.0 \\
\hline 0.330 & 67.5 & -60 & 9.3 \\
\hline 0.330 & 45.0 & -60 & 8.9 \\
\hline 0.330 & 22.5 & -60 & 9.3 \\
\hline 0.430 & 0.0 & -60 & 4.1 \\
\hline 0.430 & -22.5 & -60 & 4.1 \\
\hline 0.430 & -45.0 & -60 & 4.2 \\
\hline 0.430 & -67.5 & -60 & 4.2 \\
\hline 0.430 & -90.0 & -60 & 4.2 \\
\hline 0.430 & -112.5 & -60 & 4.3 \\
\hline 0.430 & -135.0 & -60 & 4.3 \\
\hline 0.430 & -157.5 & -60 & 4.2 \\
\hline 0.430 & 180.0 & -60 & 4.4 \\
\hline 0.430 & 157.5 & -60 & 4.4 \\
\hline 0.430 & 135.0 & -60 & 4.3 \\
\hline 0.430 & 112.5 & -60 & 4.3 \\
\hline 0.430 & 90.0 & -60 & 4.3 \\
\hline 0.430 & 67.5 & -60 & 4.4 \\
\hline 0.430 & 45.0 & -60 & 4.2 \\
\hline 0.430 & 22.5 & -60 & 4.3 \\
\hline 0.630 & 0.0 & -60 & 4.5 \\
\hline 0.630 & -22.5 & -60 & 1.5 \\
\hline 0.630 & -45.0 & -60 & 1.5 \\
\hline 0.630 & -67.5 & -60 & 1.5 \\
\hline 0.630 & -90.0 & -60 & 1.5 \\
\hline 0.630 & -112.5 & -60 & 1.6 \\
\hline 0.630 & -135.0 & -60 & 1.6 \\
\hline 0.630 & -157.5 & -60 & 1.6 \\
\hline
\end{tabular}


Table C.18 Tooth Brush - Electric

$60 \mathrm{~Hz}$ RMS Resultant Fields

Distance Horizontal Vertical $60 \mathrm{~Hz}$

Distance Horizontal Vertical $60 \mathrm{~Hz}$

meters angle deg angle deg mGauss

meters angle deg angle deg mGauss

\begin{tabular}{|l|r|r|r|}
\hline 0.630 & 180.0 & -60 & 1.6 \\
\hline 0.630 & 157.5 & -60 & 1.6 \\
\hline 0.630 & 135.0 & -60 & 1.6 \\
\hline 0.630 & 112.5 & -60 & 1.6 \\
\hline 0.630 & 90.0 & -60 & 1.6 \\
\hline 0.630 & 67.5 & -60 & 1.7 \\
\hline 0.630 & 45.0 & -60 & 1.6 \\
\hline 0.630 & 22.5 & -60 & 1.6 \\
\hline 1.000 & 0.0 & -60 & 0.4 \\
\hline 1.000 & -22.5 & -60 & 0.4 \\
\hline 1.000 & -45.0 & -60 & 0.4 \\
\hline 1.000 & -67.5 & -60 & 0.4 \\
\hline 1.000 & -90.0 & -60 & 0.4 \\
\hline 1.000 & -112.5 & -60 & 0.4 \\
\hline
\end{tabular}

\begin{tabular}{|r|r|r|r|}
\hline 1.000 & -135.0 & -60 & 0.4 \\
\hline 1.000 & -157.5 & -60 & 0.4 \\
\hline 1.000 & 180.0 & -60 & 0.4 \\
\hline 1.000 & 157.5 & -60 & 0.4 \\
\hline 1.000 & 135.0 & -60 & 0.4 \\
\hline 1.000 & 112.5 & -60 & 0.4 \\
\hline 1.000 & 90.0 & -60 & 0.4 \\
\hline 1.000 & 67.5 & -60 & 0.4 \\
\hline 1.000 & 45.0 & -60 & 0.4 \\
\hline 1.000 & 22.5 & -60 & 0.4 \\
\hline 0.127 & 0.0 & -90 & 177.2 \\
\hline 0.227 & 0.0 & -90 & 33.7 \\
\hline 0.427 & 0.0 & -90 & 5.6 \\
\hline
\end{tabular}

C.18-7 


\section{RESULTANT RMS MAGNETIC FIELD DENSITY \\ ELECTRIC TOOTHBRUSH \\ HORIZONTAL SLICE THROUGH CENTER OF APPLIANCE}

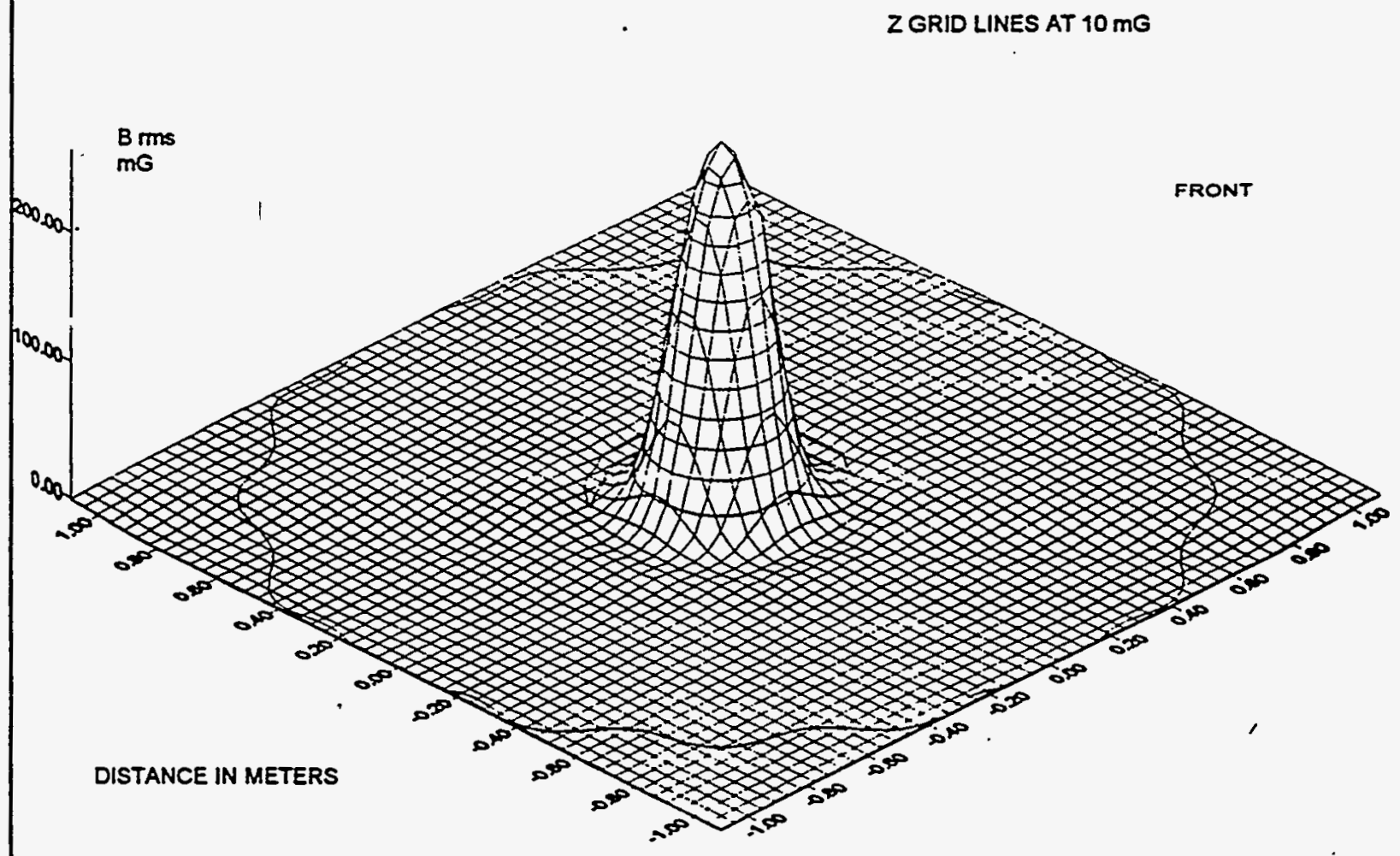

Figure C.18.1 Fundamental Resultant RMS Magnetic Field Density - Electric Toothbrush.

C. $18-8$ 


\section{C.19 Vacuum Sweeper - Upright}

Appliance Tested: A upright style vacuum sweeper as found in the home Manufacturer: SSS

Model: 017

Rated current: 10.0 Amperes

Appliance Code: 3253

Conditions for Testing: The unit was running without load

Measured voltage and current: 116.1 Volts and 8.14 Amperes.

Magnetic Field Summary:

$60 \mathrm{~Hz}$ RMS resultant level at $30 \mathrm{~cm}$ from front surface: $18.6 \mathrm{mG}$

RMS resultant low surface level: $176 \mathrm{mG}$

RMS resultant high surface level: $5157 \mathrm{mG}$ front

Highest recorded surface levels:

60 Hertz RMS resultant: $4711 \mathrm{mG}$

180 Hertz RMS resultant: $2049 \mathrm{mG}$

300 Hertz RMS resultant: $377 \mathrm{mG}$

420 Hertz RMS resultant: $129 \mathrm{mG}$

Maximum recorded RMS resultant in the $3-30 \mathrm{kHz}$ range: $5.9 \mathrm{mG}$

For the appliance test set, the front of the unit was located at 0 degrees on the test table. The handle was removed.

Summary of characteristics: The magnetic fields were relatively strong. 
Table C.19 Vacuum Sweeper - Upright $60 \mathrm{~Hz}$ RMS Resultant Fields

Distance Horizontal Vertical $60 \mathrm{~Hz}$ meters angle deg angle deg mGauss

\begin{tabular}{|r|r|r|r|}
\hline 0.089 & 0.0 & 90 & 1505.3 \\
\hline 0.189 & 0.0 & 90 & 247.0 \\
\hline 0.389 & 0.0 & 90 & 28.1 \\
\hline 1.000 & 0.0 & 90 & 1.8 \\
\hline 0.165 & 0.0 & 60 & 58.6 \\
\hline 0.165 & -22.5 & 60 & 86.7 \\
\hline 0.165 & -45.0 & 60 & 121.6 \\
\hline 0.165 & -67.5 & 60 & 169.1 \\
\hline 0.165 & -90.0 & 60 & 228.6 \\
\hline 0.165 & -112.5 & 60 & 304.4 \\
\hline 0.165 & -135.0 & 60 & 364.3 \\
\hline 0.165 & -157.5 & 60 & 396.7 \\
\hline 0.165 & 180.0 & 60 & 397.6 \\
\hline 0.165 & 157.5 & 60 & 402.6 \\
\hline 0.165 & 135.0 & 60 & 307.2 \\
\hline 0.165 & 112.5 & 60 & 195.5 \\
\hline 0.165 & 90.0 & 60 & 111.6 \\
\hline 0.165 & 67.5 & 60 & 53.7 \\
\hline 0.165 & 45.0 & 60 & 22.4 \\
\hline 0.165 & 22.5 & 60 & 43.8 \\
\hline 0.265 & 0.0 & 60 & 82.8 \\
\hline 0.265 & -22.5 & 60 & 81.7 \\
\hline 0.265 & -45.0 & 60 & 79.5 \\
\hline 0.265 & -67.5 & 60 & 75.6 \\
\hline 0.265 & -90.0 & 60 & 72.1 \\
\hline 0.265 & -112.5 & 60 & 77.2 \\
\hline 0.265 & -135.0 & 60 & 90.8 \\
\hline 0.265 & -157.5 & 60 & 104.3 \\
\hline 0.265 & 180.0 & 60 & 108.8 \\
\hline 0.265 & 157.5 & 60 & 97.7 \\
\hline
\end{tabular}

Distance Horizontal Vertical $60 \mathrm{~Hz}$ meters angle deg angle deg mGauss

\begin{tabular}{|r|r|r|r|}
\hline 0.265 & 135.0 & 60 & 83.9 \\
\hline 0.265 & 112.5 & 60 & 75.5 \\
\hline 0.265 & 90.0 & 60 & 75.5 \\
\hline 0.265 & 67.5 & 60 & 76.9 \\
\hline 0.265 & 45.0 & 60 & 82.6 \\
\hline 0.265 & 22.5 & 60 & 87.2 \\
\hline 0.465 & 0.0 & 60 & 18.8 \\
\hline 0.465 & -22.5 & 60 & 18.4 \\
\hline 0.465 & -45.0 & 60 & 17.6 \\
\hline 0.465 & -67.5 & 60 & 16.2 \\
\hline 0.465 & -90.0 & 60 & 14.9 \\
\hline 0.465 & -112.5 & 60 & 15.4 \\
\hline 0.465 & -135.0 & 60 & 17.2 \\
\hline 0.465 & -157.5 & 60 & 19.0 \\
\hline 0.465 & 180.0 & 60 & 19.6 \\
\hline 0.465 & 157.5 & 60 & 18.7 \\
\hline 0.465 & 135.0 & 60 & 16.9 \\
\hline 0.465 & 112.5 & 60 & 15.5 \\
\hline 0.465 & 90.0 & 60 & 15.5 \\
\hline 0.465 & 67.5 & 60 & 16.3 \\
\hline 0.465 & 45.0 & 60 & 18.0 \\
\hline 0.465 & 22.5 & 60 & 19.5 \\
\hline 1.000 & 0.0 & 60 & 1.9 \\
\hline 1.000 & -22.5 & 60 & 2.0 \\
\hline 1.000 & -45.0 & 60 & 2.0 \\
\hline 1.000 & -67.5 & 60 & 1.9 \\
\hline 1.000 & -90.0 & 60 & 1.7 \\
\hline 1.000 & -112.5 & 60 & 1.6 \\
\hline 1.000 & -135.0 & 60 & 1.8 \\
\hline 1.000 & -157.5 & 60 & 2.9 \\
\hline
\end{tabular}


Table C.19 Vacuum Sweeper - Upright $60 \mathrm{~Hz}$ RMS Resultant Fields

Distance Horizontal Vertical $60 \mathrm{~Hz}$ meters angle deg angle deg mGauss

\begin{tabular}{|r|r|r|r|}
\hline 1.000 & 180.0 & 60 & 1.9 \\
\hline 1.000 & 157.5 & 60 & 1.9 \\
\hline 1.000 & 135.0 & 60 & 1.8 \\
\hline 1.000 & 112.5 & 60 & 1.5 \\
\hline 1.000 & 90.0 & 60 & 1.7 \\
\hline 1.000 & 67.5 & 60 & 1.8 \\
\hline 1.000 & 45.0 & 60 & 1.9 \\
\hline 1.000 & 22.5 & 60 & 2.0 \\
\hline 0.178 & 0.0 & 30 & 311.7 \\
\hline 0.178 & -22.5 & 30 & 304.5 \\
\hline 0.178 & -45.0 & 30 & 297.7 \\
\hline 0.178 & -67.5 & 30 & 239.9 \\
\hline 0.178 & -90.0 & 30 & 266.5 \\
\hline 0.178 & -112.5 & 30 & 369.1 \\
\hline 0.178 & -135.0 & 30 & 666.7 \\
\hline 0.178 & -157.5 & 30 & 1203.5 \\
\hline 0.178 & 180.0 & 30 & 1373.0 \\
\hline 0.178 & 157.5 & 30 & 910.4 \\
\hline 0.178 & 135.0 & 30 & 447.6 \\
\hline 0.178 & 112.5 & 30 & 317.8 \\
\hline 0.178 & 90.0 & 30 & 308.9 \\
\hline 0.178 & 67.5 & 30 & 313.5 \\
\hline 0.178 & 45.0 & 30 & 331.5 \\
\hline 0.178 & 22.5 & 30 & 341.3 \\
\hline 0.278 & 0.0 & 30 & 88.8 \\
\hline 0.278 & -22.5 & 30 & 87.8 \\
\hline 0.278 & -45.0 & 30 & 86.8 \\
\hline 0.278 & -57.5 & 30 & 85.4 \\
\hline 0.278 & -90.0 & 30 & 88.4 \\
\hline 0.278 & -112.5 & 30 & 109.9 \\
\hline
\end{tabular}

Distance Horizontal Vertical $60 \mathrm{~Hz}$ meters angle deg angle deg mGauss

\begin{tabular}{|c|c|c|c|}
\hline 0.278 & -135.0 & 30 & 148.5 \\
\hline 0.278 & -157.5 & 30 & 159.9 \\
\hline 0.278 & 180.0 & 30 & 163.1 \\
\hline 0.278 & 157.5 & 30 & 178.4 \\
\hline 0.278 & 135.0 & 30 & 135.7 \\
\hline 0.278 & 112.5 & 30 & 96.3 \\
\hline 0.278 & 90.0 & 30 & 83.3 \\
\hline 0.278 & 67.5 & 30 & 82.1 \\
\hline 0.278 & 45.0 & 30 & 88.4 \\
\hline 0.278 & 22.5 & 30 & 94.0 \\
\hline 0.478 & 0.0 & 30 & 21.8 \\
\hline 0.478 & -22.5 & 30 & 21.1 \\
\hline 0.478 & -45.0 & 30 & 19.5 \\
\hline 0.478 & -67.5 & 30 & 16.9 \\
\hline 0.478 & -90.0 & 30 & 15.1 \\
\hline 0.478 & -112.5 & 30 & 18.0 \\
\hline 0.478 & -135.0 & 30 & 24.8 \\
\hline 0.478 & .157 .5 & 30 & 32.0 \\
\hline 0.478 & 180.0 & 30 & 33.4 \\
\hline 0.478 & 157.5 & 30 & 31.2 \\
\hline 0.478 & 135.0 & 30 & 22.8 \\
\hline 0.478 & 112.5 & 30 & 16.8 \\
\hline 0.478 & 90.0 & 30 & 16.0 \\
\hline 0.478 & 67.5 & 30 & 17.9 \\
\hline 0.478 & 45.0 & 30 & 20.7 \\
\hline 0.478 & 22.5 & 30 & 22.9 \\
\hline 1.178 & 0.0 & 30 & 1.8 \\
\hline 1.178 & -22.5 & 30 & 1.9 \\
\hline 1.178 & -45.0 & 30 & 1.9 \\
\hline 1.178 & -67.5 & 30 & 1.7 \\
\hline
\end{tabular}


Table C.19 Vacuum Sweeper - Upright $60 \mathrm{~Hz}$ RMS Resultant Fields

Distance Horizontal Vertical $60 \mathrm{~Hz}$

meters angle deg angle deg mGauss

\begin{tabular}{|r|r|r|r|}
\hline 1.178 & -90.0 & 30 & 1.2 \\
\hline 1.178 & -112.5 & 30 & 1.5 \\
\hline 1.178 & -135.0 & 30 & 1.9 \\
\hline 1.178 & -157.5 & 30 & 2.0 \\
\hline 1.178 & 180.0 & 30 & 2.0 \\
\hline 1.178 & 157.5 & 30 & 2.1 \\
\hline 1.178 & 135.0 & 30 & 1.9 \\
\hline 1.178 & 112.5 & 30 & 1.3 \\
\hline 1.178 & 90.0 & 30 & 1.2 \\
\hline 1.178 & 67.5 & 30 & 1.6 \\
\hline 1.178 & 45.0 & 30 & 1.8 \\
\hline 1.178 & 22.5 & 30 & 1.8 \\
\hline 0.178 & 0.0 & 0 & 169.3 \\
\hline 0.178 & -22.5 & 0 & 166.2 \\
\hline 0.178 & -45.0 & 0 & 163.3 \\
\hline 0.178 & -67.5 & 0 & 152.5 \\
\hline 0.178 & -90.0 & 0 & 146.4 \\
\hline 0.178 & -112.5 & 0 & 195.1 \\
\hline 0.178 & -135.0 & 0 & 338.7 \\
\hline 0.178 & -157.5 & 0 & 568.5 \\
\hline 0.178 & 180.0 & 0 & 660.7 \\
\hline 0.178 & 157.5 & 0 & 500.5 \\
\hline 0.178 & 135.0 & 0 & 273.4 \\
\hline 0.178 & 112.5 & 0 & 170.9 \\
\hline 0.178 & 90.0 & 0 & 157.4 \\
\hline 0.178 & 67.5 & 0 & 164.2 \\
\hline 0.178 & 45.0 & 0 & 178.8 \\
\hline 0.178 & 22.5 & 0 & 183.8 \\
\hline 0.278 & 0.0 & 0 & 61.1 \\
\hline 0.278 & -22.5 & 0 & 59.8 \\
\hline
\end{tabular}

Distance Horizontal Vertical $60 \mathrm{~Hz}$

meters angle deg angle deg mGauss

\begin{tabular}{|c|c|c|c|}
\hline 0.278 & -45.0 & 0 & 57.4 \\
\hline 0.278 & -67.5 & 0 & 51.7 \\
\hline 0.278 & -90.0 & 0 & 47.4 \\
\hline 0.278 & -112.5 & 0 & 60.9 \\
\hline 0.278 & -135.0 & 0 & 94.7 \\
\hline 0.278 & -157.5 & 0 & 125.1 \\
\hline 0.278 & 180.0 & 0 & 131.8 \\
\hline 0.278 & 157.5 & 0 & 128.7 \\
\hline 0.278 & 135.0 & 0 & 88.9 \\
\hline 0.278 & 112.5 & 0 & 56.8 \\
\hline 0.278 & 90.0 & 0 & 49.6 \\
\hline 0.278 & 67.5 & 0 & 53.1 \\
\hline 0.278 & 45.0 & 0 & 60.5 \\
\hline 0.278 & 22.5 & 0 & 64.8 \\
\hline 0.478 & 0.0 & 0 & 17.2 \\
\hline 0.478 & -22.5 & 0 & 16.6 \\
\hline 0.478 & -45.0 & 0 & 15.3 \\
\hline 0.478 & -67.5 & 0 & 13.1 \\
\hline 0.478 & -90.0 & 0 & 11.4 \\
\hline 0.478 & -112.5 & 0 & 14.2 \\
\hline 0.478 & -135.0 & 0 & 20.8 \\
\hline 0.478 & -157.5 & 0 & 27.9 \\
\hline 0.478 & 180.0 & 0 & 30.3 \\
\hline 0.478 & 157.5 & 0 & 28.3 \\
\hline 0.478 & 135.0 & 0 & 20.8 \\
\hline 0.478 & 112.5 & 0 & 14.1 \\
\hline 0.478 & 90.0 & 0 & 11.8 \\
\hline 0.478 & 67.5 & 0 & 13.1 \\
\hline 0.478 & 45.0 & 0 & 15.8 \\
\hline 0.478 & 22.5 & 0 & 17.7 \\
\hline
\end{tabular}


Table C.19 Vacuum Sweeper - Upright $60 \mathrm{~Hz}$ RMS Resultant Fields

Distance Horizontal Vertical $60 \mathrm{~Hz}$ meters angle deg angle deg mGauss

\begin{tabular}{|r|r|r|r|}
\hline 1.178 & 0.0 & 0 & 1.5 \\
\hline 1.178 & -22.5 & 0 & 1.6 \\
\hline 1.178 & -45.0 & 0 & 1.5 \\
\hline 1.178 & -67.5 & 0 & 1.2 \\
\hline 1.178 & -90.0 & 0 & 1.0 \\
\hline 1.178 & -112.5 & 0 & 1.1 \\
\hline 1.178 & -135.0 & 0 & 1.6 \\
\hline 1.178 & -157.5 & 0 & 1.7 \\
\hline 1.178 & 180.0 & 0 & 1.7 \\
\hline 1.178 & 157.5 & 0 & 1.8 \\
\hline 1.178 & 135.0 & 0 & 1.7 \\
\hline 1.178 & 112.5 & 0 & 1.2 \\
\hline 1.178 & 90.0 & 0 & 0.9 \\
\hline 1.178 & 67.5 & 0 & 1.1 \\
\hline 1.178 & 45.0 & 0 & 1.5 \\
\hline 1.178 & 22.5 & 0 & 1.6 \\
\hline 0.279 & 0.0 & -30 & 73.4 \\
\hline 0.279 & -22.5 & -30 & 72.3 \\
\hline 0.279 & -45.0 & -30 & 71.2 \\
\hline 0.279 & -67.5 & -30 & 65.8 \\
\hline 0.279 & -90.0 & -30 & 64.3 \\
\hline 0.279 & -112.5 & -30 & 83.4 \\
\hline 0.279 & -135.0 & -30 & 121.3 \\
\hline 0.279 & -157.5 & -30 & 167.2 \\
\hline 0.279 & 180.0 & -30 & 184.8 \\
\hline 0.279 & 157.5 & -30 & 164.8 \\
\hline 0.279 & 135.0 & -30 & 121.2 \\
\hline 0.279 & 112.5 & -30 & 82.8 \\
\hline 0.279 & 90.0 & -30 & 66.7 \\
\hline 0.279 & 67.5 & -30 & 67.0 \\
\hline
\end{tabular}

Distance Horizontal Vertical $60 \mathrm{~Hz}$ meters angle deg angle deg mGauss

\begin{tabular}{|r|r|r|r|}
\hline 0.279 & 45.0 & -30 & 73.9 \\
\hline 0.279 & 22.5 & -30 & 78.5 \\
\hline 0.379 & 0.0 & -30 & 31.2 \\
\hline 0.379 & -22.5 & -30 & 30.4 \\
\hline 0.379 & -45.0 & -30 & 28.7 \\
\hline 0.379 & -67.5 & -30 & 25.7 \\
\hline 0.379 & -90.0 & -30 & 24.1 \\
\hline 0.379 & -112.5 & -30 & 31.1 \\
\hline 0.379 & -135.0 & -30 & 44.5 \\
\hline 0.379 & -157.5 & -30 & 59.0 \\
\hline 0.379 & 180.0 & -30 & 64.2 \\
\hline 0.379 & 157.5 & -30 & 58.6 \\
\hline 0.379 & 135.0 & -30 & 44.9 \\
\hline 0.379 & 112.5 & -30 & 31.0 \\
\hline 0.379 & 90.0 & -30 & 25.0 \\
\hline 0.379 & 67.5 & -30 & 25.7 \\
\hline 0.379 & 45.0 & -30 & 29.6 \\
\hline 0.379 & 22.5 & -30 & 32.6 \\
\hline 0.579 & 0.0 & -30 & 10.8 \\
\hline 0.579 & -22.5 & -30 & 10.5 \\
\hline 0.579 & -45.0 & -30 & 9.5 \\
\hline 0.579 & -67.5 & -30 & 8.1 \\
\hline 0.579 & -90.0 & -30 & 7.4 \\
\hline 0.579 & -112.5 & -30 & 9.6 \\
\hline 0.579 & -135.0 & -30 & 13.4 \\
\hline 0.579 & -157.5 & -30 & 17.1 \\
\hline 0.579 & 180.0 & -30 & 18.3 \\
\hline 0.579 & 157.5 & -30 & 16.9 \\
\hline 0.579 & 135.0 & -30 & 13.4 \\
\hline 0.579 & 112.5 & -30 & 9.5 \\
\hline
\end{tabular}


Table C.19 Vacuum Sweeper-Upright $60 \mathrm{~Hz}$ RMS Resultant Fields

Distance Horizontal Vertical $60 \mathrm{~Hz}$ meters angle deg angle deg mGauss

\begin{tabular}{|r|r|r|r|}
\hline 0.579 & 90.0 & -30 & 7.6 \\
\hline 0.579 & 67.5 & -30 & 8.1 \\
\hline 0.579 & 45.0 & -30 & 9.8 \\
\hline 0.579 & 22.5 & -30 & 11.1 \\
\hline 1.279 & 0.0 & -30 & 1.3 \\
\hline 1.279 & -22.5 & -30 & 1.3 \\
\hline 1.279 & -45.0 & -30 & 1.1 \\
\hline 1.279 & -67.5 & -30 & 1.0 \\
\hline 1.279 & -90.0 & -30 & 0.8 \\
\hline 1.279 & -112.5 & -30 & 0.9 \\
\hline 1.279 & -135.0 & -30 & 1.4 \\
\hline 1.279 & -157.5 & -30 & 1.5 \\
\hline 1.279 & 180.0 & -30 & 1.5 \\
\hline 1.279 & 157.5 & -30 & 1.5 \\
\hline 1.279 & 135.0 & -30 & 1.4 \\
\hline 1.279 & 112.5 & -30 & 1.1 \\
\hline 1.279 & 90.0 & -30 & 0.8 \\
\hline 1.279 & 67.5 & -30 & 0.8 \\
\hline 1.279 & 45.0 & -30 & 1.0 \\
\hline 1.279 & 22.5 & -30 & 1.3 \\
\hline 0.305 & 0.0 & -60 & 49.6 \\
\hline 0.305 & -22.5 & -60 & 48.2 \\
\hline 0.305 & -45.0 & -60 & 47.5 \\
\hline 0.305 & -67.5 & -60 & 44.7 \\
\hline 0.305 & -90.0 & -60 & 46.3 \\
\hline 0.305 & -112.5 & -60 & 56.9 \\
\hline 0.305 & -135.0 & -60 & 71.5 \\
\hline 0.305 & -157.5 & -60 & 85.3 \\
\hline 0.305 & 180.0 & -60 & 85.5 \\
\hline 0.305 & 157.5 & -60 & 77.5 \\
\hline
\end{tabular}

Distance Horizontal Vertical $60 \mathrm{~Hz}$ meters angle deg angle deg maauss

\begin{tabular}{|c|c|c|c|}
\hline 0.305 & 135.0 & -60 & 63.5 \\
\hline 0.305 & 112.5 & -60 & 51.5 \\
\hline 0.305 & 90.0 & -60 & 46.5 \\
\hline 0.305 & 67.5 & -60 & 48.2 \\
\hline 0.305 & 45.0 & -60 & 50.8 \\
\hline 0.305 & 22.5 & -60 & 53.2 \\
\hline 0.405 & 0.0 & -60 & 21.4 \\
\hline 0.405 & -22.5 & .60 & 20.9 \\
\hline 0.405 & -45.0 & .60 & 20.2 \\
\hline 0.405 & -67.5 & .60 & 19.2 \\
\hline 0.405 & -90.0 & -60 & 19.8 \\
\hline 0.405 & -912.5 & -60 & 23.7 \\
\hline 0.405 & -135.0 & -60 & 29.0 \\
\hline 0.405 & -157.5 & -60 & 34.0 \\
\hline 0.405 & 180.0 & -60 & 34.8 \\
\hline 0.405 & 157.5 & -60 & 32.6 \\
\hline 0.405 & 135.0 & -60 & 27.7 \\
\hline 0.405 & 112.5 & -60 & 22.8 \\
\hline 0.405 & 90.0 & -60 & 19.9 \\
\hline 0.405 & 67.5 & -60 & 19.0 \\
\hline 0.405 & 45.0 & -60 & 20.7 \\
\hline 0.405 & 22.5 & -60 & 22.3 \\
\hline 0.605 & 0.0 & -60 & 7.1 \\
\hline 0.605 & -22.5 & -60 & 7.0 \\
\hline 0.605 & -45.0 & -60 & 6.8 \\
\hline 0.605 & -67.5 & -60 & 6.4 \\
\hline 0.605 & .90 .0 & -60 & 6.4 \\
\hline 0.605 & -112.5 & -60 & 7.4 \\
\hline 0.605 & -135.0 & .60 & 8.8 \\
\hline 0.605 & -157.5 & .60 & 10.1 \\
\hline
\end{tabular}




\section{Table C.19 Vacuum Sweeper - Upright}

$60 \mathrm{~Hz}$ RMS Resultant Fields

Distance Horizontal Vertical $60 \mathrm{~Hz}$

meters angle deg angle deg mGauss

\begin{tabular}{|r|r|r|r|}
\hline 0.605 & 180.0 & -60 & 10.4 \\
\hline 0.605 & 157.5 & -60 & 10.0 \\
\hline 0.605 & 135.0 & -60 & 8.9 \\
\hline 0.605 & 112.5 & -60 & 7.5 \\
\hline 0.605 & 90.0 & -60 & 6.6 \\
\hline 0.605 & 67.5 & -60 & 6.2 \\
\hline 0.605 & 45.0 & -60 & 6.8 \\
\hline 0.605 & 22.5 & -60 & 7.4 \\
\hline 1.000 & 0.0 & -60 & 1.5 \\
\hline 1.000 & -22.5 & -60 & 1.4 \\
\hline 1.000 & -45.0 & -60 & 1.4 \\
\hline 1.000 & -67.5 & -60 & 1.2 \\
\hline 1.000 & -90.0 & -60 & 1.2 \\
\hline 1.000 & -112.5 & -60 & 1.3 \\
\hline
\end{tabular}

Distance Horizontal Vertical $60 \mathrm{~Hz}$ meters angle deg angle deg mGauss

\begin{tabular}{|r|r|r|r|}
\hline 1.000 & -135.0 & -60 & 1.5 \\
\hline 1.000 & -157.5 & -60 & 1.8 \\
\hline 1.000 & 180.0 & -60 & 1.7 \\
\hline 1.000 & 157.5 & -60 & 1.7 \\
\hline 1.000 & 135.0 & -60 & 1.7 \\
\hline 1.000 & 112.5 & -60 & 1.3 \\
\hline 1.000 & 90.0 & -60 & 1.2 \\
\hline 1.000 & 67.5 & -60 & 1.0 \\
\hline 1.000 & 45.0 & -60 & 1.4 \\
\hline 1.000 & 22.5 & -60 & 1.4 \\
\hline 0.114 & 0.0 & -90 & 453.1 \\
\hline 0.214 & 0.0 & -90 & 101.4 \\
\hline 0.414 & 0.0 & -90 & 18.4 \\
\hline
\end{tabular}




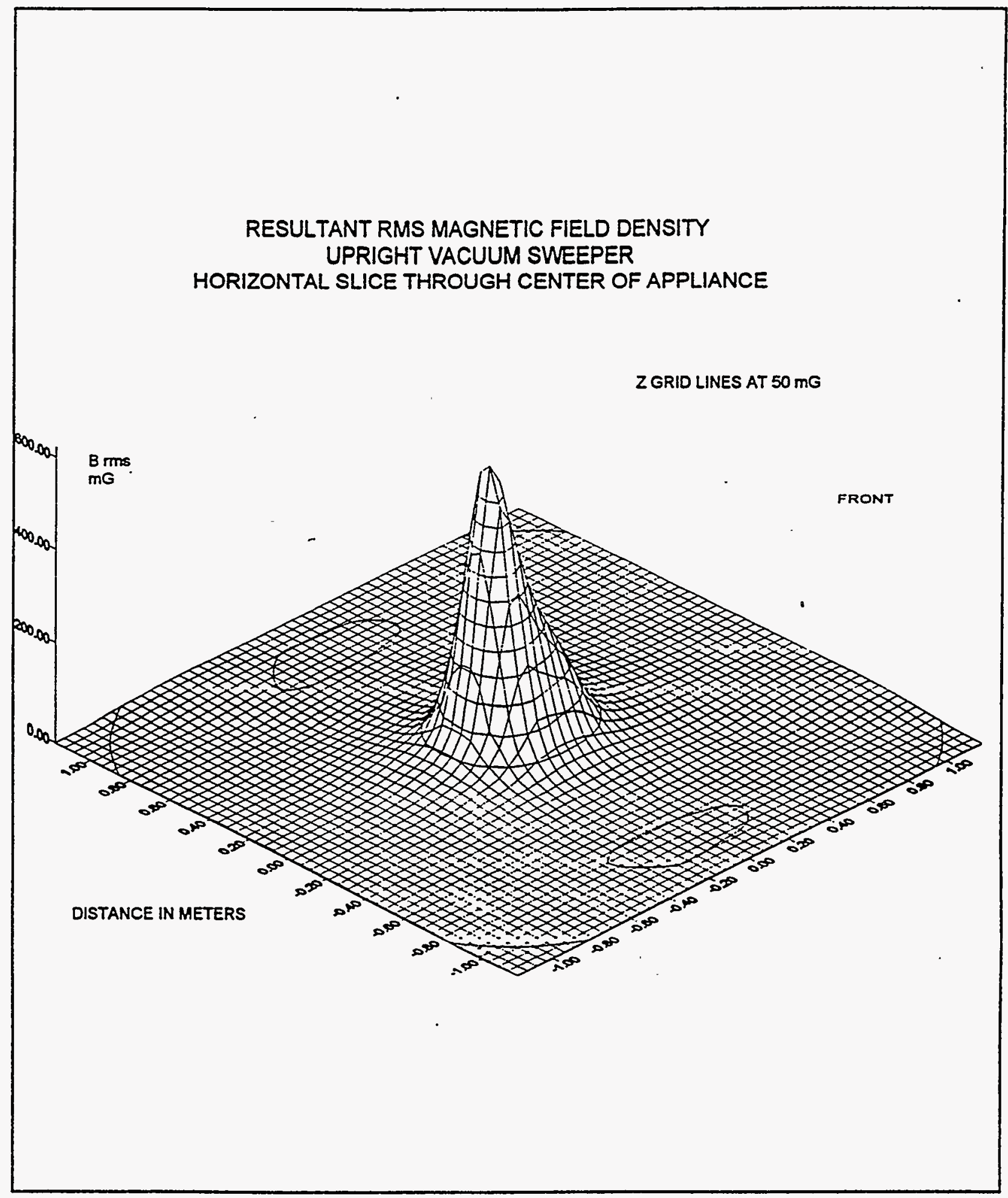

Figure C.19.1 Fundamental Resultant RMS Magnetic Field Density - Upright Vacuum Sweeper. 


\section{C.20 Video Display Terminal}

Appliance Tested: A video display terminal used with office or home computer equipment.

Manufacturer: TTT

Model: 018

Appliance Code: 1242

Conditions for Testing: The unit was running with a laptop computer eight feet away.

Measured voltage and current: 119.6 Volts and 0.048 Amperes.

Magnetic Field Summary:

$60 \mathrm{~Hz}$ RMS resultant level at $30 \mathrm{~cm}$ from front surface: $0.9 \mathrm{mG}$

RMS resultant high surface level: $115 \mathrm{mG}$ top

Highest recorded surface levels:

22.5 Hertz RMS resultant: $104 \mathrm{mG}$

60 Hertz RMS resultant: $37.3 \mathrm{mG}$

120 Hertz RMS resultant: $18.3 \mathrm{mG}$

180 Hertz RMS resultant: $12.7 \mathrm{mG}$

240 Hertz RMS resultant: $9.1 \mathrm{mG}$

300 Hertz RMS resultant: $7.1 \mathrm{mG}$

360 Hertz RMS resultant: $6.1 \mathrm{mG}$

420 Hertz RMS resultant: $5.5 \mathrm{mG}$

Maximum recorded RMS resultant in the $30-300 \mathrm{kHz}$ range: $22.6 \mathrm{mG}$

Maximum recorded RMS resultant in the $3-30 \mathrm{kHz}$ range: $4.23 \mathrm{mG}$

For the appliance test set, the front of the unit was located at 180 degrees on the test table.

Summary of characteristics: The strongest magnetic field was not at the $60 \mathrm{~Hz}$ power frequency but was at $22.5 \mathrm{Hertz}$. This field would not have been recorded with magnetic meters having bandwidths above $30 \mathrm{~Hz}$. 
Table C.20 Video Display Terminal $60 \mathrm{~Hz}$ RMS Resultant Fields

Distance Horizontal Vertical $60 \mathrm{~Hz}$ meters angle deg angle deg mGauss

\begin{tabular}{|c|c|c|c|}
\hline 0.191 & 0.0 & 90 & 17.1 \\
\hline 0.291 & 0.0 & 90 & 3.0 \\
\hline 0.491 & 0.0 & 90 & 1.1 \\
\hline 1.000 & 0.0 & 90 & 0.1 \\
\hline 0.229 & 0.0 & 60 & 9.5 \\
\hline 0.229 & -22.5 & 60 & 10.3 \\
\hline 0.229 & -45.0 & 60 & 10.8 \\
\hline 0.229 & -67.5 & 60 & 10.3 \\
\hline 0.229 & -90.0 & 60 & 9.2 \\
\hline 0.229 & -112.5 & 60 & 7.8 \\
\hline 0.222 & -135.0 & 60 & 5.8 \\
\hline 0.2229 & -157.5 & 60 & 5.3 \\
\hline 0.2229 & 180.0 & 60 & 5.3 \\
\hline 0.222 & 157.5 & 60 & 6.5 \\
\hline 0.229 & 135.0 & 60 & 8.1 \\
\hline 0.229 & 112.5 & 60 & 9.4 \\
\hline 0.229 & 90.0 & 60 & 10.3 \\
\hline 0.229 & 67.5 & 60 & 10.5 \\
\hline 0.229 & 45.0 & 60 & 10.2 \\
\hline 0.229 & 22.5 & 60 & 9.4 \\
\hline 0.329 & 0.0 & 60 & 3.6 \\
\hline 0.329 & -22.5 & 60 & 3.8 \\
\hline 0.329 & -45.0 & 60 & 4.1 \\
\hline 0.329 & -67.5 & 60 & 4.1 \\
\hline 0.329 & -90.0 & 60 & 3.7 \\
\hline 0.329 & -112.5 & 60 & 3.2 \\
\hline 0.329 & -135.0 & 60 & 2.8 \\
\hline 0.329 & -157.5 & 60 & 2.4 \\
\hline 0.329 & 180.0 & 60 & 2.4 \\
\hline 0.329 & 157.5 & 60 & 2.8 \\
\hline
\end{tabular}

Distance Horizontal Vertical $60 \mathrm{~Hz}$

meters angle deg angle deg mGauss

\begin{tabular}{|r|r|r|r|}
\hline 0.329 & 135.0 & 60 & 3.2 \\
\hline 0.329 & 112.5 & 60 & 3.6 \\
\hline 0.329 & 90.0 & 60 & 4.0 \\
\hline 0.329 & 67.5 & 60 & 4.0 \\
\hline 0.329 & 45.0 & 60 & 3.8 \\
\hline 0.329 & 22.5 & 60 & 3.6 \\
\hline 0.529 & 0.0 & 60 & 1.0 \\
\hline 0.529 & -22.5 & 60 & 0.9 \\
\hline 0.529 & -45.0 & 60 & 1.0 \\
\hline 0.529 & -67.5 & 60 & 0.8 \\
\hline 0.529 & -90.0 & 60 & 1.0 \\
\hline 0.529 & -112.5 & 60 & 0.9 \\
\hline 0.529 & -135.0 & 60 & 0.8 \\
\hline 0.529 & -157.5 & 60 & 0.8 \\
\hline 0.529 & 180.0 & 60 & 0.7 \\
\hline 0.529 & 157.5 & 60 & 0.8 \\
\hline 0.529 & 135.0 & 60 & 0.9 \\
\hline 0.529 & 112.5 & 60 & 1.0 \\
\hline 0.529 & 90.0 & 60 & 1.1 \\
\hline 0.529 & 67.5 & 60 & 1.1 \\
\hline 0.529 & 45.0 & 60 & 1.0 \\
\hline 0.529 & 22.5 & 60 & 1.0 \\
\hline 1.000 & 0.0 & 60 & 2.6 \\
\hline 1.000 & -67.5 & 60 & 0.1 \\
\hline 1.000 & -90.0 & 60 & 0.1 \\
\hline 1.000 & -112.5 & 60 & 0.1 \\
\hline 1.000 & -135.0 & 60 & 0.1 \\
\hline 1.000 & -157.5 & 60 & 0.1 \\
\hline 1.000 & 180.0 & 60 & 0.1 \\
\hline 1.000 & 157.5 & 60 & 0.3 \\
\hline
\end{tabular}


Table C.20 Video Display Terminal $60 \mathrm{~Hz}$ RMS Resultant Fields

Distance Horizontal Vertical $60 \mathrm{~Hz}$ meters angle deg angle deg mGauss

\begin{tabular}{|r|r|r|r|}
\hline 1.000 & 112.5 & 60 & 1.4 \\
\hline 1.000 & 90.0 & 60 & 1.3 \\
\hline 1.000 & 45.0 & 60 & 0.6 \\
\hline 1.000 & 22.5 & 60 & 0.4 \\
\hline 0.305 & 0.0 & 30 & 10.4 \\
\hline 0.305 & -22.5 & 30 & 12.6 \\
\hline 0.305 & -45.0 & 30 & 13.0 \\
\hline 0.305 & -67.5 & 30 & 12.4 \\
\hline 0.305 & -90.0 & 30 & 10.9 \\
\hline 0.305 & -112.5 & 30 & 9.3 \\
\hline 0.305 & -135.0 & 30 & 6.4 \\
\hline 0.305 & -157.5 & 30 & 4.8 \\
\hline 0.305 & 180.0 & 30 & 4.1 \\
\hline 0.305 & 157.5 & 30 & 5.3 \\
\hline 0.305 & 135.0 & 30 & 7.2 \\
\hline 0.305 & 112.5 & 30 & 9.4 \\
\hline 0.305 & 90.0 & 30 & 10.9 \\
\hline 0.305 & 67.5 & 30 & 11.7 \\
\hline 0.305 & 45.0 & 30 & 12.3 \\
\hline 0.305 & 22.5 & 30 & 11.4 \\
\hline 0.405 & 0.0 & 30 & 4.6 \\
\hline 0.405 & -22.5 & 30 & 4.8 \\
\hline 0.405 & -45.0 & 30 & 4.7 \\
\hline 0.405 & -67.5 & 30 & 4.7 \\
\hline 0.405 & -90.0 & 30 & 4.3 \\
\hline 0.405 & -112.5 & 30 & 3.9 \\
\hline 0.405 & -135.0 & 30 & 3.2 \\
\hline 0.405 & -157.5 & 30 & 2.7 \\
\hline 0.405 & 180.0 & 30 & 2.3 \\
\hline 0.405 & 157.5 & 30 & 2.6 \\
\hline
\end{tabular}

Distance Horizontal Vertical $60 \mathrm{~Hz}$ meters angle deg angle deg mGauss

\begin{tabular}{|r|r|r|r|}
\hline 0.405 & 135.0 & 30 & 3.1 \\
\hline 0.405 & 112.5 & 30 & 3.8 \\
\hline 0.405 & 90.0 & 30 & 4.3 \\
\hline 0.405 & 67.5 & 30 & 4.5 \\
\hline 0.405 & 45.0 & 30 & 4.6 \\
\hline 0.405 & 22.5 & 30 & 4.6 \\
\hline 0.605 & -22.5 & 30 & 0.7 \\
\hline 0.605 & -45.0 & 30 & 0.6 \\
\hline 0.605 & -67.5 & 30 & 0.3 \\
\hline 0.605 & -90.0 & 30 & 0.7 \\
\hline 0.605 & -112.5 & 30 & 0.8 \\
\hline 0.605 & -135.0 & 30 & 0.4 \\
\hline 0.605 & -157.5 & 30 & 0.8 \\
\hline 0.605 & 157.5 & 30 & 0.5 \\
\hline 0.605 & 135.0 & 30 & 0.5 \\
\hline 0.605 & 112.5 & 30 & 0.5 \\
\hline 0.605 & 90.0 & 30 & 0.5 \\
\hline 0.605 & 67.5 & 30 & 0.7 \\
\hline 0.605 & 45.0 & 30 & 0.8 \\
\hline 1.305 & 0.0 & 30 & 0.2 \\
\hline 1.305 & -22.5 & 30 & 0.3 \\
\hline 1.305 & -45.0 & 30 & 0.4 \\
\hline 1.305 & -67.5 & 30 & 0.4 \\
\hline 1.305 & -90.0 & 30 & 0.4 \\
\hline 1.305 & -112.5 & 30 & 0.4 \\
\hline 1.305 & -135.0 & 30 & 0.3 \\
\hline 1.305 & -157.5 & 30 & 0.3 \\
\hline 1.305 & 180.0 & 30 & 0.2 \\
\hline 1.305 & 135.0 & 30 & 0.3 \\
\hline 1.305 & 112.5 & 30 & 0.4 \\
\hline
\end{tabular}


Table C.20 Video Display Terminal

$60 \mathrm{~Hz}$ RMS Resultant Fields

Distance Horizontal Vertical $60 \mathrm{~Hz}$

meters angle deg angle deg mGauss

\begin{tabular}{|c|c|c|c|}
\hline 1.305 & 90.0 & 30 & 0.4 \\
\hline 1.305 & 45.0 & 30 & 0.4 \\
\hline 0.254 & 0.0 & 0 & 8.1 \\
\hline 0.254 & -22.5 & 0 & 10.3 \\
\hline 0.254 & -45.0 & 0 & 9.6 \\
\hline 0.254 & -67.5 & 0 & 8.7 \\
\hline 0.254 & -90.0 & 0 & 7.4 \\
\hline 0.254 & -112.5 & 0 & 6.1 \\
\hline 0.254 & -135.0 & 0 & 4.5 \\
\hline 0.254 & -157.5 & 0 & 3.3 \\
\hline 0.254 & 180.0 & 0 & 2.9 \\
\hline 0.254 & 157.5 & 0 & 3.7 \\
\hline 0.254 & 135.0 & 0 & 5.0 \\
\hline 0.254 & 112.5 & 0 & 6.4 \\
\hline 0.254 & 90.0 & 0 & 7.4 \\
\hline 0.254 & 67.5 & 0 & 8.0 \\
\hline 0.254 & 45.0 & 0 & 8.8 \\
\hline 0.254 & 22.5 & 0 & 8.7 \\
\hline 0.354 & 0.0 & 0 & 2.9 \\
\hline 0.354 & -22.5 & 0 & 3.6 \\
\hline 0.354 & -45.0 & 0 & 4.2 \\
\hline 0.354 & -67.5 & 0 & 4.0 \\
\hline 0.354 & -90.0 & 0 & 3.6 \\
\hline 0.354 & -112.5 & 0 & 3.1 \\
\hline 0.354 & -135.0 & 0 & 2.2 \\
\hline 0.354 & -157.5 & 0 & 1.9 \\
\hline 0.354 & 180.0 & 0 & 1.7 \\
\hline 0.354 & 157.5 & 0 & 1.8 \\
\hline 0.354 & 135.0 & 0 & 2.5 \\
\hline 0.354 & 112.5 & 0 & 3.0 \\
\hline & & & \\
\hline 0.5 & 0 & 0 & 0 \\
\hline
\end{tabular}

Distance Horizontal Vertical $60 \mathrm{~Hz}$

meters angle deg angle deg mGauss

\begin{tabular}{|r|r|r|r|}
\hline 0.354 & 90.0 & 0 & 3.5 \\
\hline 0.354 & 67.5 & 0 & 3.7 \\
\hline 0.354 & 45.0 & 0 & 3.9 \\
\hline 0.354 & 22.5 & 0 & 3.3 \\
\hline 0.554 & 0.0 & 0 & 0.8 \\
\hline 0.554 & -22.5 & 0 & 0.9 \\
\hline 0.554 & -45.0 & 0 & 1.2 \\
\hline 0.554 & -67.5 & 0 & 1.2 \\
\hline 0.554 & -90.0 & 0 & 1.1 \\
\hline 0.554 & -112.5 & 0 & 1.0 \\
\hline 0.554 & -135.0 & 0 & 0.8 \\
\hline 0.554 & -157.5 & 0 & 0.6 \\
\hline 0.554 & 180.0 & 0 & 0.6 \\
\hline 0.554 & 157.5 & 0 & 0.6 \\
\hline 0.554 & 135.0 & 0 & 0.8 \\
\hline 0.554 & 112.5 & 0 & 1.0 \\
\hline 0.554 & 90.0 & 0 & 1.1 \\
\hline 0.554 & 67.5 & 0 & 1.2 \\
\hline 0.554 & 45.0 & 0 & 1.1 \\
\hline 0.554 & 22.5 & 0 & 0.9 \\
\hline 0.305 & 0.0 & -30 & 4.6 \\
\hline 0.305 & -22.5 & -30 & 5.1 \\
\hline 0.305 & -45.0 & -30 & 6.0 \\
\hline 0.305 & -67.5 & -30 & 6.2 \\
\hline 0.305 & -90.0 & -30 & 5.0 \\
\hline 0.305 & -112.5 & -30 & 4.4 \\
\hline 0.305 & -135.0 & -30 & 3.7 \\
\hline 0.305 & -157.5 & -30 & 2.7 \\
\hline 0.305 & 180.0 & -30 & 2.4 \\
\hline 0.305 & 157.5 & -30 & 2.7 \\
\hline
\end{tabular}


Table C.20 Video Display Terminal $60 \mathrm{~Hz}$ RMS Resultant Fields

Distance Horizontal Vertical $60 \mathrm{~Hz}$ meters angle deg angle deg mGauss

\begin{tabular}{|c|c|c|c|}
\hline 0,305 & 135.0 & -30 & 3.2 \\
\hline 0.305 & 112.5 & -30 & 3.9 \\
\hline 0.305 & 90.0 & -30 & 4.7 \\
\hline 0.305 & 67.5 & -30 & 4.9 \\
\hline 0,305 & 45.0 & -30 & 5.2 \\
\hline 0.305 & 22.5 & -30 & 5.1 \\
\hline 0.405 & 0.0 & .30 & 2.1 \\
\hline 0.405 & -22.5 & -30 & 2.2 \\
\hline 0.405 & -45.0 & .30 & 2.8 \\
\hline 0.405 & -67.5 & -30 & 2.9 \\
\hline 0.405 & .90 .0 & .30 & 2.5 \\
\hline 0.405 & -112.5 & -30 & 2.2 \\
\hline 0.405 & -135.0 & -30 & 1.9 \\
\hline 0.405 & -157.5 & -30 & 1.4 \\
\hline 0.405 & 180.0 & .30 & 1.2 \\
\hline 0.405 & 157.5 & -30 & 1.3 \\
\hline 0.405 & 135.0 & -30 & 1.7 \\
\hline 0.405 & 112.5 & -30 & 2.1 \\
\hline 0.405 & 90.0 & .30 & 2.4 \\
\hline 0.405 & 67.5 & -30 & 2.5 \\
\hline 0.405 & 45.0 & -30 & 2.5 \\
\hline 0.405 & 22.5 & -30 & 2.3 \\
\hline 0.605 & 0.0 & .30 & 0.7 \\
\hline 0.605 & -22.5 & .30 & 0.8 \\
\hline 0.605 & -45.0 & -30 & 0.9 \\
\hline 0.605 & -67.5 & .30 & 1.0 \\
\hline 0.605 & -90.0 & .30 & 1.0 \\
\hline 0.605 & -112.5 & -30 & 0.9 \\
\hline 0.605 & -135.0 & -30 & 0.7 \\
\hline 0.605 & -157.5 & -30 & 0.5 \\
\hline
\end{tabular}

Distance Horizontal Vertical $60 \mathrm{~Hz}$

meters angle deg angle deg mGauss

\begin{tabular}{|c|c|c|c|}
\hline 0.605 & 180.0 & -30 & 0.4 \\
\hline 0.605 & 157.5 & -30 & 0.6 \\
\hline 0.605 & 135.0 & -30 & 0.6 \\
\hline 0.605 & 112.5 & -30 & 0.9 \\
\hline 0.605 & 90.0 & -30 & 0.9 \\
\hline 0.605 & 67.5 & -30 & 0.9 \\
\hline 0.605 & 45.0 & -30 & 0.9 \\
\hline 0.605 & 22.5 & -30 & 0.7 \\
\hline 0.305 & 0.0 & -60 & 8.3 \\
\hline 0.305 & -22.5 & -60 & 7.1 \\
\hline 0.305 & -45.0 & -60 & 3.9 \\
\hline 0.305 & -67.5 & -60 & 4.0 \\
\hline 0.305 & -90.0 & -60 & 2.8 \\
\hline 0.305 & -112.5 & -60 & 4.3 \\
\hline 0.305 & -135.0 & -60 & 5.9 \\
\hline 0.305 & -157.5 & -60 & 5.4 \\
\hline 0.305 & 180.0 & -60 & 5.9 \\
\hline 0.305 & 157.5 & -60 & 5.4 \\
\hline 0.305 & 135.0 & -60 & 4.8 \\
\hline 0.305 & 112.5 & -60 & 4.0 \\
\hline 0.305 & 90.0 & -60 & 3.0 \\
\hline 0.305 & 67.5 & -60 & 2.9 \\
\hline 0.305 & 45.0 & -60 & 4.5 \\
\hline 0.305 & 22.5 & -60 & 8.1 \\
\hline 0.405 & 0.0 & -60 & 1.5 \\
\hline 0.405 & -22.5 & -60 & 1.5 \\
\hline 0.405 & -45.0 & -60 & 1.9 \\
\hline 0.405 & -67.5 & -60 & 1.9 \\
\hline 0.405 & -90.0 & -60 & 1.5 \\
\hline 0.405 & -112.5 & -60 & 1.4 \\
\hline
\end{tabular}




\section{Table C.20 Video Display Terminal}

\section{$60 \mathrm{~Hz}$ RMS Resultant Fields}

Distance Horizontal Vertical $60 \mathrm{~Hz}$.

meters angle deg angle deg mGauss

\begin{tabular}{|r|r|r|r|}
\hline 0.405 & -135.0 & -60 & 1.4 \\
\hline 0.405 & -157.5 & -60 & 1.2 \\
\hline 0.405 & 180.0 & -60 & 1.2 \\
\hline 0.405 & 157.5 & -60 & 1.1 \\
\hline 0.405 & 135.0 & -60 & 1.4 \\
\hline 0.405 & 112.5 & -60 & 1.4 \\
\hline 0.405 & 90.0 & -60 & 1.6 \\
\hline 0.405 & 67.5 & -60 & 1.5 \\
\hline 0.405 & 45.0 & -60 & 1.6 \\
\hline 0.405 & 22.5 & -60 & 1.5 \\
\hline 0.605 & 0.0 & -60 & 0.6 \\
\hline 0.605 & -22.5 & -60 & 0.5 \\
\hline 0.605 & -45.0 & -60 & 0.7 \\
\hline
\end{tabular}

Distance Horizontal Vertical $60 \mathrm{~Hz}$

meters angle deg angle deg mGauss

\begin{tabular}{|r|r|r|r|}
\hline 0.605 & -67.5 & -60 & 0.4 \\
\hline 0.605 & -90.0 & -60 & 0.3 \\
\hline 0.605 & -112.5 & -60 & 0.3 \\
\hline 0.605 & -135.0 & -60 & 0.6 \\
\hline 0.605 & -157.5 & -60 & 0.4 \\
\hline 0.605 & 180.0 & -60 & 0.4 \\
\hline 0.605 & 157.5 & -60 & 0.4 \\
\hline 0.605 & 135.0 & -60 & 0.4 \\
\hline 0.605 & 67.5 & -60 & 0.4 \\
\hline 0.605 & 22.5 & -60 & 0.5 \\
\hline 0.229 & 0.0 & -90 & 10.0 \\
\hline 0.329 & 0.0 & -90 & 3.7 \\
\hline
\end{tabular}




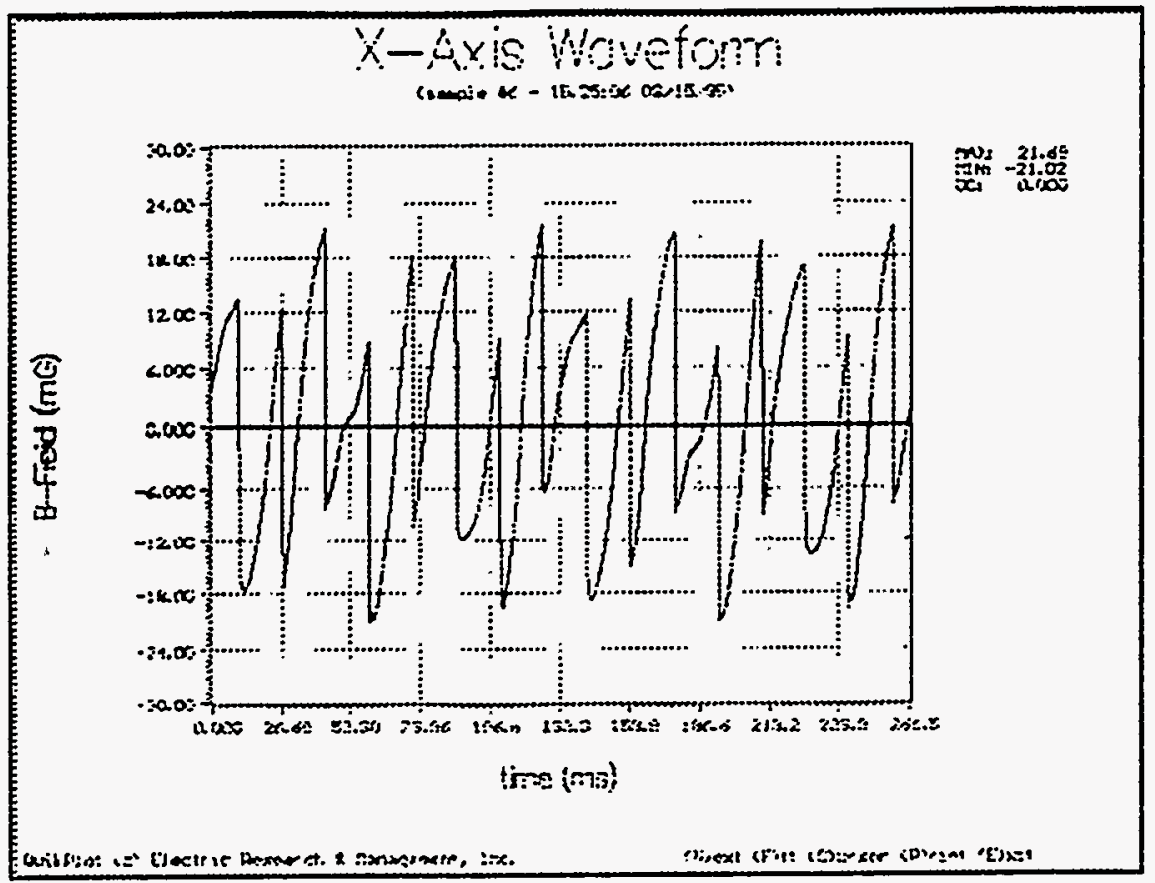

Figure C.20.1 $X$ axis waveform at bottom of Video Display Terminal showing the high rates of rise and the $22.5 \mathrm{~Hz}$ Frequency.

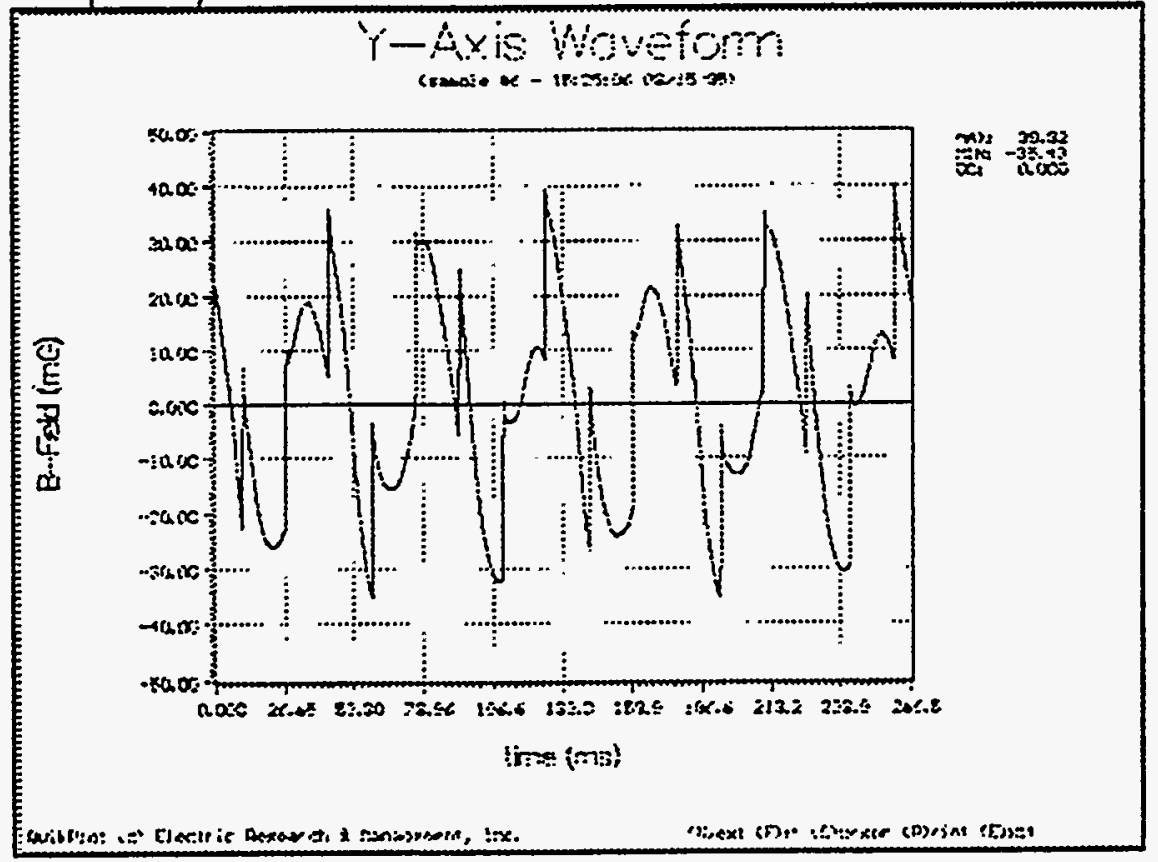

Figure C.20.2 The $y$ axis waveform at the bottom of the Video Display Terminal 


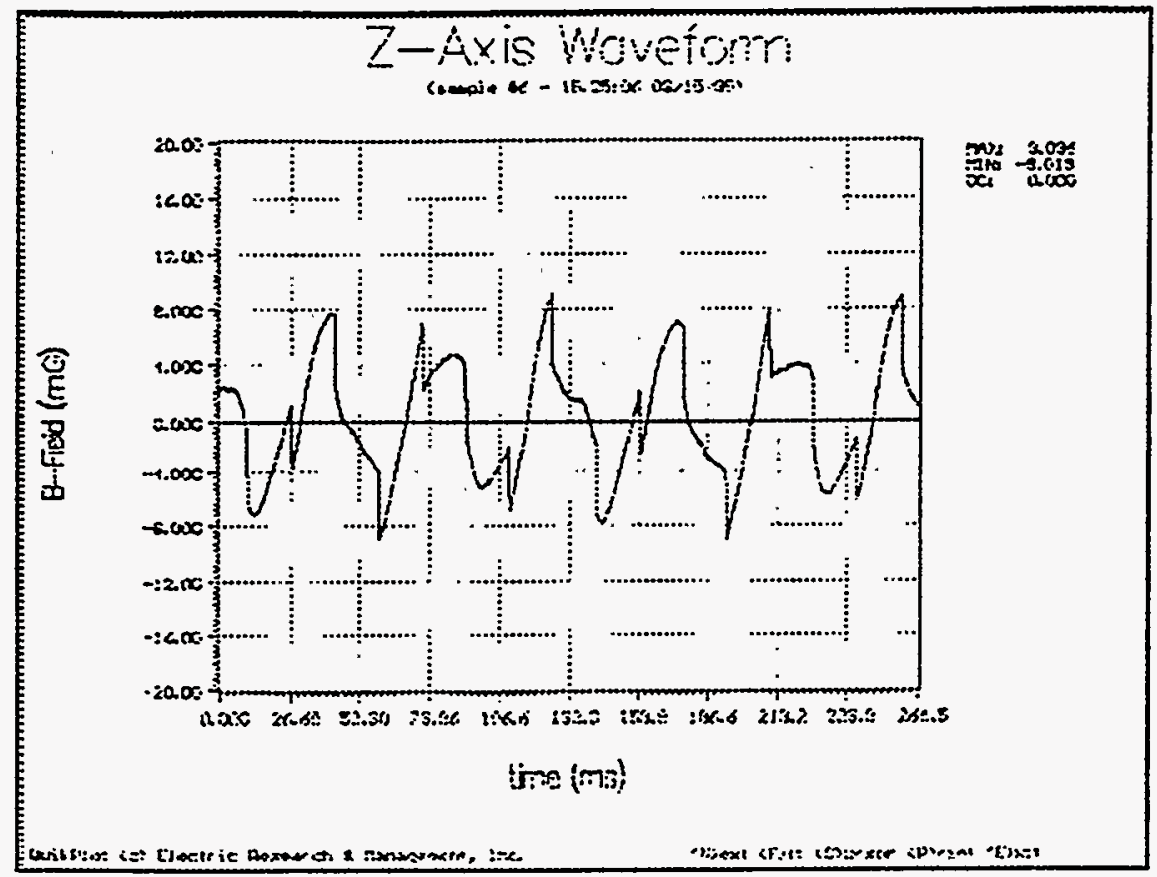

Figure C.20.3 The $z$ axis at the bottom of the Video Display Terminal 


\section{RESULTANT RMS MAGNETIC FIELD DENSITY CRT MONITOR \\ HORIZONTAL SLICE THROUGH CENTER OF APPLIANCE`}

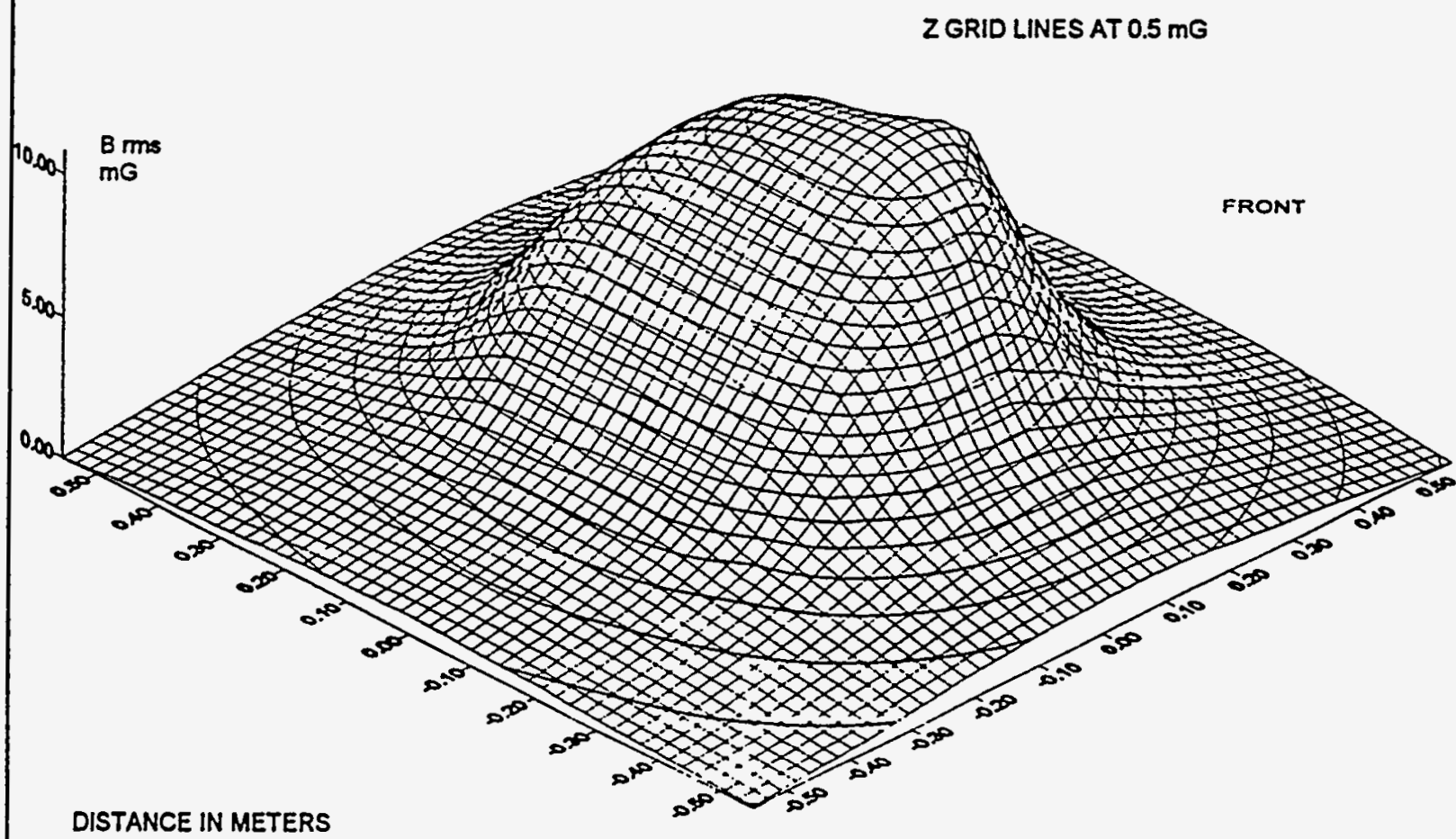

Figure C.20.4 Fundamental Resultant RMS Magnetic Field Density - CRT Monitor. 
Appendix D In-Situ Test Data

Introduction

The testing of in-situ appliances was attempted in two specific locations. The first location was a large uninterruptible power supply (UPS) utilized for backup power in a medium-sized computer room where approximately 15 high-resolution computer terminals with high resolution-printers and scanners were located. The second was an inverter to change dc to ac for use with protection and communication equipment. The inverter was located in a large hydro-electric plant.

The data taken seems minimal compared to the volume of data available for the appliances from the appliance test set. However, the data was taken while the equipment was in place and working. No movement of desks, personnel, walls or any other equipment nearby was allowed in either situation. The data was obtained without special measuring jigs to properly position the transducers. Only a staff was used. The concept of having specific jigs to position the transducers resulted from this early work.

The data taken was stored as wave-capture information with a data window of one cycle of the $60 \mathrm{~Hz}$ waveform and with 128 samples per cycle. Each axis was recorded and stored separately. The sample were taken sequentially and the load of the appliance was assumed constant over the measurement period.

\section{D.1 The Uninterruptible Power Supply}

The measurements of the uninterruptible power supply were made while work in the computer room was in progress. There could be no extensive gridding of the measurements as most of the grid would be at work station locations. Also the size of the appliance was about 36 inches wide, 32 inches deep and 72 inches high 10.9 by 0.8 by 1.8 meters). The size did not allow spherical coordinates to be utilized since the ceiling was about 7.5 feet high $(2.3$ meters) with a raised floor. Rectangular coordinates were chosen. The limited grid was chosen to thread between desks and down the two aisles.

The waveforms demonstrated that the magnetic fields were rich in harmonics with the $11^{\text {th }}$ harmonic dominate. Figure D.1.10 shows a representative wave form taken at the back left edge approximately 12 inches $(30 \mathrm{~cm})$ from the back of the appliance even with the left side. Table D.1.1 gives the data for the rms, the fundamental, the third, the fifth and the eleventh harmonics taken at the various coordinates. The table also provides the polarization number for the fundamental, the third, the fifth and the eleventh harmonics. It can be seen from these values and the graphs that are made at the 43 inch level that the polarization can have a significant value 
removed from the appliance if there are multiple magnetic sources within the appliance which are spatially separated. A polarization of 1 indicates that the magnetic field vector $B$ is spinning in a circle. A polarization of $O$ indicates that $B$ is not spinning but is linear as it increases and decreases throughout the cycle. For small appliances, the location of the source of the harmonics is typically the same as for the fundamental and the relationship between the fundamental magnitude and the harmonic magnitude is fixed. In this appliance, the harmonic magnetic fields are not generated in the exact same places as the fundamental and the relationships of the harmonic and fundamental magnitudes are not fixed. 


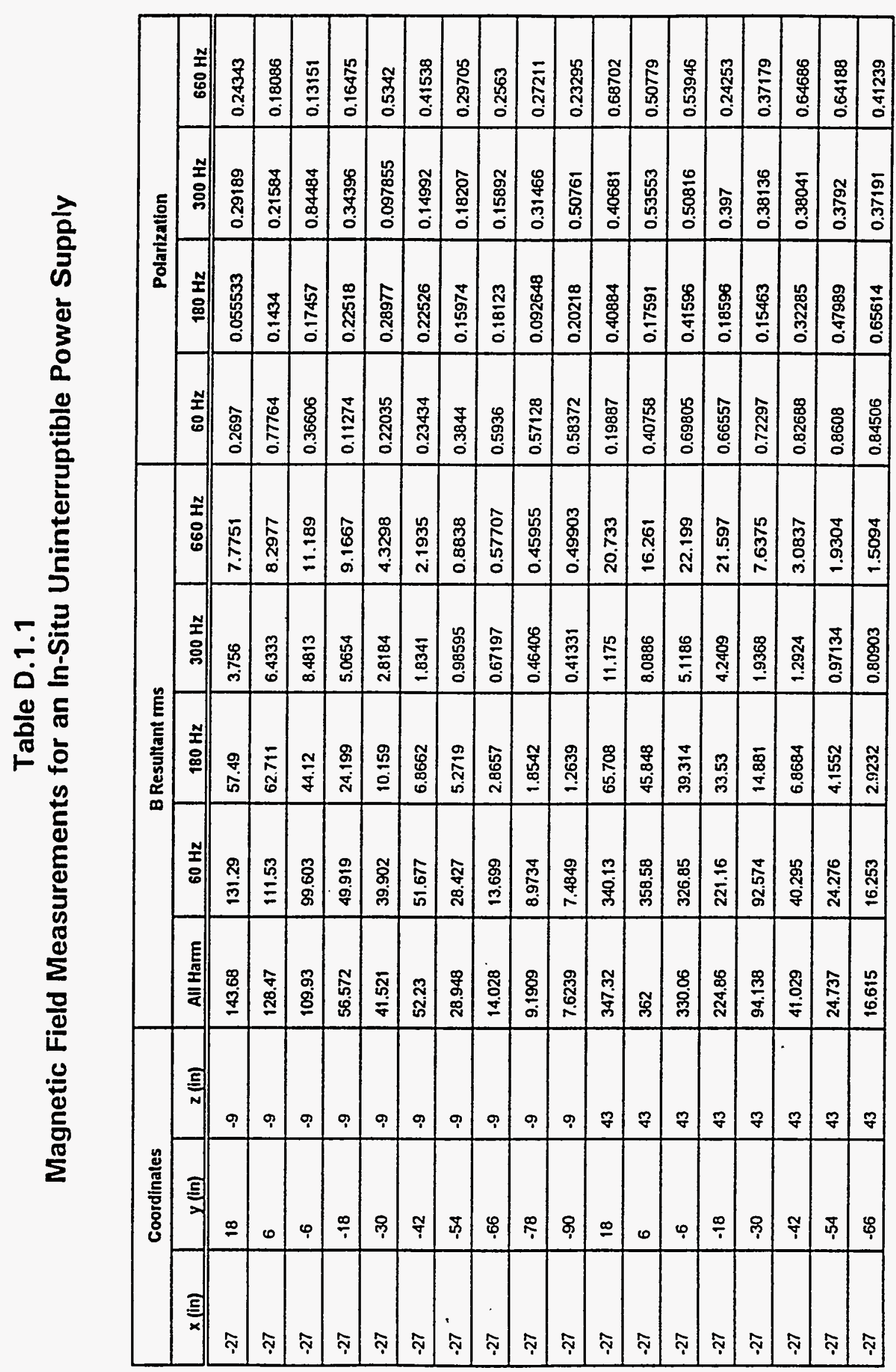




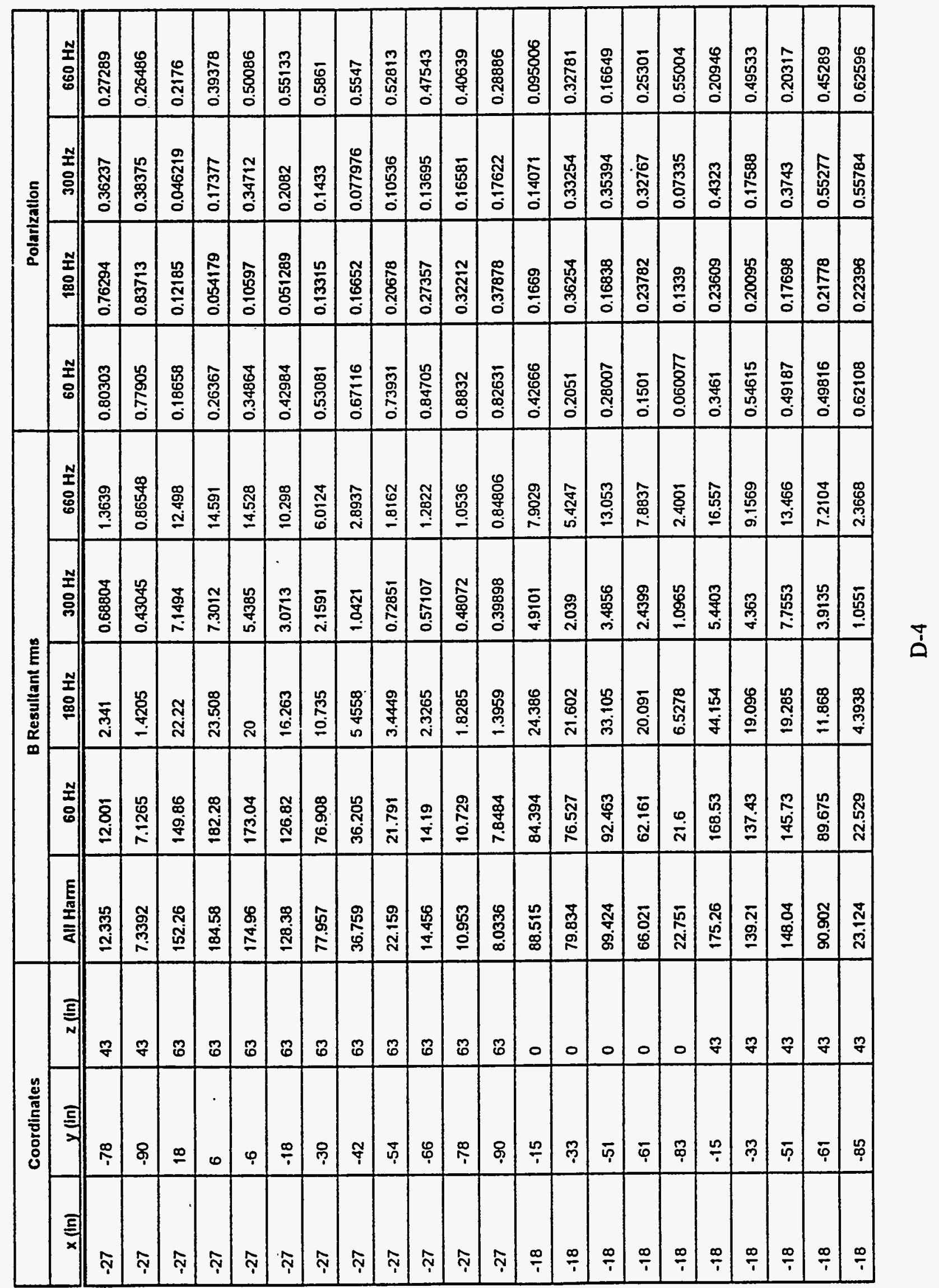




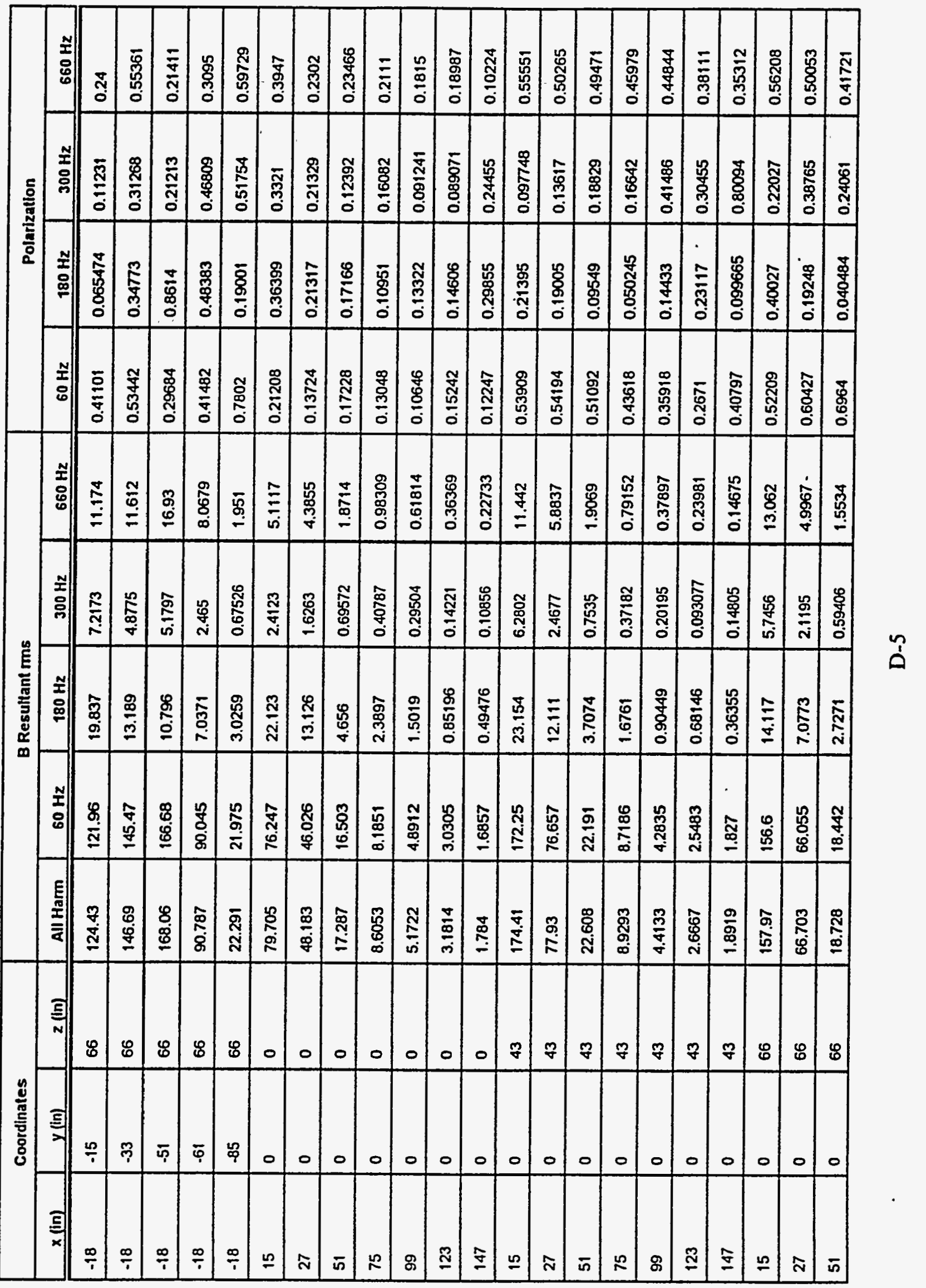




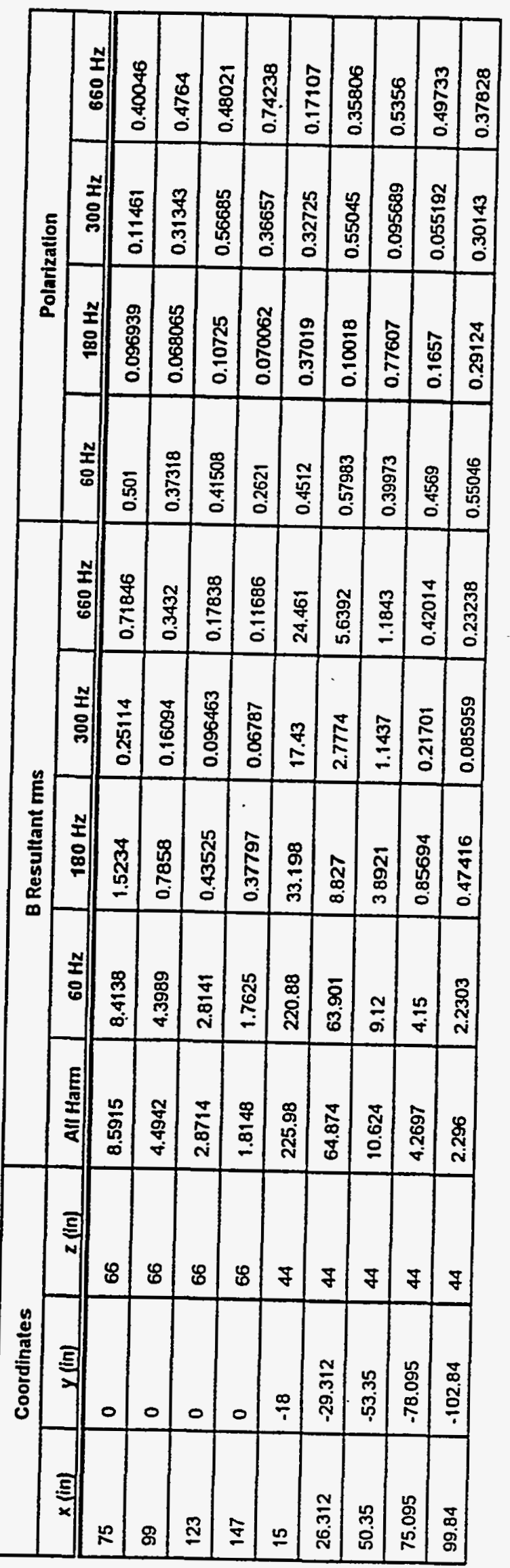




\section{RMS RESULTANT MAGNETIC FIELD DENSITY UPS EQUIPMENT}

HORIZONTAL SLICE AT 43 INCHES FROM FLOOR

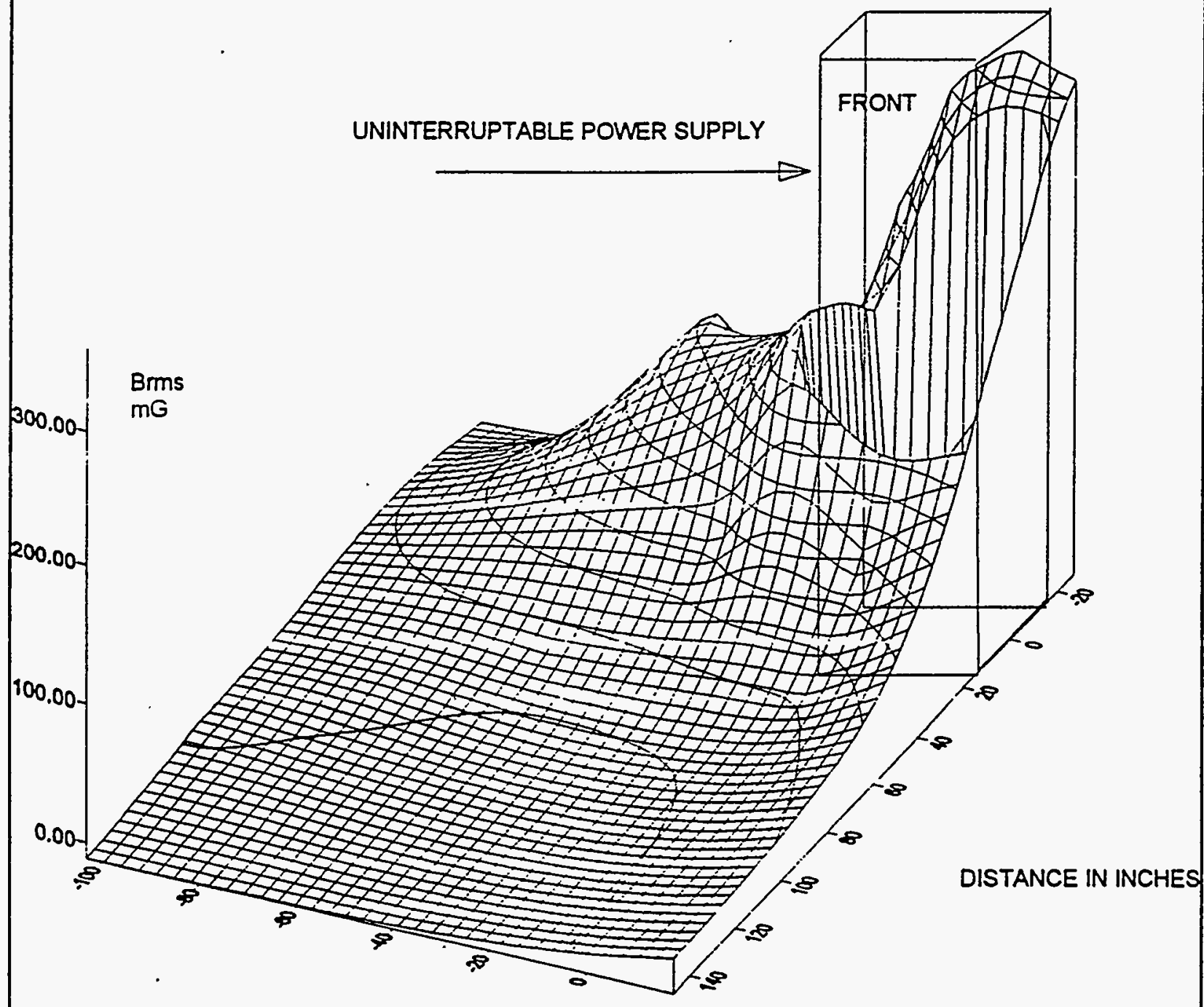

Figure D.1.1 RMS Resultant Magnetic Field Density - UPS Equipment. 


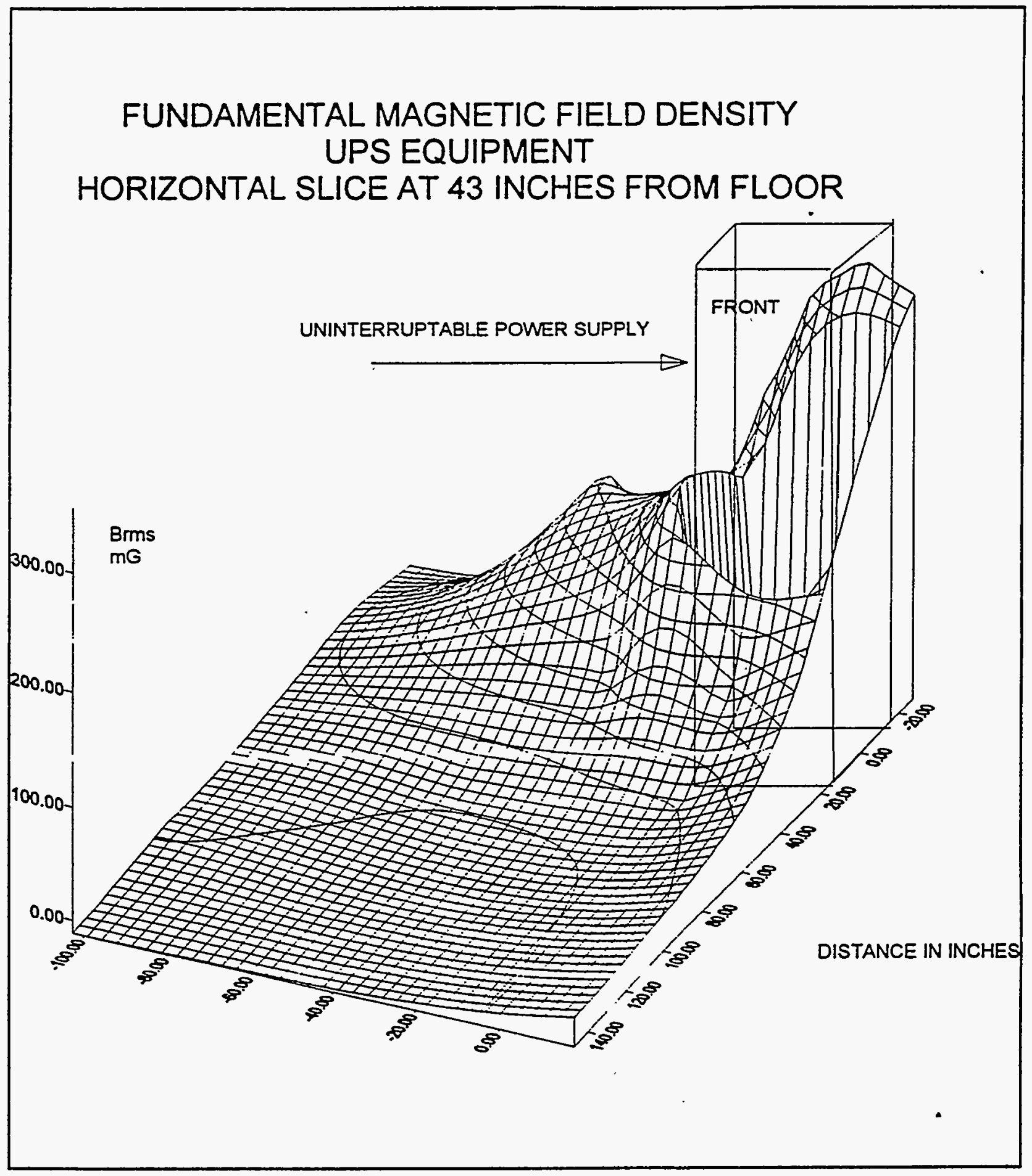

Figure D.1.2 Fundamental Magnetic Field Density - UPS Equipment. 


\section{THIRD HARMONIC MAGNETIC FIELD DENSITY UPS EQUIPMENT \\ HORIZONTAL SLICE AT 43 INCHES FROM FLOOR}

b.

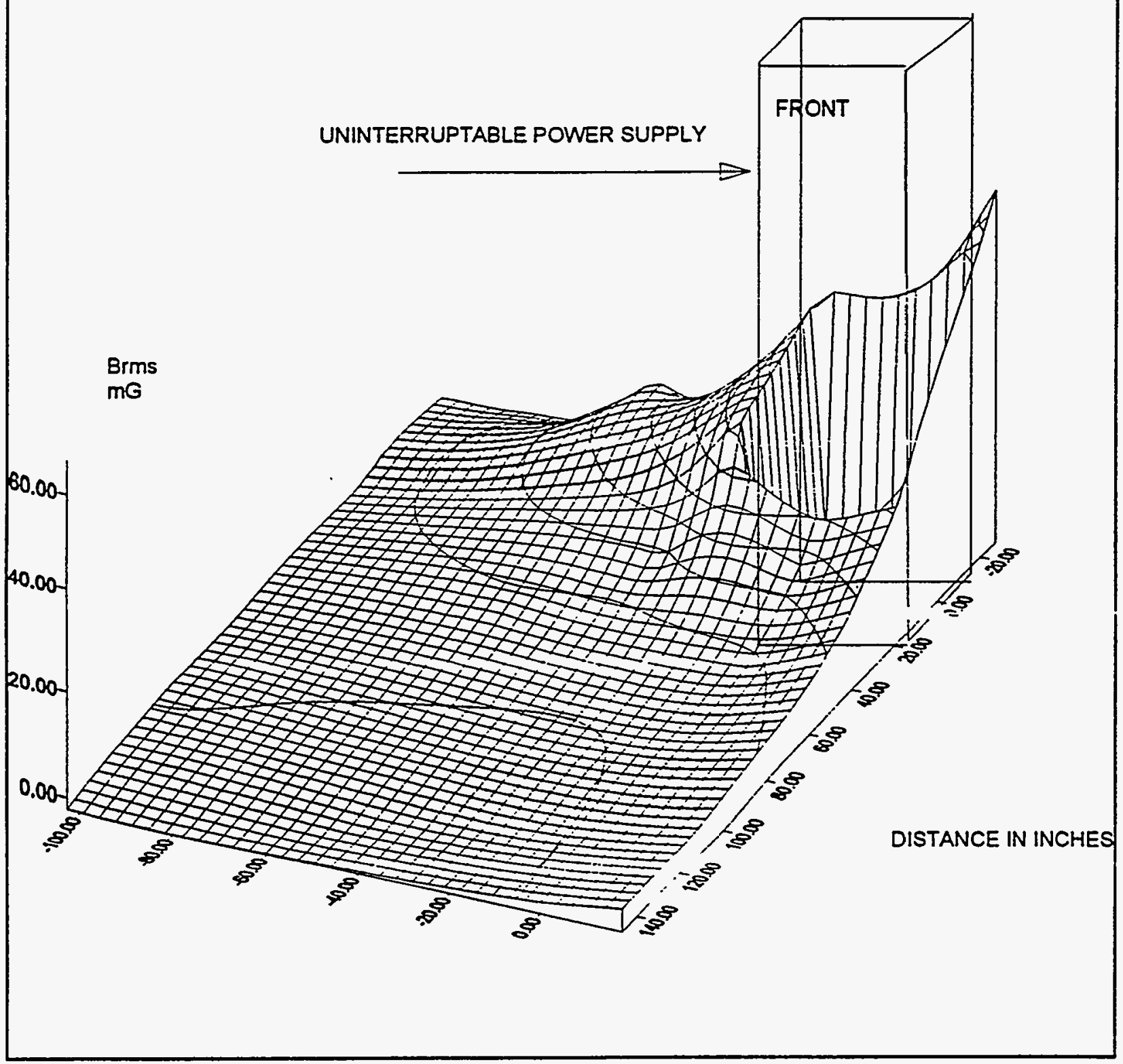

Figure D.1.3 Third Harmonic Magnetic Field Density - UPS Equipment. 


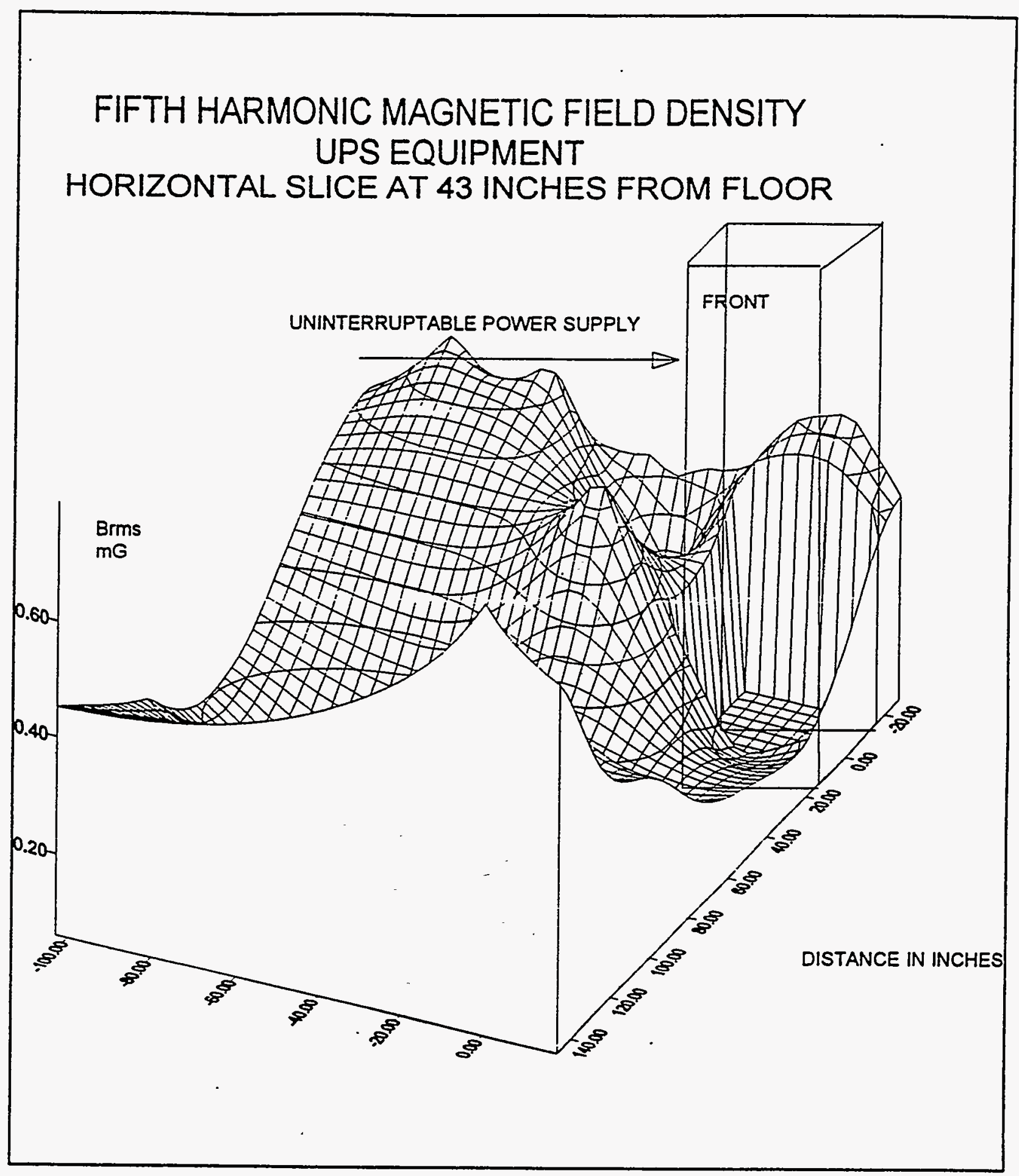

Figure D.1.4 Fifth Harmonic Magnetic Field Density - UPS Equipment. 


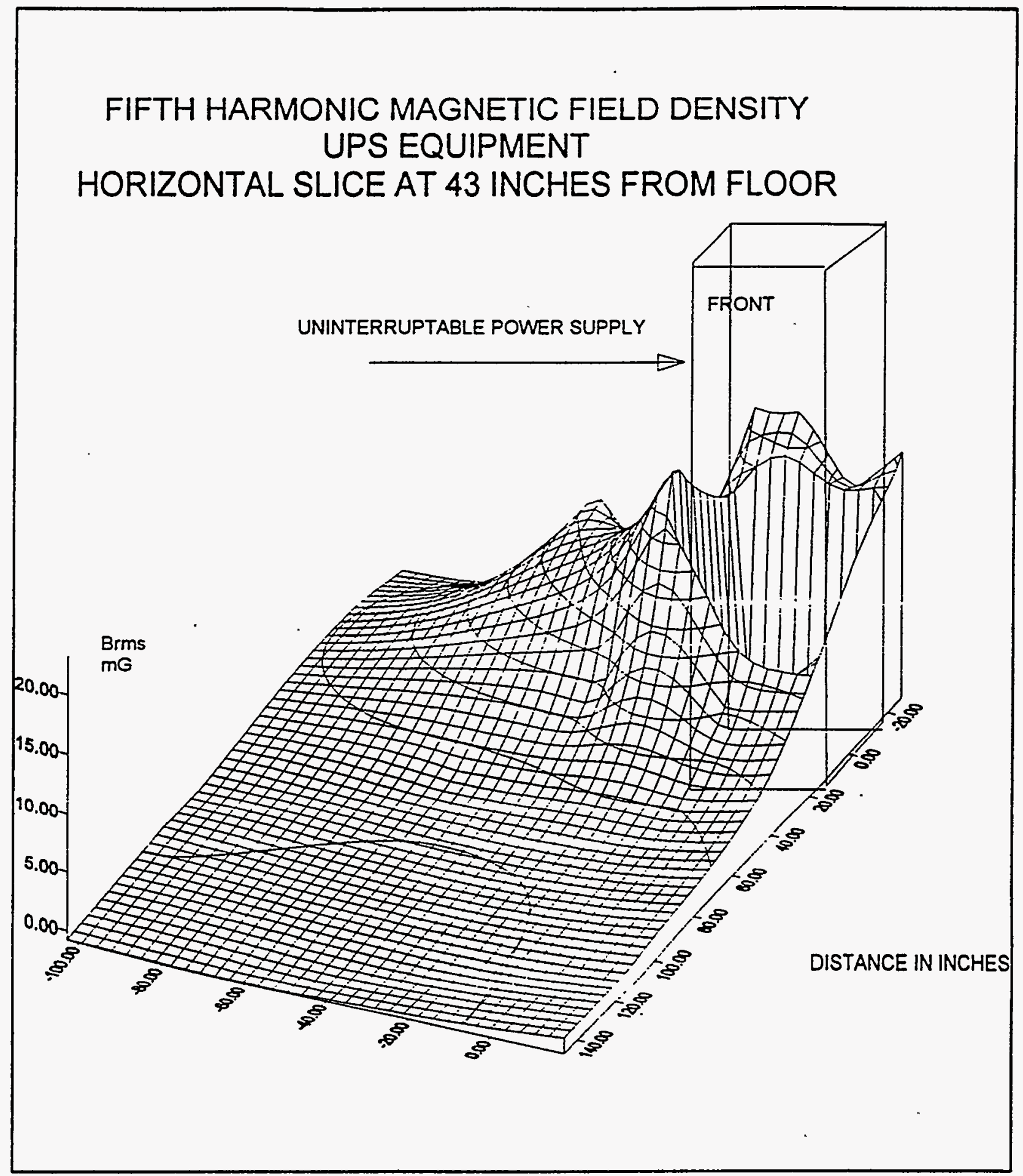

Figure D.1.5 Fifth Harmonic Magnetic Field Density - UPS Equipment.

D-11 


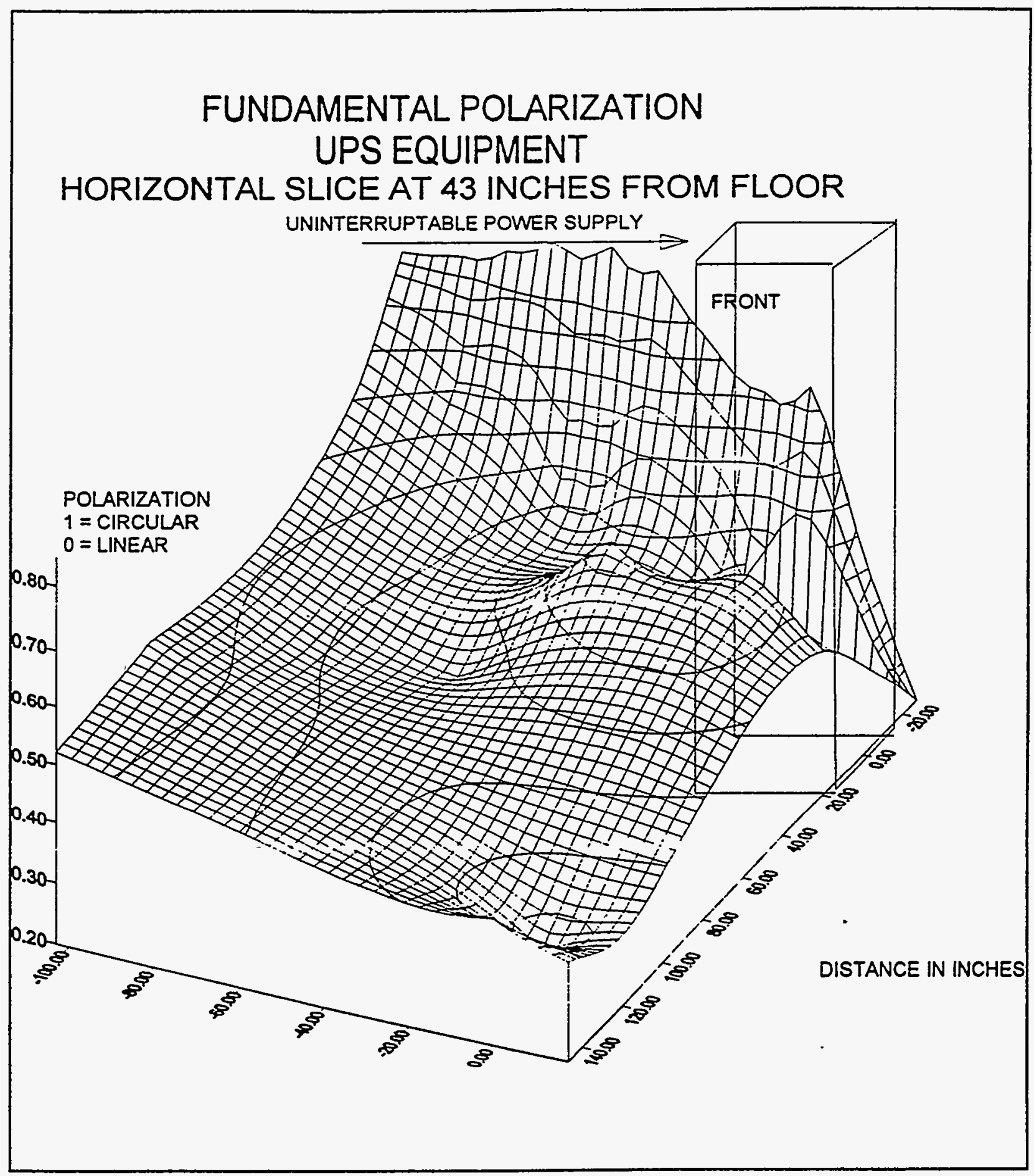

Figure D.1.6 Fundamental Polarization - UPS Equipment. 


\section{THIRD HARMONIC POLARIZATION UPS EQUIPMENT HORIZONTAL SLICE AT 43 INCHES FROM FLOOR}

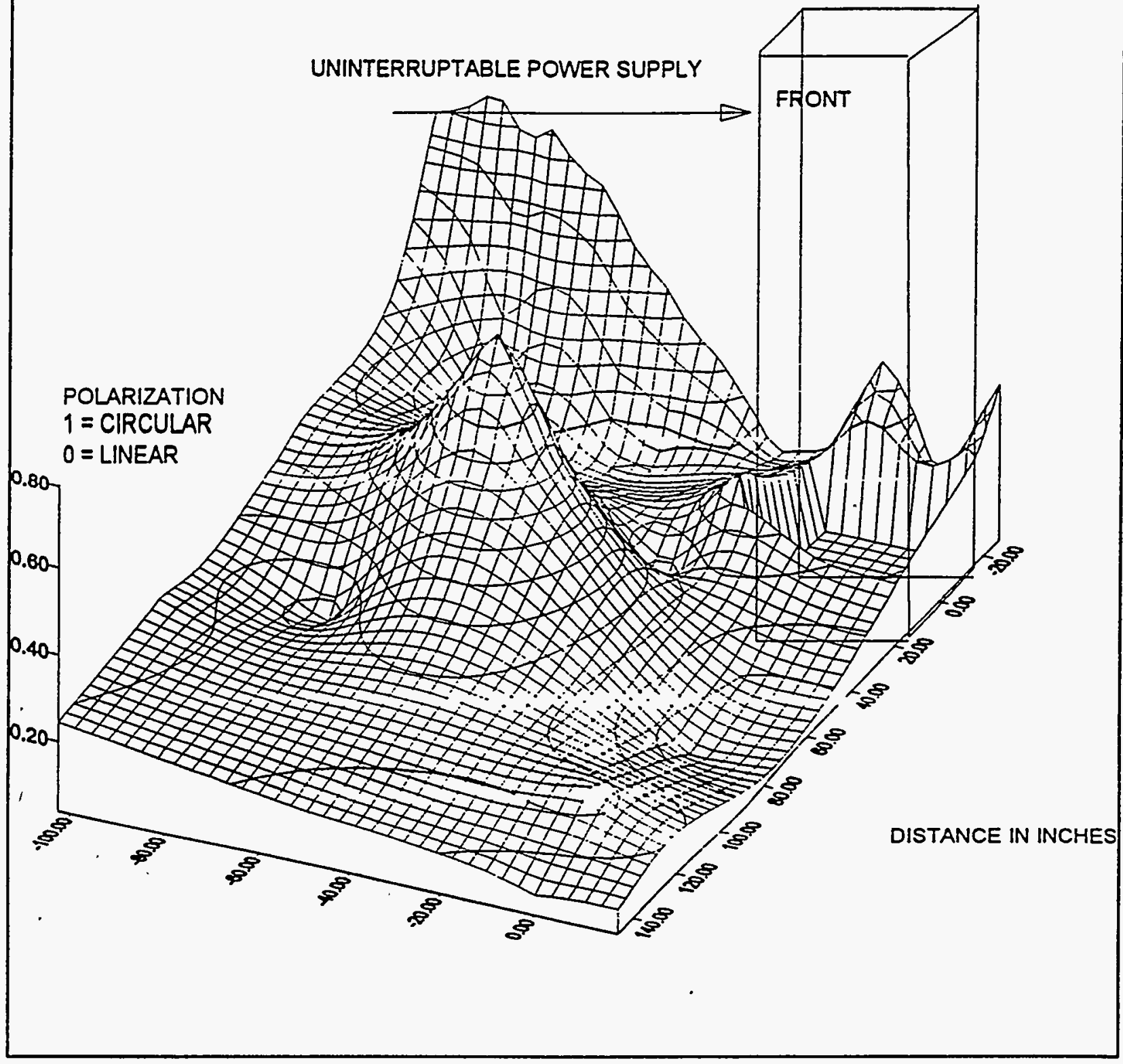

Figure D.1.7 Third Harmonic Polarization - UPS Equipment. 


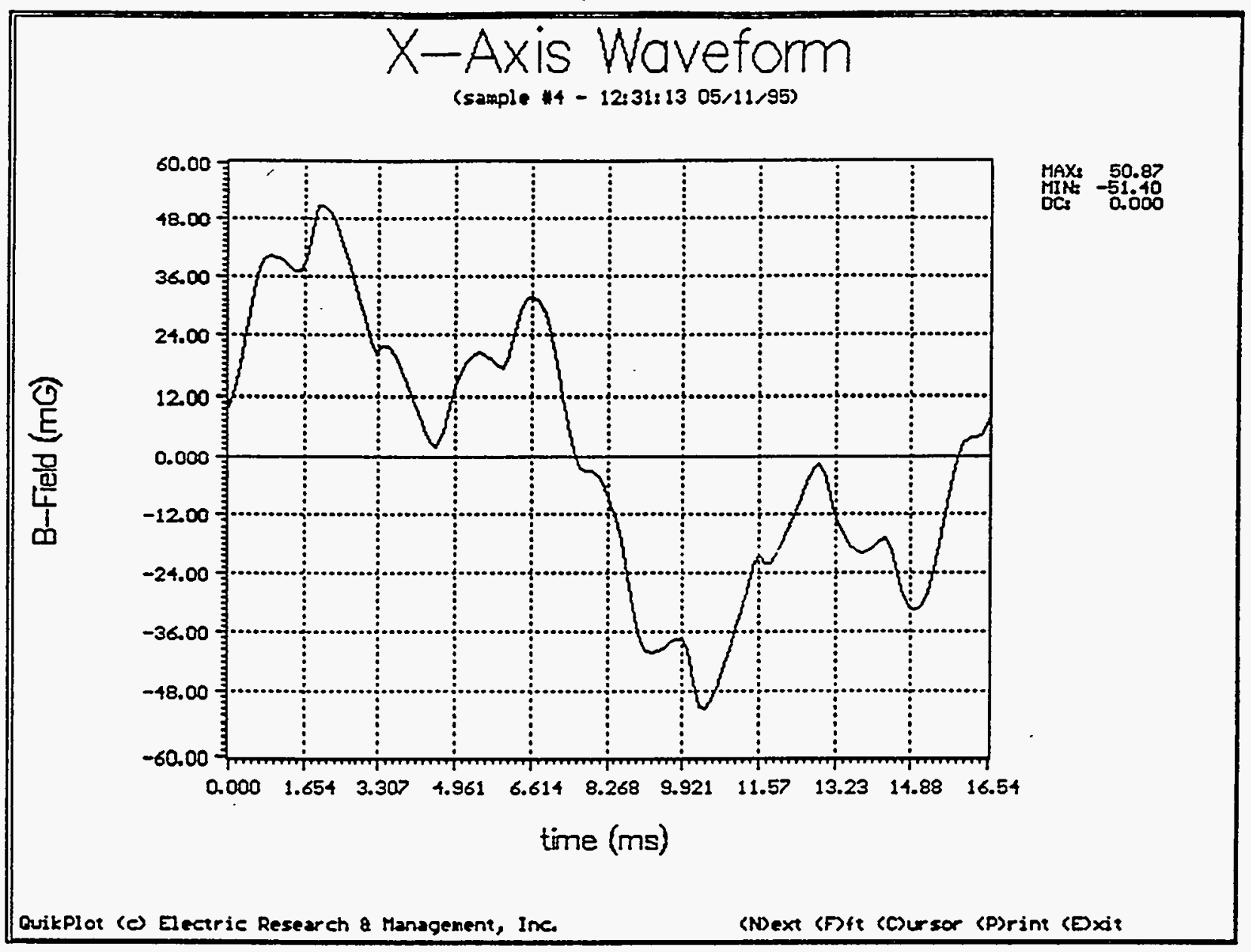

Figure D.1.8 Waveform of the Probe $X$ Axis at Location $-27,-18,-9$. 


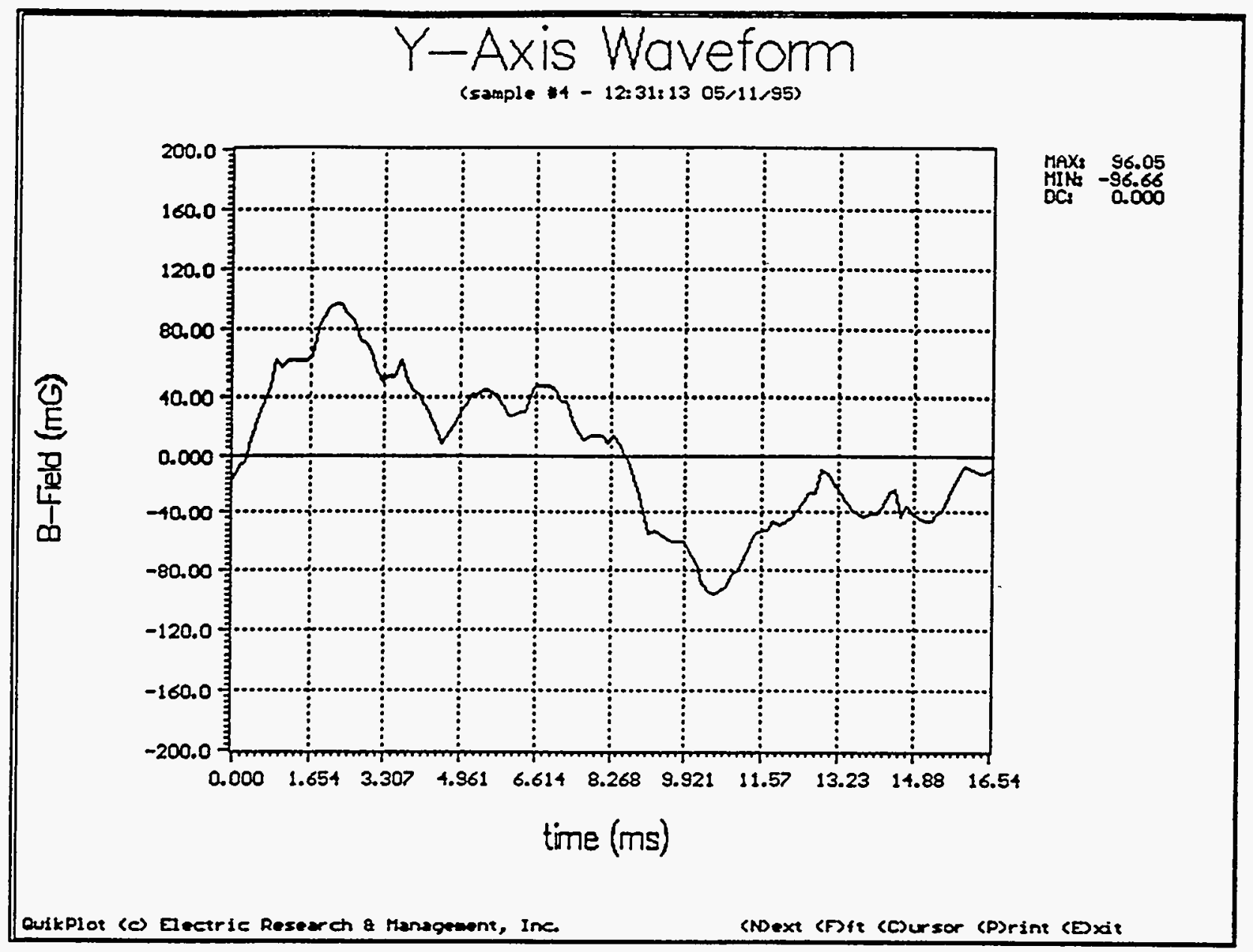

Figure D.1.9 Waveform of the Probe $Y$ Axis at Location -27,-18,-9. 


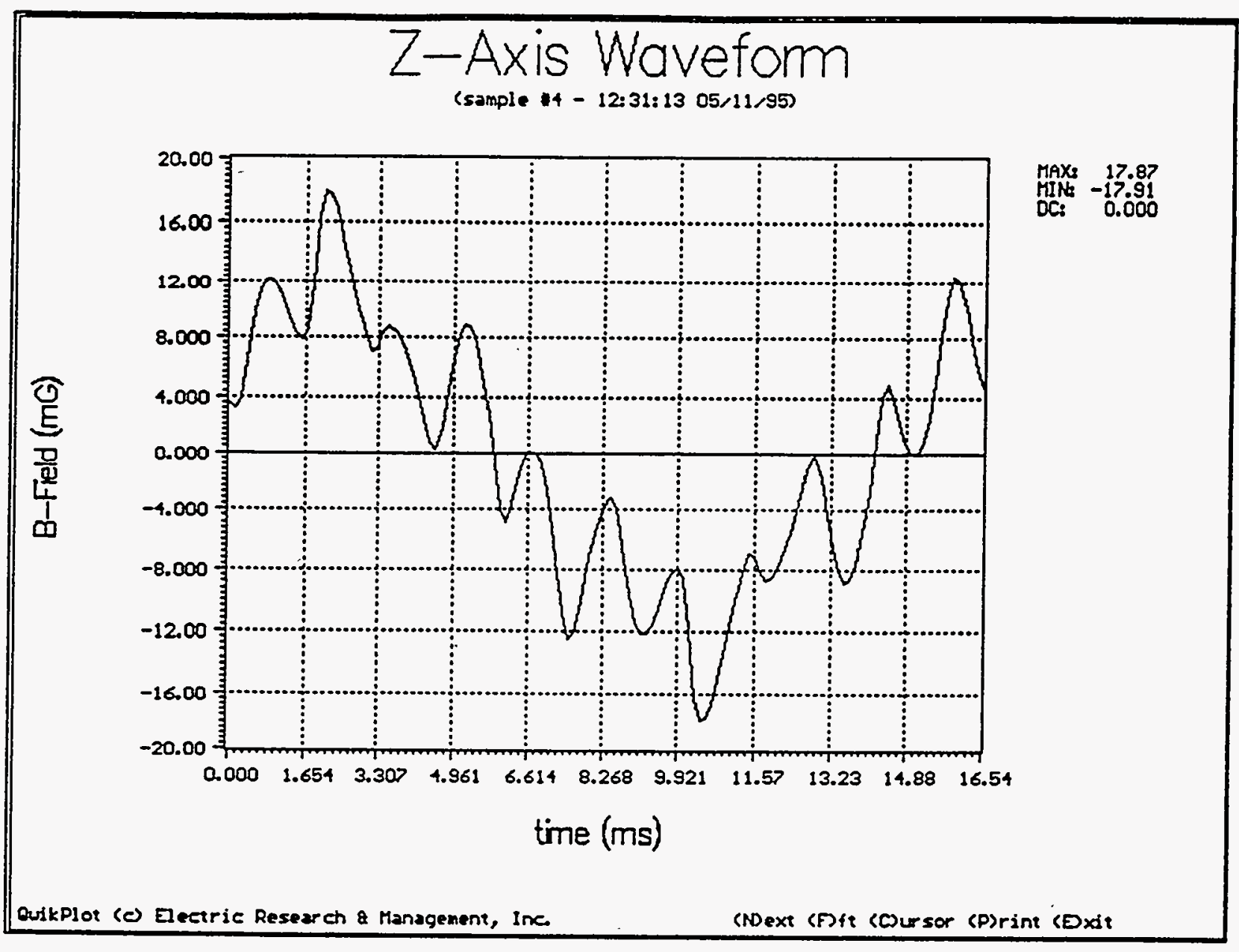

Figure D.1.10 Waveform of the Probe $Z$ Axis at Location $-27,-18,-9$. 


\section{D.2 An Inverter}

The inverter was located in one of the equipment rooms of a large hydroelectric power plant. It was mounted in an electronics equipment rack designed for 19 inch front panels. One side was very near a wall and the back was close to another wall. A testing bench was located about 2 feet in front of the rack. The only accessible side of this device was the right side. A point was chosen about three feet in elevation at approximately the center of the appliance. A set of spherical coordinate measurements were made. Each group of measurements along a ray of the sphere consisted of 5 measurements. The rays were horizontal to the right, horizontal to the front, horizontal at an angle of 45 degrees between the front and right, and a ray at an angle of 45 degrees upward from the horizontal perpendicular to the right side of the unit. The purpose of the measurements was to determine the possibility of using spherical coordinates in an in-situ environment. The result was that such measurements could be made only if a set of jigs were created ahead of time so that the location of the measurements could be more precisely determined. When there is limited access to the equipment, Cartesian coordinates are more easily utilized in measurements. The Table D.2.1 shows the horizontal slice at the three foot elevation.

Typical for high power equipment which is connected to three phase sources, the polarization for the fundamental and harmonics is strong. This device also exhibited very strong harmonic activity in the even harmonics. Since there were switching circuits, large rates-of-change of magnetic field were seen. One of the largest was 200 Gauss per second. If the fundamental amplitude alone was used to estimate the rate of change, the fundamental would be expected to have an amplitude of 425 milligauss. The actual fundamental magnitude was 144 milligauss indicating a rate of change of 77 Gauss per second. This is indicative of the estimation errors which occur when using rms amplitudes to determine rate-of-change. Figures D.2.1 through D.2.5 show the levels of magnetic field for the rms magnitude, the fundamental magnitude and the harmonic magnitudes for the horizontal slice. Figures D.2.6 through D.2.9 show the polarization of the fundamental and the harmonics. 


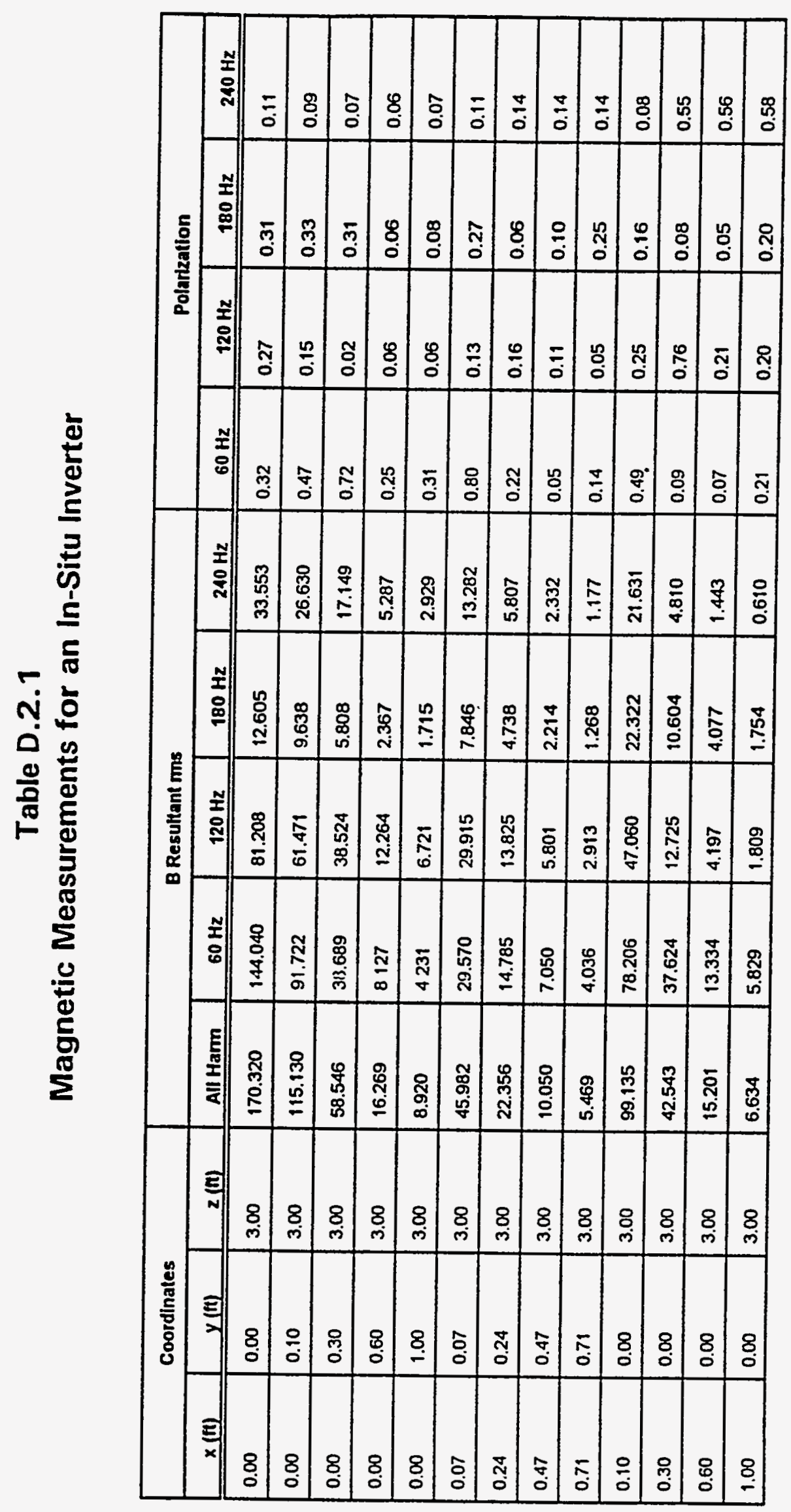




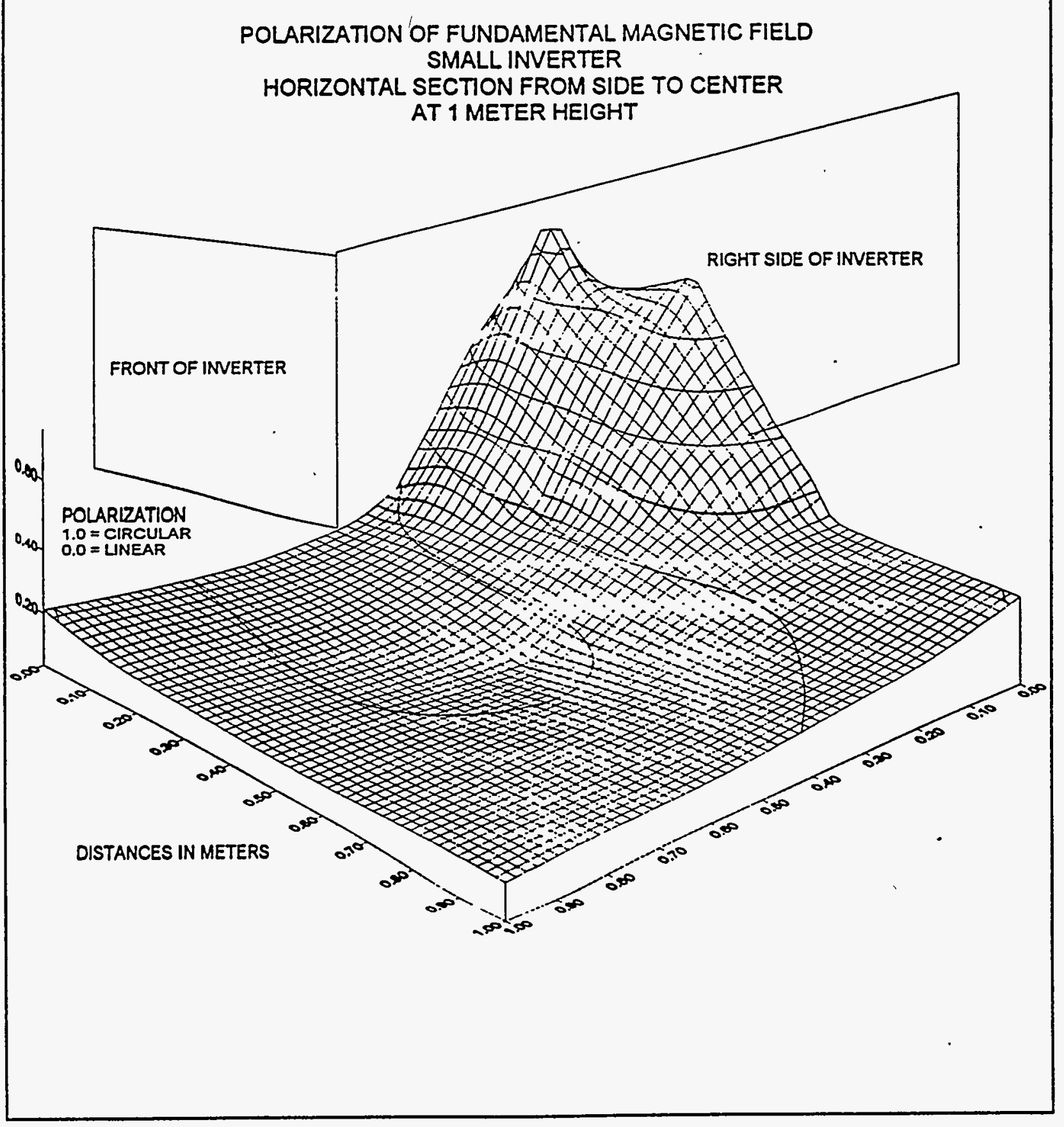

Figure D.2.1 Polarization of Fundamental Magnetic Field - Small Inverter.

D-19 


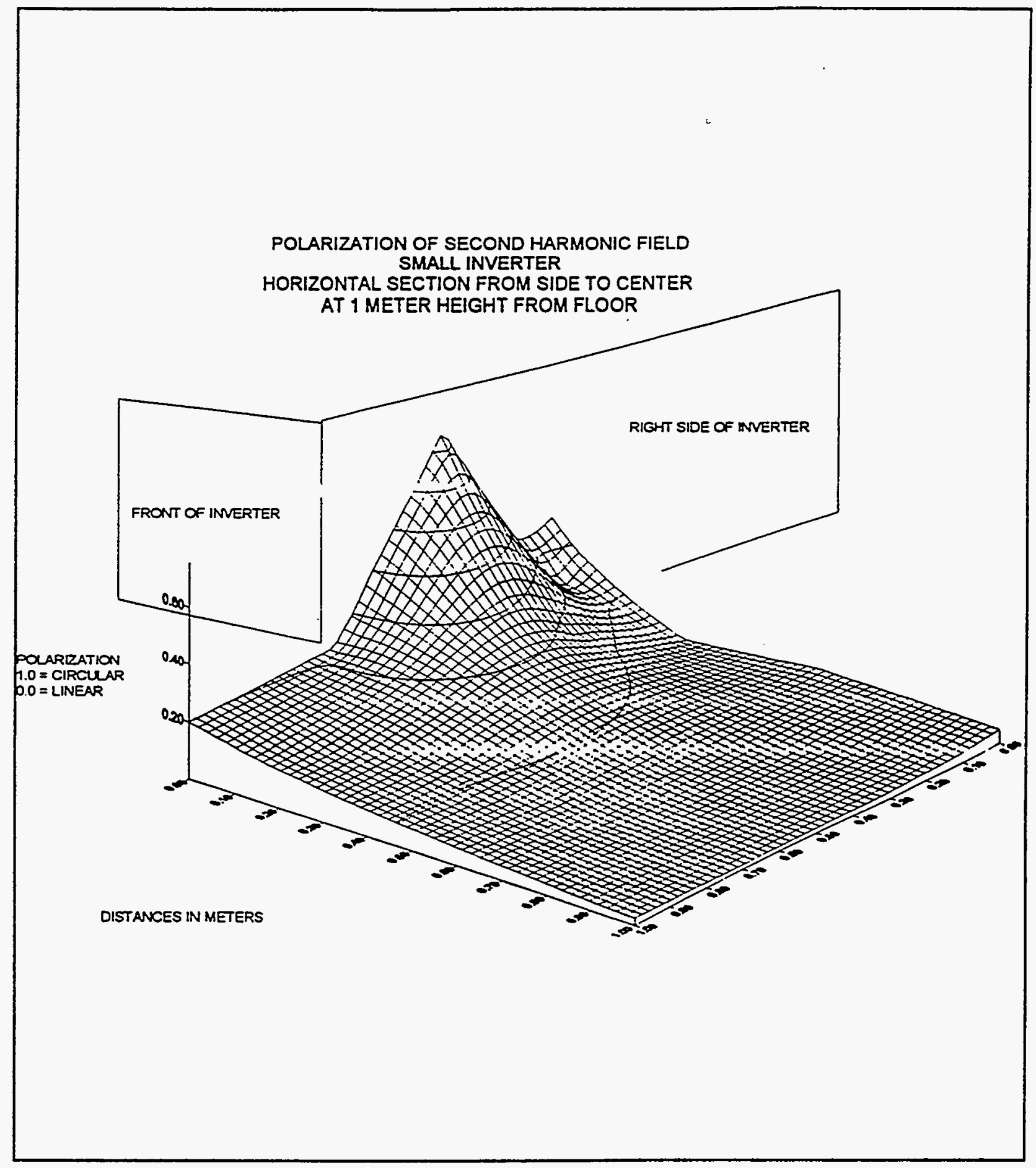

Figure D.2.2 Polarization of Second Harmonic Field - Small Inverter. 


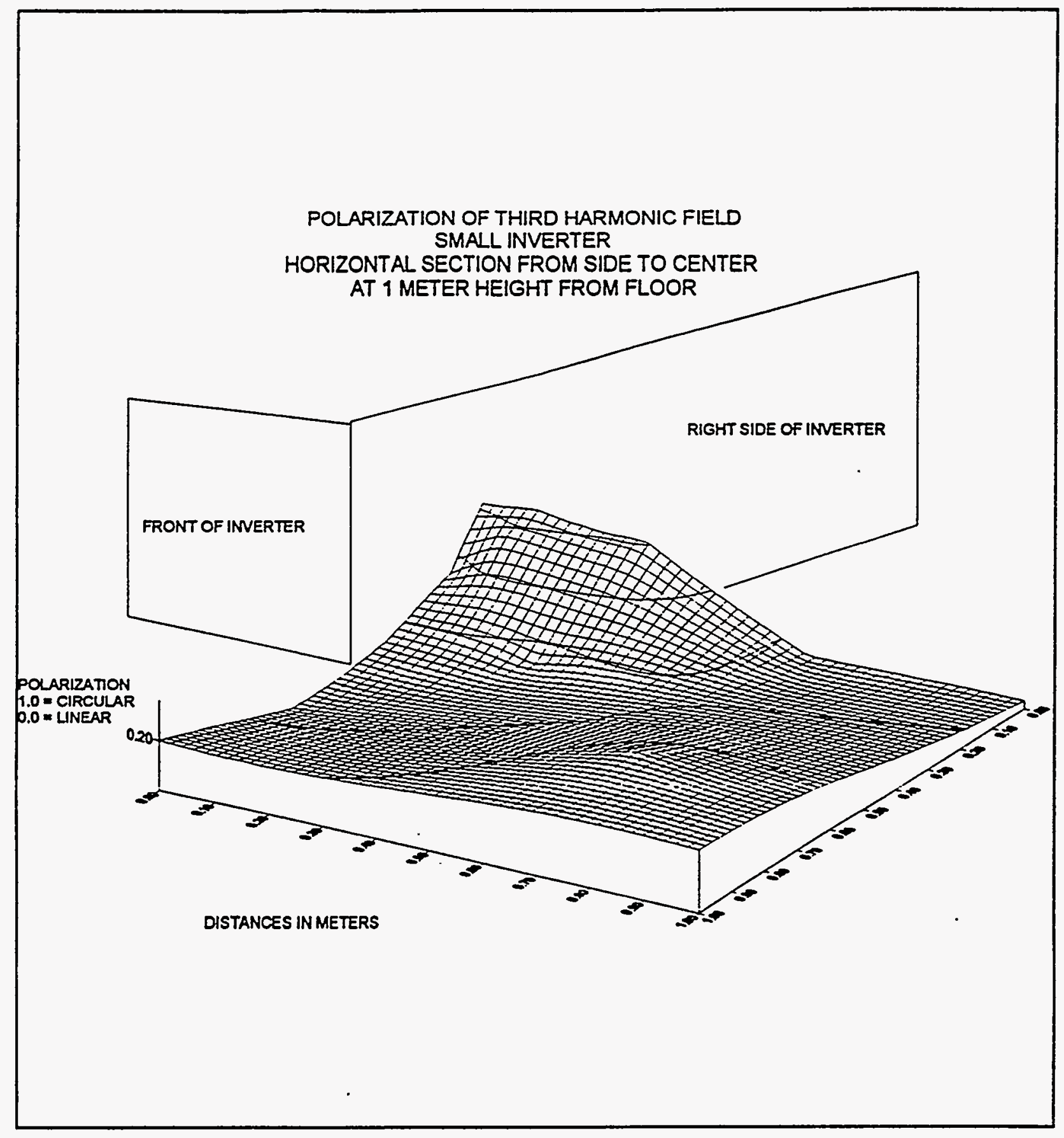

Figure D.2.3 Polarization of Third Harmonic Field - Small Inverter.

$\therefore$

D-21 


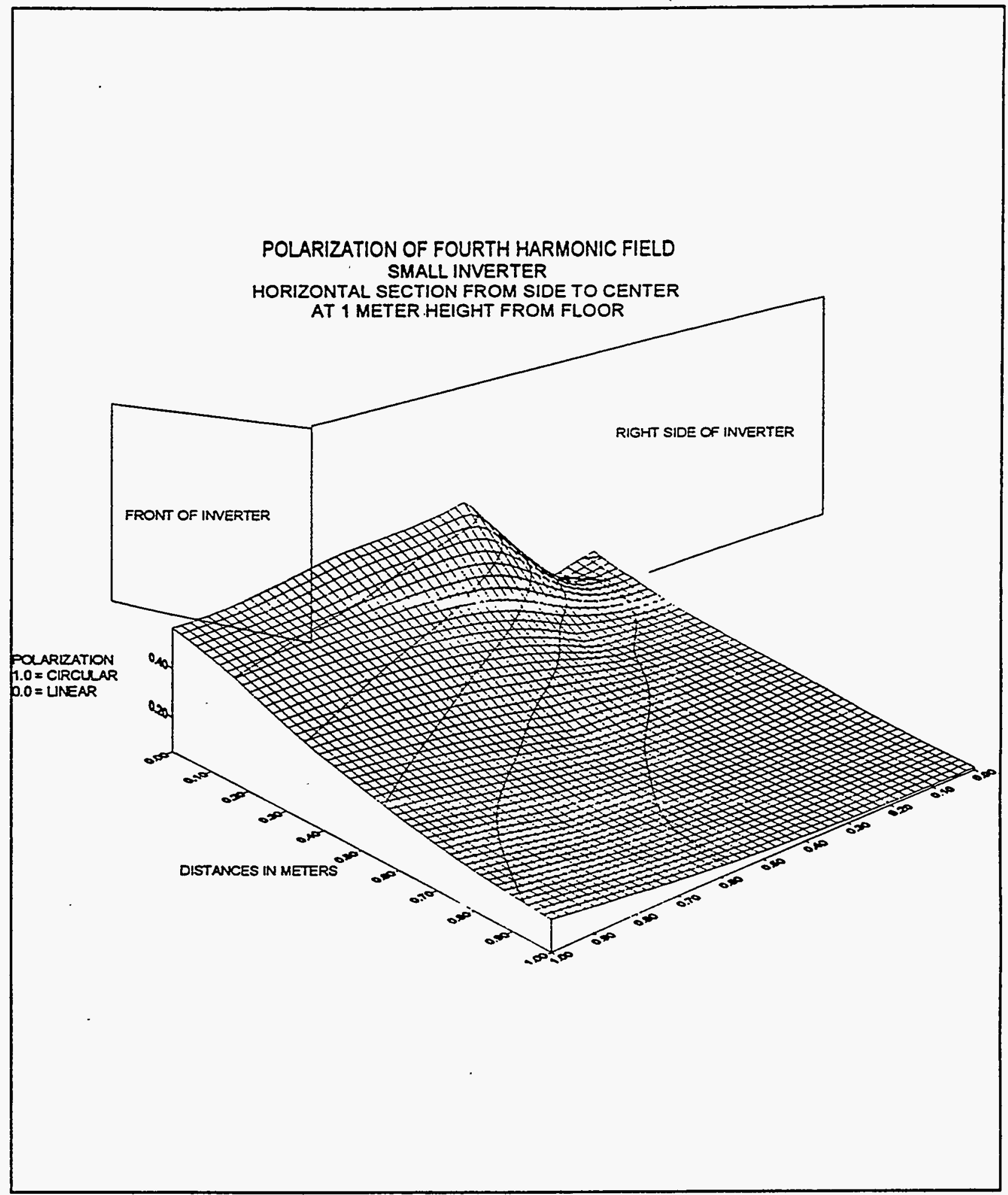

Figure D.2.4 Polarization of Fourth Harmonic Field - Small Inverter. 


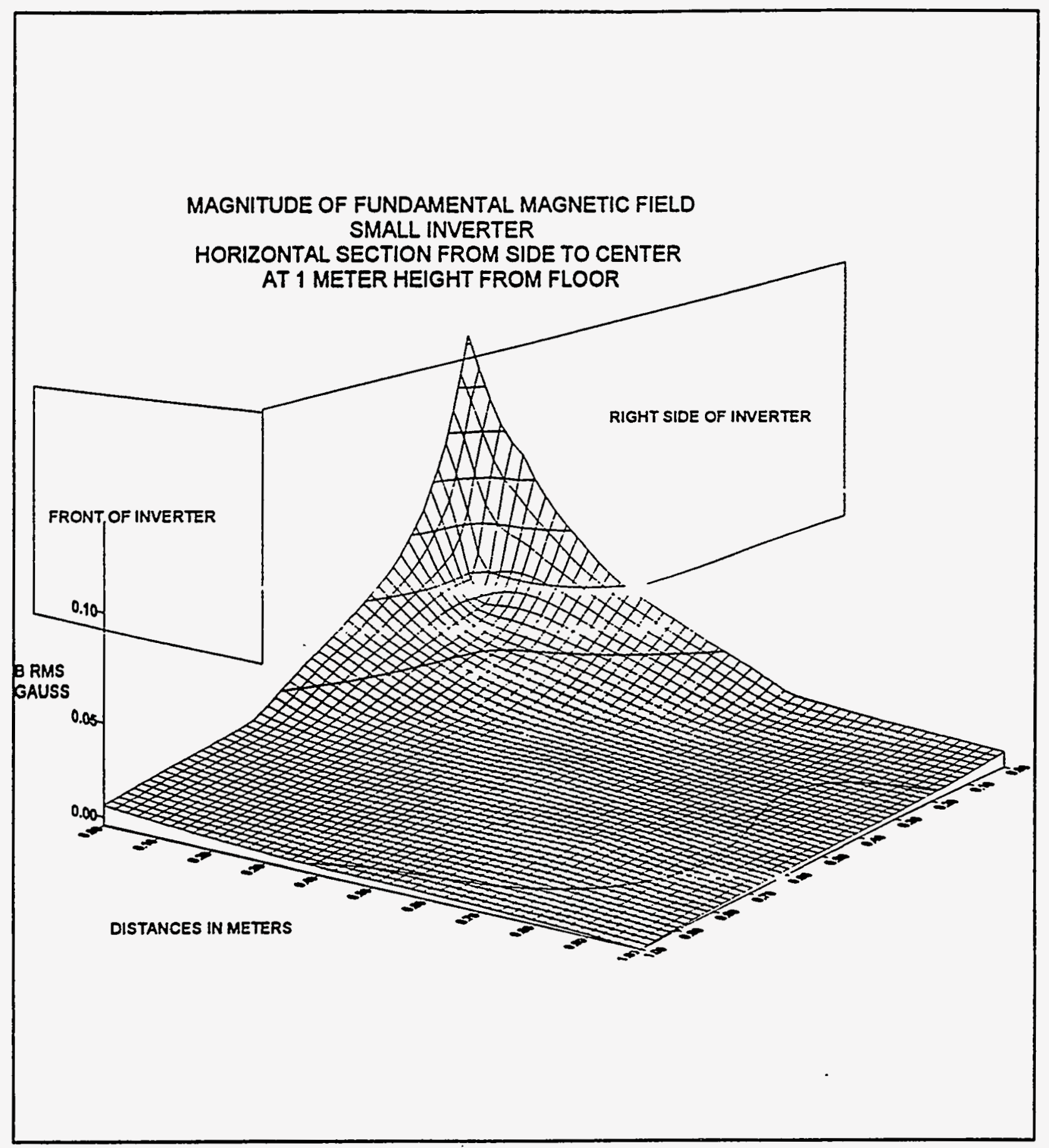

Figure D.2.5 Magnitude of Fundamental Magnetic. Field - Small Inverter. 


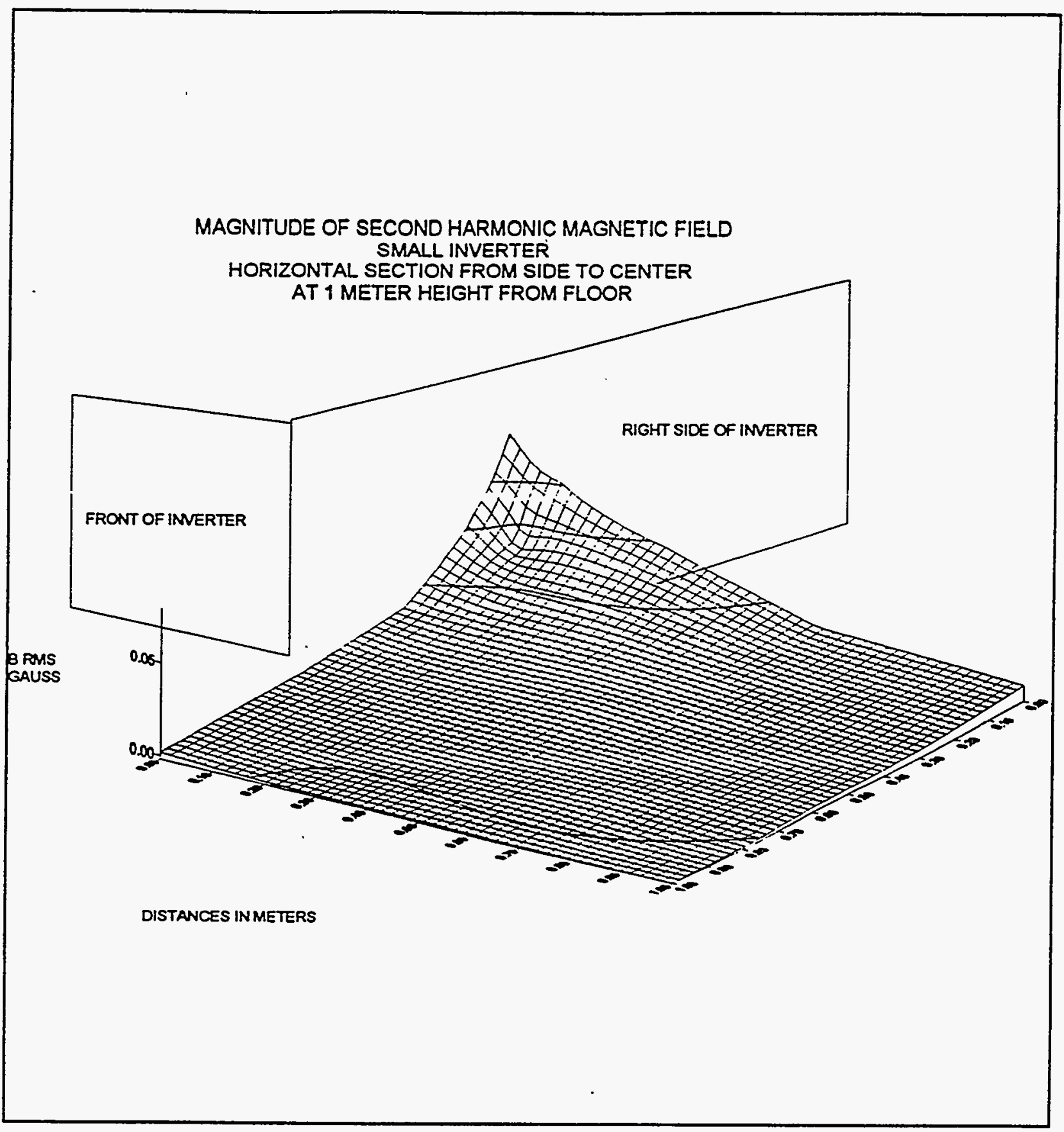

Figure D.2.6 Magnitude of Second Harmonic Magnetic Field - Small Inverter. 


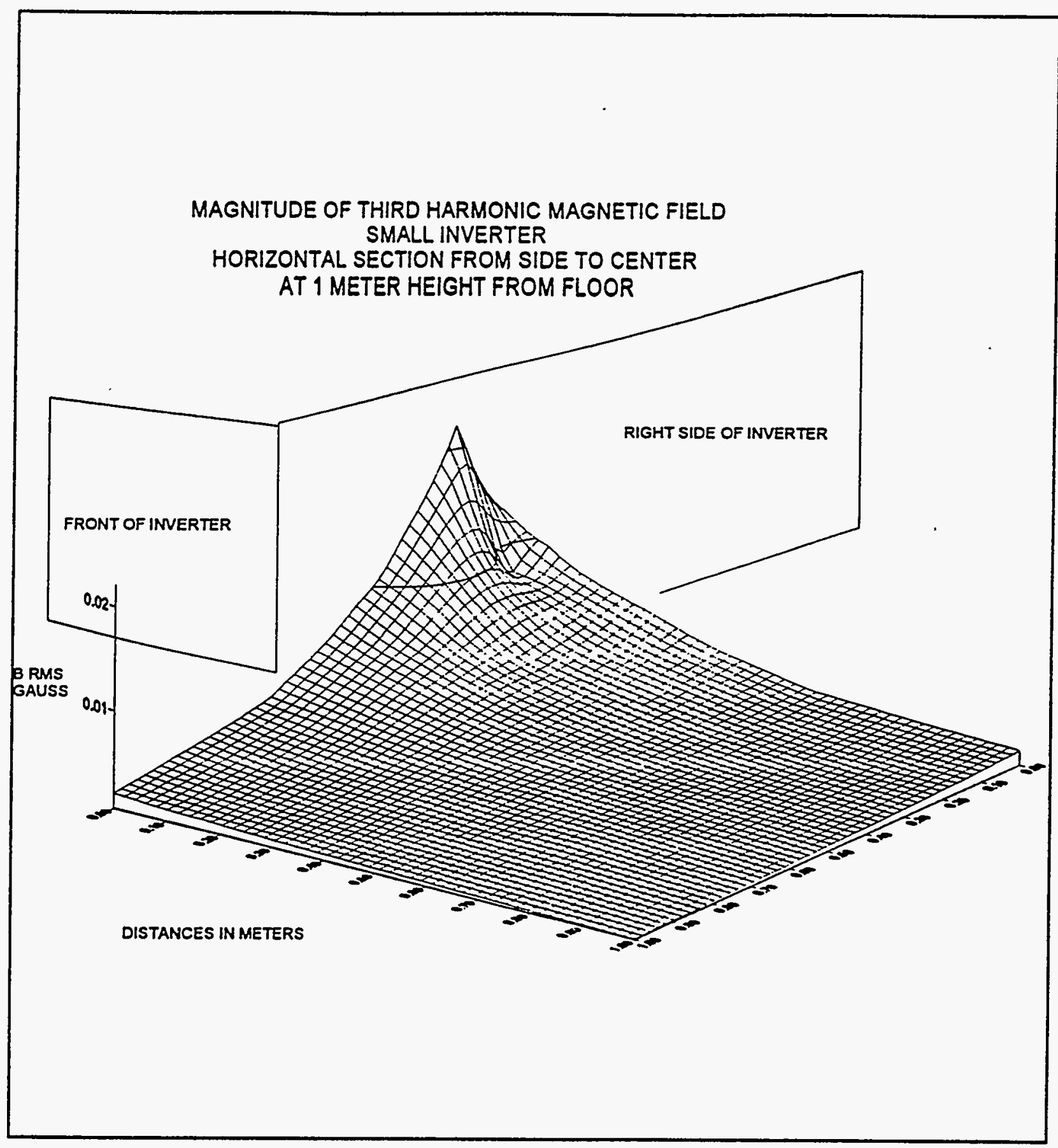

Figure D.2.7 Magnitude of Third Harmonic Magnetic Field - Small Inverter. 


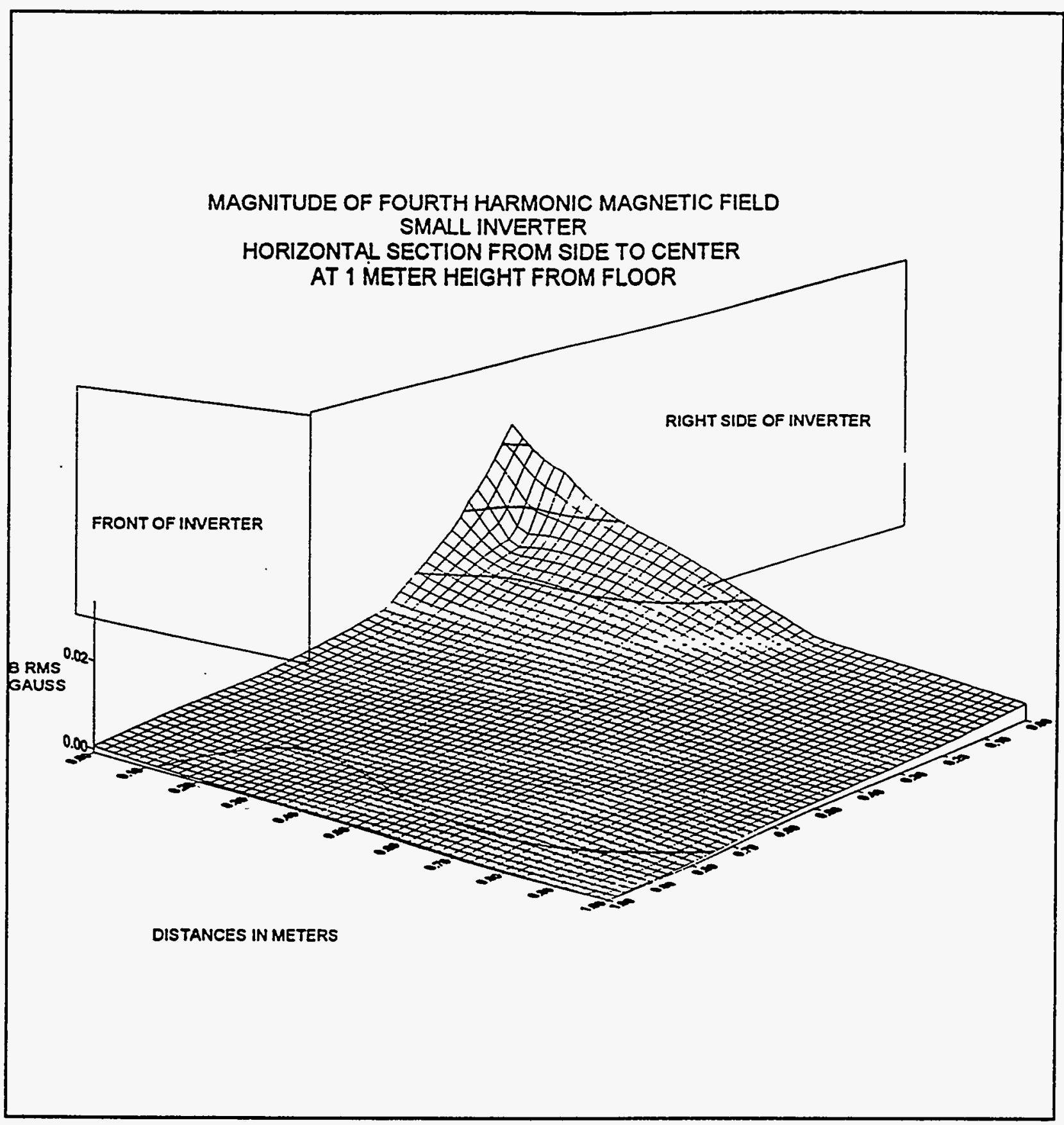

Figure D.2.8 Magnitude of Fourth Harmonic Magnetic Field - Small Inverter. 


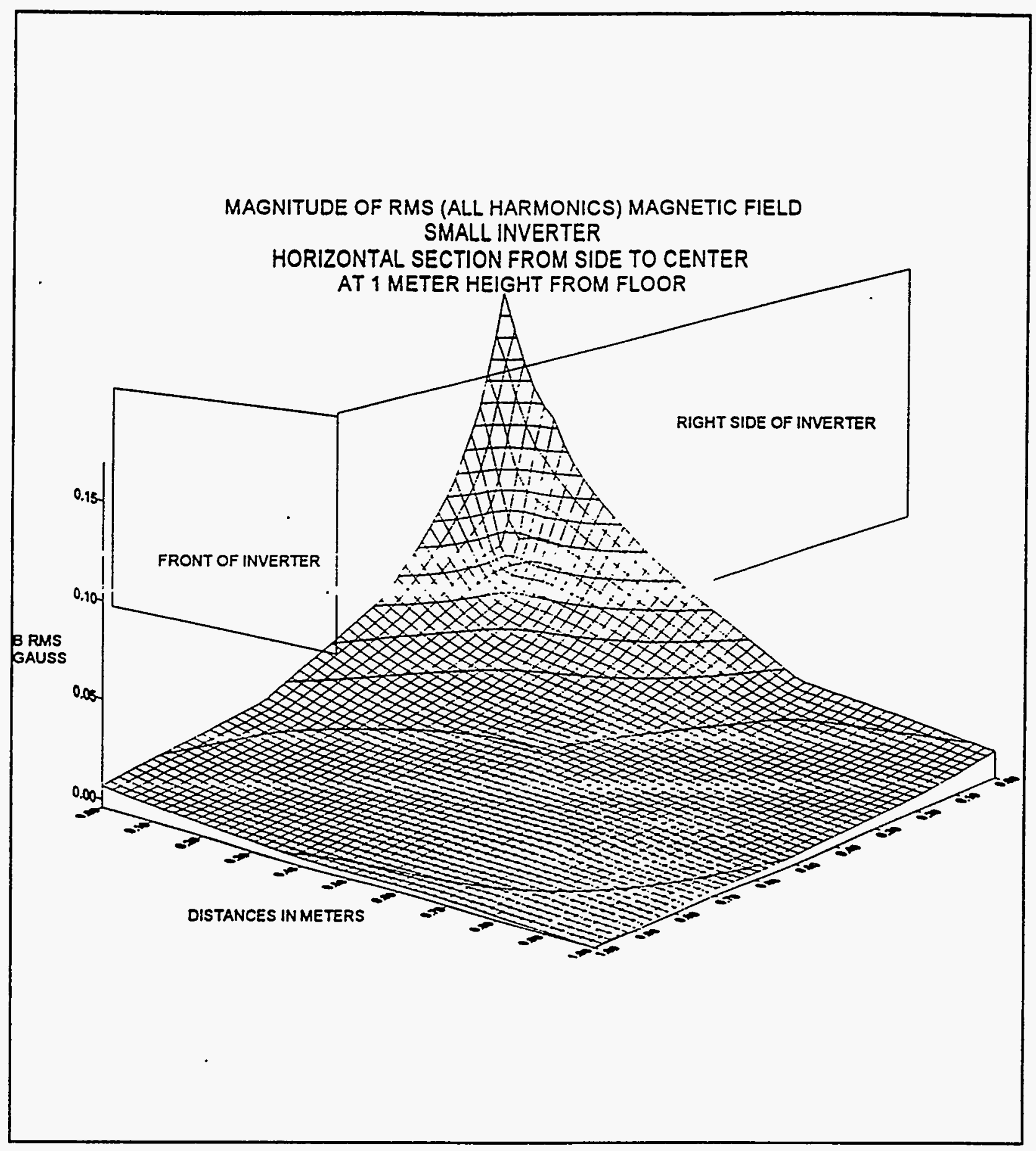

Figure D.2.9 Magnitude of RMS (All Harmonics) Magnetic Field - Small Inverter. 


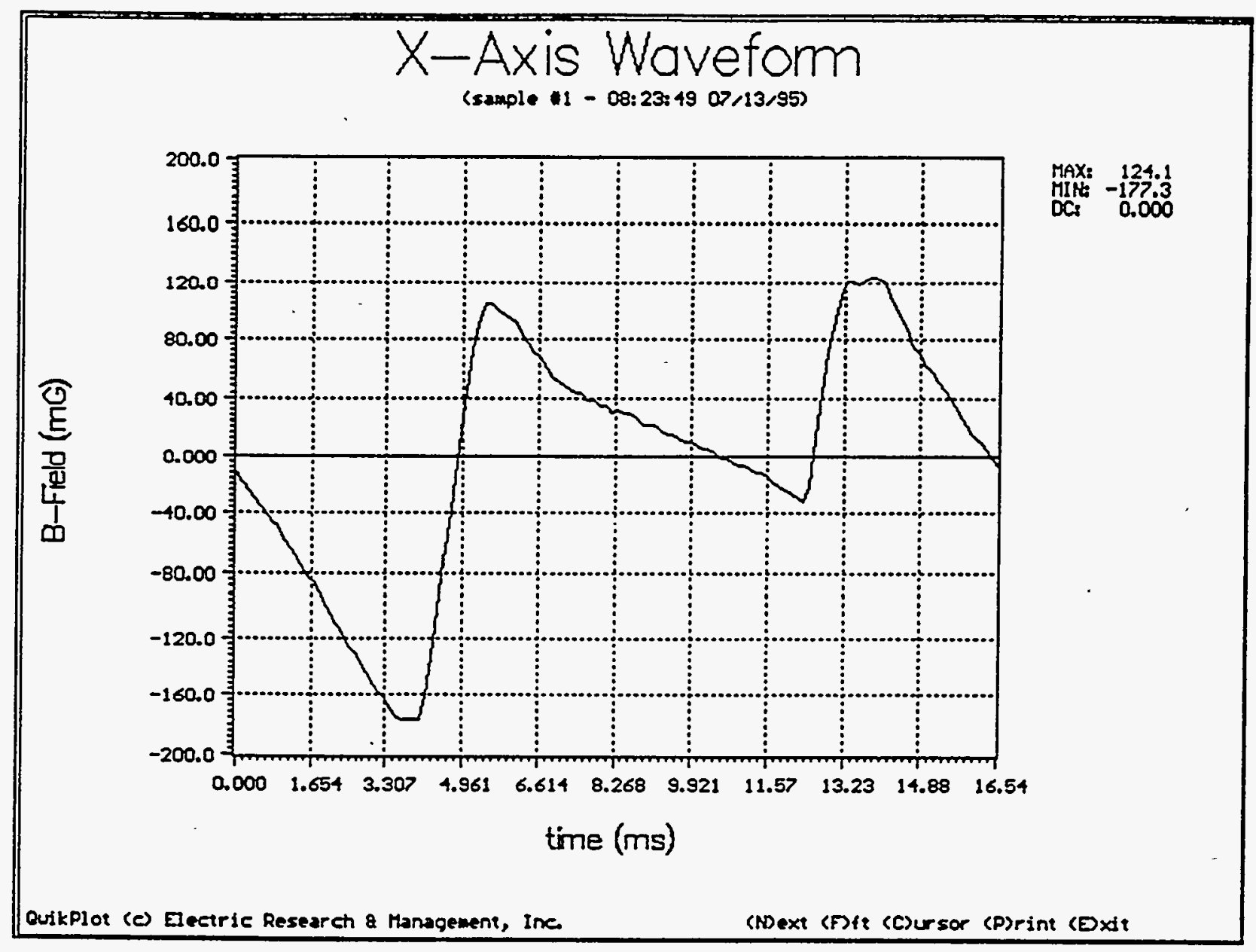

Figure D.2.10 Waveform of the Probe $X$ Axis Showing a dB/dt of 227 gauss/second at Location 0,0,3. 


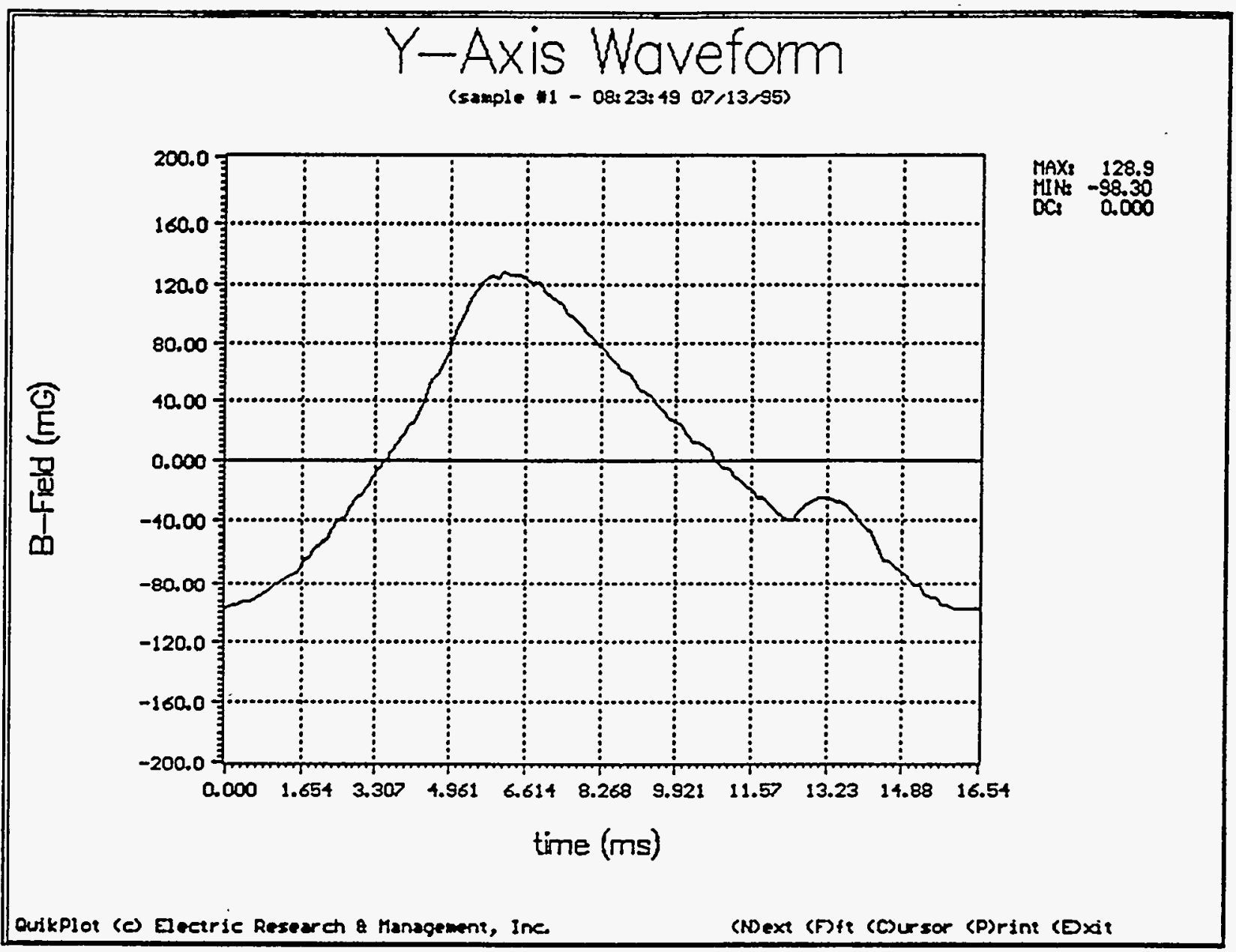

Figure D.2.11 Waveform of the Probe $Y$ Axis at Location $0,0,3$. 


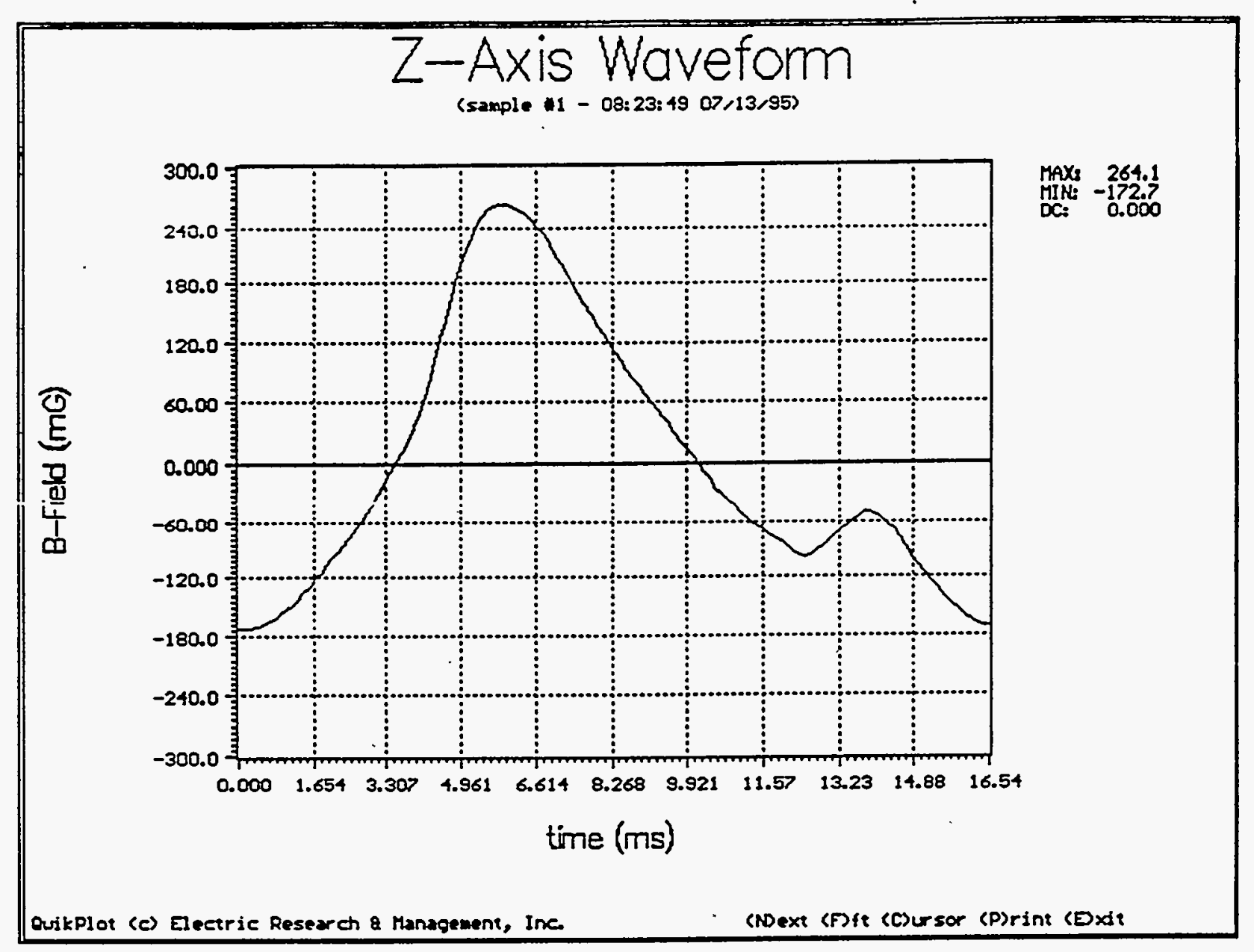

Figure D.2.12 Waveform of the Probe $Z$ Axis at Location 0,0,3. 